Irene Giesen-Netzer

Implementierung

von Rücknahme- und

Recyclingsystemen bei

Gebrauchsgütern 


\section{Implementierung von Rücknahme- und Recyclingsystemen bei Gebrauchsgütern}

Angesichts der Erlassung des Kreislaufwirtschaftsgesetzes und produktbezogener Rücknahmeverordnungen sehen sich Hersteller von langlebigen Gebrauchsgütern gezwungen, Rücknahme- und Recyclingsysteme für Altprodukte zu implementieren. Dabei stehen ihnen verschiedene organisatorische Gestaltungsformen zur Verfügung. Auch bestehen hinsichtlich der Realisation Handlungsoptionen, die mit verschiedenen Auswirkungen verbunden sind. Die Arbeit verfolgt das Ziel, bezüglich dieser Problemstellungen eine wissenschaftlich fundierte Entscheidungsunterstützung $\mathrm{zu}$ geben. Dabei wird das Implementierungsverhalten der Hersteller erfaßt und anhand von situativen Kontextfaktoren erklärt. Darüber hinaus werden die Erfolgswirkungen unterschiedlichen Implementierungsverhaltens untersucht, um Implikationen für eine zielführende Vorgehensweise der Implementierung von Rücknahme- und Recyclingsystemen ableiten zu können.

Irene Giesen-Netzer wurde 1966 in Isselburg geboren. Nach einer Banklehre in Bocholt studierte sie ab 1989 Betriebswirtschaftslehre an der Universität in Münster. 1993 beendete sie ihr Studium mit dem Abschluß als Diplom-Kauffrau. Anschließend arbeitete sie zunächst als Marketingleiterin an der Europäischen Umweltakademie in Borken, bevor sie wissenschaftliche Mitarbeiterin am Institut für Marketing in Münster wurde. 
Implementierung von Rücknahmeund Recyclingsystemen bei Gebrauchsgütern 


\section{SCHRIFTEN ZU \\ MARKETING UND MANAGEMENT}

Herausgegeben von Prof. Dr. Dr. h.c. Heribert Meffert

Band 36

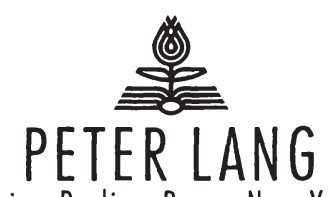

Frankfurt am Main - Berlin. Bern. New York - Paris . Wien

Irene Giesen-Netzer and Universität Münster - 9/8-3-631-75058-2

Downloaded from PubFactory at 01/11/2019 08:44:49AM

via free access 


\section{Irene Giesen-Netzer}

\section{Implementierung von
Rücknahme- und Recyclingsystemen \\ Implementierung von
Rücknahme- und Recyclingsystemen bei Gebrauchsgütern}


Die Deutsche Bibliothek - CIP-Einheitsaufnahme

Giesen-Netzer, Irene:

Implementierung von Rücknahme- und Recyclingsystemen bei Gebrauchsgütern / Irene Giesen-Netzer. - Frankfurt am Main ; Berlin ; Bern ; New York ; Paris ; Wien : Lang, 1998

(Schriften zu Marketing und Management ; Bd. 36)

Zugl.: Münster (Westfalen), Univ., Diss., 1998

ISBN 3-631-33777-9

Open Access: The online version of this publication is published on www.peterlang.com and www.econstor.eu under the international Creative Commons License CC-BY 4.0. Learn more on how you can use and share this work: http://creativecommons. org/licenses/by/4.0.

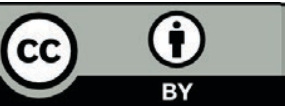

This book is available Open Access thanks to the kind support of ZBW - Leibniz-Informationszentrum Wirtschaft.

Gedruckt auf alterungsbeständigem, säurefreiem Papier.

\author{
D 6 \\ ISSN 0176-2729 \\ ISBN 3-631-33777-9 \\ ISBN 978-3-631-75058-2 (eBook) \\ (C) Peter Lang GmbH \\ Europäischer Verlag der Wissenschaften \\ Frankfurt am Main 1998 \\ Alle Rechte vorbehalten.
}

Das Werk einschließlich aller seiner Teile ist urheberrechtlich geschützt. Jede Verwertung außerhalb der engen Grenzen des

Urheberrechtsgesetzes ist ohne Zustimmung des Verlages unzulässig und strafbar. Das gilt insbesondere für Vervielfältigungen, Übersetzungen, Mikroverfilmungen und die Einspeicherung und Verarbeitung in elektronischen Systemen.

Printed in Germany 124567 


\section{Meinen Eltern, Frithjof und Nils Bastian}


Irene Giesen-Netzer and Universität Münster - 978-3-631-75058-2 Downloaded from PubFactory at 01/11/2019 08:44:49AM via free access 


\section{Vorwort des Herausgebers}

Unter dem Leitbild des Sustainable Development oder einer dauerhaft umweltgerechten Entwicklung wurde bereits Mitte der achtziger Jahre die Abkehr von einer Durchlaufwirtschaft zu einer Kreislaufwirtschaft diskutiert. Allerdings wurden von betriebswirtschaftlicher Seite zum einen die Operationalität des Konzepts als problematisch angesehen sowie zum anderen die Standortfrage Deutschland neu gestellt, da durch die umweltbezogenen Maßnahmen ein höherer Kostendruck für Unternehmen erwartet wurde. Nicht zuletzt aus diesem Grund sahen sich die Träger der staatlichen Umweltpolitik in Deutschland aufgefordert, rechtliche Vorschriften für Unternehmen zu erlassen.

Das Abfallgesetz wurde vor diesem Hintergrund 1986 novelliert und unterschied erstmals Abfallvermeidung, -verwertung und -beseitigung. Diese Rechtsnorm wurde zum Kreislaufwirtschaftsgesetz weiterentwickelt, welches am 7. Oktober 1996 in Kraft trat. Diese Entwicklungen in Verbindung mit der am 1. April 1998 wirksam gewordenen Altautoverordnung und weiteren zu erwartenden produktbezogenen Rücknahmeverordnungen veranlaßten Hersteller von Gebrauchsgütern seither, sich mit der Gestaltung von Rücknahme- und Recyclingsystemen für Altprodukte auseinanderzusetzen, die eine unternehmensseitige Antwort auf die Gesetzesinhalte darstellen. Die Ansätze der Hersteller zur organisatorischen Gestaltung von Rücknahme- und Recyclingsystemen reichen dabei von herstellereigenen Rücknahme- und Recyclingsystemen bis hin zur Externalisierung nahezu aller damit verbundenen Aufgaben.

Eine intensive Auseinandersetzung der betriebswirtschaftlichen Forschung mit der herstellerseitigen Implementierung von Rücknahme- und Recyclingsystemen hat bislang kaum stattgefunden. Vielmehr ist eine getrennte Erforschung von Implementierungsaspekten einerseits und umweltbezogenen Problemstellungen andererseits festzustellen. Intensiv diskutiert wurde bereits seit den siebziger Jahren die Implementierung von Operations Research-Systemen und Management Science-Projekten, bevor seit den achtziger Jahren nicht-technische Implementierungsobjekte Gegenstand der Untersuchung waren. Zentrales Ergebnis aller Arbeiten ist die Erkenntnis, daß die Implementierung maßgeblich für den Erfolg der jeweilig umzusetzenden Implementierungsobjekte ist. Daneben fand eine extensive Diskussion von ökologieorientierten Problemstellungen statt, die bis heute an- 
hält und sich in der rasch wachsenden betriebswirtschaftlichen Literatur zum Themenkreis des Umweltmanagement manifestiert.

Vor diesem Hintergrund ist die Zielsetzung der vorliegenden Arbeit zu sehen, die das Implementierungsverhalten der Hersteller von Gebrauchsgütern zu erfassen und anhand situativer Kontextfaktoren zu erklären sucht. Darüber hinaus werden die Erfolgswirkungen unterschiedlichen Implementierungsverhaltens untersucht, um Implikationen für eine zielführende Vorgehensweise bei der Implementierung von Rücknahme- und Recyclingsystemen ableiten zu können.

Hierzu findet zunächst eine umfassende Analyse des Implementierungsobjektes Rücknahme- und Recyclingsysteme statt. Auf der Basis eines mehrdimensionalen Operationalisierungsansatzes werden anschließend die Gestaltungsoptionen der Implementierung von Rücknahme- und Recyclingsystemen identifiziert, daraufhin zu zeitlichen, hierarchie- und intensitätsbezogenen Implementierungsdimensionen verdichtet und einer empirischen Überprüfung unterzogen. Auf Grundlage der nachgewiesenen Implementierungsdimensionen lassen sich Implementierungstypen bilden, die sich hinsichtlich der Merkmalsausprägungen in ihrem Implementierungsverhalten mit hoher diskriminatorischer Güte unterscheiden.

Um Erklärungsansätze für die Unterschiedlichkeit der ermittelten Typen zu gewinnen, wurde vor dem Hintergrund kontingenztheoretischer Erkenntnisse eine Analyse der rechtlichen, netzwerkbezogenen, aufgabenspezifischen, internen unternehmensbezogenen und individualbezogenen Einflußfaktoren durchgeführt. Dabei vermittelt die Untersuchung der Vielzahl der Einflußfaktoren interessante Aufschlüsse über relevante Kontextfaktoren der Hersteller, die Rücknahme- und Recyclingsysteme implementieren. So hat z.B. der Produktwert einen großen Einfluß auf das Implementierungsverhalten bei Rücknahme- und Recyclingsystemen, womit die intensive Diskussion um den Begriff der "Wertschöpfungskreisläufe“ gerechtfertigt erscheint. Zudem sind beispielsweise weniger die Strategien in der Einzelbetrachtung als vielmehr das Zusammenspiel von wettbewerbs- und umweltbezogenen Strategien wesentliche Faktoren, die das Implementierungsverhalten hinsichtlich aller Implementierungsdimensionen beeinflussen. 
Die Untersuchung der Erfolgswirkungen findet auf Basis der subjektiven Erfolgseinschätzungen der Entscheidungsträger statt. Während bei der Erreichung der Implementierungsziele z.T. signifikante Unterschiede zwischen den Implementierungstypen auf den Ebenen der Analyse (Netzwerk-, Unternehmens- und Individualebene) feststellbar sind, erweist sich die Beurteilung bei den ökologischen und den ökonomischen Zielen als schwieriger. Bei der ökologischen Zielerreichung weist lediglich das Ziel der Wiederverwertung - welches einen wichtigen Stellenwert für Rücknahme- und Recyclingsysteme darstellt - signifikante Unterschiede auf. Auch der ökologische Erfolg zeigt lediglich tendenziöse Unterschiede zwischen den Implementierungstypen. Die empirischen Befunde bestätigen gleichwohl einen insgesamt positiven Zusammenhang zwischen dem relativen Implementierungserfolg und dem relativen ökonomischen Erfolg.

Insgesamt stellt die vorliegende Untersuchung eine Bereicherung der wissenschaftlichen Arbeiten auf einem neuen, komplexen und interdisziplinären Forschungsfeld dar und gibt aufschlußreiche Hinweise für die Realisierung von Rücknahme- und Recyclingsystemen. Die besondere Stärke der Arbeit liegt in der Verknüpfung theoretischer Strukturierung mit einer empirischen Bestandsaufnahme zum Implementierungsverhalten von Rücknahme- und Recyclingsystemen. Es bleibt zu wünschen, daß diese Veröffentlichung in Wissenschaft und Praxis auf eine breite Resonanz stößt. 


\section{Vorwort der Verfasserin}

Um nicht nur der umweltbezogenen rechtlichen Legalität, sondern auch der gesellschaftlichen Legitimität seitens verschiedener Anspruchsgruppen (z.B. Kunden, Umweltschutzgruppen als Meinungsführer) gerecht zu werden, implementieren Hersteller von Gebrauchsgütern Rücknahme- und Recyclingsysteme. Darüber hinaus fassen einige Hersteller Rücknahme- und Recyclingsysteme als Chance auf, die überwiegend darin gesehen wird, eigene, noch werthaltige Produkte wiederzuerhalten, damit ökonomischen Vorteil zu erzielen und gleichzeitig die ökologische Umwelt von den Altprodukten zu entlasten.

Allerdings bestehen zum einen hinsichtlich der organisatorischen Ausgestaltung dieser Systeme verschiedene Entscheidungsalternativen, die von einer Internalisierung nahezu aller rücknahme- und recyclingsystembezogenen Aktivitäten (Hierarchieform) bis hin zu einer weitgehenden Externalisierung dieser Aktivitäten (Marktform) und aller dazwischenliegenden Formen reichen können. Zum anderen ergeben sich hinsichtlich der Realisierung der Rücknahme- und Recyclingsysteme verschiedene Handlungsoptionen, die sich im Implementierungsverhalten der Hersteller zeigen. Diese Handlungsalternativen sind mit verschiedenen Auswirkungen verbunden, die sich in der Erreichung der Implementierungsziele sowie der ökologischen und ökonomischen Ziele niederschlagen.

Die vorliegende Arbeit verfolgt das Ziel, eine wissenschaftlich fundierte Entscheidungsunterstützung in bezug auf die genannten Fragestellungen in einer recht frühen Phase der Implementierungsaktivitäten bei Herstellern von Gebrauchsgütern zu leisten. Diese Erkenntnisziele scheinen insbesondere vor dem Hintergrund der bestehenden Forschungsdefizite hinsichtlich der Implementierungsaktivitäten bei Rücknahme- und Recyclingsystemen anstrebenswert zu sein. Zu diesem Zweck werden zunächst Gestaltungsansätze und Ziele von Rücknahme- und Recyclingsystemen bei Gebrauchsgüterherstellern untersucht, bevor Dimensionen der Implementierung von Rücknahme- und Recyclingsystemen und Implementierungstypen hergeleitet und empirisch überprüft werden. Darauf aufbauend werden der Einfluß von politisch-rechtlichen Faktoren sowie Einflußfaktoren auf Netzwerkebene, Unternehmensebene und Individualebene untersucht, um Erklärungsansätze für das Implementierungsverhalten bei Rücknahme- und Recyclingsystemen zu generieren. Abschließend werden der Implementierungserfolg sowie der ökologi- 
sche und ökonomische Erfolg der herstellerseitigen Implementierung von Rücknahme- und Recyclingsystemen untersucht.

Die Erstellung der Arbeit war nur mit der Unterstützung zahlreicher Personen möglich. Hier gilt mein besonderer Dank zunächst meinem akademischen Lehrer, Herrn Prof. Dr. Dr. h.c. Heribert Meffert. Er regte bereits frühzeitig die Themenstellung an, förderte umfassend die empirische Untersuchung und schließlich die Fertigstellung der Arbeit. Herrn Prof. Dr. Wolfgang Ströbele sei für die Übernahme des Zweitgutachtens gedankt. Auch Herrn Prof. Dr. Manfred Kirchgeorg möchte ich danken, der nicht nur zu ausführlichen inhaltlichen Diskussionen bereit war und äußerst wertvolle Hinweise geleistet hat, sondern auch die empirische Untersuchung betreut hat.

Der empirische Teil meiner Arbeit stützt sich auf eine Befragung von etwa 100 Herstellern von langlebigen Gebrauchsgütern. Da die persönlichen Interviews bis zu vier Zeitstunden in Anspruch nahmen, möchte ich den Vertretern dieser Hersteller nachdrücklich danken. Durch ihre Diskussions- und Hilfsbereitschaft konnte die Qualität und letztlich die Durchführung der empirischen Untersuchung sichergestellt werden. Hervorheben möchte ich dabei die Mitarbeiter der Hersteller Bauknecht, Gromathic, Kaldewei, Rank Xerox, Siemens-Nixdorf und Volkswagen, die in zahlreichen intensiven Gesprächen zusätzlich bereit waren, die Fallstudienerstellung zu ermöglichen.

Darüber hinaus gilt mein Dank allen aktuellen und ehemaligen Kolleginnen und Kollegen sowie Freundinnen und Freunden, die mich während meiner Arbeit entlastet und vielfältig unterstützt haben. Für die perfekte Erstellung der Abbildungen sei Herrn cand. rer. pol. Christoph Sitzer gedankt, der sich durch eine unerschütterliche Geduld und Hilfsbereitschaft ausgezeichnet hat. Besonders hervorheben möchte ich jedoch an dieser Stelle den Einsatz derjenigen Frauen, die sich über den langen Zeitraum von rund einem Jahr so liebevoll um meinen inzwischen eineinhalbjährigen Sohn Nils Bastian gekümmert haben. Neben Eva Franke sind hier insbesondere Andrea Schwenke, Ruth Schüler, Hildegard Twenter und Birgit Zenker zu nennen, die es mir immer wieder ermöglicht haben, auch dann an der Arbeit zu schreiben, wenn Nils Bastian nicht schlief. Auch Nils Bastian möchte ich danken, da er mit seinem freundlichen Wesen und seiner Unbekümmertheit die Arbeit mehr unterstützt hat, als er vermuten könnte. 
Ganz besonderer Dank gilt auch meinen Eltern und Schwiegereltern, die insbesondere in der Schußphase der Arbeit meinen Sohn und mich ganze Wochen betreut haben. Zudem haben meine Eltern mich in allen Phasen meiner Ausbildung umfassend und liebevoll unterstützt und damit die Grundlage für die Erstellung dieser Arbeit ermöglicht. Sie haben mir die Freiheit eigener Entscheidungen gelassen und mir zusammen mit meiner Schwester Uta ihr Verständnis und ihre Hilfe entgegengebracht.

Schließlich ist es mir ein besonderes Anliegen, mich bei meinem großartigen Mann Frithjof Netzer bedanken. Er hat mir immer wieder mit seiner Fachkompetenz hilfreich zur Seite gestanden und mir wertvolle Anregungen im Fortlauf der Arbeit gegeben. Ohne seine Unterstützung in fachlicher wie in persönlicher Hinsicht, seinen motivierenden Zuspruch und sein Verständnis wäre der erfolgreiche Abschluß dieser Arbeit nicht denkbar gewesen.

Münster, im April 1998

Irene Giesen-Netzer 


\section{Inhaltsverzeichnis}

A. Rücknahme- und Recyclingsysteme als Herausforderung an die umweltorientierte Unternehmensführung.......................................... 1

1. Umweltpolitische Rahmenbedingungen als Entstehungsgrund für Rücknahme- und Recyclingsysteme

2. Stellenwert der Implementierung von Rücknahme- und Recyclingsystemen für Hersteller von Gebrauchsgütern 11

3. Rücknahme- und Recyclingsysteme als Objekt der Implementierung.......... 17

4. Ziel und Gang der Untersuchung ............................................... 32

B. Analyse des Implementierungsverhaltens bei Rücknahme- und Recyclingsystemen .............................................................................. 36

1. Gestaltungsansätze und Ziele von Rücknahme- und Recyclingsystemen bei Gebrauchsgütern

1.1 Ansätze zur organisatorischen Gestaltung von Rücknahme- und Recyclingsystemen 36

1.2 Konstitutive Merkmale von Rücknahme- und Recyclingsystemen ........ 47

1.3 Ziele der Implementierung von Rücknahme- und Recyclingsystemen... 52

2. Dimensionen der Implementierung von Rücknahme- und Recyclingsystemen 66

2.1 Implementierungsdimensionen von Forschungsansätzen

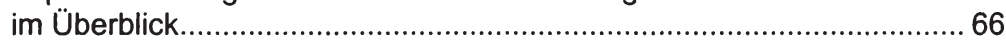

2.2 Zeitliche Dimensionen der Implementierung ................................... 71

2.3 Hierarchiebezogene Implementierungsdimensionen ......................... 76

2.4 Intensitätsbezogene Implementierungsdimension ............................. 81

2.4.1 Aufgaben bei der Implementierung von Rücknahme- und Recyclingsystemen 82

2.4.2 Identifikation betroffener Funktionen der Hersteller und Operationalisierung der Intensitätsdimension 90

2.5 Integration der Implementierungsdimensionen von Rücknahme- und Recyclingsystemen ....

2.6 Konfirmatorische Faktoranalyse zur Überprüfung der hergeleiteten Implementierungsdimensionen

3. Implementierungstypen bei Rücknahme- und Recyclingsystemen ............. 103

3.1 Ermittlung der Anzahl unterschiedlicher Implementierungstypen......... 103

3.2 Beschreibung und Konsistenzprüfung der Implementierungstypen ..... 107

3.3 Diskriminanzanalytische Überprüfung der Güte der Clusterlösung und Ermittlung der zentralen Implementierungsdimensionen 
C. Einflußfaktoren und Erfolgswirkungen des Implementierungsverhaltens von Rücknahme- und Recyclingsystemen........................... 119

1. Erklärungsansätze für das Implementierungsverhalten bei Rücknahme-

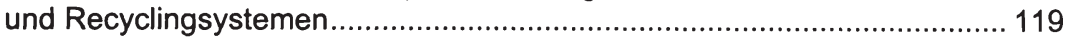

1.1 Bezugsrahmen der Einflußfaktoren und Methodik der Analyse .......... 119

1.2 Einfluß politisch-rechtlicher Faktoren auf das Implementierungs-

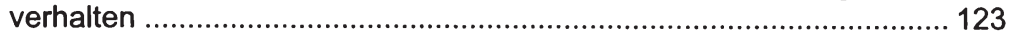

1.3 Einflußfaktoren auf der Netzwerkebene ........................................... 133

1.4 Einflußfaktoren der Umsetzung von Rücknahme- und Recyclingsystemen auf Unternehmensebene ............................................. 143

1.4.1 Aufgabenspezifische Einflußfaktoren ......................................... 143

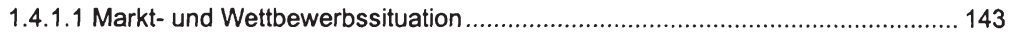

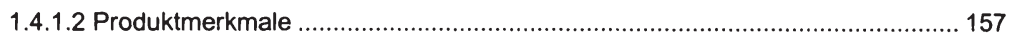

1.4.2 Interne unternehmensbezogene Einflußfaktoren ........................ 165

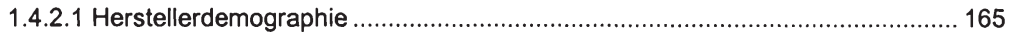

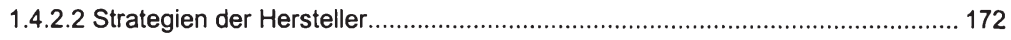

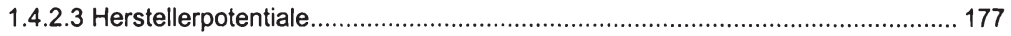

1.5 Einflußfaktoren der Durchsetzung von Rücknahme- und Recyclingsystemen auf der Individualebene................................................... 195

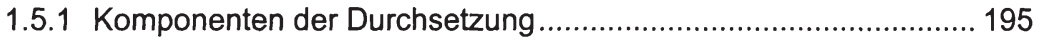

1.5.2 Instrumente zur Durchsetzung von Rücknahme- und Recycling-

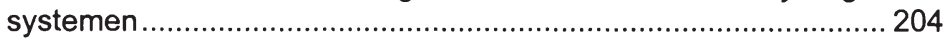

2. Erfolgswirkungen der Implementierung von Rücknahme- und

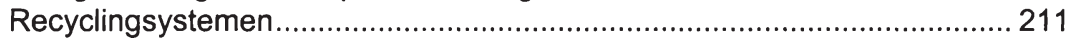

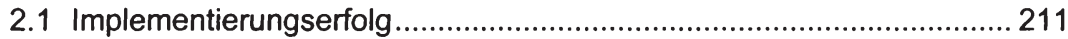

2.2 Ökologischer und ökonomischer Erfolg......................................... 217

D. Zusammenfassung und Implikationen der Untersuchungsergebnisse über Rücknahme- und Recyclingsysteme ............................................ 224

1. Zusammenfassende Würdigung der Untersuchungsergebnisse ................ 224

2. Implikationen für Hersteller langlebiger Gebrauchsgüter............................ 230

3. Auswirkungen auf die zukünftige theoretische und empirische Forschung . 232

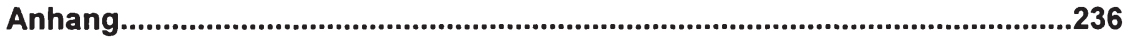

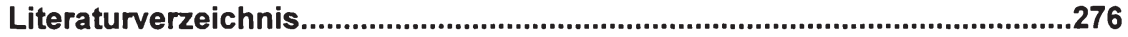




\section{Abbildungsverzeichnis}

Abb. 1: Intensität der Auseinandersetzung mit dem Kreislaufwirtschaftsgesetz .

Abb. 2: Wichtige Ablehnungsgründe für produktbezogene Rücknahmeund Recyclingsysteme

Abb. 3: Systematisierung von Implementierungsbegriffen und deren Zuordnung zu Forschungsrichtungen ................................................. 18

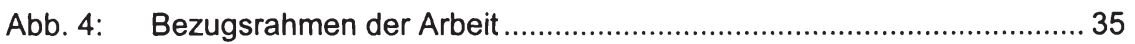

Abb. 5: Institutionelle Ausgestaltungsformen von Rücknahme- und

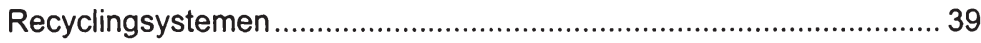

Abb. 6: Vorteile von kooperativen Rücknahme- und Recyclingsystemen ........ 43

Abb. 7: Allgemeine Implementierungsziele ................................................... 54

Abb. 8: Implementierungsziele von Rücknahme- und Recyclingsystemen ...... 58

Abb. 9: Ökonomische und ökologische Ziele von Rücknahme- und

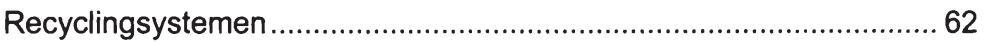

Abb. 10: Systematisierung von Einflußfaktoren auf den Implementierungserfolg.

Abb. 11: Vergleich zwischen „Programmatic"-Ansatz und " Market-Back"-Ansatz

Abb. 12: Aufgabenverteilung bei der Gestaltung von Rücknahme- und Recyclingsystemen 84

Abb. 13: Stellenwert der Implementierungsaufgaben bei Herstellern langlebiger Gebrauchsgüter

Abb. 14: Identifikation der betroffenen Funktionen der Herstellerunternehmen

Abb. 15: Modell der identifizierten Implementierungsdimensionen bei

Rücknahme- und Recyclingsystemen

Abb. 16: Konfirmatorische Faktoranalyse für die Implementierungsdimensionen

Abb. 17: Q-Plot der standardisierten Residuen 
Abb. 18a: Mittelwertprofile der identifizierten Implementierungstypen bei Rücknahme- und Recyclingsystemen............................................. 106

Abb. 18b: Mittelwerte identifizierten Implementierungstypen bei Rücknahme- und Recyclingsystemen.

Abb. 19: Mittelwertabweichungen der clusterbildenden Merkmale der ermittelten Implementierungstypen vom Gesamtmittelwert

Abb. 20: Empirischer Bezugsrahmen der Einflußfaktoren des Implementierungsverhaltens.

Abb. 21a: Entwürfe von Rücknahmeverordnungen langlebiger Gebrauchsgüter

Abb. 21b: Entwürfe von Rücknahmeverordnungen langlebiger Gebrauchsgüter

Abb. 22: Kontingenzanalyse zum Zusammenhang zwischen der herstellerseitigen Erwartung einer Rücknahmeverordnung und den Implementierungstypen

Abb. 23: Zusammenhang zwischen institutionellen Ausgestaltungsformen des Rücknahme- und Recyclingsystems und Implementierungstypen..... 136

Abb. 24: Zusammenhang zwischen der herstellerseitigen Einbringung von Ressourcen in das Rücknahme- und Recyclingsystem und Implementierungstypen

Abb. 25: Zusammenhang zwischen Absatzmarktsituation und Implementierungstypen

Abb. 26: Kontingenzanalytische Untersuchung des Zusammenhangs zwischen Existenz und Art von Rücknahme- und Recyclingsystemen in der Branche und Implementierungstypen 152

Abb. 27: Zusammenhang zwischen Beschaffungsmarktsituation und Implementierungstypen 155

Abb. 28: Zusammenhang zwischen Neupreisen, Absatzform sowie der Produktkomplexität und Implementierungstypen 160

Abb. 29: Zusammenhang zwischen Produktkategorie sowie -spezifität und Implementierungstypen 
Abb. 30: Kontingenzanalyse zum Zusammenhang zwischen Unternehmensgröße und Implementierungstypen.

Abb. 31: Zusammenhang zwischen Branche und Implementierungstypen ..... 171

Abb. 32: Ökologische Auswirkungen von Wettbewerbsstrategien

Abb. 33: Zusammenhang zwischen Strategie und Implementierungstypen .... 175

Abb. 34a: Organisatorische Maßnahmen zur Implementierung von Rücknahmeund Recyclingsystemen differenziert nach Implementierungstypen. 180

Abb. 34b: Organisatorische Maßnahmen zur Implementierung von Rücknahmeund Recyclingsystemen differenziert nach Implementierungstypen .. 181

Abb. 35a: Organisatorische Anbindung der Maßnahmen differenziert nach Implementierungstypen

Abb. 35b: Organisatorische Anbindung der Maßnahmen differenziert nach Implementierungstypen 185

Abb. 36: Zusammenhang zwischen Kultur und Implementierungstypen 189

Abb. 37: Zusammenhang zwischen Informationssystemen und Implementierungstypen

Abb. 38: Zusammenwirken der Umsetzung und Durchsetzung zur Erreichung der Anforderungen der Implementierung von Rücknahme- und Recyclingsystemen 196

Abb. 39: Zusammenhang zwischen Schwerpunktsetzungen bei den Durchsetzungsaufgaben und Implementierungstypen 202

Abb. 40: Zusammenhang zwischen Förderung des "Kennens und Verstehens" und "Könnens" bei den Mitarbeitern und Implementierungstypen ..... 206

Abb. 41: Zusammenhang zwischen Förderung des "Wollens" bei den Mitarbeitern und Implementierungstypen 209

Abb. 42: Erreichung der Implementierungsziele 213

Abb. 43: Ökologische Zielerreichung 218

Abb. 44: Ökonomische Zielerreichung 
Abb. 45: Postionierung der Implementierungstypen anhand des Status der Implementierung und der institutionellen Ausgestaltungsformen von Rücknahme- und Recyclingsystemen

Abb. 46: Synoptische Darstellung des Strategie-ImplementierungsZusammenhangs

Abb. 47: Positionierung der Implementierungstypen anhand der relativen Erfolgswirkungen 
$\mathrm{XIX}$

\section{Tabellenverzeichnis}

Tab. 1: Neuerungen im Kreislaufwirtschafts- und Abfallgesetz......................... 6

Tab. 2: Übersicht über ausgewählte Implementierungsdefinitionen ................. 17

Tab. 3: Überblick über Arten des Recycling .................................................. 23

Tab. 4: Gestaltungsspielräume der Implementierung von Rücknahme- und

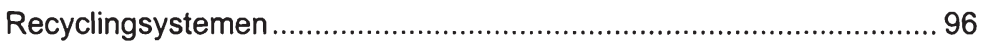

Tab. 5: Bestimmung der Clusterzahl mit dem Fusionierungsprozeß des

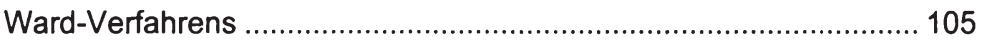

Tab. 6: Klassifikationsmatrix der diskriminanzanalytischen Untersuchung der Implementierungstypen .............................................................. 115

Tab. 7: Ermittlung der zentralen Implementierungsdimensionen nach der schrittweisen Diskriminanzanalyse

Tab. 8: Veränderung der Denkweise vom Einzelunternehmen zum Netzwerkunternehmen. 


\section{Abkürzungsverzeichnis}

a.a.O.

Abb.

Abs.

AGFI

Aufl.

Bd.

BFuP

bspw.

bzw.

ca.

DBW

d.h.

Diss.

e.V.

einschl.

erg.

erw.

EWG

f.

ff.

FAZ

FQS

FREI

GFI

ggf.

Hrsg. am angegebenen Ort

Abbildung

Absatz

Adjusted goodness of fit-Index

Auflage

Band

Betriebswirtschaftliche Forschung und Praxis

Beispielsweise

beziehungsweise

circa

Die Betriebswirtschaft

das heißt

Dissertation

eingetragener Verein

einschließlich

ergänzte

erweiterte

Europäische Wirtschaftsgemeinschaft

folgende

fortfolgende

Frankfurter Allgemeine Zeitung

Fehlerquadratsumme

FesterREcycling Initiative

Goodness of fit-index

gegebenenfalls

Herausgeber 
i.e.S.

i.d.R.

i.S.

IT

i.V.m.

i.w.S.

IWT

KD

$\mathrm{KFZ}$

KrW-IAbfG

Marketing ZFP

neubearb.

No.

NP

$\mathrm{Nr}$.

o.V.

RMR

S.

St.

SPSS

$\mathrm{t}$

Tab.

u.

u.a.

u.a.m.

u.ä.

überarb. im engeren Sinne

in der Regel

im Sinne

Informationstechnologie

in Verbindung mit

im weiteren Sinne

Institut für Wissenschafts- und Technikforschung

Kundendienst

Kraftfahrzeug

Kreislaufwirtschafts- und Abfallgesetz

Marketing Zeitschrift für Forschung und Praxis

neubearbeitete

Number

Neupreis

Nummer

ohne Verfasser

Root mean square residual-index

Seite

Sankt

Superior Performing Software System

Tonnen

Tabelle

und

und andere

und anderes mehr

und ähnliches

überarbeitete 
u.s.w.

UWF

verb.

vgl.

VO

vollst.

VW

WiSt

WISU

WSK

WZB

z.B.

ZfB

$\mathrm{ZfbF}$

zfo

z.T.

ZVEI und so weiter

Umweltwirtschaftsforum

verbesserte

vergleiche

Verordnungen

Vollständig

Volkswagen

Wirtschaftswissenschaftliches Studium

Das Wirtschaftsstudium

Wertschöpfungskreisläufe

Wissenschaftszentrum Berlin

zum Beispiel

Zeitschrift für Betriebswirtschaft

Zeitschrift für die betriebswirtschaftliche Forschung

Zeitschrift Führung und Organisation

zum Teil

Zentralverband Elektrotechnik und Elektroindustrie 


\section{A. Rücknahme- und Recyclingsysteme als Herausforderung an die umweltorientierte Unternehmensführung}

\section{Umweltpolitische Rahmenbedingungen als Entstehungsgrund für Rücknahme- und Recyclingsysteme}

Seit den 80er Jahren beeinflußt das Thema des Umweltschutzes die Entwicklung von Branchen und Unternehmen sehr intensiv. Dennoch unterblieb häufig eine offensive Auseinandersetzung seitens der Unternehmen, obwohl sie sich zunehmend dramatischen Entwicklungen der Umweltschutzproblematik gegenübersahen. Sich verschärfende rechtliche Regelungen (Umwelthaftung, Umweltstrafrecht) verdeutlichten Herstellern, Handel und Entsorgungsunternehmen zudem neue betriebswirtschaftliche Risikodimensionen. ${ }^{1}$

Gleichzeitig waren die Voraussetzungen für eine neue Phase der staatlichen Umweltpolitik, Umweltbelastungen zu vermeiden und zu vermindern, bis Ende der 80er Jahre günstig, da sich die gesamtwirtschaftliche Entwicklung in einer Wachstumsphase befand und zudem sozialpolitische Konflikte trotz relativ hoher Arbeitslosigkeit nicht im Vordergrund standen. Auch die bis dato eher defensive Haltung der Unternehmen und Gewerkschaften gegenüber dem Umweltschutz relativierte sich und führte zur Entwicklung und Umsetzung unternehmerischer umweltbezogener Konzepte. Die Umweltverantwortung des Management sowie die Zunahme des Umweltbewußtseins in der deutschen Bevölkerung führten dazu, daß eine Überwindung des Konfliktes zwischen ökonomischer Entwicklung, Sozialverträglichkeit und ökologischer Verträglichkeit realisierbar schien. ${ }^{2}$ Neben der praktischen Annahme umweltbezogener Fragestellungen befaßte sich zunehmend die Wirtschaftswissenschaft ${ }^{3}$ mit der Integration des Umweltschutzes in

Vgl. Meffert, H., Kirchgeorg, M., Marktorientiertes Umweltmanagement: Grundlagen und Fallstudien, 3. überarb. und erw. Aufl., Stuttgart 1997, S. 3ff.

2 Vgl. Der Rat von Sachverständigen für Umweltfragen, Umweltgutachten 1994: Für eine dauerhaft-umweltgerechte Entwicklung, Stuttgart 1994, S. 177.

3 Die Betriebswirtschaftslehre zeigte zunächst stärkere Berührungsängste als die Volkswirtschaftslehre, den Umweltschutz in Theorie und Praxis einzubeziehen, da dessen Operationalităt und Kompatibilität zu wettbewerbsstrategischen Grundsätzen als problematisch angesehen wurde. Inzwischen setzt sich die Betriebswirtschaft aufgrund des wachsenden Drucks der ökologischen Herausforderungen sehr intensiv mit Fragen des Umweltschutzes auseinander. Vor dem Hintergrund einer Befragung mit Professoren und Praxisvertretern erläutert Meffert, daß eine neue Qualităt in der interdisziplinären Zusammenarbeit notwendig ist, um die entstehenden Zukunftsaufgaben zu bewältigen. Vgl. Meffert, H., Stand und Perspektiven des Umweltmanagement in der betriebswirtschaftlichen Forschung und Lehre, in: Weber, J. (Hrsg.), Umweltmanagement, Aspekte einer umweltbezogenen Unternehmensführung, Stuttgart 1997, S. $5 \mathrm{ff}$. 
gesamt- und einzelwirtschaftliche Belange, welches sich in der exponentiell ansteigenden Anzahl ökologiebezogener Literatur ausdrückte. Der Schwerpunkt der Abhandlungen lag in den 80er Jahren im Bereich der Abfallproblematik auf der Entsorgung und Verwertung von Abfällen, bevor in den 90er Jahren zunehmend der Vermeidungsaspekt in den Vordergrund trat. ${ }^{4}$

Kerngedanke der Bemühungen um den Umweltschutz von Wissenschaft, Praxis und Politik waren Bestrebungen, die sogenannte Kreislaufwirtschaft auf den Weg zu bringen und umzusetzen. ${ }^{5}$ Dieses wurde häufig unter dem Begriff Sustainable Development subsumiert, welches als Leitbild die Überwindung des Gegensatzes zwischen Ökonomie und Ökologie beinhaltet. Dabei steht die Aufrechterhaltung der ökologischen Funktionen im Mittelpunkt der Betrachtung. ${ }^{6}$ Die Weltkommissi-

Zur Diskussion der Wettbewerbswirkungen von verschiedenen Instrumenten der Umweltpolitik vgl. Bonus, H., Umweltschutz und Wettbewerb aus ökonomischer Sicht, in: 12. Trierer Kolloquium zum Umwelt- und Technikrecht, Umweltschutz und Wettbewerb, Trier 1996, S. $21 \mathrm{ff}$.

Vgl. Corsten, H., Götzelmann, F., Abfallvermeidung und Reststoffverwertung - eine produktund verfahrensorientierte Analyse, in: Betriebswirtschaftliche Forschung und Praxis, 1992, Heft 2, S. 102ff. Unter dem Begriff Vermeidung wird eine grundsätzliche Unterlassung einer Handlung verstanden, die mit nachteiligen Folgen verbunden ist. Die partielle Vermeidung (Verminderung) wird ebenfalls unter diesem Begriff subsumiert, so daß die absolute Vermeidung einen Spezialfall der Verminderung darstellt. In der Literatur hat sich dennoch der Begriff der Abfallvermeidung durchgesetzt.

Im Gegensatz zur Vermeidung stellt die Verwertung eine Maßnahme dar, die im Anschluß an die Produktnutzung stattfindet. Die energetische Verwertung beinhaltet eine Abfallverbrennung unter Gewinnung von Energie, wobei die Energieausbeute im Vordergrund steht. Bei der stofflichen Verwertung werden Reststoffe in einen Produktions- und Konsumprozeß zurückgeführt. Vgl. Müller, H., Industrielle Abfallbewältigung, Entscheidungsprobleme aus theoretischer Sicht, Wiesbaden 1991, S. 66.

5 Die Kreislaufwirtschaft betrachtet im Gegensatz zur "Durchlaufwirtschaft" den gesamten Lebenszyklus eines Produktes, d.h. neben der Produktion und Nutzung werden auch die späteren Phasen des Produktes, wie das Recycling, die Entsorgung und der erneute Einsatz der entstehenden Reststoffe oder Altproduktteile, mit in die einzelwirtschaftliche Betrachtung einbezogen (Betrachtung des Produktes "von der Wiege bis zur Wiege“).

6 Vgl. Barbier, E. B., The Concept of Sustainable Development, in: Environmental Conservation, Nr. 2, 1987, S. 105.

Die Funktionen des ökologischen Systems (Versorgungs-, Produktions- und Trägerfunktion) dürfen dabei von dem ökonomischen System nur insoweit beansprucht werden, wie es die Regenerationsraten und Assimilationskraft der Natur zulassen, um dabei eine "Circular Economy“ zu gewährleisten. Vgl. Pearce, D., Turner, R. K., Economics of Natural Resources and the Environment, New York/Harvester Wheatsheaf 1990, S. $40 \mathrm{ff}$.

Die thermodynamischen Sätze unterstützen die These der notwendig werdenden Kreislaufwirtschaft. Erster Hauptsatz: eingesetzte Ressourcen können nur umgewandelt und zerstreut werden, sie werden aber letztlich irgendwo im ökologischen System als Belastung wieder auftreten (Trägerfunktion der Natur). Zweiter Hauptsatz: In einem geschlossenen System strebt die Entropie als Maß für die Verfügbarkeit von Energie einem Maximum zu. Demnach wird der Reinheitsgrad von Energie- und Ressourcenbeständen immer geringer, womit die Verwertbarkeit sinkt. Vgl. Brenk, A., Moderne umweltpolitische Konzepte: Sustainable Development und ökologisch-soziale Marktwirtschaft, Diskussionspapier Nr. 3 des Instituts für Verkehrswissenschaft an der Universität Münster, Münster 1991, S. $12 \mathrm{ff}$. 
on für Umwelt und Entwicklung setzte 1987 mit ihrem Bericht ein wichtiges Signal für die dabei postulierte Verantwortungsethik der Wirtschaftssubjekte. Sustainable Development beinhaltet eine Entwicklung, die den Bedürfnissen der Gegenwart entspricht, ohne künftige Generationen in ihrer Fähigkeit zu beeinträchtigen, ihre eigenen Bedürfnisse zu befriedigen. ${ }^{?}$

Das Abfallgesetz unterschied in der novellierten Fassung vom 27. August $1986^{8}$ erstmalig Abfallvermeidung, -verwertung und -beseitigung, ohne eine Prioritätenreihenfolge festzulegen. Dem folgten nachgesetzliche Regelwerke in Form der Technischen Anweisung Abfall, Verpackungsverordnung und anderen Regularien, die zum einen zu einer stärkeren Berücksichtigung von Umweltschutzmaßnahmen führten und zum anderen Verteuerungen in der Abfallbeseitigung nach sich zogen. ${ }^{9}$ Dieses führte dazu, daß illegale Abfallexporte und der (legale) "Mülltourismus" deutlich zunahmen und durch rechtlich unscharfe Begrifflichkeiten wie "Reststoffe" und "Wirtschaftsgüter" noch unterstützt wurden. ${ }^{10}$ Ausgehend von der Diskussion um Sustainable Development und den beschriebenen Regelungsdefiziten wurde das Abfallgesetz zum Kreislaufwirtschafts- und Abfallgesetz (KrW-IAbfG) von der Regierung weiterentwickelt und am 24. Juni 1994 vom Bundestag beschlossen, bevor es am 7. Oktober 1996 in Kraft trat. ${ }^{11}$

Wesentliche Neuerungen des Kreislaufwirtschaftsgesetzes betreffen den Abfallbegriff, die Pflichtenhierarchie der Abfallbehandlung und die erweiterte Produkt-

7 Vgl. Weltkommission für Umwelt und Entwicklung, Unsere gemeinsame Zukunft, der Brundtland-Bericht der Weltkommission für Umwelt und Entwicklung, Hauff, V. (Hrsg.), Greven 1987, S. 46.

8 Das Abfallgesetz löste das Abfallbeseitigungsgesetz vom 7. Juni 1972 ab, welches in erster Linie auf eine Ordnung der Abfallbeseitigung zielte.

$9 \quad 1987$ trat die Einheitliche Europäische Akte in Kraft, die den bis dahin eindeutig wirtschaftlichen Gedanken des EWG-Vertrags um Umweltschutzziele erweiterte.

10 Vgl. Ruchay, D., Kreislaufwirtschaft und Rücknahmeverordnungen - Umweltpolitische Ziele und Status der Umsetzung, in: Meffert, H., Wagner, H., Backhaus, K. (Hrsg.), Marktorientierte Unternehmensführung in der Kreislaufwirtschaft - Kostenmanagement oder Wettbewerbsprofilierung?, Dokumentationspapier Nr. 106 der Wissenschaftlichen Gesellschaft für Marketing und Unternehmensführung e.V., Münster 1996, S. 5.

Reststoffe und Wirtschaftsgüter bilden keine Abfälle im rechtlichen Sinne und unterliegen daher keiner abfallrechtlichen Exportbeschränkung.

11 Vgl. Bundesminister für Umwelt, Naturschutz und Reaktorsicherheit, Gesetz zur Vermeidung von Rückständen, Verwertung von Sekundärrohstoffen und Entsorgung von Abfällen (Kreislaufwirtschafts- und Abfallgesetz - KrW-/AbfG), Bonn im März 1993 (im folgenden nur noch als KrW-/AbfG zitiert) und vgl. Bundesminister für Umwelt, Naturschutz und Reaktorsicherheit, Bundesrat verabschiedet Kreislaufwirtschaftsgesetz, Pressemitteilung Nr. 49/94, Bonn 1994. 
verantwortung der Hersteller, Be- und Verarbeiter sowie Vermarkter. Der Abfallbegriff des Abfallgesetzes von 1986 definierte Abfälle als bewegliche Sachgegenstände, deren sich der Besitzer entledigen will oder deren geordnete Beseitigung zur Wahrung des Allgemeinwohls geboten ist (subjektive Abfalldefinition). ${ }^{12}$ Der erweiterte Abfallbegriff des Kreislaufwirtschaftsgesetzes hingegen definiert Abfälle als alle beweglichen Sachen, „derer sich ihr Besitzer entledigt, entledigen will oder entledigen muß“. ${ }^{13}$

Die Pflichtenhierarchie legt fest, daß Abfälle in erster Linie zu vermeiden sind und sofern dieses nicht möglich ist, sind ihre Menge und Schädlichkeit zu vermindern. ${ }^{14}$ In zweiter Linie sind Abfälle entweder stofflich zu verwerten oder zur Gewinnung von Energie (energetische Verwertung) zu nutzen. Grundsätzlich gilt dabei, daß eine möglichst hochwertige Verwertung durchgeführt werden sollte. Bei der Entscheidung, ob eine stoffliche oder energetische Verwertung den Vorrang erhalten soll, ist die umweltverträglichere Alternative vorzuziehen. Abfälle, die weder vermieden noch verwertet werden können, sind dauerhaft von der Kreislaufwirtschaft auszuschließen und zur Wahrung des Wohls der Allgemeinheit zu beseitigen. Zudem sind Abfälle im Inland zu beseitigen, und die bei der Behandlung und Ablagerung der Abfälle anfallenden Energien oder Abfälle sollen so weit wie möglich genutzt werden. ${ }^{15}$

12 Vgl. Ruchay, D., Kreislaufwirtschaft und Rücknahmeverordnungen - Umweltpolitische Ziele und Status der Umsetzung, a.a.O., S. 5 f.

$13 \S 3$ Abs. $1 \mathrm{KrW}-/ \mathrm{AbfG}$.

Die Entledigung ist anzunehmen, wenn der Besitzer den Sachgegenstand einer Verwertung oder Beseitigung zuführt oder die tatsächliche Sachherrschaft über sie aufgibt. Der Entledigungswille liegt vor, wenn Stoffe nicht Zweck einer Handlung waren oder ihre übliche Zweckbestimmung entfällt, ohne daß ein neuer Verwendungszweck unmittelbar an ihre Stelle tritt. Die Zweckbestimmung richtet sich nach der Auffassung des Besitzers unter Berücksichtigung der Verkehrsanschauung (Bsp.: alte Badewanne nicht als Gartenteich verwendbar). Ferner ist die Abfalldefinition durch die Aufzählung im Anhang I des KrW-/AbfG, die international gilt, ergänzt. Vgl. Ruchay, D., Kreislaufwirtschaft und Rücknahmeverordnungen - Umweltpolitische Ziele und Status der Umsetzung, a.a.O., S.6.

Der Besitzer muß sich einer Sache entledigen (Entledigungszwang), wenn sie entsprechend ihrer üblichen Zweckbestimmung nicht mehr verwendet wird oder das Wohl der Allgemeinheit (Gefährdungspotential) beeinträchtigt ist.

Zur Diskussion des objektiven versus subjektiven Abfallbegriffs vgl. Matschke, M., Lemser, B., Entsorgung als betriebliche Grundfunktion, in: Betriebswirtschaftliche Forschung und Praxis, Heft 2, 1992, S. $88 \mathrm{f}$. 
Die notwendigen Verordnungen zur verpflichtenden Umsetzung der erweiterten Produktverantwortung des Kreislaufwirtschaftsgesetzes sollen laut Koalitionsvereinbarung für die 13. Legislaturperiode des Deutschen Bundestages vorgelegt werden. Es handelt sich insbesondere um die Produktbereiche Altautos, Elektronikschrott, Batterien und Kompost, wobei Selbstverpflichtungen der Unternehmen Vorrang vor gesetzlichen Regelungen haben sollen. ${ }^{16}$ Die erweiterte Produktverantwortung urnfaßt die gemeinsame Verantwortlichkeit von Herstellern, Be- und Verarbeitern, Vermarktern sowie eingeschränkt der Konsumenten für die Organisation der Sammlung und Rückführung, für die Sicherstellung der Verwertung, für die Schaffung von Sekundärrohstoff- bzw. Sekundärproduktmärkten sowie für die Finanzierung der Produktentsorgung. ${ }^{17}$ Dabei können die Verpflichteten Dritte mit der Erfüllung dieser Aufgaben beauftragen, wobei die Verantwortlichkeit jedoch unberührt bleibt. ${ }^{18}$ Die wesentlichen Neuerungen des Kreislaufwirtschaftsgesetzes sind in Tabelle 1 zusammengefaßt.

16 Vgl. Ruchay, D., Kreislaufwirtschaft und Rücknahmeverordnungen - Umweltpolitische Ziele und Status der Umsetzung, a.a.O., S. 7. Eine Selbstverpflichtung von Wirtschaftssubjekten ist rechtlich verbindlich, auch wenn eine Rechtsverordnung für diesen Bereich noch nicht erlassen wurde.

Unter dem Begriff der Entsorgung wird in Gesetzestexten - so wie er an dieser Stelle verstanden wird - die stoffliche Verwertung, das Gewinnen von Stoffen oder Energien aus Abfall sowie das Ablagern und die hierzu notwendigen Maßnahmen gefaßt. Abfälle, die nicht verwertet werden, sind zu beseitigen. Danach ist die Beseitigung die Deponierung, Verbrennung oder sonstige Beseitigung. Vgl. Abfallgesetz vom 27.8.1986, Bundesgesetzblatt I, 1986, S. 1410, §1 Abs. 2 und vgl. KrW-/AbfG.

In der betriebswirtschaftlichen Literatur wird in der Regel die Entsorgung mit der Beseitigung gleichgesetzt. Diesem Begriffsverständnis soll hier gefolgt werden.

18 Der Dritte muß über die erforderliche Zuverlässigkeit verfügen, die er durch ein Abfallwirtschaftskonzept belegen muß. Dieses enthält Angaben über Art, Menge und Verbleib der Abfälle, Angaben über die getroffenen und geplanten Maßnahmen zur Abfallbehandlung sowie die Darlegung der vorgesehenen Entsorgungswege für die năchsten fünf Jahre einschließlich der notwendigen Standort- und Anlagenplanung sowie ihrer zeitlichen Abfolge. Vgl. §16 Abs. 3 KrW-IAbfG. 


\begin{tabular}{|c|c|c|}
\hline $\begin{array}{l}\text { Wesentliche } \\
\text { Neuerungen }\end{array}$ & Inhalt & Einzelheiten \\
\hline $\begin{array}{l}\text { Abfallbegriff } \\
\$ 3\end{array}$ & $\begin{array}{l}\text { Objektivierung des Abfallbe- } \\
\text { griffs bei beweglichen Sach- } \\
\text { gegenständen }\end{array}$ & $\begin{array}{l}\text { Abfälle liegen vor bei: } \\
\text { Entledigung, } \\
\text { Entledigungswille, } \\
\text { Entledigungszwang, wenn Stoffe nicht Zweck } \\
\text { der Handlung waren oder Zweckbestimmung } \\
\text { entfällt. } \\
\text { Zusätzlich die im Anhang I des KrW-/AbfG } \\
\text { aufgeführten Kategorien. } \\
\end{array}$ \\
\hline $\begin{array}{l}\text { Pflichten- } \\
\text { hierarchie } \\
\text { \$4 Abs. 1, 2, } 3 \\
\text { \$5 Abs. 2, } 4 \\
\text { \$6 Abs. 1, } 2\end{array}$ & $\begin{array}{l}\text { Oberziel: Abfallvermeidung } \\
\text { Stoffliche und energetische } \\
\text { Verwertung haben Vorrang } \\
\text { vor anderen abfallwirtschaftli- } \\
\text { chen Maßnahmen } \\
\text { Stoffliche und energetische } \\
\text { Verwertung grundsätzlich } \\
\text { gleichrangig } \\
\text { Vermeidung } \\
\text { vor Verwertung } \\
\text { vor Beseitigung }\end{array}$ & $\begin{array}{l}\text { Vorgesehene Maßnahmen der Vermeidung: } \\
\text { Anlageninterne Kreislaufführung von Stoffen } \\
\text { Abfallarme Produktgestaltung } \\
\text { Beeinflussung des Konsumentenverhaltens } \\
\text { - Bei stofflicher Verwertung stehen Maßnah- } \\
\text { men der Abfallnutzung und nicht der Beseiti- } \\
\text { gung eines Schadstoffpotentials im Vorder- } \\
\text { grund } \\
\text { - Eine energetische Verwertung muß Mindest- } \\
\text { kriterien erfüllen: } \\
\text { ausreichender Energiegehalt (11.000 kj/kg), } \\
\text { ausreichende Energieausbeute (>75\% Feu- } \\
\text { erwirkungsgrad), } \\
\text { ganzjährige Energienutzung, } \\
\text { Entsorgungsmöglichkeit der Restabfälle } \\
\text { Abweichung möglich, wenn Vermeidung und } \\
\text { Verwertung technisch nicht möglich und wirt- } \\
\text { schaftlich unzumutbar sind sowie wenn kein } \\
\text { Markt vorhanden oder nicht schaffbar ist } \\
\end{array}$ \\
\hline $\begin{array}{l}\text { erweiterte Pro- } \\
\text { dukt- } \\
\text { verantwortung } \\
\$ 16 \text { Abs. } 1 \\
\text { \$22 } \\
\text { \$24 Abs. } 2\end{array}$ & $\begin{array}{l}\text { Rücknahme-, Verwertungs- } \\
\text { und Finanzierungspflicht für } \\
\text { Hersteller, Be- und Verarbei- } \\
\text { ter sowie Vermarkter } \\
\text { Rückgabepflicht für Konsu- } \\
\text { menten }\end{array}$ & $\begin{array}{l}\text { - Rechtlich verpflichtend kann die Produktver- } \\
\text { antwortung nur durch Rechtsverordnungen } \\
\text { durchgesetzt werden } \\
\text { - Dritte können zur Verwertung und Beseiti- } \\
\text { gung beauftragt werden (Pflicht eines Abfall- } \\
\text { wirtschaftskonzeptes), Verantwortlichkeit } \\
\text { bleibt beim Auftraggeber } \\
\text { - Abfallbilanz bei mehr als } 2000 \mathrm{~kg} \text { besonders } \\
\text { überwachungsbedürftiger Abfälle oder bei } \\
\text { mehr als } 2000 \text { t überwachungsbedürftiger } \\
\text { Abfälle }\end{array}$ \\
\hline
\end{tabular}

Tab. 1: Neuerungen im Kreislaufwirtschafts- und Abfallgesetz

Rechtlich bindend - und damit relevant für die Unternehmen - werden Rücknahme- und Rückgabepflichten allerdings erst durch die Möglichkeit der Bundesregierung, nach Anhörung der beteiligten Kreise und mit Zustimmung des Bundesrates Rechtsverordnungen zu erlassen. ${ }^{19}$ Die im Entwurf vorliegenden Rücknahmeverordnungen beziehen sich - nach bereits bestehenden Verordnungen zur Rück- 
gabe von Altöl oder Verpackungen - in erster Linie auf langlebige Gebrauchsgüter. So liegen Entwürfe für Elektro- und Elektronikprodukte (für die Informationstechnologien existiert ein separater Verordnungsentwur ${ }^{20}$ ) und für Altautos vor. ${ }^{21}$

Einige Hersteller haben bereits Rücknahme- und Recyclingsysteme eingeführt und verhalten sich damit proaktiv in bezug auf die Rechtsverordnungen. Andere planen solche Systeme, um sie in nächster Zeit umsetzen zu können und dem Inkrafttreten der Verordnungen vorzubeugen. Unter Rücknahme- und Recyclingsystemen sind dabei im folgenden produktbezogene Stoffkreisläufe zu subsumieren, die sich mit der Rückführung von Altprodukten (bzw. Rückständen) in den Produktions- bzw. Konsumprozeß befassen. ${ }^{22}$

In der Praxis sind vor allem Systeme für langlebige Gebrauchsgüter zu beobachten, ${ }^{23}$ die daher Gegenstand der vorliegenden Untersuchung sind. ${ }^{24}$ Eine Fokussierung auf den Bereich von langlebigen Gebrauchsgütern liegt - neben den erläuterten gesetzlichen Rahmenbedingungen - im hohen Wertschöpfungspotential dieser Güter, d.h. der in Geldeinheiten gemessene Neuproduktwert liegt i.d.R.

20 Es ist zu erwarten, daß weitere „Unterverordnungen“ zur Elektro- und Elektronikschrottverordnung entstehen, da die Produkte, die der Elektro- und Elektronikbranche zuzuordnen sind, sehr vielfältig sind und daher sehr heterogene Anforderungen an die Rücknahme und das Recycling stellen.

Weitere Verordnungsentwürfe liegen für Batterien und Kompost vor.

21 Bereits im Mai 1997 billigte der Bundesrat mit kleinen Änderungen die Altautoverordnung. Nach genauer Klärung der Bestimmungen tritt sie damit zum 1. April 1998 in Kraft. Vgl. Bundesminister für Umwelt, Naturschutz und Reaktorsicherheit, Weg frei für das Inkrafttreten der deutschen Altautoverordnung, Pressemitteilung vom 20.1.98, S. 1. Eine eingehende Begriffserläuterung schließt sich in Kapitel $A 3$ an.

Neben den Rücknahme- und Recyclingsystemen für langlebige Gebrauchsgüter sind in der Praxis Systeme für Verpackungen (insbesondere unter dem Dualen System Deutschland) festzustellen. Dabei ergeben sich auch aus dem Kreislaufwirtschaftsgesetz Anknüpfungspunkte zur Novellierung der Verpackungsverordnung, die insbesondere helfen soll, die "Trittbrettfahrer" beim Dualen System Deutschland auszuschließen. Vgl. zu näheren Ausführungen Ruchay, D., Kreislaufwirtschaft und Rücknahmeverordnungen - Umweltpolitische Ziele und Status der Umsetzung, a.a.O., S. 7 f.

24 Vgl. unter anderem die Rücknahme- und Recyclingsysteme von Rank Xerox, Volkswagen und Bauknecht, Siemens-Nixdorf und der Fenster-Reycling-Initiative (FREI). Vgl. Meffert, H., Kirchgeorg, M., Marktorientiertes Umweltmanagement, a.a.O., Fallstudie Rank Xerox, S. 693743 und vgl. Fallstudie Volkswagen, Fallstudie Bauknecht, Fallstudie Siemens-Nixdorf, FREI (Fenster-Recycling-Initiative) -Fallstudie, unveröffentlichte Fallstudien des Instituts für Marketing, Münster 1997.

Da sich aus der Betrachtungsperspektive der Hersteller durch Einführung von Rücknahmeund Recyclingsystemen insbesondere organisatorische Probleme ergeben, wird diesem Problembereich besondere Beachtung gewidmet. Die anderen Möglichkeiten der Problemanalyse von Rücknahme- und Recyclingsystemen wären zum einen die technische und zum anderen die stoffliche Betrachtung von Rücknahme- und Recyclingsystemen. 
hoch und wird insbesondere durch die Produktion, Logistik, Marketing und Kundendienst des Herstellers und/oder des Vertreibers erworben. Durch diesen Tatbestand kann es sich insbesondere für den Hersteller lohnen, eigene Altprodukte zurückzuerhalten, um diese dann im Ganzen oder in Teilen weiterzunutzen bzw. deren Materialien wieder einzusetzen. In diesem Zusammenhang wird in der betriebswirtschaftlichen Literatur auch vom Wertschöpfungskreislauf gesprochen. ${ }^{25}$ In Anlehnung an Porter wird die Wertschöpfung als Gewinnspanne aufgefaßt, ${ }^{26}$ wobei wettbewerbsstrategisch relevante Aktivitäten gesucht, werden, die einen wahrnehmbaren, wichtigen und dauerhaften Wettbewerbsvorteil über den gesamten Produktlebenszyklus aufbauen sollen. ${ }^{27}$

Ein weiterer Grund für die hohe Bedeutung von langlebigen Gebrauchsgütern für Rücknahme- und Recyclingsysteme liegt darin, daß die Öffentlichkeit diese Güter verstärkt mit der Umweltproblematik in Zusammenhang bringt und diese im besonderen Interesse der Medien und Umweltschutzorganisationen stehen ${ }^{28} \mathrm{Ur}$ -

25 Vgl. Meffert, H., Kirchgeorg, M., Ökologisches Marketing, Erfolgsvoraussetzungen und Gestaltungsoptionen, in: UWF, Heft 1, 1995, S. 21ff.; vgl. Meffert, M., Sustainable Development: Thesen zur betriebswirtschaftlichen Alternative, in: Wissenschaftliche Gesellschaft für Marketing und Unternehmensführung e.V. (Hrsg.), Sustainable Development als Leitbild einer umweltbewußten Unternehmensführung, Dokumentation einer Vortragsveranstaltung am 22. Juni 1992 an der Westfälischen Wilhelms-Universität zu Münster, Münster 1992, S.33 und vgl. Kirchgeorg, M., Kreislaufwirtschaft - neue Herausforderungen an das Marketing, a.a.O., S. $16 \mathrm{ff}$.

Der Porter'sche Bergriff steht damit im Gegensatz zum klassischen Wertschöpfungsbegriff (Umsatzerlöse abzüglich Vorleistungen).

Vgl. Kirchgeorg, M., Kreislaufwirtschaft - neue Herausforderungen an das Marketing, a.a.O., S. 17.

Stoffkreisläufe können demnach als Wertschöpfungskreisläufe gekennzeichnet werden, wenn durch „(1) die Wiederverwendung und Weiterverwendung von im ersten (primären) Herstellungsprozeß produzierten Produktkomponenten im Rahmen einer erneuten Produktion über den Materialwert hinaus die Wertschöpfungsbestandteile erhalten bleiben, wodurch im zweiten Einsatzprozeß Kostenvorteile realisiert werden können; (2) die Wiederverwertung und Weiterverwertung von Sekundärrohstoffen gleiche und höhere Wertschöpfungspotentiale erschlossen werden können als beim Einsatz von neuen Rohstoffen; (3) eine integrierte Optimierung von vorwärts- und rückwärtsgerichteten Prozeßstufen Wertschöpfungspotentiale erschlossen werden können, d.h. Kostensenkungs- und/oder Erlössteigerungspotentiale gleichzeitig im Beschaffungs-, Produktions- oder Marketingbereich als auch im Demontage- oder Recyclingbereich realisiert werden." Kirchgeorg, M., Kreislaufwirtschaft - neue Herausforderungen an das Marketing, a.a.O., S. 23 f.

28 Die Identifikation der langlebigen Gebrauchsgüter mit der Umweltproblematik seitens der Konsumenten bedeutet nicht gleichzeitig, daß aus Herstellersicht die ökonomischen Chancen einer ökologieorientierten Profilierung generell als positiv zu beurteilen sind. In der Elektrobranche ist beispielsweise lediglich denjenigen ökologieorientierten Produktanforderungen, die einen deutlichen und gut kommunizierbaren Individualnutzen bieten, eine gewisse Kaufrelevanz beizumessen. Vgl. Ceyp, M. H., Ökologieorientierte Profilierung im vertikalen Marketing dargestellt am Beispiel der Elektronikbranche, Frankfurt am Main u.a. 1996, S. $123 f$. 
sache dafür sind zum einen die hohen Mengen und der Wert der anfallenden Schrottmengen. ${ }^{29}$ Zum anderen werden die Schrottmengen für den privaten Nutzer unmittelbar erfahrbar, da Elektrogeräte wie auch Automobile eine große Verbreitung haben und nahezu in allen Haushalten zu finden sind. ${ }^{30}$ Somit sind private (Letzt)nutzer am Ende des Produktlebenszyklus mit dem Entsorgungsproblem der Geräte konfrontiert, und es besteht eine hohe individuelle Betroffenheit. Dabei kann vermutet werden, daß die Probleme der Rückgabe und des Recycling für den Konsumenten mit zunehmender Annäherung an das Produktlebensende wichtiger werden. Insbesondere dann, wenn er das Altprodukt nicht in den Hausmüll entsorgen oder zum Sperrmüll stellen darf. ${ }^{31}$ Darüber hinaus enthalten Automobile sowie Elektro- und Elektronikschrott hohe Schadstoffpotentiale ${ }^{32}$ und sind unter abfallwirtschaftlichen Gesichtspunkten nicht hausmüllgeeignet. ${ }^{33}$

Andererseits wird eine Nichterfüllung gewisser Umweltstandards seitens der Konsumenten kritisiert, so daß diese m.E. zu Hygienefaktoren werden, dessen Erfüllung zwar keine positive Auswirkungen auf das Kaufverhalten ausübt, deren Nichterfüllung jedoch sanktioniert wird. Herzberg beschreibt die Hygienefaktoren in der Zwei-Faktoren-Theorie der Arbeitsmotivation, die bei einer negativen Ausprägung zur Arbeitsunzufriedenheit führt und bei einer positiven Ausprägung zu einer Reduzierung der Unzufriedenheit. Im Gegensatz dazu sind Motivatoren bei positiver Ausprägung Zufriedenheitsstifter. Vgl. Herzberg, F., Mausner, B., Snydermann, B. B., The Motivation to Work, New York, London, Sydney 1959.

Der Zentralverband Elektrotechnik gibt an, daß ca. 1,5 Mio. Tonnen Elektro- und Elektronikschrott in Deutschland pro Jahr anfallen (66\% Konsumgüter, $33 \%$ Investitionsgüter). Davon werden bisher nur ca. 100.000 Tonnen fachgerecht entsorgt. Der weitaus größere Anteil wird bisher verbrannt oder auf Deponien entsorgt. Experten schätzen dabei den Wert der nicht genutzten Wertstoffe auf ca. 6-10 Mrd. DM ein. Vgl. o.V., Computerrecycling, Edelschrott, in: Stern, Heft 25, 1995, S. 48 und vgl. Fallstudie Siemens-Nixdorf, a.a.O.

2,5 Mio. Personenkraftwagen fielen in den letzten Jahren jährlich zur Entsorgung an. Lastkraftwagen (ca. 100.000) und Busse (ca. 5.000 jährlich), deren Entsorgung keine besonderen Probleme bereitet, fallen nicht unter die Altautoverordnung. Vgl. Bundesminister für Umwelt, Naturschutz und Reaktorsicherheit, Entwurf über die Entsorgung von Altautos, Bonn, Stand vom 27. Januar 1994, Erläuterungen zu den Vorschriften im einzelnen §3.

Zudem sind langlebige Gebrauchsgüter für den Konsumenten in der Regel High-InvolvementProdukte, da sie einen relativ hohen Anschaffungspreis haben und damit einem längeren Kaufentscheidungsprozeß unterliegen als Produkte mit einem geringen Verkaufspreis.

31 Besteht für den Konsumenten ein „Entsorgungsproblem“ für sein Altprodukt (z.B. ein Entsorgungsverbot für das Altprodukt in den Hausmüll), wird er darüber hinaus vermutlich das Kriterium der Rücknahme durch den Hersteller oder Handel in der nächsten Kaufentscheidung (Wiederkauf) stärker berücksichtigen.

Beispielsweise sei bei Automobilen an dieser Stelle auf Öle, Fette und Schmierstoffe hingewiesen, bei Computern auf Bildröhren und bei Kühlschränken auf FCKW-haltige Kältemittel.

33 Dieses wird aber beispielsweise bei Personalcomputern häufig nicht beachtet, da diese zum einen aufgrund der relativ geringen Größe bequem in den normalen Hausmüll entsorgt werden können und zum anderen wegen der sehr kurzen Innovationszyklen regelmäßig ersetzt werden. Lag die Erstnutzungsdauer von Computern in den 50er Jahren noch bei durchschnittlich 10 Jahren, liegt sie heute bei durchschnittlich 4,5 Jahren - bei sehr innovativen Produkten in Einzelfällen bereits bei weniger als zwei Jahren. Vgl. Burgdorf, P., Computerwiederverwendung und -recycling: Erfahrungen bei Siemens-Nixdorf, Vortrag 1995, S. 1. 
Als Hauptadressaten des Kreislaufwirtschaftsgesetzes werden die Hersteller gesehen, da diese nach dem Verursacherprinzip für die Fertigung der Produkte und damit letztlich auch für die Entstehung der entsprechenden Altprodukte verantwortlich sind. 


\section{Stellenwert der Implementierung von Rücknahme- und Recycling- systemen für Hersteller von Gebrauchsgütern}

Bisher wird von Unternehmen noch ein hoher Prozentsatz von Altprodukten gar nicht erfaßt und entsorgt ${ }^{34}$. Findet bereits eine Erfassung statt, werden z.T. auch verwertbare Abfälle aus Altprodukten (Produktabfälle) entsorgt. ${ }^{35}$ Automobile beispielsweise haben zwar gemessen am Gewicht eine relativ hohe Recyclingquote von $75 \%{ }^{36}$, die restlichen $25 \%$ werden jedoch i.d.R. als Schreddermüll auf Deponien verbracht (450.000 Tonnen in Deutschland im Jahr), obwohl es sich um weitestgehend weiterverwertbare Ressourcen wie Kunststoffe, Glas und Gummi handelt. ${ }^{37}$ Daraus kann abgeleitet werden, daß der Stellenwert der Gestaltung und Implementierung von Rücknahme- und Recyclingsystemen bei Herstellerunternehmen in der Praxis nicht die gleiche Bedeutung erlangt hat wie in der theoretischen Auseinandersetzung und in der Diskussion um das Kreislaufwirtschaftsgesetz. Eine empirische Untersuchung, die vom Institut für Marketing von November

34 Es fallen im Jahr in Deutschland ca. 1,5 Mio. $t$ gebrauchter elektrischer und elektronischer Geräte an. Davon sind ca. 600.000 t ausgediente Investitionsgüter und 900.000 t Geräte, die in privaten Haushalten anfallen. Die großen Haushaltsgeräte $(500.000 \mathrm{t})$ stellten $56 \%$, die Fernsehgeräte $17 \%(150.000 \mathrm{t})$ und die Geräte der Unterhaltungselektronik $11 \%(100.000 \mathrm{t})$ der ausgedienten Gebrauchsgüter. Die kleinen Haushaltsgeräte tragen $8 \%(72.500)$ zum Abfallaufkommen elektronischer Konsumgüter bei. Insbesondere Fernsehgeräte und Kleingeräte werden nach Ablauf ihrer regulären Gebrauchsdauer in den Haushalten noch relativ lange an anderer Stelle weitergenutzt oder aufbewahrt, bevor sie entsorgt werden. Daher sind die wirklich zurücklaufenden Mengen deutlich geringer als die durch Neuverkäufe in den Markt gebrachten Mengen. Vgl. Zentralverband Elektrotechnik- und Elektroindustrie e.V. (Hrsg.), Lösungskonzept der deutschen Elektroindustrie für die Verwertung und Entsorgung elektrotechnischer und elektronischer Geräte, ZVEI-Memorandum zum Entwurf einer „Elektronik-SchrottVerordnung “, Frankfurt am Main 1993, A-9.

35 Auch Produktionsabfälle - die nicht Gegenstand der vorliegenden Arbeit sind - , die an den Markt abgegeben werden (externe Verwertung), werden noch in großen Teilen entsorgt, obwohl sie noch verwertbar wären. Betrachtet man beispielsweise das Produktionsgütergewerbe und das Grundstoffgewerbe zusammen, welches - bezogen auf die Rückstandsmenge - der bedeutendste Teilbereich des verarbeitenden Gewerbes ist, fielen hier $1987 \mathrm{ca}$. 45,5 Millionen Tonnen Abfälle an, von denen jedoch nur $33,4 \%$ zur Verwertung abgegeben wurden. Insgesamt wurden im verarbeitenden Gewerbe $42,3 \%$ und im gesamten produzierenden Gewerbe $21,3 \%$ verwertet Vgl. Schwarz, E., Unternehmensnetzwerke im Recycling-Bereich, Wiesbaden 1994, S. $53 f$.

Die Recyclingquote wird definiert als der Anteil des recycelten Materials pro Jahr dividiert durch den Gesamtverbrauch im Jahr. Vgl. Stahlmann, V., Umweltorientierte Materialwirtschaft: das Optimierungskonzept für Ressourcen, Recycling und Rendite, Wiesbaden 1988, S. 143.

Stahlmann weist neben der Definition darauf hin, daß das Recyclingpotential je nach Branche und Art der Stoffumwandlung unterschiedlich groß ist. Geringe Recyclingquoten können durch eine weitreichende Kuppelproduktion bedingt sein, während hohe Schrott- und Abfallvolumina, die nur zu geringem Wert verkauft werden können oder hohe Entsorgungskosten verursachen, die Dringlichkeit des Recycling unterstreichen. Daher müssen weitere Kennzahlen wie der Schrottverkaufserlös oder Energiekennzahlen zur umfassenden Interpretation ergänzend herangezogen werden. Vgl. Stahlmann, V., Umweltorientierte Materialwirtschaft, a.a.O., S.143f.

${ }^{37}$ Vgl. Zimmermann, H., Automobilrecycling im Verbund, PREUSSAG-Konzept eines flächendeckenden Netzes von Kfz-Demontagezentren, in: UWF, Heft 4, 1995, S. 44. 
1995 bis Januar 1996 durchgeführt wurde, ${ }^{38}$ bestätigt diese Erkenntnis. ${ }^{39}$ Die im folgenden dargestellten Untersuchungsergebnisse geben Aufschluß über die Einschätzungen der Auswirkungen des Kreislaufwirtschaftsgesetzes auf mittelständische Unternehmen. ${ }^{40}$

Auf die Frage, wie gut sich die Unternehmen über das Kreislaufwirtschaftsgesetz informiert fühlen, gaben $59 \%$ an, daß sie gar nicht bis kaum über das Gesetz in Kenntnis gesetzt sind, wobei die Unternehmen bis 100 Mitarbeiter die größten Informationsdefizite aufweisen (bis zu $83 \%$ ). Knapp $80 \%$ der befragten Unternehmen haben sich daher kaum bis gar nicht mit dem Kreislaufwirtschaftsgesetz auseinandergesetzt (vgl. Abb. 1). Lediglich $20 \%$ setzen sich „ein wenig" bis „intensiv“ mit dem Kreislaufwirtschaftsgesetz auseinander und haben ein Rücknahme- und Recyclingsystem eingerichtet bzw. planen dieses. Die Antwortmöglichkeit der sehr intensiven Auseinandersetzung wurde sogar nie angegeben.

Die Ursachen der geringen Auseinandersetzung mit dem Kreislaufwirtschaftsgesetz können in den relativ günstigen Transport- und Entsorgungskosten sowie den relativ geringen Rohstoff- und Energiekosten gesehen werden. Diesen Kosten sind die relativ hohen Kosten für die Rücknahme und das Recycling von Produkten gegenüber zu stellen. ${ }^{41}$ Erst wenn steigende Entsorgungsgebühren sowie unsichere Entsorgungsmöglichkeiten einerseits und kostengünstigere Recyclingtechnologien andererseits die Verwertung von Rückständen (im Vergleich zur

38 Die Konzeption der empirischen Untersuchung und die Auswertung wurden von Dr. Manfred Kirchgeorg und der Verfasserin (beide Institut für Marketing in Münster) vorgenommen. Die Telefonate wurden mit speziell geschulten Interviewern durchgeführt. Die Auswahl der Unternehmen erfolgte zufallsgestützt. Dabei wurden diejenigen Führungskräfte und Mitarbeiter ermittelt, die sich im Unternehmensalltag mit dem Themenkomplex auseinandersetzen.

Im Rahmen der Untersuchung wurden Unternehmen mit einer Betriebsgröße von bis zu 500 Mitarbeitern aus den Branchen Möbelverarbeitung, Elektro- und Elektronikgeräte sowie Kunststoffverarbeitung und Metallbau ausgewählt. Knapp 60\% der Stichprobe bildeten Unternehmen mit weniger als 100 Mitarbeitern. Bei insgesamt 400 Telefonkontakten betrug die Beteiligungsquote ca. 55\%, womit 219 Interviews zur Auswertung vorlagen.

Teile der Ergebnisse der Telefonbefragung mittelständischer Unternehmen sind nachzulesen in: Meffert, H., Kirchgeorg, M., Giesen-Netzer, I., Die meisten mittelständischen Betriebe haben sich noch nicht auf die Veränderungen vorbereitet, in: Blick durch die Wirtschaft, 17.7.1996, S. 8 sowie Kirchgeorg, M., Giesen-Netzer, I., WSK-Infobrief, Institut für Marketing, Projektgruppe Wertschöpfungskreisläufe (Hrsg.), Ausgabe 2, 1995, S. $2 \mathrm{ff}$.

Die Ergebnisse erheben keinen Anspruch auf Repräsentativität, lassen jedoch grundsätzliche Tendenzaussagen $\mathrm{zu}$.

41 Vgl. u.a. Stahlmann, V., Umweltorientierte Materialwirtschaft, a.a.O., S. 186f.; Schwarz, E., Unternehmensnetzwerke im Recycling-Bereich, a.a.O., S. 80 und vgl. FREI-Fallstudie, a.a.O. 
Entsorgung) als "wirtschaftliche" Alternative erscheinen lassen, ${ }^{42}$ werden Unternehmen vermutlich stärker bestrebt sein, Rücknahme- und Recyclingsysteme einzurichten bzw. sich an einem solchen zu beteiligen. ${ }^{43}$ In den nächsten fünf Jahren wird mit einer Verdoppelung der durchschnittlichen Entsorgungskosten gerechnet ${ }^{44}$ welche die Einführung von Rücknahme- und Recyclingsystemen wahrscheinlich begünstigen wird. ${ }^{45}$ Als weitere Gründe für die derzeit überwiegende Entsorgung von Abfällen werden in der Literatur zudem mangelhafte betriebliche Organisation, unvollständige Informationen über Verwertungsalternativen ${ }^{46}$ sowie z.T. noch fehlende Technologien angeführt.

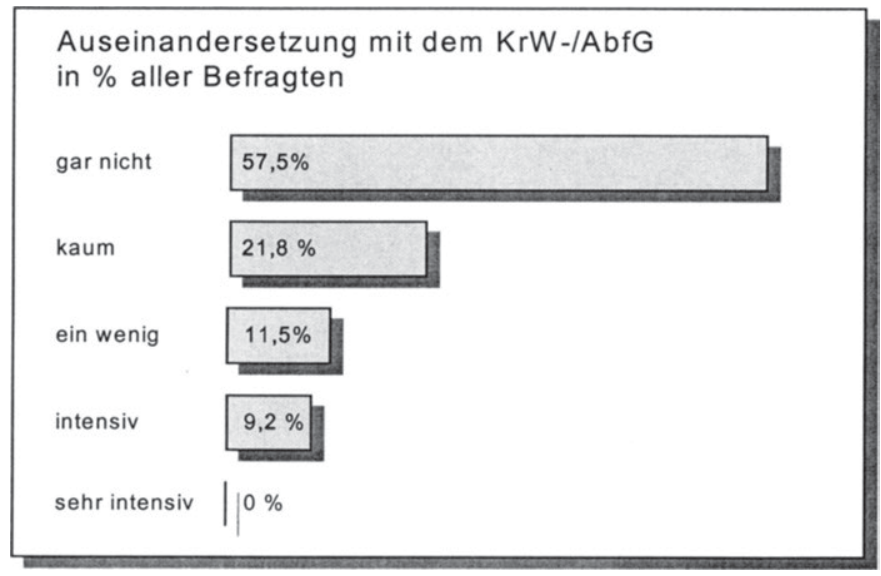

Abb. 1: Intensität der Auseinandersetzung mit dem Kreislaufwirtschaftsgesetz

$42 \quad$ An dieser Stelle wird der Bezug zum Kreislaufwirtschaftsgesetz deutlich, nach dem eine Abweichung von der Prioritätenrangfolge (Vermeidung vor Verwertung vor Entsorgung) zulässig ist, wenn dieses wirtschaftlich nicht zumutbar ist. Vgl. §5 Abs. $4 \mathrm{KrW}$ - /AbfG.

43 Im Zeitraum von 1975 bis 1992 erhöhten sich die Aufwendungen für Umweltmaßnahmen im produzierenden Gewerbe in Deutschland um etwa 250\%. Der Anteil der Entsorgungskosten an den Herstellkosten liegt im Durchschnitt aller Branchen bei etwa 3\%, wobei die Chemische Industrie und die Energieversorger den höchsten Anteil aufweisen. Der Anteil der Entsorgungskosten an den Herstellkosten in verschiedenen Branchen beträgt im einzelnen in Prozent: Energieversorgung: 7,9\%, Chemische Industrie: 5,5\%, Automobilindustrie: $3,0 \%, \mathrm{Ma}-$ schinenbau: 1,4\% und im Durchschnitt aller Branchen 2,9\%. Vgl. Voegele, A., Entsorgungslogistik: Von der logistischen Kette zum logistischen Kreis, in: Beschaffung aktuell, Heft 11, 1993, S. 40.

44 Vgl. Voegele, A., Entsorgungslogistik, a.a.O., S. 39.

45 Vgl. u.a. Stahlmann, V., Umweltorientierte Materialwirtschaft, a.a.O., S. $186 f$.

46 Einige Recycling-Informationssysteme und Handbücher über Recyclingverfahren werden jedoch bereits angeboten, um die Informationsdefizite auszugleichen. Vgl. z.B. die Abfallwirtschaftsdatenbank des Umweltbundesamtes in Berlin (AWIDAT) oder DORIS-DornierRecyclinginformationssystem sowie das Recycling-Handbuch des Umweltbundesamtes über Verfahren, Hersteller und Anwender. 
Im Ergebnis der empirischen Untersuchung des Instituts für Marketing werden ähnliche Barrieren für die Umsetzung von Rücknahme- und Recyclingsystemen genannt. Insbesondere werden Zeitprobleme, Informationsdefizite und die zu erwartenden Kosten- und Wettbewerbsnachteilen hervorgehoben (vgl. Abb. 2). ${ }^{47}$ Vor allem bei kleinen und mittleren Unternehmen reichen Zeit- und Personalkontingente kaum aus, um sich mit dieser Thematik auseinanderzusetzen. Größere Unternehmen verfügen hier über deutliche Vorteile, da diese zum Teil eigene Abteilungen für Umweltschutz- und Abfallaufgaben eingerichtet haben.

Akzeptanzprobleme beim Handel oder beim Kunden werden hingegen aus Herstellersicht weniger als Ursache dafür angeben, kein Rücknahme- und Recyclingsystem einrichten zu wollen. Die Erfahrungen mit der hohen Sammelbereitschaft der Bevölkerung bei Verpackungsabfällen im Rahmen der Einführung des Dualen Systems in Deutschland haben gezeigt, daß diesbezüglich kaum kundenbezogene Akzeptanzprobleme zu erwarten sind. Bei handelsbezogenen Akzeptanzproblemen sehen die Hersteller kaum Barrieren, da auch Vertreiber durch das Kreislaufwirtschaftsgesetz in die Pflicht genommen werden, Rücknahme- und Recyclingsysteme einzuführen. Darüber hinaus besteht die Möglichkeit für den Handel, diejenigen Kosten, die aus der Umsetzung der Systeme entstehen, zumindest teilweise auf den Kunden umzuwälzen. ${ }^{48}$

Demzufolge scheint es, daß sich die meisten mittelständischen Unternehmen des produzierenden Gewerbes noch nicht auf die Veränderungen vorbereitet haben, die sich durch die Anforderungen des Kreislaufwirtschaftsgesetzes ergeben. Daher ist eine Diskussion der Problemwahrnehmung bezüglich Rücknahmeund Recyclingsysteme erforderlich. Eine Auseinandersetzung mit den gesetzlichen Bestimmungen für die Herstellerunternehmen stellt zum einen mittelfristig eine Notwendigkeit dar, die spätestens mit Inkrafttreten der Rechtsverordnungen

47 Bei der Analyse der Ergebnisse dieser Studie ist hervorzuheben, daß sich die befragten Unternehmen überwiegend nicht mit dem Kreislaufwirtschaftsgesetz auseinandergesetzt haben und diese daher lediglich die Gründe nennen konnten, warum sie keine produktbezogenen Rücknahme- und Recyclingsysteme einrichten oder sich daran beteiligen wollen bzw. dieses in nächster Zeit nicht planen. Sie verfügen also überwiegend über keine eigenen Erfahrungen mit Rücknahme- und Recyclingsystemen.

48

Bei Haushaltsgeräten empfiehlt der Hersteller Bauknecht den Händlern explizit, die Kosten den Konsumenten in Rechnung zu stellen, da die Leistung im Rahmen der Rücknahme als zusätzlicher Service gegenüber dem Kunden und als Profilierungsmöglichkeit insbesondere bei den Fachhändlern eingestuft werden kann. Vgl. Fallstudie Bauknecht, a.a.O. 
in verbindliches Handeln umgesetzt werden muß. ${ }^{49}$ Zum anderen ermöglicht eine frühzeitige proaktive - d.h. vor Inkrafttreten der Verordnungen - Einbeziehung rechtlicher Bestimmungen in die Unternehmenspraxis die Ausnutzung eines eigenen Gestaltungsspielraums und damit eine ökonomisch und ökologisch sinnvolle Lösung. Diesen Zeitraum sollten Unternehmen im Sinne eines strategischen Fensters als Chance begreifen.

keine ausreichenden Zeitkontingente verfügbar

unzureichende Informationen über Verordnungsentwürfe

hohe Betriebskosten

unzureichende Informationen

über Altproduktmengen, die zurückzunehmen sind

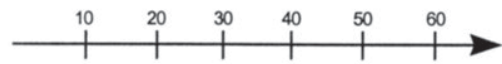

Wettbewerbsnachteile

(z.B. gegenüber Importprodukten)

Koordination komplexer

Strukturen und Systeme

fehlendes Kow-how im eigenen Unternehmen

unzureichende Informationen über Recyclingtechnologien

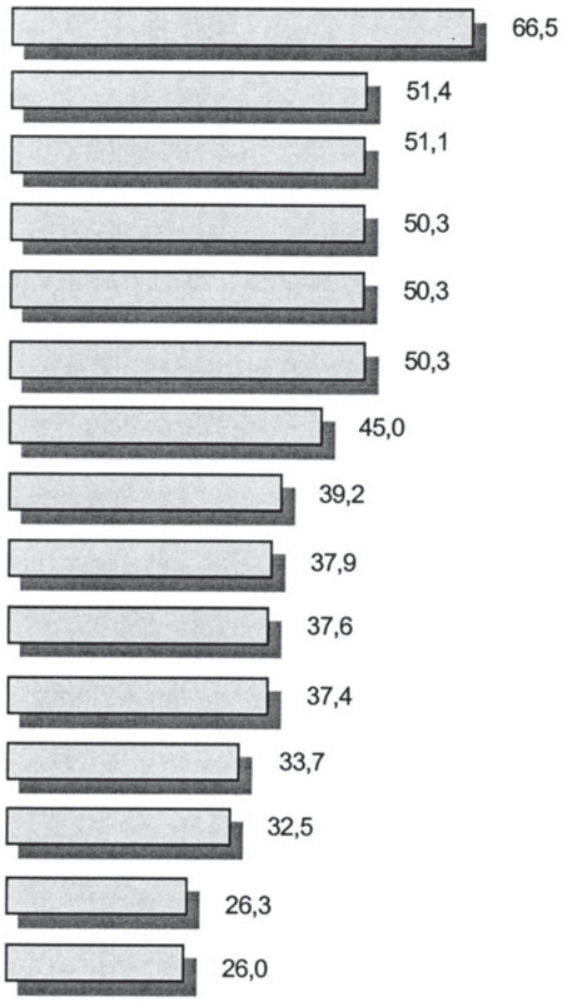

hohe Investitionen

Abhängigkeit von Systempartnem (z.B. Verwertungsunternehmen)

fehlende Unterstützung von seiten der Verbände, IHK u.a.

Akzeptanzprobleme beim Kunden

fehlende gesetzl. Vorgaben

durch Verordnungen

Probleme bei der Auswahl geeigneter Kooperationspartner

Akzeptanzprobleme beim Handel

26,0

Abb. 2: Wichtige Ablehnungsgründe für produktbezogene Rücknahme- und Recyclingsysteme

49 Die gesetzlichen Ansprüche sind zwar zum jetzigen Zeitpunkt noch nicht rechtsbindend, haben jedoch bereits deklaratorischen Charakter. 
Vor diesem Hintergrund erscheint es wichtig, Erfahrungen von Unternehmen, die Rücknahme- und Recyclingsysteme bereits realisiert haben bzw. gerade dabei sind, diese einzuführen, näher zu analysieren und Hinweise für diejenigen Unternehmen zu liefern, die sich bisher noch nicht intensiv mit dieser Thematik auseinandergesetzt haben. Angesichts der Komplexität des Untersuchungsgegenstandes befaßt sich die vorliegende Arbeit daher mit der Implementierung von Rücknahme- und Recyclingsystemen, d. h. dem Prozeß der Verwirklichung eines gedanklich formulierten Konzepts ${ }^{50}$ in unternehmerischem Handeln.

Die Implementierungsproblematik von Rücknahme- und Recyclingsystemen bei Herstellern langlebiger Gebrauchsgüter ist einerseits aufgrund des Prozesses der Implementierung, der als Voraussetzung für eine erfolgreiche Durchführung eines solches Systems betrachtet wird, von besonderer Bedeutung. ${ }^{51}$ Andererseits ist die intensive Auseinandersetzung mit der Implementierung von Rücknahme- und Recyclingsystemen sinnvoll, da in weiten Teilen in der betriebswirtschaftlichen Literatur die Diskussion der Implementierungsproblematik vernachlässigt wird. ${ }^{52}$ Es wird deutlich, „daß Umsetzungsprobleme schon seit über 30 Jahren die Diskussion bestimmen, jedoch bis heute nicht befriedigend gelöst werden konnten“. ${ }^{33}$ Die konkreten Implementierungsprobleme entstehen dabei insbesondere aus zwei Gründen. Die Implementierung von Rücknahme- und Recyclingsystemen erfolgt einerseits unternehmensindividuell, so daß keine allgemeingültigen "Erfolgsrezepte“ für die Implementierung gegeben werden können. Darüber hinaus varriieren die Implementierungsobjekte, d.h. der Gegenstand der Implementierung, was eine Übertragung von Erfahrungen erschwert. Damit ist bereits ein Teil der Diskussion der Implementierung und der Kennzeichnung des Implementierungsobjekts Rücknahme- und Recyclingsysteme vorgezeichnet.

50 Vgl. Kolks, U., Strategieimplementierung: ein anwenderorientiertes Konzept, Wiesbaden 1990, S. 77.

51 Vgl. Meffert, H., Marketing-Management: Analyse - Strategie - Implementierung, Wiesbaden 1994, S. $361 \mathrm{ff}$.

52 Maßstäbe setzen u.a. Ansoff, H. I., Implanting strategic Management, Englewood Cliffs u.a. 1984, Bonoma, T. V., Der Marketing-Vorsprung: wie man Marketingstrategien sofort erfolgreich in die Praxis umsetzt, Landsberg 1986, Kolks, U., Strategieimplementierung, a.a.O.; Hilker, J., Marketingimplementierung: Grundlagen und Umsetzung am Beispiel ostdeutscher Unternehmen, Wiesbaden 1993; Lehner, J. M., Implementierung von Strategien: Konzeption unter Berücksichtigung von Unsicherheit und Mehrdeutigkeit, Wiesbaden 1996.

53 Hilker, J., Marketingimplementierung, a.a.O., S. 2. Diese Aussage bezieht sich zwar nicht auf die Implementierung von Rücknahme- und Recyclingsystemen, sondern auf Marketing(strategien), gilt jedoch angesichts der relativ neuen Problematik hier um so mehr. 


\section{Rücknahme- und Recyclingsysteme als Objekt der Implementierung}

Betrachtet man die zur Implementierung vorgelegten Definitionsansätze (vgl. Tab. 2), kann festgestellt werden, lassen sich vier unterschiedliche Sichtweisen identifizieren (vgl. Abb. 3).

\begin{tabular}{|c|c|}
\hline Autoren & Definitionen von Implementierung \\
\hline Ansoff $1984^{3 x}$ & $\begin{array}{l}\text { "Implementation is the process of causing the firm to behave in accordance } \\
\text { with the purposes, guidelines and strategies." }\end{array}$ \\
\hline Bonoma $1986^{55}$ & $\begin{array}{l}\text { Implementierung als die Realisierung von Strategien } \\
\text { Marketing(strategie)implementierung als praktische Umsetzung des Marke- } \\
\text { ting in Aktionen, Programme, Systeme und Grundsatzbestimmungen }\end{array}$ \\
\hline Greenley $1987^{56}$ & $\begin{array}{l}\text { "Implementation is a transition from planning to actual "doing", requiring a } \\
\text { change from following a sequence of planning stages to executing a range } \\
\text { of activities" }\end{array}$ \\
\hline Meffert 1988 & $\begin{array}{l}\text { Implementierung (des strategischen Marketing) ist "die Umsetzung globaler } \\
\text { und mehrdimensionaler Denkkonzepte in konkretes praktisches Handeln." }\end{array}$ \\
\hline Clauss $1989^{58}$ & $\begin{array}{l}\text { „Implementierung sind die im Zusammenhang mit der Lösung eines kon- } \\
\text { kreten Problems stehenden Aktivitäten (Interaktionen, Handlungen), die eine } \\
\text { Ausweitung der Gruppe beteiligter Personen, ein Eingehen auf die Bedür- } \\
\text { nisse der jeweiligen Adressaten und eine Abstimmung dieser Bedürfnisse } \\
\text { mit den sachlichen Kriterien des Problemlösens beinhalten." }\end{array}$ \\
\hline Kolks $1990^{50}$ & $\begin{array}{l}\text { Implementierung ist die Umsetzung und Durchsetzung von Maßnahmen- } \\
\text { programmen. }\end{array}$ \\
\hline Kotler $1991^{80}$ & $\begin{array}{l}\text { Marketing implementation is the process that turns marketing plans into } \\
\text { action assignments and ensures that such assignments are executed in a } \\
\text { manner that accomplishes the plan's stated objectives. }\end{array}$ \\
\hline Lehner $1996^{64}$ & $\begin{array}{l}\text { "Strategieimplementierung ist die Suche nach Ressourcen, nach deren Ein- } \\
\text { satzbedingungen und der bedingte Einsatz dieser Ressourcen zur Errei- } \\
\text { chung der strategischen Ziele" }\end{array}$ \\
\hline
\end{tabular}

Tab. 2: Übersicht über ausgewählte Implementierungsdefinitionen

\footnotetext{
54 Ansoff, H. I., Implanting strategic Management, a.a.O., S. 260.

55 Vgl. Bonoma, T. V., Der Marketing-Vorsprung, a.a.O., S. 18 u. S. $44 f$.

56 Greenley, G. E., The Strategic and Operational Planning of Marketing, London u.a. 1986, S. 209.

57 Meffert, H., Anforderungen an die Strategie-Implementierung, in: Meffert, H. (Hrsg.), Strategische Unternehmensführung und Marketing, Wiesbaden 1988, S. 142.

58 Clauss, M., Die Strategie der Implementierung in der Unternehmung, Freiburg 1989, S. 19.

59 Vgl. Kolks, U., Strategieimplementierung, a.a.O., S. 79.

60 Kotler, P., Marketing-Management - Analysis, Planning, Implementation and Control, 7. Aufl., Englewood Cliffs 1991, S. 724. Auch in Kotler, P., Bliemel, F., Marketing-Management, Analyse, Planung, Umsetzung und Steuerung, 8. vollst. neu bearb. und erw. Aufl., Stuttgart 1995, S. 1143. In ähnlicher Weise definiert auch Meffert die Implementierung von Strategien. Vgl. Meffert, H., Marketing-Management, a.a.O., S. 363.
}

61 Lehner, J. M., Implementierung von Strategien, a.a.O., S. 79. 


\begin{tabular}{|c|c|c|c|}
\hline Implementierung als... & Autoren & Inhalt & Theoriefokus \\
\hline $\begin{array}{l}\text {...Gestaltung der } \\
\text { Organisation }\end{array}$ & $\begin{array}{l}\text { Chandler (1962) } \\
\text { Hrebiniak (1984) }\end{array}$ & $\begin{array}{l}\text { Zur Strategie muß die passen- } \\
\text { de Struktur gefunden werden; } \\
\text { „structure follows strategy“ } \\
\text { Einbeziehung von Rollen und } \\
\text { Belohnungssystemen }\end{array}$ & $\begin{array}{l}\text { Organisations- } \\
\text { theorie } \\
\text { Führungs- } \\
\text { konzepte }\end{array}$ \\
\hline $\begin{array}{l}\text {...Führung und } \\
\text { Kommunikation }\end{array}$ & $\begin{array}{l}\text { Kirsch/Esser/Gabele } \\
(1979) \\
\text { Ansoff (1984) } \\
\text { Clauss (1989) }\end{array}$ & $\begin{array}{l}\text { Gestaltungsmaßnahmen und } \\
\text { Pläne werden kommuniziert, } \\
\text { um Interessengruppen zur } \\
\text { Unterstützung des geplanten } \\
\text { Wandels zu bewegen }\end{array}$ & $\begin{array}{l}\text { Geplanter } \\
\text { organisatorischer } \\
\text { Wandel }\end{array}$ \\
\hline $\begin{array}{l}\text {...zunehmende } \\
\text { Detaillierung und } \\
\text { Operationalisierung }\end{array}$ & $\begin{array}{l}\text { Bonoma (1986) } \\
\text { Greenley (1987) } \\
\text { Meffert (1988) } \\
\text { Kolks }(1990) \\
\text { May (1995) } \\
\end{array}$ & $\begin{array}{l}\text { Allgemeine Zielsetzungen bzw. } \\
\text { Richtungsformulierungen ge- } \\
\text { langen über taktische Pläne zur } \\
\text { Umsetzung }\end{array}$ & $\begin{array}{l}\text { Führungs- } \\
\text { konzepte }\end{array}$ \\
\hline $\begin{array}{l}\text {...Prozeß zur } \\
\text { Zielerreichung }\end{array}$ & $\begin{array}{l}\text { Kotler }(1991) \\
\text { Hilker }(1993) \\
\text { Meffert }(1994) \\
\text { Lehner }(1996)^{62}\end{array}$ & $\begin{array}{l}\text { Vorhandene Pläne bzw. Kon- } \\
\text { zeptionen müssen durch kon- } \\
\text { kretes Handeln umgesetzt } \\
\text { (Unternehmenspotentiale) und } \\
\text { durchgesetzt (Individualebene) } \\
\text { werden }\end{array}$ & $\begin{array}{l}\text { Motivations- } \\
\text { psychologie } \\
\text { Organisations- } \\
\text { entwicklung }\end{array}$ \\
\hline
\end{tabular}

Abb. 3: Systematisierung von Implementierungsbegriffen und deren Zuordnung zu Forschungsrichtungen

Nach der ersten Sichtweise wird die Implementierung als Gestaltung von Organisationen verstanden, womit i.d.R. die Frage der Anpassung der Organisationsstruktur an die neu formulierte Strategie angesprochen wird. Chandlers These "structure follows strategy" ${ }^{43}$ ist dabei Gegenstand einer Vielzahl von Beiträgen zur Implementierungsproblematik geworden, die sich überwiegend mit der Eignung von klassischen Unternehmensstrukturen wie funktionaler oder objektorientierter Organisation sowie der Matrixorganisation für die entsprechende Strategie auseinandersetzt. ${ }^{64}$ Diese Betrachtung schließt z.T. auch die Definition von Rollen der Führenden und Geführten sowie die Konstruktion von Belohnungssystemen ein. ${ }^{65}$ Theoretische Grundlage dieses Begriffsverständnisses bilden zum einen die Organisationstheorie und zum anderen Führungskonzepte.

62 Der Prozeßbezug wird aus der vorgelegten Definition von Lehner zwar nicht deutlich, im weiteren Verlauf der Ausführungen geht er jedoch explizit darauf ein. Vgl. z.B. Lehner, J. M., Implementierung von Strategien, a.a.O., S. 30.

63 Vgl. Chandler, A. D., Strategy and Structure, Cambridge, Mass. 1962, S. 15.

64 Vgl. Meffert, H., Marketing-Management, a.a.O., S. 368.

65 Vgl. z.B. Hrebiniak, L. G., Joyce, W. F., Implementing Strategy, New York 1984, S. $187 f$. 
Zum zweiten wird Implementierung als Problem der Führung und Kommunikation aufgefaßt. Demgemäß müssen Pläne und Gestaltungsmaßnahmen kommuniziert werden, um Interessengruppen zur Unterstützung des Wandels zu bewegen. ${ }^{66}$ Dieses wird insbesondere bei den Ansätzen des geplanten Wandels diskutiert, bei denen die Individualebene sehr deutlich in den Fokus der Betrachtung gerückt wird. Wird die Implementierung in einer dritten Sichtweise als zunehmende Detaillierung und Operationalisierung verstanden, ist die Frage angesprochen, wie allgemeine Zielsetzungen und Richtungsformulierungen über taktische Pläne umgesetzt werden können. Diesem Gedanken liegt die Grundvorstellung der deduktiven Ableitbarkeit von Mikroschritten aus einer übergeordneten Vorgabe zugrunde. ${ }^{67}$ Gefolgt wird dem entscheidungsorientierten Ansatz, in dessen Denkweise von einer allgemeinen Zielsetzung und Richtungsformulierung Strategien abgeleitet werden, die dann über taktische Maßnahmen zur Umsetzung gelangen. Da dieser Prozeß zumeist entlang der Unternehmenshierarchie verläuft, handelt es sich i.d.R. um einen „Top-to-down-Ansatz", nach dem die Detailplanung an untergeordneten Ebenen geschieht. ${ }^{68}$ Zugrunde liegen wiederum Führungskonzepte.

Schließlich wird die Implementierung als Prozeß zur Zielerreichung aufgefaßt, wobei vorliegende Pläne bzw. Konzeptionen in die Unternehmensstruktur, systeme und -kultur umgesetzt sowie auf der Individualebene durchgesetzt werden müssen. Insbesondere werden hier Erkenntnisse der Organisationsentwicklung (Unternehmensebene) und Motivationspsychologie (Individualebene) angesprochen.

Die Organisationsentwicklung ${ }^{69}$ beinhaltet Fragen nach dem Management des Wandels. Daher ist die Implementierung auch im Zusammenhang von Forschun-

\footnotetext{
66

Vgl. Ansoff, H. I., Implanting strategic Management, a.a.O., S. 241.

Vgl. Lehner, J. M., Implementierung von Strategien, a.a.O., S. 25.

68

Bonoma spricht von einem „Wasserfallphänomen“, nach dem die Strategie und die Implementierung in der Unternehmenshierarchie von oben nach unten geformt und weitergegeben werden, und verwendet einen Springbrunnen in einem japanischen Garten als Alegorie, bei dem das Wasser vom höheren in das niedrigere Auffangbecken fließt. Vgl. Bonoma, T. V., Der Marketing-Vorsprung, a.a.O., S. $25 f$. on" bzw. "Organisatorisches Lernen" diskutiert. Bereits Studien der frühen verhaltenswissenschaftlichen Entscheidungstheorie (menschliches Entscheidungsverhalten) liegt die Annahme zugrunde, daß sich Organisationen adaptiv rational verhalten, indem sie schrittweise aus Erfahrungen lernen. Damit wird ein evolutorischer Fortschritts- und Verbesserungsprozeß durch organisatorisches Lernen unterstellt. Vgl. Kieser, A., Kubicek, H., Organisationstheorien I,
} 
gen über den geplanten Wandel zu analysieren, so daß sich Überschneidungen zu der zweiten Sichtweise ergeben. ${ }^{70}$ Der geplante Wandel wird als Reorganisation von Unternehmen aufgefaßt, die tiefgreifende Veränderungen mit sich bringt. ${ }^{71}$ Dabei findet ein Rückgriff auf die bewußte Willensbildung und Steuerungsaktivitäten der beteiligten Akteure statt, welche die Aufmerksamkeit auf das Phänomen der Führung lenken. ${ }^{72}$ Neben diesen Fragen stehen auch Fragen der Handhabung von Konflikten und Anpassungswiderständen im Rahmen von Implementierungsaktivitäten im Fokus der Betrachtungen. ${ }^{73}$ Daher wird bei der Diskussion um Implementierungsverhalten auf Erkenntnisse der Führung ${ }^{74}$ und Motivation $^{75}$ von In-

Wissenschaftstheoretische Anforderungen und kritische Analyse klassischer Ansätze, in: Bolte, K. M., u.a. (Hrsg.), Sozioökonomie, Band 514/l, Stuttgart u.a. 1978, S. 143.

Durch das organisatorische Lernen, dessen Begrifflichkeit insbesondere in den 80er Jahren geprägt wurde, sollen die Unternehmen Fortschrittsfähigkeit und die Fähigkeit zur „Selbsttransfusion“ sicherstellen. Dabei werden drei Typen des organisatorischen Lernens unterschieden: Organisationstransformation, Organisationsentwicklung und Organisationsänderung. Vgl. Sattelberger, T., Die lernende Organisation im Spannungsfeld von Strategie, Struktur und Kultur, in: Die lernende Organisation: Konzepte für eine neue Qualität der Unternehmensentwicklung, Sattelberger, T. (Hrsg.), 3. Aufl., Wiesbaden 1996, S. $14 f f$.

Zu gleichen Ergebnissen kommen auch Hilker und Clauss. Vgl. Hilker, J., Marketingimplementierung, a.a.O., S. 10 und vgl. Clauss, M., Die Strategie der Implementierung in der Unternehmung, a.a.O., S. 200ff.

Vgl. Kirsch, W., Esser, W. M., Gabele, E., Das Management des geplanten Wandels von Organisationen, Stuttgart 1974, S. 3.

Hier liegt ein Wandel 2. Ordnung vor, der im Gegensatz zum Wandel 1. Ordnung lediglich eine inkrementale Modifikation der Arbeitsweise einer Organisation beinhaltet. Vgl. zur genaueren Darstellung Staehle, W. H., Management, a.a.O., S. 849ff.

Vgl. Kirsch, W., Esser, W. M., Gabele, E., Das Management des geplanten Wandels von Organisationen, a.a.O., S. $20 \mathrm{ff}$.

73 Vgl. Hilker, J., Marketingimplementierung, a.a.O., S. 10 und vgl. Kirsch, W., Esser, W. M., Gabele, E., Das Management des geplanten Wandels von Organisationen, a.a.O., S. 18f.

„Führung ist eine personenbezogene Handlung, bei der einzelne Personen oder Personenmehrheiten (Führende) auf andere Personen (Geführte) einwirken, um diese zu einem zielentsprechenden Handeln zu veranlassen." Heinen, E., Betriebswirtschaftliche Führungslehre, ein entscheidungsorientierter Ansatz, in: Heinen, E. (Hrsg.), Entscheidungsorientierte betriebswirtschaftliche Studien, Bd. 2, Wiesbaden 1978, S. 31. Diesem verhaltensbezogenen Führungsbegriff, den Heinen in Anlehnung an die angelsächsische Organisationstheorie begründet hat, wird ein managementorientierter Führungsbegriff gegenübergestellt. Der verhaltensbezogene Begriff wird als personenbezogene Handlung auf andere Personen erfaßt, um diese zu einem zielentsprechenden Handeln zu veranlassen, während der managementorientierte Begriff Planung, Organisation, Durchsetzung und Kontrolle der betrieblichen Leistungsprozesse in den Mittelpunkt der Betrachtung stellt.

75 Motivationstheorien beschreiben Motivation als Voraussetzung für zielorientiertes Verhalten und gelten deshalb aus Managementperspektive als Hauptansatzpunkt für leistungssteigernde Beeinflussungsmöglichkeiten. Vgl. Staehle, W. H., Management, a.a.O., S. 204.

Führung wird als Verhalten beschrieben, welches Verhalten anderer verändert. Dabei finden neben der Beeinflussung von Einstellungen und Verhalten von Einzelpersonen, Interaktionen in und zwischen Gruppen statt, um bestimmte Ziele zu erreichen. Vgl. ebenda, S. 308. 
dividuen zurückgegriffen, d.h. auf die Organisationspsychologie und damit auf das Verhalten von Individuen und Gruppen. ${ }^{76}$

Zur Systematisierung der Implementierung können fünf Bezugsebenen herangezogen werden: der Akteurbezug, der Objektbezug, der Ebenen- und Prozeßbezug sowie der Wirkungsbezug. Den Akteurbezug greifen alle vorgelegten Definitionen nicht explizit auf, sondern gehen aufgrund ihres Objektbezugs davon aus, daß Akteure des produzierenden Gewerbes, d.h. Herstellerunternehmen, bereits entwickelte Verhaltenspläne zu implementieren haben. Im Rahmen dieser Arbeit sollen demzufolge die Unternehmensmitglieder von Herstellerunternehmen genauer untersucht werden und in Anlehnung an die Erkenntnisse der Promotorenforschung" explizit bei der Implementierung von Rücknahme- und Recyclingsystemen in die Betrachtung aufgenommen werden. ${ }^{78}$

Beim Objektbezug besteht Einigkeit darüber, daß die Implementierung sich auf umfassende Änderungen bezieht, die durch ein bewußtes Handel ${ }^{79}$ herbeigeführt werden sollen. Umfassende Änderungen betreffen in diesem Zusammenhang für die Unternehmen Entscheidungen, die umfassend neue Denkstrukturen und Handlungsoptionen zur Folge haben - z.B. bezüglich der strategischen Ausrichtung des Unternehmens ${ }^{80}$ oder bedeutender Problemlösungen. ${ }^{81}$ Im folgenden wird die Grundthese vertreten, daß die Integration von Rücknahme- und Recyclingsystemen umfassende Änderungen im Unternehmen mit sich bringt, da die Rücknahme und das Recycling von Altprodukten unternehmens- und funktionsübergreifende Aktivitäten erfordert, um das Kreislaufprinzip anwenden zu können. Ob diese umfassenden Änderungen im Sinne von Ad-hoc-Entscheidungen

76 Zur exakten begrifflichen Analyse des Begriffs der Führung vgl. Meurer, J., Führung von Franchisesystemen: Erklärungsansätze, Verhaltens- und Erfolgswirkungen auf Grundlage einer empirischen Führungstypologie, Wiesbaden 1997, S. $19 \mathrm{ff}$. Vgl. z.B. Witte, E., Organisation für Innovationsentscheidungen, Göttingen 1973.

$78 \mathrm{Vgl}$. insbesondere die Hierarchiedimension der Implementierung in Kapitel B 2.3.

79 Vgl. Clauss, M., Die Strategie der Implementierung in der Unternehmung, a.a.O., S. 4. Bei der Theorie des geplanten Wandels findet ebenfalls „ein Rückgriff auf die bewußten Willensbildungs- und Steuerungsaktivitäten der beteiligten Aktoren" statt. Vgl. Kirsch, W., Esser, W. M., Gabele, E., Das Management des geplanten Wandels von Organisationen, Stuttgart 1974, S. 20. mentierung von Strategien, a.a.O., S 7ff., Meffert, H., Marketing-Management, a.a.O., S. $24 \mathrm{ff}$. 
oder im Sinne von geplanten und konzeptionierten Plänen durchgeführt werden, soll dabei keine Rolle spielen. ${ }^{82}$

Aufgrund der - im Sinne des deskriptiven Strategiebegriffs verstandenen - strategischen Bedeutung von Rücknahme- und Recyclingsystemen ist es besonders wichtig, das Implementierungsobjekt "Rücknahme- und Recyclingsysteme“ implementierungsgerecht zu erfassen. ${ }^{83}$ Dieses beinhaltet eine einfache, klare und knappe Formulierung der konzeptionellen Idee, damit sie von allen Mitarbeitern verstanden und akzeptiert wird, ${ }^{84} \mathrm{~d}$. h. die Konzeption muß logisch und ausführbar sein. ${ }^{85}$ Diesem Vorgehen muß die Definition des Implementierungsobjekts „Rücknahme- und Recyclingsystem“ vorausgehen.

Die Tätigkeit der „Rücknahme“ deutet auf die (Selbst-)Verpflichtung der Akteure hier Hersteller - hin, die Altprodukte wieder in ihren Verfügungsbereich zurückzuführen. Die Rücknahme umfaßt neben Holsystemen der Hersteller, bei denen die Altprodukte bei Kunden abgeholt werden, auch Bringsysteme, die die Rückgabe durch den Kunden an den Hersteller oder den Handel beinhalten. Der Begriff „Verfügungsbereich“ des Herstellers bedeutet, daß die Hersteller Kenntnis darüber haben, welchen Weg die Produkte im logistischen System nehmen und

82 Zur Übertragung der Erkenntnisse der Strategieimplementierung auf die Implementierung von Rücknahme- und Recyclingsystemen wird der deskriptive Strategiebegriff zugrunde gelegt, der Strategien insbesondere ausgehend von den Wirkungen der Handlungen definiert. D.h. unabhängig davon, ob die Hersteller selbst inre Entscheidungen als strategisch interpretieren und behandeln, wird bei Vorliegen bestimmter Muster von Entscheidungen von einer Strategie gesprochen. Damit wird unter einer Strategie nicht nur ein Verhaltensplan, sondern darüber hinaus eine „retrospektives Konstrukt" verstanden, welches einen signifikanten Einfluß auf die Erfolgsposition und die dahinterstehenden Unternehmensziele hat. Vgl. Schreyögg, G., Unternehmensstrategie, Grundfragen einer Theorie strategischer Unternehmensführung, Berlin, New York 1984, S. 148.

Vgl. zur weiteren Diskussion des deskriptiven Strategiebegriffs: Mintzberg, H., Patterns in strategy Formation, in: Management Science, Nr. 9, S. 934ff.; Mintzberg, H., McHugh, A., Strategy Formation in Adhocracy, in: Administrative Science Quarterly, June 1985, S. $160 \mathrm{ff}$. und Mintzberg, H., Strategie als Handwerk, in: Strategie, die brillianten Beiträge der weltbesten Experten, Montgomery, C., Porter, M. E. (Hrsg.), Wien 1996, S. 459ff.

Hrebiniak und Joyce verlangen für die Implementierung nachvollziehbares, beeinflußbares, situativ anpassungsfähiges und rationelles Vorgehen, um unnötigen Widerstand zu vermeiden. Auch sollte die Komplexität gering gehalten werden, damit - aufgrund der begrenzten Auffassungsgabe von Entscheidungsträgern - keine Überforderung stattfindet. Vgl. Hrebiniak, L. G., Joyce, W. F., Implementing Strategy, a.a.O., S. 5ff.

Vgl. Levitt, T., Marketing Imagination. Die unbegrenzte Macht des kreativen Marketing, Landsberg/Lech 1984, S. 166. 
schließt damit die Beauftragung Dritter mit der Rücknahme, z.B. Verwerter oder Kommunen, ein.

Der Recyclingbegriff ${ }^{\text {b }}$ hat in der Literatur bisher noch keine einheitliche Abgrenzung erfahren und wird mit vielfältigen Inhalten genutzt. Tabelle 3 zeigt in Anlehnung an Kirchgeorg ${ }^{87}$ verschiedene Abgrenzungsebenen des Recycling, die Ausprägungen und die dazu entstanden Begrifflichkeiten. ${ }^{88}$

\begin{tabular}{|c|c|c|c|}
\hline Abgrenzung & Ausprägungen & \multicolumn{2}{|c|}{ Recyclingbegriff } \\
\hline $\begin{array}{l}\text { nach dem } \\
\text { Objekt }\end{array}$ & $\begin{array}{ll}\begin{array}{l}\text { Produktions- } \\
\text { abfälle }\end{array} & \begin{array}{l}\text { Konsum- } \\
\text { abfälle }\end{array} \\
\end{array}$ & $\begin{array}{l}\text { Mikro- } \\
\text { recycling }\end{array}$ & $\begin{array}{r}\text { Makro- } \\
\text { recycling }\end{array}$ \\
\hline $\begin{array}{l}\text { nach der Anzahl der } \\
\text { Institutionen }\end{array}$ & $\begin{array}{l}\text { ein } \\
\text { Unternehmen }\end{array} \begin{array}{r}\text { mehrere } \\
\text { Unternehmen }\end{array}$ & $\begin{array}{l}\text { einstufiges } \\
\text { internes } \\
\text { Recycling }\end{array}$ & $\begin{array}{r}\leftrightarrow \text { mehrstufiges } \\
\text { externes } \\
\text { Recycling }\end{array}$ \\
\hline $\begin{array}{c}\text { nach eingesetzten } \\
\text { Recyclingtechnologien }\end{array}$ & $\begin{array}{lr}\text { spezielle } \leftrightarrow & \begin{array}{r}\text { keine } \\
\text { Technologie }\end{array} \\
\text { spezielle } \\
\text { Technologie }\end{array}$ & $\begin{array}{l}\text { indirektes } \\
\text { Material- } \\
\text { Recycling } \\
\text { Verwertung }\end{array}$ & $\leftrightarrow \begin{array}{r}\text { direktes } \\
\text { Produkt- } \\
\text { Recycling } \\
\text { Verwendung }\end{array}$ \\
\hline $\begin{array}{l}\text { nach dem } \\
\text { Einsatzort }\end{array}$ & $\begin{array}{l}\text { gleicher } \quad \leftrightarrow \text { verschiedener } \\
\text { Einsatzort } \quad \text { Einsatzort }\end{array}$ & $\begin{array}{l}\text { Primäres } \\
\text { Recycling } \\
\text { (Wieder-) }\end{array}$ & $\leftrightarrow \begin{array}{r}\text { Sekundäres } \\
\text { Recycling } \\
\text { (Weiter-) }\end{array}$ \\
\hline $\begin{array}{l}\text { nach Erhalt } \\
\text { der Stoffe }\end{array}$ & $\leftrightarrow \quad \begin{array}{r}\text { Stoff } \\
\text { vernichtend }\end{array}$ & $\begin{array}{l}\text { stoffliches } \\
\text { Recycling } \\
\text { Verwertung }\end{array}$ & $\begin{array}{r}\leftrightarrow \text { energetisches } \\
\text { Recycling } \\
\text { Verwertung }\end{array}$ \\
\hline
\end{tabular}

Tab. 3: Überblick über Arten des Recycling

Wird das Recycling nach dem Objekt abgegrenzt, wird gefragt, um welche Art von Abfall es sich handelt. Handelt es sich um Konsumabfall, wird das Recycling als

86 Zur Diskussion der Historie und der Definition von Recycling vgl. Rautenstrauch, C., Betriebliches Recycling, eine Literaturanalyse, in: ZfB-Ergänzungsheft 2, 1993. Eine wissenschaftliche Auseinandersetzung mit dem Thema wird auf das Jahr 1955 (insbesondere Riebel, P., Die Kuppelproduktion, Köln 1955) datiert. Dabei wurde die Einbeziehung von ${ }_{n}$ sekundären Rohstoffen" zunächst ausschließlich ökonomisch begründet, bevor in den 70er Jahren die ökologische Perspektive zunehmend in den Vordergrund trat.

${ }^{87}$ Vgl. Kirchgeorg, M., Unternehmensstrategische Gestaltungsprobleme von Stoffkreisläufen, a.a.O., Anhang 2 (Autorenspezifische Würdigung von Strategieansătzen zum Recycling) S. $11 \mathrm{ff}$.

Die betriebswirtschaftlichen Forschungsansätze zur Kreislaufwirtschaft werden detailliert beschrieben in: Kirchgeorg, M., Kreislaufwirtschaft, a.a.O., S. 8ff. Hier unterscheidet Kirchgeorg die sektorale Betrachtung der betriebswirtschaftlichen Problemstellung in der Abfallwirtschaft, Auseinandersetzungen im Rahmen der Behandlung genereller Konzepte zum Umweltmanagement, primär technische und produktionswirtschaftliche Beiträge zur Berücksichtigung des Recycling sowie absatzwirtschaftliche Auseinandersetzungen mit Recyclingkonzepten aus angebots- und nachfragerorientierter Sicht. 
Makrorecycling klassifiziert, geht es um Produktionsabfall, als Mikrorecycling. Diese Unterscheidung ist von Bedeutung, da konsumbezogene Abfälle häufig für Unternehmen mit größeren Problemen verbunden sind als produktionsinterne Abfälle. Diese treten insbesondere bei langlebigen Gebrauchsgütern auf, da dort neben Qualitäts-, Kollektions-, Vermischungs- und Kundenproblemen bei kurzlebigen Gütern zusätzlich ein Zeitproblem auftritt, welches die Problemlösung auf Jahre hinaus verzögert. ${ }^{89}$ Aus diesem Grunde sind die Erkenntnisse, die aus der betriebswirtschaftlichen Diskussion der Konzepte der Verwertung produktionsinterner Abfälle der 50er Jahre nur bedingt übertragbar. Ist am Recyclingprozeß nur ein Unternehmen beteiligt, wird in einstufiges, wenn mehrere Unternehmen beteiligt sind in mehrstufiges Recycling unterschieden (Unterscheidung nach der Anzahl beteiligter Institutionen).$^{90}$ Auch diese Unterscheidung ist für die Implementierung von Rücknahme- und Recyclingsystemen von hoher Relevanz, da mit zunehmender Anzahl von Unternehmen der Koordinationsaufwand steigt und die Komplexität der Aufgabe zunimmt.

Weitere Untergliederungen sind zum einen die Abgrenzung nach der eingesetzten Technologie, nach der direktes Recycling (Verwendung = ohne den Einsatz spezieller Recyclingtechnologie) und indirektes Recycling (Verwertung = mit dem Einsatz spezieller Recyclingtechnologie) getrennt wird. ${ }^{91}$ Diese Abgrenzung hat für die Werterhaltung der Altprodukt(teile) und den Investitionsbedarf bei der Implementierung der Rücknahme- und Recyclingsysteme Relevanz. Dahinter steht die Überlegung, daß spezielle Recyclingtechnologien zwar Investitionen in der Einführungsphase notwendig machen, aber eine höherwertige Verwertung oder Verwendung von Altproduktteilen ermöglichen und damit die Investition und die Betriebskosten des Recycling (über)kompensieren können. Zum anderen wird nach dem Einsatzort untergliedert, d.h. ob die recycelten Abfälle in denselben Produktions- bzw. Anwendungsbereich oder in einen neuen Prozeß zugeführt werden.

89 Besonderheiten langlebiger Gebrauchsgüter: 1. Abfall entsteht erst nach Jahren, 2. Abfall entsteht dezentral außerhalb des Unternehmens, 3. Abfall entsteht mit unbekannter Qualität, 4. Abfall entsteht in komplexer stofflicher Zusammensetzung, 5. Abfall entsteht beim Kunden. Vgl. Kirchgeorg, M., Giesen-Netzer, I., WSK-Infobrief, Institut für Marketing, Projektgruppe Wertschöpfungskreisläufe (Hrsg.), Ausgabe 4, 1996, S. 4 lich die unterschiedlichen Recyclingdefinitionen in der wissenschaftlichen Literatur. Vgl. Kleinaltenkamp, M., Recycling-Strategien, Berlin 1985, S. 49ff. und S. $71 \mathrm{ff}$. 114. 
Demzufolge werden primäres und sekundäres Recycling unterschieden. ${ }^{92}$ Dieses hat wiederum Implikationen für die Rückführung oder Vermarktung der entstehenden Recyclate. Aus der Kombination der Merkmale "Technologie" und "Einsatzort" ergeben sich verschiedene Recyclingarten: Wiederverwendung, Weiterverwendung, Wiederverwertung und Weiterverwertung. ${ }^{93}$

Schließlich kann nach stofflichem und energetischem Recycling unterschieden werden. Beim stofflichen Recycling bleibt das Material bestehen, während das energetische Recycling die Verbrennung von Abfällen mit dem primären Zweck der Energiegewinnung beinhaltet. ${ }^{94} \mathrm{Da}$ die energetische Verwertung jedoch keine werterhaltende Maßnahme darstellt und diese Verwertungsalternative insbesondere bei kurzlebigen Gütern angewendet wird, wird diese Recyclingart im folgenden nicht weiter untersucht.

Im Rahmen dieser Arbeit ist der Recyclingbegriff in einem generischen Sinne für alle stofflichen Rückkoppelungsprozesse anzuwenden. Daher wird Recycling als die Rückführung von stofflichen Altprodukten oder Rückständen in Produktions- bzw. Konsumprozesse verstanden. ${ }^{95}$

92 Jäger stellt jedoch aufgrund preistheoretischer Überlegungen fest, daß Recycling unter den Annahmen der vollständigen Konkurrenz nicht unbedingt zu Einsparungen sondern zu vermehrten Abbau von Ressourcen führen kann. Daher fordert er, daß ein verstärktes Recycling stets mit Maßnahmen zu koppeln ist, die eine Reduzierung der Abbaukosten der in das Recycling eingehenden Primärressourcen bewirken. Vgl. Jaeger, K., Eine ökonomische Theorie des Recycling, in: Kyklos, Heft 29, 1975, S. $660 f f$.

93 Für die Wiederverwendung, bei der ohne den Einsatz einer speziellen Technologie das Altprodukt oder Teile davon in den gleichen Produktions- oder Anwendungsbereich wieder eingesetzt werden, ist der Markt für Gebrauchtcomputer als Beispiel zu nennen. Dort wird der Computer - nach einer genauen Qualitäts- und Funktionskontrolle durch das Werk - am Markt wieder angeboten. Bei der Weiterverwendung werden beispielsweise Computerchips ausgebaut und der Spielwarenindustrie angeboten, die diese in einer Form einsetzt, die nicht so hohe Qualitätsanforderungen stellt wie der primäre Einsatzzweck (z.B. Sprechpuppen o.ä.). Die Wiederverwertung kann in der Form erfolgen, daß das entstehende Kunststoffrecyclat wieder in den Produktionsprozeß integriert wird und daraus neue Computergehäuse entstehen. Die Verhüttung von Metallen schließlich ist als Beispiel für die Weiterverwertung anzuführen, da die Hütten die neuen Metalle unterschiedlichen Abnehmer verkaufen. Vgl. Fallstudie SiemensNixdorf, a.a.O., die alle Arten des Recycling durchführen.

Eine energetische Verwertung nach dem KrW-/AbfG liegt vor, "wenn 1. der Heizwert des einzelnen Abfalls, ohne Vermischung mit anderen Stoffen, mindestens $11.000 \mathrm{kj} / \mathrm{kg}$ beträgt, 2. ein Feuerwirkungsgrad von mindestens $75 \%$ erzielt wird, 3 . entstehende Wärme selbst genutzt oder an Dritte abgegeben wird und 4. die im Rahmen der Verwertung anfallenden weiteren Abfälle möglichst ohne weitere Behandlung abgelagert werden können." Vgl. §6 Abs. $2 \mathrm{KrW}$ IAbfG. Zusätzlich hat sich der Begriff der thermischen Verwertung gebildet, der jedoch lediglich die Verbrennung von Abfall und damit die Beseitigung anstrebt. Daher wird im Rahmen dieser Arbeit in dem Fall nicht von thermischer Verwertung, sondern von Verbrennung gesprochen.

95 In der neueren Literatur ist eine Tendenz festzustellen, den Recyclingbegriff in dieser weiten Form zu fassen. Vgl. z.B. Schwarz. E. J., Unternehmensnetzwerke im Recycling-Bereich, Irene Giesen-Netzer and Üniversität Münster - 978-3-631-75058-2 
Der Systembestandteil des Implementierungsobjektes „Rücknahme- und Recyclingsysteme" steht schließlich im engen Zusammenhang mit dem Recycling. Der aus dem Griechischen stammenden Begriff des „Recycling“ deutet bereits auf die Rückführung („Re-“) von Objekten in Kreisläufe (,Kyklos“=Kreis) hin. Ökologiebezogenes Ziel solcher Rückführungskreisläufe ist die Entlastung der ökologischen Umwelt, um die Funktionen derselben aufrechtzuerhalten. Diese Funktionen können - aus einer systemtheoretischen Betrachtungsweise ${ }^{96}$ heraus - anhand eines dynamischen Regelkreismodells "Circular Economy" dargestellt werden, bei dem ein ökonomisches System (Produktionssektor, Konsumbereich, Recycling) und ein ökologisches System (erneuerbare und nicht erneuerbare Ressourcen) im Mittelpunkt der Betrachtung stehen. ${ }^{97}$ Das ökonomische System stellt über die Produktion den Konsum sicher und ist für den Reststoffanfall und die Nutzung der Ressourcen verantwortlich. Das ökologische System gewährleistet über die natürlichen Ressourcen die Versorgung und Produktion (Versorgungs- und Produktionsfunktion) und übernimmt mit der Aufnahme des Abfalls die Trägerfunktion. Um diese Funktionen wahrzunehmen muß das ökologische System gewisse Anforderungen erfüllen: Die Abbaurate der Ressourcen darf die Regenerationsrate und die Reststoffaufnahme darf die Assimilationskraft nicht übersteigen. Gleich-

a.a.O., S. 18 und Stahlmann, V., Umweltorientierte Materialwirtschaft, a.a.O, S. 198. Diesem Vorgehen soll auch im Rahmen der vorliegenden Arbeit gefolgt werden.

Die Unternehmen werden in der Systemtheorie als offene, zielgerichtete sozio-technische Güter-Umsatz-Systeme gekennzeichnet, wobei die Offenheit eines Unternehmens die Umweltverbundenheit zum Ausdruck bringt, die sich - neben Marktbeziehungen - auch auf die ökologische Umwelt als Subsystem der Makroumwelt bezieht. Vgl. Meffert, H., Kirchgeorg, M., Marktorientiertes Umweltmanagement, a.a.O., S. 60.

97 Vgl. Pearce, D., Turner, R. K., Economics of Natural Resources and the Environment, New York, Harvester Wheatsheaf 1990, S. 40ff. und vgl. Barbier, E. B., The Concept of Sustainable Development, a.a.O., S. $188 \mathrm{ff}$.

Zur Berechnung von anderen ökonomischen Modellen von Rücknahmeverpflichtungen vgl. Clausen, H., Wacker, H., Rücknahmeverpflichtungen als intertemporäres Allokationsproblem, in: Jahrbuch für Wirtschaftswissenschaften, Bd. 48, Heft 1, 1998 (im Druck). Gesellschaftliche Zielvorstellungen werden in diesen Modellen den zentralen Planungsbehörden zugewiesen. Bei der Berechnung werden optimale Preispfade für die Primärressource und die Deponienutzung sowie für die in die Sekundärproduktion einzusetzenden Mengen ermittelt.

Clausen und Wacker zeigen auf, inwieweit direkte Unvollkommenheiten des Marktes durch Rücknahmeverpflichtungen (ergänzt durch andere Instrumente) korrigierbar sind: 1. Regelung der Verfügungsrechte (Rücknahmeverpflichtungen so ändern, daß die Kosten der Entsorgung und Verwertung neu definiert werden), 2. Schaffung "künstlicher Märkte" (Verpflichtung von Abfallnachfragern (öffentliche Entsorgungsträger) und Regelung des Zutritts von Anbietern), 3. Preisbildung nach dem Kostendeckungsprinzip im Entsorgungssektor auflösen. Vgl. Clausen, H., Wacker, H., Okonomische Modellierung von Rücknahmeverpflichtungen, in: Herbold, R., u.a. (Hrsg.), Unterwegs in die Kreislaufwirtschaft, interdisziplinäre Beiträge, IWT- Papier 16, Institut für Wissenschafts- und Technikforschung, Universität Bielefeld, 1997, S. 34ff. (zugleich Volkswirtschaftlicher Diskussionsbeitrag Nr. 241, Westfälische Wilhelms-Universität Münster), S. 10. 
zeitig wird als notwendig erachtet, eine Entlastung der ökologischen Funktionen durch die Gestaltung der Rücknahme- und Recyclingsysteme zu erzielen. Dabei sollen die aus den Produktions- und Konsumprozessen anfallenden Abfälle erneut als Inputfaktoren in dem ökonomischen System eingesetzt werden. ${ }^{98}$

Neben den ökologischen Zielsetzungen des Recycling untergliedern sich die ökonomische Zielsetzungen des Recycling in Mengen- und Wertziele. Dementsprechend soll durch das Recycling eine zumindest temporäre Verminderung der Rohstoffverbrauchsmengen ermöglicht werden. Die Wertziele beinhalten die Möglichkeit der Vermeidung/Verringerung von Entsorgungskosten und die Nutzung von zusätzlichen Erlösmöglichkeiten (Verkauf von Altprodukt(teilen) oder/und Recyclingprodukten) sowie Einsparungen, die durch den Wiedereinsatz von recyclierten Produkt(teilen) erreicht werden können. ${ }^{99}$ Vor dem Hintergrund der analysierten Begriffsbestandteile ist das Implementierungsobjekt

„Rücknahme- und Recyclingsysteme“ als von (Hersteller-) Unternehmen einzurichtender (produktbezogener) Stoffkreislauf zu definieren, der die Rückführung von Altprodukten (bzw. Rückständen) in den Produktions- bzw. Konsumprozeß zum Inhalt hat und der verschiedene Gestaltungsoptionen hinsichtlich der Recyclingarten und der institutionellen Ausgestaltung zur Erfüllung der ökonomischen und ökologischen Ziele offenläßt.

Nachdem der Objektbezug der Implementierung als hinreichend erläutert angesehen werden kann, gilt es, die Ebenen der Implementierung von Rücknahme- und Recyclingsystemen zu systematisieren. Der Ebenenbezug umfaßt die "Adressaten" der Implementierung, die zum einen Objekte und zum anderen Subjekte sein können. Einige Autoren greifen explizit die Veränderungen in der Unternehmensstruktur und den -systemen auf (Objekte), ${ }^{100}$ andere hingegen be-

98 Vgl. Kirchgeorg, M., Unternehmensstrategische Gestaltungsprobleme von Stoffkreisläufen, a.a.O., S. 140ff.

Vgl. Rautenstrauch, C., Betriebliches Recycling, a.a.O., S. 91. Rautenstrauch weist darauf hin, daß vor dem Recycling eine Gegenüberstellung von Recyclingkosten (Aufbereitung. Transaktion, Planung) und -erlösen (Verkauf der entstehenden Recyclinggüter) stattfinden muß. Dabei können auch umweltschutzinduzierte Mindererlöse auftreten.

Rautenstrauch beschreibt weiterhin ein ökonomisches Zeitziel, nach dem die Verweildauer einzelner Recyclinggüter erhöht werden soll, um beschaffungsbedingte Unsicherheiten zu absorbieren. Dieses ist m.E. nicht sinnvoll, da durch eine Erhöhung der Verweildauer von Recyclinggütern im Unternehmen eine zusätzliche Lagerhaltung aufgebaut werden müßte, die mit zusätzlichen Kosten verbunden wäre. Die Ausgleichung der Mengenströme der Recyclinggüter wäre demgegenüber ein ökonomisch sinnvolles Ziel zur Verringerung der beschaffungsbedingten Unsicherheit. Dieses spiegelt sich jedoch bereits im Mengenziel wider.

100 Vgl. Kirsch, W., Esser, W. M., Gabele, E., Das Management des geplanten Wandels von Organisationen, a.a.O. 
schäftigen sich überwiegend mit den betroffenen Individuen, die ihr Handeln entsprechend der neuen Verhaltenspläne anpassen sollen (Subjekte). ${ }^{101}$ Schließlich gibt es Autoren, die beide Ebenen - die Unternehmensebene (Unternehmensstruktur, -kultur und -systeme) und die Individualebene - trennen, um die Bedeutung beider Implementierungsnotwendigkeiten hervorzuheben. Dabei wird zum einen zwischen der Umsetzung auf der Unternehmensebene und der Durchsetzung auf der Individualebene unterschieden. ${ }^{102}$ Unter Umsetzung wird die Spezifizierung des globalen Verhaltensplans sowie die Anpassung von Unternehmensstruktur, -systemen und -kultur subsumiert. Unter Durchsetzung wird die Schaffung von Akzeptanz für die Konzeption bei den betroffenen Unternehmensmitgliedern verstanden. ${ }^{103}$

Die Durchsetzung auf der Individualebene wird häufig als Hauptproblem ${ }^{104}$ der Implementierung gesehen und kann in die Teilprobleme "Kennen und Verstehen“, „Können“ sowie „Wollen" untergliedert werden. ${ }^{105}$

- Kennen und Verstehen impliziert, daß die Betroffenen ${ }^{106}$ über den Inhalt und die Zielsetzung des Rücknahme- und Recyclingsystems in der Weise informiert sind, daß sie die Problemstellung erfassen können.

- Können heißt für die Betroffenen, daß sie zum einen aufgrund der eigenen Fähigkeiten und zum anderen aufgrund der ausreichenden organisatorischen Bedingungen allen Anforderungen, die mit der Implementierung des Rücknahme- und Recyclingsystems zusammenhängen, gerecht werden.

- Wollen spricht die Motivation der Betroffenen an, d.h. alle mit dem Rücknahme- und Recyclingsystem zusammenhängenden Aktivitäten werden mit einer bestimmten Intensität verfolgt und dieses wird über eine gewisse Zeitdauer hin aufrechterhalten. ${ }^{107}$

Der Unterscheidung in Unternehmens- und Individualebene soll im Rahmen dieser Arbeit gefolgt werden. Darüber hinaus muß diesem internen Fokus

\footnotetext{
101 Vgl. Clauss, M., Die Strategie der Implementierung in der Unternehmung, a.a.O.

102 Vgl. Meffert, H., Marketing-Management, a.a.O., S. 362f. und vgl. Kolks, U., Strategieimplementierung, a.a.O. und vgl. Hilker, J., Marketingimplementierung, a.a.O., S. 13.

103

104

Vgl. Meffert, H., Marketing-Management, a.a.O., S. 362.

Vgl. Kolks, U., Strategieimplementierung, a.a.O., S. 70,

Vgl. Hilker, J., Marketingimplementierung, a.a.O., S. 14ff.

Mit den „Betroffenen“ sind im Rahmen dieser Arbeit diejenigen Mitarbeiter angesprochen, die mit Aufgaben betraut werden, die mit der Implementierung von Rücknahme- und Recyclingsystemen zusammenhängen. Vgl. Kapitel B.2.4.2.

Vgl. zum genaueren Gegenstandsbereich der Motivationstheorie Frese, E., Organisationstheorie: Stand und Aussagen aus betriebswirtschaftlicher Sicht, Wiesbaden 1990, S. 51.
} 
(intraorganisational) aufgrund der besonderen Problematik der Implementierung von Rücknahme- und Recyclingsystemen eine dritte Betrachtungsebene hinzugefügt werden: die Netzwerkebene (interorganisational). ${ }^{108}$ Es wird von einem Netzwerk gesprochen, da externe, d.h. über die Grenzen des Herstellers hinaus bestehende Akteure dazu beitragen, die Funktionsfähigkeit eines Rücknahmeund Recyclingsystems sicherzustellen. Z.B. können Verwerter oder Wettbewerber, die als Systempartner eingebunden sind, Einfluß auf die Gestaltung und Implementierung des Rücknahme- und Recyclingsystems nehmen. ${ }^{109}$

Hinsichtlich des Prozeßbezugs der Implementierung wird deutlich, daß die Implementierung nicht zeitpunktbezogen ist, sondern als Abfolge von miteinander verknüpften Handlungen und Ereignissen verstanden wird. ${ }^{110}$ In der Literatur ist allerdings umstritten, wie die Abgrenzung des Implementierungsprozesses erfolgen soll, d.h. ob sich die Implementierung auf den gesamten Änderungsprozeß von der Initiierung bis hin zur Kontrolle bezieht oder vielmehr nur auf eine Phase

108 Short und Venkatraman gehen so weit, daß das Neugestalten der Netzwerke eines Unternehmens, welches die Rolle der Unternehmen und ihre Schlüsselprozesse in einen größeren Zusammenhang stellt, die größere strategische Bedeutung hat, als die internen Schlüsselgrößen (Effizienz und Effektivität) zu verbessern. Dieses verdeutlichen die Autoren an der Fallstudie Baxter's ASPS system. Vgl. Short, J. E., Beyond Business Redesign, Redefining Buisiness Network, in: Sloan Management Review, Nr. 4, 1992, S. 7ff. (Zum Begriff des Netzwerkes vgl. Kapitel B 1.1.1.)

Auch ist hier an die hohe Bedeutung des Konsumenten zu denken, der durch sein Nutzungsund Entsorgungsverhalten maßgeblich mitbestimmt, ob und in welchem Zustand ein Altprodukt den Hersteller wieder erreicht. Der Begriff des „Prosumenten“ findet daher in diesem Zusammenhang Anwendung. Vgl. Kirchgeorg, M., Kreislaufwirtschaft - neue Herausforderungen an das Marketing, a.a.O., S. 20. Im Verlaufe der vorliegenden Arbeit werden jedoch nur Systempartner i.e.S. als Netzwerkebene betrachtet.

In der Literatur findet sich eine Vielzahl von Versuchen, die Aktivitäten zu klassifizieren, die in einem Veränderungsprozeß anfallen. Dabei werden zum einen Aktivitäten zusammengefaßt, die sich funktional gruppieren lassen, und zum anderen werden solche Gruppen gebildet, die jeweils eine Abfolge aus zeitlich-sequentieller Perspektive darstellen. Kirsch, Esser, Gabele listen allein 21 verschiedene Phasenschemata auf. Vgl. Kirsch, W., Esser, W. M., Gabele, E., Das Management des geplanten Wandels von Organisationen, a.a.O., S. 36ff.

Ansoff unterscheidet z.B. drei Phasen: 1. „Planning establishes purposes, guidelines, strategies and constraints for the firm," 2. „implementation is the process of causing the firm to behave in accordance with the purposes, guidelines and strategies" und 3. "Control evaluates the organisation's performance and determines the needed adjustments in planning and implementation." Vgl. Ansoff, H. I., Implanting strategic Management, a.a.O., 241ff. Ansoff unterscheidet je nach Umweltsituation des Unternehmens verschiedene Kreislăufe, die mit zunehmend turbulenter Umwelt komplexer werden, wobei der innere "Implementierungskreis“ in jeder Umweltsituation die dominante Managementaktivităt darstellt.

Byars beschreibt ebenfalls einen rückgekoppelten Prozeß, der sich in die Phasen Zielsetzungsprozeß, Strategieselektion und Strategieimplementierung untergliedert, wobei sich alle Phasen nochmals in Teilphasen aufgliedern. Vgl. Byars, L. L., Strategic Management: Planning and Implementation, Concepts and Cases, Second Edition, New York u.a. 1987, S 4ff. 
des gesamten Prozesses. Insbesondere wird diskutiert, wie die Planung bzw. Formulierung der Strategie im Verhältnis zur Implementierung zu behandeln ist. ${ }^{111}$

In diesem Zusammenhang wird ein enger und ein weiter Begriff der Implementierung unterschieden. Der enge Implementierungsbegriff beinhaltet ausschließlich eine Phase des Änderungsprozesses, während sich der weite Begriff auf den ganzen Prozeß bezieht und damit als Anpassungsvorgang der Handlungsbedingungen unter einer längerfristigen Perspektive aufgefaßt wird. ${ }^{112}$ Im Rahmen der vorliegenden Arbeit wird ein Mittelweg bezüglich des Begriffsverständnisses zugrunde gelegt, d.h. die Planungsphase des Rücknahme- und Recyclingsystems wird als Implementierung aufgefaßt und als Teil eines gesamten Änderungsprozesses begriffen. Erste Initiierungsaktivitäten, die lediglich einer Information und Orientierung dienen, fallen dagegen nicht unter dieses Implementierungsverständnis. Die Implementierung wird in zwei Phasen untergliedert: in die Planungsbzw. Einführungsphase und die Realisationsphase des Rücknahme- und Recyclingsystems. ${ }^{113}$ Dieses liegt in der Annahme begründet, daß zum Beginn des Implementierungsprozesses andere Personen und Abteilungen in den Prozeß eingebunden sind als in einer späteren Phase. Die Begründung läßt sich mit Hilfe motivationstheoretischer Ansätze finden, nach denen bei anstehenden umfangreichen Neuerungen der Wunsch der Mitarbeiter groß ist, am Status quo festzuhalten und daher zunächst der Wille ${ }^{114}$ geschaffen werden muß, an der Implementierung des Rücknahme- und Recyclingsystems mitzuwirken. ${ }^{115}$ Ein Beispiel ist die

111 Kotler erläutert in diesem Zusammenhang, daß die Strategie das "Was" und das "Wie" der Aktivitäten klärt, während die Implementierung das "Wer", „Wo“, "Wann" und "Wie“ bewältigt. Vgl. Kotler, P., Bliemel, F., Marketing-Management, a.a.O., S. 1143.

112 Vgl. Clauss, M., Die Strategie der Implementierung in der Unternehmung, a.a.O., S. 11.

Hrebiniak und Joyce fassen die Implementierung im weiten Begriffsverständnis als Planung und Organisation auf, da sich beide Bereiche aufeinander beziehen und jeweils Implikationen für den anderen haben. Vgl. Hrebiniak, L. G., Joyce, W. F., Implementing Strategy, New York 1984 , S. 9.

Darüber hinaus wird darauf hingewiesen, daß der gesamte Änderungsprozeß wie auch nur die Implementierungsphase im engen Sinne als rückgekoppelter Prozeß ablaufen sollte, da sich nach der jeweiligen Kontrollphase eine Diskussion über eventuell neue Änderungsbedarfe ergibt.

113 Knopf unterscheidet neben Anfangs-, Planungs- und Konsolidierungsstadium ebenfalls ein „Frühes Implementierungsstadium“ und ein „Spätes Implementierungsstadium“. Vgl. Knopf, R., Dimension des Erfolgs von Reorganisationsprozessen, Mannheim 1976.

114 Voraussetzung für "Wollen" sind auch hier die beiden Komponenten „Kennen und Verstehen" und „Können“.

115 Das Durchbrechen emotionaler Barrieren wird häufig als schwieriger angesehen als die Überwindung finanzieller Restriktionen. Es wird versucht, insbesondere dann an „bewährtem Verhalten" festzuhalten wenn das Verhalten in der Vergangenheit zy Erfolg geführt hat. Es liegt rrene Giesen-Netzer and Universitat Vunster - 978-3-63 1-75058-2 
Überzeugungsleistung in der Einführungsphase, die durch die Vorgesetzten oder gar die Unternehmensleitung stattfinden kann und die im späteren Verlauf nicht mehr so notwendig ist wie zu Beginn des Prozesses.

Der Wirkungsbezug der Implementierung schließlich beinhaltet die Frage, wie der Erfolg der Implementierung erfaßt und gemessen wird. Auf der einen Seite werden auf das Individuum bezogene Erfolgsindikatoren wie der Anwendungsgrad der Strategie innerhalb der Strategieumgebung und damit die Akzeptanz und die Änderung des Handelns beschrieben. ${ }^{116}$ Auf der anderen Seite werden Erfolgsindikatoren im Sinne von Zielerreichungen herangezogen, die sich auf allen Bezugsebenen der Implementierung definieren lassen. ${ }^{117}$ Im Rahmen der vorliegenden Arbeit wird dem Ansatz gefolgt, den Erfolg daran zu messen, in welchem Ausmaß die Hersteller die im Zusammenhang mit dem Rücknahme- und Recyclingsystem gesetzten Ziele erreicht (Effektivität). ${ }^{118}$ Dabei werden individualbezogene, unternehmensbezogene und netzwerkbezogene Zielerreichungen analysiert. ${ }^{119}$

die Bestrebung vor, das Erreichte zu sichern und nicht durch eine Änderung des bisherigen Verhaltens das Risiko eines relativen Leistungsabfalls einzugehen. Vgl. zu weiteren Ausführungen über Grundlagen der Personal- und Organisationsentwicklung Wottawa, H., Gluminski, I., Psychologische Theorien für Unternehmen, in: Schuler, H. (Hrsg.), Schriftenreihe Wirtschaftspsychologie, Göttingen 1995, S. 295f.

Vgl. Kolks, U., Strategieimplementierung, a.a.O., S. 111.

Meffert unterscheidet Durchsetzungs- und Umsetzungsziele sowie Durchführungsziele (z.B. Kostenziele, termin- und durchführungsorientierte Ablaufziele) und erfaßt damit die Ziele auf der Individual- und der Unternehmensebene. Vgl. Meffert, H., Marketing-Management, a.a.O., S. $362 \mathrm{ff}$.

Byars erläutert qualitative Zielsetzungen (z.B. „Unterstützt die Implementierung der Strategie die anderen Ziele und Strategien des Unternehmens?", „Wie ist der Fit zu umweltbezogenen Trends und zu den eigenen Ressourcen?") und quantitative Zielsetzungen (z.B. Return on investment, Wachstum, Gewinn), die mit der tatsächlich erreichten Leistung nach der Implementierung abgeglichen werden müssen. Vgl. Byars, L. L., Strategic Management, a.a.O., S. $170 \mathrm{ff}$.

Hrebiniak und Joyce weisen auf die Notwendigkeit hin, die aktuellen Leistungsdaten aufzunehmen, die vor der Implementierung einer Strategie bestehen, um diese dann anschließend mit den Daten vergleichen zu können, die im Anschluß an die Strategieimplementierung vorliegen. Vgl. Hrebiniak, L. G., Joyce, W. F., Implementing Strategy, a.a.O., S. $204 f$. 


\section{Ziel und Gang der Untersuchung}

Die erste deskriptive Zielsetzung der Arbeit liegt - aufgrund der komplexen intraund interorganisationalen Problemstellungen des Implementierungsobjektes - in der Erfassung der organisatorischen Gestaltungsformen und der damit verbundenen Ziele von Rücknahme- und Recyclingsystemen. Eine weitere deskriptive Zielsetzung besteht in der theoriegeleiteten ${ }^{120}$ Erfassung des Implementierungsverhaltens von Herstellern langlebiger Gebrauchsgüter und in der Ableitung von Implementierungsmustern ${ }^{121}$, die eine Erkennung von Gemeinsamkeiten und Unterschieden zwischen dem Verhalten der Hersteller ermöglichen. Dieses Vorgehen beruht auf der Annahme, daß sich Dimensionen der Implementierung finden lassen, die als Grundlage zur Bildung von Implementierungsmustern genutzt werden können.

Aus den Implementierungsmustern sollen Implementierungstypen abgeleitet werden, auf denen eine explikative Zielsetzung der vorliegenden Arbeit aufbaut. Diese besteht in der Entwicklung eines Erklärungsansatzes für die Herausbildung der Implementierungstypen. Dabei wird untersucht, ob und ggf. welchen Einfluß netzwerkbezogene, unternehmensbezogene sowie individualbezogene Kontingenzfaktoren auf die Implementierungstypen besitzen. Zusätzlich wird untersucht, welche Erfolgswirkungen die Implementierungstypen besitzen.

Eine eher normative Zielsetzung besteht darin, aus den gewonnenen Erkenntnissen über erfolgreiche bzw. nicht erfolgreiche Implementierung von Rücknahmeund Recyclingsystemen seitens der Hersteller Gestaltungsansätze zur Implementierung von Rücknahme- und Recyclingsystemen abzuleiten. Dazu werden Ansätze zur Operationalisierung des Implementierungserfolgs von Rücknahme- und Recyclingsystemen systematisiert und empirisch überprüft. Aus diesen übergeordneten Zielen ergibt sich folgender Gang der Untersuchung:

\footnotetext{
120 Die theoriegeleitete Erfassung des Implementierungsverhaltens umfaßt in diesem Zusammenhang eine literaturgestützte Analyse, die insbesondere auf Theorien der Organisation und der Führung sowie spezieller Implementierungsliteratur beruht, um Erkenntnisse daraus auf die spezielle Implementierungsproblematik bei Rücknahme- und Recyclingsystemen übertragen zu können.

121 Durch einen Ähnlichkeitsvergleich zwischen Profilen werden Cluster gebildet und typische (Implementierungs-)Muster innerhalb der Cluster gesucht. Dabei ist ein Profil ein Satz von Daten eines Untersuchungsobjektes und kennzeichnet damit die Ausprägung bestimmter Variablen bzw. Dimensionen (Dimensionen werden aus der Verdichtung von Variablen gebildet). Vgl. Scholz, Chr., Strategisches Management: Ein integrativer Ansatz, Berlin/ New York 1987, S. $77 \mathrm{ff}$.
} 
1. Analyse und Systematisierung der organisatorischen Gestaltungsformen und Ziele von Rücknahme- und Recyclingsystemen (Kapitel B 1).

2. Entwicklung von theoriegeleiteten Begründungen der Implementierungsdimensionen, die Grundlage zur Ermittlung der Implementierungstypen sind. Die Implementierungsdimensionen stellen dabei aggregierte Gestaltungsspielräume der Herstellerunternehmen dar, innerhalb derer sich das Implementierungsverhalten der Hersteller abbildet. Diese Gestaltungsspielräume sind hinsichtlich ihrer Existenz und ihrer Ausprägungen bei Rücknahme- und Recyclingsystemen zu überprüfen (Kapitel B 2). ${ }^{122}$

3. Systematisierung der Einflußfaktoren von Implementierungsmustern für Rücknahme- und Recyclingsysteme. Es sind netzwerkbezogene, unternehmensbezogene und individualbezogene Einflüsse zu berücksichtigen und deren Wirkungsbeziehungen in einem empirischen Bezugsrahmen zu verdichten. Parallel werden diese Wirkungsbeziehungen empirisch überprüft und Aussagen über Bestimmungsfaktoren der Ausprägung von Implementierungsmustern bzw. über die Ausgestaltung der Implementierungsdimensionen abgeleitet. (Kapitel C 1).

4. Analyse der Verhaltens- und Erfolgswirkungen verschiedener Implementierungsmuster unter Berücksichtigung des situativen Kontextes von Rücknahmeund Recyclingsystemen langlebiger Gebrauchsgüter. Auch bei diesem Forschungsschwerpunkt sind die Verhaltens- und Erfolgswirkungen der Implementierungsmuster und deren instrumentelle Ausgestaltung zunächst theoriegeleitet zu überprüfen und anschließend empirisch zu validieren. Hier ist insbeson-

122 Die Überprüfung der hergeleiteten Implementierungstypen anhand empirischer Daten erfolgt mit Hilfe multivariater Analysemethoden. Dabei sind die Implementierungstypen auf innere Konsistenz zu überprüfen, wobei angesichts des Stichprobenumfangs ein forschungstechnisch exploratives Vorgehen gewählt wurde (Kapitel B 3).

Die empirische Überprüfung der in Kapitel $B$ und $C$ ermittelten Hypothesen, die jeweils in die Kapitel integriert wird, erfolgt auf Grundlage einer Untersuchung, die das Institut für Marketing im Auftrag der Bundesstiftung Umwelt in den Jahren 1995/96 durchgeführt hat. Dabei wurden 101 persönliche Interviews bei Herstellern langlebiger Gebrauchsgüter geführt denen ein 43seitiger Fragebogen zugrunde lag. Die Untersuchung konzentrierte sich auf die Branchen Automobil $(12 \%)$, Möbel (12\%), Elektro- und Elektronikgeräte $(36 \%)$ und Bauzubehör bzw. Chemie (29\%). Hinsichtlich der Größenverteilung zählen $44 \%$ der befragten Hersteller zu den kleinen und mittelständischen Betrieben und $56 \%$ zu den Großunternehmen mit über 500 Mitarbeitern. Es wurden ausschließlich Unternehmen befragt, die bereits ein Rücknahme- und Recyclingsystem realisiert hatten und somit bereits Implementierungserfahrungen vorweisen konnten (Realisationsphase der Implementierung $=71 \%$ ) oder solche Unternehmen, die die Einführung von Rücknahme- und Recyclingsystemen konkret planten und somit bereits relevante Umsetzungs- und Durchsetzungsaspekte erkannt hatten (Einführungsphase $=29 \%$ ). Zum genauen empirischen Design und Methodik der empirischen Untersuchung vgl. Anhang 2.1 . 
dere zu analysieren, ob bestimmte Implementierungsmuster eine situationsspezifische Vorteilhaftigkeit aufweisen. Dabei sollen praxisrelevante Implikationen für die Implementierung von Rücknahme- und Recyclingsystemen abgeleitet werden (Kapitel C 2).

5. Auf Basis der erarbeiteten Ergebnisse werden Implikationen für die praktische Ausgestaltung der Implementierung von Rücknahme- und Recyclingsystemen für Hersteller langlebiger Gebrauchsgüter abgeleitet. Darauf aufbauend ergeben sich Schlußfolgerungen für weitere wissenschaftliche Untersuchungen (Kapitel D).

Abbildung 4 faßt die grundlegenden Einflußfaktorengruppen, die Verhaltens- bzw. Gestaltungsparameter und Wirkungsgrößen sowie ihre möglichen Wirkungsbeziehungen in einem kontingenztheoretischen Bezugsrahmen zusammen. 


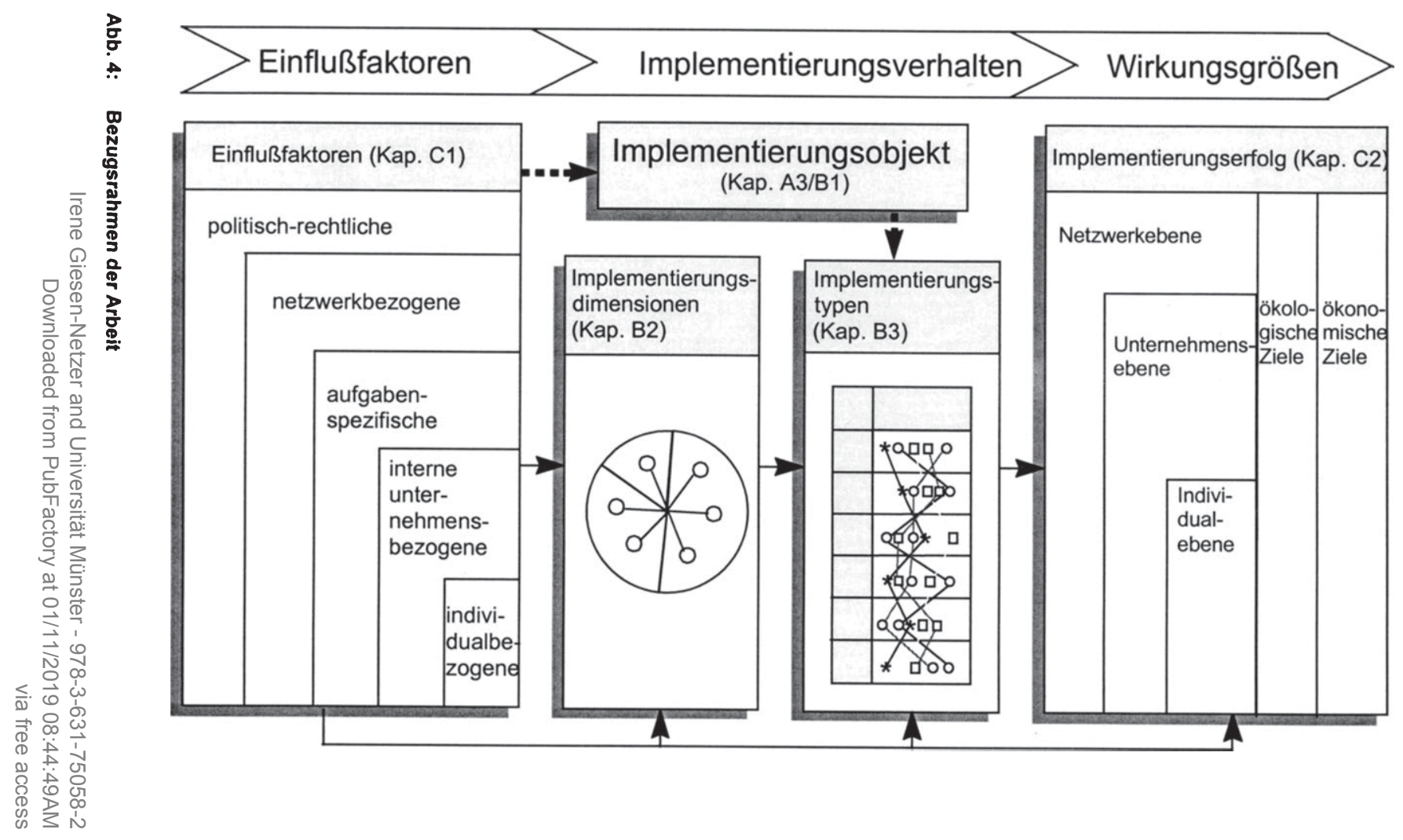




\section{B. Analyse des Implementierungsverhaltens bei Rücknahme- und Recyclingsystemen}

\section{Gestaltungsansätze und Ziele von Rücknahme- und Recyclingsyste- men bei Gebrauchsgütern}

\subsection{Ansätze zur organisatorischen Gestaltung von Rücknahme- und Re- cyclingsystemen}

Um das Implementierungsobjekt näher zu beschreiben, werden die institutionellen Ausgestaltungsformen von Rücknahme- und Recyclingsystemen analysiert. In Anlehnung an die Transaktionskostentheorie ${ }^{123}$ bewegen sich diese zwischen zwei Extremen: Dem Markt, bei dem das Unternehmen alle Aufgaben Dritten überträgt und zum anderen der Hierarchie, bei der alle Aufgaben in einem firmeneigenen System durchgeführt werden. ${ }^{124}$

Folgt man (vereinfachend) der neoklassischen Markttheorie oder dem Ansatz der institutionellen Ökonomie, zeichnet sich der Markt durch ökonomische Aktivitäten aus, in der beliebige Marktteilnehmer eine genau spezifizierte Leistung austauschen, dessen Koordination ausschließlich auf Grundlage von Preisen erfolgt. ${ }^{125}$

123 Zur kritischen Diskussion der Erkenntnisse der Neuen Institutionenökonomik (insbesondere von R. H. Coase und D. C. North) auf die Umweltpolitik vgl. Bonus, H., Institutionen und Institutionelle Ökonomik, Anwendungen für die Umweltpolitik, Volkswirtschaftliche Diskussionsbeiträge, Nr. 231, Münster 1996, S. $11 \mathrm{ff}$. Nach Bonus sind insbesondere die Wirkungen der Transaktionskosten auf die Umweltökonomie bisher nur ansatzweise herausgearbeitet worden. Vgl. ebenda, S. $18 \mathrm{ff}$.

In diesem Zusammenhang werden der Markt oder die Hierarchie auch als Koordinationsform oder Organisationsform bezeichnet. Vgl. Meyer, M., Ökonomische Organisation der Industrie: Netzwerkarrangements zwischen Markt und Unternehmung, Wiesbaden 1995, S. 137 oder vgl. Sydow, J., Strategische Netzwerke: Evolution und Organisation, Wiesbaden 1992, S. $130 \mathrm{f}$. Da es sich genaugenommen jedoch um institutionelle Arrangements auf vertragstheoretischer Basis handelt, wird in der vorliegenden Arbeit jeweils von der institutionellen Ausgestaltung gesprochen. Vgl. Kieser, A. (Hrsg.), Organisationstheorien, 2. überarb. Aufl., Stuttgart/ Berlin/ Köln 1995, S. 214ff.

Diese institutionellen Arrangements lassen sich auf die klassische Make-or-Buy-Entscheidung zurückführen. Vgl. Dubois, A., Changing Activity Structures - A new View of Make-or-Buy Decisions, in: Institut de Recherche de l'entreprise (Hrsg.), Business Networks in an international Context: Recent Research Developments, Proceedings of the 8th IMP Conference, 1992, S. 70ff. und vgl. Kirchgeorg, M., Ziele, Strategien und Organisationskonzepte von Recycling- und Rücknahmesystemen für langlebige Gebrauchsgüter, in: Meffert, H., Wagner, H., Backhaus, K. (Hrsg.), Marktorientierte Unternehmensführung in der Kreislaufwirtschaft - Kostenmanagement oder Wettbewerbsprofilierung, Dokumentationspapier Nr. 106 der Wissenschaftlichen Gesellschaft für Marketing und Unternehmensführung e.V., Münster 1996, S. 11-25, S.19.

Die Neoklassische Theorie geht von der Annahme des vollkommenen Marktes aus, während die Neue Institutionenökonomie den unvollkommenen Markt annimmt und zwei Fragestellungen im Mittelpunkt der Betrachtung stehen: 1. Wie ist die gegebene institutionelle Infrastruktur zu erklären? 2. Durch welche institutionelle Lösungen kann ein bestimmtes Koordinationsproblem am effizientesten gelöst werden? Vgl. Kaas, K. P., Marketing und Neue Institu- 
Zudem sind marktliche Beziehungen flüchtig und idealtypisch kompetitiv. Bezogen auf die Gestaltung von Rücknahme- und Recyclingsystemen würde dieses bedeuten, daß Herstellerunternehmen alle Aufgaben externalisieren und diejenigen Partner, die im Rahmen des Rücknahme- und Recyclingsystems Leistungen für sie erbringen, ausschließlich nach Preisen auswählen. ${ }^{126}$

Bei der Hierarchie hingegen substituieren Weisungen der Unternehmensleitung an die Organisationsmitglieder idealtypisch jede marktliche Koordination. ${ }^{127}$ Hierarchische Beziehungen sind auf Dauer angelegt und idealtypisch kooperativ. ${ }^{128}$ Alle Aufgaben der Implementierung von Rücknahme- und Recyclingsystemen würden bei dieser institutionellen Ausgestaltungsform vom Unternehmen selbst durchgeführt (internalisiert).

Die beiden institutionellen Ausgestaltungsformen der Hierarchie und des Marktes sind in Reinform in der Praxis nicht festzustellen. ${ }^{129}$ Die realen Phänomene befinden sich vielmehr in einer intermediären Position zwischen diesen beiden Extremformen. ${ }^{130}$ Dabei können herstellereigene Systeme, kooperative Systeme

tionenökonomik, in: Kaas, K. P. (Hrsg.), Kontrakte, Geschäftsbeziehungen, Netzwerke: Marketing und neue Institutionenökonomik, Frankfurt am Main 1995, S. 3.

Die Neoklassische (mikroökonomische) Theorie geht demgegenüber von einer vorhandenen Infrastruktur aus, bei der keine Ineffizienz auftritt, und wird daher auch als „organisationsloser" Ansatz gekennzeichnet. Vgl. Meyer, M., Ökonomische Organisation der Industrie, a.a.O., S. 21. Im folgenden beschreibt Meyer die Fortentwicklung der (volkswirtschaftlichen)Theorien bis hin zur Erklärung der formalen Organisation (Hierarchie) von Unternehmen.

Die Marktteilnehmer verhalten sich gemäß der Annahme der Neuen Institutionenökonomie grundsătzlich begrenzt rational und opportunistisch. Zudem sind sie gleichberechtigt in ihren Handlungen und weitgehend von einander unabhängig. Vgl. Sydow, J., Strategische Netzwerke: a.a.O., Wiesbaden 1992, S. 98.

In der Regel wird dann von Hierarchie gesprochen, wenn sich zentrale Führungspositionen bilden, die die Aktivităten im Hinblick auf die Organisationsziele koordinieren. Vgl. Meyer, M., Ökonomische Organisation der Industrie, a.a.O., S. 55.

Vgl. Sydow, J., Strategische Netzwerke, a.a.O. , S. 98.

Kaas spricht im Zusammenhang der Hierarchie von der inneren Organisation eines Unternehmens, die den Markt als Koordinationsmechanismus "total" ablöst. Das Marketing ordnet er als Schnittstelle zwischen beiden Extremen ein. Vgl., Kaas, K. P., Marketing zwischen Markt und Hierarchie, in: Kaas, K. P. (Hrsg.), Kontrakte, Geschäftsbeziehungen, Netzwerke: Marketing und neue Institutionenökonomik, Frankfurt am Main 1995, S. $20 \mathrm{ff}$.

Vgl. Meyer, M., Okonomische Organisation der Industrie, a.a.O., S. 73.

130

Sydow unterscheidet die beiden institutionellen Ausgestaltungsformen der Hierarchie und des Marktes bei der Bildung strategischer Netzwerke und legt einen (interorganisations-) theoretischen Ansatz dar, der die Evolution und Organisation strategischer Netzwerke zu erklären sucht. „Ein strategisches Netzwerk stellt eine auf die Realisierung von Wettbewerbsvorteilen zielende, polyzentrische, gleichwohl von einer oder mehreren Unternehmen strategisch geführte Organisationsform ökonomischer Aktivitäten zwischen Markt und Hierarchie dar, die sich durch komplex-rezipoke, eher kooperative denn kompetitive und relativ stabile BeziehunIrene Giesen-Netzer and Universität Munster - 978-3-631-75058-2 
und Verträge mit Verwertern unterschieden werden. ${ }^{131}$ Das eigene System entspricht am ehesten der Hierarchieform, während bei einem Vertrag mit Verwertern nahezu klassische Vertragsbeziehungen vorliegen, die durch eine kurze Dauer, die präzise Bestimmung des Gegenstandes und der Konditionen der Transaktion sowie eine begrenzte persönliche Interaktion der Vertragspartner chararkterisiert werden können. ${ }^{132}$ Bei den kooperativen Systemen, die i.d.R. auf längerfristigen Beziehungen zwischen den Systempartnern aufbauen, werden darüber hinaus Gemeinschaftsunternehmen, gemeinsame Entwicklung eines Systems mit Verwertern und Branchenkooperationen unterschieden, die sich idealtypisch durch zunehmende Kompetitivität und abnehmende Kooperation auszeichnen (vgl. Abb. $5) .{ }^{133}$

gen zwischen rechtlich selbständigen, wirtschaftlich jedoch zumeist abhängigen Unternehmen auszeichnet." Sydow, J., Strategische Netzwerke, a.a.O., S. 82 und vgl. $127 \mathrm{ff}$.

Meyer ergänzt diese Definition um den Aspekt, daß eine solche Koalition auf gegenseitigem Einvernehmen beruht und i.d.R. auf Basis von institutionellen Arrangements geregelt wird. Vgl. Meyer, M., Ökonomische Organisation der Industrie, a.a.O., S. 136.

Im Rahmen dieser Arbeit findet der Begriff des strategischen Netzwerkes Anwendung für Kooperationen, die eine (oder mehrere) zentrale Unternehmung(en) (hub firm) als strategische(n) Führer haben. Dieses wird aus den Ausführungen von Jarillo deutlich, der dieses als wesentliches Merkmal für strategische Netzwerke herausstellt. Vgl. Jarillo, J. C., On stategic networks, in: Strategic Management Journal, Heft 1, 1988, S. 32. Darüber hinaus wird von Netzwerkebene gesprochen, wenn es um Transaktionen und Koordinationsbedarfe geht, die über die Grenzen des Unternehmens hinaus gehen und nur in Kooperation mit den anderen beteiligten Systempartnern des Rücknahme- und Recyclingsystems bewältigt werden können. Dieses setzt keine strategische Führung durch eine einzelne Unternehmung voraus, kann wechselnde Interorganisationsbeziehungen aufweisen und besitzt eine eher emergente, informale Struktur und Kultur. Wenn es sich dabei zusätzlich um eine Kooperation zwischen kleinen und mittleren Betrieben handelt, die räumlich auf eine Region konzentriert sind, handelt es sich um regionale Netzwerke. Vgl. Sydow, J., Netzwerkorganisation, Interne und externe Restrukturierung von Unternehmungen, in: WiSt, Heft 12, 1995, S. 630ff.

131 Richardson versucht 1972 zu erklären, unter welchen Bedingungen drei institutionelle Ausgestaltungsformen (Unternehmung, Kooperation, Markt) eine effektive Koordinationsform darstellen und begründet dieses mit der Ähnlichkeit der Aktivitäten auf der einen Seite und der Komplementarität auf der anderen Seite. Vgl. Richardson, G. B., The Organisation of Industry, in: Economic Journal, No. 2, 1972, S. 888ff.

Vgl. Kieser, A. (Hrsg.), Organisationstheorien, a.a.O., S. 214.

133 Dabei muß hervorgehoben werden, daß es sich hierbei lediglich um einen Strukturierungsansatz handelt und je nach Ausgestaltung des Rücknahme- und Recyclingsystems sich die Lösung mehr oder weniger kooperativ bzw. kompetitiv darstellt und daß eine klare Trennung der unterschiedenen Formen nicht immer möglich ist.

Bronder und Pritzl beispielsweise unterscheiden - wie in der vorliegenden Arbeit - drei Formen: eine selbständige Lösung innerhalb der eigenen Unternehmenshierarchie, eine Lösung über den Markt oder eine Mischform. Vgl. Bronder, Chr. Pritzl, R., Strategische Allianzen zur Steigerung der Wettbewerbsfähigkeit, in: io Management Zeitschrift, Heft 5, 1991, S. 27. Ähnlich auch Sydow, der Unternehmensnetzwerke als intermediäre institutionelle Ausgestaltungsformen zwischen Markt und Hierarchie darstellt. Vgl. Sydow, J., Unternehmensnetzwerke, in: Corsten, H., Reiß, M. (Hrsg.), Handbuch Unternehmensführung: Konzepte - Instrumente - Schnittstellen, Wiesbaden 1995, S. 160. 


\begin{tabular}{|c|c|c|}
\hline Hierarchie & $\begin{aligned} \rightarrow & \text { Zunehmende Kompetitivität } \rightarrow \\
& \text { Abnehmende Kooperation }\end{aligned}$ & Markt \\
\hline
\end{tabular}

\begin{tabular}{|c|c|c|}
\hline $\begin{array}{c}\text { Eigenes System } \\
19,5 \%\end{array}$ & $\begin{array}{c}\text { Kooperative Systeme } \\
65 \%\end{array}$ & $\begin{array}{c}\text { Vertrag mit Ver- } \\
\text { wertern } \\
15,5 \%\end{array}$ \\
\hline
\end{tabular}

\begin{tabular}{|c|c|c|}
\hline $\begin{array}{c}\text { Gemein- } \\
\text { schafts- } \\
\text { unternehmen }\end{array}$ & $\begin{array}{c}\text { Gemeinsame } \\
\text { Entwicklung } \\
\text { eines Systems } \\
\text { mit Verwertern } \\
1 \%\end{array}$ & $\begin{array}{c}\text { Branchen- } \\
\text { kooperationen }\end{array}$ \\
\hline \multicolumn{3}{|c|}{ Mischsysteme 48\% } \\
\hline
\end{tabular}

Abb. 5: Institutionelle Ausgestaltungsformen von Rücknahme- und Recyclingsystemen

Die empirischen Ergebnisse zeigen, daß 19,5\% der befragten Hersteller überwiegend alle Aufgaben selbst durchführen und damit ein eigenes System realisieren. ${ }^{134} 15,5 \%$ haben einen Vertrag mit Verwertern, in dem sie die Verwertungsaufgaben für ihre Produkte an Verwerter delegieren, ohne eine intensivere Kooperation einzugehen. Dieses kommt der Marktform sehr nahe. ${ }^{135}$

Nahezu $65 \%$ der befragten Herstellerunternehmen kooperieren mit unterschiedlichen Systempartnern, die dann Aufgaben wie z.B. Rücknahme, Rückführungslogistik, Demontage sowie Verwertungsprozesse oder Erschließung von Sekundärrohstoffmärkten übernehmen. Die Form des Gemeinschaftsunternehmens - im internationalen Bereich auch Joint Ventures genannt - wählten lediglich $1 \%$ der gesamten Stichprobe, d.h. es wird von zwei oder mehreren unabhängigen Gesell-

Schneidewind klassifiziert ökologisch orientierte Kooperationen von Unternehmen nach vertikalen Kooperationen, horizontalen Kooperationen und gesellschaftsbezogenen Kooperationen. Zu den vertikalen Kooperationen gruppiert er die Markt- und die Hierarchieform. Vgl. Schneidewind, U., Ökologisch orientierte Kooperationen aus betriebswirtschaftlicher Sicht, in: UWF, Heft 4, 1995, S. 17f. Dieses ist m.E. nach nur bedingt sinnvoll, da in der Marktform nur eine vertragliche Bindung und damit lediglich eine Geschäftsbeziehung vorliegt, die eine klare Abgrenzung von Ressourcen und den Austausch von Leistung gegen Geld beinhaltet. Die vertikale Kooperation hat hingegen - bezogen auf den Verhandlungsgegenstand - den Einsatz gemeinsamer Ressourcen mit einem gemeinsamen Ziel zum Gegenstand. Auch Hierarchie innerhalb des Unternehmens wird mit "Kooperation" nicht treffend beschrieben.

Bei herstellereigenen Systemen werden auch Verwerter im geringen Umfang für ausgewählte Aufgaben eingesetzt. Die Verwerter stehen dabei in einem Auftragsverhältnis und haben keinen (kaum einen) Einfluß auf die Gestaltung des Rücknahme- und Recyclingsystems.

Stellt man sich "Markt“, "Netzwerk“ und "Hierarchie" auf einem Kontinuum vor, erfolgt die Absicherung von Unsicherheit bei der Marktform durch Verträge, die in Richtung einer Kooperation durch Vertrauen und Reputation nach und nach abgelöst werden. Wird jetzt weiter in Richtung "Hierarchie“" organisiert, wird zunehmend durch gemeinsames Eigentum abgesichert. Vgl. Meyer, M., Ókonomische Organisation der Industrie, a.a.O., S. 92. 
schafterunternehmen ein rechtlich selbständiges Unternehmen gemeinsam gegründet oder erworben, mit dem (Rücknahme- und Recycling-)Ziele und Aufgaben im gemeinsamen Interesse der Gesellschafterunternehmen durchgeführt werden. ${ }^{136}$

$5 \%$ der Herstellerunternehmen beteiligten sich an einer Branchenkooperation (branchenweites System) zur Lösung von Rücknahme- und Recyclingaufgaben. Ziel einer Branchenkooperation, an der sich Unternehmen der gleichen Produktions- und Handelsstufe beteiligen, ist die gemeinsame Nutzung von Ressourcen und Kapazitäten. ${ }^{137}$ Bei einer Branchenkooperation zur Gestaltung von Rücknahme- und Recyclingsystemen werden in der Regel jedoch auch ein oder mehrere Verwerter (in einem Auftragsverhältnis) einbezogen, um Verwertungsaufgaben durchzuführen. Die besondere Bedeutung solcher (überwiegend) horizontalen Kooperationen liegt im Aufbau von branchenspezifischen Goodwill-Potentialen bei gesellschaftlichen Anspruchsgruppen. ${ }^{138}$ Darüber hinaus kann eine Branchenkooperation auch als Instrument zur Stärkung der Verhandlungsposition gegenüber dem Gesetzgeber genutzt werden. Die Entwürfe zur Altauto- und Elektronikschrottverordnung wurden beispielsweise von den Branchenverbänden angefertigt und anschließend den Behörden als Vorschlag vorgelegt. Neben einem einheitlichen Verhalten gegenüber den Behörden liegt das Ziel eines branchenweiten Vorgehens darin, die Verhandlungsgewinne eines einzelnen Unternehmens auch den übrigen beteiligten Unternehmen zugute kommen zu lassen. ${ }^{139}$

136 In diesem Fall wird auch von Equity Joint Ventures gesprochen, denen Contractual Joint Ventures als rein vertragliche Form gegenüberstehen. Vgl. zur Begriffsbestimmung von Joint Ventures Schubert, W., Küting, K., Unternehmenszusammenschlüsse, München 1981, S. 219.

137 Zweck der Branchenkooperation bei der Bildung von Rücknahme- und Recyclingsystemen ist i.d.R. die Koordinierung oder gemeinsame Durchführung bestimmter Funktionen, ohne jedoch den Wettbewerb zu beeinträchtigen. Vgl. zu weiteren Details horizontaler Kooperationen Wöhe, G., Einführung in die Allgemeine Betriebswirtschaftslehre, 17. überarb. und erw. Aufl., München 1990, S. $407 f$.

Im Gegensatz zur Branchenkooperation haben horizontale strategische Allianzen zwischen Wettbewerbern das Ziel, sich durch den Ausgleich von spezifischen Stärken und Schwächen Vorteile gegenüber Konkurrenten zu verschaffen. Vgl. Meyer, M., Ökonomische Organisation der Industrie, a.a.O., S. 137.

138 Vgl. Corsten, H., Götzelmann, F., Das Kooperationsprinzip in umweltpolitischer und unternehmerischer Sicht, in: WISU, Heft 5, 1991, S. 377.

Diese Zielsetzung insbesondere bei den untersuchten langlebigen Gebrauchsgütern Automobil und Elektronikschrott virulent, da sie aufgrund der hohen Abfallmengen und der Betroffenheit der privaten Verbraucher sehr stark in die öffentliche Diskussion gelangt sind. Vgl. dazu die Ausführungen in Kapitel A 1.

139 Vgl. Corsten, H., Götzelmann, F., Das Kooperationsprinzip in umweltpolitischer und unternehmerischer Sicht, a.a.O., S. $377 \mathrm{f}$. 
Schließlich entwickeln $11 \%$ der Herstellerunternehmen gemeinsam mit Verwertern ein Rücknahme- und Recyclingsystem. ${ }^{140}$ Dabei werden den Verwertern in der Regel die gesamten Recyclingaufgaben übertragen. Die Verwerter wiederum geben ein Feed-back an die Herstellerunternehmen bezüglich der Konstruktion und Materialzusammensetzung, damit diese im Sinne eines Recycling gegebenenfalls angepaßt werden können. ${ }^{141}$ Voraussetzung für eine solche Kooperation ist eine dauerhafte Input-Output-Beziehung, damit für den Abfallnehmer eine gesicherte Zuführung des Input und für den Rückstandslieferant eine gesicherte Entsorgung bzw. eine Zuführung von Sekundärrohstoffen (Recyclaten) oder Altproduktteilen gegeben ist. ${ }^{142}$

Lediglich $17 \%$ der befragten Gesamtunternehmen sind eindeutig bestimmten Kooperationsformen zuordenbar, während $48 \%$ aller Unternehmen kooperative Systeme organisieren, die eine Mischform der aufgezeigten Kooperationsformen darstellen. Dieses kann mit dem Versuch der Unternehmen begründet werden, die Vorteile verschiedener Formen von Rücknahme- und Recyclingsystemen miteinander zu kombinieren bzw. die Nachteile einzelner Formen zu kompensieren. Sucht man nach Ursachen für die hohe Bedeutung von kooperativen Lösungen bei der Schaffung eines Rücknahme- und Recyclingsystems - insbesondere bei kleinen und mittelständischen Unternehmen -, liegen diese zum einen in dem von Herstellern vermuteten hohen Investitionsbedarf und zum anderen in den schwierigen Prozeßabläufen für Unternehmen und damit verbundenen komplexen or-

140 Bei der Lösung der gemeinsamen Entwicklung eines Rücknahme- und Recyclingsystems mit Verwertern handelt es sich zwischen Herstellern und Verwertern um eine vertikale Zusammenarbeit, bei der aufeinanderfolgende Produktions- und Handelsstufen miteinander verzahnt werden. Vgl. Wöhe, G., Einführung in die Allgemeine Betriebswirtschaftslehre, a.a.O., S. 408.

Die betrachtete Zusammenarbeit kann am ehesten als rückwärts- und gleichzeitig vorwärtsgerichtete Kooperation betrachtet werden, d.h. von der Endstufe einer Produktion (Herstellerunternehmen) ist sie auf die vorgelagerte (Verwerter) und die nachgelagerte (Verwerter) Stufe gerichtet. Der Verwerter ist ein "Mischtyp“, da er sowohl Endabnehmer der Altprodukte ist (nachgelagert) als auch durch die Lieferung von Sekundärrohstoffen (Recyclat) oder Fertigteilen (Altproduktteile) an die Herstellerunternehmen vorgelagert als Lieferant fungiert.

141 Vgl. Fallstudie Bauknecht, a.a.O.

142 Die FensterREcyclinglnitative (FREI) hat in Ihrer Branchenkooperation gleichzeitig einen „Verwerter" (Bayer) eingebunden, um die genannten Ziele erfüllen zu können. Vgl. FREI-Fallstudie, a.a.O.

Hier ergeben sich neue Herausforderungen für ein Beschaffungsmarketing bei den Herstellern, um die Sekundärrohstoffe in gewünschter Qualität, Menge, Zeitpunkt und am richtigen Ort zu haben. 


\section{ganisatorischen Anpassungsproblemen. ${ }^{143}$ Der Koordinationsbedarf zwi-} schen den System- und Marktpartnern, der aufgrund der Kontrollnotwendigkeit der Produkte über den gesamten Produktlebenszyklus im Rahmen von Rücknahmeund Recyclingsystemen besteht, ist darüber hinaus recht hoch und daher scheinbar am besten in Kooperationen zu lösen. ${ }^{144}$ Bei langlebigen Gebrauchsgütern ist die Koordination besonders schwierig, da der Wert der Produkte erheblich von der Nutzung durch den Konsumenten abhängt. Damit gewinnt die herstellerseitige Kontrollmöglichkeit über das Produkt und über dessen konsumentenseitige Nutzung an Relevanz. ${ }^{145}$ Tendenziell können dabei Produkte im Investitionsgüterbereich besser überwacht werden als im Konsumgüterbereich.

Werden die Probleme der Rücknahme und des Recycling in quantitative, qualitative, räumliche, zeitliche sowie technologische und wirtschaftliche Probleme unterteilt, ergeben sich Lösungsansätze durch kooperative Systeme (vgl. Abb. 6). ${ }^{146}$

Einem kooperativen System stehen wahrscheinlich von Beginn an größere Mengen für ein Recycling zur Verfügung, so daß damit Skaleneffekte beim Betrieb einer Recyclinganlage genutzt werden können. Bezüglich der qualitativen Dimension können durch frühzeitige Absprachen zwischen Zulieferern, Abnehmern und Mitbewerbern Materialien und Verarbeitungstechniken abgesprochen werden, um

143 Vgl. Hansen, U., Raabe, T., Dombrowsky, B., Die Gestaltung des Konsumgüterrecycling als strategische Netzwerke, in: UWF, Heft 1, 1995, S. 62 und vgl. Horneber, M., Innovatives Entsorgungsmanagement: Methoden und Instrumente zur Vermeidung und Bewältigung von Umweltbelastungsproblemen, in: Pfeiffer, W. (Hrsg.), Innovative Unternehmensführung: Planung, Durchführung und Kontrolle von Innovationen, Band 24, Göttingen 1994, S. 3ff. Horneber bezieht diese Argumentation auf internes Recycling und spricht in diesem Zusammenhang von einem Wertschöpfungsnetzwerkproblem (innerhalb des Unternehmens treten an verschiedenen Stellen Abfälle auf) und vom Zeitproblem des innovativen Entsorgungsmanagement, wobei unter Entsorgung Abfallvermeidung, -verwertung und -beseitigung gefaßt werden.

$144 \mathrm{Vgl}$. Schneidewind, U., OKologisch orientierte Kooperationen aus betriebswirtschaftlicher Sicht, a.a.O., S. 16 .

145 Inzwischen wird daran gearbeitet, daß die Geräte ihre eigene Nutzung selbst dokumentieren. Bei Kopierern ist es bereits üblich, die Gesamtzahl der getätigten Kopien abzulesen. Bei Automobilen ist der Kilometerstand ein Indiz für die mehr oder weniger intensive Nutzung. Diese Formen der Kontrolle sagen aber noch nichts über die Art des Gebrauchs der Geräte. Daher wird versucht, genauere Angaben zu ermitteln - beispielsweise durch die Selbstdokumentation von Überhitzung bei Kopierern.

146 Vgl. Hansen, U., Raabe, T., Dombrowsky, B., Die Gestaltung des Konsumgüterrecycling als strategische Netzwerke, a.a.O., S. 64.

Ein ähnliche Strukturierung weist auch Krcal auf, der die Notwendigkeit von Kooperationen durch den Mengenaspekt, den Zeitaspekt und den Materialqualitätsaspekt begründet. Vgl. Krcal, H.-Chr., Wirkungsbeziehungen produktbezogener Umweltschutzmaßnahmen als Beweggrund zwischenbetrieblicher Zusammenarbeit, in: UWF, Heft 4, 1995, S. $22 \mathrm{ff}$. 
eine einfachere Demontage und bessere Verwertungsmöglichkeiten sicherzustellen.

\begin{tabular}{|l|l|l|}
\cline { 3 - 3 } \multicolumn{2}{l|}{} & $\begin{array}{l}\text { Vorteile von kooperativen Rücknahme- } \\
\text { und Recyclingsystemen }\end{array}$ \\
\hline $\begin{array}{l}\text { Quantitative } \\
\text { Probleme }\end{array}$ & $\begin{array}{l}\text { Zu geringe Altproduktmengen für } \\
\text { den einzelnen Produzenten }\end{array}$ & $\begin{array}{l}\text { Erreichung von Mindestmengen für einen } \\
\text { effizienten Betrieb von Recyclinganlagen } \\
\text { (Skaleneffekte) }\end{array}$ \\
\hline $\begin{array}{l}\text { Qualitative } \\
\text { Probleme }\end{array}$ & $\begin{array}{l}\text { Qualitative Vielfalt in bezug auf } \\
\text { Produktbestandteile, Materialien } \\
\text { und Verarbeitungstechniken } \\
\text { (Befestigung, Verbindungen) }\end{array}$ & $\begin{array}{l}\text { Einfachere Demontage und bessere Ver- } \\
\text { wertungsmöglichkeiten durch frühzeitige } \\
\text { Absprache zwischen Zulieferern, Abneh- } \\
\text { mern und Wettbewerbern hinsichtlich der } \\
\text { Materialien und Verfahren }\end{array}$ \\
\hline $\begin{array}{l}\text { Räumliche } \\
\text { Probleme }\end{array}$ & $\begin{array}{l}\text { Räumliche Verteilung der Altpro- } \\
\text { dukte unbekannt unterscheidet } \\
\text { sich u.U. deutlich von den unter- } \\
\text { nehmenseigenen gesteuerten } \\
\text { Distributionsorten }\end{array}$ & $\begin{array}{l}\text { Ausgleich durch gemeinsame Nutzung } \\
\text { von Einrichtungen verschiedener Anbieter }\end{array}$ \\
\hline $\begin{array}{l}\text { Zeitliche } \\
\text { Probleme }\end{array}$ & $\begin{array}{l}\text { Zeitlich unbekannter Anfall der } \\
\text { Altprodukte/kaum beeinflußbare } \\
\text { Frequenz des Anfalls }\end{array}$ & $\begin{array}{l}\text { Verstetigung durch Ausweitung der An- } \\
\text { zahl der Zugenommenen Altprodukte }\end{array}$ \\
\hline $\begin{array}{l}\text { Technologische } \\
\text { und wirtschaftli- } \\
\text { che Probleme }\end{array}$ & $\begin{array}{l}\text { Know how und finanzielle Res- } \\
\text { sourcen reichen zur Entwicklung } \\
\text { der notwendigen Technologien } \\
\text { nicht aus }\end{array}$ & $\begin{array}{l}\text { Verteilung von Risiko und Investitionen auf } \\
\text { mehrere Träger/ zügigere Verbesserung } \\
\text { der Technologien }\end{array}$ \\
\hline
\end{tabular}

Abb. 6: Vorteile von kooperativen Rücknahme- und Recyclingsystemen

(Quelle: in Anlehnung an: Hansen, U., Raabe, T., Die Gestaltung des Konsumgüterrecycling als strategische Netzwerke, a.a.O., S. 65)

Durch die gemeinsame Nutzung von Rücknahmestellen kann die räumliche Verteilung der Altprodukte ausgeglichen werden. Zudem besteht durch die gemeinsame Einrichtung von Rücknahmestellen ein geringeres individuelles Investitionsrisiko bei den Kooperationspartnern. Das Zeitproblem umfaßt den Tatbestand, daß die Altprodukte erst am Ende ihres Lebenszyklus wieder in den Kreislauf zurückgelangen und damit ggf. Produkte zum Recycling anfallen, die ohne Berücksichtigung der Recyclingerfordernisse konstruiert, produziert und abgesetzt worden sind. Diese produktbezogene Argumentation bei der Weiterverwendung gilt insofern insbesondere bei langlebigen Gebrauchsgütern, als eine Hochrüstung oder Reparatur bei Verschleißerscheinungen nach einer sehr langen Nutzungszeit

147 Eine weitere Option zur Verstetigung des Anfalls der Altprodukte wäre die "Risikostreuung“ durch unterschiedliche Bezugsquellen. Diese Option ist aber i.d.R. nur dann möglich, wenn nicht bereits schon alle Händler in das Rücknahme- und Recyclingsystem eingebunden sind. 
(bei Waschmaschinen beispielsweise bis zu 20 Jahren) nicht mehr sinnvoll ist. ${ }^{148}$ Die Ausweitung der Anzahl zurückgenommener Altprodukte durch eine Kooperation kann zu einer zeitlichen Verstetigung der anfallenden Mengen führen und damit einen geringeren Lagerhaltungsbedarf verursachen. Schließlich können die notwendigen technologischen und wirtschaftlichen Aufwendungen und Risiken, die durch die Entwicklung von Recyclingtechnologien entstehen, auf mehrere Systempartner verteilt werden. ${ }^{149}$

Mögliche Nachteile bei kooperativen Rücknahme- und Recyclingsystemen sind für Hersteller insbesondere im opportunistischen Verhalten ${ }^{150}$ der Kooperationspartner zu sehen. Die Unsicherheit im Hinblick auf die kommende Gesetzgebung sowie die geringe Transparenz und die Dynamik der sich noch konstituierenden Recyclingmärkte werden besonders hoch eingeschätzt und unterstützen damit einerseits die Gefahr des opportunistischen Verhaltens. ${ }^{151}$ Andererseits vermögen solche situativen Faktoren gleichzeitig kooperative Lösungen in Form von Netzwerkorganisationen zu fördern, da damit Transaktionskosten gesenkt und eine höhere Effektivität und Effizienz erreicht werden können. ${ }^{152}$ Zudem ermöglichen Netzwerke, den Kunden Produkte und Dienste zu offerieren, die der Hersteller alleine nicht anbieten könnte. ${ }^{153}$ Dadurch schaffen die Hersteller zusammen mit den Systempartnern eine gemeinsame Ausgangsposition zur Erhaltung und/oder Erzielung von Wettbewerbsvorteilen. Dabei kommt es zu einer Auflösung traditio-

148 Vgl. Stahel, W., Langlebigkeit von Gütern, Materialrecycling, Ressourceneffizienz, in: Kreislaufwirtschaft statt Abfallwirtschaft: optimierte Nutzung und Einsparung von Ressourcen durch Öko-Leasing und Servicekonzepte, Hockerts, K. u.a. (Hrsg.), Ulm 1994, S. $93 \mathrm{ff}$.

149 Vgl. Hansen, U., Raabe, T., Dombrowsky, B., Die Gestaltung des Konsumgüterrecycling als strategische Netzwerke, a.a.O., S. 64.

150 Neben dem Eigeninteresse der Partner kommt es zu opportunistischem Verhalten, das durch „Zurhilfenahme von List“ egoistische Interessen durchzusetzen versucht. Dadurch entstehen Transaktionskosten. Vgl. Meyer, M., Ökonomische Organisation der Industrie, a.a.O., S. 78.

151 Vgl. Hansen, U., Raabe, T., Dombrowsky, B., Die Gestaltung des Konsumgüterrecycling als strategische Netzwerke, a.a.O., S. 65.

152 Vgl. dazu die Ausführungen von Meyer, die den Beitrag der Transaktionskostentheorie zur Erklärung der Organisation von Hersteller-Zulieferersystemen erläutert. Vgl. Meyer, M., Ökonomische Organisation der Industrie, a.a.O., S. 96ff.

153 Vgl. Bronder, Chr. Pritzl, R., Strategische Allianzen zur Steigerung der Wettbewerbsfähigkeit, a.a.O., S. 27.

Die Rücknahme von Altprodukten wird z.T. als zusätzliche Dienstleistung angeboten. Dieses ist aus Konsumentensicht insbesondere dann von Bedeutung, wenn die Rückgabe sich aufgrund der Produktspezifika (Größe, Gewicht, Verschmutzung u.ä.) als schwierig gestaltet. Dieses gilt auch für die Rückgabe von PVC-Fenstern, weshalb die Rücknahme der alten Produkte daher schon häufig Bestandteil der Verhandlungsbasis bei Kaufverträgen ist. Vgl. FREIFallstudie, a.a.O. 
neller Branchengrenzen. ${ }^{154}$ Zum einen werden Hersteller z.T. in der Entsorgungswirtschaft und zum anderen werden Entsorgungsunternehmen als Hersteller und Lieferanten von Sekundärrohstoffen tätig. So hat sich die Wettbewerbsintensität in der Entsorgungswirtschaft durch den verstärkten Markteintritt branchenfremder Unternehmen wie Energieversorgungsunternehmen ${ }^{155}$ und die Vorwärts- und Rückwärtsintegration von Herstellern - genannt seien beispielhaft die Verwertungskonzepte der Automobilindustrie - stark erhöht. ${ }^{156}$

Die Gestaltung eines herstellereigenen Rücknahme- und Recyclingsystems erscheint demgegenüber dann sinnvoll, wenn bestimmte Bedingungen erfüllt sind. Wichtige Bedingungen liegen in der Verfügbarkeit der Recyclingtechnologie bei den Herstellern sowie im Vorhandensein der Abfälle in geeigneten Qualitäten, zeitlichen Zyklen sowie in ökonomisch vertretbaren Entfernungen. Darüber hinaus erscheint eine Prüfung notwenig, ob die Recyclingkosten die alternativen Kosten des Einsatzes primärer Inputfaktoren in Verbindung mit den internalisierten Kosten der Abfallentsorgung nicht überschreiten. ${ }^{157}$ Die Wiederverwendung von Altgeräten (z.B. der Verkauf von Gebrauchtcomputern am Markt oder im eigenen Unternehmen) oder die Weiterverwendung von Altgeräteteilen (Verkauf von Altcomputerchips an die Spielwarenindustrie) könnten zudem bei einem herstellereigenen Rücknahme- und Recyclingsystem einen zusätzlichen Ertrag erwirtschaften. ${ }^{158}$ Eine weitere Zielsetzung kann in der Erreichung positiver Imageeffekte liegen, die ausschließlich das eigene Unternehmen betreffen. Darüber hin-

154 Vgl. Bronder, Chr. Pritzl, R., Strategische Allianzen zur Steigerung der Wettbewerbsfähigkeit, a.a.O., S. 27.

Die Verwerter haben vielfältigere Aufgaben zu bewältigen als noch vor einigen Jahren, da neben den traditionellen Verwertungsaufgaben andere Aufgaben wie die Beschaffung von Abfällen, die Vermarktung von Recyclaten (Bsp. Kompost) und die Produktion (REA-Gips) hinzugekommen sind. Vgl. Pues, C., Marketing in der Entsorgungswirtschaft, in: IFM-News, Meffert, H. (Hrsg.), Heft 2, 1995, S. 13 f.

1551993 wurde beispielsweise eine Abfallverwertungsanlage in Augsburg in Betrieb genommen, die durch öffentliche Gebietskörperschaften und einen Partner der Energiewirtschaft gegründet wurde. Vgl. Drobek, F. K., Public-Private-Partnership am Beispiel der Abfallverwertungsanlage Augsburg, in: Energiewirtschaftliche Tagesfragen, Heft 5, 1994, S. $273 \mathrm{ff}$.

Die Volkswagen AG hat beispielsweise in Zusammenarbeit mit der PREUSSAG ein Rücknahme- und Recyclingsystem aufgebaut, welches auch „klassische“ Aufgaben der Entsorger wie die Trockenlegung und Demontage beinhaltet. Vgl. VW-Fallstudie, a.a.O.

157 Vgl. Hansen, U., Raabe, T., Dombrowsky, B., Die Gestaltung des Konsumgüterrecycling als strategische Netzwerke, a.a.O., S. 63.

Vgl. Fallstudie Siemens-Nixdorf, a.a.O.

Die Organisation herstellereigener Rücknahme- und Recyclingsysteme ist darüber hinaus besonders zu empfehlen, wenn für die Kontrolle des Verbleibs keine Mittler und damit "Margenkiller" erforderlich sind. Vgl. Fallstudie Rank Xerox, a.a.O. 
aus ist zu prüfen, inwieweit die Integration von Altproduktteilen in die Produktion von Neugeräten möglich und effektiv erscheint, da der Wiedereinsatz ganzer Altproduktteile in die Neuproduktion der Geräte (z.B. Einsatz von Altlüftern in Kopierern) eine eventuelle Werterhaltung ermöglicht und damit Neuproduktionskosten senkt. Dieses Vorgehen könnte insbesondere dann möglich sein, wenn die neuen Geräte nicht in das Eigentum des Besitzers übergehen, sondern lediglich die Nutzung der Geräte verkauft wird. ${ }^{159}$

Gründe für die Auslagerung weitestgehend aller mit dem Rücknahme- und Recyclingsystem zusammenhängenden Aktivitäten in Form eines Vertrages zwischen Herstellern und Verwertern werden zum einen in der Konzentration der Herstellerunternehmen auf die eigenen Kernkompetenzen gesehen. ${ }^{160}$ Diese Konzentration auf die eigenen Kernkompetenzen wird insbesondere bei kleinen und mittelständischen Unternehmen notwendig sein, da dort die Mitarbeiter noch weniger zeitliche Freiräume haben, zusätzliche Rücknahme- und Recyclingaufgaben zu übernehmen. Zum anderen ist es das Ziel einer (klassischen) vertraglichen Gestaltung des Rücknahme- und Recyclingsystems, eine möglichst preisgünstige und kompetente Lösung zu finden, die über den Markt geregelt wird. Die Herstellerunternehmen, die sich für eine solche "marktnahe" Lösung entscheiden, verlagern damit weitgehend die Risiken - und Chancen - auf die Systempartner, die mit der Gestaltung von Rücknahme- und Recyclingsystemen zusammenhängen. Im Rahmen solcher Systeme bestehen für Herstellerunternehmen zudem weniger die Notwendigkeit und der Anreiz, recyclinggerechte Produkte zu konzipieren, da das

159 Dieses Vorgehen ist bei Kopierern möglich, da diese insbesondere im gewerblichen Bereich nicht verkauft werden, sondern Kopierleistungen und Wartung Gegenstände der Verträge sind. Vgl. Fallstudie Rank Xerox, a.a.O.

Vgl. Fallstudie Bauknecht, a.a.O.

Die Konzentration auf die eigenen Kernkompetenzen wird zudem argumentativ genutzt, um die Bildung von Netzwerken zu begründen. Meyer schreibt, daß sich bei hoher Veränderlichkeit der Umwelt, Heterogenität der Produkte und hoher Wettbewerbsintensität ein Unternehmen auf seine speziellen Fähigkeiten in der Wertschöpfungskette konzentrieren muß, um sich Wettbewerbsvorteile verschaffen zu können. Dazu muß ein Unternehmen den hohen Grad an vertikaler Integration reduzieren und seine Kooperationsfähigkeit steigern. Vgl. Meyer, M., Ökonomische Organisation der Industrie, a.a.O., S. 125 f.

Padberg fordert ebenfalls die Orientierung am „Wertschöpfungssystem“ eines Netzes von Unternehmen statt einer Konzentrierung auf die Wertschöpfung des Einzelunternehmens, wobei sich jedes Unternehmen auf seine eigenen Kernkompetenzen konzentrieren kann. Danach muß das Optimierungsziel nicht mehr länger an der „individuellen Höchstleistung“, sondern an dem „Erfolg des gesamten“ (Netzwerk-), Teams“ ausgerichtet werden. Vgl. Padberg, A., Partnerschaften entlang der Wertschöpfungskette, Formelle und informelle Netze von Einzelunternehmen: Eigene Schwächen durch Zusammenarbeit mit anderen kompensieren, in: Blick durch die Wirtschaft, 11.3.1996, S. 11. 
Problem der Verwertung dem Entsorger komplett übertragen wird und diese teilweise weniger an Bauteilen als vielmehr an Materialfraktionen interessiert sind, wodurch ein Kostendruck auf die Hersteller entsteht. Ein weiteres Risiko bei dieser institutionellen Ausgestaltungsform von Rücknahme- und Recyclingsystemen besteht in der Schaffung von Konkurrenten in Form der Partner, die ihrerseits einen Gebrauchtmarkt für die Altprodukt(teil)e schaffen können. ${ }^{161}$

Angesichts der vielfältigen institutionellen Ausgestaltungsformen der Rücknahmeund Recyclingsysteme ist zu diskutieren, ob Rücknahme- und Recyclingsysteme überhaupt als ein in sich geschlossenes Implementierungsobjekt analysiert werden können. ${ }^{162}$

\subsection{Konstitutive Merkmale von Rücknahme- und Recyclingsystemen}

Eine alleinige Bestimmung des Implementierungsobjektes aus organisationstheoretischen Gesichtspunkten scheint für die Implementierungsproblematik bei Rücknahme- und Recyclingsystemen zu kurz zu greifen. Vielmehr ist eine Analyse hinsichtlich des Implementierungsverhaltens durchzuführen, aus der hervorgeht, ob von „einem“ Implementierungsobjekt gesprochen werden kann. Sofern sich ähnliche Verhaltensmuster in allen oben dargestellten institutionellen Ausgestaltungsformen von Rücknahme- und Recyclingsystemen nachweisen lassen, die im Zuge der Implementierung evident werden, kann von einem konsistenten Objektbezug ausgegangen werden. ${ }^{163}$

161 Bei der Kooperation des Haushaltsgeräteherstellers Bauknecht mit dem Verwerter Rethmann wird vertraglich festgelegt, daß Rethmann keine Altteile oder Gebrauchtgeräte vermarkten darf. Hintergrund dieser Vereinbarung ist die Verhinderung von Konkurrenz durch Rethmann, die durch die Entstehung eines Gebrauchtgerätemarktes Bauknecht schaden könnte. Vgl. Fallstudie Bauknecht, a.a.O.

Bei Automobilen ist das Interesse der Schrotthändler an den Bauteilen der Automobile inzwischen zu einem Problem für die Hersteller geworden. Die Schrotthändler kaufen alte PKW auf und bauen alle Teile aus, die mit Gewinn verkauft werden können. Z.T. werden diese in Katalogen nach Herstellern und Modellen geordnet als Gebrauchtteile am Markt angeboten. Dadurch erhalten die Hersteller z.T. nur noch die "komplett ausgeschlachteten“ Automobile zurück, die ihrerseits nur mit hohen Kosten (und geringen Wertschöpfungspotentialen) recycelt und entsorgt werden können.

162 Auch eine Strategie umfaßt i.d.R. als mehrdimensionales Konstrukt unterschiedliche Formen und Ausgestaltungsmöglichkeiten, wird aber in ihrer Gesamtheit als Implementierungsobjekt verstanden.

163 Es wird dabei jedoch berücksichtigt, daß die institutionellen Ausgestaltungsformen Einfluß auf das Verhalten der Unternehmen bei der Implementierung ausüben. Sie werden daher als Einflußfaktoren auf der Netzwerkebene erläutert. Vgl. Kapitel C 1.3.

Kirchgeorg beschreibt die Form der Durchsetzung von Strategien (individuell/kooperativ) als umweltorientierte strategische Option. Vgl. Kirchgeorg. M., Okologieorientiertes Unterneh- 
Die Annahme "eines" Implementierungsobjektes kann mit den konstitutiven Merkmalen begründet werden, die allen Rücknahme- und Recyclingsystemen zugrunde liegen. Die konstitutiven Merkmale von Rücknahme- und Recyclingsystemen umfassen nach Kirchgeorg - unter Einbeziehung von naturwissenschaftlichen Erkenntnissen - vier Charakteristika: ${ }^{164}$

1. Rückkopplung (Systembezug),

2. Geschlossenheit,

3. Objektbezug und

4. Zielbezug.

Der Begriff der Rückkopplung, der aus der Regelungstheorie stammt, beschäftigt sich mit der Struktur und Regelung von Systemen. Es liegt dann eine Rückkopplung vor, wenn der Output eines Systems wieder als Input zurückfließt, welches unter dem Begriff des Recycling zusammengefaßt wird. Hierbei sind zwei wichtige Einschränkungen hinsichtlich einer vollständigen Rückkopplung zu betrachten. Zum einen erfordert die Beschreibung der Rückkopplung eine genaue Abgrenzung des Systems und zum anderen sind bei ökologischen Systemen die Sätze der Thermodynamik zu berücksichtigen. ${ }^{165}$ Die Systemgrenzen sind von Relevanz, weil sie festlegen, welche Systeme (Unternehmen) ${ }^{166}$ welche Inputs und Outputs in die Betrachtung einbeziehen. Werden die Systemgrenzen weit gefaßt, muß beispielsweise bei den Auswirkungen der ökonomischen Tätigkeit auf den Ressourcenverbrauch im ökologischen System nicht nur die Produktion bei Herstellern und Zulieferern berücksichtigt werden, sondern auch die Primärgewinnung

mensverhalten: Typologien und Erklärungsansätze auf empirischer Grundlage, in: Meffert, $H$., Steffenhagen, H., Freter, H. (Hrsg.), Schriftenreihe Unternehmensführung und Marketing, Band 24, Wiesbaden 1990, S. 45. In der vorliegenden Arbeit wird im Gegensatz dazu die Frage nach der kooperativen versus der individuellen Lösung als Einflußfaktor auf die Implementierung betrachtet, da diese nicht das Verhalten bei der Um- und Durchsetzung selbst, sondern die Form der Rücknahme- und Recyclingsysteme betrifft.

$\mathrm{Vgl}$. zu den folgenden Ausführungen Kirchgeorg, der die vier konstitutiven Merkmale von Stoffkreisläufen detailliert herausarbeitet. Vgl. Kirchgeorg, M., Unternehmensstrategische Gestaltungsprobleme von Stoffkreisläufen, a.a.O., S. 44ff. Stoffkreisläufe hängen eng mit dem Begriff der Rücknahme- und Recyclingsysteme zusammen. Der Begriff der Rücknahme- und Recyclingsysteme weist jedoch auf die unternehmerische Umsetzung im Sinne einer Organisation der Stoffkreisläufe hin.

165 Vgl. auch in Kapitel A 3 die Erläuterung zu der Verknüpfung von ökonomischen und ökologischen Systemen.

166 Die Unternehmung kann als System gesehen werden und ist damit eine Menge von Elementen, die durch bestimmte Beziehungen miteinander verknüpft sind. Die Elemente lassen sich ihrerseits als Systeme bzw. Subsysteme des Systems "Betriebswirtschaft" interpretieren. Vgl. Heinen, E., Einführung in die Betriebswirtschaftslehre, 6. verb. und erw. Aufl., Wiesbaden 1977, S. 53. 
des Rohstoffs. Da in der vorliegenden Arbeit Rücknahme- und Recyclingsysteme im Fokus der Betrachtung stehen, sollen die Aktivitäten aller beteiligten Partner gemeinsam die relevanten Systemgrenzen darstellen, d.h. die Systemgrenzen variieren mit der Anzahl und Art (Branche, Wertschöpfungsstufe) der beteiligten Partner.

Bei den ökologischen Systemen stellen die thermodynamischen Sätze eine physische Einschränkung der Rückkopplung dar. Laut dem ersten thermodynamischen Satz können in einem geschlossenen System weder Energie noch Materie erzeugt oder vernichtet werden, d.h. alle Prozesse sind Umwandlungs- und nicht Verbrauchsprozesse. Demzufolge können eingesetzte Ressourcen nur umgewandelt und zerstreut werden, sie werden aber letztlich irgendwo im ökologischen System wieder auftauchen. Der erste Hauptsatz alleine deutet auf eine theoretisch unendlich mögliche Kreislaufführung hin und stimmt damit sehr optimistisch für die Implementierung von Rücknahme- und Recyclingsystemen. Dieser erste Satz wird jedoch durch den zweiten Hauptsatz der Thermodynamik wesentlich eingeschränkt. Dieser besagt, daß in einem geschlossenen System die Entropie einem Maximum zustrebt. ${ }^{167}$ Der Reinheitsgrad von Energiebeständen - und analog dazu von Ressourcenbeständen - wird aufgrund dieses Entropiesatzes immer geringer, womit die Qualität der Ressourcen und damit ihre Verwertbarkeit sinken. ${ }^{168}$

Die Sätze der Thermodynamik weisen gleichzeitig auf das zweite Merkmal der Geschlossenheit ${ }^{169}$ hin, nach dem offene, geschlossene und abgeschlossene Systeme unterschieden werden. Bei einem offenen System ist ein Austausch von Materie und Energie mit dem übergeordneten System möglich, während bei einem geschlossenen System nur ein Energieaustausch möglich ist. Bei einem abgeschlossenen System schließlich ist gar kein Austausch mehr möglich. Ein ökologisches System - wie die Erde - wird aufgrund der Sonneneinstrahlung und der Wärmeabgabe an den Weltraum als geschlossenes System eingestuft. Ein Rücknahme- und Recyclingsystem kann als offenes System charakterisiert werden, da

\footnotetext{
167 Die Entropie ist ein Maß für die Verfügbarkeit von Energie, wobei geringe Entropie konzentrierte Verfügbarkeit von Energie bedeutet. Vgl. Schreiner, M., Umweltmanagement in 22 Lektionen: ein ökonomischer Weg in eine ökologische Wirtschaft, 2. überarb. Aufl., Wiesbaden 1991, S. 64.

Vgl. Brenk, A., Moderne umweltpolitische Konzepte, a.a.O., S. 12ff.

169

Vgl. Kirchgeorg, M., Unternehmensstrategische Gestaltungsprobleme von Stoffkreisläufen, a.a.O., S. 51 .
} 
Materie und Energien in den Produktionsprozeß zurückgelangen können, wenn auch auf höherer Entropiestufe als zuvor.

Neben den physikalischen Einschränkungen der Geschlossenheit durch die thermodynamischen Sätze können noch weitere Grenzen von Rücknahme- und Recyclingsystemen unterschieden werden: ${ }^{170}$

- informationsbezogene,

- technische,

- ökologische,

- ökonomische Grenzen und

- psychologisch-verhaltensbezogene.

Informationsbezogene Grenzen bestehen darin, daß die Herstellerunternehmen z.T. keine Informationen darüber besitzen, wo die Altprodukte über den Distributionsprozeß hingelangt sind bzw. wo sich diese nach der Nutzung durch den Letztbesitzer befinden. ${ }^{171}$ Dieses Problem ist bei langlebigen Gebrauchsgütern besonders im privaten Bereich virulent, da dort zum einen die Zeitdauer der Nutzung durch die Unternehmen nicht prognostizierbar ist und zum anderen langlebige Gebrauchsgüter z.T. an andere private Personen weitergegeben werden. ${ }^{172}$ Technische Grenzen beschreiben die Nichtverfügbarkeit von technologischen Problemlösungen über den Reproduktionsprozeß des Abfalls. Hier ist an die begrenzte Demontierbarkeit und Trennung verschiedener Grundstoffe gedacht.

170 Rautenstrauch unterscheidet technische, ökonomische, ökologische und psychologische Grenzen (vgl. Rautenstrauch, C., Betriebliches Recycling, a.a.O., S. 91f.) während Meffert und Kirchgeorg thermodynamische, toxiologische, ökonomische, informationsbezogene, technologische Grenzen unterscheiden (vgl. Meffert, H., Kirchgeorg, M., Ziele und Strategien zur Gestaltung von produktbezogenen Rücknahme- und Recyclingsystemen - ausgewählte For-

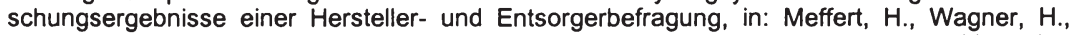
Backhaus, K. (Hrsg.), Arbeitspapier Nr. 107 der Wissenschaftlichen Gesellschaft für Marketing und Unternehmensführung, Münster 1997, S. 31).

171 Vgl. Kirchgeorg, M., Unternehmensstrategische Gestaltungsprobleme von Stoffkreisläufen, a.a.O., S. 54 .

172 Bei Neukauf eines Personalcomputers beispielsweise wird der alte Computer innerhalb der Familie oder im Freundeskreis für eine Sekundärnutzung weitergegeben (beispielsweise für Computerspiele). Ähnlich ist dieses bei Fernsehgeräten. Im Gegensatz dazu können Hersteller im gewerblichen Bereich leichter feststellen, wo sich die Altgeräte befinden. Dieses ist bei Computern im gewerblichen Bereich schon bereits aufgrund der Größe der Geräte - z.T. handelt es sich um Mainframes -, der hohen Anzahl oder aufgrund von Miet- oder Leasingverträgen möglich. 
Ökologische Grenzen hingegen umfassen den Sachverhalt, daß die Rücknahme und das Recycling einen Energie- und Materialeinsatz erfordern. Bei der Rücknahme fallen beispielsweise Transportenergien an, während bei einem Recycling häufig ein Zusatz von Primärstoffen zur Qualitätsverbesserung der Produktionsgrundlage notwendig wird. ${ }^{173}$ Eine genaue Analyse der ökologischen negativen Auswirkungen im Vergleich zu den ökologischen positiven Auswirkungen ist daher dringend erforderlich. Die ökologischen Grenzen hängen eng mit den ökonomischen Grenzen zusammen, und auch hier kann eine genaue Analyse der ökonomischen Kosten und des Nutzens dazu führen, eine weitergehende Rückkopplung zu unterlassen. Rücknahme- und recyclingbezogene Informationssuchkosten sowie Investitions- und Betriebskosten sind dabei den Erlösen des Recycling gegenüberzustellen. ${ }^{174}$

Schließlich sind die konsumentenseitig psychologisch-verhaltensbezogenen Grenzen der Geschlossenheit von Rücknahme- und Recyclingsystemen zu berücksichtigen, die im wesentlichen durch das beobachtbare Kaufverhalten sichtbar werden. Zum einen können exogene und zum anderen endogene Bestimmungsfaktoren erklären, warum Konsumenten Produkte aus Sekundärrohstoffen bzw. Produkte mit Gebrauchtgeräteteilen nicht kaufen (wollen). ${ }^{175}$ Ein exogener Bestimmungsfaktor, der besondere Relevanz hat, wird in wahrgenommenen Qualitätsbarrieren gesehen. D.h. Recyclingprodukte werden vom Konsumenten häufig als qualitativ minderwertig angesehen, so daß die Käufer oftmals mit einer gewissen Zurückhaltung reagieren. ${ }^{176}$ Diese Grenzen erklären, warum Rücknahme- und

\footnotetext{
173 Vgl. Rautenstrauch, C., Betriebliches Recycling, a.a.O., S. 92.

174 Gewinnsteigerungen können insbesondere durch Kosteneinsparungen im Rohstoffbereich oder im Kundendienst sowie durch den Verkauf von Gebrauchtgeräte(teilen) erzielt werden.

175 Monhemius erklärt die Divergenz zwischen Umweltbewußtsein und des Kaufverhalten anhand von exogenen und endogenen Bestimmungsfaktoren des ökologischen Kaufverhaltens. Die exogenen Variablen umfassen produktbezogene, situationsbezogene, allgemeine Umwelt und Demographie, während die endogenen Besimmungsfaktoren in intrapersonelle und interpersonelle Faktoren untergliedert werden. Unter den intrapersonellen Faktoren werden die Motivstruktur, das Risikoempfinden, lerntheoretische sowie dissonanztheoretische Erklärungen herangezogen. Vgl. zur detaillierten Analyse Monhemius, K. Chr., Umweltbewußtes Kaufverhalten von Konsumenten: ein Beitrag zur Operationalisierung, Erklärung und Typologie des Verhaltens in Kaufsituationen, Frankfurt am Main u.a. 1992. Diese Bestimmungsfaktoren sind folglich ebenfalls dafür verantwortlich, daß Rücknahme- und Recyclingsysteme nur bedingt geschlossen werden können.
} 
Recyclingsysteme nicht vollständig geschlossen werden können und damit Abfallbeseitigungsprozesse auch in einer Kreislaufwirtschaft ihre Berechtigung finden. ${ }^{177}$

Die Merkmale des Objektbezugs und mehrdimensionalen Zielbezugs wurden bereits bei den Bezugsebenen der Implementierung von Rücknahme- und Recyclingsystemen erläutert. ${ }^{178}$ In der vorliegenden Arbeit bilden Altprodukte, die nach ihrer Verwendungsphase durch den Konsumenten wieder als Inputfaktoren in den Produktionsprozeß von Herstellerunternehmen gelangen sollen, die Objekte der Untersuchung. ${ }^{179}$ Zudem verfolgen die Hersteller mit der Implementierung von Rücknahme- und Recyclingsystemen bestimmte Ziele. ${ }^{180}$

Da den Akteuren ein zielorientiertes Verhalten unterstellt werden kann, schließt sich eine Analyse der Ziele der Implementierung von Rücknahme- und Recyclingsystemen an.

\subsection{Ziele der Implementierung von Rücknahme- und Recyclingsystemen}

Die umfassenden Änderungen, die mit der Implementierung von Rücknahme- und Recyclingsystemen verbunden sind, können - insbesondere bei herstellereigenen Systemen - nur sukzessive erreicht werden und bedürfen einer sorgfältigen Zielund Maßnahmenplanung. ${ }^{181}$ Ziele werden dabei als Vorzugszustände definiert, die

177 Vgl. Meffert, H., Kirchgeorg, M., Ziele und Strategien zur Gestaltung von produktbezogenen Rücknahme- und Recyclingsystemen, a.a.O., S. 14.

$178 \mathrm{Vgl}$. zu folgenden Ausführungen Kirchgeorg, M., Unternehmensstrategische Gestaltungsprobleme von Stoffkreisläufen, a.a.O., S. 55ff.

179 Die Analyse kann auf mehreren Aggregationsebenen erfolgen, d.h. auf der Ebene von Produkten, von Bauteilegruppen und Komponenten oder auf der Ebene von stofflichen Fraktionen. Kirchgeorg spricht daher in diesem Zusammenhang von produktbezogenen Stoffkreisläufen. Vgl. Kirchgeorg, M., Unternehmensstrategische Gestaltungsprobleme von Stoffkreisläufen, a.a.O., S. 56.

Kirchgeorg weist darauf hin, daß sich durch diese Betrachtung mehrere Kreisläufe bei der Gestaltung eines Rücknahme- und Recyclingsystems für ein Altprodukte bilden können. Bei einem Automobil entstehen z.B. Komponentenkreisläufe für Reifen (runderneuerte Reifen), für Motorteile (Schrotthändler) oder Produktkreisläufe (Gebrauchtwarenmarkt). Vgl. Kirchgeorg, M., Unternehmensstrategische Gestaltungsprobleme von Stoffkreisläufen, a.a.O., S. 56.

Dieses grenzt zum einen diejenigen Systeme aus, die evolutorisch gewachsen und somit natürlich entstanden sind und zum anderen diejenigen Rücknahme- und Recyclingsysteme, die zwar künstlich entstanden sind, aber über anonyme Märkte koordiniert werden und daher keine herstellerbezogenen Zielsetzungen verfolgen. Die Mehrdimensionalität impliziert, daß neben ökonomischen und ökologischen Zielsetzungen auf der Unternehmensebene auch netzwerkbezogene und individualbezogene Zielsetzungen im Rahmen der vorliegenden Arbeit zu untersuchen sind.

181 Bei der „ad hoc“-Implementierung von Rücknahme- und Recyclingsystemen im Sinne einer emergenten Strategie werden ebenfalls zielorientierte Entscheidungen getroffen. Nur ist sich Irene Giesen-Netzer and Universität Münster - 978-3-631-75058-2 
durch den Einsatz bestimmter Maßnahmen erreicht werden sollen ${ }^{182}$. Implementierungsziele erfüllen wichtige Funktionen, da die Ziele bei operationaler Formulierung den jeweiligen Zielerreichungsgrad (Effizienz) ${ }^{183}$ aufzeigen können und eine hinreichende Ableitung der zu erfüllenden Teilaufgaben ermöglichen. Während der Implementierung dienen sie der Fortschrittskontrolle. ${ }^{184}$

Es existieren eigenständige Implementierungsziele, die zunächst unabhängig vom Implementierungsobjekt bestehen. Diese Implementierungsziele lassen sich neben dem Oberziel einer "hinreichenden Anwendung der Strategie" in Systemziele $^{185}$ und Durchführungsziele untergliedern (vgl. Abb. 7). ${ }^{186}$ Systemziele beschreiben die Erwartungen an die Ergebnisse der Implementierung der Strategie („Was soll erreicht werden?“), während sich die Durchführungsziele auf die Vorgehensweise („Wie soll vorgegangen werden?") beziehen. ${ }^{187}$

Die Systemziele werden in Durchsetzungsziele und in Umsetzungsziele unterteilt. Durchsetzungsziele streben eine Akzeptanzerzielung auf der Individualebene an, d.h. das Denken und Handeln der von der Implementierung betroffenen Mitarbeiter und Personen wird zu beeinflussen versucht. Hier steht die Beeinflussung der

der Akteur in der Regel nicht bewußt daß es sich um Entscheidungen mit strategischen Auswirkungen handelt.

182 Vgl. zu der Zieledefinition Meffert, H., Marketing: Grundlagen marktorientierter Unternehmensführung: Konzepte-Instrumente-Praxisbeispiele; mit neuer Fallstudie VW-Golf, 8., vollst. neubearb. und erw. Aufl., Wiesbaden 1998, S. 74. Heinen weist darauf hin, daß Ziele in drei Dimensionen zu konkretisieren sind: nach Inhalt, angestrebtem Ausmaß und dem zeitlichen Bezug. Vgl. Heinen, E., Einführung in die Betriebswirtschaftslehre, a.a.O., S. 99ff.

183 Die organisatorische Effektivität bezieht sich darauf, daß eine Organisation die "richtigen" Ziele anstrebt, während die organisatorische Effizienz den Grad der Zielerreichung beinhaltet. Vgl. Schulte-Zurhausen, M., Organisation, München 1995, S. 4. Die Effizienz als Output zu Input bemessen, wird z.B. in der Zielgröße der Kostenwirtschaftlichkeit deutlich. Beispielsweise soll mit möglichst geringen Geldeinheiten (Input) gewährleistet werden, daß eine bestimmte Anzahl von Altprodukten recycelt werden kann (Output). Mit der Erfassung der Effizienz wird demnach zwangsläufig auch die Effektivität berücksichtigt.

Zur Analyse der Effizienz der Implementierung von Rücknahme- und Recyclingsystemen vgl. Kapitel C 2.

Vgl. Kolks, U., Strategieimplementierung, a.a.O., S. $108 f$.

Vgl. dazu die Ausführungen zum Zusammenhang zwischen Implementierungszielen und implementierungsobjektspezifischen Zielen in diesem Kapitel.

Vgl. Meffert, H., Marketing-Management, a.a.O., S. 363 f.

Kolks unterscheidet in Systemziele und Vorgehensziele der Implementierung, die die gleiche inhaltliche Bedeutung wie die von Meffert umfassen. Vgl. Kolks, U., Strategieimplementierung, a.a.O., S. 108ff.

Vgl. Kolks, U., Strategieimplementierung, a.a.O., S. 109. 
Einstellung, die als Ursache von Widerständen identifiziert wird, im Vordergrund (Einstellungsakzeptanz). ${ }^{188}$

\begin{tabular}{|c|c|c|c|c|}
\hline & \multicolumn{3}{|c|}{ Oberziel } & \\
\hline & \multicolumn{3}{|c|}{ Hinreichender Anwendungsgrad der Strategie } & \\
\hline & 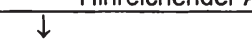 & \multicolumn{3}{|c|}{$\downarrow$} \\
\hline \multicolumn{2}{|c|}{ Systemziele } & \multicolumn{3}{|c|}{ Durchführungsziele } \\
\hline Durchsetzung & Umsetzung & ökonomische & technische & soziale \\
\hline $\begin{array}{ll}\text { - } & \text { Kennen und } \\
\text { Verstehen } \\
\text { - Können } \\
\text { - Wollen }\end{array}$ & $\begin{array}{l}\text { - Spezifizieren der } \\
\text { Strategie } \\
\text { - Anpassung der } \\
\text { Unternehmens- } \\
\text { potentiale } \\
\text { - Struktur } \\
\text { - Systeme } \\
\text { - Kultur }\end{array}$ & $\begin{array}{l}\text { - Sachkosten } \\
\text { - Personalkosten } \\
\text { - Fremd- } \\
\text { leistungen }\end{array}$ & $\begin{array}{l}\text { - Termine } \\
\text { - Ablauf }\end{array}$ & $\begin{array}{l}\text { - Stil } \\
\text { - Partizipations- } \\
\text { grad } \\
\text { - Kooperation }\end{array}$ \\
\hline
\end{tabular}

Abb. 7: Allgemeine Implementierungsziele

(Quellen: in ähnlicher Form in: Kolks, U., Strategieimplementierung, a.a.O., S. 111f. und Meffert, H., Marketing-Management, a.a.O., S. 364)

Die „Kennen und Verstehen-“ sowie die „Können-“ Zielsetzung ${ }^{189}$ werden aufgrund der Erlernbarkeit der Einstellung als Voraussetzung für die Durchsetzung angesehen. ${ }^{190}$ Dementsprechend sollen die Ziele, Maßnahmen, Erfolgswahrscheinlichkeit sowie die unmittelbaren Konsequenzen der Strategie für die einzelnen betroffenen Funktionsbereiche und Mitarbeiter offengelegt werden, um Unsicherheiten und eine damit verbundene kognitive und emotionale Ablehnung bezüglich der Neuerungen abzubauen. ${ }^{191}$ Die konative Komponente der Einstellung wird insbesondere in den Unterzielen des "Könnens" und "Wollens" der Strategie deutlich. Die Fähigkeiten müssen demnach durch die entsprechende Motivation zu einem zielorientierten Handeln führen. Darüber hinaus sollen die organisatorischen Bedingungen ein zielorientiertes Handeln ermöglichen. Damit sind bereits die Umsetzungsziele angesprochen, die die Umgebung des Implementierungsobjektes

188 Einstellung wird als (erlernte) innere Prädisposition eines Individuums definiert, auf bestimmte Stimuli der Umwelt relativ dauerhaft positiv oder negativ zu reagieren. Die Einstellung hat drei Komponenten: die affektive Komponente (gefühlsmäßige Einschätzung), die kognitive Komponente (subjektives Wissen über das Objekt) und die konative Komponente (Handlungstendenz, Verhaltensabsicht). Vgl. Meffert, H., Marketing, a.a.O., S. 75 und S. 113f. Vgl. dazu auch die Ausführungen über den Ebenenbezug der Implementierung in Kapitel A 3. Steinle fordert spezielle Informationsveranstaltungen (Kennen und Verstehen) zum Implementierungsobjekt, um eine Aktivierung und eine positive Einstellung der Mitarbeiter zu erreichen. Vgl. Steinle, C., Führungskonzepte und ihre Implementation, in: Kieser, A., u.a. (Hrsg.), Handwörterbuch der Führung, Stuttgart 1987, S. 586.

Vgl. Kolks, U., Strategieimplementierung, a.a.O., S. 110. 
(Unternehmensebene) betreffen, d.h. wie die Unternehmenspotentiale ${ }^{192}$ anzupassen sind, um die Neuerungen umsetzen zu können. Zudem ist mit der Spezifizierung der Strategie festzulegen, in welchem Ausmaß die zunächst global formulierten Vorgaben im Verlauf der Implementierung zu präzisieren sind. ${ }^{193}$

Durchführungsziele beschreiben die möglichst effiziente Vorgehensweise bei der Implementierung von Verhaltensplänen. Kolks teilt diese in ökonomische, technische und soziale Teilziele ein. ${ }^{194}$ Die ökonomischen Durchführungsziele beinhalten die monetären Sollvorgaben der Implementierung wie die Personal- und Sachkosten sowie die Kosten für Fremdleistungen z.B. für externe Berater. Technische Durchführungsziele sind Ablauf- und Terminziele, die im Rahmen der Strategiespezifizierung abgeleitet wurden. ${ }^{195}$ Der anzustrebende Führungsstil und der Grad der Partizipation der betroffenen Mitarbeiter sowie der Kooperationsgrad sind beispielhafte Ziele, die die sozialen Durchführungsziele bilden.

Werden Implementierungsziele isoliert von der zugrunde liegenden Konzeption betrachtet, sind sie für jedes Implementierungsvorhaben gültig und bleiben ohne die implementierungsobjektspezifischen Ziele zu allgemeingültig. Übertragen auf das Implementierungsobjekt des Rücknahme- und Recyclingsystems ergibt sich als oberstes Implementierungsziel die Realisation der Rücknahme- und Recyclingsysteme, d.h. eine hinreichende Anwendung bzw. Nutzung des geschaffenen Systems. Eine hinreichende Anwendung der Rücknahme- und Recyclingsysteme kann dabei (beispielhaft) nach mehreren Zielinhalten operationalisiert ${ }^{196}$ werden.

- Erschließung neuer Umsatzmöglichkeiten,

- Imagevorteile beim Kunden,

- Erfüllung von gesetzlichen Vorschriften,

- Ressourceneinsparungen,

192 Zur Analyse der Unternehmenspotentiale Struktur, Systeme und Kultur als Einflußfaktoren der Implementierung von Rücknahme- und Recyclingsystemen vgl. Kapitel C 1.4.2.3.

193 Vgl. Meffert, H., Marketing-Management, a.a.O., S. 364.

194 Vgl. Kolks, U., Strategieimplementierung, a.a.O., S. $112 f$.

185 Grimmeisen spricht in diesem Zusammenhang davon, daß neben die Effektivität der Implementierung im Sinne der Zielerreichung eine Effizienz der Implementierung im Sinne einer Wirtschaftlichkeit hinzutritt. Vgl. Grimmeisen, M., Perspektiven eines Implementierungscontrolling, in: zfo, Heft 5, 1995, S. 290.

196 Eine Operationalisierung der Ziele erfordert neben den Zielinhalten die Festlegung des Zielausmaßes, des Zeitbezugs und des Bereichsbezugs. Vgl. Meffert, Marketing, a.a.O., S. $81 \mathrm{ff}$. 
- Erfassungsquote der eigenen Altprodukte,

- Verwertungsquote der eigenen Altprodukte,

- Deponiermenge sowie

- Substitution von Problemstoffen u.a.

Angesichts dieser Operationalisierung einer "hinreichenden Anwendung“ stellt sich die Frage in welchem Verhältnis die Implementierungsziele ${ }^{197} \mathrm{zu}$ den Zielen des Rücknahme- und Recyclingsystems ${ }^{198}$ stehen. Die Kriterien zur Operationalisierung weisen darauf hin, daß der Anwendungsgrad gleichzeitig die "erfolgreiche" Implementierung von Rücknahme- und Recyclingsystemen impliziert. Folglich läßt sich eine erfolgreiche Implementierung auch an den Zielen der zugrunde gelegten Strategie - soweit operational definierbar - beurteilen. Implementierungsziele dienen damit dem Oberziel der „erfolgreichen“ Implementierung von Rücknahme- und Recyclingsystemen. ${ }^{199}$ Sie sind demnach Mittel zur Erreichung der Ziele, die die Akteure bei der Gestaltung des Rücknahme- und Recyclingsystems als erfolgreich definieren (Mittel-Zweck-Beziehung). ${ }^{200}$

Die Messung des Erfolgs im Sinne der zu erreichenden Zielsetzung der Implementierung kann daher zum einen in bezug auf die Implementierungsziele und zum anderen in bezug auf die implementierungsobjektspezifischen Ziele (ökonomischer und ökologischen Erfolg) erfolgen. Es läßt sich jedoch nur schwer ermitteln, aufgrund welcher Tatsache - der Konzeptionierung oder/und der Imple-

197 Kolks erläutert, daß die Systemziele in einem komplementären Verhältnis zu einander stehen. Demzufolge bestehen zum einen innerhalb der Durchsetzungsziele außer der Mittel-ZweckBeziehung in vertikaler Richtung eine Komplementarität zu den Umsetzungszielen. So setzt beispielsweise das „Können einer Strategie“ ein hinreichendes „Verstehen“ voraus. Zum anderen ist ein Mindestmaß an Kennen, Verstehen und Können Voraussetzung zur Erreichung der Umsetzungsziele. Diese beeinflussen dann wieder positiv die Durchsetzungsziele. Vgl. Kolks, U., Strategieimplementierung, a.a.O., S. $113 f$.

Ziele von Rücknahme- und Recyclingsystemen bilden den Ausgangspunkt für die gesamte Planung der Rücknahme- und Recyclingsysteme und sind Voraussetzung für die Kontrolle (Soll-Ist-Analysen).

199 Galbraith und Kazanjian definieren als Oberziel der Implementierung „The Concept of fit“, d.h. alle Dimensionen im Unternehmen wie die Struktur, die Systeme, die Ressourcenallokation und die Mitarbeiter und Führungskräfte müssen an den Strategiezielen ausgerichtet werden. Vgl. Galbraith, J. R., Kazanjian, R. K., Strategy Implementation, Structure, Systems and Process, 2. Aufl., St. Paul u.a. 1986, S. 108ff. Dieses bestätigt m.E. die Mittel-Zweck-Beziehung zwischen Implementierungszielen und Strategiezielen.

200 Eine Mittel-Zweck-Beziehung geht von der Annahme aus, daß sich die Ziele ersetzen können und eine Komplementaritätsbeziehung vorliegt. Aussagen über Komplementarität sind nur unter Bezugnahme auf das Entscheidungsfeld und den Entscheidungsträger möglich. Vgl. Heinen, E., Einführung in die Betriebswirtschaftslehre, a.a.O., S. 108f. 
mentierung - der Erfolg oder Mißerfolg bei der Gestaltung der Rücknahme- und Recyclingsysteme eingetreten ist (Zurechnungsproblem). Danach kann sich der Erfolg des zugrunde liegenden Rücknahme- und Recyclingsystems erst zieladäquat zeigen, wenn die Implementierung entsprechend erfolgt ist. Andererseits fehlt ohne eine Definition der Ziele des Rücknahme- und Recyclingsystems die Orientierung für die Implementierung.

Implementierungsziele können auf der Netzwerk-, der Unternehmens- und der Individualebene festgelegt werden. Die Zielbildung erfolgt im Rahmen eines politischen Prozesses, wobei verschiedene Interessen die Ziele beeinflussen. Dabei kann es zu Zielsetzungsdefekten kommen, weil aufgrund von mehreren konfiktären Zielsetzungen keine operationale, eindimensionale Zielfunktion existiert. ${ }^{201}$ Die Komplexität des Zielbildungsprozesses bei der Implementierung von Rücknahmeund Recyclingsystemen wird darüber hinaus dadurch erhöht, daß je Ebene unterschiedliche Zielhierarchien ${ }^{202}$ bzw. -systeme vorliegen. ${ }^{203}$

Um die Durchsetzungsziele idealerweise zu erreichen, sollte das Zielsystem des Individuums mit den Unternehmenszielen sowie die Unternehmensziele mit den Netzwerkzielen in Übereinstimmung gebracht werden. Eine hohe Funktionsfähigkeit eines Systems wäre demzufolge nur dann gewährleistet, wenn das "Eigeninteresse“ der Subsysteme so gestaltet ist, daß ihr Zusammenwirken den gewünschten Gesamteffekt sichert. ${ }^{204}$ Dieses gilt in gleicher Weise innerhalb der Suborganisationssysteme (Unternehmen) sowie für die Mitarbeiter. Es ist darüber hinaus wichtig, die Ziele und Bedeutung der individuellen Beiträge zur Zielerreichung für die Mitarbeiter transparent zu machen, da erst dann der einzelne Mitarbeiter abschätzen kann, welche Handlungen für den Gesamtzusammenhang angemessen und effizient sind. Dieses ist aus motivationstheoretischer Sicht not-

201 Vgl. Adam, D., Planung und Entscheidung: Modelle - Ziele - Methoden, mit Fallstudien und Lösungen, 4., vollst. überarb. u. erw. Aufl., Wiesbaden 1996, S. $11 f$.

Maslow stellt die Bedürfnishierarchie beim Individuum vor, nach der zunächst primäre (physiologische) Bedürfnisse befriedigt werden müssen, bevor sekundäre Bedürfnisse (Sicherheit, soziale Bedürfnisse, Wertschätzung, Selbstverwirklichung) befriedigt werden wollen. Diese Vorgehensweise ist zwar nur ein Idealbild, veranschaulicht jedoch in übersichtlicher Weise die Bandbreite der Bedürfnisse. Vgl. Heinen, E., Betriebswirtschaftliche Führungslehre, ein entscheidungsorientierter Ansatz, in: Heinen, E. (Hrsg), Entscheidungsorientierte betriebswirtschaftliche Studien, Bd. 2, Wiesbaden 1978, S. 27. systeme der beteiligten Partner möglichst vor der Gründung desselben in Übereinstimmung gebracht, während dieses bei den anderen Formen während der Implementierung geschieht.

Vgl. Wottawa, H., Gluminski, I., Psychologische Theorien für Unternehmen, a.a.O., S. 49. 
wendig, da der Anreiz ein bestimmtes Verhalten zu zeigen größer ist, desto eher die eigenen Ziele erreicht werden können. ${ }^{205}$

\begin{tabular}{|c|c|}
\hline Bezugsebenen & Implementierungsziele von Rücknahme- und Recyclingsystemen \\
\hline $\begin{array}{c}\text { Individualebene } \\
\text { Vertikale } \\
\text { Durchsetzungsziele }\end{array}$ & $\begin{array}{l}\text { - Kennen und Verstehen } \\
\text { - Denken in Kreisläufen } \\
\text { - Können } \\
\text { - Eigenengagement } \\
\text { - Verbesserungsvorschläge } \\
\text { - Wollen } \\
\text { - Zufriedenheit mit dem System } \\
\text { - Wertschätzung von Altprodukt(teilen) } \\
\text { - Wertschätzung der mit der Umsetzung von Rücknahme- und } \\
\text { Recyclingsystem betrauten Mitarbeiter }\end{array}$ \\
\hline $\begin{array}{l}\text { Unternehmensebene } \\
\text { Umsetzungsziele }\end{array}$ & $\begin{array}{l}\text { - Spezifizierung der Konzeption des Rücknahme- und Recyclingsystems } \\
\text { - Stellenwert der Ableitung operativer Maßnahmen } \\
\text { - Rücknahme- und recyclingsystemkonforme Anpassung der } \\
\text { - Struktur } \\
\text { - Systeme } \\
\text { - Kultur }\end{array}$ \\
\hline $\begin{array}{l}\text { Netzwerkebenehori- } \\
\text { zontale } \\
\text { Durchsetzungsziele }\end{array}$ & $\begin{array}{l}\text { - Zufriedenheitsgrad aller Systempartner } \\
\text { - Vertrauensvolle Zusammenarbeit } \\
\text { - Konfliktfreie Zusammenarbeit }{ }^{206}\end{array}$ \\
\hline
\end{tabular}

Abb. 8: Implementierungsziele von Rücknahme- und Recyclingsystemen

Abbildung 8 zeigt die Implementierungsziele ${ }^{207}$ von Rücknahme- und Recyclingsystemen auf. Auf der Individualebene sind vertikale Durchsetzungsziele zu operationalisieren. Es kann von vertikalen Durchsetzungszielen gesprochen werden, weil die Implementierung von Rücknahme- und Recyclingsystemen entlang der Linie innerhalb des Unternehmens stattfindet. ${ }^{208}$ Das Element „Kennen und Ver-

205 Wottawa und Gluminski sprechen von der Zielkongruenz zwischen Mitarbeitern und Unternehmen als ein nicht vollständig erreichbares, aber stets anzustrebendes Ideal. Vgl. Wottawa, H., Gluminski, I., Psychologische Theorien für Unternehmen, a.a.O., S. 54ff.

Heinen erläutert die Anreiz-Beitrags-theoretischen Grundlagen der Organisationsmitgliedschaft, nach denen Menschen Mitglieder von Organisationen werden, da diese Beteiligungsanreize bieten. Persönliche Ziele, wahrgenommene Verhaltensmöglichkeiten und vermutete Auswirkungen sind dabei die Bestimmungsfaktoren, die zu rollenkonformem Verhalten führen. Vgl. Heinen, E., Betriebswirtschaftliche Führungslehre, a.a.O., S. 23f.

206 Bei der Zielsetzung der konfliktfreien Zusammenarbeit wird die produktive Wirkung von Konflikten zur Problemlösung zunächst vernachlässigt. Vgl. Wottawa, H., Gluminski, I., Psychologische Theorien für Unternehmen, a.a.O., S. $115 \mathrm{ff}$.

207 Auf Durchführungsziele wird im weiteren nicht eingegangen, da diese nicht scharf genug von den Zielen des Rücknahme- und Recyclingsystems zu trennen sind, die noch in diesem Kapitel erläutert werden. Die Kostenwirtschaftlichkeit von Rücknahme- und Recyclingsystemen überschneidet sich beispielsweise mit den ökonomischen Durchführungszielen.

208 Die Richtung der Implementierung wird innerhalb der Hierarchieebene genauer analysiert. Vgl. Kapitel B 2.3. 
stehen" kann dabei mit der Denkhaltung der Mitarbeiter in bezug auf das Rücknahme- und Recyclingsystem (von der Durchlaufwirtschaft zur Kreislaufwirtschaft) gemessen werden. ${ }^{209}$ Eigenengagement und die Vorlage von Verbesserungsvorschlägen sagen etwas über das "Können" der Mitarbeiter aus. „Wollen“ schließlich kann mit der Zufriedenheit mit dem System sowie der Wertschätzung von Altprodukt(teilen) und der Personen, die mit dem Umsetzungsprozeß betraut sind, operationalisiert werden. Eine zweifelsfreie Abgrenzung dieser Zielinhalte ist angesichts der Interdependenzen zwischen den Durchsetzungszielen jedoch kaum möglich. ${ }^{210}$

Die unternehmensbezogenen Umsetzungsziele können durch die Umsetzung von Strategien in operative Maßnahmen sowie durch den Stellenwert der Anpassung der Organisationsstruktur, der Unternehmenskultur und der Informationssysteme gemessen werden. Durch Erfüllung dieser Aufgaben wird diese Zielsetzung konkretisiert. Daher werden die umsetzungsbezogenen Ziele durch die Analyse der Aufgaben ${ }^{211}$ besser beschrieben.

Schließlich sind horizontale, netzwerkbezogene Durchsetzungsziele zu untersuchen. Aufgrund der hohen Anzahl kooperativer Systeme bei der Implementierung von Rücknahme- und Recyclingsystemen nimmt die Netzwerkebene einen wichtigen Stellenwert ein. Die Zielvorstellungen der beteiligten Unternehmen müssen in Einklang gebracht und Aufgaben für die einzelnen Unternehmen müssen gemeinschaftlich spezifiziert werden. Diese Zusammenarbeit findet i.d.R. auf den gleichen Hierarchieebenen statt, weshalb dieses als horizontale Durchsetzung eingeordnet werden kann.

209 Die empirische Analyse der Ziele erfolgt im Kapitel C 2, das den Erfolg von Rücknahme- und Recyclingsystemen verdeutlicht.

210 Über die Interdependenzen zwischen den Elementen „Kennen und Verstehen“, „Können“ und "Wollen“ gibt es eine Vielzahl von Motivationstheorien, die zum einen zu erklären versuchen, welche spezifischen Faktoren den Menschen zur Arbeit motivieren (Inhaltstheorien, z.B. Maslow 1943, Herzberg 1966) und zum anderen sich auf die Frage konzentrieren, wie Arbeitsverhalten gesteuert wird (Prozeßtheorien, z.B. Vroom 1964, Lawler 1973).

211 Vgl. Kapitel B 2.4.1.

Vgl. zu einer ähnlichen Feststellung und Vorgehensweise vgl. Kolks, U., Strategieimplementierung, a.a.O., S. 111 f. 


\section{Wendet man sich den implementierungsobjektspezifischen Zielsetzungen zu,} sind ökonomische, psychographische und ökologische Ziele ${ }^{212}$ für Rücknahmeund Recyclingsysteme zu unterscheiden. ${ }^{213}$ Die ökonomischen Zielsetzungen ${ }^{214}$ werden in monetären Größen gemessen. In der Literatur wird bei der Gestaltung von Rücknahme- und Recyclingsystemen überwiegend die Kostenwirtschaftlichkeit diskutiert, ${ }^{215}$ während Wertschöpfungsdiskussionen oder die Thematisierung von Erlöserzielung durch die Rücknahme und das Recycling von Altprodukten eher die Ausnahme darstellen. Grund hierfür besteht in der noch immer recht groBen Unsicherheit der Hersteller darüber, welche Auswirkungen umweltschutzbezogene Tätigkeiten und damit auch die Implementierung von Rücknahme- und Recyclingsystemen auf die ökonomischen Zielgrößen besitzen. ${ }^{216}$ Dieser Unsicherheit kann durch eigene Erfahrungsziele entgegengewirkt werden, so daß auch Erfahrungsziele als ökonomische Zielsetzung eine Rolle spielen können.

Schließlich sind psychographische Ziele mit der Implementierung von Rücknahme- und Recyclingsystemen verbunden. Diese beinhalten insbesondere den

212 Meffert unterscheidet ökonomische und psychographische Marketingziele. Ökonomische Ziele sind monetäre Zielgrößen, die sich auf beobachtbare Ergebnisse von Kaufentscheidungsprozessen zurückführen lassen. Psychographische Ziele hingegen knüpfen an mentalen Prozessen des Käufers an und haben eine psychische Wirkung. Vgl. Meffert, H., Marketing, a.a.O., S. $74 f$.

213 Vgl. zu einer Darstellung der Ziele zur Gestaltung von Rücknahme- und Recyclingsystemen Meffert, H., Kirchgeorg, M., Ziele und Strategien zur Gestaltung von produktbezogenen Rücknahme- und Recyclingsystemen, a.a.O., S. 13ff.

Zur Messung des Erfolgs wird in unternehmensbezogenen und netzwerkbezogenen Erfolg unterteilt, da davon ausgegangen werden kann, daß das Gesamtsystem weitere und z.T. andere Erfolgsgrößen anstrebt. Beispielhafte Ziele auf der Netzwerkebene sind: Konkurrenzfähigkeit des Gesamtsystems, Ausreichende Anreize für alle Systempartner, Kostenwirtschaftlichkeit des Gesamtsystems, Auslastung der Systemkapazitäten, Kostenwirtschaftlichkeit des Gesamtsystems, Gutes Image des Rücknahme- und Recyclingsystems bei den Kunden. Diese differenzierte Betrachtung erfolgt in Kapitel C 2.

214 Auch Galbraith und Kazanjian ziehen „klassische“ ökonomische Zielsetzungen zur Beurteilung der Strategieimplementierung heran wie z.B. Return on assets, Return on investment und Wachstum. Vgl. Galbraith, J. R., Kazanjian, R. K., Strategy Implementation, a.a.O., S. $28 f f$.

215 Die Diskussion ging dabei zunächst weitestgehend davon aus, wie die Entsorgungskosten möglichst minimiert werden konnten. Vgl. Steven, Effizienz von betrieblichen Entsorgungsprozessen, in: Betriebswirtschaftliche Forschung und Praxis, Heft 2, 1992, S. 120. Horneber erläutert daher, daß die Kostenbetrachtung allein nicht ausreicht, sondern auch die erzielbaren Erlöse in die Überlegungen Eingang finden müssen. Vgl. Horneber, M., Innovatives Entsorgungsmanagement, a.a.O., S. 200f. Ähnlich argumentiert auch Stölzle, W., Ansätze zur Erfassung der Umweltschutzkosten in der betriebswirtschaftlichen Kostenrechnung, in: Zeitschrift für Umweltpolitik und Umweltrecht, Heft 4, 1990, S. 394.

216 Je nach wirtschaftlicher Lage einerseits und Schärfe der umweltpolitischen Anforderungen andererseits stehen bei der Beziehung zwischen Umweltschutz und wirtschaftlicher Entwicklung mehr konfliktbetonende oder mehr harmonieorientierte Argumente im Vordergrund. Vgl. Der Rat von Sachverständigen für Umweltfragen, Umweltgutachten 1996, S. 86f. 
Wunsch der Hersteller, sich in den Augen der Konsumenten als Unternehmen zu profilieren, welches sich der umweltbezogenen Produktverantwortung bewußt ist und sich entsprechend verhält. Damit sind Imageziele, die mit der Gestaltung von Rücknahme- und Recyclingsystemen verbunden sind, angesprochen, die gleichzeitig die Legitimität des unternehmerischen Handelns abzusichern helfen. Voraussetzung hierzu ist auch die Legalität des unternehmerischen Handelns, die durch die Zielsetzung der Vorbeugung gegenüber gesetzlichen Rücknahmeverordungen zum Ausdruck kommt.

Bei ökologischen Zielsetzungen werden input- und outputbezogene Zielgrößen unterschieden, die sich an den Oberzielen der Ressourcenschonung und der Abfallverringerung orientieren. ${ }^{217}$ Auf der Herstellerebene können diese Oberziele weiter differenziert werden. Neben den Erfassungszielen können insbesondere Verwendungs- und Verwertungsziele angestrebt werden. D.h. die Hersteller streben auf der einen Seite eine Erhöhung (z.B. im Vergleich zum Vorjahr) der anzahl- oder gewichtsmäßigen Rückführung der Altprodukte in das Rücknahme- und Recyclingsystem an. Auf der anderen Seite sollen die Altprodukte zu höheren Anteilen im eigenen Unternehmen (im Kundendienst oder in der Neuproduktion) eingesetzt werden oder dem Gebraucht(stoff)markt zur Verfügung gestellt werden, womit gleichzeitig eine Deponierung der Altprodukte verringert wird.

Werden die empirischen Ergebnisse bezüglich der implementierungsobjektspezifischen Zielsetzungen betrachtet, zeigt die Abb. 9, daß bei der Einrichtung von Rücknahme- und Recyclingsystemen die ökonomischen Ziele ${ }^{218}$ mit großem Abstand wichtiger sind als die ökologischen Ziele. Dieses erscheint vor dem Hintergrund der Erwerbswirtschaftlichkeit der betrachteten Hersteller verständlich, bei der stets die monetäre Sicherung im Vordergrund stehen muß, um in deren Rahmen ökologische Zielsetzungen verfolgen zu können. Innerhalb der ökonomischen Ziele steht die Kostenwirtschaftlichkeit an erster Stelle. ${ }^{219}$ Dieses ist ins-

$217 \S 1 \mathrm{KrW}-/ \mathrm{AbfG}$ "Zweck des Gesetzes ist die Förderung der Kreislaufwirtschaft zur Schonung der natürlichen Ressourcen und die Sicherung der umweltverträglichen Beseitigung von $A b$ fällen."

218 Die psychographischen und ökonomischen Zielsetzungen wurden unter den ökonomischen zusammengefaßt, um die Bedeutung der ökologischen Ziele besser verdeutlichen zu können.

219 Damit erfährt die Diskussion um die hohe Bedeutung der Kostenwirtschaftlichkeit in der Literatur eine realwissenschaftliche Begründung. Vgl. Meffert, H., Kirchgeorg, M., Ziele und Strategien zur Gestaltung von produktbezogenen Rücknahme- und Recyclingsystemen, a.a.O., S. 17. 
besondere vor dem Hintergrund verständlich, daß es sich in der Befragung ausschließlich um solche Unternehmen handelt, die freiwillige Lösungen realisiert haben und sich mit dieser Internalisierung der Abfallentsorgungskosten dem international hohen Kostenwettbewerb mit Unternehmen ohne internalisierte Abfallentsorgungskosten-Komponente stellen müssen.

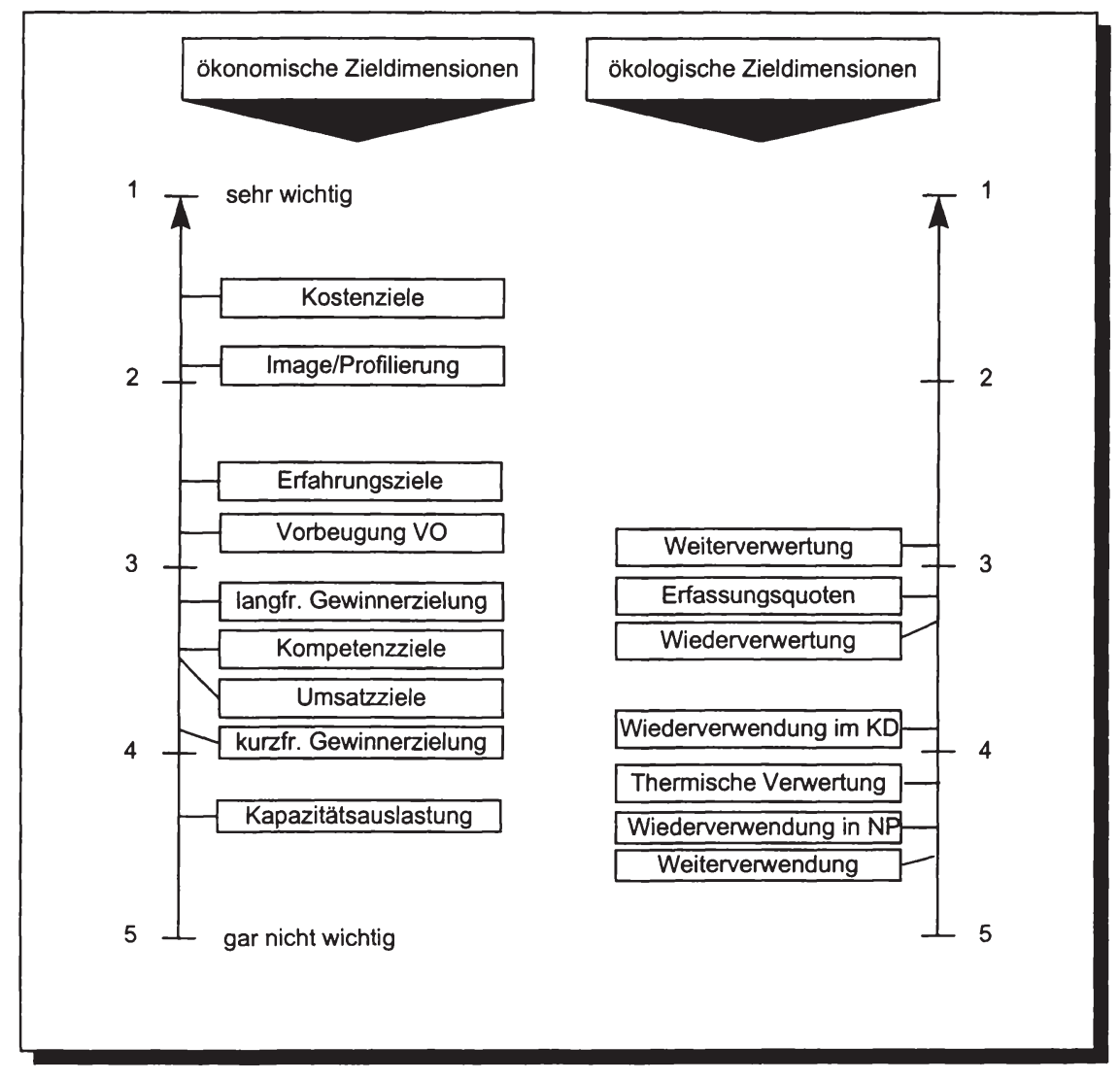

Abb. 9: Ökonomische und ökologische Ziele von Rücknahme- und Recyclingsystemen (Quelle: Kirchgeorg, M., Giesen-Netzer, I., WSK-Infobrief, Institut für Marketing, Projektgruppe Wertschöpfungskreisläufe (Hrsg.), Ausgabe 3, 1996, S. 2)

Nach den Kostenzielen wird den markt- und wettbewerbsgerichteten Image- und Profilierungszielen eine hohe Bedeutung beigemessen. Die Hersteller erhoffen sich über die Rücknahme und das Recycling der Altgeräte ein Verbesserung des 
Marken- und Unternehmensimage bei den Käufern und damit Chancen zur Wettbewerbsprofilierung. ${ }^{220}$ Die Hersteller können mit dieser Leistung für den Kunden einen Zusatznutzen erbringen, dem sie damit das „Entsorgungsproblem" abnehmen und gleichzeitig das Gefühl geben, verantwortungsvoll zu handeln. ${ }^{221}$

Wird ein Rücknahme- und Recyclingsystem als Branchenlösung organisiert, ist die Profilierung für die einzelnen Hersteller im Wettbewerb weniger gegeben. Doch auch in diesem Fall kann ein solches System von hoher Relevanz sein, um Goodwill-Potentiale und damit Imageziele bei Anspruchsgruppen aufzubauen und abzusichern, wie auch am Beispiel des PVC deutlich wird. ${ }^{222}$ In der PVC-FensterBranche ist die Einrichtung von Rücknahme- und Recyclingsystemen sogar notwendig geworden, um den Absatz zu sichern. ${ }^{223}$ In solchen Fällen kann die Gestaltung eines Rücknahme- und Recyclingsystems Signalfunktion haben und den Anspruchsgruppen die Bemühung der Hersteller um den Umweltschutz verdeutlichen und damit die Marktattraktivität erhöhen. ${ }^{224}$

Die nächsten wichtigen ökonomischen Ziele sind Erfahrungsziele und Vorbeugung gegenüber den gesetzlichen Rücknahmeverordnungen. Die Erfahrungsziele beinhalten den Wunsch der Hersteller, aus dem Umgang mit den Altprodukten zu lernen. Dieses kann dann zu Kostenvorteilen in der Demontage und Produktion

220 Bisher finden markt- und wettbewerbsbezogene Zielsetzungen wenig Berücksichtigung, die damit auf eine Vernachlässigung des Erfolgspotentials solcher Systeme hindeutet. Vgl. Meffert, H., Kirchgeorg, M., Ziele und Strategien zur Gestaltung von produktbezogenen Rücknahme- und Recyclingsystemen, a.a.O., S.15f.

Je nachdem, wie die Rücknahme für den Konsumenten bepreist (Rücknahme kostenlos oder kostenpflichtig oder Rücknahmeprämien) ist, ergeben sich zusätzliche Differenzierungspotentiale.

221 Bei der Vergabe von öffentlichen Aufträgen wird die Zusatzleistung der Rücknahme und des Recycling bereits teilweise honoriert, d.h. diejenigen Hersteller werden bevorzugt, die ein Rücknahme- und Recyclingsystem vorweisen können. Siemens-Nixdorf konnte diese Tatsache bei dem Verkauf von Personalcomputern an Behörden als Wettbewerbsvorteil nutzen.

Erkenntnisse über die krebserzeugende Wirkung des PVC Vorproduktes Vinylchlorid (VC) löste in den $80 \mathrm{er}$ Jahren eine direkte Betroffenheit bei allen PVC verarbeitenden Herstellern aus. Inzwischen konnte der Restgehalt bei PVC-Fenstern und fast allen anderen PVC-Produkten teilweise bis unter die Nachweisgrenze gesenkt werden. Trotzdem wird PVC auch heute noch als krebserregender Stoff in der Offentlichkeit diskutiert.

So kam es in den 80er Jahren wegen starker Vorbehalte beim Einsatz von PVC-Fenstern in der Verwendungs- und Entsorgungsphase in zahlreichen Verwaltungen und Kommunen zu Bauförderungsstops bezüglich der Verwendung von PVC in öffentlichen Gebäuden und im sozialen Wohnungsbau (z.B. Hessen, Berlin). Die Vergabe von Fördermitteln wurde für PVCFenster später wieder freigegeben, weil die PVC-Hersteller ein Recycling garantieren, eine Rücknahmegarantie (Berlin) aussprechen und die Verwendung von Schwermetallen ausschließen. Vgl. FREI- Fallstudie, a.a.O.

Vgl. FREI- Fallstudie, a.a.O. 
führen, wenn die Unternehmen eigene Systeme einführen. Werden Mischpreise im Rahmen von Kooperationen vereinbart, haben diejenigen Unternehmen, die auf besondere Recyclingfähigkeit der Produkte Wert legen, allerdings keinen besonderen Kostenvorteil, da z.B. der individuelle produktbezogene Zeitvorsprung bei der Demontage nicht dem herstellenden Unternehmen zukommt, sondern allen Herstellern gemeinsam. Das Ziel der Vorbeugung gegenüber Rücknahmeverordnungen deutet darauf hin, daß die Herstellerunternehmen ihre Freiheitsgrade im ökologiebezogenen Handeln bewahren wollen, um möglichst effiziente Lösungen zu finden.

Erst danach folgen die langfristigen Gewinnziele, Kompetenz- und Umsatzziele. Dieses mag damit zusammenhängen, daß sich die Rücknahme- und Recyclingsysteme noch relativ am Anfang ihrer Entwicklung befinden. Die Kapazitätsauslastung schließlich ist das öko5nomische Ziel, welches die geringste Bedeutung aufweist. Dieses hängt mit der überwiegend kooperativen Gestaltung der Rücknahme- und Recyclingsysteme zusammen, bei der die Aufgaben der Verwertung überwiegend an Entsorger abgegeben werden und daher kein individueller herstellerspezifischer Vorteil generiert werden kann.

Wendet man sich den ökologischen Zielsetzungen von Rücknahme- und Recyclingsystemen zu, die die Arten des Recycling widerspiegeln, zeigt sich, daß diese lediglich eine mittlere bis geringe Bedeutung bei Herstellerunternehmen einnehmen. Dieses birgt die Gefahr, daß Unternehmen möglicherweise Rücknahme- und Recyclingsysteme bevorzugen, die "nur" kostenwirtschaftlich sind, sich aber langfristig suboptimal in bezug auf die ökologischen Ziele darstellen. ${ }^{225}$ Eindeutige Dominanz haben die Verwertungsziele, wobei der Einsatz von Sekundärrohstoffen außerhalb des eigenen Unternehmens präferiert wird. Die Erzielung höherer Erfassungsquoten ist ein relativ wichtiges Ziel im Rahmen der ökologischen Zielsetzungen. Dieses mag darin begründet liegen, daß zum einen die bisher

225 Im Kreislaufwirtschaftsgesetz ist vorgesehen, daß bei „wirtschaftlicher Unzumutbarkeit“ und "technischer Unmöglichkeit" sich die Prioritätenreihenfolge (vermeiden vor verwerten vor beseitigen) ändern kann. §5 Abs. $4 \mathrm{KrW}-/$ AbfG. Dieses birgt m.E. die Gefahr, daß die Unternehmen keinen Anreiz sehen, die technischen Verfahren oder die Wirtschaftlichkeit zu verbessern, da sie damit den einfacheren Weg der „end-of-pipe“- Lösungen gehen können, d.h. die Hersteller übertragen die Aufgabe der Verwertung an einen Entsorger, der die Altprodukte zerlegt und zerkleinert, um die Sekundärrohstoffe am Markt weiter zu veräußern. 
(noch) niedrigen Quoten von 5-15\% ${ }^{226}$ es kaum ermöglichen, von geschlossenen Systemen zu sprechen und Erfahrungen bezüglich ökonomischer und ökologischer Auswirkungen zu machen. Zum anderen sind hohe Erfassungsquoten Voraussetzung für die Zufriedenheit aller beteiligten Partner, da der relativ hohe Organisations- und Koordinationsaufwand gerechtfertigt werden muß. Verwendungsziele von aufbereiteten Altgeräteteilen spielen bisher kaum eine Rolle. Wenn solche Ziele verfolgt werden, werden diese Altteile am ehesten im Kundendienst eingesetzt, um dem Kunden auch bei relativ alten Geräten noch Ersatzteile liefern zu können. Die Möglichkeit der Nutzung der Wertschöpfung der Altprodukte ist insofern bisher bei Herstellern eher die Ausnahme. ${ }^{227}$

226 Die Erfassungsquote hängt wesentlich von drei Einflußfaktoren ab, die nicht überschneidungsfrei sind: 1. Der Art der Überlassung der Leistung (Verkauf, Leasing, Mieten) und 2. der Größe des Altproduktes und 3. ob es sich um ein Investitions- oder Konsumgut handelt. Dabei ist die Erfassung tendenziell um so höher, je mehr es sich um ein Mietgeschäft handelt, je größer das Altprodukte und je häufiger es sich um ein Investitionsgut handelt.

Stahel diskutiert unter dem „Konzept der Zurverfügungstellung” die Auswirkungen verschiedener Nutzungsalternativen auf die Faktoren Risikoträger, Dauerhaftigkeitsinteresse und Abfallverantwortlichkeit. Er unterscheidet vier Optionen: 1. Eigentümer ist Benutzer (Kauf), Träger des Risikos ist der (private) Nutzer, der nur beschränkt an der Dauerhaftigkeit interessiert ist. Die Abfälle sind verstreut, die Allgemeinheit ist verantwortlich. 2. Eigentümer ist Betreiber. Der Betreiber trägt die Risiken. Auch hier sind die Abfälle verstreut, und die Allgemeinheit verantwortlich. 3. Eigentümer ist Betreiber und Instandhalter. Alle Risiken werden zwischen Hersteller und Betreiber ausgehandelt. Der Instandhalter ist wegen der Betriebskosten an Dauerhaftigkeit interessiert. Es entstehen konzentrierte Abfälle bei gleichzeitiger Internalisierung der Kosten. 4. Eigentümer ist Hersteller, Instandhalter und Betreiber (Der Kunde ist lediglich Mieter). Hersteller ist an Dauerhaftigkeit interessiert. Abfälle entstehen konzentriert bei gleichzeitiger Internalisierung der Kosten und Abfallvermeidung. Vgl. Stahel, W., Langlebigkeit von Gütern, Materialrecycling, Ressourceneffizienz, a.a.O., Ulm 1994, S. $62 f f$.

Zu branchenspezifischen Unterschieden vgl. die Abbildungen im Anhang 1.1 bis Anhang 1.4. Daraus geht hervor, daß beispielsweise die Automobilindustrie ein deutlich höheres Interesse an höheren Erfassungsquoten hat als die anderen Branchen, da hier teilweise bereits herstellerbezogene Kapazităten aufgebaut wurden, die nicht ausgelastet werden konnten. 


\section{Dimensionen der Implementierung von Rücknahme- und Recycling- systemen}

Unter Implementierungsdimensionen wird ein für die Bildung von Implementierungstypen verwendetes Merkmal des Implementierungsverhaltens verstanden. ${ }^{228}$ Sie sind als aggregierte Aktionsparamenter des Implementierungsverhaltens aufzufassen und werden aus der Verdichtung von Verhaltensvariablen gebildet. ${ }^{22}$

\subsection{Implementierungsdimensionen von Forschungsansätzen im Überblick}

Zunächst ist zu untersuchen, welche Dimensionen der Implementierung, d.h. welche Verhaltens- und Handlungsoptionen den Herstellern bei der Implementierung von Rücknahme- und Recyclingsystemen grundsätzlich zur Verfügung stehen. Verschiedene, bereits bestehende theoriegeleitete und empirische Forschungsansätze zur Implementierung können dazu wichtige Grundlagen und damit eine Strukturierung bereitstellen, die hinsichtlich ihrer Übertragbarkeit auf Rücknahmeund Recyclingsysteme überprüft werden müssen.

Im wesentlichen soll dazu auf eine Untersuchung von Pfohl, Kunz und Linn ${ }^{230}$ zurückgegriffen werden, die 22 bis 1988 vorliegende empirische Untersuchungen zur Implementierung systematisieren. ${ }^{231}$ Dabei unterscheiden die Autoren fünf

228 In der Organisationstheorie wird in Analogie dazu der Begriff der Strukurdimensionen verwendet, wobei von ein- oder mehrdimensionalen Strukturen gesprochen wird. Dabei wird der Frage nachgegangen, ob die Zerlegung eines Systems in mehrere Teilsysteme nach einem Kriterium oder mehreren Kriterien erfolgt. Vgl. Frese, E., Grundlagen der Organisation: Konzept Prinzipien - Strukturen, 6., überarb. Aufl., Wiesbaden 1995, S. 164. Dieses dient der Erfassung der realen Organisationsstrukturen nach Art und Ausmaß. Gleichzeitig weisen die Dimensionen auf Verhaltensmöglichkeiten der Organisationsmitglieder hin.

Bei diesem Begriffsverständnis müssen die Implementierungsdimensionen nicht unabhängig voneinander sein. Aufgrund der Komplexitätsreduktion wäre die Verwendung von möglichst wenigen und unabhängigen Dimensionen zwar wünschenswert, es kann aber nicht ausgeschlossen werden, daß einzelne Parameter miteinander korrelieren. Vgl. Kieser, A., Kubicek, H., Organisation, 3. Aufl., Berlin, New York 1992, S. 191.

Vgl. Pfohl, H.-Chr., Kunz, T., Linn, N., Implementierung, a.a.O., S. $10 \mathrm{ff}$.

Zur detaillierten Darstellung der Untersuchung der technischen Hochschule Darmstadt vgl. auch Linn, N., Die Implementierung vertikaler Kooperationen: theoretische Konzeption und erste empirische Ergebnisse zum Prozeß der Ausgliederung logistischer Teilaufgaben, Frankfurt am Main u.a. 1989.

231 Die Auswahl der Arbeiten aus der Implementierungsliteratur wurde folgendermaßen begründet: Aus der deutschsprachigen Literatur wurden empirische und theoretische Untersuchungen ausgewählt, die Veränderungen im nicht-technischen Bereich behandeln. Zusätzlich wurde die Arbeit von Knopf stellvertretend für die Schriften der Reihe „Planungs- und organisationswissenschaftliche Schriften" ausgewählt. Die US-amerikanischen Arbeiten umfassen ausschließlich Untersuchungen, die aus dem zweiten Tagungsband der Konferenzen über die Implementierung von Operations Research-Systemen und Management Science-Projekten in Pittsburgh stammen Dabei wurden nur Arbeiten ausgewählt, die neben einem empirischen rrene Giesen-Netzer and Universitatt Munster'- 978-3-631-75058-2 
Gruppen unabhängiger Variablen der Implementierung, die aus der Abbildung 10 deutlich werden. ${ }^{232}$ Gleichzeitig beschreibt die Abbildung, zu welchen Ergebnissen die Forschungsansätze gekommen sind, d.h. welche unabhängigen Größen einen signifikanten Einfluß auf den Implementierungserfolg haben. ${ }^{233}$

Die objektspezifischen Dimensionen zählen nach Pfohl, Kunz und Linn zu den das Implementierungsobjekt beschreibenden Erfolgsdeterminanten, ${ }^{234}$ d.h. die Qualität und die Charakteristika der Konzeption des Rücknahme- und Recyclingsystems müßten demnach den Implementierungserfolg beeinflussen. Diese Sichtweise macht eine Trennung der Güte der Implementierung auf der einen Seite von der Güte der Konzeptionierung auf der anderen Seite nicht mehr möglich. Dieses gilt um so mehr, wenn - z.B. aufgrund fehlenden rechtlichen Drucks bei der Implementierung von Rücknahme- und Recyclingsystemen - kein Verhaltensplan bei den Herstellern vorliegt. Wenn der Erfolg einer Implementierung darüber hinaus objektabhängig ist, dann muß die konkrete Situation „Einführung eines Rücknahme- und Recyclingsystems" betrachtet werden, d.h. die (erfolgsspezifische) Vergleichbarkeit von Untersuchungen über Implementierungsverhalten ist nur bei den gleichen Implementierungsobjekten möglich. ${ }^{235}$

Teil auch einen theoretischen Teil beinhalten. Außerdem wurden zwei aus den 80er Jahren stammende Buchveröffentlichungen von Lucas und Schultz/Ginzberg einbezogen. (Gerl 1975, Kempf 1984, Knopf 1975, Beyss 1983, Falkenberg/Scholz 1984, Schulz/Ginzberg/Lucas 1984, Witte 1973, Lucas 1979 und 1981, Dienstbach 1972, Gebert 1974, Bean, u.a. 1975, Narasimhan/Schroeder 1979, Galbraith 1979, Hentschke 1979, Larréche 1979, Bean/Radnor 1979, Alter 1979, Ginzberg 1979, Hammond III 1979, Zmund/McLaughlin/Might 1984, DeSanctis 1984) Vgl. Linn, N., Die Implementierung vertikaler Kooperationen, a.a.O., S. $58 f$.

Pfohl, Kunz und Linn beschreiben als abhängige Variable den Erfolg der Implementierung, den sie in objektive und individuelle Leistungsmerkmale sowie in prozeßbezogene Kriterien untergliedern. Vgl. Pfohl, H.-Chr., Kunz, T., Linn, N., Implementierung, a.a.O., S. 8f.

Bezüglich der Operationalisierung des Implementierungserfolgs vgl. die Ausführungen in Kapitel $\mathrm{B} 1.3$ und $\mathrm{C} 2$.

234 Vgl. z.B. Linn, N., Die Implementierung vertikaler Kooperationen, a.a.O., S. 68.

235 Im folgenden wird daher von einer Einbeziehung der objektspezifischen Dimensionen abgesehen. 


\begin{tabular}{|c|c|c|c|c|c|c|c|c|}
\hline $\begin{array}{c}\text { Abhängige Variablen } \\
\text { Unabhängige Varia- } \\
\text { blen }\end{array}$ & $\begin{array}{c}\text { Be- } \\
\text { wirkte } \\
\text { Leistung }\end{array}$ & $\begin{array}{c}\text { Be- } \\
\text { wirkter } \\
\text { Wandel }\end{array}$ & $\begin{array}{c}\text { Voll- } \\
\text { zugs- } \\
\text { grad }\end{array}$ & $\begin{array}{c}\text { Prozeß- } \\
\text { effizienz }\end{array}$ & $\begin{array}{c}\text { Benut- } \\
\text { zungs- } \\
\text { häufigkeit }\end{array}$ & $\begin{array}{c}\text { Ver- } \\
\text { haltens- } \\
\text { änderung }\end{array}$ & $\begin{array}{c}\text { Zufrie- } \\
\text { denheit }\end{array}$ & $\begin{array}{c}\text { Erfolgs- } \\
\text { einschät- } \\
\text { zung }\end{array}$ \\
\hline
\end{tabular}

Objektspezifische Dimensionen

\begin{tabular}{|l|l|l|l|l|l|l|l|l|}
\hline funktionale Qualität & & & & & & & & \\
\hline Konsequenzen & & & & & & & & \\
\hline Objektcharakteristiken & & & & & & & & \\
\hline
\end{tabular}

Prozeßspezifische Dimensionen

\begin{tabular}{|l|l|l|l|l|l|l|l|l|}
\hline Situation & & & & & & & & \\
\hline Vorgehensweise & & & & & & & & \\
\hline Zeitfaktoren & & & & & & & & \\
\hline Unterstützung Experten & & & & & & & & \\
\hline
\end{tabular}

Gruppenspezifische Dimensionen

\begin{tabular}{|l|l|l|l|l|l|l|l|l|}
\hline Größe Expertengruppe & & & & & & & & \\
\hline $\begin{array}{l}\text { Größe Betroffenen- } \\
\text { gruppe }\end{array}$ & & & & & & & & \\
\hline Gruppendruck & & & & & & & & \\
\hline
\end{tabular}

\section{Organisationsspezifische Dimensionen}

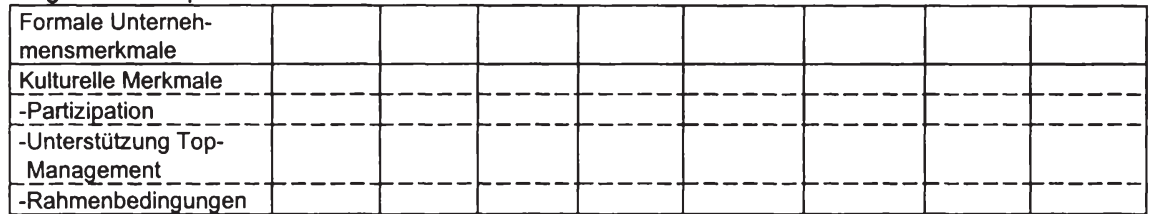

\section{Personenspezifische Dimensionen}

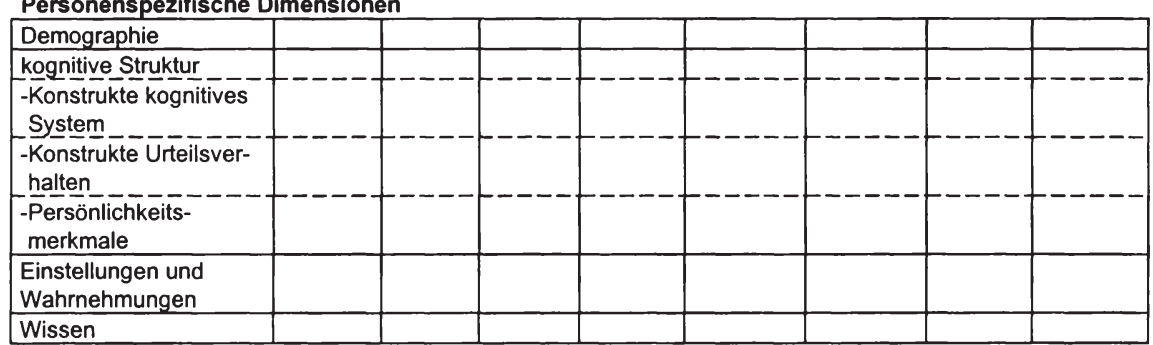

signifikanter Einfluß auf den Erfolg (höchstes Signifikanzlevel $p<0,05$ )

Abb. 10: Systematisierung von Einflußfaktoren auf den Implementierungserfolg (Quelle: in Anlehung an Pfohl, H.-Chr., Kunz, T., Linn, N., Implementierung, a.a.O., S. 29)

Bei den prozeßspezifischen Dimensionen, die insbesondere die „Situation“237 und die "Vorgehensweise ${ }^{\text {"238 }}$ der Reorganisationsprozesse charakterisieren, sind

236 Bei der Analyse zwischen Einflußfaktoren und Implementierungsverhalten ist das Implementierungsverhalten, weiches in den Implementierungsdimensionen zum Ausdruck kommt, zunächst die abhängige Variablen und die Einflußfaktoren die unabhängigen Variablen. Vgl. Kapitel C 1. In der nachgelagerten Analyse zwischen Implementierungsverhalten und Implementierungserfolg verändert sich die Betrachtungsweise grundlegend. Dort gehen das Implementierungsverhalten als unabhängige Variable und der Erfolg als abhängige Variable in die Betrachtung ein. Vgl. Kapitel C 2. Dieser zweite Analyseschritt wird in der Abbildung dargestellt. 
die zeitlichen Dimensionen zwar bei den vorgelegten Untersuchungen nicht signifikant für den Implementierungserfolg. Sie sollen jedoch im Rahmen der vorliegenden Arbeit besonders analysiert werden, da untersucht werden soll, ob die Hersteller Wettbewerbsvorteile durch eine frühzeitige Implementierung (Timingstrategie) von Rücknahme- und Recyclingsystemen generieren können. Grundlage der Überlegungen ist hier, daß die Implementierung von Rücknahmeund Recyclingsystemen die Wertschöpfungsstruktur im Unternehmen beeinflußt. ${ }^{239}$ Zudem gehen neuere theoretisch geleitete Ausführungen davon aus, daß Zeitaspekte der Implementierung zu wenig diskutiert wurden ${ }^{240}$ und von dem Faktor Zeit als Gestaltungsgröße ein Einfluß auf den Implementierungserfolg ausgeht. ${ }^{241}$ Die zeitlichen Dimensionen der Implementierung werden daher als relevante Verhaltensdimensionen der Implementierung gekennzeichnet.

Die gruppenspezifischen Dimensionen weisen insgesamt darauf hin, mit welcher Intensität die Unternehmung das Konzept verfolgt. Die vom Unternehmen eingesetzte "Manpower“ wird durch diese Größen verdeutlicht (intensitätsmäßige Dimensionen der Implementierung). Diese Dimension ist insbesondere vor dem Hintergrund der verschiedenen institutionellen Gestaltungsformen von

${ }^{237}$ Die Situation umfaßt die Randbedingungen der Implementierung und kann zunächst von den Akteuren nicht oder nur sehr langsam geändert werden. Vgl. Pfohl, H.-Chr., Kunz, T., Linn, N., Implementierung, a.a.O., S. 22f. Aus diesem Grunde wird die Situation nicht als Implementierungsdimension erfaßt sondern in Kapitel C 1.4.1.1 als Einflußfaktor untersucht.

Unter der „Vorgehensweise“ werden beispielsweise die Verwendung von Kosten-NutzenAnalysen, die Praktizierung von Projektgruppenarbeit oder die Anzahl der verwendeten formalen Verwaltungsverfahren verstanden. Da diese Kriterien bereits Maßnahmen darstellen, werden sie nicht als Implementierungsdimension erfaßt, sondern dienen im weiteren Verlauf der vorliegenden Arbeit der Beschreibung von Implementierungstypen.

Die Unterstützung der Experten hingegen wird im Rahmen einer hierarchiebezogenen Implementierungsdimension analysiert.

Gerade bei der Analyse von Herstellern langlebiger Gebrauchsgüter muß überprüft werden, ob Hersteller Altprodukt(teile) in die Produktion einbeziehen und dadurch Produktwerte erhalten, um die Kosten der Vorprodukte zu senken oder zusätzliche Leistungen (Gebrauchtgütermarkt; Einsatz im Kundendienst) anzubieten. Vgl. dazu auch die Ausführungen in Kapitel A 1.

Lediglich drei (Knopf, Gerbert, Bean u.a.) der 22 ausgewählten Untersuchungen analysierten die Zeitfaktoren bei der Implementierung. Vgl. Pfohl, H.-Chr., Kunz, T., Linn, N., Implementierung, a.a.O., S. $22 f$.

241 Vgl. Zeyer, U., Zeitaspekte der Implementierung aktueller Managementkonzepte, in: Zeitschrift für Organisation, Heft 5, 1995, S. 283. Zeyer führt aus, daß eine zu frühe Implementierung aufgrund von z.B. unausgereiften Konzepten zu einem Fehlschlag führen kann. Eine zu späte Implementierung hingegen kann einen Verlust von Wettbewerbspositionen verursachen. Ist die Implementierung zu langsam, birgt sie die Gefahr der Versandung. Werden Implementierungsobjekte zu schnell durchgeführt, kann dieses zu einer "Kollapsreaktion" im Unternehmen führen. Vgl. ebenda. 
Rücknahme- und Recyclingsystemen bei deren Implementierung von Relevanz. Bei den organisations- und personenspezifischen Dimensionen hingegen handelt es sich z.T. um beschreibende Merkmale oder Einflußfaktoren auf die Implementierung, da beispielsweise die Unternehmensgröße oder demographische Merkmale nichts über das Implementierungsverhalten selbst aussagen und damit strenggenommen keine Aktionsparameter darstellen. Anders ist dieses bei den „kulturellen Merkmalen" der organisationsspezifischen Dimensionen wie etwa dem Partizipationsverhalten und bei der Unterstützung durch das Topmanagement. Die beiden letztgenannten Größen betreffen jedoch eher das Implementierungsverhalten in der Hierarchie, so daß sie exakter unter dem Begriff „Hierarchiedimensionen" erfaßt werden können. Vor diesem Hintergrund kann damit zunächst festgehalten werden, daß mit der Einteilung in zeitliche, hierarchie- und intensitätsbezogene Verhaltensdimensionen ein tragfähiger Ansatz zur Klassifizierung von Implementierungsdimensionen besteht.

Die Herstellerunternehmen haben im Rahmen dieser Implementierungsdimensionen die Möglichkeit zu handeln. ${ }^{242}$ Dieser Handlungsspielraum weist - aus forscherischer Perspektive - auf die Möglichkeit hin, spezifische Verhaltensmuster der Implementierung und damit unterscheidbare Implementierungstypen zu identifizieren. Vor diesem Hintergrund kann eine Basishypothese ${ }^{243}$ formuliert werden, die im weiteren Verlauf der vorliegenden Arbeit noch zu spezifizieren ist, indem die Ausgestaltungsmöglichkeiten für jede einzelne Implementierungsdimension zu untersuchen sind.

242 Je Hersteller kann damit ein individuelles Verhaltens-Profil abgeleitet werden. Vgl. zum Begriff der Profile und Muster Scholz, Chr., Strategisches Management, a.a.O., S. 78.

243 Basishypothesen werden zur ersten Orientierung bei noch ungenauen Vermutungen über die strukturellen Zusammenhänge in der Realität formuliert. Im Gegensatz dazu werden Tendenzhypothesen formuliert, wenn Vermutungen über konkrete Beziehungen zwischen abhängigen und unabhängigen Variablen bestehen. Vgl. Schanz, G., Zwei Arten von Empirismus, in: $\mathrm{ZfbF}, 1975, \mathrm{~S}$. 325 und vgl. Kopp, M., Hypothesenformulierung in der Absatzforschung, Betriebswirtschaftliche Schriften, Heft 60, Berlin 1972, S. $537 \mathrm{ff}$.

Empirische Hypothesen kennzeichnen in empirischen Untersuchungen getestete Vermutungen über die realen Zusammenhänge ohne Gesetzescharakter, während nomologische Hypothesen sich bereits in der Realität umfassend bewährt haben. Vgl. Schanz, G., Methodologie für Betriebswirte, 2., überarb. und erw. Aufl., Stuttgart 1988, S. 27. Hypothesen sind darüber hinaus nicht zwingend als Bedingungssätze im Sinne von Wenn-Dann-Aussagen zur formulieren. Vgl. Andritzky, K., Die Operationalisierbarkeit von Theorien zum Konsumentenverhalten, in: Schriften zum Marketing, Band 4, Berlin 1976, S. 17.

In der vorliegenden Arbeit wird aufgrund des noch geringen Kenntnisstandes über die Implementierung von Rücknahme- und Recyclingsystemen und des Hypothesentests anhand nur einer relativ kleinen Stichprobe ausschließlich mit empirischen Basishypothesen gearbeitet. 
$\mathrm{H}_{\mathrm{IV} 1}$ : Es lassen sich Ausprägungen zeitlicher, intensitätsmäßiger sowie hierarchiebezogener Implementierungsdimensionen erkennen, die eine Identifikation von Implementierungstypen bei Rücknahme- und Recyclingsystemen ermöglichen.

Die Implementierungsdimensionen sind einer unmittelbaren Beobachtung und Erfassung in der Realität nicht zugänglich. Daher werden zur Operationalisierung der Implementierungsdimensionen Variablen abgeleitet, die die einzelnen Dimensionen beschreiben können und deren Beobachtung möglich ist. ${ }^{244}$

\subsection{Zeitliche Dimensionen der Implementierung}

Das Timing soll als erst zeitliche Implementierungsdimension Gegenstand der Betrachtung sein. Diese Größe ist eine zeitpunktbezogene Dimension, und gibt an zu welchem Zeitpunkt die Hersteller (in Relation zu den Wettbewerbern) mit Implementierungsaktivitäten begonnen haben. Das Timing kann dabei Ausprägungen zwischen "früh“ und "spät" annehmen. ${ }^{245}$

Unter Timing wird auch die Planung des Markteintrittszeitpunkts verstanden, der im Hinblick auf bestimmte Merkmale der Marktsituation bestimmt wird. Als strategische Option können beispielsweise drei Typen abgeleitet werden: Pioniere, frühe Folger und späte Folger. ${ }^{246}$ Diesem Verständnis wohnt ein geplantes strategi-

244 Vgl. zu einer ähnlichen Vorgehensweise bei der Operationalisierung von Führungsdimensionenen Meurer, J., Führung von Franchisesystemen, a.a.O., S. 70ff.

Die Ableitung von Meßvorschriften als zweiter Teilschritt der Untersuchung erfolgt im Rahmen der empirischen Untersuchung. Andritzky bezeichnet diesen Schritt als empirischmeßtechnische Operationalisierung im Gegensatz zu einer sprachlich-semantischen Operationalisierung. Vgl. Andritzky, K., Die Operationalisierbarkeit von Theorien zum Konsumentenverhalten, a.a.O., S. 20ff.

Alle in der Stichprobe erfaßten Hersteller verhalten sich proaktiv in bezug auf das Kreislaufwirtschaftsgesetz, da sie sich bereits vor Inkraftreten desselben entweder konzeptionell oder bereits implementierungsmäßig mit Rücknahme- und Recyclingsystemen auseinandersetzen. Die Gesamtheit der proaktiven Unternehmen läßt sich wiederum in wettbewerbsbezogen frühe und späte Implementierer unterteilen. Kirchgeorg unterscheidet nach dem Zeitpunkt der Konzeptionsentwicklung bzw. der Maßnahmenrealisierung ein ökologiebezogenes reaktives oder proaktives Unternehmensverhalten. Reaktives Verhalten von Unternehmen ist dabei dadurch gekennzeichnet, daß Umweltschutzerfordernisse erst aufgrund bestimmter Sachzwänge heraus im Unternehmen berücksichtigt werden. Sachzwänge entstehen aufgrund einer unternehmensbezogenen aktuellen Betroffenheitssitutation z.B. durch erlassene Umweltgesetze oder markt- oder gesellschaftsbezogene Umweltschutzforderungen. Proaktives Verhalten hingegen zeichnet sich dadurch aus, daß Unternehmen bereits auf erste "schwache Signale“ im Vorfeld ökologiebezogene Erfordernisse in ihren Strategiekonzepten antizipieren. Vgl. Kirchgeorg, M., Okologieorientiertes Unternehmensverhalten, a.a.O., S. 40ff. strategischen Marketingplanung unter besonderer Berücksichtigung des Zeitaspektes, Wiesbaden 1988, S. $51 \mathrm{ff}$. Zu einem ähnlichen Vorgehen vgl. Schnaars, St. P., When Entering 
sches Vorgehen im Sinne einer präskriptiven Strategiefindung inne. Das Timing der Implementierung hängt damit wesentlich von dem Zeitpunkt ab, an dem die Planung des Rücknahme- und Recyclingsystems realisiert wird und damit von der Qualität der Planung („wie schnell wird geplant und wie halten sich Akteure an die Planung ?") und von der Markt- und Wettbewerbssituation, die den Realisationszeitpunkt verschieben können. Konkret kann das Timing der Implementierung der Rücknahme- und Recyclingsysteme beispielsweise an den Realisationszeitpunkt von Gesetzes- und Verordungsentwürfen oder an die Aktivität von Wettbewerbern gebunden sein. ${ }^{247}$ Damit kann von einem derivativen (abgeleiteten) Timingbegriff gesprochen werden. Wird ein Rücknahme- und Recyclingsystem im Sinne der deskriptiven Strategie verstanden, werden auch „Ad-hoc-Strategien“ einbezogen. Aber auch hier bestimmt sich das Timing der Implementierung in Abhängigkeit von der gleichzeitigen Gestaltung des Rücknahme- und Recyclingsystems.

Die Bedeutung des Timing liegt darin, daß es vermutlich einen „optimalen Zeitpunkt" gibt, wann die Implementierungsprozesse von den Herstellern eingeleitet werden sollten, d.h. es ergeben sich in Abhängigkeit vom Zeitpunkt der Implementierung Konsequenzen für den Implementierungserfolg. ${ }^{248}$ Während eine zu späte Implementierung einen Verlust von Wettbewerbsvorteilen mit sich bringen kann, ${ }^{249}$ kann eine zu frühe Implementierung die Gefahr bergen, daß zugrunde

Growth Markets, Are Pioneers better than Poachers?, in: Business Horizons, March-April 1986, S. $27 \mathrm{ff}$.

Bereits 1967 leiteten Ansoff und Stewart ähnliche Strategietypen technologieorientierter Entscheidungen ab: „First to market", "Follow the leader", "Application“, "Mee too“. Vgl. Ansoff, H. I., Stewart, H. M., Strategies for a Technology-based business, in: Harvard Business Review, Nov.-Dec. 1967, S. $73 \mathrm{ff}$.

Vgl. Kapitel C 1.2 und C 1.4.1.1.

248 Knopf u.a. versuchen diesen optimalen Zeitpunkt zu operationalisieren, indem sie davon ausgehen, daß dieser vorliegt, wenn die Mehrheit den Reorganisationsprozeß startet. Sie unterscheiden dabei Frühadopter (Innovatoren), die Mehrheit und Spätadopter (Nachzügler). Die Bestätigung dieser Hypothese ist jedoch je nach Implementierungsobjekt zu differenzieren und in den meisten Fällen widerlegt worden, d.h. Frühadopter oder späte Adopter sind erfolgreicher als die Mehrheit. Vgl. Knopf, R. H., U.a., Die Effizienz von Reorganisationsprozessen aus der Sicht der Praxis, München 1976, S. 65ff.

Als Ursache vermuten Knopf u.a. zwei Einflußfaktoren, die den zeitpunktbezogenen Erfolg (im Sinne eines Vollzugrades bzw. einer Prozeßeffizienz) beeinflussen: Innovationsbereitschaft der Implementierer und das spezifische Implemenierungs-Know-how. Vgl. ebenda, S. 70.

249 Vgl. Zeyer, U., Zeitaspekte der Implementierung aktueller Managementkonzepte, a.a.O., S. 283.

Ein frühzeitiges Beobachten und Erkennen von Chancen und Risiken bietet hingegen die Möglichkeit, die erforderlichen Maßnahmen und Vorbereitungen rechtzeitig einzuleiten. Vgl. Kern, W., Die Zeit als Dimension betriebswirtschaftlichen Denkens und Handelns, in: DBW, Heft 1, 1992, S. 46. 
liegende Konzepte noch unausgereift sind oder die Ziele, die mit dem Rücknahme- und Recyclingsystem verfolgt werden sollen, den Akteuren noch nicht klar sind.

Zudem besteht die Gefahr, daß bei einer sehr frühen Implementierung eines Rücknahme- und Recyclingsystems seitens der Hersteller trotz einer bestehenden Selbstverpflichtung eine Rücknahmeverordnung erlassen wird, deren Anforderungen nicht mit dem entsprechenden Rücknahme- und Recyclingsystem übereinstimmen. Ist dieses der Fall, müssen die Hersteller ihr Rücknahme- und Recyclingsystem erneut verändern. Darüber hinaus ist eine Selbstverpflichtung rechtlich bindend, d.h. für alle internalisierten Kosten der Entsorgung sind die Hersteller verantwortlich, ${ }^{250}$ während diejenigen Hersteller, die auf die Rücknahmeverordnung warten, bis zu dem Zeitpunkt keinerlei Pflichten eingehen. ${ }^{251}$ Schließlich ist auf die Gefahr von "Trittbrettfahrern" hinzuweisen, die sich zwar nicht an den Aufbaukosten des Rücknahme- und Recyclingsystems beteiligen, jedoch an dem Nutzen partizipieren wollen. ${ }^{252}$ Auf der anderen Seite können jedoch durch frühzeitige Erfahrungen mit der Implementierung von Rücknahme- und Recyclingsystemen Wissensvorsprünge entstehen, die als Wettbewerbsvorteil durch die Hersteller genutzt werden können.

Beim Timing spielen zum einen die praktische und zum anderen die konzeptionelle Auseinandersetzung mit dem Recycling vor Inkrafttreten des Kreislaufwirtschaftsgesetzes ${ }^{253}$ eine Rolle. Durch die konzeptionelle Auseinandersetzung wird die Möglichkeit der Hersteller verdeutlicht, den noch bestehenden Handlungsspielraum vor der Verabschiedung der Rücknahmeverordnungen auszunutzen bzw.

250 \$26 KrW-/AbfG. „Hersteller oder Vertreiber, die Abfälle aufgrund einer Rechtsverordnung... oder freiwillig zurücknehmen, unterliegen den Pflichten“ des Kreislaufwirtschaftsgesetzes.

Die Automobilindustrie übergab im Februar 1996 eine Selbstverpflichtung an die Bundesregierung. Diese tritt erst in Kraft, wenn eine ergänzende Altautoverordnung besteht. Vgl. Deutscher Bundestag, Zustimmungsbedürftige Verordnung über die Entsorgung von Altautos und die Anpassung straßenverkehrsrechtlicher Vorschriften, Drucksache 13/5998 vom 7.11.96.

251 Da sich alle Hersteller in der Stichprobe proaktiv bezüglich des Kreislaufwirtschaftsgesetzes verhalten, trifft diese Argumentation nur auf Hersteller außerhalb der Stichprobe zu bzw. auf solche Hersteller, die zum Zeitpunkt der Befragung ein Rücknahme- und Recyclingsystem planten und gleichzeitig die Realisierung der Planung vom Zeitpunkt des Inkrafttretens der jeweiligen Rücknahmeverordnung abhängig machen.

In diesem Zusammenhang wird die Umwelt als öffentliches Gut oder Kollektivgut bezeichnet. Das Problem entsteht dadurch, daß jedermann dieses Gut wegen des Nicht-Ausschlußprinzips kostenlos konsumieren kann und sich daher nicht an den Kosten beteiligen möchte. Vgl. Wicke, L., Umweltökonomie, a.a.O., S. $41 \mathrm{ff}$. 
deren Verabschiedung sogar zu verhindern. Zum anderen deutet diese Größe auf das Problembewußtsein der Hersteller bezüglich der Rücknahme und des Recycling hin. So ist es denkbar, daß sich Hersteller theoretisch sehr früh mit der Problematik auseinandergesetzt haben, sich jedoch bewußt entscheiden, das Rücknahme- und Recyclingsystem erst später in die Praxis umzusetzen. Dieses ist beispielsweise aufgrund der gesetzlichen Unsicherheiten oder der Komplexität des langlebigen Produktes möglich. ${ }^{254}$

Eine weitere bedeutende zeitliche Implementierungsdimension ist der Zeithorizont. ${ }^{255}$ Diese Dimension umfaßt die Dauer der Implementierung eines Rücknahme- und Recyclingsystems durch einen Hersteller, wobei die Zeithorizonte zwischen „kurzen“ und „langen“ Zeiträumen variieren können. ${ }^{256}$ Diese Größe ist demzufolge eine prozeßbezogene Dimension der Implementierung von Rücknahmeund Recyclingsystemen. ${ }^{257}$ Zeyer unterscheidet in diesem Zusammenhang eine

254 Vgl. dazu Kapitel C 1.2 und C 1.4.1.2.

255 Zeitpunkte als Lageangaben und Zeitdauern als Längenangaben werden als Basiszeitgrößen unternehmerischer Handlungen betrachtet. Vgl. z.B. Clauss, M., Die Strategie der Implementierung in der Unternehmung, a.a.O., S. 101ff.

Andere zeitliche Abgrenzungen wie die Abfolge der Schritte des Implementierungsverlaufs oder die zeitliche Reihung von verschiedenen Implementierungsprojekten werden nicht erfaßt, weil sie zum einen idealtypisch und nur schwer abgrenzbar sind oder zum anderen für die spezifische Fragestellung der Implementierung von Rücknahme- und Recyclingsysteme nur beschränkt relevant sind, da in es in der vorliegenden Betrachtung bei den jeweiligen Herstellerunternehmen nur um ein „Implementierungsprojekt“ geht.

Eine Operationalisierung der Zeitdimensionen erfolgt nach folgenden Variablen vgl. auch die Auszüge aus dem Fragebogen im Anhang 3.1:

\begin{tabular}{ll}
\hline $\begin{array}{l}\text { Zeitliche Implementierungs- } \\
\text { dimensionen }\end{array}$ & Variablen \\
\hline Timing & Beschäftigung mit Recycling vor der Verabschiedung des \\
& KrW-/AbfG \\
& Zeitpunkt der praktischen Auseinandersetzung mit dem \\
& Rücknahme- und Recyclingsystem: \\
& Beginn der Planungsphase (bzw. Pilotphase) \\
& Dauer von Beginn der Planungsaktivitäten bis hin zur Pilot- \\
Zeithorizont & oder Realisationsphase \\
\hline
\end{tabular}

Die Indikatoren der zeitlichen Implementierungsdimensionen wurden konkret mit Jahreszahlen erfaßt. Diese Vorgehensweise ermöglicht objektivierte (wettbewerbsbezogene) Aussagen darüber, was eine frühe(bzw. späte) oder eine lange (bzw. kurze) Implementierung darstellt. Vgl. auch Anhang 2.2, der eine beispielhafte Operationalisierung der Zeitdimensionen enthält.

Kern äußert sich über die Zeit als Dimension betriebswirtschaftlichen Handelns und Denkens: „Eine jede Handlung bedarf eben kürzerer oder längerer Zeitspannen (Zeitbedarfe), um einen Zustand in einen anderen - angestrebten - Zustand zu überführen. Ein jeder solcher Zustand ist jedoch zeitpunktbezogen, d.h. ein nur für diesen Augenblick geltender Status." Kern, W., Die Zeit als Dimension betriebswirtschaftlichen Denkens und Handelns, a.a.O., S. 41.

Im weiteren konstatiert Kern, daß betriebswirtschaftliche Erklärungsansätze den zeitraumbezogenen Betrachtungsweisen zu wenig Beachtung schenken und damit Zeitabläufe vernachlässigt werden. Vgl. ebenda, S. $42 \mathrm{ff}$. 
revolutionäre, gestufte und evolutionäre Implementierung. Bei einer revolutionären Implementierung ist die Implementierungsgeschwindigkeit aufgrund des kurzen Implementierungszeitraums hoch, während sie bei der evolutionären aufgrund des langen Zeitraums der Implementierung gering ist. Eine gestufte Implementierung liegt zwischen diesen beiden Extremen. Eine revolutionäre Implementierung entsteht dabei häufig durch einen wahrgenommenen Leidensdruck der Unternehmen. ${ }^{258}$

Die besondere Bedeutung des Zeithorizonts liegt darin, daß diese Größe zum einen Informationen über die Vorgehensweise der Hersteller bei der Implementierung von Rücknahme- und Recyclingsystemen zu geben vermag. Zum anderen wird der Zeithorizont in Abhängigkeit von der Ausgestaltung des Rücknahme- und Recyclingsystems selbst variieren. ${ }^{259}$ Tendenziell werden dabei kurzfristige Betrachtungen primär auf die Durchführung von Implementierungsmaßnahmen zielen, während langfristige Betrachtungen sich über den geschätzten Zeithorizont der gesamten Veränderung erstrecken. ${ }^{260}$ Knopf u.a. sprechen in diesem Zusammenhang von mehreren Phasen der Implementierung, ${ }^{261}$ die einen Einfluß auf den Erfolg der Implementierung haben. Auch hier gilt es "ein Optimum zu finden zwischen „zu viel“ und „zu knapp vorgegebener Zeit"262, da bei zu reichlich vorgegebener Zeit die beteiligten Personen keinen Anreiz verspüren, sich entsprechend

258 Vgl. Zeyer, U., Zeitaspekte der Implementierung aktueller Managementkonzepte, a.a.O., S. 287f. Übertragen auf die Implementierung von Rücknahme- und Recyclingsystemen, kann der Erlaß einer Rücknahmeverordnung einen solchen Leidensdruck ausüben.

Hier ist z.B. an die Komplexität des Rücknahme- und Recyclingsystems, die Ressourcen im Unternehmen oder die strategische Grundhaltungen der Hersteller gedacht. Diese beispielhaften Größen werden in Kapitel C 1.3 und C 1.4.2.2 als Einflußfaktoren auf die Implementierung untersucht.

260 Vgl. Zeyer, U., Zeitaspekte der Implementierung aktueller Managementkonzepte, a.a.O., S. 283.

261 Sie unterscheiden eine Initiierungsphase, in der erste Aktivitäten und rudimentäre Planungsaktivitäten stattfinden, eine Planungsphase, in der konkrete Aktivitäten stattfinden, und eine Implementierungsphase, die die Durch- und Umsetzung im engeren Sinne umfaßt. Vgl. Knopf, R. H., u.a., Die Effizienz von Reorganisationsprozessen aus der Sicht der Praxis, a.a.O., S. 73f. Eine abschließende Konsolidierungsphase, in der das erneute "refreezing" stattfindet, schließt den Prozeß ab.

Da die Initiierungsphase ein „oft langer Gärungsprozeß in der Geschäftsleitung“ (ebenda, $S$. 75) und sehr schwer faßbar ist, werden in der vorliegenden Arbeit die beiden weiteren Phasen der Implementierung unter den Begriffen der Einführungs- und Realisationsphase untersucht. Die Einführungsphase beinhaltet (in Analogie zu Knopf) detaillierte Planungen des Systems und erste Aktivitäten der Implementierung, während die Realisationsphase die Durchund Umsetzung des Rücknahme- und Recyclingsystems umfaßt. Die Konsolidierungsphase wird empirisch nicht weiter analysiert, da auch hier die Operationalisierung nur ungenau möglich ist. 
intensiv und frühzeitig zu engagieren und bei zu knapper Zeit das Auftreten von Widerständen beobachtet werden konnte. ${ }^{263}$

\subsection{Hierarchiebezogene Implementierungsdimensionen}

Als erste hierarchiebezogene Implementierungsdimension wird die Interventionsebene der Implementierung von Rücknahme- und Recyclingsystemen Gegenstand der Betrachtung sein. Dabei wird untersucht, welche Hierarchieebene für die Implementierung des Rücknahme- und Recyclingsystems verantwortlich ist und von welcher Hierarchieebene aus der Prozeß der Implementierung angestoBen wird. ${ }^{264}$

Die Interventionsebene ist für die Implementierung von Rücknahme- und Recyclingsystemen relevant, da die Art und die Anzahl der involvierten Aktoren den Verlauf der Implementierung bestimmen. ${ }^{265}$ Hilker unterscheidet in Anlehnung an Narver und Slater in diesem Zusammenhang den „Programmatic“-Ansatz, der dadurch gekennzeichnet ist, daß alle geplanten Veränderungen unternehmensweit vom Top-Management geplant und eingeführt werden, und den "MarketBack"-Ansatz, der sich durch ein schrittweises und ergebnisorientiertes Vorgehen auszeichnet (Vgl. Abb. 11). ${ }^{266}$ Der Programmatic-Ansatz ist dabei mit dem top-todown-Ansatz zu vergleichen, der von „oben nach unten“ alle Entscheidungen und Maßnahmen bezüglich der Implementierung durchführt. Auf diese Weise wird versucht, nachhaltig die notwendige Unterstützung durch das Top-Management zu erreichen, ${ }^{267}$ das nicht nur in der Einführungsphase einbezogen wird, sondern eine

263 Gebert erläutert in diesem Zusammenhang, daß die Unternehmen ein gewisses Maß an Rezeptivität (Aufnahmefähigkeit) erreicht haben sollten, um konkrete Maßnahmen einleiten zu können. Vgl. Gebert, D., Organisationsentwicklung, Probleme des geplanten organisatorischen Wandels, in: Bolte, K., u.a. (Hrsg.), Sozioökonomie, Bd. 6, Stuttgart 1976, S. 121. Knopf u.a. wollen diese Rezeptivität in der Initiierungsphase erreichen. Vgl. Knopf, R. H. u.a., Die Effizienz von Reorganisationsprozessen aus der Sicht der Praxis, a.a.O., S. 77.

264 Die Konkretisierung der betroffenen Akteure hinsichtlich der abteilungs- bzw. funktionsbezogenen Zuordnung erfolgt bei der Intensitätsdimension in Kapitel B 2.4.2.

265 Vgl. Knopf, R. H., u.a., Die Effizienz von Reorganisationsprozessen aus der Sicht der Praxis, a.a.O., S. 80. Knopf u.a. differenzieren beim Verhalten der Akteure nach den involvierten Personen und nach Aktivitäten, wobei sie feststellen, daß die Akteure selbst die Ursache für Aktivitäten sind. Vgl. ebenda, S. 80. Daher werden in der vorliegenden Arbeit nicht die Implementierungsaktivitäten als Implementierungsdimension untersucht.

Vgl. Hilker, J., Marketingimplementierung, a.a.O., S. 229ff. und vgl. Narver, J. C., Slater, S. F., Becoming more Market Oriented: An Exploratory Study of the Pragrammatic and Market-BackApproaches, Report Nr. 91-128 des Marketing Science Institute, Cambridge, Mass. 1991, S. $7 \mathrm{ff}$.

267 Vgl. Meffert, H., Kirchgeorg, M., Marktorientiertes Umweltmanagement, a.a.O., S. 396. 
direkte Veranwortungsübernahmen im ganzen Implementierungsprozeß übernimmt.

\begin{tabular}{|c|c|c|}
\hline Merkmale & „Programmatic“-Ansatz & „Market-Back“-Ansatz \\
\hline $\begin{array}{l}\text { Beziehung zwischen } \\
\text { Unternehmensführung } \\
\text { und -teilbereichen }\end{array}$ & $\begin{array}{l}\text { Entwicklung von Implementie- } \\
\text { rungsstrategien von der Unter- } \\
\text { nehmensführung } \\
\text { Keine explizite Berücksichtigung } \\
\text { von Erfahrungen von Unterneh- } \\
\text { mensteilbereichen }\end{array}$ & $\begin{array}{l}\text { Entwicklung von Implementie- } \\
\text { rungsstrategien für Unterneh- } \\
\text { mensteilbereiche } \\
\text { Gesammelte Erfahrungen wer- } \\
\text { den für eine schrittweise Aus- } \\
\text { dehnung des Prozesses auf } \\
\text { das gesamte Unternehmen ge- } \\
\text { nutzt }\end{array}$ \\
\hline $\begin{array}{l}\text { Auslöser des } \\
\text { Implementierungs- } \\
\text { prozesses }\end{array}$ & $\begin{array}{l}\text { Insbesondere Erfahrungen des } \\
\text { Top-Management oder anderer } \\
\text { Unternehmen }\end{array}$ & Konkrete Unzulänglichkeiten \\
\hline Hauptverantwortlichkeit & $\begin{array}{l}\text { Von Linienmanager delegiert an } \\
\text { interne und externe Berater }\end{array}$ & $\begin{array}{l}\text { Linienmanager (von internen und } \\
\text { externen Beratern unterstützt) }\end{array}$ \\
\hline $\begin{array}{l}\text { Ansätze zur Verhaltensän- } \\
\text { derung }\end{array}$ & $\begin{array}{l}\text { Beobachtung, Lesen, Ausbildung, } \\
\text { Statements der Unterneh- } \\
\text { mensphilosophie }\end{array}$ & $\begin{array}{l}\text { Praxisorientiertes "Trial and Er- } \\
\text { ror" (anschließende Schulungen } \\
\text { und Unternehmensphilosophie) }\end{array}$ \\
\hline Planungsgrad & Detaillierte Planungen notwendig & $\begin{array}{l}\text { Festlegung eines „critical path“, } \\
\text { Zielvorgaben erst dann, wenn die } \\
\text { Betroffenen Veränderungsbedarf } \\
\text { konkretisieren können }\end{array}$ \\
\hline Handlungsorientierung & $\begin{array}{l}\text { Aktivitätszentiert } \\
\text { (richtige Maßnahmen werden für } \\
\text { eine langfristige Strategie einge- } \\
\text { setzt) }\end{array}$ & $\begin{array}{l}\text { Ergebnisorientiert } \\
\text { (vereinbarte Ziele sollen von den } \\
\text { verantwortlichen Mitarbeitern } \\
\text { erreicht werden) }\end{array}$ \\
\hline
\end{tabular}

Abb. 11: Vergleich zwischen „Programmatic"-Ansatz und „Market-Back“-Ansatz (Quelle: in Abwandlung an: Hilker, J., Marketingimplementierung, a.a.O., S. 234.

Der „Market-Back“-Ansatz hingegen umfaßt neben der Bottom-up-Orientierung, d.h. die verantwortlichen Mitarbeiter steuern von „unten nach oben“, insbesondere das schrittweise Vorgehen (Step-by-step-Prinzip), indem sich die Veränderung ausgehend von einem Teilbereich der Unternehmens (Referenzeinheit) schrittweise auf die anderen Bereiche ausdehnt. Darüber hinaus besteht die Hauptverantwortlichkeit der zu realisierenden Veränderungen bei den Linienmanagern, die ggf. durch interne und externe Berater unterstützt werden. Daran wird deutlich, daß die Ernennung von Fachexperten bei diesem Ansatz eine wichtige Rolle spielt.

Werden die Merkmale des Market-Back-Ansatzes auf die Implementierung von Rücknahme- und Recyclingsystemen angewendet, kann beispielsweise eine $\mathrm{Pi}$ lotphase sinnvoll sein, die erste Erfahrungen mit dem System ermöglicht, um an- 
schließend (mit eventuell notwendigen Verbesserungen) die eigentliche Realisation durchzuführen. Weiterhin ist es - auf Abteilungen des Herstellers bezogen möglich, daß aufgrund der Erfahrungen mit der Rücknahme und dem Recycling der Altprodukte die Einkaufsabteilung verpflichtet wird, bestimmte Stoffe zu ersetzen oder daß im Produktionsbereich auf die leichtere Demontierbarkeit der Altprodukte umgestellt wird. Über den beschriebenen "Market-Back"-Ansatz hinaus ist bei der Implementierung von Rücknahme- und Recyclingsystemen auch die Nutzung der Erfahrungen der Partner auf der Netzwerkebene (z.B. des Systempartners Verwerter) möglich. ${ }^{268}$

Als weitere hierarchiebezogene Implementierungsdimension hat die Partizipation der Mitarbeiter im Sinne der Integration der Organisationsmitglieder in den Implementierungsprozeß die Zielsetzung, die drei Teilbereiche des „Kennen und Verstehens“, des „Könnens“ und des "Wollens" der Individualebene zu fördern. ${ }^{269}$ Damit soll erreicht werden, daß die Mitarbeiter das Rücknahme- und Recyclingsystem als ein von dem Hersteller selbst gewolltes (und gestaltetes) und nicht als ein von internen oder externen Beratern aufoktroyiertes System begreifen. ${ }^{270}$ Dazu

268 Zur Operationalisierung der Interventionsebene wurden 7 Variablen herangezogen vgl. auch den Auszug aus dem Fragebogen im Anhang 3.1:

\begin{tabular}{ll}
\hline Implementierungsdimension & Variablen \\
\hline Interventionsebene & 1. Direkte Verantwortungsübernahme auf der Geschäfts- \\
& leitungsebene \\
2. Top-to-down-Prinzip \\
3. Bottom-up-Prinzip \\
4. Step-by-step-Prinzip \\
5. Persönliche Anweisungen der Vorgesetzten \\
6. Ernennung eines Fachexperten \\
7. Einbeziehung der Geschäftsleitung in der Einführungs- \\
phase
\end{tabular}

Vgl. dazu die Ausführungen in Kapitel A 3.

Gebert beschreibt zwei Zielsetzungen der Integration der Organisationsangehörigen: a) die Lernfähigkeit der Organisation soll erhöht werden („Können“) b) Die Akzeptanz („Wollen“) und Angemessenheit (,.Verstehen) der Maßnahmen für die Organisation sollen erhöht werden. Vgl. Gebert, D., Organisationsentwicklung, Probleme des geplanten organisatorischen Wandels, a.a.O., S. 84 .

Gebert weist bei der Diskussion der „Können“-Komponente darauf hin, daß die gesamten Aktivităten im Rahmen einer Veränderung überwiegend bei den Organisationsmitgliedern selbst liegen sollen und weniger bei (internen oder externen) Beratern, da sonst eine "spezifische Abhängigkeit" vom Berater entsteht. Gebert vergleicht dieses mit einem Arzt-Patienten-Verhältnis, bei dem im Krankheitsfall eine Medizin vom Arzt verlangt wird, statt zunächst selbst zu versuchen, mit der Krankheit fertig zu werden. Vgl. ebenda, S. 84f.

270 Clauss weist darauf hin, daß die Partizipation eng mit der Dauer der Implementierung zusammenhängt, da er davon ausgeht, daß die „effektive Einflußmöglichkeit“ der Betroffenen mit zunehmender Dauer zunimmt. Er geht so weit, daß er das Kriterium „Partizipationsgrad“ und „Dauer des Implementierungsprozesses“ integriert. um daraus Strategien abzuleiten, die er als rene Giesen-Netzer and Universitat Munster - 978-3-631-75058-2 
können zum einen Zielvereinbarungen zwischen Vorgesetzten und Mitarbeitern stattinden und zum anderen Mitbestimmungsmöglichkeiten der Mitarbeiter hinsichtlich Entscheidungen im Implementierungprozeß eingeräumt werden. Eine Koordination über Richtlinien und Handbücher deutet hingegen auf eine relativ standardisierte Abwicklung der Implementierung hin, die den Mitarbeitern geringere Freiräume bei der Implementierung der Rücknahme- und Recyclingsysteme läßt und eine Partizipation erschwert.

Aufgrund des psychologischen Phänomens, daß Menschen dazu neigen, den Status Quo aufrechterhalten zu wollen, ist Überzeugungsarbeit zu leisten, um Veränderungen durchzusetzen. Dieses ist gerade bei umweltbezogenen Problemstellungen wie der Implementierung von Rücknahme- und Recyclingsystemen von besonderer Bedeutung, weil z.T. Vorurteile gegen engagierte Umweltaktivitäten bestehen. Die Mitarbeiter könnten im Unternehmensumfeld befürchten, ihr Ansehen durch ein hohes Engagement bei der Implementierung des Systems zu verlieren. Für Mitarbeiter, die umweltbezogene Aufgaben engagiert wahrnehmen oder sogar ausschließlich mit solchen Aufgaben betraut werden, besteht vermutlich auch die Gefahr, mit ihrem Aufgabenfeld wenig anerkannt zu werden, da Umweltschutzmaßnahmen häufig überwiegend mit Kosten verbunden werden und damit eine Absicherung des Aufgabenfeldes nur in Abhängigkeit anderer gewinnbringender Abteilungen gewährleistet werden kann. Um diese Vorurteile abzubauen, kann eine Partizipation der Mitarbeiter bei der Implementierung von Rücknahmeund Recyclingsystemen hilfreich sein.

Die Promotorenstruktur als letzte hierarchiebezogene Implementierungsdimension umfaßt diejenigen Personen, die den Prozeß der Implementierung von Rücknahme- und Recyclingsystemen aktiv fördern. ${ }^{271}$ Dabei handelt es sich um interne Organisationsmitglieder, die Inhaber von prozeßbezogenen Energien sind und sich in Macht- und Fachpromotoren unterscheiden lassen. ${ }^{272}$ Auch dieser Teil der hierarchischen Implementierungsdimension zielt auf das "Kennen und Verstehen“,

gedrängte und gestreckte Implementierung bezeichnet. Vgl. Clauss, M., Die Strategie der Implementierung in der Unternehmung, a.a.O., S. 90ff.

271 Witte weist darauf hin, daß es sich beim Promotorenmodell nicht um ein organisatorisches Konzept handelt, welches die aufbauorganisatorische Unternehmensorganisation ersetzt, sondern um ein Konzept, welches hinzutritt und mit der dauerhaften Organisation verträglich sein muß. Vgl. Witte, E., Organisation für Innovationsentscheidungen, a.a.O., S. 14.

Vgl. auch Knopf, R. H., u.a., Die Effizienz von Reorganisationsprozessen aus der Sicht der Praxis, a.a.O., S. $100 \mathrm{ff}$. 
„Kennen“ und „Wollen“ ab. Machtpromotoren fördern den Implementierungsprozeß durch ihr hierarchisches Potential innerhalb der Aufbauorganisation und können Opponenten des Nichtwollens mit Sanktionen belegen. ${ }^{273}$ Es ist von Vorteil für den Prozeß, wenn der Machtpromotor hochrangig - im Idealfall Mitglied der höchsten Ebene - ist oder zumindest deren Unterstützung genießt, da das glaubwürdige hierarchische Potential dort am höchsten ist. Fachpromotoren sind diejenigen Personen im Unternehmen, die den Implementierungsprozeß durch objektspezifisches Fachwissen intensiv fördern, wobei für die zu fördernde Aktivität keine oder nur eine unwesentliche hierarchische Macht zur Verfügung steht. ${ }^{274}$

Zur Überwindung von Fähigkeitsbarrieren kann auch ein (organisations)externer Berater das notwendige Fachwissen im Zusammenhang der Implementierung von Rücknahme- und Recyclingsystemen liefern. ${ }^{275}$ Dabei sprechen für die Einbeziehung von externen Beratern insbesondere das überbetriebliche Expertenwissen, die Objektivierung der Sachverhalte sowie der Mut Außenstehender, ungewöhnliche Lösungen vorzuschlagen. ${ }^{276}$ Angesichts der Komplexität von Rücknahme- und Recyclingsystemen und der Bewältigung neuer hinzukommender Aufgaben könnten sie dabei die Organisationsmöglichkeiten für Rücknahme- und Recyclingsysteme eruieren, die Konsequenzen analysieren und die Unterstützung bei der Implementierung im Sinne einer Prozeßberatung leisten.

273 Dienstbach unterscheidet sieben mögliche Reaktionen der betroffenen Mitarbeiter auf geplante Änderungen zu reagieren: 1. Volle Anpassungsbereitschaft, 2. Beschränkte Anpassungsbereitschaft, 3. Indifferenz, 4. Passiver Widerstand, 5. Opposition, 6. Ausscheiden und 7. Vorläufig keine Reaktion. Im Fall der Opposition muß mit heftigen Gegenmaßnahmen gerechnet werden. Vgl. Dienstbach, H., Die Anpassung der Unternehmungs-Organisation, München 1968, S. $183 \mathrm{ff}$.

274 Vgl. Witte, E., Organisation für Innovationsentscheidungen, a.a.O., S. 17ff.

Die Personalunion von Macht- und Fachpromotor bezeichnet diejenige Person, die einen Innovationsprozeß sowohl durch hierarchisches Potential als auch durch objektspezifisches Fachwissen aktiv fördert.

275 Donckels untersucht 900 kleine und mittelständische Betriebe hinsichtlich der Netzwerkbildung in Belgien und stellt dabei fest, daß Leistungen von externen Beratern meist Fachwissen betreffen $(89 \%)$ und daß $83 \%$ der befragten Unternehmen mit den Leistungen zufrieden gewesen seien. Vgl. Donckels, R. von, Profil und Determinanten der Netzbildung bei Klein- und Mittelunternehmen in Belgien, in: Zeitschrift für Klein- und Mittelunternehmen, Heft 1/4, 1993, S. 41.

Vgl. Meffert, H., Kirchgeorg, M., Marktorientiertes Umweltmanagement, a.a.O., S. 395.

Klein konstatiert, daß externe Berater Innovationsprozesse fördern. Dieses begründet er mit drei Ansätzen: 1. Eigenschaftserklärung: Fachwissen und Erfahrung des Beraters, 2. Informationserklärung: Quantität und Qualität der Informationsversorgung durch externen Berater nimmt zu, 3. Organisationserklärung: Berater steht außerhalb des Organisationsprozesses und ist interessenunabhängig. Vgl. Klein, K., Die Konsultation externer Berater in Innvovationsprozesse: Ein Beitrag zur empirischen Theorie der Unternehmung, München 1972, S. $63 \mathrm{ff}$. 
Die Bedeutung der Promotoren bei der Implementierung von Rücknahme- und Recyclingsystemen liegt darin, daß personenbezogene Barrieren der Durchsetzung entsprechend durch personenbezogene Energien in Form von Promotoren überwunden werden können. ${ }^{277}$ Darüber hinaus scheint ein Promotorengespann ${ }^{278}$ positiv beeinflussen zu können, in welcher Geschwindigkeit und in welchem Ausmaß die Ziele der Implementierung eines Rücknahme- und Recyclingsystems erreicht werden können. ${ }^{279}$

\subsection{Intensitätsbezogene Implementierungsdimension}

Die intensitätsbezogene Implementierungsdimension geht der Frage nach, mit welchem Ausmaß und welcher Zahl die Herstellerunternehmen die Abteilungen bzw. Funktionen in ihrem Unternehmen bei der Implementierung des Rücknahmeund Recyclingsystems einbeziehen ${ }^{280}$. Wichtig erscheint dabei eine Differenzie-

277 Insbesondere soll der Machtpromotor Willensbarrieren und der Fachpromotor Fähigkeitsbarrieren zu überwinden helfen. Vgl. Knopf, R. H., u.a., Die Effizienz von Reorganisationsprozessen aus der Sicht der Praxis, a.a.O., S. 101 und vgl. Witte, E., Organisation für Innovationsentscheidungen, a.a.O., S. 15.

278 Es wird von Promotorengespann gesprochen, um zu verdeutlichen, daß beide aufeinander angewiesen sind, d.h. sie haben keine Verpflichtung zur Zusammenarbeit, sondern verbünden sich notwendigerweise, weil sie die Neuerung wollen. Vgl. Witte, E., Organisation für Innovationsentscheidungen, a.a.O., S. 21.

Vgl. Witte, E., Organisation für Innovationsentscheidungen, a.a.O., S. 55.

Zur Operationalisierung der Partizipation und der Promotorenstruktur wurden jeweils drei Variablen herangezogen; vgl. auch den Auszug aus dem Fragebogen im Anhang 3.1:

\begin{tabular}{ll}
\hline Implementierungsdimension & Variablen \\
\hline Partizipation & 1. Zielvereinbarungen zwischen Vorgesetzten und Mitar- \\
& 2. Meitern \\
Promotorenstimmungsmöglichkeiten der Mitarbeiter \\
3. Koordination über Richtlinien und Handbücher \\
4. Hierarchieebene des wichtigsten Fachpromotors \\
5. Hierarchieebene des wichtigsten Machtpromotors \\
6. Einbeziehung eines externen Beraters
\end{tabular}

Knopf u.a. analysieren ebenfalls die Intensität der Beteiligung von Akteuren und Abteilungen an Veränderungsprozessen. Vgl. Knopf, R. H., U.a., Die Effizienz von Reorganisationsprozessen aus der Sicht der Praxis, a.a.O., S. $81 \mathrm{ff}$. Bei den Akteuren untersuchen sie neben der funktionsbezogenen Intensität u.a. die hierarchische Zuordnung sowie die Rolle von Beratern. Diese personenbezogenen Aspekte werden in der vorliegenden Arbeit im Rahmen der hierarchiebezogenen Implementierungsdimension untersucht.

Gebert unterscheidet Extensität und Intensität von Implementierungsmaßnahmen. Unter Extensität versteht er die Reichweite der Maßnahmen, d.h. bei beispielsweise vollständiger Extensität wird die Gesamtheit der Personen (bzw. der Funktionen) in den Prozeß integriert. Intensităt hingegen bezeichnet er als Tiefendimension der Maßnahmen, d.h. - auf personenbezogene Prozesse bezogen - den Grad der emotional-affektiven Einbeziehung oder Betroffenheit. Vgl. Gebert, D., Organisationsentwicklung, Probleme des geplanten organisatorischen Wandels, a.a.O., S. $116 \mathrm{ff}$. In der vorliegenden Arbeit werden Intensität und Extensität unter Irrene Giesen-Netzer and Universität Münster - 978-3-631-75058-2 
rung zwischen der Einführungsphase und der Realisationsphase der Systeme, da zu untersuchen ist, ob die Intensität in beiden Phasen variiert.

Voraussetzung hierzu ist zunächst die sachliche Analyse der Aufgaben, die bei der Implementierung und Gestaltung eines Rücknahme- und Recyclingsystems zu übernehmen sind. Zusätzlich ist von Interesse, welche Aufgaben die Hersteller häufig selbst durchführen und welche von den Systempartnern übernommen werden.

\subsubsection{Aufgaben bei der Implementierung von Rücknahme- und Recycling- systemen}

Bei der Implementierung und Gestaltung von Rücknahme- und Recyclingsystemen können zum einen rücknahme- und recyclingsystemspezifische Aufgaben identifiziert werden, die zum einen von Systempartnern des Herstellers übernommen werden und zum anderen solche Aufgaben, die eine Veränderung der „klassischen“ Funktionen im Herstellerunternehmen mit sich bringen. Darüber hinaus existieren Implementierungsaufgaben, die von den Herstellern bewältigt werden müssen. Die Koordination dieser beiden Aufgabenbereiche wird gleichzeitig als "Schlüsselfaktor auf dem Weg zu integrierten Lösungen“ bezeichnet, ${ }^{281}$ weil damit die Funktionsfähigkeit der Rücknahme- und Recyclingsysteme gewährleistet werden kann. ${ }^{282}$

Die Aufgaben bei der Implementierung von Rücknahme- und Recyclingsystemen können sich am Wertschöpfungskreislauf orientieren, der die "klassische" Wertschöpfungskette erweitert. Als weitere Wert(schöpfungs)elemente treten neben die Beschaffung, Produktion und den Absatz die "Verwendung des Produkts" und

dem Begriff der Intensität zusammengefaßt, da mit der Erfassung der Intensitätsdimension die Extensität (Anzahl der Abteilungen) mit abgebildet und durch die Partizipationsdimension ein Teil der „Tiefendimension" erfaßt wird.

281 Meffert, H., Kirchgeorg, M., Ziele und Strategien zur Gestaltung von produktbezogenen Rücknahme- und Recyclingsystemen, a.a.O., S. 32.

Eine integrierte Lösung bedeutet in diesem Zusammenhang die Gestaltung und Implementierung eines Rücknahme- und Recyclingsystems als „homogenes Gesamtsystem“ mit unterschiedlichen Systempartnern.

282 In diesem Zusammenhang wird auch von "Schnittstellen“ gesprochen, die es zu managen gilt. Eine Schnittstelle entsteht, wenn aufgrund art- oder mengenmäßigen Arbeitsteilung zwischen zwei (oder mehr) Einheiten ein Koodinationsbedarf existiert. Unter dem Begriff des Schnittstellenmanagement werden Kommunikations- und Entscheidungsaktivitäten subsumiert, die zu einer Abstimmung zwischen den Einheiten beitragen. Zur näheren Erläuterung des Schnittstellenmanagement vgl. Frese, E., Grundlagen der Organisation, a.a.O., S. 124ff. 
das "Recycling“, die zwischen der Beschaffung und dem Absatz stehend, den Kreislauf vervollständigen ${ }^{283}$ Die implementierungsobjektbezogenen Aufgaben beziehen sich insbesondere auf die Verwendungs- und die Recyclingphase der Produkte und lassen sich in primäre Aufgaben und in sekundäre Aufgaben einteilen. ${ }^{284}$ Primäre Aufgaben bei der Gestaltung von Rücknahme- und Recyclingsystemen sind eher technischer Art und können in Rücknahme, Logistik, Recycling, Deponierung und die Entwicklung von Recyclingtechnologien unterteilt werden. Sekundäre Aufgaben haben einen funktionsübergreifenden Charakter und erfüllen eher administrative Funktionen. Dabei können die Planung, die Kontrolle, die Koordination und die Öffentlichkeitsarbeit unterschieden werden. Bei einer Beteiligung von mehreren Systempartnern werden abgestimmte Verhaltensweisen auf vertikaler und horizontaler ${ }^{285}$ sowie lateraler Ebene notwendig, d.h. Zulieferer, Handel, Verwerter und der Wettbewerb können in die Implementierung eines Rücknahme- und Recyclingsystems einbezogen werden. ${ }^{286}$

Welche implementierungsobjektbezogene Aufgaben die Hersteller - laut der Befragung - selbst durchführen oder externalisieren und damit anderen Systempartnern ${ }^{287}$ übertragen wird aus der Abb. 12 deutlich. ${ }^{288}$

283 Vgl. Meffert, M., Sustainable Development, a.a.O., S. 33ff.

284 Die Einteilung von Funktionen in primäre und sekundäre nimmt Porter im Zusammenhang mit der Diskussion der Wertkette vor. Porter zählt die Beschaffungslogistik, Produktion, Absatz, Marketing/Vertrieb und Kundendienst zu den primären Funktionen während er die sekundären Funktionen als unterstützende Aktivitäten definiert, die Beschaffung, Forschung und Entwicklung, Personal und Unternehmensinfrastruktur umfassen. Vgl. Porter, M. E., Competitive Advantage, Creating and Sustaining Superior Performance, New York, London 1985, S. $45 \mathrm{ff}$.

Die Gliederung der Wertschöpfungskette eines Unternehmens deckt sich nicht mit den Standardkategorien der Kostenartenrechnung und nicht mit der üblichen Funktionseinteilung. Vielmehr werden die Unternehmensaktivitäten im Hinblick auf ihren Beitrag zur Befriedigung der Kundenwünsche untersucht, um Gewinnpotentiale offenzulegen. Vgl. Kreikebaum, H., Strategische Unternehmensplanung, 5. Aufl., Stuttgart, Berlin, Köln 1993, S. $93 f$. Vgl., Meffert, H., Sustainable Development, a.a.O., S. $35 f f$.

286 Darüber hinaus sind bei der Implementierung einer Branchenkooperation noch Verhaltensweisen mit anderen Systempartnern wie z.B. Verbänden abzustimmen.

287 Auf die Wahl der Systempartner wird in Kapitel C 1.3 eingegangen.

288 Bei der an die Hersteller gerichtete Frage nach der Übernahme der Aufgaben durch die unterschiedlichen Systempartner waren Mehrfachnennungen möglich. 


\begin{tabular}{|l|c|c|c|c|c|}
\hline $\begin{array}{c}\text { Systempartner } \\
\text { Primäre Aufgaben }\end{array}$ & Zulieferer & Hersteller & Handel & Verwerter & Wettbewerb \\
\hline Rücknahme & & 43 & 40 & 34 & 4 \\
\hline Logistik & & 27 & 18 & 37 & 4 \\
\hline Zerlegung, Demontage & & 32 & & 60 & \\
\hline Aufarbeitung & & 34 & & 17 & \\
\hline Wiederverwertung & $8^{\star \star}$ & 31 & & 20 & \\
\hline Wiederverwendung & & 28 & & 3 & \\
\hline Weiterverwertung & 8 & 11 & & 56 & \\
\hline Weiterverwendung & & 11 & & 17 & \\
\hline Thermische Verwert. & & 4 & & 34 & \\
\hline Deponierung & & 8 & & 52 & \\
\hline $\begin{array}{l}\text { Entwicklung von Recy- } \\
\text { clingtechnologien }\end{array}$ & 9 & 43 & & 53 & 9 \\
\hline Sekundäre Aufgaben & & & & & \\
\hline Planung des Systems & & 75 & 4 & 29 & 14 \\
\hline Verwaltung & & 63 & & 29 & 4 \\
\hline Kontrolle & 76 & & 21 & 5 \\
\hline Koordination & 72 & & 14 & 7 \\
\hline Öffentlichkeitsarbeit & & 62 & & 53 & 10 \\
\hline
\end{tabular}

=Führt Aufgaben am häufigsten durch

* $\quad=$ Mehrfachantworten waren möglich $\square=$ =ührt Aufgaben am zweithäufigsten durch

** Angaben in Prozent der Stichprobe

Abb. 12: Aufgabenverteilung bei der Gestaltung von Rücknahme- und Recyclingsystemen (Quelle: in Anlehnung an: Meffert, H., Kirchgeorg, M., Ziele und Strategien zur Gestaltung von produktbezogenen Rücknahme- und Recyclingsystemen, a.a.O, S. 34)

Ein ausgeprägtes Kooperationspotential besteht demnach bei den primären Aufgaben, wobei Verwerter am häufigsten Aufgaben wie die Zerlegung (60\%), Verwertung (bis zu 56\%) und Deponierung (52\%) im hohen Maße übernehmen. ${ }^{289}$ Auch die Entwicklung von Recyclingtechnologien wird mit $53 \%$ von den Verwertern durchgeführt. ${ }^{290}$ Allerdings geschieht dieses häufig in Abstimmung mit den

${ }^{289}$ Krcal untersucht (theoretisch) die Wirkungsbeziehungen zwischen den beteiligten Partnern Vorlieferanten, Lieferanten, Hersteller und Entsorger produktbezogener Stoffkreisläufe. Er geht davon aus, daß die Sammlung, Demontage, Verwertung und Deponierung Aufgaben der Entsorger sind und leitet $a b$, daß diese eine Standardisierung der Entsorgungsprozesse erreichen müssen, um Entsorgungsmengen umfassend bewältigen zu können. Auch beschreibt Krcal, daß es Aufgabe der Verwerter sei, Sekundärmaterial in der Produktionsprozeß der Lieferanten zurückzuführen, um den Kreislauf schließen zu können. Vgl. Krcal, H.-Chr., Wirkungsbeziehungen produktbezogener Umweltschutzmaßnahmen als Beweggrund zwischenbetrieblicher Zusammenarbeit, a.a.O., S. 28ff. Diese theoretischen Ausführungen konnten mit der empirischen Analyse in der idealtypischen Form nicht untermauert werden, die Tendenz der Aussagen wird jedoch durch Abb. 14 durchaus bestätigt.

290 Die Entwicklung von (Produktions- oder Produkt-)technologien wird im Gegensatz dazu als sekundäre Aufgabe im Rahmen der Wertkette eingestuft. Vgl. Porter, M. E., Competitive Advantage, a.a.O., S. $45 \mathrm{ff}$. 
Herstellern, weshalb $43 \%$ der Hersteller angeben, daß sie die Forschungs- und Entwicklungsarbeiten für Recyclingtechnologien durchführen (zweithäufigste Nennung). Immerhin wird noch mit $9 \%$ angegeben, daß sich die Wettbewerber ebenfalls an der Entwicklung von Recyclingtechnologien beteiligen. ${ }^{291}$

Die Hersteller übernehmen überwiegend die sekundären Aufgaben wie Planungs-, Koordinations- und Kontrollfunktionen. Auch die Öffentlichkeitsarbeit für die Recyclingaktivitäten wird von $62 \%$ der Hersteller selbst durchgeführt. ${ }^{292}$ Aufgrund der bisher noch geringen Erfassungsquoten bei Altgeräten besteht bei der (Rückführungs-)Logistik noch keine klare Aufgabenverteilung zwischen den Systempartnern, sondern es werden dem Kunden teilweise mehrere Möglichkeiten angeboten: So kann der Konsument die herstellereigenen Vertriebs- und Kundendienste ${ }^{293}$ oder die Handelsinfrastruktur ${ }^{294}$ nutzen. Darüber hinaus bestehen Bring-

291 Evan und Olk untersuchen Forschungs- \& Entwicklungskonsortien von Wettbewerbern bei USamerikanischen Unternehmen und geben dort als Entstehungsgründe insbesondere Kosten der Forschung und Entwicklung an sowie einen Strategiewechsel der einzelnen Mitglieder und damit die Nutzbarmachung von Know-how anderer Mitglieder. Vgl. Evan, W. M., Olk, P., R\&D Consortia: A New U.S. Organisational Form, in: Sloan Management Review, Nr. 1, 1990, S. $43 f$.

Bei der Zusammenarbeit der Forschung und Entwicklung von Recyclingtechnologien sind ebenfalls die Kosten Ursache der Zusammenarbeit. Darüber hinaus spielen auch markt- und wettbewerbsbezogene Überlegungen eine Rolle. So geben immerhin $50 \%$ der in der vorliegenden Untersuchung befragten Hersteller an, die Auswirkungen dieser Aufgabe auf die Wettbewerbsposition sei sehr hoch bis hoch. Nur knapp ein Viertel der Hersteller meinen, die Forschungs- und Entwicklungsaufgaben für Recyclingtechnolgien haben keine oder kaum Auswirkungen auf die Wettbewerbsposition.

292 Die Öfentlichkeitsarbeit hat für die befragten Hersteller die höchste Bedeutung für die Wettbewerbsposition (Mittelwert 2,13 bei einer Fünferskala). 69,5\% der Hersteller bekunden dementsprechend, daß die Offentlichkeitsarbeit sehr wichtig bzw. wichtig ist. Dahinter steht der Wunsch der Hersteller, eine Marktprofilierung durch die Implementierung des Rücknahmeund Recyclingsystems anzustreben. Nach der Öffentlichkeitsarbeit folgen die (Rückführungs)Logistik (Mittelwert 2,31) und die Forschung und Entwicklung für die Recyclingtechnologie (Mittelwert 2,78) bei der Beurteilung der Wichtigkeit für die Wettbewerbsposition. Am wenigsten wichtig werden die Deponierung und die thermische Verwertung eingeschätzt.

293 Bei der Siemens-Nixdorf AG nimmt der Kundendienst beispielsweise die alten Mainframes bei der Montage der neuen mit ins Werk zurück. Vgl. Fallstudie Siemens-Nixdorf, a.a.O.

Bei Rank Xerox werden die Altkopierer ebenfalls vom eigenen Vertrieb abgeholt. Bei Großgeräten kann der Kunde (im Investitionsgütergeschäft), der das Gerät häufig nur besitzt, anrufen, falls Störungen auftreten. Der Kundendienst entscheidet dann, ob das Altgerät komplett ausgewechselt oder repariert wird. Bei kleinen Kopierern besteht die Möglichkeit, das Altgerät direkt an den Hersteller zu senden. Vgl. Fallstudie Rank Xerox, a.a.O.

Bauknecht weist den Handel explizit darauf hin, daß ein Rücknahme- und Recyclingsystem für Hausgeräte in Kooperation mit Rethmann existiert und legt dem Handel nahe, dieses als zusätzliche Serviceleistung den Kunden anzubieten.

Opel-Vertragshändler hingegen zahlen dem Letztbesitzer für Gebrauchtwagen ohne Katalysator und mit einem Mindestalter von 10 Jahren eine Abwrackprämie von bis zu 3000,- DM Irene Giesen-Netzer and Universität Münster - 978-3-631-75058-2 
und Holsysteme von Entsorgern, ${ }^{295}$ in denen der Letztnutzer seine Altprodukte übergeben kann. Der Handel ist dementsprechend laut der Aussagen der Hersteller mit $40 \%$ in die Rücknahme der Altgeräte und mit $18 \%$ in die Logistik der Altprodukte eingebunden. ${ }^{296}$ Stölzle und Jung fordern im Zusammenhang mit der (Rückführungs-)Logistik ein strategisches Vorgehen, da davon die Realisierung von Kostensenkungs- und Differenzierungpotential für alle beteiligten Systempartner abhängt. ${ }^{297}$ Sie vergleichen den Rückführungservice mit dem Lieferservice und leiten in Anlehnung an die "4 R's“ der versorgungsorientierten Logistik vier Komponenten ab: Danach soll die Rückführungslogistik den richtigen Abfall in Art und Menge zum richtigen Zeitpunkt am richtigen Ort sowie im richtigen Zustand abnehmen und abliefern. ${ }^{298}$

Die Zulieferer werden bisher kaum in Rücknahme- und Recyclingsysteme eingebunden. Wenn sie im Rahmen der Systeme aktiv sind, so unterstützen sie die

beim Kauf eines neuer Corsa oder Combo. Ford bietet einen ähnlichen Anreiz bei seinem Modell Fiesta. Vgl. o.V., Opel zahlt Abwrackprämie, in: Handelsblatt, 21.3.1995, S. 23.

Ziel des Zentralverbandes der Elektrotechnik- und Elektronikindustrie e.V. (ZVEI) ist es, die Kommunen auch weiterhin über die Sperrgutabfuhr für die Rücknahme von Elektro- und Elektronikgeräten einzubinden. Vgl. Zentralverband Elektrotechnik- und Elektroindustrie e.V. (Hrsg.), Lösungskonzept der deutschen Elektroindustrie für die Verwertung und Entsorgung elektrotechnischer und elektronischer Geräte, ZVEI-Memorandum zum Entwurf einer "Elektronik-Schrott-Verordnung", a.a.O., S. 7 und A-11ff.

296 Raabe diskutiert ebenfalls drei Möglichkeiten der Redistribution: 1. Direkter Redistributionsweg durch den Hersteller, 2. Redistribution in Zusammenarbeit zwischen Hersteller und Handel und 3. Redistribution in Verbindung mit dem Einsatz von spezialisierten Redistibutionsmittlern (Verwerter) und nimmt eine gegenüberstellende Bewertung aus Herstellersicht hinsichtlich der Arbeitsteilung, der verbraucherseitigen Akzeptanz sowie der Nutzbarkeit und Kosten vor. Raabe kommt dabei zu dem Schluß, daß ein direktes Redistributionssystem sich nur im Investitionsgütergeschäft bei gleichzeitigem Direktvertrieb lohnt und räumt den Kooperationen zwischen Herstellern und Verwertern die größten Chancen ein. Vgl. Raabe, T., Die ElektronikSchrott-Verordnung: Perspektiven einer aktiven, herstellerseitigen Redistributionspolitik, in: Jahrbuch der Absatz- und Verbrauchsforschung, 1993, S. 297ff.

297 Stölzle und Jung gehen davon aus, daß dieser Rückführungsservice für die Kunden einen ähnlich wichtigen Stellenwert einnehmen wird wie der Lieferservice. Vgl. Stölzle, W., Jung, K. P., Strategische Optionen der Entsorgungslogistik zur Realisierung von Kreislaufwirtschaftskonzepten, in: UWF, Heft 1, 1994, S. 33. In der vorliegenden Befragung geben jedoch nur $26,2 \%$ der Hersteller an, daß sie die Rückführungslogistik als wettbewerbsrelevant einschätzen. $30,7 \%$ der Hersteller meinen, daß diese Aufgabe keinen oder kaum Einfluß auf die Wettbewerbsposition hat.

Ziems und Koschay argumentieren für die strategische Bedeutung der Rückführungslogistik mit den Kostensenkungspotentialen von 10-30\% als Wettbewerbsvorteil, die durch eine optimierte Rückführungslogistik erzielt werden können. Vgl. Ziems, D., Koschay, V., Innerbetriebliche Entsorgungslogistik, in: UWF, Heft 1, 1996, S. 38. Auch die "klassische Logistik“ ist bei vielen Unternehmen noch verbesserungsbedürttig. Laut einer Studie von A.T. Kearney verfügen nur $4 \%$ aller Unternehmen über "optimale“ Logistikabläufe. Vgl. o.V., Schnittstellenfrei, in: Ernährungswirtschaft, Heft 2, 1995, S. 17.

298 Vgl. Stölzle, W., Jung, K. P., Strategische Optionen der Entsorgungslogistik zur Realisierung von Kreislaufwirtschaftskonzepten, a.a.O., S. 33ff. 
Entwicklung von Recyclingtechnologien oder sind in der Lage, Stoffe zu verwerten, die sie anschließend den Herstellern wieder zur Verfügung stellen. Auch der Wettbewerb wird aus der Sicht der befragten Hersteller kaum in die Systemgestaltung eingebunden. Dieses ist aufgrund der kartellrechtlichen Auseinandersetzungen bei Branchenlösungen jedoch nicht verwunderlich. ${ }^{299}$ Die direkte Zusammenarbeit zwischen Wettbewerbern beschränkt sich daher i.d.R. auf die Planung des Systemdesigns. Indirekt arbeiten die Wettbewerber jedoch über die Entsorgungsunternehmen, die gleichsam als "Clearingstellen" fungieren, zusammen. ${ }^{300}$ Die Entsorgungsunternehmen bieten den unterschiedlichen Wettbewerbern in dieser Funktion die von innen ausgearbeiteten Branchenkonzepte an und schließen mit jedem einzelnen Wettbewerber individuelle Verträge ab.

Die mit den rücknahme- und recyclingsystemspezifischen Aufgaben zu verbindenden Implementierungsaufgaben können in Anlehnung an die Umsetzungsziele $^{301}$ strukturiert werden. Die Implementierungsaufgaben, die als Basiselement des Implementierungsprozesses bezeichnet werden ${ }^{302}$, wurden in zehn Items operationalisiert, die die Spezifizierung der Konzeption der Rücknahme- und Recyclingsysteme sowie die Anpassung der Unternehmenspotentiale beinhalten (vgl. Abb. 13).

Der Strategiespezifizierung, die durch den Stellenwert der Ableitung operativer Maßnahmen erfaßt wird, wird mit einem Mittelwert von 2,66 eine wichtige bis mittelmäßige Bedeutung beigemessen. Eine weitergehende Analyse der Häufigkeiten zeigt, daß viele Hersteller dieser Aufgabe eine sehr wichtige (oder wichtige) Beurteilung zumessen $(51,2 \%)$, andere Hersteller jedoch dieser Aufgabe keine oder kaum eine Bedeutung beimessen (26,2\%). Letztere sind entweder Hersteller, die keine Konzeption ihres Rücknahme- und Recyclingsystems im Sinne einer Planung durchgeführt haben oder solche Hersteller, die durch schrittweises Vor-

299 Vgl. Zentralverband Elektrotechnik- und Elektroindustrie e.V. (Hrsg.), Lösungskonzept der deutschen Elektroindustrie für die Verwertung und Entsorgung elektrotechnischer und elektronischer Geräte, a.a.O., S. A-8.

Vgl. Meffert, H., Kirchgeorg, M., Ziele und Strategien zur Gestaltung von produktbezogenen Rücknahme- und Recyclingsystemen, a.a.O., S. 35.

301 Vgl. Kapitel B 1.3.

Die durchsetzungsbezogenen Aufgaben werden im Rahmen der individualbezogenen Einflußfaktoren diskutiert, da hier die Frage nach strukturbezogenen Aufgaben der Einbindung von Funktionen und Abteilungen im Vordergrund steht. Vgl. Kapitel C 1.5.

Vgl. Kolks, U., Strategieimplementierung, a.a.O., S.114. 
gehen nach und nach Maßnahmen ableiten und dadurch keinen großen Aufgabenkomplex „Strategiespezifizierung“, sondern mehrere Teilkomplexe zu bewältigen haben.

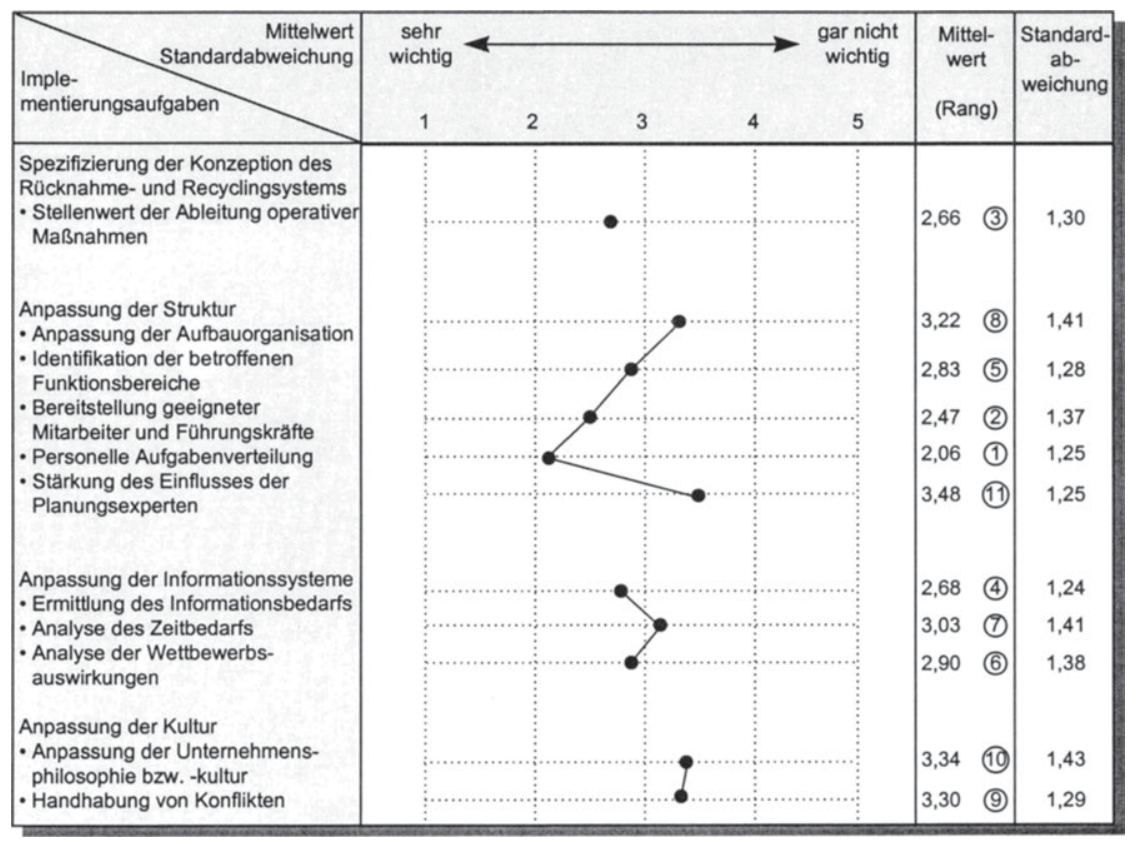

Abb. 13: Stellenwert der Implementierungsaufgaben bei Herstellern langlebiger Gebrauchsgüter

Bei der Anpassung der Struktur an die Erfordernisse des Rücknahme- und Recyclingsystems ist die Organisationsstruktur, die als Menge von Regelungen für die Aktivitäten der Mitarbeiter verstanden wird, ${ }^{303}$ so zu gestalten, daß der Implementierungsprozeß gewährleistet werden kann. Dabei sind zeitlich befristete, multipersonale interdisziplinäre organisatorische Maßnahmen und auf Dauer angelegte Veränderungen in der Organisationsstruktur (Organisationsform) zu unterscheiden. ${ }^{304}$ Es fällt auf, daß bei der Implementierung von Rücknahme- und Recycling-

303 Vgl. Kieser, A., Kubicek, H., Organisation, a.a.O., S. 15.

304 Als weitere Klassifikationsmerkmale von Organisationsstrukturen unterscheidet Frese:1. Unipersonelle oder multipersonelle Strukturausrichtung, 2. Eindimensionale oder mehrdimen-

Irene Giesen-Netzer and Universität Münster - 978-3-631-75058-2 
systemen personenbezogene Maßnahmen wie die Bereitstellung geeigneter Mitarbeiter und Führungskräfte sowie die personelle Aufgabenverteilung von hoher Wichtigkeit für die befragten Hersteller sind. Dem folgt mit hoher bis mittelmäßiger Bedeutung die Identifikation der Funktionsbereiche, die die Koordination im Unternehmen und mit den Systempartnern übernehmen sollen. Die Anpassung der Aufbauorganisation, d.h. beispielsweise die Schaffung einer zusätzlichen Abteilung oder die strukturelle Veränderung von Abteilungen, spielt nur eine untergeordnete Rolle. Dieses spricht dafür, daß die Hersteller die Implementierungsaufgaben überwiegend mit ihren bestehenden Strukturen wahrnehmen. Die Stärkung des Planungsexperten (Fachpromotors), also derjenigen Person(en), die ggf. die Konzeption des Rücknahme- und Recyclingsystems ausgearbeitet hat (haben), ist relativ unwichtig und nimmt den letzten Rang der Wichtigkeit aller Implementierungsaufgaben ein. Dieser Befund läßt die Interpretation zu, daß entweder kaum Pläne entwickelt wurden oder die Planungspersonen direkt mit der Implementierung (i.V.m. einer Durchsetzungsbefugnis) betraut wurden und daher keine Stärkung des Einflusses notwendig war. Eine weitere Erklärung besteht darin, daß die Implementierung die Planung komplett ablöst und andere Personen damit betraut werden.

Die Informationssysteme umfassen die Analyseinstrumente zur Planung, Steuerung und Kontrolle der Implementierung. ${ }^{305}$ Während die Planungssysteme systematisch Informationen für zukünftige Unternehmensentscheidungen liefern, dienen Steuerungssysteme der gegenwärtigen Entscheidungshilfe. Kontrollsysteme schließlich ermöglichen eine ex-post Analyse des vergangenen Unternehmensverhaltens, um Abweichungen von den Zielsetzungen zu erhalten und ggf. Anpassungsmaßnahmen durchzuführen. Bei der rücknahme- und recyclingsystembezogenen Anpassung der Informationssysteme wird der Ermittlung des Informationsbedarfs der relativ höchste Stellenwert eingeräumt. Dieses ist nicht überraschend, da das Implementierungsobjekt sehr komplex ist und die Koordination mit den Systempartnern vielfältige Informationsströme erfordert. Die Analyse der wettbewerbsbezogenen Auswirkungen und des Zeitbedarfs der Implementierung nimmt hingegen nur einen mittelmäßigen Stellenwert ein.

sionale Strukturausrichtung, 3. Traditionelle oder computergestützte Strategieausrichtung. Vgl. Frese, E., Grundlagen der Organisation, a.a.O., S. $170 \mathrm{ff}$. 
Die Anpassung der Unternehmenskultur, die die gemeinsamen geteilten Werte der Mitarbeiter zum Ausdruck bringt, ${ }^{306}$ wird von den Herstellern als mittel- bis kaum wichtig eingeschätzt. Hier ergibt eine weitere Analyse der Häufigkeiten, daß eine Polarisierung stattfindet und die Hersteller kaum eine mittelmäßige Bewertung abgegeben haben $(9,6 \%)$. Sie entscheiden sich entweder dafür, die Kultur fast $(24,1 \%)$ oder völlig $(28,9 \%)$ zu vernachlässigen oder aber sie messen der Anpassung der Unternehmenskultur einen wichtigen oder sehr wichtigen Stellenwert zu (27,3\%). Diejenigen Hersteller, die der Anpassung der Kultur wenig Bedeutung beimessen, bergen auch solche Hersteller, die in ihrer Kultur bereits von jeher sehr umweltorientiert waren und daher keinen Anpassungsbedarf sehen.

Aus den bisherigen Ausführungen kann gefolgert werden, daß die Hersteller in unterschiedlichem Ausmaß Aufgaben der Gestaltung und Implementierung von Rücknahme- und Recyclingsystemen übernehmen. Diese Aufgaben werden von verschiedenen Abteilungen durchgeführt, die zunächst identifiziert werden müssen.

\subsubsection{Identifikation betroffener Funktionen der Hersteller und Operationali- sierung der Intensitätsdimension}

Die rücknahme- und recyclingsystemspezifischen sowie die Implementierungsaufgaben werden - so weit sie nicht externalisiert werden - in den klassischen Funktionen (einschließlich der Umweltschutzabteilung ${ }^{307}$ ) des Herstellers durchgeführt. Die Anpassungserfordernisse erstrecken sich dabei über den gesamten Produktlebenszyklus der (Alt-)Produkte und damit über alle Wertkettenaktivitäten der Hersteller, d.h. angefangen von der recyclinggerechten Produktkonstruktion in der Forschung und Entwicklung über den Einkauf und die Produktion bis hin zum Controlling und Marketing. ${ }^{308}$ Abb. 14 zeigt, wie die befragten Hersteller die Betroffenheit der Funktionen durch die beschriebenen Aufgaben einschätzen. ${ }^{309}$

\footnotetext{
306 Vgl. Frese, E., Grundlagen der Organisation, a.a.O., S. $148 \mathrm{ff}$.

Meffert erläutert, daß die Kultur das Verhalten der Mitarbeiter beeinflußt und damit auch den Ablauf der internen Prozesse. Vgl. Meffert, H., Marketing-Mangement, a.a.O., S. 371f. Damit kann die Kultur als Einflußfaktor des Implementierungsverhaltens betrachtet werden. Vgl. zur Operationalisierung der Unternehmenskultur Kapitel C 1.4.2.3. 


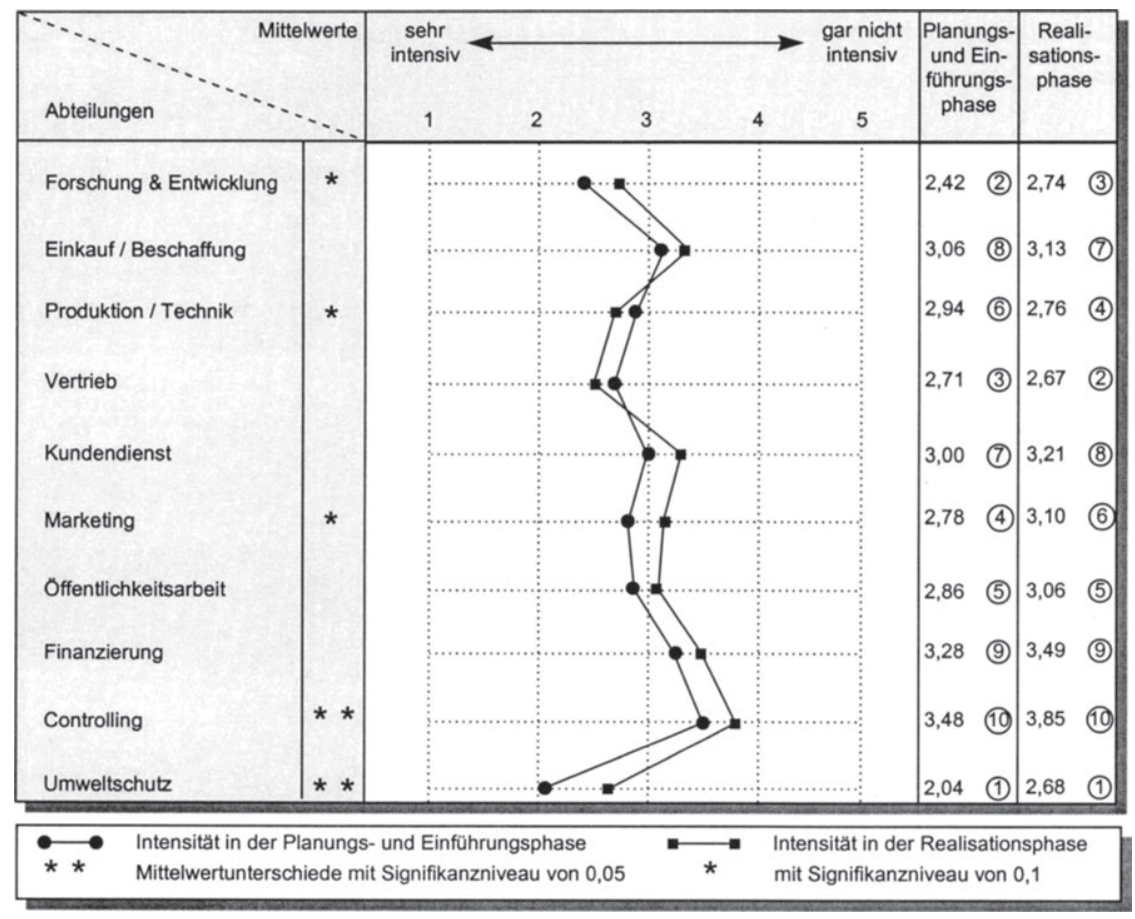

Abb. 14: Identifikation der betroffenen Funktionen der Herstellerunternehmen

Auffallend an diesem Profil ist zunächst, daß die Umweltschutzabteilung bei den Herstellern durch die Implementierung von Rücknahme- und Recyclingsystemen am meisten betroffen ist. In der Einführungsphase ist die Umweltschutzabteilung stärker involviert als in der Realisationsphase, was darauf schließen läßt, daß die konzeptionelle Ausarbeitung überwiegend der umweltbezogenen Kompetenz zugesprochen wird. Die Forschung und Entwicklung, die sich mit der Produktkonzeption befaßt, ist diejenige Abteilung, die in der Einführungsphase den zweitwichtigsten Stellenwert einnimmt. Hier fallen Aufgaben an, die Produkte so zu konzipieren oder zu verändern, daß sie den Anforderungen des Rücknahme- und Recyclingsystems entsprechen. Dieses kann bei einer Verwertung der langlebigen

Bereits in den $80 \mathrm{er}$ Jahren wurden funktionsübergreifende Ansätze des ökologieorientierten Unternehmensverhaltens diskutiert. Vgl. Kirchgeorg, M., Ökologieorientiertes Unternehmensverhalten, a.a.O., S. 21ff. und die dort angegebene Literatur.

Vgl. zur genauen Fragestellung die Auszüge aus dem Fragebogen in Anhang 3.1. 
Gebrauchsgüter in der Ermittlung einer verwertungsgerechten Materialzusammensetzung (Reduktion der Stoffvielfalt, Werkstoffverträglichkeit) liegen und bei einer Verwendung in der leichteren Demontierbarkeit ${ }^{310}$ (Trennbarkeit, Zugänglichkeit, Normung und Typisierung, Modulbauweise $\left.{ }^{311}\right)$. Zusätzlich müssen Einsatzmöglichkeiten für Sekundärprodukte oder -materialien gefunden sowie Qualitätsanforderungen definiert werden, ${ }^{312}$ wenn die Hersteller diese wieder ins eigene Unternehmen zurückführen möchten. In der Realisationsphase nimmt die Forschungs- und Entwicklungsabteilung den dritten Rang in der Betroffenheit ein. Hier hat diese Abteilung die Aufgabe, erste Erfahrungen und Feedbacks der Verwertung und Verwendung in die Produktkonzeption umzusetzen. ${ }^{313}$

Der Vertrieb hat mit einem Mittelwert von 2,71 in der Einführungsphase und einem Mittelwert von 2,67 in der Realisationsphase ebenfalls einen relativ hohen Betroffenheitsgrad durch die Implementierung des Rücknahme- und Recyclingsystems. Dieses kann darin begründet liegen, daß die Rücknahme der Altgeräte insbesondere bei investiven langlebigen Gebrauchsgütern durch den Vertrieb organisiert wird und z.T. Gegenstand der Verträge ist. ${ }^{314}$ Die Mittelwertunterschiede in den Implementierungsphasen sind nicht signifikant unterschiedlich, womit deutlich wird, daß der Vertrieb in der Einführungsphase ebenso eingebunden ist (z.B. In-

310 Im Produktionstechnischen Zentrum Berlin wird die Demontage technischer Konsumgüter wie Computer und Fernseher an die Produktspezifika angepaßt. Dabei sollen automatisierte flexible Recyclingprozesse entwickelt werden, die eine Verringerung der Deponierung von Sondermüll bei der Shredderung der Geräte ermöglichen. Vgl. o.V., Neue Wege bei der Demontage von Monitoren durch flexible Recyclingprozesse, in: Handelsblatt, 10.3.1995, S.7.

Vgl. zu den beispielhaften Maßnahmen Krcal, H.-Chr., Wirkungsbeziehungen produktbezogener Umweltschutzmaßnahmen als Beweggrund zwischenbetrieblicher Zusammenarbeit, a.a.O., S. $26 f f$.

312 Vgl. Krcal, H.-Chr., ebenda, S. 25.

313 Die Beschaffungs- und Einkaufsabteilung hat nur einen relativ geringen Betroffenheitsgrad während der Implementierung des Rücknahme- und Recyclingsystems. Dieses mag darin begründet liegen, daß die Maßnahme der „Beschaffung und Einsatzmöglichkeit von umweltschonenden Roh-, Hilfs- und Betriebsstoffen" insbesondere auf die vorgelagerten Vorlieferanten und Lieferanten wirkt. Wenn diese eine Stoffsubstitution nicht ermöglichen, werden andere (Vor-)Lieferanten vom Hersteller ausgewählt. Vgl. zu dieser Argumentation ebenda, S. 24. Dieses ist m.E. plausibel, da lediglich weitere (materialbezogene) Anforderungen an den bereits bestehenden Anforderungskatalog bei der Bestellung hinzugefügt werden.

Backhaus äußert zudem, daß insbesondere im Bereich der Automobilindustrie die Zulieferer von den Herstellern abhängig sind. Dieses wird mit der "Integralqualität" von Komponenten begründet, d.h. diese sind genau auf das Auto zugeschnitten ("lock-in-Effekt"). Daher können Automobilhersteller häufig die Ausgestaltung der Beziehung bestimmen. Vgl. Backhaus, K., Marketingschnittstellen, in: Specht, G. (Hrsg.), Marketingschnittstellen, Stuttgart 1989, S. $288 f f$.

Vgl. Fallstudie Siemens-Nixdorf, a.a.O. 
formationen über das Rücknahme- und Recyclingsystem sowie geänderte Produktanforderungen) wie in der Realisationsphase (z.B. Rücknahme).

In der Produktion bzw. Technik ${ }^{315}$ ist in der Realisationsphase mit (signifikant) höheren Anforderungen (Rang 4) im Rahmen der Implementierung zu rechnen als in der Einführungsphase (Rang 6). Hier entstehen neue Herausforderungen dadurch, daß bei einer Wiederverwertung und -verwendung Sekundärrohstoffe und Altteile der langlebigen Gebrauchsgüter in den Produktionsprozeß integriert werden $^{316}$ und technische Anforderungen gewährleistet sein müssen. Dem Marketing $^{317}$ hingegen wird in der Einführungsphase ein deutlich höherer Stellenwert beigemessen (Mittelwert: 2,78, Rang 4) als in der Realisationsphase (Mittelwert 3,1, Rang 6). Eine mögliche Ursache besteht darin, daß bereits im Vorfeld insbesondere der Fit zwischen den Marketingstrategien einerseits und dem Rücknahme- und Recyclingsystem andererseits geprüft werden muß. Auch die Überprüfung der Komplementarität einer Qualitätsführerschaft einerseits mit "gebrauchten Geräten" andererseits, ${ }^{318}$ sowie die Überprüfung des Absatzpotentials für solche Produkte sind Aufgaben, die das Marketing vermutlich in der Einführungsphase durchführt.

Betrachtet man die am wenigsten betroffenen Abteilungen, sind dieses die Finanzabteilung und das Controlling. Mögliche Ursachen liegen darin, daß sich die Rücknahme- und Recyclingsysteme häufig noch in frühen Phasen befinden und daher ein effektives Controlling nur schwer möglich ist und zum andern anfallende Kosten häufig nicht eindeutig der Implementierung des Rücknahme- und Recyclingsystems zurechenbar sind.

Wie intensiv die Hersteller mit ihren eigenen Funktionen von der Einrichtung des Rücknahme- und Recyclingsystems betroffen sind, kann empirisch als globale

315 Auf der Envitec - Internationale Fachmesse für Umweltschutz- und Entsorgungstechnologien wurden 1995 hauptsächlich Technologien für den nachsorgenden Umweltschutz ausgestellt. Vgl. o.V., Gute, teure Technik ist schwer verkäuflich, in: Handelsblatt, 14.6.1995, S. B1. Das läßt darauf schließen, daß weniger die Hersteller in der Produktion als die Verwerter für die Verwertung von Abfällen zuständig ist. Beweggrund zwischenbetrieblicher Zusammenarbeit, a.a.O., S. 255. 
Größte über die Anzahl der (sehr intensiv und intensiv) einbezogenen Funktionen in der Einführungs- und Realisationsphase des Systems bei den Herstellern operationalisiert werden. Diese Anzahl an Funktionen wird im weiteren als die Intensitätsdimension der Implementierung von Rücknahme- und Recyclingsystemen bezeichnet, da dieses Merkmal darauf hinweist, wie hoch die eingesetzten personellen und sachlichen Ressourcen der Hersteller bei der Implementierung von Rücknahme- und Recyclingsystemen sind. ${ }^{319}$

Damit ist die Ableitung der Implementierungsdimensionen bei Rücknahme- und Recyclingsystemen abgeschlossen. Bevor eine empirische Überprüfung der Dimensionen stattfindet, soll eine Zusammenführung derselben in ein Gesamtmodell stattfinden.

\subsection{Integration der Implementierungsdimensionen von Rücknahme- und Recyclingsystemen}

In den vorhergehenden Kapiteln wurden insgesamt sechs Dimensionen des Implementierungsverhaltens hergeleitet, die anhand von 18 Variablen operationalisiert wurden. Abbildung 15 faßt diese Implementierungsdimensionen im Überblick zusammen.

319 Die Intensitätsdimension wird über zwei Variablen gemessen vgl. dazu auch den Auszug aus dem Fragebogen im Anhang 3.1:

\begin{tabular}{ll}
\hline Implementierungsdimension & Variablen \\
\hline Intensität & 1. Anzahl der ((sehr) intensiv) einbezogenen Abteilungen in \\
der Einführungsphase
\end{tabular}




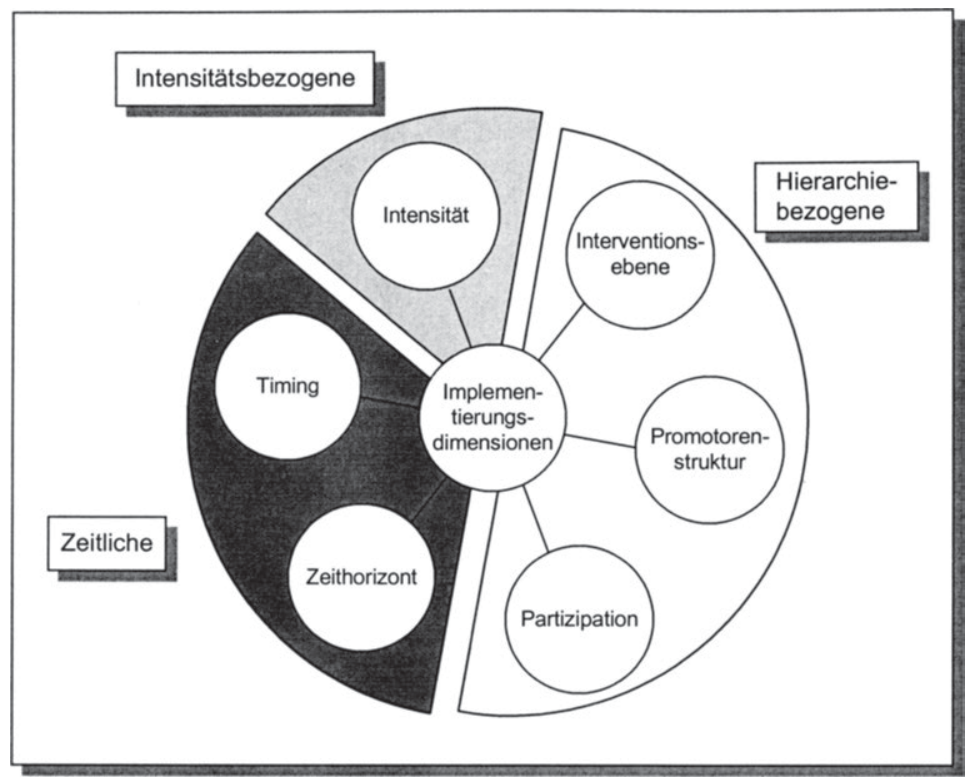

Abb. 15: Modell der identifizierten Implementierungsdimensionen bei Rücknahme- und Recyclingsystemen

Dieses Vorgehen präjudiziert die Anwendung der konfirmatorischen Faktoranalyse zwecks empirischer Überprüfung der Existenz dieser Dimensionen. ${ }^{320}$ Dem Konstrukt liegt damit folgende Basishypothese zugrunde:

\begin{tabular}{|ll|}
\hline $\mathrm{H}_{\mathrm{IV} 2}:$ & $\begin{array}{l}\text { Ausgehend von einer Analyse des Implementierungsverhaltens lassen sich zeitliche, in- } \\
\text { tensitätsbezogene und hierarchiebezogene Dimensionen identifizieren, die sich in sechs } \\
\text { Implementierungsdimensionen (Faktoren) bei Rücknahme- und Recyclingsystemen un- } \\
\text { terteilen. }\end{array}$ \\
\end{tabular}

Weiterhin ist die Fragestellung von zentraler Relevanz, welche der Implementierungsdimensionen bei der Implementierung von Rücknahme- und Recycling-

320 Die konfirmatorische Faktoranalyse ist ein strukturprüfendes Verfahren, das durch theoretisch fundierte Überlegungen gewonnene Annahmen über die Anzahl von Faktoren, die Beziehungen zwischen den Faktoren und zwischen Faktoren und Variablen empirisch überprüft. Die explorative Faktoranalyse ist hingegen ein hypothesenbildendes Verfahren. Vgl. Ost, F., Faktorenanalyse, in: Fahrmeir, L., Hamerle, A. (Hrsg.), Multivariate statistische Verfahren, Berlin, New York, de Gruyter 1984, S. 639ff. und Backhaus, K., U.a., Multivariate Analysemethoden, Eine anwendungsorientierte Einführung, 8., verb. Aufl., Berlin, u.a.1996, S. $407 \mathrm{ff}$.

Vgl. auch Anhang 2.1. 
systemen die höchste Bedeutung hat und welche von eher geringerem Stellenwert ist. Gleichzeitig ist zu klären, ob sich die Hersteller bei der Implementierung von Rücknahme- und Recyclingsystemen tatsächlich in Implementierungstypen einteilen lassen. Die Herleitung von Implementierungstypen erfolgt durch eine Zusammenfassung der Hersteller zu Gruppen mit ähnlichen Antwortprofilen (Muster), die untereinander hinsichtlich der Ausprägungen der Implementierungsdimensionen differieren (Typen). Voraussetzung für die Bildung von Implementierungstypen sind herstellerbezogene Gestaltungsspielräume in den Implementierungsdimensionen. ${ }^{321}$ Folgende Plausibilitätsüberlegungen führen zu der Annahme, daß die Gestaltungsspielräume je Implementierungsdimension unterschiedlich ausgeprägt sind. ${ }^{322}$

\begin{tabular}{|l|l|l|l|}
\cline { 2 - 4 } \multicolumn{1}{c|}{} & $\begin{array}{l}\text { Implementie- } \\
\text { rungs- } \\
\text { dimensionen }\end{array}$ & $\begin{array}{l}\text { voraussichtlich } \\
\text { er Gestaltungs- } \\
\text { spielraum }\end{array}$ & \multicolumn{1}{|c|}{ Begründung } \\
\hline Zeitliche & Timing & hoch bis mittel & $\begin{array}{l}\text { Pioniere im „rechtsfreien“ Raum befragt, da } \\
\text { Verordnungen erst später eingeführt werden. }\end{array}$ \\
\cline { 2 - 5 } & Zeithorizont & hoch bis mittel & $\begin{array}{l}\text { Aufgrund des frühzeitigen Beginns (Pioniere) } \\
\text { der Implementierung stehen lange Zeiträume } \\
\text { für die Implementierung zur Verfügung. }\end{array}$ \\
\hline $\begin{array}{llll}\text { Hierarchie- } \\
\text { bezogene }\end{array}$ & $\begin{array}{l}\text { Interventions- } \\
\text { ebene }\end{array}$ & hoch bis mittel & $\begin{array}{l}\text { Rücknahme- und Recyclingsysteme sind } \\
\text { i.d.R. unterschiedlich kooperativ ausgeprägt. } \\
\text { Daher ist die Einbeziehung der Geschäftslei- } \\
\text { tung bei der Einführung unterschiedlich. }\end{array}$ \\
\cline { 2 - 4 } & Partizipation & hoch bis mittel & $\begin{array}{l}\text { Unternehmen mit unterschiedlicher Führungs- } \\
\text { philosophie befragt. }\end{array}$ \\
\cline { 2 - 4 } & Promotoren- & gering bis mittel & $\begin{array}{l}\text { Dokumentierte Erfahrungen sind gering. Ei- } \\
\text { gene Erfahrungen von hoher Bedeutung. } \\
\text { Bei befragten Pionieren Stellenwert von } \\
\text { Rücknahme- und Recyclingsystemen hoch. }\end{array}$ \\
\hline $\begin{array}{l}\text { Intensitäts- } \\
\text { bezogene }\end{array}$ & Intensität & gering bis mittel & $\begin{array}{l}\text { „Wesen“ von Rücknahme- und Recycling- } \\
\text { systemen auf funktionsübergreifende Ansätze } \\
\text { ausgelegt. } \\
\text { Hersteller langlebiger Gebrauchsgüter wer- } \\
\text { den in ähnlicher Art und Anzahl Abteilungen } \\
\text { einbeziehen. }\end{array}$ \\
\hline
\end{tabular}

Tab. 4: Gestaltungsspielräume der Implementierung von Rücknahme- und Recyclingsystemen

321 Zu einer ähnlichen Argumentation bezogen auf die Gestaltungsspielräume bei der Bildung von Führungstypen vgl. Meurer, J., Führung von Franchisesystemen, a.a.O., S. 91.

Empirisch kann das durch die Skalierung und die Beantwortung der Fragestellungen zwischen eins und fünf erfaßt werden.

322 Diese Plausibilitätsüberlegungen sollen zunächst nur begründen, warum angenommen wird, daß sich Typen bilden lassen und diese sich insbesondere hinsichtlich spezifischer Implementierungsdimensionen unterscheiden. Eine empirische und vollständigere Diskussion erfolgt jedoch in Kapitel B 3. 
Aus der Tabelle 4 wird deutlich, daß vermutlich insbesondere bei den zeitlichen Dimensionen und den hierarchiebezogenen Dimensionen der Interventionsebene und der Partizipation hohe Gestaltungsspielräume bei der Implementierung von Rücknahme- und Recyclingsystemen bestehen. Diese Überlegungen erfolgen daraus, daß sich die befragten Hersteller in einer noch ungeklärten Rechtslage befinden, d.h. es wurden solche Hersteller befragt, die bereits vor Inkrafttreten des Kreislaufwirtschaftsgesetzes mit der Implementierung von Rücknahme- und Recyclingsystemen begonnen haben. Die herstellerbezogene Entscheidung über das Timing und die Dauer bleibt also den unternehmensindividuellen Präferenzen überlassen.

Einschränkungen hinsichtlich der Gestaltungsspielräume der Hersteller bei der Implementierung von Rücknahme- und Recyclingsystemen ergeben sich aufgrund der Plausibilitätsüberlegungen hauptsächlich für die Promotorenstruktur und für die Intensitätsdimension. Mögliche Ursachen könnten zum einen in der Dominanz von Pionieren der Implementierung von Rücknahme- und Recyclingsystemen in der vorliegenden Untersuchung liegen. Dieses Vorgehen führt dazu, daß voraussichtlich in solchen Unternehmen umweltbezogene Fragestellungen hoher Relevanz sind und Promotoren in hohem Maße einbezogen werden. Zum anderen ist das "Wesen" von Rücknahme- und Recyclingsystemen als mögliche Erklärung heranzuziehen. D.h. ein Rücknahme- und Recyclingsystem ist i.d.R. als „Kreislauf” mit funktionsübergreifendem Ansatz darauf ausgelegt, eine gewisse (relativ hohe) Anzahl von Abteilungen einzubeziehen, welches den Gestaltungsspielraum bei der Intensitätsdimension nur mittel bis gering erscheinen läßt. Diese Überlegungen drücken sich in folgender Basishypothese aus:

\begin{tabular}{ll}
\hline $\mathrm{H}_{\mathrm{IV}}: \quad \begin{array}{l}\text { Die Implementierungsdimensionen liefern aufgrund ihrer spezifischen Gestaltungsspiel- } \\
\text { räume signifikant unterschiedliche Erklärungsbeiträge für die Typenbildung und } \\
\text {-trennung bei der Implementierung von Rücknahme- und Recyclingsystemen. }\end{array}$ \\
\hline
\end{tabular} 


\subsection{Konfirmatorische Faktoranalyse zur Überprüfung der hergeleiteten Implementierungsdimensionen}

Um die Dimensionen der Implementierung einer empirischen Überprüfung zugänglich zu machen, war es notwendig, sie und ihre zugrunde liegenden beobachtbaren Variablen in ein Modell der konfirmatorischen Faktoranalyse zu überführen. Voraussetzung für die Ermittlung von verläßlichen Schätzparametern ${ }^{323}$ ist dabei die Identifizierbarkeit des Modells. ${ }^{324}$ Die Identifizierbarkeit des Modells wird üblicherweise über die Freiheitsgrade des Modells gemessen. ${ }^{325}$ Diese (notwendige) Bedingung ist bei dem vorliegenden Modell mit 121 Freiheitsgraden $^{326}$ erfüllt. Für die Parameterschätzung wurde das iterative Schätzverfahren der ungewichteten kleinsten Quadrate (ULS) gewählt. Dieses ermöglicht zum einen die simultane Verwendung aller Informationen aus der empirischen Korrelationsmatrix für die Schätzung der Parameter. Zum anderen setzt ULS keine Normalverteilung der Ausgangsvariablen voraus. ${ }^{327}$ Die Ergebnisse der konfirmatorischen Faktoranalyse sind in der Abb. 16 ersichtlich.

323 Das Meßmodell der konfirmatorischen Faktoranalyse gibt an, wie latente Variablen (Implementierungsdimensionen) durch beobachtete Variablen erfaßt wird. Die Beziehungen zwischen diesen Variablen und innerhalb der latenten Variablen müssen geschätzt werden. Zusätzlich werden Residualvariablen, d.h. Meßfehler bei den beobachtbaren Variablen geschätzt. Vgl. Backhaus, K., u.a., Multivariate Analysemethoden, a.a.O., S. 414f.

324 Das Identifikationsproblem besteht darin zu zeigen, daß jeder unbekannte Parameter durch Elemente der Varianz-Kovarianz-Matrix bestimmt werden kann. Vgl. zum Identifikationsproblem die Ausführungen von Förster, F., u.a., Der LISREL-Ansatz der Kausalanalyse und seine Bedeutung für die Marketing-Forschung, in: ZfB, Heft 4, 1984, S. $353 f$.

$t<t^{*}$, wobei gilt: $t=A n z a h l$ der zu schätzenden Paramenter, $t^{*}=(q)(q+1) / 2, q=A n z a h l$ der $x$ Variablen.

Allerdings ist damit lediglich die notwendige Bedingung der Identifizierbarkeit erfüllt. Da hinreichende Identifikationsbedingungen für LISREL-Modelle nicht vorliegen, müßte jeder einzelne Modellparameter algebraisch geprüft werden, welches sich als sehr mühsam erweist. Vgl. Förster, F., u.a., Der LISREL-Ansatz der Kausalanalyse und seine Bedeutung für die Marketing-Forschung, a.a.O., S 353f. und Fritz, W., Marketing-Management und Unternehmenserfolg: Grundlagen und Ergebnisse einer empirischen Untersuchung, 2., überarb. und erg. Aufl., Stuttgart 1995, S. 122f. Daher wird im folgenden der üblichen Vorgehensweise in der Literatur gefolgt und nur die notwendige Bedingung überprüft.

Fritz erläutert, daß sich für einen Stichprobenumfang zwischen $n=100$ und $n=200$ das ULSVerfahren anbietet, da das Verfahren bei Stichproben dieser Größenordnung robuste Schätzergebnisse liefern kann. Dieses wurde durch Simulationsstudien nachgewiesen. Vgl. Fritz, W., Marketing-Management und Unternehmenserfolg: Grundlagen und Ergebnisse einer empirischen Untersuchung, a.a.O., S. $118 \mathrm{f}$. 


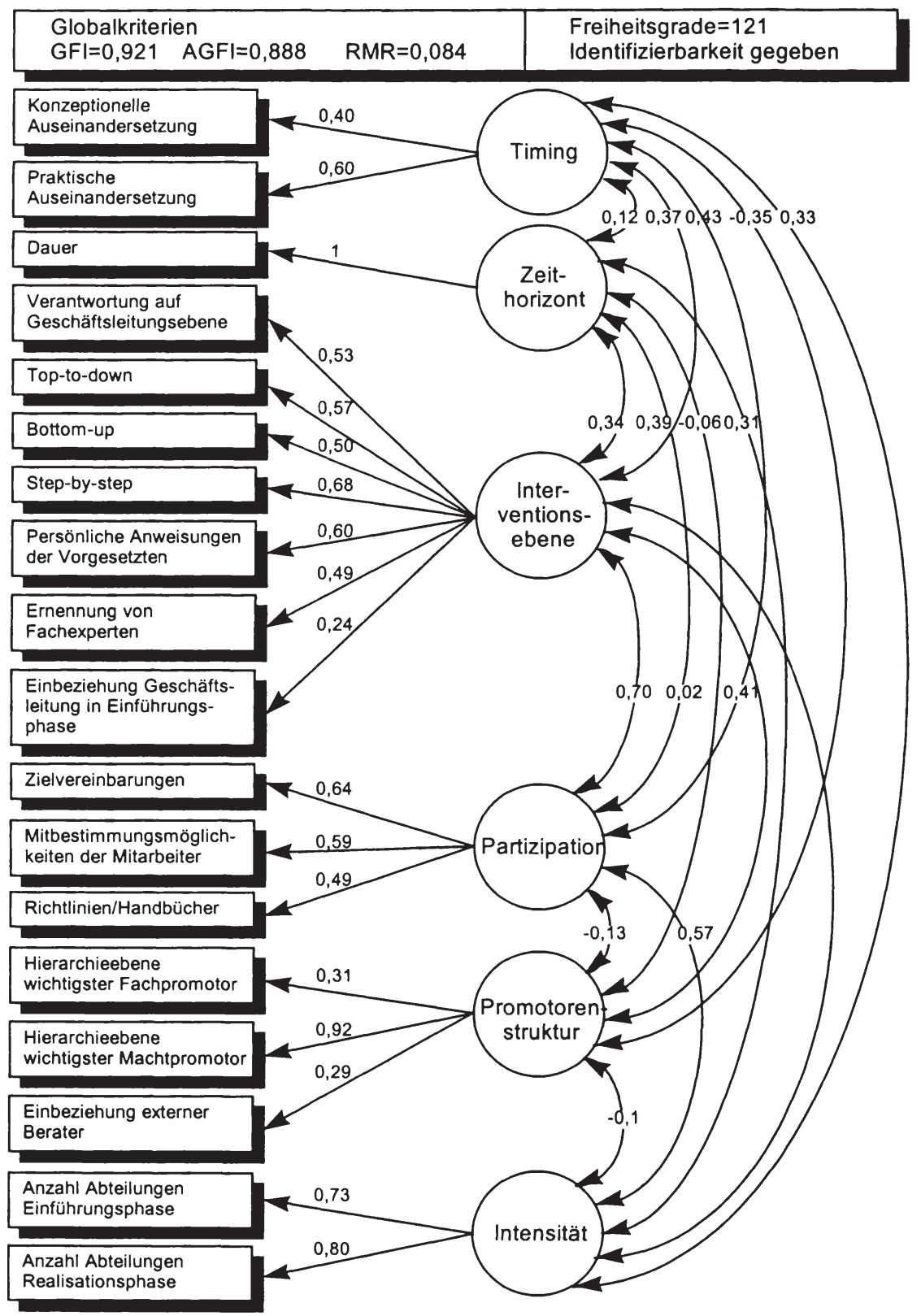

Abb. 16: Konfirmatorische Faktoranalyse für die Implementierungsdimensionen 
Als Gütemaße zur Beurteilung der Gesamtstruktur des Modells werden drei zur globalen Anpassungsgüte, auch bei vollständigen LISREL-Modellen verwendete Fit-Maße herangezogen. ${ }^{328}$ Diese globalen Gütemaße sind der Anpassungsindex ("Goodness of fit-index" (GFI)), der adjustierte Anpassungsindex („adjusted goodness of fit-Index" (AGFI)) und der mittlere Residualkoeffizient ("root mean square residual"-Index (RMR)). ${ }^{329}$ Der GFI mißt die relative Menge an Kovarianz und Varianz, die insgesamt vom Modell erfaßt wird. Der AGFI ist ebenso ein Maß für die Varianz, die vom Modell erklärt wird, berücksichtigt aber darüber hinaus die Zahl der Freiheitsgrade. Der GFI und der AGFI liegen zwischen Null und Eins. Je mehr sie sich an Eins annähern, desto besser ist der Fit des Modells. ${ }^{330}$ Mit den Werten des GFI von 0,912 und des AGFI von 0,888 zeigen diese globalen Maße eine gute Anpassungsgüte der Modellstruktur.

Der RMR bezieht sich auf die Residualvarianzen, die nicht von dem Modell erklärt werden können und muß daher immer in bezug auf die beobachtbaren Varianzen und Kovarianzen gesehen werden. ${ }^{331}$ Je mehr sich der RMR Null annähert, desto weniger Varianz wird nicht erklärt, d.h. desto besser ist die Anpassungsgüte des Modells. Mit einem RMR-Wert von 0,084 bleibt lediglich ein Varianz- und Kovarianzanteil von $8,4 \%$ der Stichprobe unerklärt. ${ }^{332}$

328 Das Chi-Quadrat Maß stellt in der LISREL-Praxis das am häufigsten verwendete und zugleich problematischste Maß der Anpassung der Modelle dar, da die Ausprägung wesentlich von der Stichprobengröße abhängig ist (tendenziell wächst die Ablehnung des Modells mit zunehmender Größe). Daher soll hier der Chi-Quadrat-Wert nur ergänzend betrachtet werden. Dabei gilt es, den Quotient von Chi-Quadrat Wert zur Anzahl der Freiheitsgrade zu betrachten. Die Quotientwerte sollten dabei Werte annehmen, die unter 10 liegen (teilweise werden in der LISRELPraxis auch Werte unter 2 gefordert). Vgl. dazu die Ausführungen von Fritz und die dort angegebene Literatur: Fritz, W., Marketing-Management und Unternehmenserfolg: Grundlagen und Ergebnisse einer empirischen Untersuchung, a.a.O., S. 125f. In dem vorliegenden Fall liegt der Wert bei 189/121=1,6, womit dieser Wert die Anforderungen erfüllt.

Der GFI und AGFI besitzen den Vorteil, daß sie im Gegensatz zum Chi-Quadrat-Wert unabhängig vom Stichprobenumfang und relativ robust gegen Verletzungen der Multinormalverteilungsannahme sind. Vgl. Pfeifer, A., Schmidt, P., Lisrel: Die Analyse komplexer Strukturgleichungsmodelle, Stuttgart, New York 1987, S. 37.

330 Vgl. Backhaus, K., u.a., Multivariate Analysemethoden, a.a.O., S. $399 f$.

331 Vgl. Pfeifer, A., Schmidt, P., Lisrel: Die Analyse komplexer Strukturgleichungsmodelle, a.a.O., S. $37 f$.

332 In der Forschungspraxis werden Kausalmodelle i.d.R. dann akzeptiert, wenn die GFI- und AGFI- Werte größer als 0,9 sind und der RMR einen Wert kleiner als 0,1 aufweist. Vgl. Fritz, W., Marketing-Management und Unternehmenserfolg: Grundlagen und Ergebnisse einer empirischen Untersuchung, a.a.O., S. 126. 
Die bisher dargestellten Gütekriterien geben jedoch keine Auskunft über Teilstrukturen des Modells. ${ }^{333}$ Daher muß anhand von Detailkriterien überprüft werden, wie die Anpassungsgüte von Teilstrukturen zu beurteilen ist und ob Teile des Modells ggf. schlecht spezifiziert wurden ${ }^{334}$ Dazu werden die standardisierten Residuen der einzelnen Variablen, die in einem Q-Plot dargestellt werden können, herangezogen.

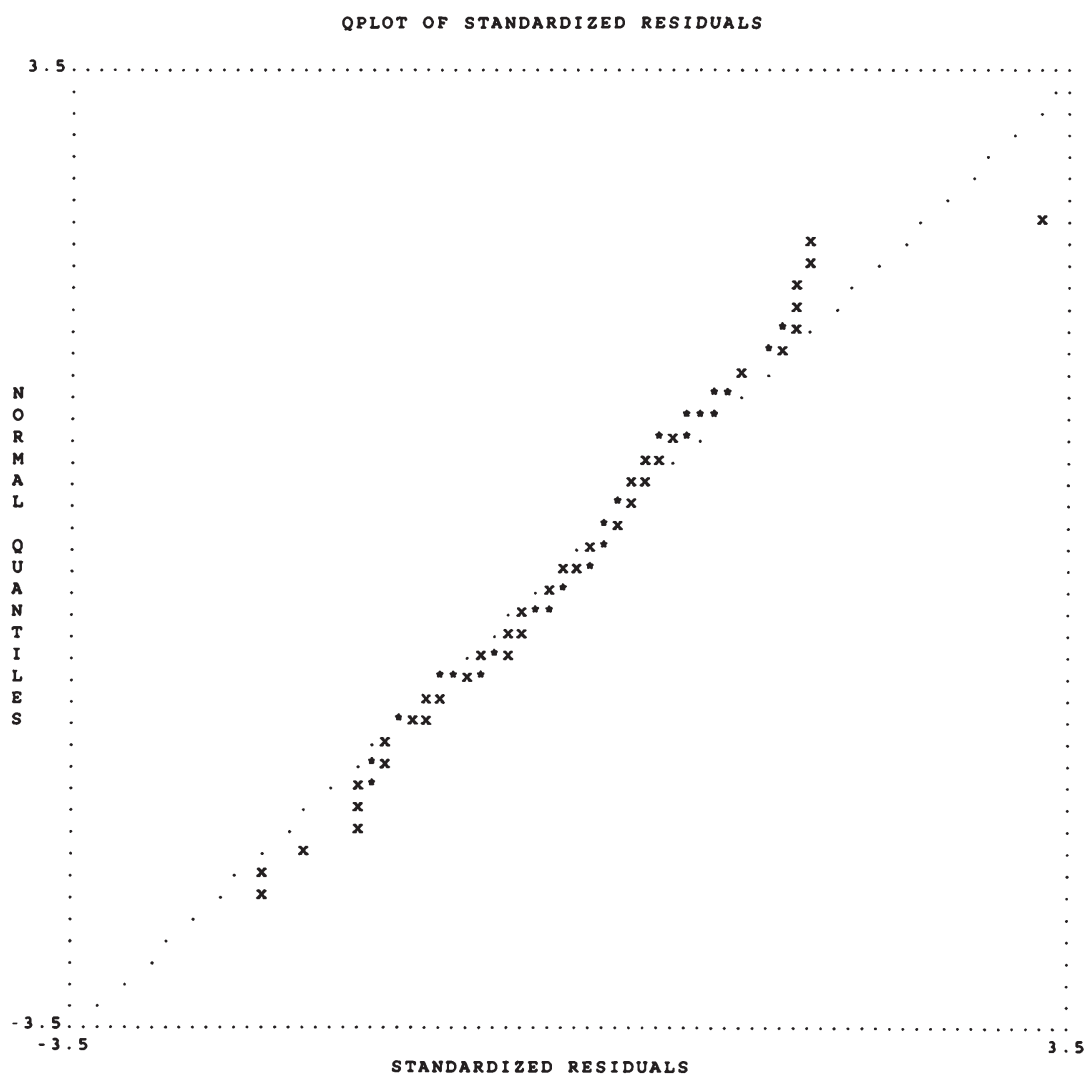

Abb. 17: Q-Plot der standardisierten Residuen

333 Zu einer kritischen Beurteilung der Global- und Detailkriterien vgl. Fritz, W., Marketing-Management und Unternehmenserfolg: Grundlagen und Ergebnisse einer empirischen Untersuchung, a.a.O., S. $121 \mathrm{ff}$ und S. 128. 
Beim Q-Plot werden die standardisierten Residuen gegen die Quantille der Normalverteilung geplottet. Liegen die sich ergebenden Punkte auf einer senkrechten Geraden, so liegt der absolut beste Fit vor, liegen sie auf einer horizontalen Geraden, so bedeutet dieses den absolut schlechtesten Fit. Ein noch zu akzeptierender Fit ergibt sich, wenn die Koordinatenwerte der standardisierten Residuen ungefähr entlang der Diagonalen im Q-Plot verlaufen. ${ }^{335}$ Dieser akzeptable Fit wird, wie aus der Abb. 17 ersichtlich, im vorliegenden Fall erreicht.

Bei der konfirmatorischen Faktoranalyse werden Abhängigkeiten zwischen den verschiedenen Faktoren zugelassen, während bei der explorativen Faktoranalyse möglichst unabhängige Faktoren erzielt werden sollen. ${ }^{336}$ Dennoch dürfen die Faktoren auch bei der konfirmatorischen Faktoranalyse nicht zu stark korrelieren, um eine Unterscheidbarkeit der Implementierungsdimensionen zu ermöglichen. Diese Eigenständigkeit der Implementierungsdimensionen kann anhand der Korrelationskoeffizienten beurteilt werden. Im vorliegenden Modell ergeben sich mit sechs Implementierungsdimensionen 15 Korrelationskoeffizienten. In der Literatur besteht hinsichtlich der Eigenständigkeit und damit der Diskriminanzvalidität ${ }^{337}$ keine eindeutige Meinung. Die Forschungspraxis sieht die Diskriminanzvalidität hingegen als gegeben an, wenn die Koeffizienten kleiner als eins sind und damit nicht vollständig miteinander korrelieren. ${ }^{338}$ Alle Korrelationskoeffizienten liegen bis auf zwei Ausnahmen unter 0,5. Am höchsten korreliert die Interventionsebene mit der Partizipation mit einem Wert von 0,7 sowie die Intensitätsdimension mit der Partizipation mit einem Wert von 0,57. Damit kann die Anforderung der Diskriminanzvalidität als erfüllt angesehen werden.

Als Ergebnis kann die Basishypothese $\boldsymbol{H}_{\mathrm{IV} 2}$ bestätigt werden. Es existieren demnach zeitliche, intensitäts- und hierarchiebezogene Dimensionen bei der Implementierung von Rücknahme- und Recyclingsystemen, die als eigenständig betrachtet werden können. Damit haben sich die theoretischen Überlegungen an der Realität bewährt. Vor diesem Hintergrund ist es möglich, auf Grundlage der ermittelten Implementierungsdimensionen Implementierungstypen zu bilden.

\footnotetext{
335 Vgl. ebenda, S. 403.

336 Vgl. Backhaus, K., u.a., Multivariate Analysemethoden, a.a.O., S. $407 f$.

337 Zum Begriff der Diskriminanzvalidität vgl. Fritz, W., Marketing-Management und Unternehmenserfolg: Grundlagen und Ergebnisse einer empirischen Untersuchung, a.a.O., S. 137f. 


\section{Implementierungstypen bei Rücknahme- und Recyclingsystemen}

\subsection{Ermittlung der Anzahl unterschiedlicher Implementierungstypen}

Können bei zwei (oder mehr) Herstellern gleiche Verhaltensmuster beobachtet werden, so bilden diese einen identischen Implementierungstyp. ${ }^{339}$ Für die Bildung derartiger in sich homogener, aber untereinander heterogener Gruppen von Objekten (Hersteller) anhand der zugehörigen Merkmalsausprägungen (Implementierungsdimensionen) stellt die Clusteranalyse eine geeignetes Verfahren dar. ${ }^{340}$ Die Gruppenbildung bei der Clusteranalyse erfolgt aus methodischen Gründen auf Variablenbasis, da die konfirmatorische Faktoranalyse im Gegensatz zur explorativen Faktoranalyse keine Faktorwerte berechnet, die als Grundlage der Clusteranalyse verwendet werden können.

Bezüglich des Fusionierungsalgorithmus wird auf hierarchische Verfahren ${ }^{341}$ zurückgegriffen, welche eine schrittweise Zusammenfassung von Objekten vormehmen. Dieses hat den Vorteil, daß aufgrund der Entwicklung der Fehlerquadratsumme der Verlauf der Clusterbildung erkennbar bleibt sowie die angemessene Anzahl der Cluster ermittelt werden kann. ${ }^{342}$ Dabei wird ein zweistufiges Vorgehen gewählt. Zunächst werden mit Hilfe des single-linkage-Verfahrens Ausreißer eliminiert, bevor mit dem Ward-Verfahren die eigentliche Gruppenbildung durch-

339 Diese Zusammenfassung zu Gruppen von Herstellern mit ähnlichen (Implementierungs)mustern, die dann als Typen bezeichnet werden, erfolgt unter Rückgriff auf die typologische Methode in der Betriebswirtschaftslehre. Vgl. Knoblich, H., Die typologische Methode in der Betriebswirtschaftslehre, in WiSt, Heft 4, 1972, S. $141 \mathrm{ff}$.

340 Vgl. z.B., Kaufmann, H., Pape, H., Clusteranalyse, in: Fahrmeir, L., Hamerle, A. (Hrsg.), Multivariate statistische Verfahren, Berlin, New York, de Gryter 1984, S. 371; Backhaus, K., u.a., Multivariate Analysemethoden, a.a.O., S. 262 und Meffert, H., Marketingforschung und Käuferverhalten, Marketingforschung und Käuferverhalten, 2. vollst. überarb. und erw. Aufl., Wiesbaden 1992, S. 267.

341 Partitionierende Verfahren hingegen zeichnen sich dadurch aus, daß auf Grundlage einer vorgegebenen Clusterzahl die Zuordnung der Objekte in einem iterativen Prozeß vorgenommen wird. Vgl. Bleymüller, J., Multivariate Analyse für Wirtschaftswissenschaftler, Manuskript, Münster 1989, S. $163 \mathrm{ff}$.

Vgl. Meffert, H., Marketingforschung und Käuferverhalten, a.a.O., S. 273. Der Nachteil der hierarchischen Verfahren besteht darin, daß eine einmal vorgenommene Clusterzuordnung innerhalb des weiteren Fusionierungsprozesses nicht noch einmal überprüft wird, so daß suboptimale Gruppenzuordnungen entstehen können.

Neben hierarchischen und partitionierenden Verfahren existieren noch graphentheoretische und Optimierungsverfahren, die jedoch nur selten angewendet werden. Vgl. zu einer Übersicht und einer Beschreibung z.B. Backhaus, K., u.a., Multivariate Analysemethoden, a.a.O., S. $281 \mathrm{ff}$. und Kaufmann, H., Pape, H., Clusteranalyse, a.a.O., S. 387ff. 
geführt wird. ${ }^{343}$ Das single-linkage-Verfahren vereinigt im ersten Schritt die Objekte, die sich am ähnlichsten sind und neigt daher zur Kettenbildung, d.h. diejenigen Elemente werden zuletzt zugeordnet, die am wenigsten in die Cluster passen. ${ }^{344}$ Damit ist es in der Lage, Ausreißer in einer Objektmenge zu erkennen. ${ }^{345}$ Bei der vorliegenden Untersuchung entstehen bei der Anwendung des singlelinkage-Verfahrens lediglich zwei Ausreißer, so daß 99 Fälle in die Clusterbildung einbezogen werden konnten. Damit zeigt sich, daß nur 2 der befragten Hersteller individuelle Implementierungsmuster aufweisen, die nicht herstellerübergreifend festgestellt werden können. Das anschließende Ward-Verfahren vereinigt Objekte, die die Varianz in einer Gruppe möglichst wenig erhöhen. Dadurch werden möglichst homogene Gruppen gebildet. ${ }^{346}$ Eine Untersuchung von Bergs hat gezeigt, daß das Ward-Verfahren in den meisten Fällen sehr gute Partitionen findet und die Elemente den Gruppen richtig zuordnet. Somit kann das Verfahren unter bestimmten Bedingungen als sehr guter Fusionierungsalgorithmus angesehen werden. $^{347}$

Die Anzahl der Cluster kann mit dem Elbow-Kriterium ermittelt werden ${ }^{348}$, welches die graphische Darstellung der Abweichung der Fehlerquadratsumme zu der Zahl der Cluster beinhaltet. Die optimale Clusterzahl liegt dort, wo eine geringere Gruppenzahl zu einem erheblichen Anwachsen der Fehlerquadratsumme führt. ${ }^{349}$ Tabelle 5 zeigt die Entwicklung der Fehlerquadratsumme für die letzten 10 Stufen des Fusionierungsprozesses. Dabei wird deutlich, daß sich ab dem Übergang der 4- zur 5-Clusterlösung die Zuwächse der Fehlerquadratsumme jeweils in Bereichen ab $6 \%$ (oder mehr) bewegen, während sie zuvor sich in Bereichen um $5 \%$ oder weniger angesiedelt sind. Daher erscheint eine 5-Clusterlösung sinnvoll. ${ }^{350}$

343 Backhaus u.a. empfehlen dieses Vorgehen, um eine bestmögliche Clusteranalyse zu erhalten. Vgl. Backhaus, K., u.a., Multivariate Analysemethoden, a.a.O., S. $299 f$.

344 Vgl. Backhaus, K., u.a., Multivariate Analysemethoden, a.a.O., S. $287 f$. und S. $290 f$.

345 Im Dendrogramm werden die Fehlerquadratsummen auf einer Skala von 0 bis 25 normiert. In der vorliegenden Untersuchung wurden alle Fälle ab dem Wert von 20 entfernt. Vgl. zu einer ähnlichen Vorgehensweise Meurer, J., Führung von Franchisesystemen, a.a.O., S. 112.

346 Vgl. Backhaus, K., u.a., Multivariate Analysemethoden, a.a.O., S. 292.

347 Vgl. Bergs, S., Optimalität bei Cluster-Analysen: Experimente zur Bewertung numerischer Klassifikationsverfahren, Münster 1981, S. 96f.

348 Zu einer ausführlichen Darstellung verschiedener Möglichkeiten der Ermittlung der Clusterzahl vgl. Kaufmann, H., Pape, H., Clusteranalyse, a.a.O., S. 406ff. und ebenda, S. 454ff.

349 Vgl. Meffert, H., Marketingforschung und Käuferverhalten, a.a.O., S. $277 f$.

350 Die Wahl der 5-Clusterlösung wurde zusätzlich durch die Analyse des Dendrogramms, in dem der Fusionierungsprozeß graphisch abgebildet ist, sowie durch die sinnvolle Interpretierbarkeit der Ergebnisse unterstützt. Vgl. zur der Empfehlung der Hinzuziehung dieser beiden Kriterien Irene Giesen-Netzer and Universität Münster - 978-3-631-75058-2 


\begin{tabular}{|c|c|c|}
\hline Zahl der Cluster & $\begin{array}{c}\text { Fehlerquadart- } \\
\text { summe (FQS) }\end{array}$ & FQS in Prozent \\
\hline$\ldots$ & $\ldots$ & $\ldots$ \\
10 & 1299,8 & 3,6 \\
9 & 1347,1 & 4,9 \\
8 & 1412,9 & 5,0 \\
7 & 1483,8 & 4,9 \\
6 & 1556,1 & $\mathbf{5 , 1}$ \\
\hline $\mathbf{5}$ & $\mathbf{1 6} 36,1$ & $\mathbf{5 , 9}$ \\
\hline $\mathbf{4}$ & $\mathbf{1 7 3 2 , 8}$ & 7,0 \\
3 & 1853,7 & 7,9 \\
2 & 2000,0 & 10,2 \\
\hline 1 & 2204,7 & \\
\hline
\end{tabular}

Tab. 5: Bestimmung der Clusterzahl mit dem Fusionierungsprozeß des Ward-Verfahrens

Die Abbildungen 18a und 18b zeigen die entstandenen Cluster im Überblick. Dabei fällt auf, daß die Clustergröße relativ homogen ist, wobei das größte Cluster $24,2 \%$ und das kleinste Cluster $11,1 \%$ der Stichprobe umfassen. Weiterhin zeigt sich an den differierenden Mittelwerten der Cluster, daß einige clusterbildende Merkmale stark, andere dagegen weniger stark trennen und damit deutliche Unterschiede zwischen den Implementierungstypen vorliegen. ${ }^{351}$

Backhaus, K., u.a., Multivariate Analysemethoden, a.a.O., S. 325f. Eine Analyse des Dendrogramms zeigt, daß sich Cluster 5 und Cluster 1 frühzeitig bilden, bevor das 3 . Cluster im weiteren Verlauf in Cluster 2, 3 und 4 aufgeteilt wird. Die graphische Darstellung dieses Prozesses findet sich im Anhang 2.3 der Arbeit. 


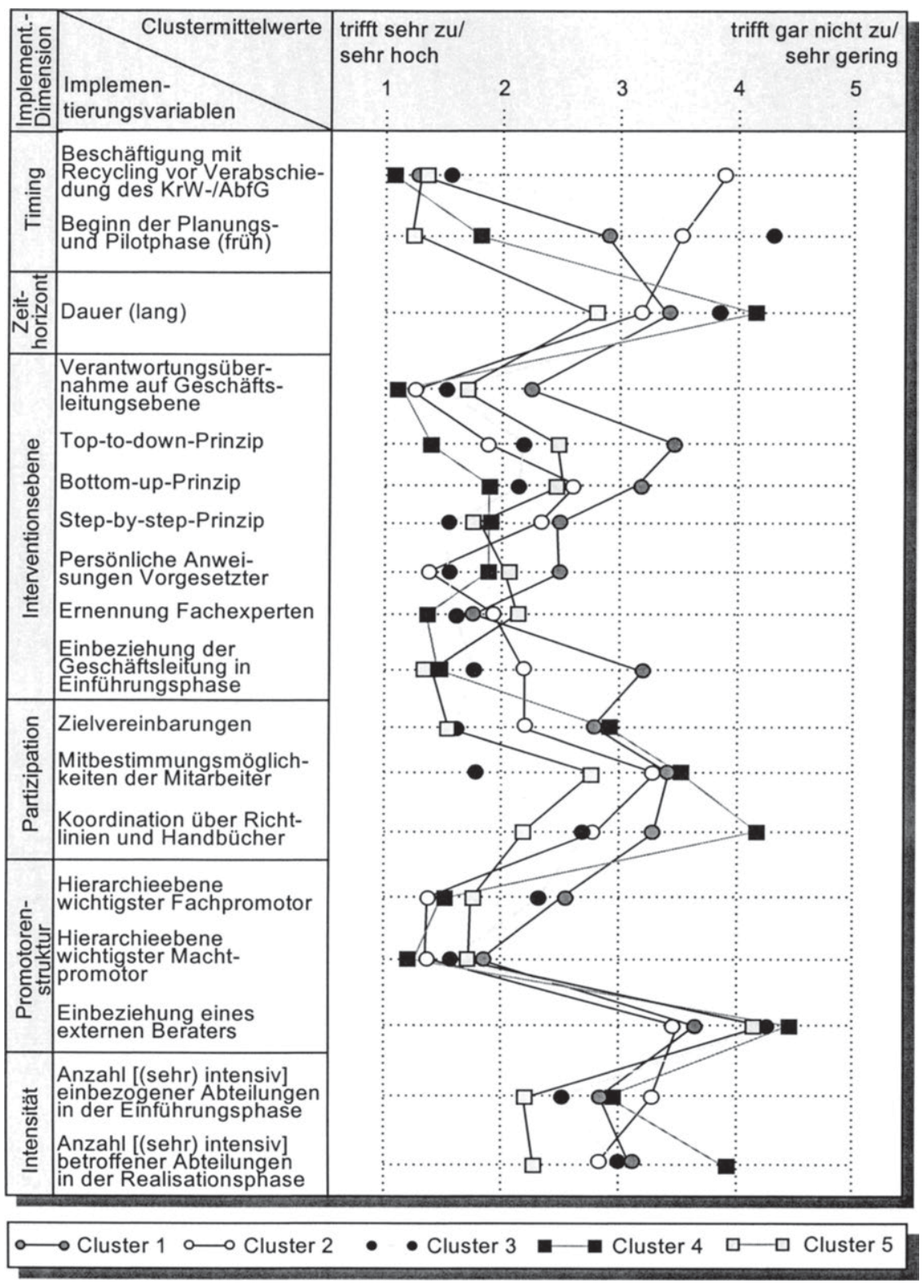

\section{Abb. 18a: Mittelwertprofile der identifizierten Implementierungstypen bei Rücknahme- und Recyclingsystemen}

Irene Giesen-Netzer and Universität Münster - 978-3-631-75058-2

Downloaded from PubFactory at 01/11/2019 08:44:49AM 


\begin{tabular}{|c|c|c|c|c|c|c|}
\hline \multirow{2}{*}{ 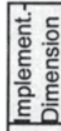 } & \multirow{2}{*}{$\begin{array}{l}\text { Clustermittelwerte } \\
\text { Implemen- } \\
\text { tierungsvariablen }\end{array}$} & \multirow{2}{*}{\begin{tabular}{|r|} 
Cluster I \\
$n=24$ \\
$24,2 \%$ \\
\end{tabular}} & \multirow{2}{*}{ Cluster II } & \multirow{2}{*}{ Cluster III } & \multirow{2}{*}{ Cluster IV } & \multirow{2}{*}{ Cluster V } \\
\hline & & & & & & \\
\hline \multirow{2}{*}{ 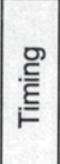 } & $\begin{array}{l}\text { Beschäftigung mit } \\
\text { Recycling vor Verabschie- } \\
\text { dung des KrW-/AbfG }\end{array}$ & 1,28 & 3,94 & 1,58 & 1,09 & 1,28 \\
\hline & $\begin{array}{l}\text { Beginn der Planungs- } \\
\text { unđ Pilotphase (früh) }\end{array}$ & 2,94 & 3,46 & 4,29 & 1,82 & 1,14 \\
\hline 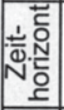 & Dauer (lang) & 3,34 & 3,08 & 3,95 & 4,16 & 2,81 \\
\hline \multirow{7}{*}{ 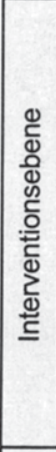 } & $\begin{array}{l}\text { Verantwortungsüber- } \\
\text { nahme auff Geschäfts- } \\
\text { leitungsebene }\end{array}$ & 2,24 & 1,29 & 1,56 & 1,18 & 1,68 \\
\hline & Top-to-down-Prinzip & 3,40 & 1,91 & 2,18 & 1,45 & 2,51 \\
\hline & Bottom-up-Prinzip & 3,15 & 2,66 & 2,13 & 1,91 & 2,64 \\
\hline & Step-by-step-Prinzip & 2,43 & 2,30 & 1,66 & 1,92 & 1,82 \\
\hline & $\begin{array}{l}\text { Persönliche Anwei- } \\
\text { sungen Vorgesetzter }\end{array}$ & 2,46 & 1,45 & 1,65 & 1,91 & 2,00 \\
\hline & Ernennung Fachexperten & 1,75 & 1,87 & 1,69 & 1,44 & 2,07 \\
\hline & $\begin{array}{l}\text { Einbeziehung der } \\
\text { Geschärtsleitung in } \\
\text { Einführungsphase }\end{array}$ & 3,08 & 2,11 & 1,76 & 1,55 & 1,48 \\
\hline \multirow{3}{*}{ 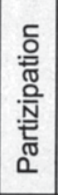 } & Zielvereinbarungen & 2,74 & 2,11 & 1,63 & 2,94 & 1,61 \\
\hline & $\begin{array}{l}\text { Mitbestimmungsmöglich- } \\
\text { keiten der Mitarbeiter }\end{array}$ & 3,39 & 3,34 & 1,84 & 3,42 & 2,81 \\
\hline & $\begin{array}{l}\text { Koordination über Richt- } \\
\text { linien und Handbücher }\end{array}$ & 3,31 & 2,86 & 2,88 & 4,09 & 2,38 \\
\hline \multirow{3}{*}{ 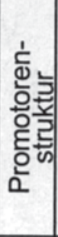 } & $\begin{array}{l}\text { Hierarchieebene } \\
\text { wichtigster Fachpromotor }\end{array}$ & 2,54 & 1,39 & 2,29 & 1,64 & 1,76 \\
\hline & $\begin{array}{l}\text { Hierarchieebene } \\
\text { wichtigster Macht- } \\
\text { promotor }\end{array}$ & 1,86 & 1,37 & 1,47 & 1,28 & 1,63 \\
\hline & $\begin{array}{l}\text { Einbeziehung eines } \\
\text { externen Beraters }\end{array}$ & 3,67 & 3,61 & 4,24 & 4,36 & 4,24 \\
\hline \multirow{2}{*}{ 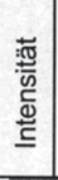 } & $\begin{array}{l}\text { Anzahl [(sehr) intensiv] } \\
\text { einbezogener Abteilunger } \\
\text { in der Einführungsphase }\end{array}$ & 2,87 & 3,21 & 2,59 & 2,91 & 2,06 \\
\hline & $\begin{array}{l}\text { Anzahl [(sehr) intensiv] } \\
\text { betroffener Abteilungen } \\
\text { in der Realisationsphase }\end{array}$ & 3,04 & 2,82 & 2,99 & 3,91 & 2,17 \\
\hline
\end{tabular}

Abb. 18b: Mittelwerte identifizierten Implementierungstypen bei Rücknahme- und Recyclingsystemen 


\subsection{Beschreibung und Konsistenzprüfung der Implementierungstypen}

Durch eine Darstellung der Abweichungen der clusterbildenden Merkmale der identifizierten Implementierungstypen vom Gesamtmittelwert wird eine Konsistenzprüfung und inhaltliche Interpretation erleichtert. ${ }^{352}$ Abbildung 19 wird daher neben Abb. 18a und 18b zur Beschreibung der fünf Implementierungstypen herangezogen. Die Benennung der Implementierungstypen erfolgt dabei bewußt plakativ, um die tendenziellen Verhaltensweisen der Hersteller bei der Implementierung von Rücknahme- und Recyclingsystemen herauszustellen.

\section{Cluster I: Expertenorientierte Implementierungsminimalisten}

Das Cluster I, welches knapp ein Viertel der gesamten Stichprobe umfaßt, hat sich vor der Verabschiedung des Kreislaufwirtschaftsgesetzes überdurchschnittlich intensiv mit dem Recycling von Rücknahme- und Recyclingsystemen befaßt. Die Implementierung erfolgte jedoch erst relativ spät im Jahre $1994 .{ }^{353}$ Die Dauer von der Planung bis zu den ersten Implementierungsaktivitäten hingegen ist durchschnittlich einzustufen und betrug ca. zwei bis drei Jahre. ${ }^{354}$

352 Vgl. auch die Abbildung im Anhang 1.5 über die Gesamtmittelwerte der clusterbildenden Variablen. Daraus wird deutlich, daß insgesamt alle befragten Hersteller wenig externe Berater bei der Implementierung von Rücknahme- und Recyclingsystemen einbeziehen. Als Grund wurde mehrfach angegeben, daß externe Berater zum Zeitpunkt der Befragung keine Erfahrungen mit diesem Aufgabenfeld hatten, aber davon auszugehen ist, daß sich dieses in Zukunft ändern wird. Weiterhin auffällig ist, daß die Einbeziehung eines Machtpromotors auf hoher Hierarchieebene von allen Befragten als wichtiges Implementierungsverhalten durchführen und eine Verantwortungsübernahme auf der Geschäftsführung für die Implementierungsaktivitäten stattfindet. An den Standardabweichungen der Variablen wird darüber hinaus deutlich, daß die Anworten der Hersteller streuen und sich daher sinnvolle Cluster bilden lassen.

Die Jahreszahlen wurden genau erfaßt. Eine Operationalisierung wurde wie folgt vorgenommen:

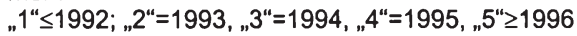

Vgl. zu einer beispielhaften Operationalisierung der Zeitdimensionen "Timing" und „Zeithorizont" den Anhang 2.2. 


\begin{tabular}{|c|c|c|c|c|c|c|}
\hline \multicolumn{2}{|c|}{$\begin{array}{lll}\text { Variablen } & \text { Ausprägungen }\end{array}$} & \multicolumn{5}{|c|}{$\begin{array}{l}\text { Abweichungen der Clustermittelwerte vom } \\
\text { Gesamtmittelwert }(n=99)\end{array}$} \\
\hline \multirow{2}{*}{ 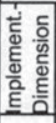 } & \multirow{2}{*}{$\begin{array}{l}\text { Implementierungs- } \\
\text { variablen }\end{array}$} & Cluster I & Cluster II & Cluster III & Cluster IV & Cluster V \\
\hline & & $24,2 \%$ & $18,2 \%$ & $17,2 \%$ & & $29,3 \%$ \\
\hline \multirow{2}{*}{ 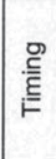 } & \begin{tabular}{|l|} 
Beschäftigung mit \\
Recycling vor Verabschie- \\
dung des KrW-/AbfG
\end{tabular} & ++ & $\cdots$ & + & +++ & ++ \\
\hline & $\begin{array}{l}\text { Beginn der Planungs- } \\
\text { und Pilotphase (früh) }\end{array}$ & - & $\cdots$ & $\cdots$ & +++ & +++ \\
\hline 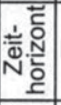 & Dauer (lang) & ○ & + & - & $\cdots$ & ++ \\
\hline \multirow{7}{*}{ 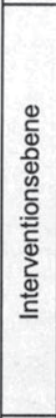 } & $\begin{array}{l}\text { Verantwortungsüber- } \\
\text { nahme auf Geschäfts- } \\
\text { leitungsebene }\end{array}$ & -- & ++ & ○ & ++ & O \\
\hline & Top-to-down-Prinzip & $\cdots$ & ++ & + & +++ & O \\
\hline & Bottom-up-Prinzip & -- & $\bigcirc$ & ++ & +++ & O \\
\hline & Step-by-step-Prinzip & - & $\bigcirc$ & ++ & $\bigcirc$ & + \\
\hline & $\begin{array}{l}\text { Persönliche Anwei- } \\
\text { sungen Vorgesetzter }\end{array}$ & - & ++ & + & O & O \\
\hline & Ernennung Fachexperten & ○ & ○ & O & ++ & O \\
\hline & $\begin{array}{l}\text { Einbeziehung der } \\
\text { Geschäftsleitung in } \\
\text { Einführungsphase } \\
\end{array}$ & $\cdots$ & ○ & + & ++ & ++ \\
\hline \multirow{3}{*}{ 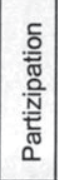 } & Zielvereinbarungen & -- & ○ & ++ & $\cdots$ & ++ \\
\hline & $\begin{array}{l}\text { Mitbestimmungsmöglich- } \\
\text { keiten der Mitarbeiter }\end{array}$ & $\cdots$ & -- & ++++ & $-\cdot$ & O \\
\hline & $\begin{array}{l}\text { Koordination über Richt- } \\
\text { linien und Handbücher }\end{array}$ & $\cdots$ & O & O & $\cdots$ & +++ \\
\hline \multirow{3}{*}{ 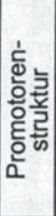 } & $\begin{array}{l}\text { Hierarchieebene } \\
\text { wichtigster Fachpromotor }\end{array}$ & O & ++ & - & + & 0 \\
\hline & $\begin{array}{l}\text { Hierarchieebene } \\
\text { wichtigster Macht- } \\
\text { promotor }\end{array}$ & - & 0 & 0 & + & 0 \\
\hline & $\begin{array}{l}\text { Einbeziehung eines } \\
\text { externen Beraters }\end{array}$ & + & + & - & - & - \\
\hline \multirow{2}{*}{ 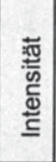 } & $\begin{array}{l}\text { Anzahl [(sehr) intensiv] } \\
\text { einbezogener Abteilunger } \\
\text { in der Einführungsphase }\end{array}$ & 0 & - & 0 & - & 0 \\
\hline & $\begin{array}{l}\text { Anzahl [(sehr) intensiv] } \\
\text { betroffener Abteilungen } \\
\text { in der Realisationsphase }\end{array}$ & 0 & 0 & 0 & $\cdots$ & +++ \\
\hline
\end{tabular}

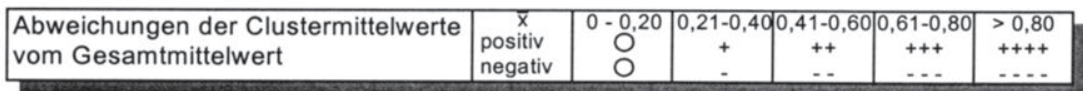

\section{Abb. 19: Mittelwertabweichungen der clusterbildenden Merkmale der ermittelten Implemen- tierungstypen vom Gesamtmittelwert}


Bezüglich der Interventions- und der Partizipationsdimensionen der Implementierung ist festzustellen, daß sich dieses Cluster insgesamt durch ein unterdurchschnittliches Aktivitätsniveau bei der Implementierung von Rücknahme- und Recyclingsystemen auszeichnet. Insbesondere die Verantwortungsübernahme auf der Geschäftsleitungsebene sowie die Einbeziehung der Geschäftsleitung in der Einführungsphase sind als relativ gering zu bezeichnen. Auch das Top-to-downPrinzip nimmt mit einem Mittelwert von 3,4 den im Vergleich niedrigsten Wert an. Diese Befunde lassen darauf schließen, daß die Geschäftsführung der Expertenorientierten Implementierungsminimalisten die Implementierung von Rücknahme- und Recyclingsystemen nicht als "Chefsache" sieht und Implementierungsaktivitäten auf ein Minimum reduziert.

Diese Überlegungen werden bestätigt, da die absolut gesehen wichtigsten Maßnahmen für diesen Implementierungstyp in dem Einsatz eines verantwortlichen Fachexperten und der Einbeziehung eines Machtpromotors auf hoher Hierarchieebene bestehen. Daraus kann gefolgert werden, daß die Aufgaben größtenteils Experten überlassen bleiben. Zwar setzt auch dieses Cluster wenig externe Berater ein (Mittelwert: 3,67), dies jedoch überdurchschnittlich häufig. Darüber hinaus liegt die Anzahl der einbezogenen Abteilungen in der Einführungsphase wie auch in der Durchführungsphase im Durchschnitt, d.h. 5 bis 6 Abteilungen waren (sehr) intensiv ${ }^{355}$ in den jeweiligen Phasen mit der Implementierung des Rücknahme- und Recyclingsystems beschäftigt.

\section{Cluster II: Späte promotorenorientierte Externalisierer}

Dem zweiten Implementierungstyp gehören $18,2 \%$ der befragten Hersteller an. Dieses Cluster hat sich vor der Verabschiedung des Kreislaufwirtschaftsgesetzes weder konzeptionell noch praktisch kaum mit dem Recycling auseinandergesetzt und daher Hersteller, die sich spät mit der Implementierung von Rücknahme- und Recyclingsystemen beschäftigen. ${ }^{356}$ Der Zeithorizont ist mit einem Wert von 3,08 leicht überdurchschnittlich lang.

355 Die Intensität wurde mit der Anzahl der einbezogenen/betroffenen Abteilungen operationalisiert:

"1“=9 und mehr Abteilungen; „2“=7und 8 Abteilungen; ${ }_{n} 3^{\prime \prime}=5$ und 6 Abteilungen; $4^{4}=3$ und 4 Abteilungen; „5“=1 und 2 Abteilungen 
Bezüglich der Interventionsebene fällt auf, daß die Verantwortungsübernahme auf der Geschäftsleitungsebene, persönliche Anweisungen als Koordinationsinstrument sowie das Top-to-down-Prinzip für dieses Cluster von besonderer Relevanz sind. Diese Merkmalsausprägungen sind konsistent zu der Aussage, daß gleichzeitig die Mitbestimmungsmöglichkeiten der Mitarbeiter eine unterdurchschnittliche Bedeutung haben. Die Einbeziehung von Fachpromotoren auf hoher Hierarchieebene hingegen ist für dieses Cluster die wichtigste Maßnahme bei der Implementierung des Rücknahme- und Recyclingsystems. Dieser Befund wirkt schlüssig zu der Tatsache, daß die Anzahl der sehr intensiv betroffenen Abteilungen in der Einführungsphase relativ am geringsten ist. Diese geringe Ausprägung der Intensitätsdimension in der Einführungsphase deutet auf die Einbeziehung unternehmensfremder Partner in dieser Phase hin. Der gleichzeitig höhere Wert in der Realisationsphase läßt darauf schließen, daß dieser Implementierungstyp ein Konzept mit durchschnittlicher Intensität realisiert, welches in der Einführungsphase - durch Einbeziehung von Systempartnern oder externen Beratern - bereits vorlag.

\section{Cluster III: Partizipativ-späte Implementierer}

Auch dieses Cluster, welches 17 Herstellerunternehmen umfaßt, zeichnet sich durch ein sehr spätes Implementierungsverhalten aus, d.h. die in diesem Cluster enthaltenen Unternehmen beschäftigen sich erst seit 1995/96 mit der Implementierung von Rücknahme- und Recyclingsystemen. Eine relativ intensive konzeptionelle Auseinandersetzung mit dem Thema Recycling fand hingegen bereits vor Verabschiedung des Kreislaufwirtschaftsgesetzes statt. Der Zeithorizont ist mit einem Mittelwert von 3,95 als kurz zu charakterisieren, d.h. der Zeitraum von den (durchschnittlichen) ersten Implementierungsaktivitäten bis zur Realisation des jeweiligen Rücknahme- und Recyclingsystems beträgt nur 1 bis 2 Jahre.

Bei den Implementierungsvariablen der Interventions- sowie der Partizipationsdimension sind insbesondere das Bottom-up-Prinzip, das Step-by-step-Prinzip sowie Zielvereinbarungen von überdurchschnittlicher Bedeutung für diesen Implementierungstyp. Darüber hinaus bilden die Mitbestimmungsmöglichkeiten der Mitarbeiter mit einem Wert von 1,84 einen zentralen Bestandteil der Implementie-

spät klassifizierte Implementierungstyp begann mit der Planung bzw. mit der Pilotphase durchschnittlich im Jahr 1995. 
rung und den nehmen wichtigsten Stellenwert im Vergleich zu allen identifizierten Implementierungstypen ein, so daß dieser Implementierungstyp als partizipativ charakterisiert werden kann. Die Macht- und Fachpromotoren sind dementsprechend auf eher niedrigen Hierarchieebenen angesiedelt. Die Intensitätsdimension dieses Implementierungstyps ist durchschnittlich ausgeprägt.

\section{Cluster IV: Autokratische Ad hoc-Externalisierer}

Dieses Cluster, welches nur $11,1 \%$ der Stichprobe repräsentiert, hat sich zwar theoretisch intensiv vor der Verabschiedung des Kreislaufwirtschaftsgesetzes mit dem Recycling auseinandergesetzt und dieses auch relativ früh in konkretes Handeln umgesetzt, aber die Zeitdauer von der Planung bis zur Realisation verläuft „ad hoc" bzw. sehr schnell.

Als autokratisch kann dieses Cluster bezeichnet werden, da die Verantwortungsübernahme auf Geschäftsleitungsebene, die Einbeziehung eines Machtpromotors sowie das Top-to-down-Prinzip die wichtigsten Verhaltensweisen bei der Implementierung von Rücknahme- und Recyclingsystemen für das Cluster darstellen. Die Mitbestimmungsmöglichkeiten der Mitarbeiter sowie Zielvereinbarungen weisen hingegen stark unterdurchschnittliche Mittelwerte auf. Auch der Standardisierungsgrad, der durch die Koordination über Richtlinien und Handbücher zum Ausdruck kommt, ist mit einem Mittelwert von 4,09 sehr gering ausgeprägt. Dieses ist jedoch aufgrund des sehr kurzen Zeithorizontes nicht überraschend.

Da die Betroffenheit der Abteilungen in der Realisationsphase am geringsten von allen betrachteten Clustern sind, liegt es nahe, daß die Unternehmen dieses Clusters viele Aktivitäten externalisieren. Diese Externalisierung findet jedoch nicht auf externe Berater statt, welches durch den niedrigen Stellenwert der Einbeziehung der externen Berater zum Ausdruck kommt. In der Einführungsphase werden hingegen leicht unterdurchschnittlich viele Abteilungen bei der Implementierung des Rücknahme- und Recyclingsystems einbezogen.

\section{Cluster V: Frühe integrierte Internalisierer}

Dieses Cluster bildet mit 29,3\% das größte Cluster. Gleichzeitig weist dieser Implementierungstyp die meisten Merkmale mit einer durchschnittlichen Ausprägung 
auf. Dennoch fallen einige Besonderheiten ins Auge, die diesen Implementierungstyp in besonderer Weise zu charakterisieren vermögen.

Die Unternehmen in diesem Cluster setzen sich nicht nur konzeptionell intensiv frühzeitig mit dem Recycling auseinander, sondern setzen dieses Verhalten auch in konkrete Maßnahmen um. Auch die Zeitdauer der Planung bis zur Realisation ist in dieser Gruppe am deutlich längsten ausgeprägt. Dieser Befund ist konsistent zu dem praktizierten Step-by-step-Prinzip, welches auf ein geplantes schrittweises Implementierungsverhalten hindeutet. In der Einführungsphase ist die Geschäftsführung intensiv in die Implementierung eingebunden.

Aufgrund der sehr frühzeitigen Implementierungsaktivitäten ist gleichzeitig die Standardisierung der Koordination der Rücknahme- und Recyclingaktivitäten am weitesten fortgeschritten, und Zielvereinbarungen mit den Mitarbeitern lassen sich überdurchschnittlich häufig feststellen. Die Anzahl der sehr intensiv betroffenen Abteilungen in der Realisationsphase ist mit einem Mittelwert von 2,17 sehr hoch, und entspricht etwa 7 bis 8 Abteilungen. Offenbar führt dieser Implementierungstyp viele Aktivitäten bei der Gestaltung des Rücknahme- und Recyclingsystems selbst durch.

Als Ergebnis der Clusteranalyse kann festgehalten werden, daß fünf Implementierungstypen identifiziert werden konnten, die die Konsistenzbedingung erfüllen. Die Benennung im Überblick:

Cluster I: Expertenorientierte Implementierungsminimalisten

Cluster II: Späte promotorenorientierte Externalisierer

Cluster III: Partizipativ-späte Implementierer

Cluster IV:Autokratische Ad hoc-Externalisierer

Cluster V: Frühe integrierte Internalisierer

Um weitergehende Interpretationen des Implementierungsverhaltens zu ermöglichen, werden im folgenden die Güte der gefundenen 5-Clusterlösung überprüft und zum anderen die Implementierungsdimensionen ermittelt, die eine zentrale Bedeutung für die (Implementierungs-) Typendifferenzierung liefern. 


\subsection{Diskriminanzanalytische Überprüfung der Güte der Clusterlösung und Ermittlung der zentralen Implementierungsdimensionen}

Hinsichtlich der Überprüfung der Güte der gefundenen 5-Clusterlösung werden die Gruppenunterschiede mit der Diskriminanzanalyse untersucht. Dieses multivariate Verfahren ${ }^{357}$ ist geeignet, zwei Fragen zu beantworten, die dem Vorgehen des Kapitels zugrunde liegen: ${ }^{358}$

1. Unterscheiden sich die Cluster hinsichtlich der clusterbildenden Indikatorvariablen (bzw. Implementierungsvariablen) signifikant voneinander?

2. Welche Variablen sind besonders relevant für die Unterscheidung der Cluster?

Zur Beantwortung der ersten Frage kann die Klassifikationsmatrix der Tabelle 6 herangezogen werden.

Die Klassifikationsmatrix stellt den Übereinstimmungsgrad der geschätzten Zugehörigkeiten zu den Implementierungstypen, die mittels vier signifikanter Diskriminanzfunktionen ermittelt wurden, ${ }^{359}$ den tatsächlichen Zuordnungen aus der Clusteranalyse gegenüber. Der Übereinstimmungsgrad zeigt die Trennschärfe zwischen den identifizierten Implementierungstypen auf. ${ }^{360}$ Mit einem Anteil korrekt klassifizierter Fälle von $95,96 \%$ wird ein gutes Ergebnis erzielt. Bei drei Clustern wurde eine Klassifikation von $100 \%$ erzielt. Lediglich bei den Expertenorientierten Implementierungsminimalisten wurden $12,6 \%$ der Stichprobe und bei den Frühen integrierten Implementierern $3,4 \%$ falsch zugeordnet. ${ }^{361}$

357 Die Diskriminanzanalyse gehört zu den strukturprüfenden Verfahren, d.h. die Anwendung erfordert, daß die Daten für die Merkmalsvariablen der Elemente und deren Gruppenzugehörigkeiten vorliegen. Vgl. Backhaus, K., u.a., Multivariate Analysemethoden, a.a.O., S. 91. In der vorliegenden Arbeit erfüllt die vorgeschaltete Clusteranalyse diese Voraussetzungen.

Vgl. zu einer ausführlichen Darstellung der Diskriminanzanalyse Fahrmeir, L., Hamerle, A., Diskriminanzanalyse, in: Fahrmeir, L., Hamerle, A. (Hrsg.), Berlin, New York, de Gruyter 1984, S. $301 \mathrm{ff}$.

$\mathrm{Vgl}$. zu diesen u.ä. Fragestellungen, die mit der Diskriminanzanalyse beantwortet werden können, Meffert, H., Marketingforschung und Käuferverhalten, a.a.O., S. 282.

Die erste Diskrimininanzfunktion erklärt $56,36 \%$ der Varianz der Variablen, während die zweite $17,48 \%$, die dritte $16,09 \%$ sowie die vierte Diskriminanzfunktion noch $10,07 \%$ der Varianz erklären. man deren Trefferquote mit derjenigen Quote vergleichen, die bei rein zufälliger Zuordnung der Elemente zu den Gruppen entstehen. Vgl. Backhaus, K., u.a., Multivariate Analysemethoden, a.a.O., S. 116. Bei fünf Gruppen ergibt sich - die Größe der Cluster unberücksichtigt - eine Zufallsquote von $20 \%$, die deutlich unter dem hier erzielten Ergebnis von $95,96 \%$ liegt. 


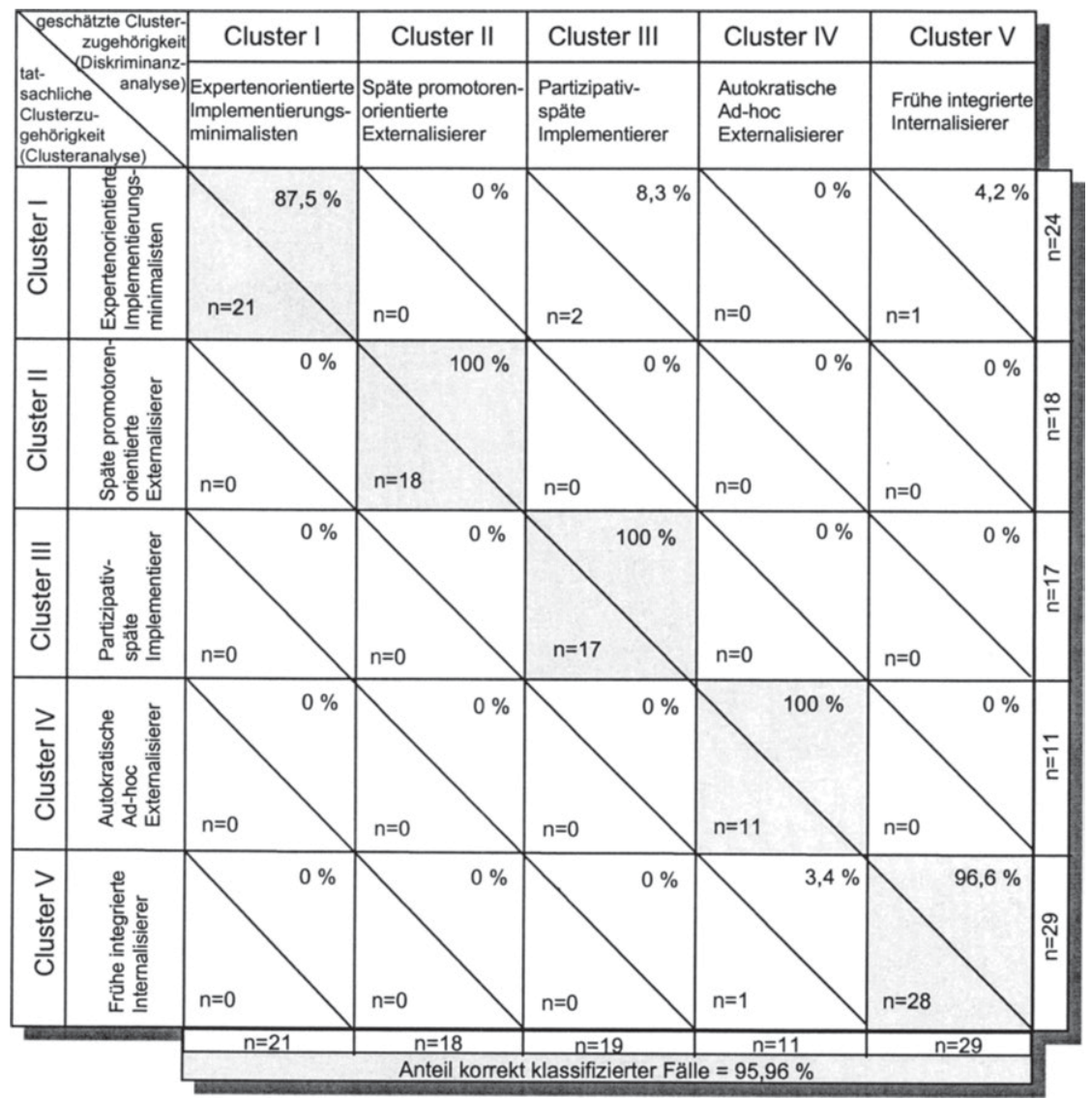

Tab. 6: Klassifikationsmatrix der diskriminanzanalytischen Untersuchung der Implementierungstypen

Als Ergebnis kann somit festgehalten werden, daß sich die Cluster hinsichtlich der Implementierungsvariablen gut unterscheiden. Damit kann die Basishypothese $H_{\mathrm{IV} 1}$ uneingeschränkt bestätigt werden, nach der sich signifikant abweichende Verhaltensmuster erkennen lassen, die es ermöglichen, verschiedene Implementierungstypen zu bilden.

Nachdem die Unterschiedlichkeit der Implementierungstypen auch statistisch nachgewiesen ist, wird die zweite Fragestellung nach den zentralen Implementie- 
rungsvariablen und -dimensionen beantwortet, die für die Diskriminierung der Implementierungstypen von besonders hoher Bedeutung sind. Dazu wird eine schrittweise Diskriminanzanalyse durchgeführt. Die schrittweise Diskriminanzanalyse $^{362}$ bezieht die Merkmalsvariablen einzeln nacheinander in die Diskriminanzfunktion ein, wobei jeweils diejenige Variable ausgewählt wird, die das Gütemaß Wilks' Lambda minimiert. ${ }^{363}$ Die Diskriminanzwirkung einer Variablen und damit ihr Bedeutungsgewicht zur Trennung kann mit dem mittleren Diskrimininanzkoeffizienten gemessen werden, der sich aus der Multiplikation der Diskriminanzkoeffizienten mit dem Eigenwert der Diskriminanz ergibt. ${ }^{364}$

Tabelle 7 zeigt, daß die schrittweise Diskriminanzanalyse zu einer Reduzierung der 18 Implementierungsvariablen auf 8 Variablen führt. Das wichtigste Trennkriterium ist nach dem mittleren Diskriminanzkoeffizienten die theoretische Auseinandersetzung mit dem Recycling vor der Verabschiedung des Kreislaufwirtschaftsgesetzes mit einer Diskriminanzwirkung von 27,62\%, gefolgt vom Top-todown-Prinzip mit 14,21\% und der Koordination der Implementierung über persönliche Anweisungen der Vorgesetzten.

Werden die Variablen den Implementierungsdimensionen zugeordnet, zeigt sich, daß insbesondere dem Timing eine hohe diskriminatorische Bedeutung zukommt. Allein diese Dimension hat eine Diskriminanzwirkung von 40,44\%. Dem folgen die Interventionsebene mit einer Diskriminanzwirkung von insgesamt $34,18 \%$ und die Partizipation mit $14,86 \%$. Diese drei Implementierungsdimensionen haben damit eine diskriminatorische Wirkung von knapp 90\%, d.h. die Implementierungstypen unterscheiden sich hinsichtlich dieser Dimensionen am stärksten. Der Zeithorizont vermag immerhin noch $10,6 \%$ der Diskriminanzwirkung zu erklären, während die Promotorenstruktur und die Intensitätsdimension keine signifikanten Diskriminanzwirkungen aufzeigen. ${ }^{365}$

\footnotetext{
362 Vgl. zu einer kurzen Darstellung der schrittweisen Diskriminianzanalyse Backhaus, K., u.a., Multivariate Analysemethoden, a.a.O., S. 124.

363 Wilks' Lambda ist ein inverses Gütemaß, d.h. kleinere Werte deuten auf eine höhere Trennkraft der Diskriminanzfunktion und umgekehrt.

364 Vgl. Meffert, H., Marketingforschung und Käuferverhalten, a.a.O., S. 286.

365 Zur Relativierung dieser Aussage muß hinzugefügt werden, daß eine Klassifikationsmatrix bei der schrittweisen Diskriminanzanalyse zu einem Anteil korrekt klassifizierter Fälle von $92,93 \%$ führt, d.h. $3 \%$ weniger richtige Clusterzuordnungen stattfinden. Vgl. dazu die Klassifikationsmatrix im Anhang 2.4.
} 


\begin{tabular}{|c|c|c|c|c|c|c|}
\hline $\begin{array}{l}\text { Implemen- } \\
\text { tierungs- } \\
\text { variablen }{ }^{1)}\end{array}$ & $\begin{array}{l}\text { Wilk's } \\
\text { Lambda }\end{array}$ & $\begin{array}{l}\text { Standard. Dis- } \\
\text { kriminanzkoeff. } \\
\text { *Eigenwert der } \\
\text { Diskriminanz- } \\
\text { funktion } \\
=\text { =mittlerer Dis- } \\
\text { kriminanzkoeff. }\end{array}$ & $\begin{array}{l}\text { Implemen- } \\
\text { tierungs- } \\
\text { variablen- } \\
\text { rang }\end{array}$ & $\begin{array}{l}\text { Diskrimi- } \\
\text { nanz- } \\
\text { wirkung } \\
\text { in } \%\end{array}$ & $\begin{array}{l}\text { Zugehörige } \\
\text { Implemen- } \\
\text { tierungs- } \\
\text { dimension }\end{array}$ & $\begin{array}{l}\text { Diskrimi- } \\
\text { nanz- } \\
\text { wirkung } \\
\text { in } \%{ }^{2)}\end{array}$ \\
\hline
\end{tabular}

\begin{tabular}{|c|c|c|c|c|c|c|}
\hline $\begin{array}{l}\text { Beschäftigung } \\
\text { mit Recycling } \\
\text { vor Ver- } \\
\text { abschiedung } \\
\text { KrW-/AbfG }\end{array}$ & 0,2588 & 5,19 & 1. & $27,62 \%$ & \multirow{2}{*}{ Timing } & \multirow{2}{*}{$40,44 \%$} \\
\hline $\begin{array}{l}\text { Beginn der } \\
\text { Planungs- } \\
\text { bzw. Pilot- } \\
\text { phase }\end{array}$ & 0,1338 & 2,41 & 4. & $12,82 \%$ & & \\
\hline Dauer & 0,0294 & 1,99 & 5. & $10,60 \%$ & $\begin{array}{l}\text { Zeithori- } \\
\text { zont }\end{array}$ & $10,60 \%$ \\
\hline $\begin{array}{l}\text { Top-to-down- } \\
\text { Prinzip }\end{array}$ & 0,0876 & 2,67 & 2. & $14,21 \%$ & \multirow{3}{*}{$\begin{array}{l}\text { Interven- } \\
\text { tions- } \\
\text { ebene }\end{array}$} & \multirow{3}{*}{$34,18 \%$} \\
\hline $\begin{array}{l}\text { Persönliche } \\
\text { Anweisungen } \\
\text { der Vorge- } \\
\text { setzten }\end{array}$ & 0,0247 & 2,41 & 3. & $12,85 \%$ & & \\
\hline $\begin{array}{l}\text { Einbeziehung } \\
\text { der Geschäfts- } \\
\text { leitung in der } \\
\text { Einführungs- } \\
\text { phase }\end{array}$ & 0,0452 & 1,32 & 7. & $7,04 \%$ & & \\
\hline $\begin{array}{l}\text { Mitbestim- } \\
\text { mungsmög- } \\
\text { lichkeiten der } \\
\text { Mitarbeiter }\end{array}$ & 0,0603 & 1,72 & 6. & $9,17 \%$ & \multirow{2}{*}{$\begin{array}{l}\text { Partizi- } \\
\text { pation }\end{array}$} & \multirow{2}{*}{$14,86 \%$} \\
\hline $\begin{array}{l}\text { Koordination } \\
\text { über Richt- } \\
\text { linien und } \\
\text { Handbücher }\end{array}$ & 0,0353 & 1,06 & 8. & $5,69 \%$ & & \\
\hline & & 18,77 & & $100 \%$ & & $100 \%$ \\
\hline
\end{tabular}

1) Reduzierte Variablenstruktrur nach schrittweiser Diskriminazanalyse
2) Beschreibt den rechnerischen Anteil zur Klassifizierung der Fälle

Tab. 7: Ermittlung der zentralen Implementierungsdimensionen nach der schrittweisen Diskriminanzanalyse 
Als Fazit kann die Basishypothese $\mathrm{H}_{\mathrm{IV} 3}$ bestätigt werden, da sich die einzelnen Implementierungsdimensionen hinsichtlich ihrer Bedeutung im Gesamtkomplex des Implementierungsverhaltens unterscheiden und die Implementierungsdimensionen aufgrund ihrer spezifischen Gestaltungsspielräume signifikant unterschiedliche Erklärungsbeiträge für die Typenbildung und -trennung liefern. Dabei werden die im Theorieteil abgeleiteten Gestaltungsspielräume durch die Einflußbereiche der implementierungsobjektspezifischen Determinanten offensichtlich genutzt. ${ }^{366}$

Im nun folgenden Hauptkapitel soll darauf aufbauend ein Erklärungsansatz für die Herausbildung der Implementierungstypen erfolgen. Dazu werden die Einflußfaktoren des Implementierungsverhaltens abgeleitet, um in einem weiteren Schritt Erfolgswirkungen des Implementierungsverhaltens zu analysieren. 


\section{Einflußfaktoren und Erfolgswirkungen des Implementierungsverhal- tens von Rücknahme- und Recyclingsystemen}

\section{Erklärungsansätze für das Implementierungsverhalten bei Rücknah- me- und Recyclingsystemen}

\subsection{Bezugsrahmen der Einflußfaktoren und Methodik der Analyse}

Um die verschiedenen Ausprägungsformen des Implementierungsverhaltens der Hersteller bei Rücknahme- und Recyclingsystemen erklären zu können, wird auf vielfältige Einflußfaktoren zurückgegriffen. ${ }^{367}$ Dabei wird neben den situativen Faktoren auch der "Handlungsspielraum“, ${ }^{368}$ der von den Organisationsgestaltern subjektiv wahrgenommen und genutzt wird, als intervenierende Variable einbezogen. ${ }^{369}$ Das Forschungsinteresse geht demnach neben der Umwelt-StrukturBeziehung zu einer Umwelt-Struktur-Verhaltensbeziehung über. ${ }^{370}$

Der empirische Bezugsrahmen der Einflußfaktoren (vgl. Abbildung 20) wird auf Grundlage des allgemeinen, umfassenden kontingenztheoretischen Bezugsrahmens hergeleitet ${ }^{371}$. Insgesamt werden acht Gruppen von Einflußfaktoren analysiert, wobei situationsexterne und -interne Faktoren unterschieden werden. Die situationsexternen Faktoren werden in eine globale $\left(\mathrm{H}_{\mathrm{GLO}}\right)^{372}$ und eine aufgaben-

367 Vgl. zu derselben Auffassung von Einflußfaktoren Kirchgeorg, M., Okologieorientiertes Unternehmensverhalten, a.a.O., S. 59. Im Gegensatz dazu sind Implementierungsvoraussetzungen Bedingungen, ohne die eine Gestaltung und Implementierung von Rücknahme- und Recyclingsystemen nicht möglich sind. Die Übergänge zwischen Voraussetzungen und Einflußfaktoren sind allerdings fließend, so daß in der vorliegenden Arbeit ausschließlich von Einflußfaktoren gesprochen wird.

Der Handlungsspielraum bezieht sich z.B. auf die subjektiv wahrgenommenen Spielräume bei der Organisationsstruktur und -kultur. Vgl. Staehle, W. H., Management, a.a.O., S. $57 \mathrm{f}$.

Vgl. ebenda, S. $53 f$.

370 Über die Tatsache, daß vielfältige situative Faktoren und Handlungsspielräume existieren, die das Unternehmensverhalten beeinflussen, besteht in der Organisationstheorie generell Einigkeit. Vgl. dazu z.B. Frese, E., Organisationstheorie: historische Entwicklung, Ansätze, Perspektiven, 2. überarb. und erw. Aufl. Wiesbaden 1992, S. 112ff.; Schreyögg, G., Unternehmensstrategie, a.a.O., S. 50f.; Staehle, W. H., Management, a.a.O., S. 47ff. Uneinigkeit hingegen besteht hinsichtlich der Auswahl der relevanten Einflußfaktoren auf das jeweilige zu untersuchende Verhalten - hier die Implementierung von Rücknahme- und Recyclingsystemen - , die zweckbestimmt zu erfolgen hat.

372 Die globale Umwelt kann in die Sphären der ökologischen, gesellschaftlichen, ökonomischen, technischen und politisch-rechtlichen Umweltbedingungen differenziert werden. Vgl. z.B. Kirchgeorg, M., OKologieorientiertes Unternehmensverhalten, a.a.O., S. 59ff.; Meffert, H., Kirchgeorg, M., Marktorientiertes Umweltmanagement, a.a.O., S. 81f. oder Schwarz, E. J., Unternehmensnetzwerke im Recycling-Bereich, a.a.O., S. 31ff. Kolks unterscheidet bei der globalen Umwelt analog dazu: 1. das wirtschaftliche Umfeld, das die generelle wirtschaftliche Entwicklung berücksichtigt, 2. das technische Umfeld und 3. das sozio-kulturelle Umfeld, das 
spezifische Umwelt gegliedert. ${ }^{373}$ Als Einflußfaktoren der aufgabenspezifischen Umwelt, die unternehmensindividuell ausgeprägt ist, werden die Markt- und Wettbewerbssituation $\left(\mathrm{H}_{\mathrm{SIT}}\right)$ sowie die Produktmerkmale $\left(\mathrm{H}_{\mathrm{PRO}}\right)$ betrachtet. Neben diesen externen Situationsvariablen werden als interne Einflußgrößen die Herstellerdemographie $\left(H_{D E M}\right)$, die Ausprägung der jeweiligen wettbewerbsund umweltbezogenen Strategie der Hersteller $\left(\mathrm{H}_{S T R}\right)$ sowie die Ausgestaltung der Unternehmenspotentiale ( $\mathrm{H}_{\text {POT }}$ ) erfaßt.

Darüber hinaus werden Einflußfaktoren auf der Netzwerkebene berücksichtigt. Diese netzwerkbezogenen Einflußfaktoren nehmen eine Zwischenposition zwischen den globalen Einflußfaktoren einerseits und aufgabenspezifishen Einflußfaktoren andererseits ein, da sie gleichermaßen für alle beteiligten Systempartner des jeweiligen Rücknahme- und Recyclingsystems gelten. Sie sind daher als systemspezifisch zu charakterisieren und stellen intermediäre Faktoren dar. Dabei ist insbesondere die Systemdemographie $\left(H_{S D E}\right) z u$ analysieren, die wesentliche Merkmale des jeweiligen Rücknahme- und Recyclingsystems umfaßt. Schließlich werden individualbezogene Einflußfaktoren $\left(H_{\mathrm{IND}}\right)$ berücksichtigt, die die Situation der (betroffenen) Mitarbeiter sowie einzelner Entscheidungsträger im Unternehmen bestimmen. ${ }^{374}$ Im Mittelpunkt der Analyse steht das Implementierungsverhalten bei Rücknahme- und Recyclingsystemen, wie es im Kapitel B konzipiert und empirisch bestätigt wurde.

die veränderten Werte der Gesellschaft, die Kaufkraft, die Altersstruktur und Ähnliches widerspiegelt sowie 4. das politische Umfeld, das die staatlichen Eingriffe sowie sich abzeichnende politische Entwicklungen beinhaltet. Vgl. Kolks, U., Strategieimplementierung, a.a.O., S. 40.

Sie können direkten Einfluß auf das Unternehmensverhalten ausüben, wenn sich aus diesen Sphären veränderte Anforderungen für die Unternehmen ergeben. Gelten die veränderten Anforderungen für alle Hersteller gleichermaßen, dann dürfte aufgrund dieser Veränderungen kein Unterschied beim Implementierungsverhalten zu messen sein. Im vorliegenden Fall besteht jedoch ein wesentlicher Unterschied im politisch-rechtlichen Umfeld der Hersteller langlebiger Gebrauchsgüter, da die inhaltliche Ausgestaltung der Rücknahmeverordnungen für die Produkte sowie der Zeitpunkt ihres Inkrafttretens variieren. Daher wird das politischrechtliche Umfeld als globaler externer Einflußfaktor des Implementierungsverhaltens in die Analyse einbezogen, während die anderen globalen Größen für alle Hersteller gleichermaßen gelten und daher nicht als Einflußfaktoren berücksichtigt werden. Gelten einige Faktoren für die Herstellerunternehmen gleichermaßen, ist kein signifikanter Erklärungsbeitrag für die Entstehung der Implementierungstypen zu erwarten. Vgl. zu einer ähnlichen Vorgehensweise Hoffmann, F., Kreder, M., Situationsabgestimmte Strukturform: Ein Erfolgspotential der Unternehmung, in: ZfbF, Nr. 6, 1985, S. 461.

373 Vgl. zu dieser Systematisierung stellvertretend Kieser, A. (Hrsg.), Organisationstheorien, a.a.O., S.161.

374 Bereits Sydow unterscheidet vier Ebenen bedeutsamer Kontingenzen für die Evolution und Organisation strategischer Netzwerke: Netzwerkumwelt, Netzwerk, Organisation, Individuum/Gruppe. Vgl. Sydow, J., Strategische Netzwerke, a.a.O., S. $283 \mathrm{ff}$. 


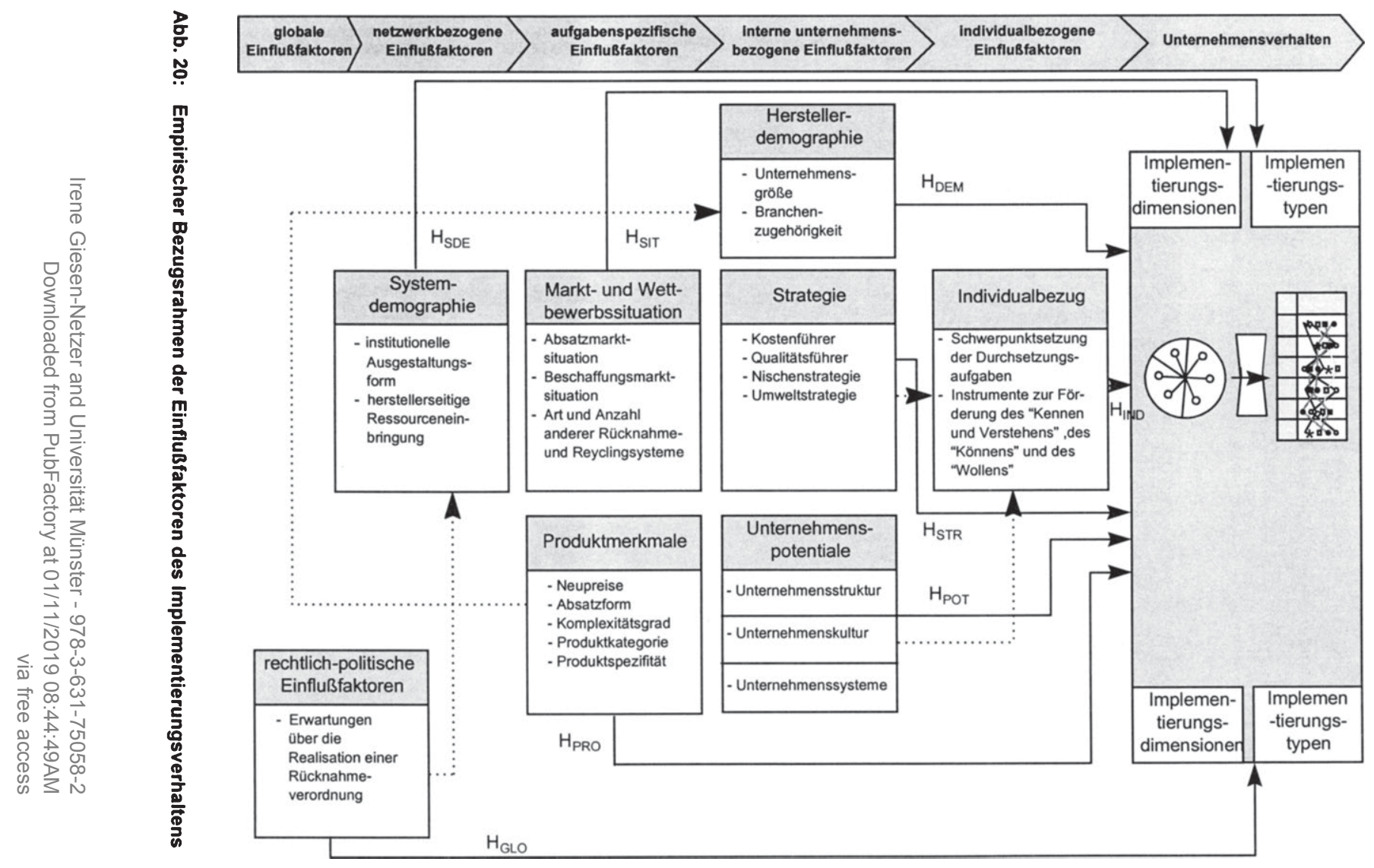


Die Untersuchung der Einflußfaktoren erfolgt gemäß der abzuleitenden Basishypothesen hauptsächlich im Hinblick auf die identifizierten Implementierungstypen. Es werden grundsätzlich keine Tendenzhypothesen bezüglich einzelner Implementierungstypen formuliert; vielmehr werden die Ausprägungen der Einflußfaktoren als clusterbeschreibende Merkmale in die Analyse einbezogen. Dieses Vorgehen wird angesichts der geringen Fallzahl gewählt, die kaum Tendenzhypothesen hinsichtlich einzelner Implementierungstypen zuläßt. Zur Überprüfung der $\mathrm{Hy}$ pothesen kommen überwiegend Mittelwertvergleiche zur Anwendung. Als Testverfahren zur Überprüfung der Signifikanz der Mittelwertunterschiede wird die einfaktorielle Varianzanalyse eingesetzt, die mit dem F-Wert ein Kriterium zur Globalbeurteilung von Mittelwertunterschieden bereitstellt. ${ }^{375}$ Zusätzlich werden multiple Mittelwertvergleichstests verwendet, die Aussagen darüber ermöglichen, ob zwischen den Implementierungstypen signifikante Unterschiede bestehen. Hier wird auf das Testverfahren von Duncan zurückgegriffen. ${ }^{36}$ Bei nominalskalierten Daten wird auf die Kontingenzanalyse zurückgegriffen. Der ChiQuadrat-Test ermöglicht dabei Aussagen darüber, ob die Einflußwirkungen signifikant sind. ${ }^{377}$

Signifikante Mittelwertunterschiede können als Indikator dafür angesehen werden, daß die jeweils untersuchten Einflußfaktoren einen Erklärungsbeitrag für das Implementierungsverhalten liefern. Allerdings ist zu berücksichtigen, daß weder die angewendeten varianz- noch die kontingenzanalytischen Verfahren Einflußrichtungen, d.h. Kausalitätsbeziehungen, erkennen lassen, sondern nur korrelative

375 Der F-Wert (Overall-F-Test) gibt bei mehreren Mittelwerten Auskunft darüber, ob sich die Mittelwerte in ihrer Gesamtheit unterscheiden, d.h. daß sich mindestens zwei Mittelwerte signifikant voneinander unterscheiden, nicht aber darüber, zwischen welchen Mittelwerten signifikante Unterschiede bestehen oder ob noch weitere signifikante Unterschiede bestehen. Vgl. Fahrmeir, L, Hamerle A., Varianz- und Kovarianzanalyse, in: Fahrmeir, L., Hamerle, A. (Hrsg.), Multivariate statistische Verfahren, Berlin, New York, de Gruyter 1984, S. 164.

376 Der multiple Mittelwertvergleich erfolgt mit einer Irrtumswahrscheinlichkeit von $\alpha<0,05$ bzw. $\alpha<0,1$. Der Duncan-Test bewirkt, daß bereits relativ kleine Mittelwertunterschiede als signifikant angesehen werden, so daß eine Unterbewertung von signifikant unterschiedlichen Mittelwertpaaren unterbleibt. Vgl. Diehl, J. M., Varianzanalyse, Frankfurt am Main 1977, S. 48ff. und vgl. Clauß, G., Finze, F.-R., Partzsch, L., Statistik für Soziologen, Pädagogen und Mediziner, Band I: Grundlagen, Frankfurt am Main 1994, S. 274ff. Zudem hat sich der Test nach Duncan bei unterschiedlichen Zellenbesetzungen als relativ robust erwiesen.

Bei der Kontingenzanalyse wird untersucht, ob die betrachteten Variablen statistisch abhängig bzw. unabhängig sind, d.h. ob ein Zusammenhang erkennbar ist. Der Chi-Quadrat-Test geht der Frage nach, ob die Assoziation in der Stichprobe zufällig auftrat oder nicht. Vgl. Backhaus, K., u.a., Multivariate Analysemethoden, a.a.O., S. 167ff. 
Beziehungen aufzeigen. ${ }^{378}$ Aufgrund der begrenzten Fallzahl und der dadurch ohnehin eher explorativen Hypothesenprüfung wurde von weitergehenden statistischen Untersuchungen, z.B. in Form von Lisrel-Analysen, verzichtet.

Abschließend sei noch einmal darauf hingewiesen, daß die Daten aufgrund der subjektiven Einschätzung der befragten Führungskräfte gewonnen wurden, d.h. die Individualebene wurde nicht direkt bei den betroffenen Mitarbeitern erfaßt. Dieser Umstand muß insbesondere bei der Einschätzung des Handlungsbedarfs hinsichtlich der Durchsetzungsaufgaben des Rücknahme- und Recyclingsystems auf der Individualebene berücksichtigt werden, ${ }^{379}$ da Verhaltensweisen wie z.B. die Mitarbeiterführung in hohem Maße individuell unterschiedlich ausgeprägt sind und Verallgemeinerungen im Sinne empirisch-induktiver Aussagen mit Einschränkungen verbunden sind.

\subsection{Einfluß politisch-rechtlicher Faktoren auf das Implementierungsver- halten}

Die branchenindividuelle Betroffenheit der Hersteller von politisch-rechtlichen Faktoren hängt von der inhaltlichen Ausgestaltung der jeweils relevanten Rücknahmeverordnung ab. Die zentralen Unterschiede zwischen Elektronikschrott-, Informationstechnologie- sowie Altautoverordnung ${ }^{380}$ werden in den Abbildungen 21a und $21 b$ deutlich.

Die Zielsetzung des Entwurfs der Elektronikschrottverordnung ${ }^{381}$ besteht demzufolge in der Vermeidung und Verringerung der Abfälle aus Elektro- und Elektronikgeräten. Dieses soll durch eine umwelt- und recyclinggerechte Konstruktion, durch leichte Reparier- und Zerlegbarkeit der Geräte sowie durch Herstellung aus umweltverträglichen und verwertbaren Materialien erreicht werden. Darüber hinaus sollen Sammelsysteme eingerichtet werden, die für den Endverbraucher leicht

\footnotetext{
378 Es liegt nur eine Vermutung über die Wirkungsrichtung der Variablen zugrunde. Vgl. ebenda, S. 57.

379 Vgl. Kapitel C 1.5.

380 Die befragten Hersteller lassen sich folgenden Branchen zuordnen: Automobil, Möbel, Elektround Elektronikgeräte, Bauzubehör und Chemie, Metall- und Maschinenbau (vgl. zum genauen Design der empirischen Befragung Anhang 2.1).

$381 \mathrm{Vgl}$. zu den folgenden Ausführungen Bundesminister für Umwelt, Naturschutz und Reaktorsicherheit, Entwurf der Verordnung über die Vermeidung, Verringerung und Verwertung von Abfällen gebrauchter elektrischer und elektronischer Geräte (Elektronik-Schrott-Verordnung), Bonn, Stand vom 15. Oktober 1992.
} 
erreichbar sind und eine hohe Rücklaufquote gewährleisten. Zurückgenommene Geräte(teile) sollen einer erneuten Verwendung oder einer Verwertung zugeführt werden. Ist dieses nicht möglich, sind die Altgeräte(teile) einer sonstigen sachgemäßen Entsorgung zuzuführen.

\begin{tabular}{|c|c|c|c|}
\hline & $\begin{array}{l}\text { Beltrariksdrottverardung } \\
\text { van } 15 \text {. Ataber } 1992\end{array}$ & $\begin{array}{l}\text { Iffarmetionsted rikverachung } \\
\text { vam20. Februar } 1996\end{array}$ & $\begin{array}{l}\text { Altaitoverordung } \\
\text { vam27. Januar } 1994\end{array}$ \\
\hline Zele & 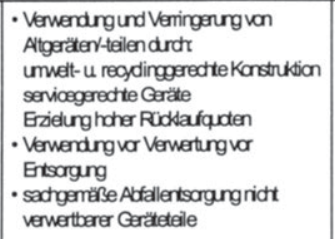 & Fơderung der Kreislaufwirtschaft & 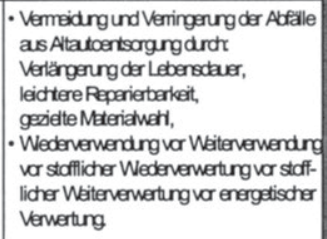 \\
\hline $\begin{array}{l}\text { Arwendings } \\
\text { bereich } \\
\text { (Zelguppen) }\end{array}$ & $\begin{array}{l}\text { Hersteller und Vertreiber van eledtrarischen } \\
\text { Gerätenund Geräteteilen }\end{array}$ & 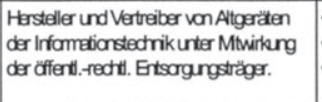 & $\begin{array}{l}\text { - Hersteller und Vertreiber } \\
\text { - Letalbesitzer } \\
\text { - Verwerter }\end{array}$ \\
\hline $\begin{array}{l}\text { Produkt- } \\
\text { kategaien }\end{array}$ & 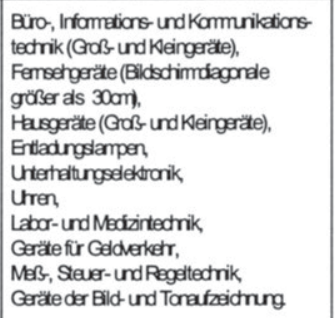 & 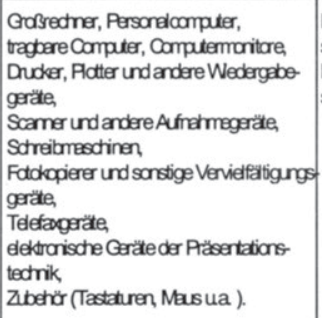 & 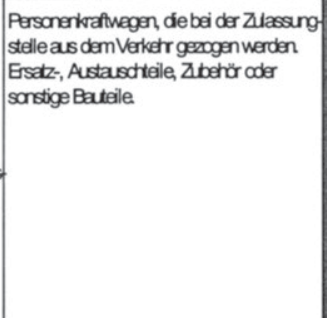 \\
\hline $\begin{array}{l}\text { Otder } \\
\text { Rudonatre }\end{array}$ & 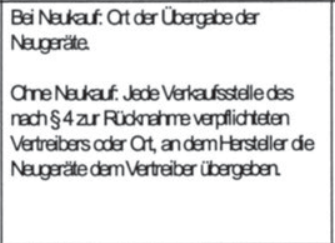 & 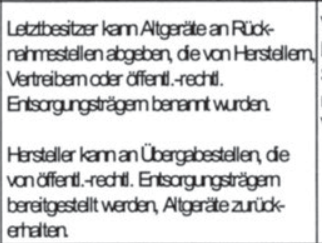 & 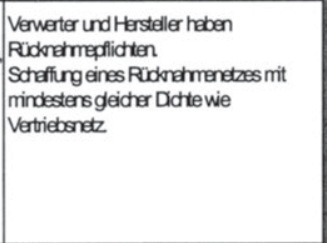 \\
\hline
\end{tabular}

Abb. 21a: Entwürfe von Rücknahmeverordnungen langlebiger Gebrauchsgüter 


\begin{tabular}{|c|c|c|c|}
\hline & $\begin{array}{l}\text { Bektrarikschrottverardiung } \\
\text { vom } 15 \text { Oktober } 1992\end{array}$ & $\begin{array}{l}\text { Informationstedrikverachung } \\
\text { van20. Februar } 1996\end{array}$ & $\begin{array}{l}\text { Altautovercichung } \\
\text { vam27. Januer } 1994\end{array}$ \\
\hline $\begin{array}{l}\text { Ridnatme } \\
\text { pflidten }\end{array}$ & 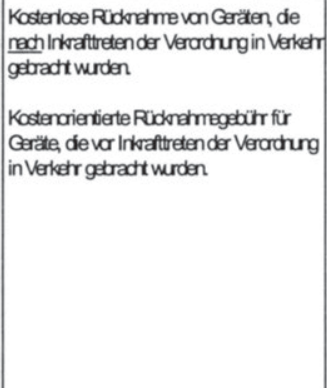 & 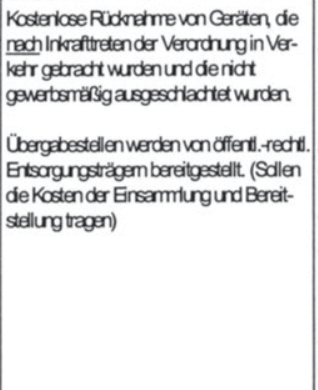 & 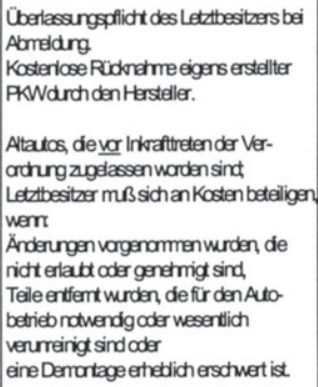 \\
\hline $\begin{array}{l}\text { Verwertungs } \\
\text { pfiditen }\end{array}$ & $\begin{array}{l}\text { Hersteller und Vertreiber übernetmen } \\
\text { Verwertungspflidten }\end{array}$ & $\begin{array}{l}\text { Hersteller und Vertreiber übernetmen } \\
\text { Verwertungsplictiten }\end{array}$ & $\begin{array}{l}\text { Hersteller und Vertreiber haben } \\
\text { Verwertungsplictiten } \\
\text { Angabe von detalierten Verwertungsiden } \\
\text { in §ु6 }\end{array}$ \\
\hline Ausnatmen & 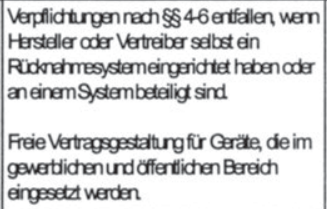 & $\begin{array}{l}\text { Freie Vertragsgestaltung fư Geräte, de in } \\
\text { Indistrie, Geverbe, freien Benfen oder } \\
\text { offertichen Erridturgen arfallen }\end{array}$ & $\begin{array}{l}\text { Lasthratwegen und Busse werden } \\
\text { auggesdiossen }\end{array}$ \\
\hline Kontrollen & & 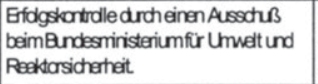 & 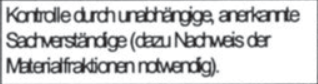 \\
\hline $\begin{array}{l}\text { Beaftraging } \\
\text { Ditter }\end{array}$ & $\begin{array}{l}\text { Zr Aufgabenefiulung künen sich He- } \\
\text { steller und Vertreiber Ditter ( } \mathrm{zB} \\
\text { Entsargungsurternetmen) bedenen }\end{array}$ & $\begin{array}{l}\text { Zr Aufgabenefululung körnen sich Her- } \\
\text { steller und Vertreiber Ditter bedienen }\end{array}$ & $\begin{array}{l}\text { Zr Aufgebenefullung kömen sich Her- } \\
\text { steller und Vertreiber Ditter bedienen }\end{array}$ \\
\hline
\end{tabular}

Abb. 21b: Entwürfe von Rücknahmeverordnungen langlebiger Gebrauchsgüter

Die Rücknahmepflichten betreffen Hersteller und Vertreiber ${ }^{382}$ elektrischer und/oder elektronischer Geräte(teile). Grundsätzlich besteht die Verpflichtung, die

382 Gemäß des Verursachungsprinzips müssen in erster Linie Hersteller Adressaten der Produktverantwortung sein. Um importierte Produkte ebenfalls dem Kreislaufwirtschaftsgesetz und den entsprechenden Rücknahmeverordnungen zu unterwerfen, ist es notwendig, auch die Vertreiber gleichermaßen in die Pflicht zu nehmen. Darüber hinaus wird durch diese doppelte Adressierung die Notwendigkeit der Konsensfindung unterstützt. Im Kreislaufwirtschaftsgesetz werden die Adressaten folgendermaßen benannt: „Wer Erzeugnisse entwickelt, herstellt, beund verarbeitet oder vertreibt, trägt zur Erfüllung der Ziele der Kreislaufwirtschaft die Produktverantwortung“. §22 Abs. $1 \mathrm{KrW} / \mathrm{AbfG}$.

In der vorliegenden Arbeit wurden ausschließlich deutsche Unternehmen befragt. Hier sind die Hauptadressaten die Hersteller der Produkte, so daß die Bearbeitung der Themenstellung ausschließlich aus deren Perspektive erfolgt. 
Geräte(teile) kostenlos zurückzunehmen, es sei denn, diese sind vor Inkrafttreten der Verordnung vertrieben worden. Die kostenpflichtige Rücknahme gilt auch nach Inkrafttreten der Verordnung, wenn der Endverbraucher ein im Ausland erworbenes Gerät in den Geltungsbereich der Verordnung (z.B. durch Import) verbracht hat oder von einem Vertreiber mit Geschäftssitz außerhalb des Geltungsbereichs der Verordnung beliefert wurde. Für Hersteller bestehen darüber hinaus Verwertungspflichten. Diese gelten nicht, wenn sie selbst ein Rücknahmesystem betreiben oder sich an einem solchen beteiligen. Zudem können sie sich zur Erfüllung ihrer Pflichten Dritter bedienen, die durch ein Gutachten bestätigen müssen, alle Pflichten entsprechend des Gesetzes erfüllen zu können.

Der Entwurf zur Informationstechnologieverordnung ${ }^{383}$ hingegen formuliert keine detaillierten Zielsetzungen, sondern legt lediglich fest, daß die Umsetzung der Verordnung das Kreislaufwirtschaftsgesetz fördern soll. Dieses impliziert jedoch die Pflichtenhierarchie und damit auch die konkrete Reihenfolge der Behandlung von Abfällen: Vermeidung vor Verwertung vor Beseitigung bzw. Entsorgung. Adressaten der Verordnung sind wie in der Elektronikschrottverordnung in erster Linie Hersteller von Informationstechnologiegeräten. Eine wesentliche Änderung gegenüber der Elektronikschrottverordnung betrifft die Beteiligung der öffentlich-rechtlichen Entsorgungsträger.

Für den Hersteller von Informationstechnologiegeräten besteht eine Pflicht zur Rücknahme, Verwertung und Beseitigung der von ihm in Verkehr gebrachten Geräte. Der Hersteller und/oder Vertreiber hat alle Kosten zu tragen, die für eine Abholung an den Übergabestellen entstehen, wenn die Geräte nach Inkrafttreten der Verordnung in den Verkehr gebracht wurden. Diese Verpflichtung entfällt bei „ausgeschlachteten“ Geräten (es fehlen z.B. Komponenten, die beim Recycling noch eine Wertschöpfung zulassen würden) und bei Geräten, die vor Inkrafttreten der Verordnung in den Verkehr gebracht wurden. Für Geräte, die nicht privaten Haushalten entstammen, bleiben die Frage der Kosten sowie die Art und der Ort der Rückgabe vertraglichen Vereinbarungen überlassen. Die Aufgabe der öffent-

383 Informationstechnologien sind als Untergruppe zu Elektro- und Elektronikgeräten zu verstehen und umfassen Geräte, die zur Datenverarbeitung und -übertragung geeignet sind (z.B. Rechner, Drucker, Schreibmaschinen, Kopierer, Telefaxgeräte).

Vgl. zu den folgenden Ausführungen Bundesminister für Umwelt, Naturschutz und Reaktorsicherheit, Entwurf der Verordnung über die Entsorgung von Geräten der Informationstechnik (IT-Geräte-Verordnung), Bonn, Stand vom 20. Februar 1996. 
lich-rechtlichen Entsorgungsträger besteht in der Sammlung bzw. Entgegennahme der gebrauchten Informationstechnologiegeräte und deren Bereitstellung in Übergabestellen sowie in der Übernahme der Kosten für diese Tätigkeiten. ${ }^{384}$ Für Hersteller und Vertreiber besteht die Möglichkeit der Beauftragung Dritter zur Übertragung ihrer Pflichten, wobei die Verantwortung bei ihnen verbleibt. Ein Ausschuß beim Bundesministerium für Umwelt, Naturschutz und Reaktorsicherheit soll die ordnungsgemäße Art, Menge, Verwertung und Beseitigung der zurückgenommenen Informationstechnologiegeräte überprüfen. ${ }^{385}$

Während bei der Informationstechnologieverordnung eine eher unscharfe Zielbeschreibung festzustellen ist, beinhaltet die Altautoverordnung ${ }^{386}$ eine detaillierte Zielsetzung. Danach sollen Abfälle aus der Altautoentsorgung ${ }^{387}$ durch eine Verlängerung der Lebensdauer, leichte Reparierbarkeit der Automobile und eine gezielte Materialwahl vermieden bzw. verringert werden. Außerdem wird die Pflichtenhierarchie weiter konkretisiert: Die Wiederverwendung ist der Weiterverwendung vorzuziehen, dem folgen stoffliche Wiederverwertung vor stofflicher Weiterverwertung und schließlich die energetische Verwertung. Erst wenn diese Möglichkeiten ausgeschöpft sind, soll eine ordnungsgemäße Entsorgung möglich sein.

384 Der Verband Deutscher Maschinen- und Anlagenbauer argumentiert, daß den öffentlichen Entsorgungsträgern die Kosten entfallen, die sie bisher für Deponierung oder sonstige Beseitigung der Geräte aufwenden mußten. Durch die arbeitsteilige Neuregelung würde folglich keine höhere Gebührenbelastung für die Endverbraucher entstehen. Vgl. Bundesminister für Umwelt, Naturschutz und Reaktorsicherheit, Begründung zur Informationstechnologieverordnung, Entwurf der Verordnung über die Entsorgung von Geräten der Informationstechnik (IT-GeräteVerordnung), a.a.O., S. 9f. Dem muß widersprochen werden, da ein Anstieg der Abfallgebühren bis zu 28\% zu erwarten ist, die der Endverbraucher zu zahlen hat, da die Deponie- und Anlagenfixkosten gleich bleiben.

Darüber hinaus ist zu bedenken, daß die Zielsetzung des Kreislaufwirtschaftsgesetzes die Externalisierung der internen ökologischen Kosten ist (vgl. Ruchay, D., Kreislaufwirtschaft und Rücknahmeverordnungen - Umweltpolitische Ziele und Status der Umsetzung, a.a.O., S. 10) und diese Kosten dem Verursacher - also Hersteller und Vertreiber - in Rechnung gestellt werden müßten. Dieses Verursacherprinzip widerspricht demnach der Lösung, daß die Kommunen weiterhin die Kosten für Sammlung und Bereitstellung übernehmen sollen.

Der Ausschuß soll aus Vertretern der Hersteller, Vertreiber, des Bundes, der Länder und der öffentlich-rechtlichen Entsorgungsträger gebildet werden und mindestens einmal jährlich zusammentreten. Vgl. Bundesminister für Umwelt, Naturschutz und Reaktorsicherheit, Begründung zur Informationstechnologieverordnung, Entwurf der Verordnung über die Entsorgung von Geräten der Informationstechnik (IT-Geräte-Verordnung), a.a.O.

$\mathrm{Vgl}$. zu den folgenden Ausführungen Bundesminister für Umwelt, Naturschutz und Reaktorsicherheit, Entwurf über die Entsorgung von Altautos, a.a.O.

387 Altautos im Sinne dieser Verordnung sind Personenkraftwagen, die aus dem Verkehr gezogen sind. Ausgeschlossen sind Lastkraftwagen und Busse. 
Den Vorschriften dieses Verordnungsentwurfes unterliegen Hersteller, Vertreiber, Letztbesitzer sowie Verwerter von Automobilen. Wie in den zuvor beschriebenen Verordnungsentwürfen haben auch hier Hersteller und Verwerter gleichermaßen Verwertungspflichten. Darüber hinaus sind die Hersteller verpflichtet, Personenkraftwagen aus eigener Produktion grundsätzlich kostenlos zurückzunehmen und ein Rücknahmenetz zu schaffen, welches der gleichen Dichte entsprechen soll wie das Vertriebsnetz. Sie können sich zur Erfüllung der Pflichten Dritter bedienen. Die Letztbesitzer haben die Pflicht, die Altautos bei der Abmeldung dem Hersteller, Vertreiber oder beauftragten Dritten oder sonstigen Verwerterbetrieben, wenn diese bestimmte Anforderungen erfüllen, zu überlassen. ${ }^{388}$ Letztbesitzer müssen sich zudem an den Kosten beteiligen, wenn Änderungen am Auto vorgenommen wurden, die nicht erlaubt oder genehmigt worden sind, Teile entfernt oder wesentlich verunreinigt worden sind, die für den Autobetrieb notwendig sind, eine Demontage wesentlich erschwert ist sowie wenn es sich um Automobile handelt, die vor Inkrafttreten der Verordnung zugelassen worden sind. Die Verwerter haben Anforderungen bezüglich der Trockenlegung, Demontage, Getrennthaltung, Wiederverwendung, Verwertung sowie der Buchführung über Material- und Stoffströme zu erfüllen. ${ }^{389}$ Außerdem müssen sie sich mindestens halbjährlich von einem unabhängigen anerkannten Sachverständigen prüfen lassen. ${ }^{390}$

388 Kritisch ist an dieser Stelle anzumerken, daß die Verwerter („normale Schrotthändler"), wenn sie bestimmte Anforderungen nicht erfüllen, aus diesem System ausgeschlossen werden und dem Endbesitzer die Möglichkeit genommen wird, ein letztes Entgelt für den gesamten Pkw (sog. Schrottwert) oder einzelne, noch wertvolle Teile zu erhalten.

Der notwendige Verwertungsnachweis eines zertifizierten Recyclingunternehmens zur endgültigen Stillegung der Altautos wird hinsichtlich des Inkrafttretens der Altautoverordnung zum 1.4.1998 diskutiert. Dementsprechend konstatiert der ADAC, daß zum einen mit 60 Betrieben zu wenig zertifizierte Recyclingunternehmen zugelassen sind und zum anderen ein erhebliches Informationsbedürfnis seitens der Nachfrager besteht. Das Bundesumweltministerium sowie der Verband der Automobilindustrie widersprechen dieser Ansicht. Sie nennen 200 zertifizierte Betriebe und 1000 weitere Annahmestellen für Altautos. Vgl. o.V., Altautoverordnung sorgt für neuen Wirbel, in: Die Welt, 7.3.1998, Online-Archiv 0307wi16.html.

Weiland analysiert aus volkswirtschaftlicher Sicht sechs Problembereiche, die sich im Rahmen der Rücknahmeverordnung ergeben. Er kommt zu dem Schluß, daß 1. Eine Rücknahmepflicht durch die Hersteller sinnvoll ist, 2. Entgelte zwischen Herstellern und Verwertern ausgehandelt werden sollten, 3. Eine Rücknahme gemeinsam durch die Händler, Verwerter und Hersteller erfolgen sollte, 4.Verwertungsquoten unter Berücksichtigung von Transaktionskosten durchaus sinnvoll sind, 5 . Hersteller und Verwerter für die Verwertung eine Kooperation anstreben sollten und 6. Der Wiedereinsatz von Altteilen in Neuprodukten nicht zu erwarten ist, während der Einsatz von Sekundärrohstoffen zunehmen wird. Vgl. Weiland, R., Rücknahme- und Entsorgungspflichten in der Abfallwirtschaft, eine institutionenökonomische Analyse der Automobilbranche, Wiesbaden 1995, S. $73 \mathrm{ff}$. 
Mit den Entwürfen der Rücknahmeverordnungen entsteht ein "Handlungszwang" für die Herstellerunternehmen, den Auflagen des Gesetzgebers Genüge zu tun. ${ }^{391}$ Neben den allgemeinen Pflichten der Verwertung enthalten die Verordnungen bereits recht detaillierte Anforderungen, die bei der Implementierung des Rücknahme- und Recyclingsystems zu berücksichtigen sind und deren konkrete Ausgestaltung für die Hersteller z.T. mit erheblichen Schwierigkeiten verbunden ist. ${ }^{392}$

Diese, die Implementierung des Rücknahme- und Recyclingsystems betreffenden politisch-rechtlichen Faktoren werden vor allem im Zusammenhang mit den verschiedenen institutionellen Ausgestaltungsformen von Rücknahme- und Recyclingsystemen diskutiert, ${ }^{393}$ d.h. es wird analysiert, unter welchen Bedingungen eher herstellereigene oder kooperative Systeme sinnvoll sind. Vernachlässigt wurde demgegenüber bisher die Frage, ob der Handlungszwang, der von den Verordnungen ausgeht, auch Auswirkungen auf das Implementierungsverhalten hat. Dieses soll untersucht und durch folgende Basishypothese zum Ausdruck gebracht werden:

391 Vgl. z.B. Raabe, T., Die Elektronik-Schrott-Verordnung, a.a.O., S. 284.

392 Beispielsweise stellt die geforderte kostenlose Rücknahme hohe Anforderungen an die Kostenrechnung der Unternehmen, die die Rücknahmemengen und -kosten abschätzen müssen, um sie dann (i.d.R.) in den Neupreis einkalkulieren zu können. Daraus entsteht eine Unsicherheit bezüglich der Höhe der einzurechnenden Kosten, die um so mehr steigt, je langlebiger das Produkt ist. Auch die Demontage der Produkte wird bisher teilweise nicht verordnungsgerecht durchgeführt. Zwar findet $z . B$. bei Automobilen eine Trockenlegung der Altfahrzeuge statt, jedoch werden nicht, wie in der Verordnung vorgesehen, die gesamten Betriebsflüssigkeiten entnommen. Vgl. Zimmermann, H., Automobilrecycling im Verbund, a.a.O., S. 45.

Zudem sind die Zusammensetzung der rücklaufenden Altprodukte wie auch ihre unterschiedliche Quantität ein zusätzlicher Unsicherheitsfaktor für die Hersteller bezüglich der Erfüllung der Verordnungen. Falke beschreibt dies am Beispiel des Unternehmens Hewlett Packard beim Computerrecycling. Vgl. Falke, R., Verantwortung für das Ganze tragen, in: Beschaffung aktuell, Heft 2, 1993, S. 34.

Bonus fordert daher, daß in der Umweltpolitik weniger Ordnungsrecht und dafür mehr Ordnungspolitik betrieben werden müßte, d.h. aus ordnungspolitischer Sicht heraus sollte der Markt durch Umweltverschmutzungsrechte so ergänzt werden, daß der Markt auch im Umweltbereich die Fähigkeit zur spontanen Selbstorganisation zurückgewinnt. Vgl. Bonus, H., Bei der Umweltpolitik geht es letzt - endlich um die Freiheit, in: Handelsblatt, 27./28.9.1996, S. 2. Eine Implementierung von Eigentumsrechten am Ressourcenbestand scheitert jedoch an den teils prohibitiv hohen (Transaktions)Kosten der Definition und der Durchsetzung solcher Rechte. Daraus folgert Ströbele, daß Fehlallokationen aus den fehlenden Eigentumsrechten und fehlende Eigentumsrechte aus hohen Transaktionskosten resultieren. Vgl. Ströbele, W., Die Nutzung regenerierbarer natürlicher Ressourcen, in: WISU, Heft 3, 1990, S. $189 f$. strategische Netzwerke, a.a.O., S. 62ff. und Raabe, T., Die Elektronik-Schrott-Verordnung, a.a.O., S. $283 \mathrm{ff}$. 


\begin{tabular}{|c|c|}
\hline $\mathrm{H}_{\mathrm{GLO} 1}:$ & $\begin{array}{l}\text { Das politisch-rechtliche Umfeld liefert durch die zu erwartenden Rücknahmeverord- } \\
\text { nungen einen Erklärungsbeitrag für die Existenz unterschiedlicher Implementierungsty- } \\
\text { pen, d.h. die Implementierungstypen unterscheiden sich signifikant hinsichtlich des jeweils } \\
\text { wahrgenommenen politisch-rechtlichen Drucks. }\end{array}$ \\
\hline
\end{tabular}

Da die vorgestellten Rücknahmeverordnungen bisher noch nicht in Kraft gesetzt wurden, ist die Frage nach der durch die Hersteller wahrgenommenen Betroffenheit relevant. Dieser subjektive Betroffenheitsbegriff umfaßt die ökologische Betroffenheit, die wahrgenommene Intensität der Ansprüche (aus den Rücknahmeverordnungen) und die damit zu erwartenden Sanktionen, sofern den Ansprüchen nicht entsprochen wird. ${ }^{394}$ Daher ist zu analysieren, welche Einflüsse der wahrgenommenen Betroffenheit auf die Implementierungsdimensionen und damit auch auf die Implementierungstypen zu erwarten sind. ${ }^{395}$

Hinsichtlich der Erwartungen eines Inkrafttretens der Rücknahmeverordnungen ist insbesondere ein Einfluß auf die zeitlichen Implementierungsdimensionen zu vermuten, da in diesen Erwartungen der Handlungsdruck zum Ausdruck kommt. Es kann davon ausgegangen werden, daß die Hersteller bei hohem wahrgenommenen zeitlichen Handlungsdruck schnell ein Rücknahme- und Recyclingsystem implementieren wollen. ${ }^{396}$ Dieses Verhalten äußert sich insbesondere in der Dimension des Zeithorizonts.

394 Vgl. Kirchgeorg, M., Ökologieorientiertes Unternehmensverhalten, a.a.O., S. 87. Kirchgeorg untersucht im Gegensatz zur vorliegenden Arbeit das gesamte Spektrum der Anspruchsgruppen bei der Analyse der ökologischen Betroffenheit der Unternehmen, d.h. neben den staatlichen Ansprüchen werden explizit Medien, Bürgerinitiativen, Mitarbeiter und marktbezogene ökologische Anspruchsgruppen einbezogen. Vgl. ebenda, S. 89.

Kirchgeorg leitet den Betroffenheitsgrad zusätzlich als objektive Größe ab. Danach kann der Betroffenheitsgrad eines Unternehmens durch das Ausmaß der durch die Sanktionen der Anspruchsgruppen verursachten Beeinträchtigungen der unternehmensbezogenen Ziele, die Anzahl der ökologischen Anspruchsgruppen und die Intensität der Umweltschutzforderungen in bezug auf den Umfang der zu internalisierenden Kosten gekennzeichnet werden. Vgl. ebenda, S. 87.

395 Generell wird die subjektiv wahrgenommene Betroffenheit der befragten Hersteller relativ höher sein als bei einer repräsentativen Befragung, weil Hersteller befragt wurden, die sich bereits mit der Gestaltung und Implementierung von Rücknahme- und Recyclingsystemen auseinandergesetzt haben.

Dies ist m.E. nach zunächst unabhängig von der institutionellen Ausgestaltungsform gültig, d.h. eine schnelle Implementierung kann durch eine hohe Externalisierung aller Aktivitäten zum Ausdruck kommen, ebenso aber durch ein hohes Tempo bei der Realisation eines eigenen Rücknahme- und Recyclingsystems. 
Die Kontingenzanalyse ${ }^{397}$ die in Abbildung 22 wiedergegeben ist, zeigt, daß über alle Implementierungstypen hinweg knapp 60\% der Hersteller eine Rücknahmeverordnung erwarten, während gut $40 \%$ keine Rücknahmeverordnung erwarten. Die Cluster weisen deutliche Unterschiede hinsichtlich der Erwartungen auf, die durch das Ergebnis des Chi-Quadrat-Tests, der eine Irrtumswahrscheinlichkeit von unter $5 \%$ aufweist, belegt werden. ${ }^{398}$ Damit kann die Basishypothese $H_{\mathrm{GLO}}$ als bestätigt angenommen werden.

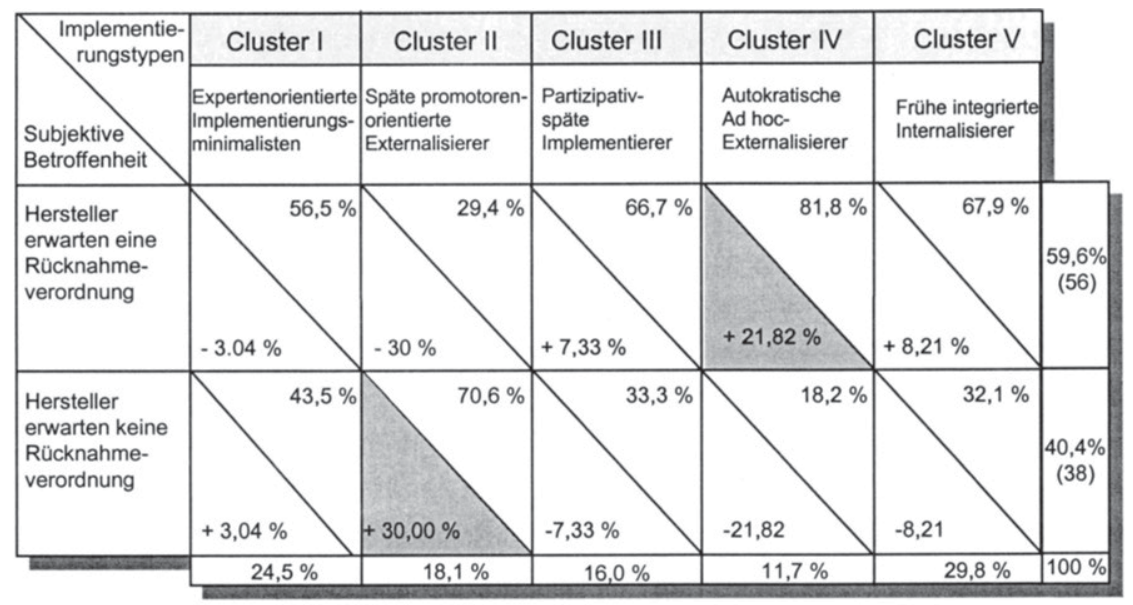

\begin{tabular}{|c|c|c|c|c|}
\hline $\begin{array}{l}\text { Chi-Quadrat-Test } \\
\text { gemäß: }\end{array}$ & $\begin{array}{l}\text { Signifikanz- } \\
\text { niveau: }\end{array}$ & $x$ & $x \%$ & $0 \%-5 \%$ \\
\hline Pearson & 0,04247 & $\begin{array}{l}\text { tatsächlicher } \\
\text { Anteil }\end{array}$ & $\begin{array}{l}\text { Abweichung } \\
\text { vom }\end{array}$ & $5,1 \%-10 \%$ \\
\hline \multirow{2}{*}{$\begin{array}{l}\text { Likelihood } \\
\text { Ratio }\end{array}$} & \multirow[t]{2}{*}{0,03938} & $\begin{array}{l}\text { Implemen- } \\
\text { tierungstyp/ }\end{array}$ & $\begin{array}{l}\text { erwarteten } \\
\text { statistischen }\end{array}$ & $10,1 \%-20 \%$ \\
\hline & & $\begin{array}{l}\text { erwarteter } \\
\text { RechtsVO }\end{array}$ & Wert & \\
\hline
\end{tabular}

\section{Abb. 22: Kontingenzanalyse zum Zusammenhang zwischen der herstellerseitigen Erwar- tung einer Rücknahmeverordnung und den Implementierungstypen}

397 Die Anwendung der Kontingenzanalyse unterliegt verschiedenen Bedingungen, damit deren Aussagefähigkeit nicht beeinträchtigt wird. Als Faustformel gilt hier, daß der Anteil der Zellen mit erwarteten Häufigkeiten, die kleiner als 5 sind, nicht mehr als $20 \%$ überschreiten dürfen. Vgl. zu weiteren Anwendungsempfehlungen, Backhaus, K., u.a., Multivariate Analysemethoden, a.a.O., S. 188.

Der auf der Likelihood-Statistik beruhende Test basiert auf der Likelihood-Schätzung und führt bei großen Stichproben zu ähnlichen Ergebnissen wie der Chi-Quadrat-Test (Pearson). Vgl. Backhaus, K., u.a., Multivariate Analysemethoden, a.a.O., S. 184. 
70\% des Clusters der Späten promotorenorientierten Externalisierer erwartet keine Rücknahmeverordnung und überschreitet damit den erwarteten statistischen Wert um $30 \%$. Offensichtlich begünstigt der nur in geringem Umfang wahrgenommene zeitliche Handlungsdruck eine eher zögerliche Implementierung. Die Dauer der Implementierungsaktivtäten ist dementsprechend überdurchschnittlich lang ausgeprägt bei einer vergleichsweise späten Plaungungs- und Pilotphase des Rücknahme- und Recyclingsystems.

Dagegen erwartet mit fast $82 \%$ ein sehr hoher Anteil der Autokratischen Ad hocExternalisierer das Eintreten einer Rücknahmeverordnung. Dieses Verhalten erscheint konsistent zu einem sehr kurzen Zeithorizont dieses Clusters. ${ }^{399}$ Die Partizipativ-späten Implementierer rechnen ebenfalls überdurchschnittlich häufig mit Rücknahme- und Recyclingsystemen, da sie einen hohen zeitlichen Handlungsdruck empfinden. Dies wird mit einem Wert von fast 2/3 aller Hersteller bestätigt.

Zunächst überraschend - wird mit den zeitlichen Implementierungsdimensionen argumentiert - mutet das Ergebnis der Frühen integrierten Internalisierer an, die mit fast $68 \%$ aller im Cluster befindlichen Hersteller eine Rücknahmeverordnung erwarten. Eine Erklärung des empirischen Befundes kann darin liegen, daß dieser Implementierungstyp aufgrund seines bestehenden Systems zum einen eine Rücknahmeverordnung erhofft, um Rechtssicherheit zu erlangen, und zum anderen durch den Erlaß einer Rücknahmeverordnung ex post eine Art „Rechtferti-

399 Eine geringe Mitbestimmungsmöglichkeit der Mitarbeiter und wenig Zielvereinbarungen zwischen Vorgesetzten und Mitarbeitern bei der Implementierung - als Dimensionen der Partizipation - unterstreichen die hohe subjektive Betroffenheit der Hersteller, da sich durch hohe Ausprägungen dieser Dimension der Abstimmungsbedarf und der Zeitbedarf erhöhen. Auch aus diesem Grunde haben die Autokratischen Ad hoc-Externalisierer hohe Erwartungen bezüglich der Realisation der Rücknahmeverordnungen.

Dieser Argumentation folgt Clauss, indem er davon ausgeht, daß der "Partizipationsgrad“ in die "Dauer des Implementierungsprozesses“ integriert werden kann. Vgl. Clauss, M., Die Strategie der Implementierung in der Unternehmung, a.a.O., S. 92. Diese Abhängigkeit zwischen den Dimensionen der Implementierung geht aus der Abbildung 16 (Kapitel B 2.6) hervor, wobei der "Zeithorizont" und die „Partizipation“ mit einem Wert von 0,39 korrelieren. Dieser Korrelationswert rechtfertigt jedoch keine Integration der beiden Dimensionen, wie Clauss es vornimmt.

Eine umgekehrte Argumentation kann für die Partizipativ-späten Implementierer herangezogen werden. Diese müßten dementsprechend keine hohen Erwartungen bezüglich der Realisation der Rücknahmeverordnungen haben. Diese Annahme steht jedoch im Gegensatz zu der sehr kurzen Implementierungsdauer, so daß bei diesem Cluster von einer hohen Erwartung bezüglich der Realisation einer Rücknahmeverordnung ausgegangen werden kann. Der Partizipationsgrad ist dementsprechend hoch, und dennoch vollzieht sich die Implementierung schnell. 
gung" für sein zurückliegendes und aktuelles Handeln erhalten möchte und dies als Erwartungshaltung ausdrückt. ${ }^{400}$

Die Expertenorientierten Implementierungsminimalisten handeln von der Erwartung der Realisation einer Rücknahmeverordnung unabhängig. ${ }^{401}$ Dieses Verhalten stimmt mit den Ausprägungen der zeitlichen Implementierungsdimension überein, die sich weder durch besonders frühes oder spätes Timing noch durch eine besonders lange oder kurze Dauer der Implementierungsaktivitäten auszeichnen.

\subsection{Einflußfaktoren auf der Netzwerkebene}

Als Einflußfaktor auf der Netzwerkebene ${ }^{402}$ wird die Systemdemographie des Rücknahme- und Recyclingsystems analysiert, die die Untersuchung des Einflusses der institutionellen Ausgestaltungsform ${ }^{403}$ und der herstellerseitigen Ressourceneinbringung in das Rücknahme- und Recyclingsystem auf das Implementierungsverhalten bzw. die -typen umfaßt. ${ }^{404}$

400 Mit einer Rücknahmeverordnung können die bisherigen Aufwendungen z.B. in Form von Zeit und Geld für die Implementierung eines Rücknahme- und Recyclingsystems im nachhinein als gerechtfertigt angesehen werden, auch wenn ursprüngliche Ziele wie z.B. Kostendeckung oder Gewinn nicht erreicht werden können.

Mit einem Wert von $43,5 \%$ erwarten die Hersteller dieses Clusters keine Rücknahmeverordnung, was nur 3,04\% über dem erwarteten statistischen Wert liegt.

Vgl. zur Anwendung der Begrifflichkeit von strategischen und regionalen Netzwerken Kapitel B 2.1.

Bei den institutionellen Ausgestaltungsformen der Rücknahme- und Recyclingsysteme wurden (hersteller)eigene Systeme als hierarchienahe Form, Verträge zwischen Herstellern und Verwertern als marktnahe Form sowie kooperative Systeme unterschieden. Bei den kooperativen Systemen werden zusätzlich die Ausgestaltungsformen der "gemeinsamen Entwicklung eines Systems mit Verwertern" sowie „Branchenkooperationen“ unterschieden. Aufgrund der geringen Fallzahl von Gemeinschaftsunternehmen wird diese Institutionelle Ausgestaltungsform nicht weiter in die Betrachtung einbezogen.

404 Sydow beschreibt die Konsequenzen der Entwicklung von Unternehmensnetzwerken auf die Rolle des Management in Unternehmensnetzwerken und erläutert dabei implementierungsrelevante Problemstellungen. Diskutiert werden z.B. Veränderungen in der Stuktur, in den Systemen, im Controlling sowie Umorientierungen bei Mitarbeitermotivationen. Vgl. Sydow, J., Unternehmensnetzwerke, a.a.O., S. 164ff. sowie Sydow, J., Netzwerkorganisation, Interne und externe Restrukturierung von Unternehmungen, in: WiSt, Heft 12, 1995, S. 631ff. In der vorliegenden Arbeit werden die Auswirkungen des speziellen Netzwerks des Rücknahme- und Recyclingsystems auf die genannten und darüber hinausgehenden Bereiche analysiert. Vgl. Kapitel C 1.4 und C 1.5. 
Zur Erklärung des Einflusses der institutionellen Ausgestaltungsform auf das Implementierungsverhalten der Hersteller ${ }^{405}$ kann der Grad der (Quasi)Externalisierung bzw. (Quasi)Internalisierung, der durch die institutionelle Ausgestaltungsform zum Ausdruck kommt, insbesondere mit der Intensitätsdimension in Zusammenhang gebracht werden. ${ }^{406}$ Es wird dann von Quasi-Externalisierung bzw. Quasi-Internalisierung gesprochen, wenn eine partielle Ausgliederung von betrieblichen Funktionen stattfindet bzw. durch eine Unternehmenskooperation eine vollständige Integration vermieden wird. ${ }^{407}$ Diese Vorgehensweise wird insbesondere aufgrund einer verstärkten Konzentration auf die eigenen Kernkompetenzen und/oder aufgrund der Internalisierung und Nutzbarmachung von Kernkompetenzen der Partner gewählt ${ }^{408}$ und gilt als Ursache für die Entstehung von Netzwerken. ${ }^{409}$ Daher wird folgende Basishypothese formuliert:

405 An dieser Stelle stellt sich die Frage, ob die institutionellen Ausgestaltungsformen der Rücknahme- und Recyclingsysteme nicht vielmehr als clusterbeschreibende Merkmale in die Analyse einbezogen werden müßten, da durchaus „Implementierungsaktivitäten“ stattfinden können, bevor sich ein Hersteller für oder gegen eine bestimmte institutionelle Ausgestaltungsform entscheidet oder die Wahl einer institutionellen Ausgestaltungsform an sich als Implementierungsentscheidung begriffen werden könnte. Dieses Argument ist jedoch zu entkräften, da sich die Implementierungsaktivitäten explizit auf die Planungs- bzw. Einführungs- und die Realisationsphase beziehen. Erste rudimentäre Aktivitäten werden in diesem Sinne nicht als Implementierung, sondern als vorgelagerte Phase verstanden. Vgl. zu dieser Ansicht auch Knopf, R. H., u.a., Die Effizienz von Reorganisationsprozessen aus der Sicht der Praxis a.a.O., S. 73f. und vgl. die Ausführungen in Kapitel B 2.2.

Dementsprechend folgt auf die Entscheidung der Geschäftsleitung für eine institutionelle Ausgestaltungsform der eigentliche Implementierungsprozeß. Dieses muß jedoch nicht allgemeingültig sein. Wenn die herstellerseitige Entscheidung darüber, daß ein Rücknahme- und Recyclingsystem durchgeführt wird, nicht zeitgleich mit der Entscheidung über die institutionelle Ausgestaltungsform getroffen wird, fängt der Implementierungsprozeß bereits mit der Entscheidung über die Durchführung an. Damit wäre die institutionelle Ausgestaltungsform clusterbeschreibendes Merkmal und Einflußfaktor zugleich. In der vorliegenden Arbeit wird sie als Einflußfaktor analysiert, da sie in jedem Fall einen Einfluß auf den Implementierungsprozeß hat, wenn z.T. auch erst in späteren Phasen. Darüber hinaus sind Wechselwirkungen beim Entstehen von Netzwerken mit den unternehmensbezogenen Variablen zu berücksichtigen. Diese Problematik wird jedoch ausgeklammert, da sich die vorliegende Untersuchung auf die Implementierungsaktivitäten der Hersteller konzentriert und damit Aktivitäten der Netzwerkpartner einer besonderen Analyse bedürfen.

$\mathrm{Zu}$ einer Bewertung der verschiedenen Institutionelle Ausgestaltungsformen eines Rücknahme- und Recyclingsystems anhand von verbraucherseitigen Kriterien (Bequemlichkeit, Anpassungsfähigkeit, Sicherheit, Wahrnehmbarkeit der Effektivität) und kanalspezifischen Entscheidungskriterien (Umfang und Qualität der Funktionswahrnehmung, Beeinflußbarkeit, kommunikative Nutzbarkeit, Image des Redistributionsweges) vgl. Raabe, T., Die Elektronik-SchrottVerordnung, a.a.O., S. $298 \mathrm{ff}$.

407 Vgl. Sydow, J., Unternehmensnetzwerke, a.a.O., S. 160 und vgl. Sydow, J., Netzwerkorganisation, a.a.O., S. 630 .

408 Vgl. Meffert, H., Netzer, F., Formen strategischer Netzwerke und Implikationen für das Marketing, in: Meffert, H., Wagner, H., Backhaus, K. (Hrsg.), Arbeitspapier Nr. 109 der Wissenschaftlichen Gesellschaft für Marketing und Unternehmensführung e.V., Münster 1997, S. 2.

Nach Rasche sichern Kernkompetenzen die Überlebensfähigkeit der Unternehmen im Wettbewerb. Vgl. Rasche, Chr., Wettbewerbsyorteile durch Kernkompetenzen; ein ressourcenoriIrene Giesen'-Netzer and Universitat Münster - 978-3-631-75058-2 
$\mathrm{H}_{\mathrm{SDE} 1}$ : Die institutionelle Ausgestaltungsform liefert einen Erklärungsbeitrag für das Implementierungsverhalten der Hersteller, d.h. bei den verschiedenen Implementierungstypen sind signifikant unterschiedlich häufig bestimmte institutionelle Ausgestaltungsformen vorzufinden.

Bezüglich des Einflusses der institutionellen Ausgestaltungsform des Rücknahmeund Recyclingsystems zeigt die Kontingenzanalyse in Abbildung 23, daß knapp $20 \%$ der Hersteller ein eigenes Rücknahme- und Recyclingsystem organisieren, bei dem sie den Großteil der Aktivitäten selbst übernehmen, ca. 15\% der befragten Hersteller einen Vertrag mit Verwertern abschließen und $65 \%$ der Hersteller sich an kooperativen Systemen beteiligen. Hinsichtlich der Unterschiede der Implementierungstypen liegen gemäß des Chi-Quadrat-Tests (mit einer Irrtumswahrscheinlichkeit von unter $5 \%$ ) signifikant unterschiedliche Typen vor, so daß die Basishypothese $\boldsymbol{H}_{\text {SDE1 }}$ bestätigt werden kann.

$42,1 \%$ derjenigen Hersteller, die ein herstellereigenes Rücknahme- und Recyclingsystem wählen, lassen sich den Frühen integrierten Internalisierern zu5ordnen, während sie nur $20 \%$ der marktnahen Lösung und $28,1 \%$ der kooperativen Systeme stellen. Damit liegt die Präferenz eigener Systeme 12,63\% über dem statistisch zu erwartenden Wert. Dieser Befund hebt die Bedeutung der unterschiedlichen Ausgestaltung der Implementierungstypen hinsichtlich der Intensitätsdimensionen in den Implementierungsphasen für die Wahl der institutionellen Ausgestaltungsform hervor. Bei der Implementierung eines herstellereigenen Systems scheint es demnach notwendig zu sein, sowohl in der Einführungs- als auch in der Realisationsphase viele Abteilungen intensiv einzubeziehen. Auch die als integriertes Verhalten gekennzeichnete Implementierung in den Hierarchiedimensionen sowie der lange Zeitbedarf und der frühe Zeitpunkt der Implementierung von Rücknahme- und Recyclingsystemen bei den Frühen integrierten Inter-

entierter Ansatz, Wiesbaden 1994, S. 149. Kernkompetenzen stellen dabei Kompetenzen dar, die durch ein Zusammenwirken von Routinen, Fähigkeiten und tangiblen Aktivposten entstehen, wobei diese extrem personengebunden sind. Vgl. ebenda, S. 145.

Vgl. Sydow, J., Unternehmensnetzwerke, a.a.O., S. 160.

Meffert und Netzer sehen die strategische Allianz, die als formalisierte, längerfristige Beziehung zu anderen Unternehmen definiert wird, mit dem Ziel der Kompensation eigener Schwächen durch Stärkenpotentiale anderer Organisationen, um die Wettbewerbsposition der Unternehmung oder der Allianzpartner langfristig zu sichern bzw. auszubauen, als einen möglichen Entstehungsgrund strategischer Netzwerke. Vgl. Meffert, H., Netzer, F., Formen strategischer Netzwerke und Implikationen für das Marketing, a.a.O., S. 3f. Nach diesem Verständnis ist ein strategisches Netzwerk die institutionelle Ausgestaltungsform und die Steuerung einer strategischen Allianz. 
nalisierern lassen es plausibel erscheinen, daß die institutionelle Ausgestaltungsform des herstellereigenen Systems bevorzugt wird.

\begin{tabular}{|c|c|c|c|c|c|c|}
\hline & $\begin{array}{l}\text { Implement.- } \\
\text { typen }\end{array}$ & Cluster I & Cluster II & Cluster III & Cluster IV & Cluster V \\
\hline $\begin{array}{l}\text { Anteil } \\
\text { der } \\
\text { Stich- } \\
\text { probe } \\
\text { in } \%\end{array}$ & $\begin{array}{c}x_{i}=\text { tatsächlicher } \\
\text { Wert } \\
\left(x_{i}\right)=\text { erwarteter } \\
\text { Wert } \\
{\left[x_{i}\right]=\text { Abweichung }}\end{array}$ & $\begin{array}{l}\text { Experten- } \\
\text { orientierte } \\
\text { Implemen- } \\
\text { tierungs- } \\
\text { minimalisten }\end{array}$ & $\begin{array}{l}\text { Späte pro- } \\
\text { motoren- } \\
\text { orientierte } \\
\text { Externa- } \\
\text { lisierer }\end{array}$ & $\begin{array}{l}\text { Partizipativ- } \\
\text { späte Im- } \\
\text { plemen- } \\
\text { tierer }\end{array}$ & $\begin{array}{l}\text { Autokra- } \\
\text { tische } \\
\text { Ad hoc- } \\
\text { Externa- } \\
\text { lisierer }\end{array}$ & $\begin{array}{c}\text { Frühe } \\
\text { integrierte } \\
\text { Interna- } \\
\text { lisierer }\end{array}$ \\
\hline \multirow[t]{2}{*}{$19,5 \%$} & \multirow[t]{2}{*}{ Eigenes System } & $\begin{array}{c}15,8 \% \\
(24,75 \%) \\
{[-8,95 \%]}\end{array}$ & $\begin{array}{c}15,8 \% \\
(17,38 \%) \\
{[-1,58 \%]}\end{array}$ & $\begin{array}{c}10,5 \% \\
(17,33 \%) \\
{[-6,83 \%]}\end{array}$ & $\begin{array}{c}15,8 \% \\
(11,6 \%) \\
{[+4,74]}\end{array}$ & $\begin{array}{c}42,1 \% \\
(29,47 \%) \\
{[+12,63]}\end{array}$ \\
\hline & & - & 0 & - & 0 & ++ \\
\hline \multirow[t]{2}{*}{$15,5 \%$} & \multirow[t]{2}{*}{$\begin{array}{l}\text { Vertrag mit Ver- } \\
\text { wertern }\end{array}$} & $\begin{array}{c}26,7 \% \\
(24,7 \%) \\
{[+2 \%]}\end{array}$ & $\begin{array}{c}40 \% \\
(17,33 \%) \\
{[+22,67 \%]}\end{array}$ & $\begin{array}{c}13,3 \% \\
(5,76 \%) \\
{[+7,54 \%]}\end{array}$ & $\begin{array}{c}0 \% \\
(25,54 \%) \\
{[-25,54]}\end{array}$ & $\begin{array}{c}20 \% \\
(26,67 \%) \\
{[-6,67]}\end{array}$ \\
\hline & & 0 & +++ & + & -.- & - \\
\hline \multirow[t]{2}{*}{$65 \%$} & \multirow[t]{2}{*}{$\begin{array}{l}\text { Kooperatives } \\
\text { System }^{410}\end{array}$} & $\begin{array}{c}26,6 \% \\
(24,57 \%) \\
{[+2,03 \%]}\end{array}$ & $\begin{array}{c}12,5 \% \\
(17,33 \%) \\
{[-4,84 \%]}\end{array}$ & $\begin{array}{c}20,3 \% \\
(17,33 \%) \\
{[+2,97 \%]}\end{array}$ & $\begin{array}{c}12,5 \% \\
(11,25 \%) \\
{[+1,25]}\end{array}$ & $\begin{array}{c}28,1 \% \\
(29,51 \%) \\
{[-1,41]}\end{array}$ \\
\hline & & 0 & 0 & 0 & 0 & 0 \\
\hline \multirow[t]{2}{*}{$\begin{array}{c}\text { davon } \\
7,8 \%\end{array}$} & \multirow[t]{2}{*}{$\begin{array}{l}\text { Branchen- } \\
\text { kooperationen }\end{array}$} & $\begin{array}{c}0 \% \\
(34,23 \%) \\
{[-34,23 \%]}\end{array}$ & $\begin{array}{c}0 \% \\
(25,71 \%) \\
{[-25,71 \%]}\end{array}$ & $\begin{array}{l}20 \% \\
(18 \%) \\
{[+2 \%]}\end{array}$ & $\begin{array}{l}60 \% \\
(12 \%) \\
{[+48 \%]}\end{array}$ & $\begin{array}{l}20 \% \\
(30 \%) \\
{[-10 \%]}\end{array}$ \\
\hline & & -- & -- & 0 & +++ & - \\
\hline \multirow[t]{2}{*}{$\begin{array}{l}\text { davon } \\
16,2 \%\end{array}$} & \multirow[t]{2}{*}{$\begin{array}{l}\text { Gemeinsame } \\
\text { Entwicklung eines } \\
\text { Systems mit Ver- } \\
\text { wertern }\end{array}$} & $\begin{array}{l}63,6 \% \\
(24,53 \%) \\
{[+39,07 \%]}\end{array}$ & $\begin{array}{l}(17,23 \%) \\
{[-17,23 \%]}\end{array}$ & $\begin{array}{c}9,1 \% \\
(17,29 \%) \\
{[-8,91 \%]}\end{array}$ & $\begin{array}{c}9,1 \% \\
(10,92 \%) \\
{[-1,82 \%]}\end{array}$ & $\begin{array}{c}18, \overline{2} \% \\
(30,03 \%) \\
{[-11,83]}\end{array}$ \\
\hline & & +++ & -- & - & 0 & -- \\
\hline
\end{tabular}

\begin{tabular}{|c|c|c|c|c|c|c|}
\hline $\begin{array}{l}\text { Chi-Quadrat- } \\
\text { Test gemäß: }\end{array}$ & $\begin{array}{l}\text { Signifikanz- } \\
\text { niveau }\end{array}$ & $x_{i}-\left(x_{i}\right)$ & $0 \%-5 \%$ & $5,1 \%-10 \%$ & $\begin{array}{c}10,1 \%- \\
20 \%\end{array}$ & $>20 \%$ \\
\hline $\begin{array}{l}\text { Pearson } \\
\text { Likelihood Ratio }\end{array}$ & $\begin{array}{l}0,0165 \\
0,0342\end{array}$ & $\begin{array}{l}\text { positiv } \\
\text { negativ }\end{array}$ & $\begin{array}{l}0 \\
0\end{array}$ & + & $\begin{array}{c}++ \\
--\end{array}$ & $\begin{array}{c}+++ \\
---\end{array}$ \\
\hline
\end{tabular}

Abb. 23: Zusammenhang zwischen institutionellen Ausgestaltungsformen des Rücknahme- und Recyclingsystems und Implementierungstypen

Der hohe Stellenwert der institutionellen Ausgestaltungsform für die Ausprägung der Intensitätsdimensionen geht auch aus dem Ergebnis bei den Branchenko-

410 Bei der Kreuztabellierung wurde die institutionelle Ausgestaltungsform des Gemeinschaftsunternehmens den Frühen integrierten Internalisierern zugeordnet. 
operationen hervor, nach dem sich $60 \%$ der als Branchenkooperation identifizierten Systeme bei den Autokratischen Ad hoc-Externalisierern finden lassen. Dieser Wert liegt $48 \%$ über dem erwarteten statistischen Wert.

Die Autokratischen Ad hoc-Externalisierer lagern Aktivitäten insbesondere in der Realisationsphase aus und ziehen auch in der Einführungsphase leicht unterdurchschnittlich wenige Abteilungen intensiv in die Implementierung des Rücknahme- und Recyclingsystems ein. Dieses Vorgehen ist offensichtlich bei der Wahl einer Branchenkooperation möglich, da sich Pioniere einer Branche bei einer Branchenkooperation zunächst zu Anfang relativ intensiv mit der Konzeptionierung des Systems auseinandersetzen, um ihre eigenen Vorstellungen einzubringen und um in der anschließenden Realisationsphase Aufgaben zu externalisieren (Intensitätsdimension).

Zudem ist beim Implementierungsverhalten der Autokratischen Ad hocExternalisierer die Verantwortungsübernahme auf der Geschäftsleitungsebene sowie die Ernennung von Fachexperten im Unternehmen hoch ausgeprägt. Diese Konstellation erscheint bei einer Kooperation zwischen Wettbewerbern aufgrund der Sensitivität der ausgetauschten Informationen konsistent (Hierarchiedimension). ${ }^{411}$ Bezüglich des Timing der Implementierung eines Rücknahme- und Recyclingsystems in Form einer Branchenlösung ist augenscheinlich ein recht früher Anfangszeitpunkt notwendig, wenn die Herstellerunternehmen bei der Konzeptionierung des Systems ihren Einfluß geltend machen wollen. ${ }^{412}$

Bei den späten promotorenorientierten Externalisierern werden Rücknahmeund Recyclingsysteme am häufigsten in Form von Verträgen mit Verwertern im-

411 Stölzle und Jung führen aus, daß das Ziel der Hersteller bei der Gestaltung von Branchenkooperationen eine Beeinflussung der Wettbewerbsbedingungen einer Branche ist, um die eigene Wettbewerbsfähigkeit zu halten bzw. zu steigern. Vgl. Stölzle, W., Jung, K. P., Strategische Optionen der Entsorgungslogistik zur Realisierung von Kreislaufwirtschaftskonzepten, a.a.O., S. 34f. Darüber hinaus reichen die Ziele solcher Kooperationen von der Abwehr einer Rücknahmeverordnung bis hin zur Schaffung und nachhaltigen Sicherung neuer Geschäftsfelder. Vgl. ebenda, S. $35 f$.

Schneidewind hebt hervor, daß eine horizontale marktliche Kooperation (wie die Branchenkooperation) nicht darauf abzielt, eine möglichst effiziente Koordination von Akteuren innerhalb gegebener marktlicher Rahmenbedingungen zu erreichen (wie eine vertikale marktliche Kooperation), sondern auf die Gestaltung eben dieser Rahmenbedingungen. Vgl. Schneidewind, U., Ökologisch orientierte Kooperationen aus betriebswirtschaftlicher Sicht, a.a.O., S. 18.

412 Einschränkend dazu muß berücksichtigt werden, daß sich Branchenkooperationen auch noch im letzten Moment bilden können. Jedoch wird es dann sinnvoll sein, zum frühen Zeitpunkt Verbündete bzw. Systempartner zu suchen, die gleich stark betroffen sind. 
plementiert (Abweichung von 22,6\% vom erwarteten statistischen Wert). Mit der Wahl dieser marktnahen Ausgestaltungsform kann der Hersteller i.d.R. in der Einführungsphase seiner Implementierung auf ein bestehendes Konzept zurückgreifen, welches inm der Verwerter anbietet. ${ }^{413}$ Dieses wird in der recht schwachen Ausprägung der Intensitätsdimension in der Einführungsphase bei den späten promotorenorientierten Externalisieren deutlich. Darüber hinaus kann der Hersteller durch diese Art der Externalisierung einen Verwerter als externen Fachexperten mit dem sehr spezifischen Problem der Rücknahme und des Recycling der Produkte beauftragen und dadurch das eigene Unternehmens-Kow-how entlasten (Hierarchiedimension). Auch ist es ihm durch die Wahl einer Vertragslösung scheinbar möglich, relativ spät mit der Implementierung des Rücknahme- und Recyclingsystems zu beginnen, da er eine Lösung „zukauft" (Zeitdimension). ${ }^{414}$

Die gemeinsame Entwicklung eines Systems mit Verwertern kommt mit 63,6\% am häufigsten bei den Expertenorientierten Implementierungsminimalisten vor. Dieses Verhalten kann insbesondere mit der Interventionsebene dieses Implementierungstyps begründet werden, da dort die Ernennung eines Fachexperten in Verbindung mit der Verantwortungsübernahme auf der Geschäftsleitungsebene die wichtigste Maßnahme darstellt. Neben dieser internen Ausrichtung kommt in der gemeinsamen Entwicklung mit Verwertern eine externe, expertenorientierte Vorgehensweise bei der Implementierung zum Ausdruck. ${ }^{415}$

413 Die Möglichkeit der Übertragung der ordnungsgemäßen Entsorgung an Dritte ist ausdrücklich im Kreislaufwirtschaftsgesetz vorgesehen und umfaßt neben der Vertragslösung alle anderen Kooperationsformen. Vgl. §16 Abs. $1 \mathrm{KrW}$-/AbfG.

Die gemeinsame Entwicklung eines Konzeptes zwischen Herstellern und Verwertern wird als kooperative institutionelle Ausgestaltungsform aufgefaßt.

414 Die institutionelle Ausgestaltungsform des Vertrages als marktliche Koordinationsform ermöglicht neben den o.g. Zielen (aufgrund der genau kalkulierbaren Kosten und der Vergabe des Auftrags an den günstigsten Verwerter) einen Beitrag zur Kostenminimierung und kann damit eine angestrebte Kostenführerschaft als Wettbewerbsstrategie unterstützen. Vgl. Stölzle, W., Jung, K. P., Strategische Optionen der Entsorgungslogistik zur Realisierung von Kreislaufwirtschaftskonzepten, a.a.O., S. 35.

415 Neben den beschriebenen institutionellen Ausgestaltungsformen von Rücknahme- und Recyclingsystemen wird in der Literatur eine Vielzahl von Merkmalen bei der Gestaltung von interorganisationalen Beziehungen untersucht. Die Diskussion geht dabei von den Ansätzen der Organisations- und Netzwerktheorie aus, nach denen das Verhalten der einzelnen beteiligten Unternehmen nach Form und Inhalt der Austauschbeziehungen untersucht wird. Dabei können strukturelle, koordinationsbezogene und kulturelle Merkmale unterschieden werden. Vgl. zu einer detaillierteren Aufbereitung der Merkmale Sydow, J., Strategische Netzwerke, a.a.O., S. 83ff. In der vorliegenden Arbeit werden diese Merkmale nicht als Einflußfaktoren auf das Implementierungsverhalten untersucht, da sie sich auf die institutionellen Ausgestaltungsformen zurückführen lassen. Vgl. auch die Analyse des Zusammenhangs zwischen institutionellen Ausgestaltungsformen und Merkmalen von Rücknahme- und Recyclingsystemen im Anhang 1.6. 
Einen weiteren wichtigen Einflußfaktor auf das Implementierungsverhalten stellt die herstellerseitige Einbringung von Ressourcen bei der Gestaltung und Implementierung eines Rücknahme- und Recyclingsystems dar, aus der die Verflechtung des Herstellers zu den Systempartnern deutlich wird. Die Bedeutung dieses Faktors wird aus dem Resource-Dependence-Ansatz deutlich, der die Entwicklung, Vermeidung und Ausnutzung von Abhängigkeiten, die aus der Ressourcenakquisition von anderen Organisationen entstehen, in den Mittelpunkt der Betrachtung stellt. ${ }^{416}$ Demnach sind die Unternehmen bestrebt, ihre eigene Abhängigkeit gering zu halten und damit das interorganisationale Machtverhältnis zu ihren Gunsten zu stabilisieren. ${ }^{417}$ Daraus folgt, daß mit zunehmender herstellerseitiger Ressourceneinbringung eine (Quasi)Internalisierung von Funktionen des Rücknahme- und Recyclingsystems einher geht. ${ }^{418}$ Vor diesem Hintergrund ist folgende Basishypothese zu formulieren:

\begin{tabular}{|c|c|}
\hline $\mathrm{H}_{\text {SDE2 }}:$ & $\begin{array}{l}\text { Die entstehenden Abhängigkeiten durch unterschiedliche Ressourceneinbringungen } \\
\text { seitens der Hersteller liefern einen Erklärungsbeitrag für die Bildung der Implementierung- } \\
\text { stypen. }\end{array}$ \\
\hline
\end{tabular}

Für die Analyse der Ressourceneinbringung der Hersteller in das jeweilige Rücknahme- und Recyclingsystem stehen 13 Variablen, die aus der Abbildung 24

416 Zu den Annahmen des Resource-Dependence-Ansatzes vgl. Gils, M. R. van, Interorganisationational relations and networks, in: Drenth, P. J. D., u.a. (Hrsg.), Handbook of work and organisational psychology, Chichester u.a. 1984, S. 1081.

Die Unternehmen können die Kontrolle über die externen Ressourcen verstärken und damit die Abhängigkeit gering halten, wenn sie den Unsicherheitsquellen entweder proaktiv begegnen (z.B. Unternehmensakquisition) oder die Unsicherheit durch Kooperation reduzieren. Des weiteren können sie versuchen, ihrerseits Abhängigkeiten zu schaffen. Vgl. zu den genauer diskutierten Möglichkeiten im Rahmen des Resource-Dependence-Ansatzes Sydow, J., Strategische Netzwerke, a.a.O., S. 196ff. und die dort angegebene Literatur.

Vgl. Sydow, J., Netzwerkorganisation, a.a.O., S. 199.

Es wird in der Literatur kritisch diskutiert, ob der Resource Dependence-Ansatz in der Lage ist. Funktionsexternalisierungen und -internalisierungen zu erkären. Hesterley, Liebeskind und Zenger gehen z.B. davon aus, daß dieser Ansatz nur die Entstehung von Hierarchien aus zuvor marktlichen Beziehungen erklären kann, da mit der (Quasi)Externalisierung Macht eingebüßt wird. Vgl. Hesterley, W. S., Liebeskind, J., Zenger, T. R., Organisational economics: An impending revolution in organization theory?, in: Academy of Management Review, 1990, S. 407. Sydow hält dem entgegen, daß auch mit der (Quasi)Externalisierung Machtziele verfolgt werden können. Vgl. Sydow, J., Strategische Netzwerke, a.a.O., S. 199. Der Argumentation von Sydow kann m.E. gefolgt werden, da z.B. Branchenkooperationen auch eingegangen werden, um den Machtbereich des eigenen Unternehmens auf die Politik (Lobbyismus) auszudehnen, zumal bei einer Branchenkooperation die Funktionsausgliederung mit anschließender Funktionswahrnehmung durch eine Netzwerkunternehmung stattfindet und der Einflußbereich des Herstellers sich dadurch erweitert. 
ersichtlich werden, zur Verfügung. ${ }^{419}$ Die Betrachtung der F-Werte führt zu dem Ergebnis, daß sich bei einem angenommenen Signifikanzniveau von $10 \% 12$ der 13 Variablen hinsichtlich der globalen Beurteilung der Mittelwerte unterscheiden. ${ }^{420}$ Nur bei der Variablen der herstellerseitigen Einbringung der Informationssysteme muß eine signifikante Unterscheidung abgelehnt werden. Damit kann die Hypothese $\mathrm{H}_{S D 2}$ angenommen werden.

Entsprechend des Ressource-Dependence-Ansatzes, der bei hoher Ressourceneinbringung eine hohe Ausprägung der Intensitätsdimension unterstellt, stellen die Frühen integrierten Internalisierer viele Ressourcen bei Implementierung des Rücknahme- und Recyclingsystems zur Verfügung. Auch die multiplen Mittelwertvergleichstests zeigen, daß sich dieses Cluster hinsichtlich aller Variablen nahezu allen anderen Clustern gegenüber signifikant unterscheidet.

Bei den Späten promotorenorientierten Externalisierern und bei den Autokratischen Ad hoc-Externalisierern hingegen erscheint eine phasenspezifisch differenzierte Betrachtung der Externalisierungsaktivitäten notwendig. ${ }^{421}$ Wird eine Unterteilung der Variablen nach den Phasen der Implementierung vorgenommen, können die Variablen Räumliche Kapazitäten (5), Annahmestellen für Altprodukte (6), Rückführungslogistik (7), Lagerhaltungsflächen (8), Demontage-Know-how (10), Know-how für stoffliches Recycling (11) und der Zugang zu Sekundärrohstoffmärkten (12) der Realisationsphase zugeordnet werden. Diese Zuordnung vollzieht sich mit der Begründung, daß die o.g. Ressourcen erst dann einzubringen sind, wenn erste Altprodukte in den Einflußbereich der Hersteller bzw. des Rücknahme- und Recyclingsystems zurückgelangen. Ein Vergleich zwischen den beiden Clustern hinsichtlich der Ressourceneinbringung zeigt, daß lediglich bei den Variablen 6, 7, 10 und 12 die Autokratischen Ad hoc-Externalisierer in der Realisationsphase weniger Ressourcen einbringen als die Späten promotorenori-

419 Bei der Betrachtung des Mittelwertprofils fällt auf, daß insbesondere das Demontage-Knowhow (mit einem relativ großen Abstand von 0,3 Punkten zur nächsten Variable Öffentlichkeitsarbeit) diejenige Ressource ist, die alle Hersteller am meisten bei der Implementierung eines Rücknahme- und Recyclingsystems einbringen. Aufgrund der Kenntnis des eigenen Produktes, dessen Materialzusammensetzung, dessen Verbindungen u.s.w. ist diese Aufgabe von dem Hersteller relativ einfach zu erfüllen. Variablen signifikant unterschiedliche Mittelwerte festgestellt werden. onsphase an Dritte ab, während die Späten Promotorenorientierten Externalisierer überwiegend Aufgaben in der Planungsphase auslagern. 
entierten Externalisierer. ${ }^{422}$ Eine Erklärung dieses Ergebnisses ist dementsprechend nicht auf Grundlage der Externalisierungsaktivitäten möglich. Eine Ursache könnte in der ungenauen Zuordenbarkeit der Variablen zu den verschiedenen Implementierungsphasen liegen. ${ }^{423}$

Die Expertenorientierten Implementierungsminimalisten bringen insgesamt über alle Variablen hinweg am wenigsten Ressourcen ein. Auch der multiple Mittelwertvergleichstest zeigt, daß sich dieses Cluster hinsichtlich fast aller Variablen signifikant gegenüber den Frühen integrierten Internalisierern und hinsichtlich einiger Variablen gegenüber den anderen Clustern unterscheidet. Dieses kann möglicherweise mit der Grundhaltung der Expertenorientierten Implementierungsminimalisten erklärt werden, die sich u.a. in der unterdurchschnittlichen Ausprägung der Partizipation der Mitarbeiter und in geringen Ausprägungen des Aktivitätsniveaus auf der Interventionsebene zeigt. Dieser Befund deutet darauf hin, daß Expertenorientierte Implementierungsminimalisten generell ein möglichst geringes Aktivitätsniveau anstreben, d.h. sie wollen in Relation zu den anderen Clustern weder viele Aufgaben externalisieren noch internalisieren, sondern verfolgen das Ziel, mit möglichst geringen Ressourcen ein Rücknahme- und Recyclingsystem zu implementieren.

422 Bei der Variablen 8, die die herstellerseitige Einbringung der Lagerhaltungsflächen zum Gegenstand hat, liegen beide betrachteten Cluster im Stichprobenmittelwert, d.h. bei ca. 3,5. Bei Variable 12 hingegen, die das Demontage Know-how der Hersteller umfaßt, bringen die Autokratischen Ad hoc-Externalisierer signifikant weniger $(\alpha<0,05)$ Ressourcen ein als die Späten promotorenorientierten Externalisierer. 


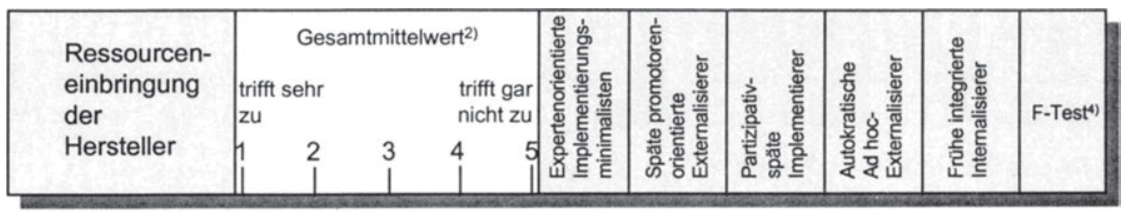

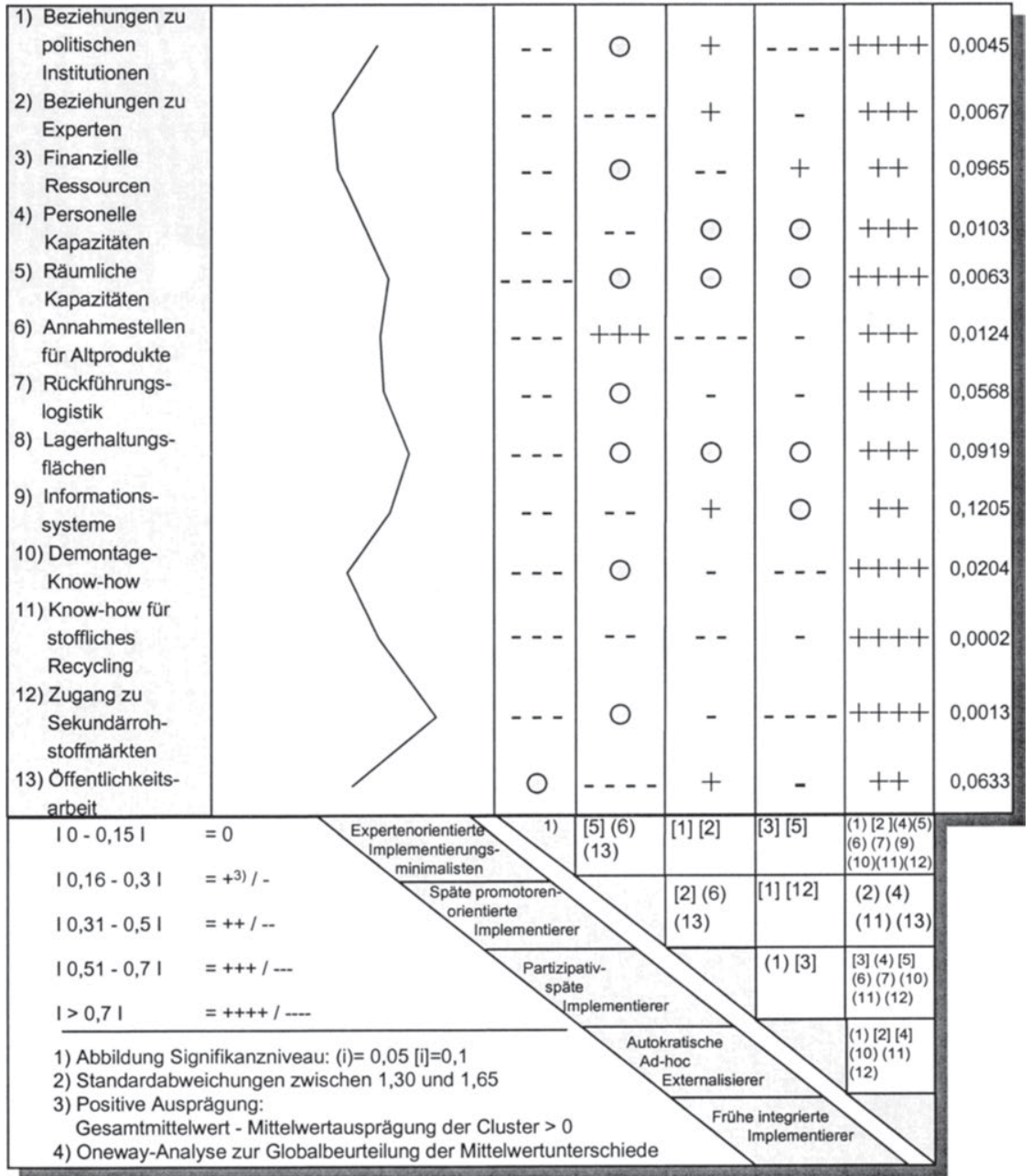
Abb. 24: Zusammenhang zwischen der herstellerseitigen Einbringung von Ressourcen in
das Rücknahme- und Recyclingsystem und Implementierungstypen 


\subsection{Einflußfaktoren der Umsetzung von Rücknahme- und Recyclingsy- stemen auf Unternehmensebene}

\subsubsection{Aufgabenspezifische Einflußfaktoren}

\subsubsection{Markt- und Wettbewerbssituation}

Die erste Gruppe der externen unternehmensbezogenen Einflußfaktoren bildet die Markt- und Wettbewerbssituation. Hier läßt sich eine Vielzahl von Untersuchungen im Rahmen der Organisationstheorie nachweisen, die unterschiedliche Variablen als Einflußfaktoren auf die Unternehmensstruktur und das -verhalten analysiert. Dabei werden insbesondere die Umweltunsicherheit (Veränderlichkeit der Situation), die Heterogenität (Anzahl und Verschiedenartigkeit der relevanten Marktteilnehmer) und die Feindlichkeit (Wettbewerbsintensität und Marktattraktivität) der Umwelt als Variablen der Markt- und Wettbewerbssituation analysiert. ${ }^{424}$

Eine verstärkte Betrachtung soll die Umweltheterogenität erfahren, da das Verhalten der Marktteilnehmer, d.h. der Kunden, des Handels und der Konkurrenz sowie das Verhalten von anderen Anspruchsgruppen wie beispielsweise Lieferanten auf den Beschaffungsmärkten, in enger Beziehung zu den umweltbezogenen Tätigkeiten der Hersteller steht. ${ }^{425}$ Die Marktteilnehmer üben als Elemente des Gesamtsystems „Rücknahme- und Recyclingsystem“ mit ihren individuellen Zielfunktionen wesentlichen Einfluß auf die Gestaltung desselben aus. ${ }^{426}$ Daher wer-

424 Vgl. z. B. Miller, D., Friesen, P. H., Momentum and Revolution in Organisational Adaptation, in: Academy of Management Journal, Nr. 4, 1980, S. 591ff. oder Kieser, A., Kubicek, H., Organisation, a.a.O., S. 371.

Auch in der vorliegenden Arbeit wird auf diese Größen zurückgegriffen, wobei die Umweltunsicherheit nicht in die Betrachtung einbezogen wird, da die Implementierung von Rücknahmeund Recyclingsystemen ein relativ neues herstellerspezifisches Problem darstellt und die organisationalen Reaktionen auf Umweltveränderungen daher zum gegenwärtigen Entwicklungsstand schwer meßbar sind. Dies wurde insbesondere in den Vorgesprächen zur empirischen Untersuchung deutlich. Die Meinungen der befragten Experten ließen eine Verstärkung der ohnehin hohen Unsicherheit erkennen, sofern nach dem Einfluß der Umweltdynamik gefragt wurde. Zu Entscheidungen unter Unsicherheit vgl. Adam, D., Planung und Entscheidung: Modelle - Ziele - Methoden, mit Fallstudien und Lösungen, 4., vollst. überarb. u. erw. Aufl., Wiesbaden 1996 sowie Bamberg, G., Coenenberg, A. G., Betriebswirtschaftliche Entscheidungslehre, 9. überarb. Aufl., München 1996.

Vgl. Kirchgeorg, M., Okologieorientiertes Unternehmensverhalten, a.a.O., S. $75 \mathrm{ff}$.

Beispielhafte Ziele sind hier: 1. Kundenziele: Bequemlichkeit des Rückgabevorganges, hohe Qualität von Produkten mit Sekundärrohstoffen bzw. von Gebrauchtprodukten, 2. Handelsziele: Nutzung von Rücknahme- und Recyclingsystemen zur Profilierung, Minimierung des zeitlichen Aufwandes und der Kosten, 3. Lieferantenziele: Verhinderung einer Konkurrenz durch „Sekundär"produkt(teil)e, 4. Wettbewerbsziele: Einführung eines auf die eigenen Strukturen abgestimmten Rücknahme- und Recyclingsystems. 
den bei der Analyse der Absatzmarktsituation insbesondere das umweltbezogene Marktteilnehmerverhalten und die Existenz von Gebrauchtmärkten für die Altprodukte zu untersuchen sein. Zusätzlich wird die Beschaffungsmarktsituation mit den Teilaspekten "Bindungsintensität zwischen Herstellern und Zulieferern“, "Art der Vorprodukte" sowie „Auswahlmöglichkeiten aus Herstellersicht" analysiert.

Die „Feindlichkeit" der Umwelt soll im Rahmen der vorliegenden Arbeit durch das Marktstadium Berücksichtigung finden. ${ }^{427}$ Während in jungen Wachstumsmärkten aufgrund einer „Aufbruchstimmung" aufgeschlossenes umweltbezogenes Unternehmensverhalten $z u$ enwarten ist, ${ }^{428}$ wird in reifen (feindlichen) Märkten weniger Zeit für neue Problemlösungen aufgewendet und neuen Experimenten gegenüber eine gewisse Vorsicht bestehen ${ }^{429}$ Gleichzeitig findet in reifen Märkten eine Konzentration auf Kernkompetenzen bei der Gestaltung von Rücknahme- und Recyclingsystemen statt, bei der die gleichzeitige Externalisierung der Aufgaben wahrscheinlich ist. ${ }^{430}$ Übertragen auf den Kontext der Implementie-

Die generelle Bereitschaft des Kunden, die Altprodukte zu sammeln (Muß-Bedingung), zu sortieren und zurückzugeben (Kann-Bedingungen), ist beispielsweise eine wesentliche Voraussetzung der Implementierung eines Rücknahme- und Recyclingsystems. Vgl. Zikmund, W. G., Stanton, W. J., Recycling Solid Wastes: A Channels-of-distribution Problem, in: Journal of Marketing, Nr. 3, 1971, S. 35. Bei Leasinggeschäften ist die Rückgabe demgegenüber nicht von der Kundenbereitschaft abhängig, da kein Eigentumsübergang stattfindet. Die Kunden werden in diesem Zusammenhang als Prosumenten bezeichnet, da sie gleichzeitig Produzent und Konsument der betrachteten Güter sind. Dies geschieht mit der Begründung, daß die Kunden insbesondere während der Nutzungsphase Einfluß auf den Wert des späteren Altproduktes ausüben. Vgl. Kirchgeorg, M., Kreislaufwirtschaft, a.a.O., S. 18ff. Darüber hinaus sollte der Kunde nicht nur als Lieferant des Altproduktes betrachtet werden. Vielmehr kann eine derartige Verbindung zwischen Kunde und Hersteller nach dem Produktkauf als Chance zur Profilierung genutzt werden. Vgl. Hansen, U., Jeschke, K., Nachkaufmarketing, in: Marketing ZFP, Heft 2, 1992, S. 88.

Das Marktstadium beruht auf dem Marktlebenszyklus eines Produktes, der sich in Einführung, Wachstums-, Reife-, Stagnations- und Degenerationsphase aufgliedert. Die Phasen können Orientierungshilfen hinsichtlich der Beurteilung des Marktpotentials und der Verhaltensalternativen geben. Vgl. Müller, N., Marketingstrategien in High-Tech-Märkten: Typologisierung, Ausgestaltungsformen und Einflußfaktoren auf der Grundlage strategischer Gruppen, Frankfurt am Main u.a. 1995, S. 58ff. Zur genauen Beschreibung des Modells des Produktlebenszyklus und der kritischen Überprüfung der Anwendbarkeit vgl. Meffert, H., Marketing, a.a.O., S. 329ff. Dies dient auch der Absicherung langfristiger Erfolgspotentiale. Vgl. Kirchgeorg, M., Ökologieorientiertes Unternehmensverhalten, a.a.O., S. 83.

429 In reifen Märkten, die i.d.R. umkämpft werden und sich durch einen hohen Sättigungsgrad auszeichnen, scheiden diejenigen Wettbewerber aus, die keine Produkt- oder Kostenvorteile realisieren können. Häufig findet zudem eine Sortimentsbereinigung bei gleichzeitigen intensiven Rationalisierungsbestrebungen in den Produktions- und Distributionsprozessen statt. Vgl. Meffert, H., Marketing, a.a.O., S. 234f.

Aggressive Preiskonkurrenz, geringe Kapazitätsauslastung des Herstellers und schrumpfende Autragsbestände kennzeichnen stagnierende und schrumpfende Märkte. Die Hersteller reagieren oftmals mit Kostenmanagement, Rationalisierungen und Fixkostenabbau. Vgl. Tacke, W., Stagnation und Rezession aus der Sicht von Unternehmen, Auswirkungen, Entwicklungen, Probleme und Problemlösungen, in: Meffert. $H$. Wagner. ${ }_{\text {. }}$. (Hrsg $)$. Marktorientierte Führung 
rung von Rücknahme- und Recyclingsystemen implizieren diese Erkenntnisse je nach Implementierungstyp entweder eine hohe Wahrscheinlichkeit von kooperativen Lösungen. Oder zum anderen eine Internalisierung von Aufgaben beim Vorliegen von jungen Märkten.

Wird nun die Umweltheterogenität näher untersucht, können die Kunden die Gestaltung und Implementierung von Rücknahme- und Recyclingsystemen in zweierlei Weise unterstützen. ${ }^{431}$ Sie können zum einen eine Pull-Wirkung dadurch erzielen, daß sie Recyclingprodukte und -materialien kaufen. Eine weitere Pull(ähnliche) Wirkung erzeugen sie, indem sie sich aktiv an der Rückgabe von Altprodukten beteiligen. ${ }^{432}$ Können idealerweise beide Effekte festgestellt werden, liegt es auch im Interesse der Hersteller, sich über diesen umweltbezogenen Aspekt zu profilieren. ${ }^{433}$ In diesem Fall wäre eine Internalisierung der Rücknahme-

in Stagnation und Rezession, Arbeitspapier Nr. 10 der Wissenschaftlichen Gesellschaft für Marketing und Unternehmensführung e.V., Dokumentation des 5. Münsteraner Führungsgesprächs, Münster 1983, S. 39ff. Zu ähnlichen Ergebnissen kommen Laakmann und Müller, die 1993375 westdeutsche Unternehmen befragten. Zusätzlich - neben Kostensenkungs- und Rationalisierungsmaßnahmen - geben die Hersteller an, Qualitätsverbesserungen, eine Intensivierung der Innovationstätigkeit sowie Kundenbindungsaktivitäten als wirksame Maßnahmen zur Bewältigung der Rezession zu ergreifen. Vgl. Laakmann, K., Müller, N., Umfrageergebnisse zu Zielen, Strategien und Maßnahmen deutscher Unternehmen in der Rezession, in: Meffert, H., Wagner, H., Backhaus, K. (Hrsg.), Marketing in der Rezession, Arbeitspapier Nr. 82 der Wissenschaftlichen Gesellschaft für Marketing und Unternehmensführung e.V., Dokumentation des Workshops vom 17. Juni 1993, Münster 1993, S. $37 \mathrm{ff}$.

Hinsichtlich umweltbezogener Maßnahmenschwerpunkte erläutert Meffert, daß in stark umkämpften Märkten die Umweltorientierung durchaus auf eine Bewährungsprobe gestellt wird, wobei insbesondere bei solchen Unternehmen Gefahr besteht, umweltbezogene Maßnahmen einzustellen, bei denen der Umweltschutz noch in den „Kinderschuhen“ steckt und noch nicht als Bestandteil der Unternehmenskultur gelebt wird. Vgl. Meffert, H., Erfolgreiches Marketing in der Rezession: Strategien und Maßnahmen in engeren Märkten; mit Erkenntnissen aus einer umfassenden Untersuchung, Wien 1994, S. 215ff.

Grundsätzlich stehen den Kunden drei Strategien zur Verfügung, um ihre (umweltbezogenen) Ziele durchzusetzen: Abwanderung, Widerspruch und Verhandlungen. Vgl. zu den Auswirkungen dieser Optionen auf die Hersteller Corsten, H., Götzelmann, F., Das Kooperationsprinzip in umweltpolitischer und unternehmerischer Sicht, a.a.O., S. 377.

432 Beim Pull-Konzept wird der Kunde direkt (ohne die Zwischenschaltung des Handels) durch den Hersteller angesprochen. Die aktive Nachfrage des Kunden beim Handel führt dazu, daß auch der Handel entsprechend der Zielsetzung der Hersteller agiert, d.h. Argumente für die Einführung von Rücknahme- und Recyclingsystemen erhält. Beim Push-Konzept wird hingegen der Handel direkt vom Hersteller durch bestimmte Anreize angesprochen, um der herstellerbezogenen Zielsetzung entsprechend zu handeln. Vgl. zur genauen Analyse von Push und Pull als Ausprägungsformen von Markendurchsetzungsstrategien Szeliga, M., Push und Pull in der Markenpolitik: ein Beitrag zur modellgestützten Marketingplanung am Beispiel des Reifenmarktes, Frankfurt am Main u.a. 1995, S. 14ff.

433 Eine konsumentenbezogene Profilierung des Herstellers kann durch die Art der gewählten Rücknahme beeinflußt werden. Im Elektronikschrott-Bereich bietet die direkte Rücknahme durch den Hersteller die größte Chance zur Profilierung, da bei einer Rücknahme durch den Handel oder Verwerter die Durchsetzung strategischer Herstellerziele beeinflußt wird. Vgl. Raabe, T., Die Elektronik-Schrott-Verordnung, a.a.O., S. 299ff. Raabe leitet in diesem ZuIrene Giesen-Netzer and Universität Münster - 978-3-631-75058-2 
und Recyclingaktivitäten sinnvoll. Sind die Kunden weder dazu bereit, die Rücknahme zu unterstützen noch Recyclingprodukte zu akzeptieren, werden die Hersteller entweder ein geringes Aktivitätsniveau bei ihrem Implementierungsverhalten zeigen und nur so viel unternehmen, daß sie bei Inkrafttreten einer gesetzlichen Regelung ein System aufweisen können oder mangels Profilierungsmöglichkeiten Rücknahme- und Recyclingaktivitäten externalisieren.

Eine Analyse des handelsbezogenen Einflusses auf die Gestaltung von Rücknahme- und Recyclingsystemen vollzieht sich vor dem Hintergrund einer Profilierungsmöglichkeit des Handels gegenüber den Kunden einerseits ${ }^{434}$ und einer Minimierung des handelsbezogenen Aufwands andererseits. Sofern die aktive Beteiligung am Rücknahme- und Recyclingsystem aus Handelssicht zu einer kundengerichteten Profilierung im Wettbewerb der Einkaufsstätten beiträgt, wäre eine Kooperation mit den Herstellern denkbar, zumal dem Handel eine wichtige Schnittstellenfunktion in Form eines ökologischen Gatekeepers zukommt. Diese Handelsfunktion kommt vor allem in den ökologiegeleiteten Listungsentscheidungen zum Ausdruck. ${ }^{435}$ Eine schriftliche Befragung aus dem Jahre 1993, bei der 120 Elektrofachhändler einen Fragebogen zur Einstellung gegenüber den Themen Umweltschutz, Umweltschutzmaßnahmen sowie absatzmarktbezogener In-

sammenhang drei verbraucherorientierte Gestaltungskriterien für Elektronikschrott ab: 1. Bequemlichkeit, 2. Anpassungsfähigkeit (Zeitpunkte des Altproduktanfalls), 3. Sicherheit. Vgl. ebenda, S. 290.

Holsystemen (die Altprodukte werden beim Kunden abgeholt) wird eine höhere mengenmäßige Erfolgswirkung zugesprochen, da damit der Bequemlichkeit des Kunden entgegengekommen wird. Darüber hinaus sind keine zusätzlichen Anreize notwendig, um den Kunden zu motivieren, die Produkte zu den Annahmestellen zu bringen. Vgl. Hansen, U., Raabe, T., Dombrowsky, B., Die Gestaltung des Konsumgüterrecycling als strategische Netzwerke, a.a.O., S. 65.

Damit bestünde gleichzeitig eine Profilierungsmöglichkeit der Hersteller gegenüber dem Handel.

Die Gatekeeper-Funktion beschreibt die Vermittlungsfunktion zwischen Produzent und Konsument sowie die Möglichkeit, Marktwege zu kontrollieren und somit Einfluß auf Teile des Verhaltens der Kunden zu nehmen. Vgl. Hauch, S., Die Rolle des Handels bei der Durchsetzung neuer Konzepte, in: Kreislaufwirtschaft statt Abfallwirtschaft: Optimierte Nutzung und Einsparung von Ressourcen durch Öko-Leasing und Servicekonzepte, Hockerts, K., u.a. (Hrsg.), Ulm 1994, S. 177. Hauch nennt als Determinanten der Umsetzung von ökologiebezogenen Aktivitäten des Handels: 1. Leistungs- und Kostenstruktur, 2., Wertorientierung und Ziele, 3. Kompetenz, 4. Konsumentenbeziehungen, 5. Lieferantenbeziehungen. Vgl. ebenda, S. $178 f$.

Ceyp untersucht die ökologieorientierten Konfliktpotentiale in vertikalen Systemen, die durch unterschiedliche Ziele und ökologieorientierte Basisstrategien entstehen und beschreibt Konflikte, die zwischen umweltaktiven Elektroherstellern und einem umweltpassiven Handelsverhalten entstehen. Demzufolge können bei der Rücknahme durch den Hersteller Unstimmigkeiten bezüglich der Häufigkeit der Abholtermine oder Mengenvorgaben bestehen. Vgl. Ceyp, M., Ökologieorientierte Profilierung im vertikalen Marketing, a.a.O., S. $31 \mathrm{ff}$. 
halte ausfüllten, kam allerdings zu dem Ergebnis, daß die ökologieorientierten Profilierungschancen im Handel als gering einzustufen sind. ${ }^{436}$ Eine geringe Profilierungsmöglichkeit i.V.m. dem durch die Rücknahme verbundenen Aufwand läßt daher auf eine geringe Kooperationswahrscheinlichkeit des Handels schließen. ${ }^{437}$ Demzufolge werden die Hersteller häufig andere Rücknahmestellen bei der Implementierung des Rücknahme- und Recyclingsystems (Hersteller selbst, Verwerter, öffentliche Entsorger) einbeziehen. Da dieses darüber hinaus für alle Implementierungstypen gleichermaßen gilt, kann vermutet werden, daß sich das Handelsverhalten nicht signifikant zwischen den vorliegenden Implementierungstypen unterscheidet.

Wird schließlich das Verhalten der Wettbewerber als Einflußfaktor auf die Implementierung von Rücknahme- und Recyclingsystemen betrachtet, ist sicherlich von Bedeutung, ob und ggf. in welcher Form sich die Wettbewerber in der Branche ebenfalls in Rücknahme- und Recyclingsystemen engagieren. ${ }^{438}$ Dabei ist zu beachten, daß die befragten Hersteller als Rücknahme- und Recycling-Pioniere nicht nur einseitig von Wettbewerbseinflüssen im Sinne von „Ruletakern“ betroffen sind. Vielmehr ist davon auszugehen, daß sie als „Rulemaker" mit ihrem Verhalten entscheidenden Einfluß auf das Wettbewerbsverhalten und damit die Wettbewerbsstruktur ausüben. Daher ist eine eindeutige Einflußrichtung nicht gegeben. Die folgende Hypothese faßt die theoretischen Überlegungen zusammen:

$\mathrm{H}_{\mathrm{SIT} 1}$ : Die Absatzmarktsituation beeinflußt - mit Ausnahme des Handelsverhaltens - das Implementierungsverhalten der Hersteller.

436 Vgl. ebenda, S. 161.

${ }^{437}$ Die Kooperationsbereitschaft des Handels hängt zum einen von den Machtverhältnissen im Distributionskanal ab, die sich auf die Zusammenarbeit im Redistributionskanal auswirken. Zum anderen hängt sie von der Einschätzung des Kosten-Nutzen-Verhältnisses ab. Vgl. Raabe, T., Die Elektronik-Schrott-Verordnung, a.a.O., S. $296 \mathrm{f}$.

Der Handel weigert sich teilweise sogar, Altprodukte zurückzunehmen, da er nicht als Zwischenstation für die Produkte dienen will. Der Bürostuhlhersteller Grammer stellt insbesondere die Frage nach der Sinnhaftigkeit eines zwischengeschalteten Handels, die bei Großprojekten nicht gegeben sei. Vgl. Wächter, F., Durchsetzbarkeit im Handel und logistische Probleme der Redistribution, in: Hockerts, K., u.a. (Hrsg.), Kreislaufwirtschaft statt Abfallwirtschaft: optimierte Nutzung und Einsparung von Ressourcen durch Öko-Leasing und Servicekonzepte, Ulm 1994, S. $195 f$.

438 Auch neu entstehende Konkurrenzbeziehungen sind zu berücksichtigen, die durch die Beauftragung Dritter mit der Rückführung und dem Recycling entstehen können. Die "Dritten“ können sich beispielsweise durch die Hochrüstung von Produktkomponenten zu Wettbewerbern im Ersatzteilgeschäft entwickeln. In der Automobilbranche wird das lukrative Ersatzteilgeschäft der Hersteller durch das Angebot von Recyclingunternehmen in dieser Weise berührt. Vgl. Kirchgeorg, M., Kreislaufwirtschaft, a.a.O., S. 22. 
Für die Erfassung und Analyse der Absatzmarktsituation werden acht Indikatoren herangezogen. Eine5 vorgeschaltete explorative Faktorenanalyse ergibt vier Faktoren, die sich wie folgt bezeichnen lassen: (1) Marktstadium bzw. Reifegrad des Marktes, (2) umweltbezogenes Marktteilnehmerverhalten, (3) kundenbezogene Akzeptanz von Recyclingprodukten sowie (4) Existenz eines Sekundärmarktes. ${ }^{439}$ Die vier Faktoren sind in der Lage, $66,9 \%$ der Varianz der Variablen zu erklären. Dabei werden unter der Umweltheterogenität das Verhalten der Marktteilnehmer, das hier den Faktoren 2 und 3 entspricht, sowie die Existenz von Gebrauchtmärkten (Faktor 4) als Einflußfaktoren auf das Implementierungsverhalten diskutiert. Die Feindlichkeit der Umwelt wird im Zusammenhang mit dem Marktstadium, das durch Faktor 1 abgebildet wird, diskutiert. Somit spiegeln sich alle diskutierten Aspekte in den Faktoren wider.

Abbildung 25 zeigt unter Zuordnung der 8 Variablen zu den explorativ ermittelten Faktoren der Absatzmarktsituation den Mittelwertvergleichstest für die fünf Implementierungstypen. Bei der Betrachtung der F-Werte fällt auf, daß nur bei der Hälfte der analysierten absatzmarktbezogenen Variablen signifikante Mittelwertunterschiede bestehen $(\alpha=0,10)$. Damit könnte zunächst vermutet werden, daß die im Zusammenhang mit der Absatzmarktsituation formulierte Hypothese abzulehnen ist. Eine differenziertere Betrachtung der Ergebnisse zeigt jedoch, welche Ursachen sich für die Ähnlichkeit der Mittelwerte finden lassen können und welche Grunde dazu führen, daß die Hypothese $\mathbf{H}_{\text {SIT1 }}$ insgesamt (eingeschränkt) bestätigt wird.

439 Die Methodik der explorativen Faktorenanalyse wird gewählt, weil keine genaue Vorstellungen über die Existenz und die Anzahl der zu ermittelnden Faktoren und damit auch relevanter Konstrukte bestehen und somit die konfirmatorische Faktorenanalyse nicht zur Anwendung kommen kann.

Die Ergebnisse der explorativen Faktorenanalyse, die mit dem Verfahren der Hauptkomponentenanalyse mit anschließender Faktorrotation durchgeführt wurde, sind im Anhang 2.5 dokumentiert. 

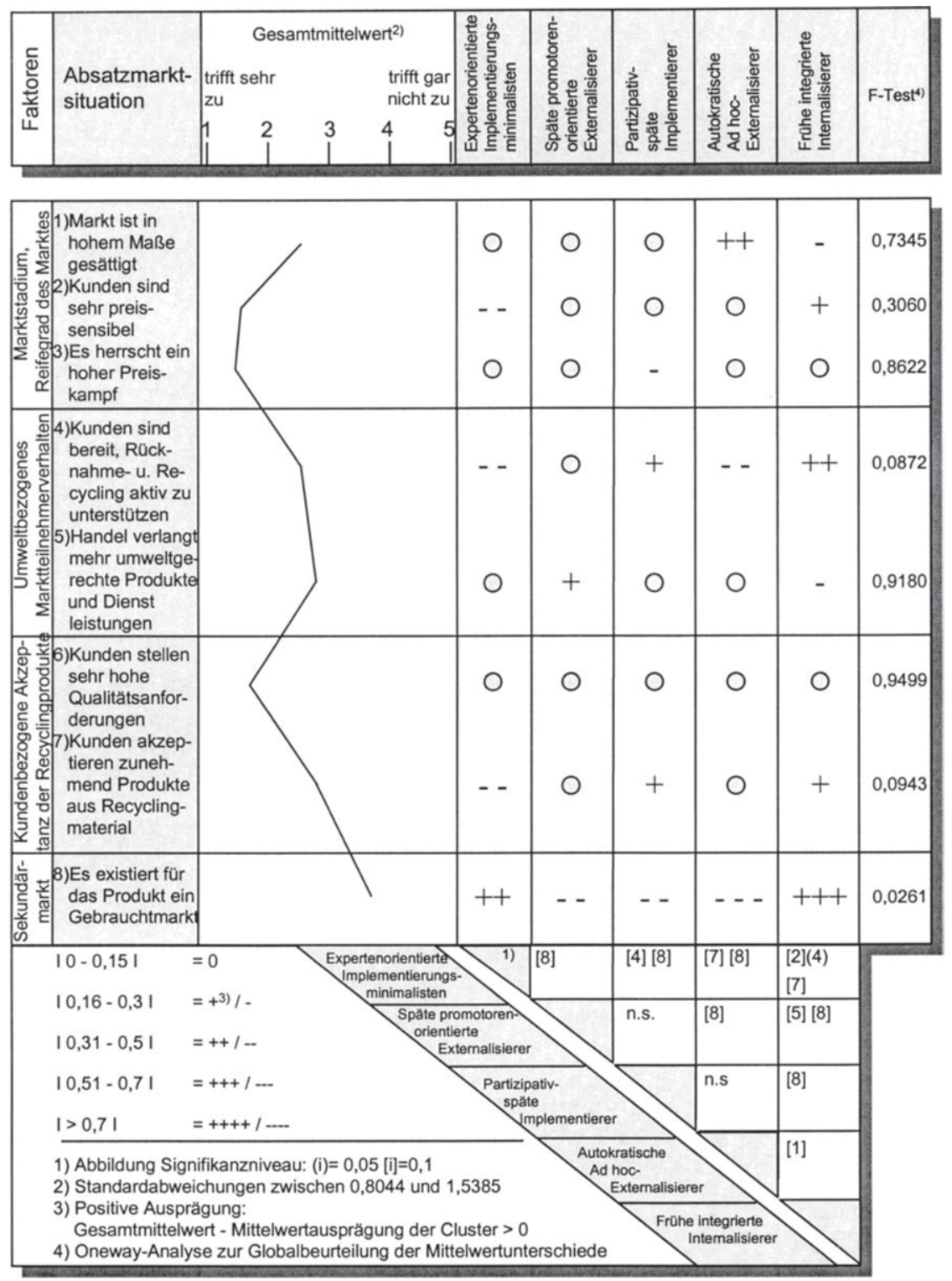

Abb. 25: Zusammenhang zwischen Absatzmarktsituation und Implementierungstypen 
Bezüglich des Marktstadiums, das sich hinsichtlich keiner Variablen signifikant unterscheidet, existieren zwei Erklärungsmöglichkeiten. Zum einen wurden Hersteller befragt, die langlebige Gebrauchsgüter herstellen und aus ausgewählten Branchen stammen. Dies kann dazu führen, daß sich die Unternehmen innerhalb einer Branche in relativ homogenen Reifegraden des Marktes befinden, sich durch die "Vermischung" der Branchen in den unterschiedlichen Implementierungstypen jedoch ein unklares Bild ergibt. Nach dieser Argumentation können derartige situative Einflüsse auf das Implementierungsverhalten der Hersteller in der Gesamtsicht aller Branchen gar nicht bestehen. ${ }^{440}$ Zum anderen mag eine Erklärung im methodischen Vorgehen zu finden sein, da insgesamt nur drei Variablen zur Verfügung stehen, um den Reifegrad des Marktes abzubilden: „Markt ist in hohem Maße gesättigt", „Kunden sind sehr preissensibel“ und „Es herrscht ein hoher Preiskampf".

Das umweltbezogene Kundenverhalten zeigt hingegen signifikante Unterschiede bei den Mittelwerten der Implementierungstypen. ${ }^{441}$ Dies gilt sowohl hinsichtlich der produktbezogenen Argumentation als auch hinsichtlich der Bereitschaft der Konsumenten, Rücknahme- und Recyclingsysteme zu unterstützen. Dieser doppelte Pull-Effekt tritt insbesondere bei den Frühen integrierten Internalisierern und bei den Partizipativ-späten Implementierern auf, während dieser Befund bei den Expertenorientierten Implementierungsminimalisten und den Autokratischen Ad hoc-Externalisierern nicht zu beobachten ist. Offenbar scheint die Profilierungsmöglichkeit bei den Kunden und deren Bereitschaft, Aktivitäten bei der Gestaltung und Implementierung von Rücknahme- und Recyclingsystemen zu unterstützen, die Internalisierung von derartigen Aktivitäten zu fördern. ${ }^{442}$

Schließlich ist die Existenz eines Gebrauchtmarktes als Einflußfaktor des Implementierungsverhaltens für die Altprodukte über alle Cluster gesehen mit einem Mittelwert von 3,77 als gering zu bezeichnen (vgl. Abb. 25). Dies läßt sich dahingehend interpretieren, daß sich die betrachteten Altprodukte entweder nur teilwei-

An dieser Stelle sei auf die Ergebnisse des Kapitels C 1.4.2.1 hingewiesen, in dem der Zusammenhang zwischen Branche und Implementierungstypen untersucht wird.

441 Die Variable „Kunden stellen sehr hohe Qualitätsanforderungen“ unterscheidet sich nicht signifikant zwischen den Clustern. Diese Variable ist keine umweltbezogene Verhaltenskomponente, sondern zeigt lediglich allgemein auf, daß sich die Hersteller in den betrachteten Branchen insgesamt sehr hohen Qualitätsanforderungen gegenübersehen.

442 Bezüglich des umweltbezogenen Handelsverhaltens zeigt die Analyse annahmegemäß keine signifikanten Mittelwertunterschiede. 
se für einen solchen Markt eignen ${ }^{443}$ oder die Marktteilnehmer diesen durchaus profitablen Markt noch nicht entdeckt haben. ${ }^{444}$ Auffällig ist der stark überdurchschnittlichen Wert bei den Frühen integrierten Internalisierern und der stark unterdurchschnittliche Wert bei den Autokratischen Ad hoc-Externalisierern. Dieses läßt grundsätzlich zwei Argumentationslinien zu: Angesichts ökonomischer Nutzenpotentiale werden Hersteller bei funktionsfähigen, renditeträchtigen $\mathrm{Ge}$ brauchtmärkten daran interessiert sein, möglichst viele Wertschöpfungsstufen zu internalisieren (Frühe integrierte Internalisierer). Im Gegenzug erscheint ein eine Externalisierung von Aktivitäten bei einem Fehlen von Gebrauchtmärkten wahrscheinlich, da kaum Opportunitätserlöse zu befürchten sind (Autokratische Ad hoc-Externalisierer). ${ }^{445}$ Damit ist die Existenz eines Gebrauchtmarktes mit der entsprechenden Nutzenkomponente in Verbindung mit dem Produktwert ein deutlicher Einflußfaktor für die Internalisierungs- und Implementierungsaktivitäten von Herstellern.

Das Verhalten der Wettbewerber geht aus Abbildung 26 hervor. ${ }^{46}$ Zunächst fällt der mit 70,59\% sehr hohe Anteil von Späten promotorenorientierten Externalisierern auf, die kein anderes Rücknahme- und Recyclingsystem in der Branche kennen, was einer Abweichung vom erwarteten statistischen Wert von über $27 \%$ entspricht. Auch bei den Autokratischen Ad hoc-Externalisierern kennen fast $12 \%$ weniger als statistisch zu erwarten andere Rücknahme- und Recyclingsysteme in der Branche. Demnach fühlen sich Hersteller nicht von den Wettbewerbern unter Druck gesetzt, frühzeitig selbst aktiv zu werden, solange sie keine oder wenige Rücknahme- und Recyclingsysteme in der Branche kennen. Dieses zeigt sich - bei einer rein wettbewerbsgerichteten Analyse - in einer späten Ausprägung der Timingdimension. ${ }^{447}$

443 Dies gilt z.B. für PVC-Fenster, die aufgrund von Verfärbungen oder speziellen Maßen nicht mehr für eine weitere Verwendung geeignet sind. Vgl. FREI-Fallstudie, a.a.O.

444 Vgl. dazu Fallstudie Siemens-Nixdorf, a.a.O.

Bei einem profitablen Markt haben sich häufig bereits Strukturen von Dritten entwickelt, die diese Profite abschöpfen. Zu denken ist z.B. an die Schrotthändler in der Automobilbranche.

445 Es wird deutlich, daß dieser Einflußfaktor auch vor dem Hintergrund des Produktwertes zu betrachten ist, da dieser als Wertkomponente des Marktpotentials maßgeblich für das Entstehen von Gebrauchtmärkten verantwortlich ist. Vgl. dazu Kapitel C 1.4.1.2.

446 Strenggenommen wird hier die Wahrnehmung der befragten Hersteller und damit die Transparenz des Marktes hinsichtlich der Kenntnis anderer Rücknahme- und Recyclingsysteme gemessen. Dementsprechend wird auch mit dem Einfluß (bekannter) bestehender Systeme auf das Implementierungsverhalten argumentiert. 


\begin{tabular}{|c|c|c|c|c|c|c|}
\hline & $\begin{array}{l}\text { Implement.- } \\
\text { typen }\end{array}$ & Cluster I & Cluster II & Cluster III & Cluster IV & Cluster V \\
\hline $\begin{array}{l}\text { Anteil } \\
\text { der } \\
\text { Stich- } \\
\text { probe } \\
\text { in } \%\end{array}$ & $\begin{array}{c}x i=\text { tatsächlicher } \\
\text { Wert } \\
(x i)=\text { erwarteter } \\
\text { Wert } \\
{[x i]=\text { Abweichung }}\end{array}$ & $\begin{array}{c}\text { Experten- } \\
\text { orientierte } \\
\text { Implemen- } \\
\text { tierungs- } \\
\text { minimalisten }\end{array}$ & $\begin{array}{l}\text { Späte pro- } \\
\text { motoren- } \\
\text { orientierte } \\
\text { Externa- } \\
\text { lisierer }\end{array}$ & $\begin{array}{l}\text { Partizipativ- } \\
\text { späte Im- } \\
\text { plemen- } \\
\text { tierer }\end{array}$ & $\begin{array}{l}\text { Autokra- } \\
\text { tische } \\
\text { Ad hoc- } \\
\text { Externa- } \\
\text { lisierer }\end{array}$ & $\begin{array}{l}\text { Frühe inte- } \\
\text { grierte } \\
\text { Interna- } \\
\text { lisierer }\end{array}$ \\
\hline \multirow[t]{3}{*}{$43,2 \%$} & \multirow{3}{*}{$\begin{array}{c}\text { Kein anderes } \\
\text { Rücknahme- und } \\
\text { Recyclingsystem } \\
\text { in der Branche } \\
\text { bekannt }\end{array}$} & $\begin{array}{c}38,10 \% \\
(43,33 \%)\end{array}$ & $\begin{array}{c}70,59 \% \\
(42,94 \%)\end{array}$ & $\begin{array}{c}47,06 \% \\
(42,94)\end{array}$ & $\begin{array}{c}54,55 \% \\
(42,73 \%)\end{array}$ & $\begin{array}{c}24,14 \% \\
(43,10 \%)\end{array}$ \\
\hline & & & & & & \\
\hline & & - & +++ & 0 & ++ & -- \\
\hline \multirow[t]{2}{*}{$13,7 \%$} & \multirow[t]{2}{*}{$\begin{array}{l}\text { Andere herstel- } \\
\text { lereigene Syste- } \\
\text { me }\end{array}$} & $\begin{array}{c}14,29 \% \\
(13,53 \%) \\
{[+0,48 \%]}\end{array}$ & $\begin{array}{c}5,88 \% \\
(13,53 \%) \\
{[-7,65 \%]}\end{array}$ & $\begin{array}{c}17,65 \% \\
(13,35 \%) \\
{[+4,12 \%]}\end{array}$ & $\begin{array}{c}18,18 \% \\
(13,64 \%) \\
{[+4,55 \%]}\end{array}$ & $\begin{array}{c}13,79 \% \\
(13,79 \%) \\
{[0 \%]}\end{array}$ \\
\hline & & 0 & - & 0 & 0 & 0 \\
\hline \multirow[t]{2}{*}{$8,4 \%$} & \multirow[t]{2}{*}{$\begin{array}{l}\text { Andere vertragli- } \\
\text { che Systeme }\end{array}$} & $\begin{array}{c}4,76 \% \\
(8,57 \%) \\
{[-3,81 \%]}\end{array}$ & $\begin{array}{c}17,65 \% \\
(8,24 \%) \\
{[+9,41 \%]}\end{array}$ & $\begin{array}{c}0 \% \\
(8,24 \%) \\
{[-8,24 \%]}\end{array}$ & $\begin{array}{c}0 \% \\
(8,18 \%) \\
{[-8,18]}\end{array}$ & $\begin{array}{c}13,79 \% \\
(8,28 \%) \\
{[+5,52]}\end{array}$ \\
\hline & & 0 & + & - & - & + \\
\hline \multirow[t]{2}{*}{$11,6 \%$} & \multirow[t]{2}{*}{$\begin{array}{c}\text { Andere } \\
\text { Branchen- } \\
\text { kooperationen }\end{array}$} & $\begin{array}{c}9,52 \% \\
(11,43 \%) \\
{[-1,90 \%]}\end{array}$ & $\begin{array}{c}5,88 \% \\
(11,76 \%) \\
{[-5,88 \%]}\end{array}$ & $\begin{array}{c}11,76 \% \\
(11,76 \%) \\
{[0 \%]}\end{array}$ & $\begin{array}{c}9,09 \% \\
(11,82 \%) \\
{[-2,72 \%]}\end{array}$ & $\begin{array}{c}17,24 \% \\
(11,72 \%) \\
{[+5,52 \%]}\end{array}$ \\
\hline & & - & - & 0 & 0 & + \\
\hline \multirow[t]{2}{*}{$23,2 \%$} & \multirow{2}{*}{$\begin{array}{l}\text { mehrere organi- } \\
\text { satorisch unter- } \\
\text { schiedliche Sy- } \\
\text { steme }\end{array}$} & $\begin{array}{c}33,33 \% \\
(23,33 \%) \\
{[+10 \%]}\end{array}$ & $\begin{array}{c}0 \% \\
(22,94 \%) \\
{[-22,94 \%]}\end{array}$ & $\begin{array}{c}23,53 \% \\
(22,94 \%) \\
{[+0,59 \%]}\end{array}$ & $\begin{array}{c}18,18 \% \\
(22,73 \%) \\
{[-4,55 \%]}\end{array}$ & $\begin{array}{c}31,03 \% \\
(23,10 \%) \\
{[+7,93]}\end{array}$ \\
\hline & & + & -- & 0 & 0 & + \\
\hline$n=95$ & & $22,1 \%$ & $17,9 \%$ & $17,9 \%$ & $11,6 \%$ & $30,5 \%$ \\
\hline
\end{tabular}

\begin{tabular}{|l|l|l|c|c|c|c|}
\hline $\begin{array}{l}\text { Chi-Quadrat } \\
\text { Test gemäß: }\end{array}$ & $\begin{array}{l}\text { Signifikanz- } \\
\text { niveau }\end{array}$ & $\mathbf{x i}-(\mathrm{xi})$ & $0 \%-5 \%$ & $5,1 \%-10 \%$ & $\begin{array}{c}10,1 \%- \\
20 \%\end{array}$ & $>20 \%$ \\
\hline $\begin{array}{l}\text { Pearson } \\
\text { Likelihood Ratio }\end{array}$ & 0,23782 & $\begin{array}{l}\text { positiv } \\
\text { negativ }\end{array}$ & 0 & + & ++ & +++ \\
\hline
\end{tabular}

Abb. 26: Kontingenzanalytische Untersuchung des Zusammenhangs zwischen Existenz und Art von Rücknahme- und Recyclingsystemen in der Branche und Implementierungstypen

me) zu tragen. Hingegen möchten viele an diesem Gut mitprofitieren, wenn andere bereit sind, die Kosten der „Produktion“ zu tragen. Zur Definition von Trittbrettfahrern vgl. Wicke, L., Umweltökonomie: eine praxisorientierte Einführung, 2. erw. u. aktualisierte Aufl., München 1989, S. 41 . 
Darüber hinaus ist offenkundig die Tendenz zur Bildung von Kooperationen angesichts mangelnder Erfahrungen anderer Unternehmen im Markt hoch, da in Kooperationen Kow-how für den Aufbau von Rücknahme- und Recyclingsystemen gewonnen werden können. Eine andere Lösungsmöglichkeit fehlenden Erfahrungswissens besteht in der Übertragung der notwendig durchzuführenden Aufgaben an Dritte, so daß bei einer geringen Durchdringung des Marktes mit Rücknahme- und Recyclingsystemen häufiger Späte promotorenorientierte Externalisierer und Autokratische Ad hoc-Externalisierer vorliegen. Auch kann die Art der (wenigen) bekannten Systeme eine Auswirkung auf das jeweilige Implementierungsverhalten ausüben. Sind beispielsweise überwiegend andere vertragliche Systeme bekannt, die mit Erfolg durchgeführt werden, ist die Wahrscheinlichkeit, ebenfalls Aktivitäten zu externalisieren, höher (imitierendes Wettbewerbsverhalten). Zudem kann die Existenz einer Branchenlösung dazu führen, daß der Hersteller sich an dieser beteiligt, um sein unternehmerisches Risiko zu senken, auch wenn er eigentlich ein anderes System präferiert hätte.

Die Frühen integrierten Internalisierer hingegen kennen zu 75\% andere Rücknahme- und Recyclingsysteme in der Branche, davon viele Branchenlösungen und andere vertragliche Systeme mit Verwertern. Diese Ergebnisse deuten darauf hin, daß bereits bestehende (insbesondere erfolgreiche) Rücknahme- und Recyclingsysteme in der Branche die Hersteller ermutigen, ihrerseits aktiv zu werden. Zum anderen sind die Frühen integrierten Internalisierer scheinbar auch überdurchschnittlich gut informiert, da Wettbewerbsbeobachtungen bei einem hohen Internalisierungsgrad der Aufgaben im Rücknahme- und Recyclingsystem von hohem Interesse sind, um eigene Systeme kontinuierlich verbessern zu können. ${ }^{448}$

Neben der Absatzmarktsituation geht - wie oben bereits dargelegt - ein wichtiger Einfluß von der Beschaffungsmarktsituation auf das Implementierungsverhalten der Herstellerunternehmen aus. ${ }^{49}$ Daher wird der empirischen Analyse folgende Basishypothese vorangestellt:

448 Der Chi-Quadrat-Test zeigt ein unklares Bild hinsichtlich der Unterschiedlichkeit der Cluster bezüglich der herstellerseitigen Wahrnehmung der Existenz anderer Rücknahme- und Recyclingsysteme in der Branche: Nach dem Chi-Quadrat-Test nach Pearson muß die Unterschiedlichkeit bei einem Signifikanzniveau von $10 \%(\alpha=0,10)$ abgelehnt werden, während der Chi-Quadrat-Test nach Likelihood Ratio mit einer noch annehmbaren Irrtumswahrscheinlichkeit von knapp über $6 \%$ die Unterschiedlichkeit bestätigt.

Durch die Beschaffung werden bereits recyclingrelvante Eigenschaften der Produkte festgelegt. Gleichzeitig können über die Beschaffung von Sekundärrohstoffen ökologische Impulse 
$\mathrm{H}_{\text {SIT2}}$ : Die Beschaffungsmarktsituation beeinflußt das Implementierungsverhalten der Hersteller.

Zur Analyse des Einflusses der Beschaffungsmarktsituation auf die Implementierung von Rücknahme- und Recyclingsystemen stehen 7 Variablen zur Verfügung (vgl. Abb. 27). Auch hier wurde eine explorative Faktorenanalyse vorgeschaltet, die diese Variablen auf drei Faktoren verdichtet und 63,1\% der Varianz der Variablen erklärt. ${ }^{450}$ Der erste Faktor, der die Bindungsintensität zwischen Herstellern und Zulieferern beschreibt, erklärt alleine knapp 27\% der Varianz der Variablen, wobei die Zusammenarbeit im Rahmen von langfristigen Verträgen sowie die gemeinsame Entwicklung von Komponenten signifikant unterschiedlich hinsichtlich der Mittelwerte $(\alpha=0,10)$ der Implementierungstypen sind.

Dabei erweisen sich insbesondere die Partizipativ-späten Implementierer und die Frühen integrierten Internalisierer als diejenigen Hersteller, die eine hohe Bindungsintensität zu den Zulieferern haben. Eine hohe Bindungsintensität zwischen Hersteller und Zulieferer läßt auf eine von Vertrauen und Offenheit geprägte Beziehung schließen und kommt daher augenscheinlich einem partizipativen Implementierungsverhalten (Partizipativ-späte Implementierer) nahe, d.h. die Partizipation bleibt nicht nur auf die Individualebene beschränkt, sondern geht offenbar über die Unternehmensgrenzen hinaus. ${ }^{451}$ Als Resultat einer hohen Bindungsintensität ist zudem scheinbar eine hohe Kompetenz in der eigenständigen oder gemeinsamen Entwicklung von Zulieferkomponenten möglich, die Zeitvorteile bei der Implementierung mit sich bringt. Darüber hinaus entsteht eine hohe Bindungsintensität augenscheinlich erst schrittweise und eher langfristig, da dieses Implementierungsverhalten bei den Partizipativ-späten Implementierern und Frühen integrierten Internalisierern überdurchschnittlich hoch ausgeprägt ist.

für Konstruktion und Produktion gegeben werden. Vgl. Eckert, W., Okologieorientierte Strategische Beschaffungsbeziehungen, Wiesbaden 1996, S. 102.

451 Falke hebt die Notwendigkeit hervor, Zulieferer eng in den Beschaffungsprozeß einzubinden, um die Berücksichtigung ökologischer Aspekte zu gewährleisten. Am Beispiel von Computern erläutert er die umweltbezogenen Kriterien, die Hewlett Packard gemeinsam mit den Zulieferern ausgearbeitet hat. Vgl. Falke, R., Verantwortung für das Ganze tragen, a.a.O., S. 34. 

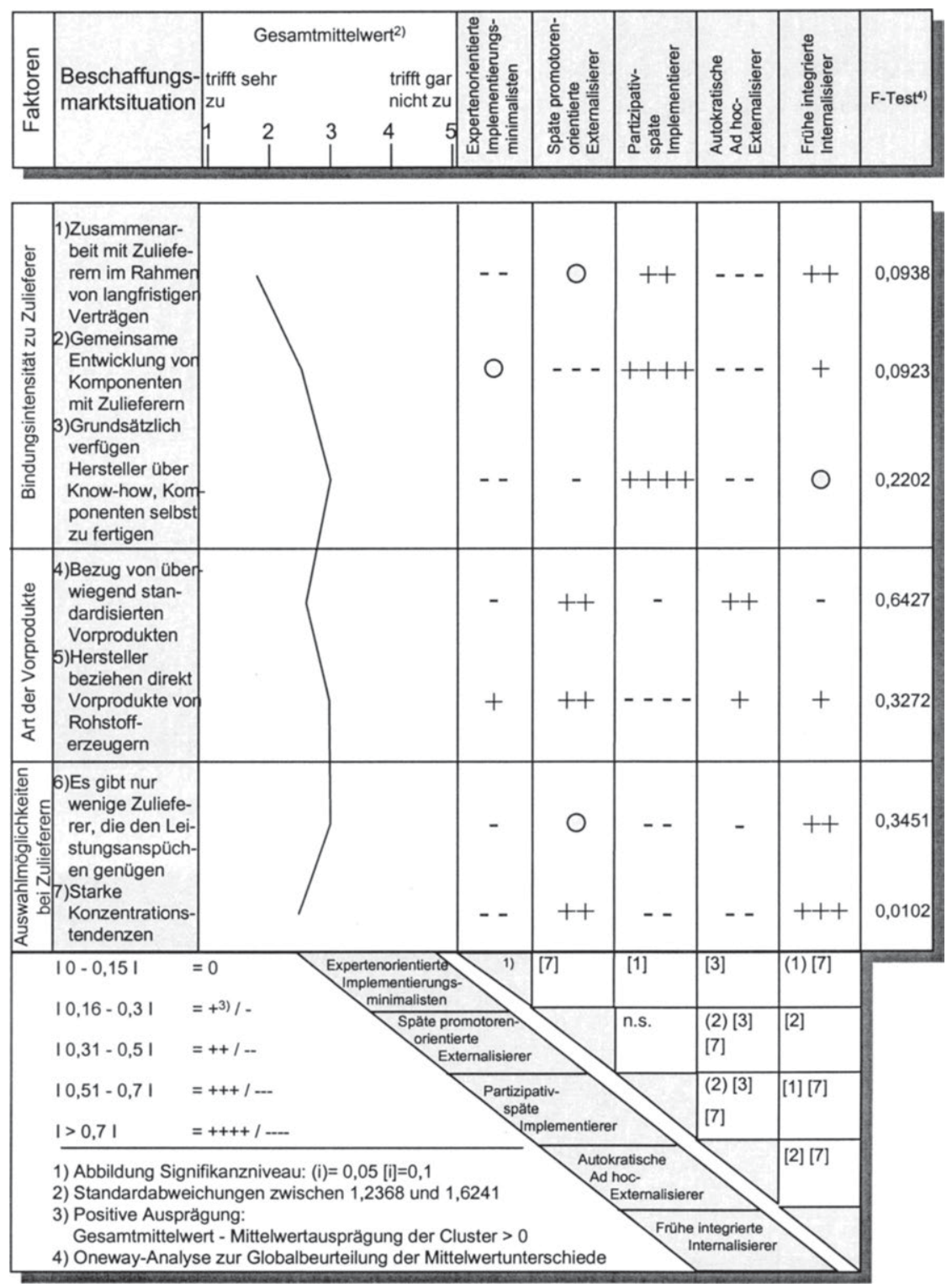

\section{Abb. 27: Zusammenhang zwischen Beschaffungsmarktsituation und Implementierungsty- pen}


Der zweite Faktor beschreibt die Art der Vorprodukte, die die Standardisierung sowie den Bezug von Vorprodukten direkt von Rohstofferzeugern umfaßt. Werden von dem Beschaffungsmarkt weitestgehend standardisierte Vorprodukte oder Rohstoffe bezogen, ist eine Internalisierung wenig wahrscheinlich, da bei einem oft mit leichter Kopierbarkeit von Produkten bzw. Prozessen einher gehenden geringen Wertschöpfungspotential auch der Anreiz dazu fehlt. Vielmehr erscheint eine Kooperationslösung oder die Externalisierung an Dritte plausibel, um Mengeneffekte zu realisieren oder Kapazitäten und Ressourcen für andere Aktivitäten freizusetzen, so daß insbesondere ein positiver Einfluß auf das Implementierungsverhalten Später promotorenorientierter Externalisierer sowie Autokratischer Ad hoc-Externalisierer vermutet werden kann. Die F-Werte zeigen demgegenüber, daß sich keiner der Implementierungstypen signifikant hinsichtlich der Art der Vorprodukte unterscheidet und der vermutete Zusammenhang sich nur in der Tendenz feststellen läßt.

Demgegenüber begünstigt eine starke Konzentration der Zulieferer (3. Faktor) deutlich ein Implementierungsverhalten, wie es die Frühen integrierten Internalisierer aufweisen. Der Faktor, der die Auswahlmöglichkeiten auf der Zuliefererseite beschreibt, besitzt einen qualitativen Aspekt, der die subjektiv vom Hersteller wahrgenommene Auswahl umfaßt („Es gibt nur wenige Zulieferer, die den Leistungsansprüchen genügen") sowie einen quantitativen Aspekt, der die objektiv zur Verfügung stehende Anzahl der möglichen Zulieferer beinhaltet. Insbesondere hinsichtlich der letzt genannten Größe unterscheiden sich die Cluster mit einem FWert von 0,0102 deutlich voneinander. Eingeschränkte Wahlmöglichkeiten - z.B. bei der Spezifität des eigenen Angebotes - begünstigen dabei offenbar die Eigenerstellung von Vorprodukten oder Einsatzstoffen und fördern ein Implementierungsverhalten wie das der Frühen integrierten Internalisierer. ${ }^{452}$ Damit kann die

452 Diese Ausführungen werden theoretisch durch den Transaktionskostenansatz gestützt. Nach diesem Ansatz ist die optimale institutionelle Ausgestaltungsform (wobei Markt und Hierarchie die Extrempunkte eines Kontinuums sind) nicht durch die Technologie oder die Produktionskosten bestimmt, sondern durch die Minimierung der Transaktionskosten, die sich in Anbahnungs-, Vereinbarungs-, Kontroll- und Anpassungskosten unterteilen. Dabei scheitern marktliche Koordinationen bei Transaktionen mit hoher Komplexität und Unsicherheit. Gleichzeitig wird das Problem des Opportunismus bei einer kleinen Zahl von Anbietern für die Durchführung der Transaktion virulent. Eine Hierarchie verliert hingegen bei sicheren Transaktionen und bei einer großen Anzahl potentieller Lieferanten ihren komparativen Vorteil. Neben vielen anderen vgl. Picot, A., Transaktionskostenansatz in der Organisationstheorie: Stand der Diskussion und Aussagewert, in: DBW, Heft 42, 1982, S. 267ff. und vgl. Williamson, O. E., Markets and hierarchies: Analysis and antitrust implication, Free Press, New York, London 1975, S. 40 . 
Basishypothese $\mathbf{H}_{\mathbf{S I T 2}}$, nach der die Beschaffungsmarktsituation das Implementierungsverhalten der Hersteller beeinflußt, insgesamt (eingeschränkt) bestätigt werden.

\subsubsection{Produktmerkmale}

Die Beschränkung der Analyse auf langlebige Gebrauchsgüter erfolgte aufgrund des im Vergleich zu kurzlebigen Verbrauchsgütern hohen Wertschöpfungspotentials dieser Produkte, das die Implementierung von Rücknahme- und Recyclingsystemen begünstigt. ${ }^{453} \mathrm{Da}$ der Umfang des Wertschöpfungspotentials aus der Höhe des Neupreises ersichtlich wird, kann dieser als Indikator genutzt werden. ${ }^{454}$

Ein sehr hoher Neupreis und damit ein hohes Wertschöpfungspotential des Produktes lassen darauf schließen, daß Hersteller bereits ohne gesetzlichen Handlungsdruck Gestaltungs- und Implementierungsansätze zur Rücknahme und zum Recycling derartiger Produkte unternehmen (Timingdimension). Die Begründung hierfür liegt in den hochwertigen Komponenten des Produktes, die idealerweise ohne erheblichen Wertverlust und ohne aufwendige Recyclingverfahren wieder in den Produktionsprozeß zurückgeführt oder auf andere Weise nutzbringend eingesetzt werden können. ${ }^{455}$ Ein niedriger Neupreis läßt demgegenüber grundsätzlich zwei Argumentationslinien zu. Zum einen ist anzunehmen, daß die Implementierung eines Rücknahme- und Recyclingsystems von geringerwertigen Produkten vor allem auf der Basis realisierbarer Mengeneffekte vorgenommen wird, da damit Kosten eingespart werden können. Demzufolge ist bei einer angestrebten Kooperationslösung eine frühzeitige Auseinandersetzung mit der Implementierung eines Rücknahme- und Recyclingsystems wahrscheinlich, die jedoch angesichts des

453 Vgl. dazu Kapitel A 1.

454 Die alleinige Betrachtung des Wertes des Neuproduktes würde bei der integrierten Betrachtungsweise eines Wertschöpfungskreislaufes eine verkürzte Sichtweise bedeuten. Eine integrierte Sichtweise bezieht auch den Wert des Altproduktes und denjenigen der Komponenten oder Stofffraktionen nach dem Recyclingprozeß, d.h. den Wert des Produktes nach seiner Verwendung, in die Überlegungen ein. Vgl. Kirchgeorg, M., Kreislaufwirtschaft - Neue Herausforderungen für das Marketing, in: Marketing ZFP, Heft 4, 1995, S. 234ff. Dieser Argumentation kann m.E. nach gefolgt werden. Dennoch kann der Neupreis eines Produktes auch unter Vernachlässigung der Kosten - erste Hinweise auf mögliche Potentiale der Werterhaltung geben, die bei geringwertigen Gütern ex definitione nicht gegeben sind.

Der technische Verschleiß von Produktkomponenten sowie die Veralterung der Produkttechnologie sind zwei wichtige Punkte, die den Wert und die Verwendbarkeit der Altprodukte erheblich beeinträchtigen. Dennoch werden hochpreisige Güter grundsätzlich eher Komponenten enthalten, die gut zu verwenden oder zu verwerten sind als geringwertige Güter. 
geringeren Wertes eher einer kurzen Zeitdauer unterliegt. Auf der anderen Seite mag ein geringer Neupreis erst spät dazu führen, daß sich Hersteller mit der Implementierung eines Rücknahme- und Recyclingsystems auseinandersetzen, da bezüglich des ökonomischen Erfolges nur relativ geringe Chancen vermutet werden.

Eng zusammenhängend mit dem Neuproduktpreis dürften die Absatzform und der Komplexitätsgrad (Anzahl der Komponenten) der Produkte sein. Die Absatzform kann bei langlebigen Gebrauchsgütern grundsätzlich in Verkauf, Vermietung und Leasing differenziert werden. ${ }^{456} \mathrm{Im}$ Fall hoher Neupreise stehen grundsätzlich alle Alternativen zur Verfügung, wobei dem Leasing vermutlich ein wichtigerer Stellenwert zukommt als bei geringerwertigen Produkten. ${ }^{457}$ Ein hoher Komplexitätsgrad des Produktes, der den hohen Neupreis rechtfertigt, dürfte entscheidend für den (hohen) Internalisierungsgrad von Implementierungsaktivitäten bei Rücknahme- und Recyclingsystemen sein. Hier können auch Kooperationslösungen unter enger Einbeziehung der Vorkomponentenlieferanten bei komplexen Produkten eine sinnvolle Lösung sein. Diese Überlegungen werden in folgenden Baishypothsen zusammengefaßt:

HPRO1: Die Höhe des Neuproduktpreises beeinflußt das Implementierungsverhalten der Hersteller.

HPR2: Die Absatzform der Produkte beeinflußt das Implementierungsverhalten der Hersteller.

456 Hockerts u.a. diskutieren die Konsequenzen des Vermietens von Produkten hinsichtlich der Förderung von Rücknahme- und Recyclingsystemen und heben hervor, daß diese Absatzform insbesondere die Abfallvermeidung fördert. Vgl. Hockerts, K., U.a., Servicekonzepte als Element einer öko-effizienten Kreislaufwirtschaft, in: Kreislaufwirtschaft statt Abfallwirtschaft: optimierte Nutzung und Einsparung von Ressourcen durch Oko-Leasing und Servicekonzepte, in: Bayreuther Initiative für Wirtschaftsökologie e.V., Universität Bayreuth (Hrsg.), Schriftenreihe, Band 1, Ulm 1994, S. $7 \mathrm{ff}$.

457 Einschränkend zu dieser Argumentation ist zu berücksichtigen, daß Leasing für den Konsumenten i.d.R. lediglich ein Finanzierungsinstrument ist. Insbesondere beim Finanzierungsleasing, bei dem der Leasingnehmer das volle Investitionsrisiko (auch Veralterung, Zerstörung, Wartung u.a.) übernimmt, liegt weniger ein Mietverhältnis vor, bei dem der Leasinggeber Eigentümer des Produktes bleibt, sondern eher ein verdeckter Teilzahlungsvertrag. Vgl. Wöhe, G., Einführung in die Allgemeine Betriebswirtschaftslehre, a.a.O., S. 858.

Auf der anderen Seite kann Leasing dazu beitragen, den Systembindungseffekt beim Investitionsgütergeschäft zu verringern. D.h., der Nachfrager hat durch den hohen Neupreis den Eindruck, „auf Gedeih und Verderb mit der getroffenen Systementscheidung verbunden“ zu sein. Durch eine Anpassung der Vertragslaufzeit an die Nutzungserfordernisse, die Austauschbarkeit der Leasingobjekte und ein Produktionskapazitätenleasing kann dieser Eindruck gemindert werden. Vgl. Backhaus, K., Investitionsgütermarketing, 5., erw. und überarb. Aufl., München 1997, S. $577 \mathrm{ff}$. 
$\mathrm{H}_{\mathrm{PRO} O}$ : Der Komplexitätsgrad der Produkte beeinflußt das Implementierungsverhalten der Hersteller.

Bei einer Betrachtung des Neupreises ergeben sich deutliche Unterschiede zwischen den Clustern (vgl. Abb. 28). ${ }^{458}$ Demzufolge haben die Frühen integrierten Internalisierer den deutlich höchsten Produktwert mit Durchschnittswerten von 54.000,- bis 284.000,- DM. Dem folgen die Expertenorientierten Implementierungsminimalisten mit Werten zwischen 10.000,- und 152.000,- DM. Demgegenüber weisen die Autokratischen Ad hoc-Externalisierer mit deutlichem Abstand den geringsten Neupreis (zwischen 280,- und 2.500,- DM) auf. Weiterhin fällt auf, daß die partizipativ-späten Implementierer die geringste Varianz beim Produktwert aufweisen und sich im Mittelfeld befinden. Mit diesem Ergebnis kann die Basishypothese $\mathrm{H}_{\mathrm{PRO1}}$ bestätigt werden. ${ }^{459}$ Offensichtlich findet damit der bereits in Kapitel A 1.1 diskutierte Begriff des Wertschöpfungskreislaufs seine empirische Legitimation. ${ }^{460}$

Bei den Frühen integrierten Internalisierern harmoniert der hohe Neuproduktpreis mit einem schrittweise-evolutorischen Vorgehen bei der Implementierung (step-by-step) bei gleichzeitiger hoher Ausprägung des Zeithorizontes sowie einer Koordination über Richtlinien und Handbücher. Zudem wird deutlich, daß Hersteller hochwertiger Produkte bestrebt sind, das in die Produkte eingebrachte Knowhow möglichst nicht aus der Hand zu geben, so daß ein hoher Internalisierungsgrad rücknahme- und recyclingbezogener Aktivitäten entsteht. Das Involvement der Geschäftsleitung ist zudem offensichtlich insbesondere in der Einführungsphase des Rücknahme- und Recyclingsystems von hochwertigen Produkten hoch, da die Umsatzbedeutung der Produkte entsprechend groß und damit von strategischer Relevanz für das Unternehmen ist.

458 Die Hersteller wurden gebeten, den jeweils niedrigsten und den höchsten Neupreis für die betrachteten Produkte zu nennen. Je nach Modell und Ausführung unterscheiden sich beispielsweise die Preise im Automobilbereich erheblich. Die angegebenen Spannen zeigen jeweils den unteren sowie den oberen durchschnittlichen Neupreis des Produktes.

459 Die späten promotorenorientierten Externalisierer haben ebenfalls einen relativ geringen unteren durchschnittlichen Einstiegspreis von etwa 8.000,- DM. Allerdings befindet sich der obere Durchschnittspreis mit einem Wert vom 77.600,- DM genau im Mittelfeld.

460 Vgl. zu dem Begriff „Wertschöpfungskreisläufe“ z.B. Kirchgeorg, M., Kreislaufwirtschaft - neue Herausforderungen an das Marketing, a.a.O., S. 16ff. 


\section{Neupreise (in DM)}

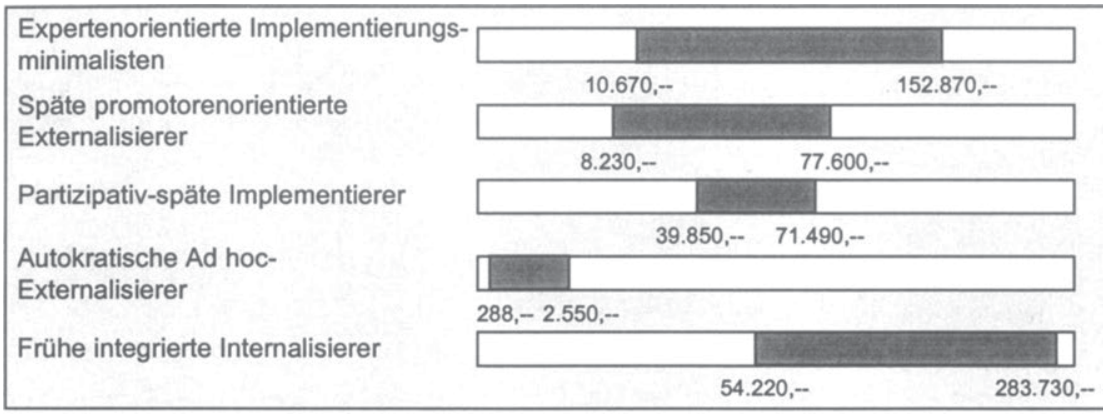

\section{Absatzform (in Prozent)}

\begin{tabular}{|c|c|c|c|}
\hline \multirow{2}{*}{$\begin{array}{l}\text { Expertenorientierte Implementierungs- } \\
\text { minimalisten }\end{array}$} & Verkauf & \multicolumn{2}{|c|}{ Vermietung Leasing } \\
\hline & $82 \%$ & $8 \%$ & $10 \%$ \\
\hline Späte promotorenorientierte & $100 \%$ & & \\
\hline Extemanaseret & & & $1 \%$ \\
\hline Partizipativ-späte Implementierer & $99 \%$ & & \\
\hline Autokratische Ad hoc- & $100 \%$ & & \\
\hline Externalisierer & & $1 \%$ & \\
\hline Frühe integrierte Internalisierer & $90 \%$ & & $9 \%$ \\
\hline
\end{tabular}

\section{Produktkopmplexität/ Anzahl Komponenten (Stück)}

Expertenorientierte Implementierungsminimalisten

Späte promotorenorientierte

Externalisierer

Partizipativ-späte Implementierer

Autokratische Ad hoc-

Externalisierer

Frühe integrierte Internalisierer

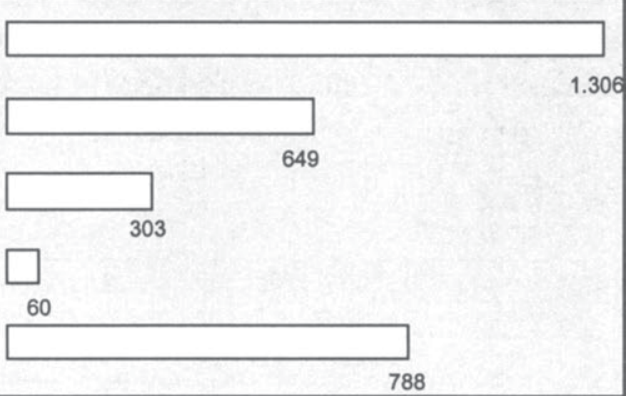

Abb. 28: Zusammenhang zwischen Neupreisen, Absatzform sowie der Produktkomplexität und Implementierungstypen ${ }^{461}$

461 Die Teststatistik zur Überprüfung, ob signifikante Unterschiede zwischen den Implementierungstypen bestehen, geht aus dem Anhang hervor. Vgl. Anhang 2.7.

Irene Giesen-Netzer and Universität Münster -978-3-631-75058-2

Downloaded from PubFactory at 01/11/2019 08:44:49AM 
Bei den Autokratischen Ad hoc-Externalisierern, die mit deutlichem Abstand Produkte mit dem geringsten Neuproduktwert fertigen, wird das Implementierungsverhalten offensichtlich auch deutlich von Produktmerkmalen beeinflußt. Der kurze Zeithorizont und die Durchsetzung der Rücknahme- und Recyclingaktivitäten über das Top-to-down-Prinzip sowie die geringe Partizipationsmöglichkeit der Mitarbeiter, die schnelle Entscheidungsprozesse sicherstellen sollen, werden vor diesem Hintergrund verständlich. Gleichzeitig kann der hohe Externalisierungsgrad der Rücknahme- und Recyclingaktivitäten erklärt werden, da dieser nicht an strategisch relevantes Know-how gebunden ist. ${ }^{462}$

Die im Zusammenhang mit dem Neupreis diskutierte Produktkomplexität und die Absatzform weisen ebenfalls deutliche Unterschiede zwischen den Clustern auf (vgl. Abb. 28). Auffallend sind die Expertenorientierten Implementierungsminimalisten, die deutlich komplexere Produkte als die Frühen integrierten Internalisierer fertigen, die an zweiter Stelle folgen. Auch hinsichtlich der Absatzform unterscheiden sich die Expertenorientierten Implementierungsminimalisten signifikant $(\alpha=0,05)$ von allen übrigen Clustern, wobei auch die Frühen integrierten Internalisierer deutlich mit den betrachteten Merkmalen differenzieren. ${ }^{463}$ Diese beiden Cluster setzen die Absatzform der Vermietung und des Leasing mit $18 \%$ bzw. 10\% (Im Gegensatz zu 0\% bzw. 1\% bei den anderen Implementierungstypen) ein und haben im Durchschnitt ca. 1.300 bzw. 790 Produktkomponenten. Damit können die Hypothesen $\mathrm{H}_{\text {PRO2 }}$ und $\mathrm{H}_{\text {PRO3 }}$ bestätigt werden.

Eine Erklärung dafür, daß die Expertenorientierten Implementierungsminimalisten eine höhere Ausprägung bei den betrachteten Produktmerkmalen (Absatzform und Produktkomplexität) aufweisen als die Frühen integrierten Internalisierer, kann vor dem Hintergrund des Wertschöpfungspotentials gefunden werden: Trotz eines relativ geringeren Neuproduktwertes (im Vergleich zu den Frühen integrierten Implementierern) hätten die Expertenorientierten Implementierungsminimalisten bei einer verstärkten Internalisierung von Rücknahme- und Recyclingaktivitäten aufgrund der höheren Produktkomplexität einen verhältnismäßig höheren Aufwand (z.B. in der Demontage und der Anzahl der Verwertungsfraktionen) zu

462 Bei niedrigen oder sehr niedrigen Neupreisen ist ein vergleichsweise geringer Komplexitätsgrad der Produkte vorzufinden, der eine Externalisierung begünstigt. Als Absatzform nimmt der Verkauf den wichtigsten Stellenwert ein, da Leasing oder Vermietung angesichts des geringen Neupreises kaum durchgeführt werden. 
bewältigen als die Frühen integrierten Internalisierer. Daher sind die ökonomischen Chancen geringer einzustufen als bei den Frühen integrierten Internalisierern, so daß die Expertenorientierten Minimalisten eher vorsichtig agieren.

In enger Verbindung mit den bisher analysierten Produktmerkmalen werden häufig auch Merkmale der Produktkategorie (Investitions- vs. Konsumgüter) ${ }^{464}$ sowie der Produktspezifität ${ }^{465}$ (Anteil der Selbst- bzw. Fremdproduktion) ${ }^{466}$ gesehen.

464 Investitionsgüter sind im Gegensatz zu Konsumgütern, die an private Konsumenten vermarktet werden, Produkte, die in Organisationen zum Einsatz gelangen. Vgl. Meffert, H., Marketing, a.a.O., S. 1115. Zu den Besonderheiten der Kaufentscheidungen von Organisationen (Multipersonalität, Multiorganisationalität) vgl. Backhaus, K., Investitionsgütermarketing, a.a.O., S. $1 \mathrm{ff}$.

Die direkte personale Interaktion zwischen Hersteller und Konsument ist das zentrale Merkmal, welches das Investitionsgütermarketing vom Konsumgütermarketing trennt. Vgl. Meffert, H., Marketing, a.a.O., S. 1116. Dieses Merkmal ist auch bei der Gestaltung und Implementierung von Rücknahme- und Recyclingsystemen von herausragender Bedeutung, da damit der Verbleib der Produkte den Herstellern bekannt ist und eine höhere Kontrolle des Lebensweges des Produktes gegeben ist. Die Rücknahme wird erleichtert, so daß Hersteller von Investitionsgütern die Rücknahmeprozesse vermutlich eher internalisieren als Hersteller von Konsumgütern.

An dieser Stelle muß berücksichtigt werden, daß die Klassifikation zwischen Konsumgütern und Investitionsgütern auf der einen Seite und von Dienstleistungen auf der anderen Seite in der betriebswirtschaftlichen Literatur kritisch diskutiert wird. Es wird angeführt, daß insbesondere Hersteller im Investitionsgütergeschäft im zunehmenden Maße auch Dienstleistungen (z.B. Kundendienst, Finanzierung) anbieten, um ein kundenorientiertes Marketing und damit einen entscheidenden Erfolgsfaktor abzusichern. Vgl. dazu die Ausführungen und die dort angegebene Literatur von Engelhardt, W. H., Kleinaltenkamp, M., Reckenfelderbäumer, M., Leistungsbündel als Absatzobjekte, Ein Ansatz zur Überwindung der Dichotomie von Sach- und Dienstleistungen, in: ZfbF, Heft 5, 1993, S. 395ff. Das Wertschöpfungspotential ist daher ausschließlich auf die rückführbaren materiellen Leistungskomponenten zu beschränken.

Das Verständnis der Spezifität von Ressourcen soll in Anlehnung an Porter und Rasche auf die Spezifität von Produkten übertragen werden. Danach können nach dem Spezialisierungsgrad generelle Faktoren (generalized factors) und problemspezifisch applizierbare Faktoren (specialized factors) unterschieden werden. Generelle Faktoren lassen sich aufgrund ihres geringen Spezialisierungsgrades in vielfältige Verwendungsfelder einordnen und sind dadurch sehr flexibel, während sich spezialisierte Faktoren nur in einigen (oder nur einem) Verwendungsfeld(ern) einsetzen lassen. Spezialisierte Faktoren haben jedoch eine höhere strategische Relevanz für Hersteller, da diese schwer imitierbare Differzierungsvorteile mit sich bringen können. Vgl. Porter, M. E., The Competitive Advantage of Nations, London, Basingstoke 1990, S. 73ff. und vgl. Rasche, Chr., Wettbewerbsvorteile durch Kernkompetenzen, a.a.O., S. $38 \mathrm{ff}$.

Das Merkmal der Produktspezifität ist jedoch nur bedingt durch den Anteil der Fremd- und Selbstproduktion abbildbar, da der Fremdbezugsanteil häufig bei über $50 \%$ der Produktionskosten liegt. Ein weiterer Trend zur Leistungstiefenreduktion besteht, da die Unternehmen bestrebt sind, schnell und flexibel auf kundenspezifische Anforderungen zu reagieren. Dadurch entsteht die Notwendigkeit einer vertrauensvollen und kooperativen Zusammenarbeit zwischen Lieferanten und Herstellern. Vgl. Wildemann, H., Kooperationen über die Wertschöpfungskette, in: Corsten, H., Reiß, M. (Hrsg.), Handbuch Unternehmensführung: Konzepte - Instrumente - Schnittstellen, Wiesbaden 1995, S. 744f. Eine Selbstproduktion von Güter(teile)n findet insbesondere dann statt, wenn Zulieferer nicht in der Lage sind, nicht standardisierte Teile zu liefern. Nicht standardisierte Teile sind i.d.R. notwendig, um Kundenwünschen entsprechen zu können, weshalb dieses Merkmal Hinweise auf die Spezifität von Produkten gibt. 
Diese Merkmale sind ebenfalls als Einflußfaktoren der Implementierung von Rücknahme- und Recyclingsystemen zu untersuchen. ${ }^{467}$ Ist das Einzelgeschäft sehr hochpreisig - wie i.d.R. im Investitionsgütergeschäft -, wird vermutlich gleichzeitig die Spezifität bzw. Individualisierung des Produktes, d.h. die Anpassung des Produktes an den Kunden, sehr hoch sein, womit der Anteil der Selbstproduktion steigt. ${ }^{468}$ Die umgekehrte Argumentation gilt für niedrige Neuproduktpreise, die häufig im Konsumgütergeschäft realisiert werden. ${ }^{469}$ Vor diesem Hintergrund werden folgende Basishypothesen formuliert: $\begin{array}{ll}\text { HPROA }_{\text {: }} & \begin{array}{l}\text { Die Produktkategorie der langlebigen Gebrauchsgüter hat Einfluß auf das Implementie- } \\ \text { rungsverhalten. }\end{array}\end{array}$

HPRO5: Die Spezifität der langlebigen Gebrauchsgüter hat Einfluß auf das Implementierungsverhalten.

Wird der Einfluß der Produktkategorie sowie -spezifität auf die unterschiedlichen Implementierungstypen empirisch analysiert (vgl. Abb. 29), zeigt sich ein relativ unsystematisches Muster. Ein Zusammenhang zwischen Investitionsgüter- bzw. Konsumgütergeschäft und der Preiskategorie besteht nicht und damit kein Einfluß auf das Implementierungsverhalten der Hersteller. Auch hinsichtlich der Produktspezifität ergibt sich kein erkennbarer Zusammenhang. D.h., der Anteil der Fremd- vs. Selbstproduktion vermag die Implementierungstypen nicht signifikant voneinander zu trennen. Das wird an der nahezu erreichten Gleichverteilung der

467 Fischer u.a., die den Aussagegehalt der Transaktionskostentheorie für das Marketing überprüfen, kommen zu der Erkenntnis, daß die Spezifität eines Produktes den Grad der Internalisierung der Distributionsleistung beeinflußt. So ist bei Standardgütern ohne Markierung (unspezifische Güter) eine vertikale Integration nur in unwesentlichem Umfang erkennbar. Vgl. Fischer, M., u.a., Marketing und neuere ökonomische Theorie: Ansätze zu einer Systematisierung, in: BFuP, Heft 4, 1993, S. 461. Dem soll hier gefolgt werden, wobei die Aussage dahingehend erweitert wird, daß die Spezifität eines Produktes auch den Internalisierungsgrad von Redistributionsleistungen und damit das intensitätsbezogene Implementierungsverhalten beeinflußt.

Raabe diskutiert den Einfluß von hochpreisigen Investitionsgütern auf die Möglichkeit der Redistribution ausschließlich durch die Hersteller (herstellereigenes Rücknahme- und Recyclingsystem). Er kommt zu dem Schluß, daß durch den unmittelbaren Kontakt zwischen Hersteller und gewerblichem Abnehmer die Anzahl der Rücknahmefälle beschränkt und überschaubar ist. Zudem hat der Hersteller die Möglichkeit, auf die Qualität der Funktionsausübung Einfluß zu nehmen. Vgl. Raabe, T., Die Elektronik-Schrott-Verordnung, a.a.O., S. 298. Insbesondere der letzte Aspekt ermöglicht ein höherwertiges Recycling und unterstützt damit die Hypothese, daß ein hoher Neuproduktpreis ein Implementierungsverhalten fördert, wie die Frühen integrierten Internalisierer es zeigen.

469 Raabe lehnt herstellereigene Systeme für Konsumgüter mit der Begründung ab, daß ein aufwendiges Sammel- bzw. Holsystem erforderlich und der kommunikative Nutzen aufgrund der Informationsüberflutung des Konsumenten gering wäre. Vgl. Raabe, T., Die Elektronik-SchrottVerordnung, a.a.O., S. 299. Folglich gilt für Konsumgüter ein hoher Grad an Externalisierung. 
Anteile von $60 \%$ zu $40 \%$ bei den Clustern 2, 3 und 4 deutlich. Damit werden die Hypothesen $\mathrm{H}_{\mathrm{PRO}}$ und $\mathrm{H}_{\mathrm{PRO} O 5}$ abgelehnt.

\section{Produktkategorie (in Prozent)}

\begin{tabular}{|c|c|c|}
\hline $\begin{array}{l}\text { Expertenorientierte Implementierung } \\
\text { minimalisten }\end{array}$ & s Investitionsqut & $\begin{array}{c}\text { Konsumgut } \\
34 \% \\
\end{array}$ \\
\hline $\begin{array}{l}\text { Späte promotorenorientierte } \\
\text { Externalisierer }\end{array}$ & $82 \%$ & $18 \%$ \\
\hline Partizipativ-späte Implementierer & $75 \%$ & $25 \%$ \\
\hline $\begin{array}{l}\text { Autokratische Ad hoc- } \\
\text { Externalisierer }\end{array}$ & $59 \%$ & $41 \%$ \\
\hline Frühe integrierte Internalisierer & $78 \%$ & $22 \%$ \\
\hline
\end{tabular}

\section{Spezifität (in Prozent)}

\begin{tabular}{|c|c|c|}
\hline & Selbstproduktion & Fremdproduktion \\
\hline $\begin{array}{l}\text { Expertenorientierte Implementierung } \\
\text { minimalisten }\end{array}$ & $48 \%$ & $52 \%$ \\
\hline $\begin{array}{l}\text { Späte promotorenorientierte } \\
\text { Externalisierer }\end{array}$ & $39 \%$ & $61 \%$ \\
\hline Partizipativ-späte Implementierer & $40 \%$ & $60 \%$ \\
\hline $\begin{array}{l}\text { Autokratische Ad hoc- } \\
\text { Externalisierer }\end{array}$ & $41 \%$ & $59 \%$ \\
\hline Frühe integrierte Internalisierer & $57 \%$ & $43 \%$ \\
\hline
\end{tabular}

Abb. 29: Zusammenhang zwischen Produktkategorie sowie -spezifität und Implementie-
rungstypen

Insgesamt geht aus den Ergebnissen hervor, daß dem Neuproduktpreis und dem damit zusammenhängenden Wertschöpfungspotential die herausragende $\mathrm{Be}$ deutung bei der Analyse der produktbezogenen Einflußfaktoren zukommt. 


\subsubsection{Interne unternehmensbezogene Einflußfaktoren}

\subsubsection{Herstellerdemographie}

Unter der Herstellerdemographie werden verschiedene Merkmale zusammengefaßt, die die Art und Tätigkeit von Unternehmen beschreiben und eine Klassifizierung nach verschiedenen Kriterien wie Größe oder Branche ermöglichen.

Im Bereich der Organisationstheorie liegen umfangreiche Untersuchungen über die organisatorischen Konsequenzen unterschiedlicher Unternehmensgrößen vor, ${ }^{470}$ deren Übertragung auf das Implementierungsverhalten möglich ist. Dabei wird die Unternehmensgröße i.d.R. durch die Zahl der Organisationsmitglieder gemessen, die bei einer Interpretation von Unternehmen als soziale Systeme mit interpersonaler Aufgabenverteilung sinnvoll erscheint. Grundsätzlich wird in den Arbeiten eine positive Korrelation zwischen Unternehmensgröße und dem Grad an Standardisierung und Formalisierung konstatiert. ${ }^{471}$ Gleichzeitig wurde festgestellt, daß mit steigender Größe der Unternehmen der Grad der Entscheidungszentralisierung ${ }^{472}$ abnimmt. Da diese Größen auch das Implementierungsverhalten bei Rücknahme- und Recyclingsystemen betriffen, kann folgende Basishypothese formuliert werden:

$\mathrm{H}_{\mathrm{DEM} 1}: \quad$ Die Unternehmensgröße beeinflußt das Implementierungsverhalten der Hersteller.

Zur Untersuchung der Unternehmensgröße wurde eine Kontingenzanalyse durchgeführt (vgl. Abbildung 30). Der Chi-Quadrat-Test zeigt, daß sich die Cluster hinsichtlich der Unternehmensgröße signifikant voneinander unterscheiden $(\alpha=0,05)$ und damit die Unternehmensgröße offensichtlich Einfluß auf das Implementierungsverhalten der Hersteller ausübt, womit die Hypothese $\mathrm{H}_{\mathrm{DEM} 1}$ bestätigt werden kann. Insgesamt befinden sich 60\% große Unternehmen mit mehr als 500 Mitarbeitern in der Stichprobe, während mittlere und kleine Unternehmen mit jeweils ca. $20 \%$ vertreten sind. Damit wird deutlich, daß größere Unternehmen ver-

\footnotetext{
470 Vgl. Frese, E., Organisationstheorie, a.a.O., S. 116 und die dort angegebene Literatur. Frese erläutert im weiteren vor allem zwei Studien: Die Untersuchung der Aston-Gruppe sowie diejenige von Blau und Schoenherr.

471 Vgl. ebenda, S. 188 und S. 122.

472 Die Entscheidungszentralisierung umfaßt die Frage nach der Verteilung von Entscheidungsaufgaben auf die Führungsebenen von Unternehmen. Vgl. zu dieser Definition Hungenberg, $H$., Zentralisation und Dezentralisation: strategische Entscheidungsverteilung in Konzernen, Wiesbaden 1995, S. 47.
} 
mutlich aufgrund ihrer besseren Ressourcenausstattung und damit auch Informationsvorsprünge sowie größerer Flexibilität, neue Projekte durchzuführen, bessere Möglichkeiten haben, Rücknahme- und Recyclingsysteme einzurichten als kleine und mittelständische Unternehmen. ${ }^{473}$

Große Unternehmen liegen insbesondere bei den Clustern der Expertenorientierten Implementierungsminimalisten und bei den Frühen integrierten Internalisierern vor. Mit jeweils $88,33 \%$ bzw. $68,97 \%$ und mit Abweichungen vom erwarteten statistischen Wert von $23,33 \%$ und $8,62 \%$ wird die Dominanz von großen Unternehmen in diesen Clustern deutlich. Das Ergebnis bei den Frühen integrierten Internalisierern bestätigt die positive Korrelation zwischen von Unternehmensgröße und dem Grad der Standardisierung und Formalisierung. Damit wird deutlich, daß die Wahrscheinlichkeit einer schriftlichen Ausarbeitung von Verfahrensregeln in Organisationen mit spezialisierten Organisationsabteilungen und hohem Koordinationsbedarf größer ist als in kleinen Organisationen ohne solche Spezialabteilungen. ${ }^{474}$ Der negative Zusammenhang der Entscheidungszentralisation und Unternehmensgröße wird bei den Expertenorientierten Implementierungsminimalisten anschaulich, da in diesem Cluster zum einen die Ernennung eines Fachexperten die wichtigste Maßnahme bei der Implementierung eines Rücknahme- und Recyclingsystems darstellt und zum anderen die Verantwortungsübernahme der Geschäftsleitung sowie das Top-to-down-Prinzip mit deutlichem Abstand zu allen übrigen Clustern am geringsten ausgeprägt ist..$^{475}$

473 Eine Befragung des Mittelstandes $(N=220)$, die im Vorfeld der persönlichen Befragung durchgeführt wurde, kam zu dem Ergebnis, daß $53 \%$ der mittelständischen Unternehmen für Großunternehmen wesentlich bessere Chancen sehen, auf das Kreislaufwirtschaftsgesetz zu reagieren als für kleinere Unternehmen. Zu Ergebnissen dieser Studie vgl. Meffert, H., Kirchgeorg, M., Ziele und Strategien zur Gestaltung von produktbezogenen Rücknahme- und Recyclingsystemen, a.a.O., S. 4 ff.

Dyckhoff und Jacobs kommen zu ähnlichen Ergebnissen bezüglich der Einrichtung von Umweltschutzeinheiten: Demzufolge haben große Unternehmen frühzeitig spezielle Umweltschutzabteilungen eingerichtet. Vgl. Dyckhoff, H., Jacobs, R., Organisation des Umweltschutzes in Industriebetrieben, in: ZfB, 1994, Heft 6, S. 723 .

475 Bezüglich des Standardisierungsgrades sind die Expertenorientierten Implementierungsminimalisten allerdings unterdurchschnittlich ausgeprägt, welches für eine eher kleinere Unternehmensgröße spräche. Allerdings kann vermutet werden, daß dies eher auf die generelle Minimierung der Implementierungsaktivitäten zurückzuführen ist als auf die Unternehmensgröße. 


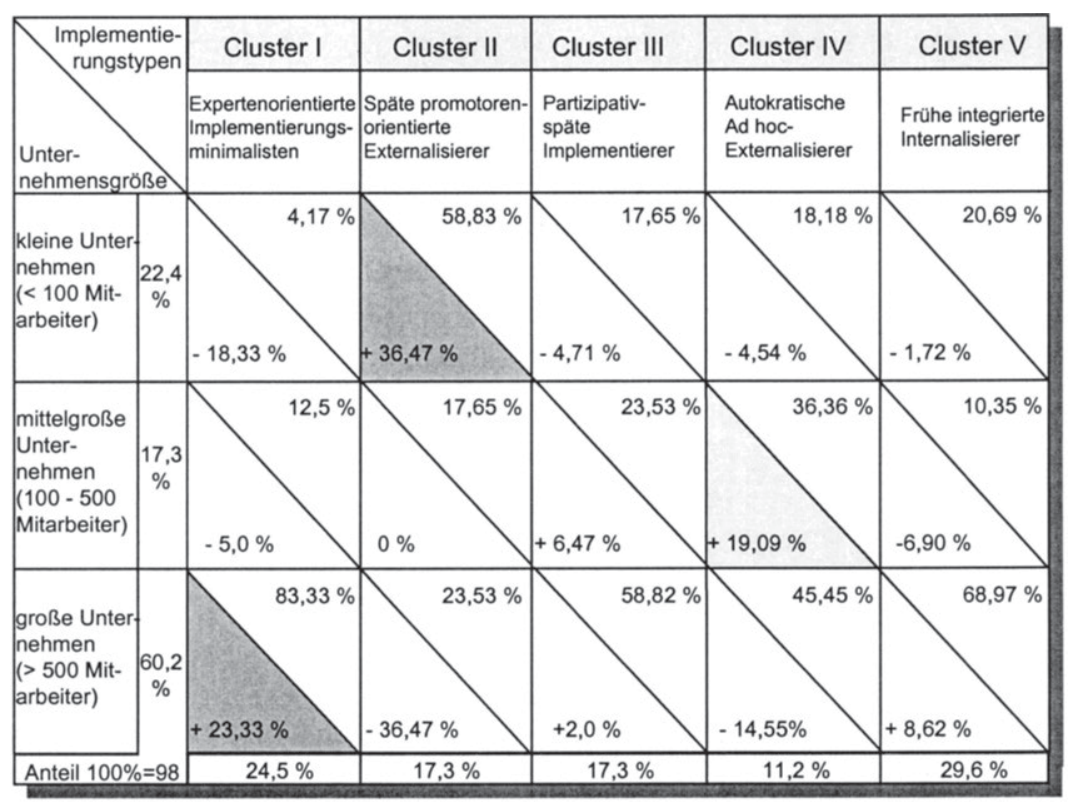

\begin{tabular}{|c|c|c|c|c|}
\hline $\begin{array}{l}\text { Chi-Quadrat-Test } \\
\text { gemäß: }\end{array}$ & $\begin{array}{l}\text { Signifikanz- } \\
\text { niveau: }\end{array}$ & X\%g & & $0 \%-5 \%$ \\
\hline & 0,00195 & $\begin{array}{l}\text { tatsächlicher } \\
\text { Anteil des }\end{array}$ & $\begin{array}{l}\text { Abweichung } \\
\text { vom }\end{array}$ & $5,1 \%-10 \%$ \\
\hline \multirow{2}{*}{$\begin{array}{l}\text { Likelihood } \\
\text { Ratio }\end{array}$} & \multirow[t]{2}{*}{0,00260} & \multirow[t]{2}{*}{$\begin{array}{l}\text { Implemen- } \\
\text { tierungstyps }\end{array}$} & \multirow{2}{*}{$\begin{array}{l}\text { erwarteten } \\
\text { statistischen } \\
\text { Wert }\end{array}$} & $10,1 \%-20 \%$ \\
\hline & & & & $>20 \%$ \\
\hline
\end{tabular}

Abb. 30: Kontingenzanalyse zum Zusammenhang zwischen Unternehmensgröße und Implementierungstypen

Das Cluster der Späten promotorenorientierten Externalisierer besteht zu fast $60 \%$ aus kleinen Herstellern. Auch hier wird der Zusammenhang zwischen Unternehmensgröße und Standardisierung sowie Entscheidungsdezentralisation unterstützt. Bei den Autokratischen Ad hoc-Externalisierern sind hingegen in Relation zu den andern Clustern - signifikant häufiger als dieses statistisch zu erwarten wäre - mittelgroße Unternehmen vertreten. Da dieses Cluster gleichzeitig viele Hersteller umfaßt, die sich an einer Branchenlösung beteiligen, kann die in der Literatur diskutierte Skepsis von mittelständischen Unternehmen gegenüber Ko- 
operationslösungen in dieser Untersuchung nicht erhärtet werden. ${ }^{476}$ Die Ergebnisse beider Cluster zeigen darüber hinaus, daß kleine und mittelständische Unternehmen scheinbar aufgrund von finanziellen oder personellen Engpässen häufig Aktivitäten externalisieren. ${ }^{477}$

Wird die Branche als weiterer Einflußfaktor betrachtet, muß zunächst kritisch angemerkt werden, daß dieser Faktor große Überschneidungen zu der Markt- und Wettbewerbssituation sowie den produktbezogenen Einflußfaktoren aufweist. Zudem wurden die Rücknahmeverordnungen branchenspezifisch formuliert, und die Hersteller orientieren sich jeweils am aktuellen Stand der Verordnungen. ${ }^{478}$ Auch freiwillige Selbstverpflichtungen, denen sich Hersteller ganzer Branchen selbst unterziehen, um Rücknahmeverordnungen zu vermeiden, ${ }^{479}$ spielen hier eine Rolle. Bezüglich der produktbezogenen Einflußfaktoren wird insbesondere der Produktwert große Überschneidungen zur Branche aufweisen, da beispielsweise in der Branche des Bauzubehörs sicherlich geringere Produkteinzelwerte möglich sind (z.B. Badewannen, Fenster) als in der Automobil- oder der Metallund Maschinenbaubranche.

Dennoch soll die Branchenzugehörigkeit als Einflußfaktor auf das Implementierungsverhalten betrachtet werden, da davon ausgegangen werden kann, daß über die Branchenzugehörigkeit die potentielle Umweltgefährdung durch die verwendeten Rohstoffe und Prozeßtechnologien der Hersteller konkretisiert wird. ${ }^{480}$

476 Vgl. Frank, G., Strategische Partnerschaften in mittelständischen Unternehmen, Wiesbaden 1994 sowie Kirchgeorg, M., Kreislaufwirtschaft - Neue Herausforderungen an das Marketing, a.a.O., S. $40 \mathrm{ff}$.

477 Kirchgeorg erläutert, daß kleinere Unternehmen gegenüber Großunternehmen betriebliche Umweltschutzinvestitionen und -aktivitäten durch die Inanspruchnahme unternehmensexterner Einrichtungen substituieren, weil dieses häufig aufgrund der Ressourcensituation nicht anders möglich ist. Vgl. Kirchgeorg, M., Okologieorientiertes Unternehmensverhalten, a.a.O., S. 212.

Kolks spricht in diesem Zusammenhang von dem notwendigen Realisationspotential, welches bei der Strategieimplementierung von den Unternehmen zur Verfügung gestellt werden muß. Dabei erläutert er das leistungswirtschaftliche, finanzielle und innovatorische Potential. Vgl. Kolks, U., Strategieimplementierung, a.a.O., S. 149ff.

Vgl. dazu die Ausführungen in Kapitel C 1.2.

Zumindest wird erwartet, daß freiwillige Selbstverpflichtungen lediglich durch "schlanke" Rücknahmeverordnungen ergänzt werden, um die Rücknahme der Produkte zu gewährleisten. Vgl. Clausen, H., Wacker, H., Ökonomische Modellierung von Rücknahmeverpflichtungen, a.a.O., 33.

480 Vgl. zu derselben Argumentation Kirchgeorg, M., Ökologieorientiertes Unternehmensverhalten, a.a.O., S. 95f. und S. 215. 
Zudem stehen einige Branchen stärker im Interesse der öffentlichen Diskussion um die Umweltfreundlichkeit als andere Branchen. ${ }^{481}$

In der Automobilbranche sind bereits lange Zeit Initiativen bekannt, Rücknahmeund Recyclingsysteme zu implementieren. Direkte, kundenbezogene Werbung mit den Rücknahme- und Recyclingsystemen verschiedener Hersteller in Verbindung mit einem Zahlungsversprechen von bis zu 3000,- DM für die Rückgabe des Altautos verdeutlichen das Interesse der Hersteller, vorhandene Systeme frühzeitig zu nutzen. ${ }^{482}$ In der Elektro- und Elektronikbranche wird ebenfalls intensiv an Verordnungen gearbeitet. Eine Initiative von Herstellern um eine branchenweite Lösung wurde allerdings vom Kartellamt aus wettbewerbsrechtlichen Gründen abgelehnt. ${ }^{43}$ Zudem wurde diskutiert, ob eine Rücknahmeverordnung für den Gesamtbereich der Elektro- und Elektronikgeräte sinnvoll sei, da dann sehr viele verschiedenartige Produkte unter eine Verordnung fielen. Als erste Konsequenz wurde bereits der Entwurf einer Informationstechnologieverordnung von entsprechenden Herstellern bzw. deren Verbänden erarbeitet.

In der Chemie- und Bauzubehörbranche sind hingegen bisher wenige Rücknahme- und Recyclingsysteme bekannt. ${ }^{484} \mathrm{Da}$ bei diesen Produkten aufgrund starker Verschmutzungen oder intensiver Nutzung relativ aufwendige Verfahren notwendig sind, um sie recyceln zu können, ist zu vermuten, daß diese Hersteller

481 Das Automobil wurde bereits in den 80er Jahren als bedeutende Schadstoffquelle für Stickoxide und bleihaltige Emissionen diskutiert. Auch das Entsorgungsproblem wurde frühzeitig erkannt. Vgl. dazu Z.B. Wiedmann, K. W. U.a., Die Überprüfung unternehmerischer Zielsysteme als Voraussetzung eines effizienten Öko-Marketing, in: Ökologisches Marketing, Brand, A., u.a., (Hrsg.), Frankfurt am Main, New York 1988, S. 113 sowie Meffert, H., Ókologieorientierte Marketing- und Werbestrategie der Marke Opel, Hamburg 1990, S. $5 f$.

Die Hersteller Opel und Ford bieten solche Summen für Altautos bei einem gleichzeitigen Kauf bestimmter Neuwagen an. Vgl. o.V., Opel zahlt Abwrackprämie, a.a.O., S. 23. In Italien subventioniert die Regierung die Verschrottung von Altautos bis zu einem Betrag von umgerechnet 2000,- DM (bei gleichzeitigem Neukauf eines Autos), um die Zahl der neuzugelassenen Autos zu erhöhen. Vgl. o.V., Italiens Regierung subventioniert den Autokauf, in: Frankfurter Allgemeine Zeitung, 2.1.1997, S. 9.

Vgl. Bauknecht-Fallstudie, a.a.O.

484

Beispiele für Rücknahme- und Recyclingsysteme in Form von Branchenkooperationen sind die FREI-Intitiative, die PVC-Fenster zurücknimmt, die AgPR (Arbeitsgemeinschaft für PVCBodenbelag Recycling) in Marl sowie die AfDR (Arbeitsgemeinschaft für PVC-DachbahnenRecycling) in Troisdorf. Vgl. dazu AgPU (Arbeitsgemeinschaft PVC und Umwelt) (Hrsg.), Renaissance eines Klassikers, in: Zukunftsfähiges Wirtschaften, Wettlauf mit der Zeit, Reporter spezial, Heft 2, 1995, S. $10 \mathrm{ff}$. 
viele Aufgaben externalisieren ${ }^{485}$ Vor diesem Hintergrund kann folgende Basishypothese verfaßt werden:

$\mathrm{H}_{\mathrm{DEM} 2}$ : In den jeweiligen Branchen liegen die identifizierten Implementierungstypen signifikant unterschiedlich häufig vor.

Angesichts des Chi-Quadrat-Tests beim Zusammenhang zwischen Branche und Implementierungstypen, der die Unterschiedlichkeit des Einflusses signifikant bestätigt, ist davon auszugehen, daß die Branche einen signifikanten Einfluß auf das Implementierungsverhalten ausübt (vgl. Abb. 31). Damit kann die Basishypothese $\mathrm{H}_{\mathrm{DEM} 2}$ bestätigt werden, die auch die Betroffenheit der Branche durch umweltbezogene Anforderungen von Anspruchsgruppen beinhaltet.

Bei den befragten Herstellern der Automobilindustrie handelt es sich zu zwei Dritteln um Frühe integrierte Internalisierer, gleichzeitig stammt dieser Implementierungstyp zu knapp 30\% aus der Automobilindustrie. Diese Befunde entsprechen Abweichungen vom erwarteten statischen Wert in Höhe von über $10 \%$ bzw. $20 \%$. Daran werden die frühzeitigen Implementierungsaktivitäten von Rücknahme- und Recyclingsystemen in der Automobilbranche deutlich. ${ }^{486}$ Die intensive Diskussion der Altautoverordnung sowie der relativ hohe Produktwert i.V.m. der Existenz eines funktionsfähigen Gebrauchtmarktes bei diesem Cluster werden vor dem Hintergrund des Branchenkontextes verständlich.

Die Partizipativ-späten Implementierer können überwiegend der Elektro- und Elektronikindustrie zugeordnet werden (über $70 \%$ dieses Clusters stammen aus dieser Branche). Offensichtlich führt damit auch die Verunsicherung der Branche über den Erlaß einer Verordnung bei gleichzeitiger Unbestimmtheit hinsichtlich Art und Umfang dazu, daß sich dieses Cluster erst spät engagiert und darüber hinaus viele Aufgaben externalisiert. Die Chemie- und Bauzubehörbranche ist bei den Autokratischen Ad hoc-Externalisierern vorherrschend. Die auch im Zusammenhang mit dem relativ geringen Produktwert diskutierten Externalisierungsaktivitäten werden vor dem Hintergrund der bisher noch geringen Anzahl an Rücknahme- und Recyclingsystemen in dieser Branche begreiflich.

485 Vgl. FREI-Fallstudie, a.a.O.

486 Zudem ergab eine Untersuchung von Dyckhoff und Jacobs, daß KFZ-Hersteller einen hohen Handlungsbedarf im Bereich der Entsorgung und des Recycling sehen. Vgl. Dyckhoff, H., Jacobs, R., Organisation des Umweltschutzes in Industriebetrieben, a.a.O., S. 721. 


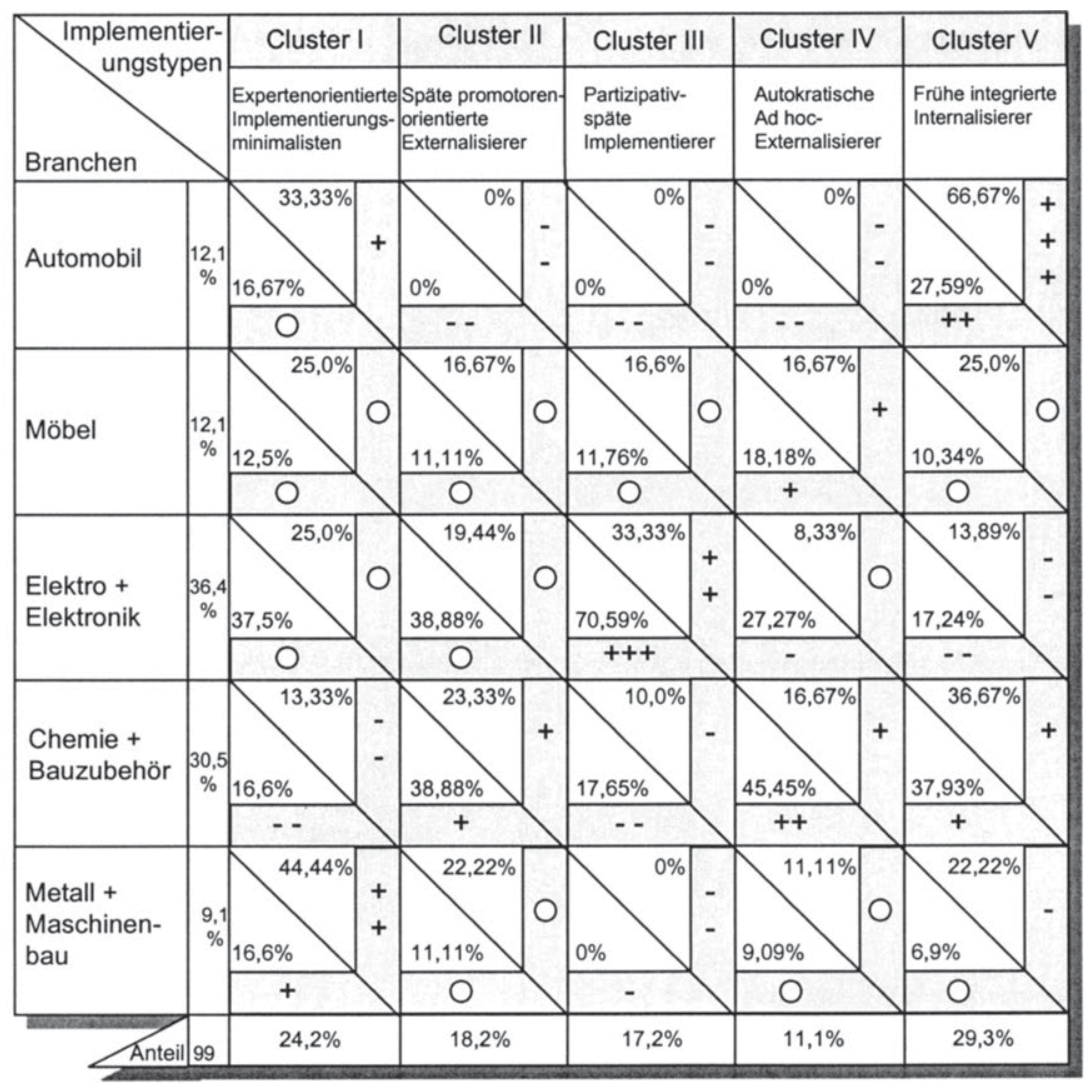

\begin{tabular}{|c|c|c|c|c|c|c|c|c|}
\hline $\begin{array}{l}\text { Chi-Quadrat-Test } \\
\text { gemăß: }\end{array}$ & $\begin{array}{l}\text { Signifikanz- } \\
\text { niveau: }\end{array}$ & $x \%$ & $\times \%$ & \begin{tabular}{|c|}
$\begin{array}{c}\text { Abweichung } \\
\text { vom er- } \\
\text { warteten Wert }\end{array}$ \\
\end{tabular} & \begin{tabular}{|l|}
$0 \%-5 \%$ \\
\end{tabular} & $5,1 \%-10 \%$ & $\begin{array}{r}10,1 \%- \\
20 \% \\
\end{array}$ & $>20 \%$ \\
\hline $\begin{array}{l}\text { Pearson } \\
\text { Likelihood } \\
\text { Ratio }\end{array}$ & 0,02901 & \begin{tabular}{|l|} 
tatsăch- \\
licher \\
Anteil des \\
Implemen- \\
tierungs- \\
typs
\end{tabular} & $\begin{array}{l}\text { tatsäch- } \\
\text { licher } \\
\text { Anteil der } \\
\text { Branche }\end{array}$ & $\begin{array}{l}\text { positiv } \\
\text { negativ }\end{array}$ & $\begin{array}{l}0 \\
0\end{array}$ & $\begin{array}{l}+ \\
-\end{array}$ & $\begin{array}{l}++ \\
--\end{array}$ & $\begin{array}{l}+++ \\
---\end{array}$ \\
\hline
\end{tabular}

Abb. 31: Zusammenhang zwischen Branche und Implementierungstypen

Ein überraschendes Ergebnis liegt bei den Expertenorientierten Implementierungsminimalisten vor, die zu knapp $45 \%$ aus Herstellern der Metall- und Maschinenbaubranche bestehen - bei einer Abweichung vom statistisch erwarteten Irene Giesen-Netzer and Universität Münster - 978-3-631-75058-2 
Wert von knapp 20\%. Eine Erklärung kann hier insbesondere im Zusammenhang mit dem hohen Produktwert einerseits und der hohen Komplexität der Produkte andererseits gefunden werden, die insbesondere Güter der Metall- und Maschinenbaubranche auszeichnet.

\subsubsection{Strategien der Hersteller}

Bei der Analyse des Einflusses der Strategien auf die Implementierung von Rücknahme- und Recyclingsystemen steht die inhaltliche Ausrichtung der Wettbewerbsstrategien im Vordergrund. ${ }^{487}$ Diese spielt eine besondere Rolle, da ein Fit zwischen dem Rücknahme- und Recyclingsystem und der Wettbewerbsstrategie wesentlich zum Aufbau eines ökologiebezogenen Wettbewerbsvorteils beiträgt, ${ }^{488}$ der wiederum als Voraussetzung für eine erfolgreiche Implementierung von Rücknahme- und Recyclingsystemen berücksichtigt werden sollte.

Zunächst stellt sich die Frage, ob und ggf. auf welche Weise sich ein Rücknahmeund Recyclingsystem mit einer Qualitätsführerschaft in Verbindung bringen läßt. Zum einen bestehen Befürchtungen der Hersteller darüber, daß Kunden Produkte mit einem Anteil von Gebrauchtteilen oder aus recyceltem Material als qualitätsmäßig minderwertig ansehen. Darüber hinaus kann das Angebot von gebrauchten Geräten, die günstiger als Neuprodukte angeboten werden, den eigenen Neuprodukten Konkurrenz bieten, d.h. es entstehen möglicherweise Kannibalisierungseffekte. Dieser Argumentation zufolge läßt sich eine Qualitätsführerschaft nicht mit einem Implementierungsverhalten bei Rücknahme- und Recyclingsystemen vereinbaren, bei dem die Hersteller viele Aktivitäten übernehmen und Altprodukt(teil)e in den eigenen Produktionsprozeß einbringen. Auf der anderen Seite spricht jedoch gerade ein integriertes Implementierungsvorgehen dafür, daß die

487 Porter leitet sogenannte generische Wettbewerbsstrategien ab (Kostenführerschaft „overall cost leadership", Qualitätsführerschaft "differentiation" und Nischenstrategie "focus"). Eine Wettbewerbsstrategie hat den Aufbau einer marktbezogenen Vorteilsposition zum Ziel, um im Wettbewerb zu bestehen. Nach Porter versucht ein Unternehmen bei einer Kostenführerschaft, die Stückkosten unter das Niveau der Konkurenten zu senken, um dann mit relativ niedrigen Preisen Wettbewerbsvorteile zu realisieren. Bei einer Qualitätsführerschaft versuchen die Unternehmen, mit Produkt- bzw. Leistungsvorteilen den differenzierten Ansprüchen der Konsumenten besser als die Wettbewerber gerecht zu werden. Die Nischenstrategie schließlich versucht, durch die Spezialisierung auf bestimmte Zielgruppen Wettbewerbsvorteile zu erzielen. Vgl. Porter, M. E., Competitive Strategy, Techniques for Analyzing Industry an Competitors, New York 1980, S. 35ff. sowie Porter, M.E., Competitive Advantage, Creating Meffert, H., Sustainable Development, a.a.O., S. 31 f. 
Hersteller bemüht sind, die Kontrolle über den Prozeß der Rücknahme und des Recycling der eigenen Produkte zu behalten, um auch dort Qualitätsstandards einhalten zu können und negative Auswirkungen auf ihren Markenwert zu vermeiden.

Bei einer Kostenführerschaft sprechen hingegen insbesondere die i.d.R. verfolgte Volumenstrategie der Hersteller sowie die geringen Serviceleistungen und die auf kurze Produktlebensdauer produzierten Produkte gegen eine umfassende, integrierte Implementierung von Rücknahme- und Recyclingsystemen. Andererseits kann vor allem durch einen hohen Standardisierungsgrad der Produkte ein Einsatz von Altprodukt(teil)en im Service oder in der Neuproduktion eine Implementierung von Rücknahme- und Recyclingsystemen unterstützen.

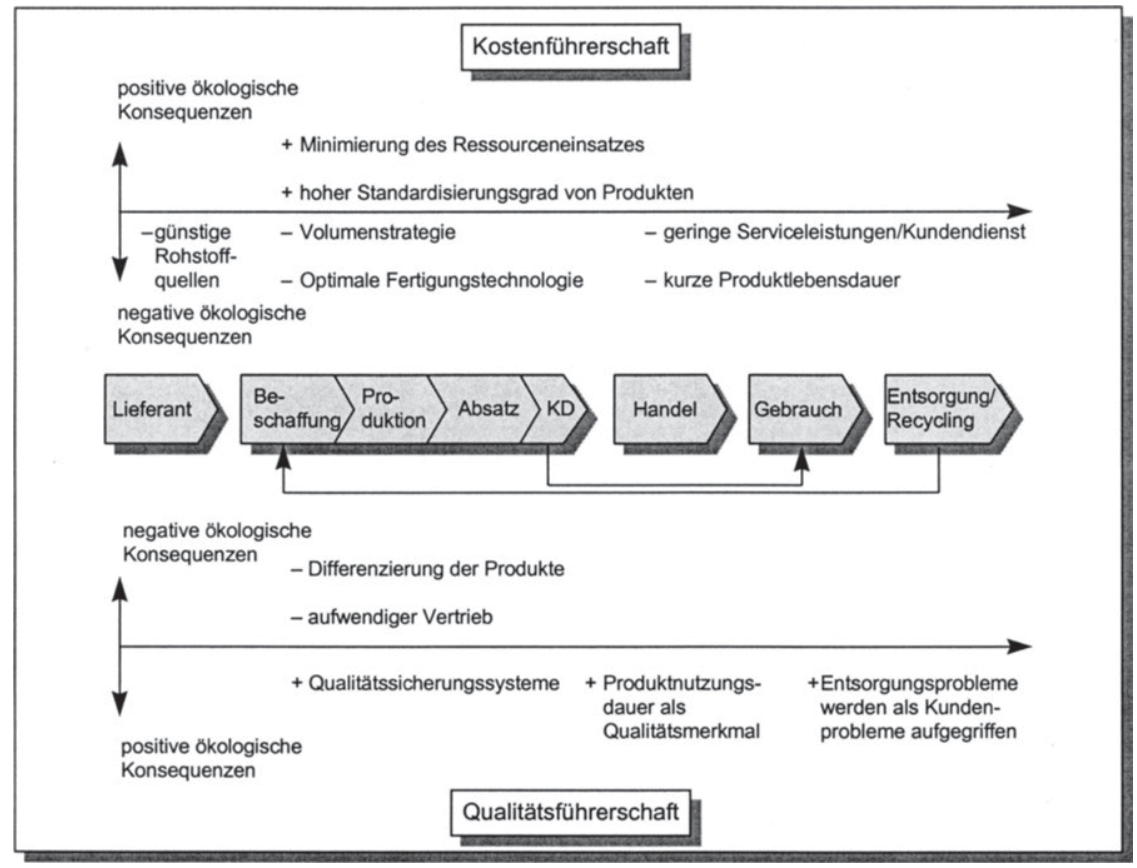

Abb. 32: Ökologische Auswirkungen von Wettbewerbsstrategien

(In Anlehnung an: Meffert, H., Sustainable Development, a.a.O., S. 34) 
Differenziert nach Kosten- und Qualitätsführer, ergeben sich ökologische Konsequenzen, die in Abbildung 32 dargestellt sind. Dabei sind erste Ansatzpunkte erkennbar, die auf die konkrete Fragestellung der Implementierung von Rücknahme- und Recyclingsystemen hinweisen.

Wird eine Nischenstrategie seitens der Hersteller verfolgt, fertigen die Hersteller für einen begrenzten Kundenkreis langlebige Gebrauchsgüter. In dieser Nische sind sie aufgrund ihrer zielgruppenspezifischen Kompetenz von anderen Wettbewerbern relativ unabhängig. Ohne Berücksichtigung des jeweils in der Nische verfolgten Wettbewerbsvorteils (Qualität vs. Kosten) läßt sich jedoch keine klare Aussage hinsichtlich eines bestimmten Implementierungsverhaltens treffen. ${ }^{489}$

Aus dieser Ambivalenz wird deutlich, daß sich das Implementierungsverhalten bei Rücknahme- und Recyclingsystemen zunächst weniger danach richten wird, welche Wettbewerbsstrategie angestrebt wird, sondern zunächst gefragt werden muß, welche umweltbezogene Strategie bereits verfolgt oder angestrebt wird..$^{490}$ Diese Ausführungen lassen sich in folgender Hypothese abbilden:

$\mathrm{H}_{\mathrm{STR} 1}$ : Das Implementierungsverhalten bei Rücknahme- und Recyclingsystemen hängt von der wettbewerbsbezogenen und umweltbezogenen Strategiekonstellation der Hersteller ab.

Abbildung 33 zeigt das Mittelwertprofil der Ausprägungen der wettbewerbs- und umweltbezogenen Strategie der Hersteller sowie die Abweichung der Cluster von den jeweiligen Mittelwerten. Die F-Werte zeigen einen signifikanten Mittelwertunterschied $(\alpha=10 \%)$ bei mindestens zwei Implementierungstypen. Insbesondere fallen zum einen die Expertenorientierten Implementierungsminimalisten und zum anderen die Späten promotorenorientierten Externalisierer auf, die sich beim mul-

An 4 Aieser Stelle wird zugleich die Kritik deutlich, die oftmals an den generischen Wettbewerbsvorteilen Porters geübt wird. Kernpunkt dieser Kritik sind die unterschiedlichen Argumentationsebenen, da sich die Nischenstrategie strenggenommen lediglich auf eine mögliche Produkt-/Marktkombination bezieht. Vgl. z.B. Meffert, H., Marketing-Management, a.a.O., S. 115.

490 Im Rahmen von offensiven Umweltstrategien bildet die Einbeziehung ökologischer Erfordernisse bei der Planung, Koordination, Durchsetzung und Kontrolle der Unternehmensaktivitäten den Ausgangspunkt, um in allen Unternehmensfunktionen mögliche Umweltbelastungen zu vermeiden oder zu vermindern. Defensive Strategien hingegen werden durch Ignorieren von Umweltproblemen und Konfrontation mit Umweltschutzforderungen deutlich. Vgl. zur genauen Begriffsbestimmung Kirchgeorg, M., Ökologieorientiertes Unternehmensverhalten, a.a.O., S. 15f. Außerdem erläutert Kirchgeorg Klassifikationsmerkmale umweltorientierter Unternehmensstrategien. Vgl. ebenda, S. 43ff. 
tiplen Mittelwertvergleichstest in drei von vier Variablen signifikant unterscheiden. Darüber hinaus belegt der multiple Mittelwertvergleichstest auch, daß sich die Frühen integrierten Internalisierer in vielen Variablen von den Späten promotorenorientierten Externalisierern und den Partizipativ-späten Implementierern signifikant unterscheiden.
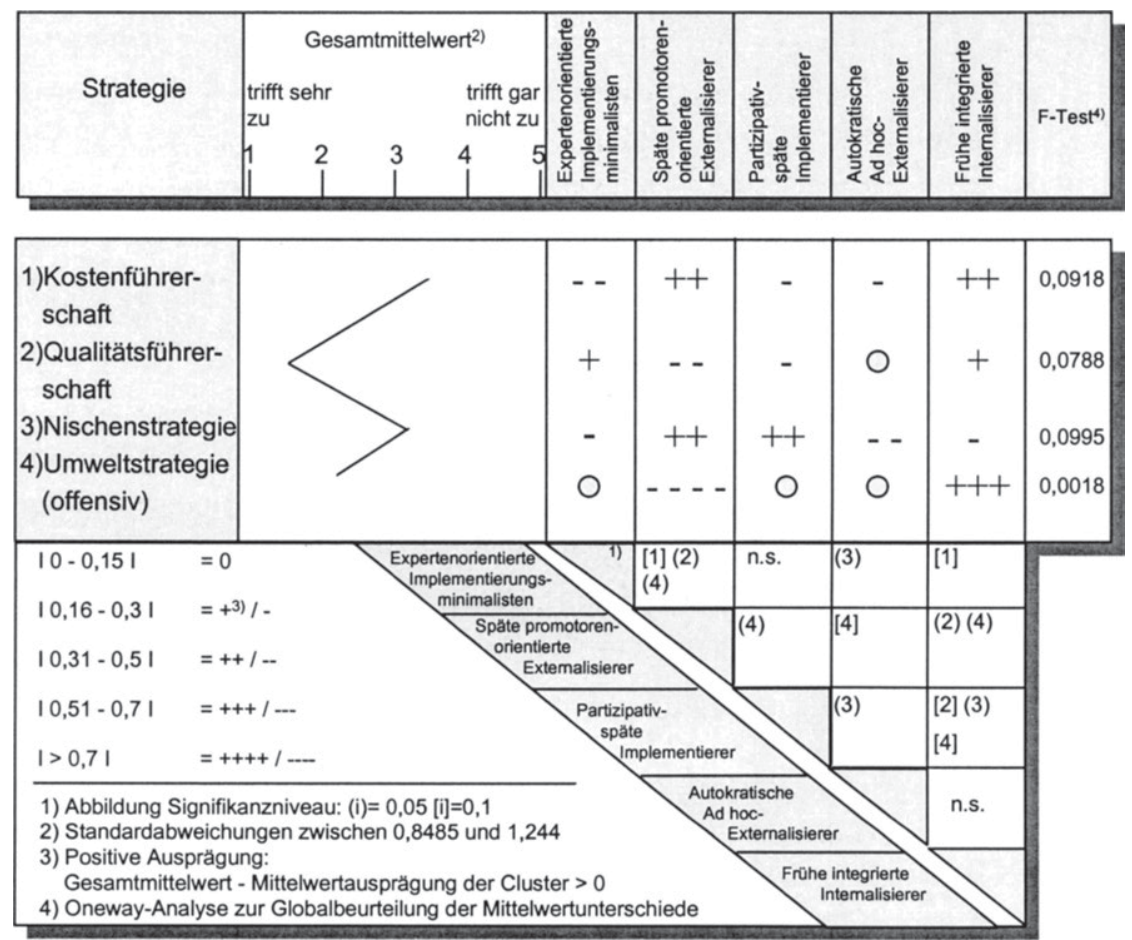

\section{Abb. 33: Zusammenhang zwischen Strategie und Implementierungstypen}

Bei einer Gesamtbetrachtung der Ausprägungen der Mittelwerte ist die sehr hohe Ausprägung der Qualitätsführerschaft mit einem Mittelwert von 1,61 bemerkenswert. Damit scheint der skizzierte Widerspruch zwischen der Qualitätsführerschaft einerseits und der Implementierung von Rücknahme- und Recyclingsystemen andererseits unbegründet zu sein. Demgegenüber ist der Zusammenhang zwischen der umweltbezogenen Strategie einerseits und der Wettbewerbsstrategie andererseits, die in ihrer Kombination das Implementierungsverhalten bei 
Rücknahme- und Recyclingsystemen beeinflussen, zu erhärten. Damit kann die Basishypothese $\mathrm{H}_{\mathrm{STR} 1}$ insgesamt voll bestätigt werden.

Eine defensive Umweltstrategie bei gleichzeitiger Kostenführerschaft (in der Nische) führt zu einem Implementierungsverhalten, wie es die Späten promotorenorientierten Externalisierer zeigen. Die Konsequenz einer derartigen Strategiekonstellation besteht scheinbar darin, daß diese Unternehmen viele Aktivitäten externalisieren, um die eigenen Unternehmensressourcen nicht zu belasten. ${ }^{491}$ Vor diesem Hintergrund wird auch einsichtig, warum diese Hersteller die Verantwortungsübernahme auf der Geschäftsführungsebene belassen und gleichzeitig nach dem Top-to-down-Prinzip handeln. Auch liegt es bei dieser Strategiekonstellation offenbar nicht im Interesse der Hersteller, frühzeitig mit der Implementierung eines Rücknahme- und Recyclingsystems zu beginnen.

Bei einer Verfolgung einer eher indifferenten Umweltstrategie, d.h. diese Unternehmen sind weder als offensiv noch als defensiv zu klassifizieren, und gleichzeitiger Qualitätsführerschaft sind häufig Expertenorientierte Implementierungsminimalisten vorzufinden. Vor dem Hintergrund der indifferenten Umweltstrategie wird das eher bedächtige Vorgehen dieses Clusters bei der Implementierung eines Rücknahme- und Recyclingsystems verständlich.

Schließlich verfolgen die Frühen integrierten Internalisierer eine offensive Umweltschutzstrategie bei gleichzeitiger Qualitätsführerschaft. ${ }^{492}$ Offensichtlich besitzt der Wunsch dieses Clusters, auch bei der Implementeirung von Rücknahme- und Recyclingsystemen Qualitätsstandards zu setzen, ein besonderes Gewicht. Auffällig ist jedoch, daß die Frühen integrierten Implementierer gleichzeitig eine Kostenführerschaft anstreben, ${ }^{493}$ wenn auch nicht im gleichen Ausmaß wie die Qualitätsführerschaft. ${ }^{494}$ Dieses Verhalten wird in der Literatur unter dem Begriff der Outpacing-Strategien diskutiert, die auch dynamische Aspekte der Wettbe-

491 Bei der Externalisierung von Aktivitäten fallen für die Hersteller vereinbarte Entgelte für Leistungen Dritter an, so daß die finanzielle Ressourcenbelastung planbar und mit relativ geringer Unsicherheit behaftet ist.

492 Als Beispiel kann die Implementierung des Rücknahme- und Recyclingsystems für Kopierer der Firma Rank Xerox angesehen werden. Vgl. Rank-Xerox Fallstudie, a.a.O.

493 Hinsichtlich der Kostenführerschaft unterscheiden sich die Frühen integrierten Internalisierer jedoch nur gegenüber Cluster 1 signifikant.

494 Der Mittelwert der Frühen integrierten Internalisierer bei der Qualitătsführerschaft liegt bei 1,38 , während er bei der Kostenführerschaft bei 3,04 liegt.

Irene Giesen-Netzer and Universität Münster - 978-3-631-75058-2 
werbsstrategien berücksichtigen. Demnach wechseln die Unternehmen in Abhängigkeit von spezifischen Wettbewerbsparametern zwischen Qualitäts- und Kostenführerschaft, um Wettbewerbsvorteile zu erzielen, so daß die klassischen eindimensionalen Wettbewerbsstrategien zu einer ganzheitlichen Strategie verschmelzen. ${ }^{495}$

\subsubsection{Herstellerpotentiale}

Nach der These, die Chandler bereits 1962 formulierte, sollte die Struktur der Strategie folgen (,structure follows strategy"). Dieser in der Literatur vehement diskutierten Annahme ging die Erfahrung Chandlers voraus, daß sich bestimmte Strategien nur in bestimmten Strukturen realisieren lassen. ${ }^{496}$ Die Implementierungsliteratur nimmt diese These auf und fordert darüber hinaus neben der Anpassung der Struktur (an die Strategie) eine Anpassung der Kultur und der Systeme - und damit der gesamten Unternehmenspotentiale. ${ }^{497}$

Grundsätzlich soll diesem Grundgedanken in der vorliegenden Arbeit gefolgt werden. Dennoch findet eine etwas andere Perspektive der Anpassung der Unternehmenspotentiale statt. Es wird gefragt, welchen Einfluß verschiedene Anpassungsmöglichkeiten auf das Implementierungsverhalten der Hersteller haben. D.h. es existiert nicht nur ein "Strategie-Potentiale"-Zusammenhang, sondern ein "Strategie-Verhalten-Potentiale“-Zusammenhang. Darüber hinaus ist zu berücksichtigen, daß eine Einflußrichtung der Potentiale auf das Implementierungsver-

495 Zu Outpacing-Strategien vgl. Gilbert, X., Strebel, P. J., Outpacing Strategies, in: IMEDE, Perspektives for Managers, Nr. 2, 1985 und Meffert, H., Marketing-Management, a.a.O., S. $116 \mathrm{ff}$. und die dort angegebene Literatur.

Chandler konnte in Zeitreihen bei vier US-amerikanischen Unternehmen nachweisen, daß sich unterschiedliche Organisationsstrukturen auf die jeweils verfolgte Wachstumsstrategie zurückführen ließen. Vgl. Chandler, A. D., Strategy and Structure, a.a.O., S. 15.

Einige Autoren kehren diese These sogar um und behaupten, daß die Organisationsstrukturen die Strategien beeinflussen. Vgl. z.B. Hall, D. J., Saias, M. A., Strategy follows Structure!, in: SMJ, Nr. 2, 1980, S. 150 und vgl. Miles, R. E., Snow, C. C., Organisational Strategy, Strucure and Process, New York 1978, S. 7 ff.

497 Vgl. z.B. Meffert, H., Marketing-Management, a.a.O., S. 368 und Hilker, J., Marketingimplementierung, a.a.O., S. 61ff. sowie die dort angegebene Literatur. Hilker analysiert zwölf Arbeiten, die zwischen 1982 und 1990 veröffentlicht wurden, zu den untersuchten Einflußfaktoren der Implementierung und hebt diejenigen Einflußfaktoren hervor, die besonders detailliert behandelt wurden. Daran wird deutlich, daß insbesondere die Struktur sowie die Kultur als Einflußfaktoren untersucht wurden. Darüber hinaus fanden jedoch auch Systeme, Personal wie auch Führung Eingang in die Einflußfaktoren. 
halten zwar richtig ist, aber auch umgekehrte Einflußrichtungen vorliegen. ${ }^{488} \mathrm{Da}$ jedoch unterstellt werden kann, daß das Implementierungsverhalten u.a. durch die Anpassung der Potentiale zum Ausdruck kommt, ist diese Einflußrichtung von höherer Priorität und wird analysiert. ${ }^{499}$

Bei der Diskussion der Organisationsstruktur ${ }^{500}$ im Zusammenhang mit der Implementierung eines Rücknahme- und Recyclingsystems geht es darum, geeignete organisatorische Maßnahmen zu finden, die den Anforderungen von Rücknahme- und Recyclingsystemen hinsichtlich der Koordination, Flexibilität sowie Entscheidungsqualität gerecht werden. ${ }^{501} \mathrm{Zu}$ diesem Zweck können zeitlich befristete, multipersonale interdisziplinäre Lösungen wie Workshops oder Projektgruppen gebildet werden und/oder auf Dauer angelegte Veränderungen in der Organisationsstruktur wie zusätzliche Stellen (Stab und/oder Linie ${ }^{502}$ ), die Integration der Aufgaben in eine Stelle oder die Schaffung eines eigenen Unternehmensbereichs durchgeführt werden. Die Anbindung solcher organisatorischen Maßnahmen er-

498 Dies folgt dem Gedanken, daß Chandlers These zwar zu deterministisch ist, grundsătzlich aber gilt. Vgl. z.B. Kolks, U., Strategieimplementierung, a.a.O., S. 131f.

499 Dabei ist zu berücksichtigen, daß Maßnahmen der Potentialanpassung aufgrund der Umsetzung des Rücknahme- und Recyclingsystems nicht nur das Implementierungsverhalten beeinflussen, sondern auch bestehende Potentiale durchaus das Implementierungsverhalten determinieren.

Die Organisationsstruktur soll hier in Anlehnung an Kieser und Kubicek verstanden werden als „Menge von Regelungen für die Aktivitäten der Organisationsmitglieder, die auf am Organisationsziel orientierten Zweckmäßigkeitsüberlegungen beruhen und durch einen offiziellen Akt oder durch Duldung autorisiert sind". Kieser, A., Kubicek, H., Organisation, a.a.O., S. 15.

Die Ablauforganisation soll im weiteren nicht untersucht werden, da sie die Abstimmung der verschiedenen Teilaktivitäten zum Inhalt hat. Dabei geht es um die inhaltliche und zeitliche Dimensionierung der einem Aufgabenträger übertragenen Aufgaben, deren effiziente Lösung ein komplexes Problem darstellt. Vgl. zu dieser Auffassung, Frese, E., Organisationstheorie, a.a.O., S. 252.

Dennoch erfordert die Einführung eines systematischen Umweltmanagment (wie auch eines Rücknahme- und Recyclingsystems, A.d.V.) häufig eine Änderung von Arbeitsabläufen, die eine hohe Motivation der Mitarbeiter benötigt. Vgl. Someren, van T. C. R., Einführung von Umweltmanagementsystemen: das Wachstumsmodell, in: UWF, Heft 6, 1994, S. 63.

501 Vgl. Kolks, U., Strategieimplementierung, a.a.O., S. 227.

Kolks leitet unterschiedliche strategieunterstützende und behindernde Strukurmerkmale ab: Spezialisierung, Koordination, Leitungssystem, Entscheidungsdelegation und Formalisierung. Vgl. ebenda, S. $132 \mathrm{ff}$.

502 Stabsstellen haben im Gegensatz zu Linienstellen keine formellen Entscheidungs- und Weisungsrechte. Stäbe haben in der Regel die Aufgabe der Entscheidungsvorbereitung und können daher als Fachpromotoren einen Implementierungsprozeß unterstützen. Vgl. Frese, E., Grundlagen der Organisation, a.a.O., S. 90 und $215 f$. 
scheint wichtig, um die grundsätzlichen Arbeitsschwerpunkte und den Integrationsgrad verschiedener Funktionen erkennen zu können. ${ }^{503}$

Kolks stellt bereits 1988 bei einer Expertenbefragung fest, daß alle Experten die Bildung von Projektgruppen als geeignete organisatorische Maßnahme zur Strategieimplementierung ansehen und darüber hinaus über $80 \%$ der Befragten die Einbindung der Geschäftsleitung als notwendig erachten. ${ }^{504}$ Daher ist zu vermuten, daß auch von den in der vorliegenden Arbeit gebildeten Clustern diese Maßnahmen oft - wenn auch in unterschiedlicher Häufigkeit - ergriffen werden. Die Integration von rücknahme- und recyclingsystemspezifischen Aufgaben in Linienstellen ist mit relativ geringem Aufwand vom Unternehmen durchführbar, weshalb zu vermuten ist, daß auch diese Maßnahme bei allen Implementierungstypen relativ wichtig ist. Die Schaffung neuer Stellen (in Form von Linien oder Stäben) deutet hingegen eher darauf hin, daß die Hersteller Expertenwissen ansammeln und nutzen wollen (interne Spezialisierung). Bei der Durchführung von Workshops, in denen sich Teilnehmer meist in mehrtägigen Veranstaltungen einem bestimmten Thema zuwenden, werden sich vermutlich solche Hersteller engagieren, die Aufgaben der Implementierung von Rücknahme- und Recyclingsystemen internalisieren wollen, da Ziele eines Workshops im Erfahrungsaustausch liegen können. Vor diesem Hintergrund wird folgende Basishypothese verfaßt:

$\mathrm{H}_{\text {POT1 }}$ : Die Anpassungen der Unternehmensstruktur beeinflussen das jeweilige Implementierungsverhalten der Hersteller.

Die Abbildungen 34a/b und 35a/b zeigen, nach Implementierungstypen differenziert, die zeitlich befristeten und die auf Dauer angelegten organisatorischen Maßnahmen sowie ihre jeweilige Anbindung an die Unternehmensfunktion.

503 Galbraith und Kazanjian beschreiben, daß die Einbeziehung von verschiedenen Abteilungen eine Rolle für den Erfolg oder den Mißerfolg einer Strategieumsetzung von Unternehmen spielt. So sei es wichtig, nicht solche Abteilungen einzubeziehen, die generell wichtig sind, sondern nur solche, die dem Problem angemessen wären. Vgl. Galbraith, J. R., Kazanjian, R. K., Strategy Implementation, a.a.O., S. $36 \mathrm{ff}$.

504 Vgl. Kolks, U., Praktische Probleme und organisatorische Lösungsansätze der Strategieimplementierung - Dokumentation einer explorativen Studie, Arbeitspapier Nr.1 des Lehrstuhls BWL II der Justus-Liebig-Universität Gießen, Gießen 1988, S. $99 \mathrm{ff}$. 
Gesamte Stichprobe (Mehrfachantworten waren möglich)

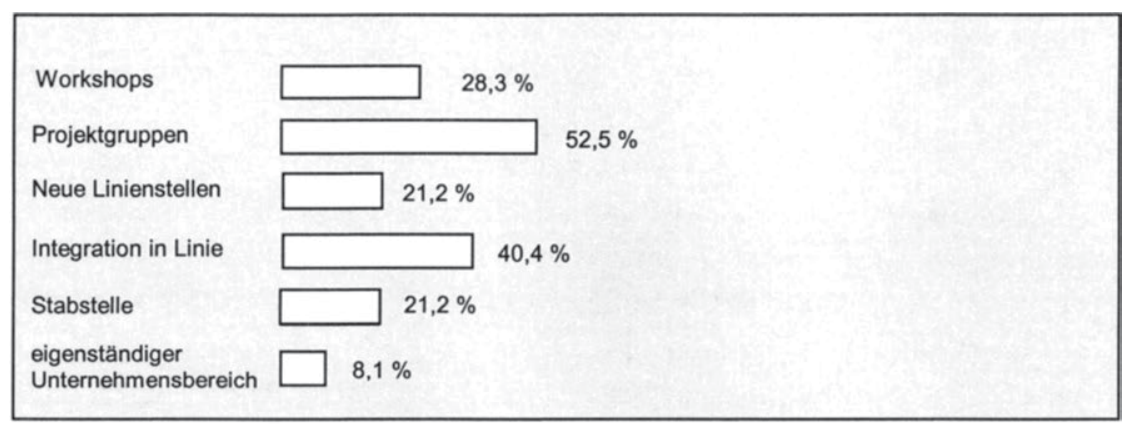

\section{Expertenorientierte Implementierungsminimalisten}

\begin{tabular}{|c|c|c|c|c|c|}
\hline Workshops & & $25 \%$ & & $\begin{array}{l}\text { Abweichung } \\
\text { statistis } \\
-3,3 \%\end{array}$ & $\begin{array}{l}\text { m erwarteten } \\
\text { en Wert }\end{array}$ \\
\hline Projektgruppen & & & $50 \%$ & $-2,5 \%$ & \\
\hline Neue Linienstellen & & $29,2 \%$ & & & $+7,9 \%$ \\
\hline Integration in Linie & & $25,0 \%$ & & $-15,4 \%$ & \\
\hline Stabstelle & & $25 \%$ & & & $+3,8 \%$ \\
\hline $\begin{array}{l}\text { eigenständiger } \\
\text { Unternehmensbereich }\end{array}$ & $8,3 \%$ & & & & $+0,2 \%$ \\
\hline
\end{tabular}

\section{Späte promotorenorientierte Externalisierer}

\begin{tabular}{|c|c|c|c|c|}
\hline & & & $\begin{array}{l}\text { Abweichung } \\
\text { statistis }\end{array}$ & $\begin{array}{l}\text { erwarteten } \\
\text { Wert }\end{array}$ \\
\hline Workshops & & $22,2 \%$ & $-6,1 \%$ & \\
\hline Projektgruppen & & $27,8 \%$ & $-25 \%$ & \\
\hline Neue Linienstellen & $5,6 \%$ & & $-15,5 \%$ & \\
\hline Integration in Linie & & $44,4 \%$ & & $+3,8 \%$ \\
\hline Stabstelle & & $16,7 \%$ & $-4,4 \%$ & \\
\hline $\begin{array}{l}\text { eigenstăndiger } \\
\text { Unternehmensbereich }\end{array}$ & $0 \%$ & & $-8,1 \%$ & \\
\hline
\end{tabular}

Abb. 34a: Organisatorische Maßnahmen zur Implementierung von Rücknahme- und
Recyclingsystemen differenziert nach Implementierungstypen 
Partizipativ-späte Implementierer

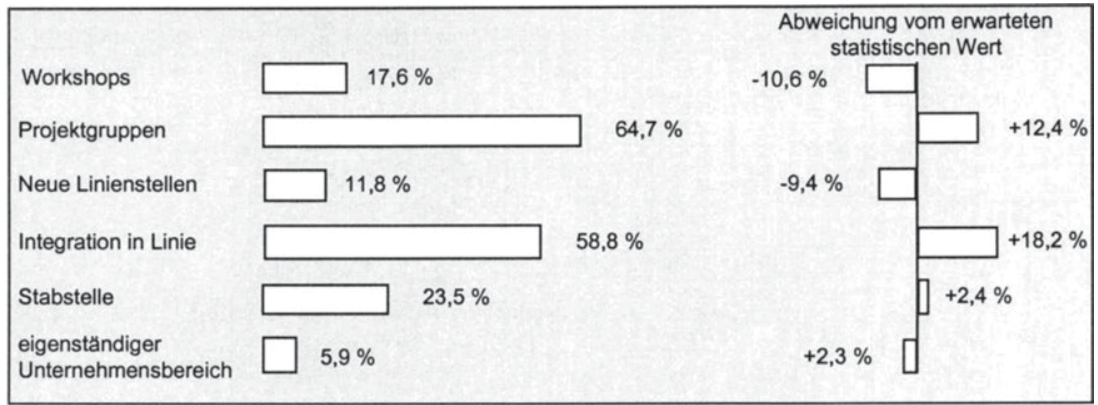

\section{Autokratische Ad hoc-Externalisierer}

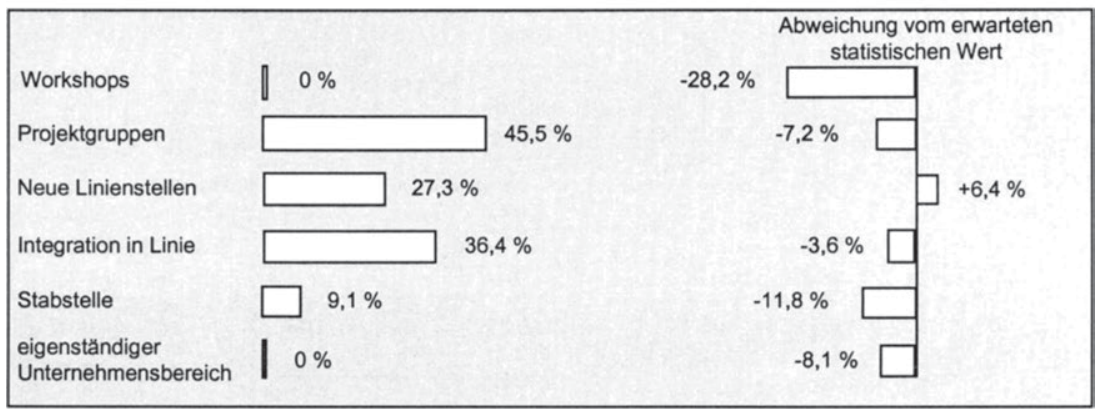

\section{Frühe integrierte Internalisierer}

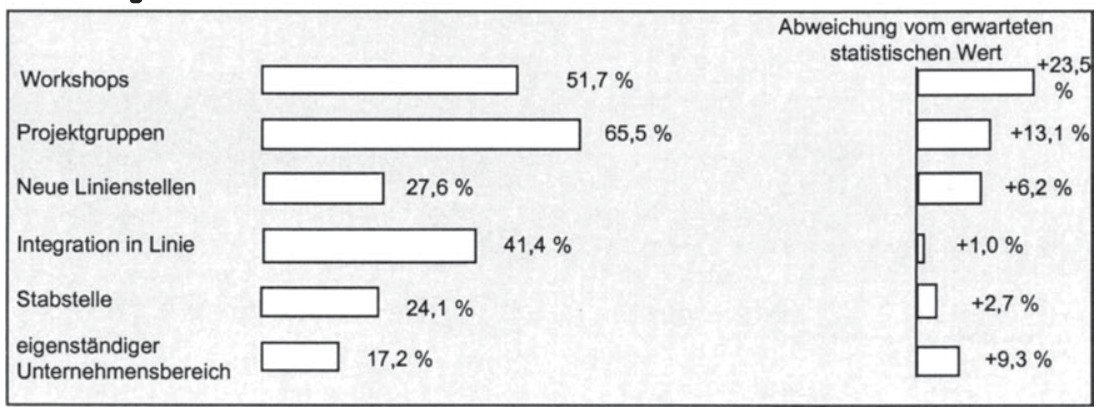

Abb. 34b: Organisatorische Maßnahmen zur Implementierung von Rücknahme- und Recyclingsystemen differenziert nach Implementierungstypen 
Aus den Abbildungen wird deutlich, daß Projektgruppen von 52,5\% aller befragten Hersteller eingesetzt werden und die organisatorischen Maßnahmen insgesamt zu $31,3 \%$ bei der Geschäftsleitung organisatorisch verankert sind. ${ }^{505}$ Hinsichtlich der Differenzierung der Implementierungstypen sind klare Unterschiede erkennbar, so

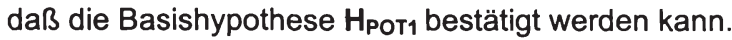

Expertenorientierte Implementierungsminimalisten richten mit knapp $8 \%$ häufiger als statistisch zu erwarten neue Linienstellen ein. Gleichzeitig werden relativ wenig $(25 \%)$ rücknahme- und recyclingsystemspezifische Aufgaben in Linien integriert. Ebenfalls läßt sich die mit Abstand höchste Anbindung der (neu geschaffenen) Linienstellen an die Geschäftsführung nachweisen, die den hohen Stellenwert der Experten für diesen Implementierungstyp unterstützt (vgl. Abb. 35a). Angesichts dieser Ergebnisse stellt sich jedoch die Frage, ob die Schaffung einer neuen Stelle mit dem Verhalten des "Minimalisten“ korrespondiert. Da diese Maßnahme nicht zwingend eine Integration und Vernetzung mit den bestehenden Strukturen bedeutet und daher durchaus eine "bequeme" und kurzfristig realisierbare Problembewältigung darstellen kann, ist diese Frage zu bejahen. ${ }^{506}$

Die Partizipativ-späten Implementierer integrieren rücknahme- und recyclingsystemspezifische Aufgaben häufiger als statistisch zu erwarten (Abweichung von $18,2 \%$ ) in bestehende Linien, während sie neue Linienstellen in geringerem Umfang einrichten. Zudem haben Projektgruppen eine extrem hohe Bedeutung und die Anbindung der organisatorischen Maßnahmen erfolgt interdisziplinär. Dieses Verhalten ergibt insgesamt ein ausgewogenes Bild, da dadurch der hohe Stellenwert der Mitarbeiter für die Partizipativ-späten Implementierer über alle Funktionen hinweg hervorgehoben wird. In diesem Sinne weist ein partizipatives Implementierungsverhalten der Hersteller auf eine Integration in die Linie hin, da damit Ent-

505 Damit werden die Ergebnisse zur Implementierung von Strategien von Kolks unterstützt. Vgl. Kolks, U., Praktische Probleme und organisatorische Lösungsansätze der Strategieimplementierung, a.a.O., S. 99ff.

Dyckhoff und Jacobs untersuchen die Institutionalisierung von Umweltschutzeinheiten im Unternehmen und stellen fest, daß sich die stärkste Korrelation mit der Unternehmensgröße ergibt. Dies begründen sie mit dem höheren Grad der Spezialisierung in großen Unternehmen. Vgl. Dyckhoff, H., Jacobs, R., Organisation des Umweltschutzes in industriebetrieben, a.a.O., S. 722f. Diese Erkenntnis unterstützt das vorliegende Ergebnis, da es sich bei Expertenorientierten Implementierungsminimalisten überwiegend um große Unternehmen handelt.

Die Bildung spezieller Umweltschutzeinheiten stellt laut Dyckhoff und Jacobs die am häufigsten ergriffene Maßnahme dar, wobei gleichzeitig die ergriffenen Umweltmaßnahmen überwiegend ausschließlich diesen Einheiten übertragen werden. Vgl. ebenda, S. 732. 
scheidungsbefugnisse dezentral auf die Mitarbeiter delegiert werden. Gleichzeitig bietet sich in diesem Cluster offensichtlich die Bildung von Projektgruppen an, da auch hier interdisziplinär aus den beteiligten Stellen konkrete Problemlösungen erarbeitet werden könnten.

Die späten promotorenorientierten Externalisierer integrieren die rücknahmeund recyclingsystembezogenen Aufgaben überwiegend in die Linie. Daran wird deutlich, daß Macht- und Fachwissen bereits bestehender Strukturen für die Implementierung eines Rücknahme- und Recyclingsystems genutzt werden. Die Veranstaltung von Workshops kann vor allem bei einer Internalisierung von Aktivitäten unterstützend eingesetzt werden. Daher führen die Späten promotorenorientierten Externalisierer weniger solche Workshops durch. Darüber hinaus fällt auf, daß dieses Cluster mit einer negativen Abweichung von $25 \%$ deutlich weniger als statistisch zu erwarten Projektgruppen einsetzt. Dieser Befund läßt ebenfalls die Interpretation zu, daß dieses Cluster weniger Interesse an unternehmensinternen Maßnahmen zeigt. Allerdings findet die Anbindung weniger an die Geschäftsleitung statt als vielmehr an den Produktionsbereich. Wird nach der möglichen Ursache gefragt, könnte diese in einer primären Fokussierung auf die technische Problemlösung bei der Implementierung von Rücknahme- und Recyclingsystemen bei diesem Implementierungstyp bestehen.

Ebenso wie die Späten promotorenorientierten Externalisierer führen Autokratische Ad hoc-Externalisierer Workshops und Projektgruppen deutlich weniger häufig durch als diejenigen Hersteller, die viele oder durchschnittlich viele Aufgaben internalisieren. Dieses ist verständlich, da die Ergebnisse der Workshops und Projektgruppen (z.B. Erstellung von Implementierungsplänen) für die eigenen internen Realisationsvorgänge der internalisierenden Hersteller von größerer Relevanz sind. Darüber hinaus verbleibt die Verantwortung für die Implementierung von Rücknahme- und Recyclingsystemen bei den Externalisierern auf der Geschäftsleitungsebene, womit die Anbindung der relevanten Stellen an die einzelnen Unternehmensfunktionen weniger ausgeprägt ist als dieses statistisch zu erwarten wäre. 
Gesamte Stichprobe (Mehrfachantworten waren möglich)

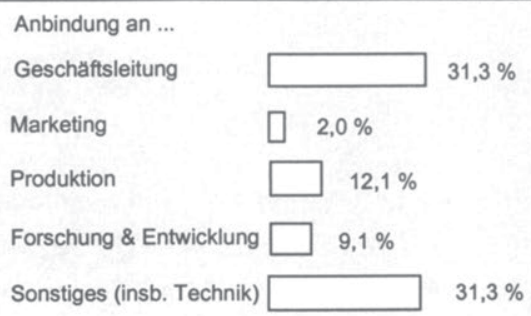

\section{Expertenorientierte Implementierungsminimalisten}

Anbindung an ...

Geschäftsleitung

Marketing

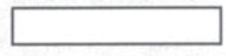

41,75

Produktion

$0 \%$

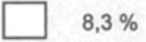

Forschung \& Entwicklung $\| 0 \%$

Sonstiges (insb. Technik)

$33,3 \%$

\section{Späte promotorenorientierte Externalisierer}

\begin{tabular}{l|l|l|l|}
\hline Anbindung an ... \\
Geschăftsleitung \\
Marketing \\
Produktion \\
Forschung \& Entwicklung \\
Sonstiges (insb. Technik)
\end{tabular}

Abb. 35a: Organisatorische Anbindung der Maßnahmen differenziert nach Implementierungstypen 
Partizipativ-späte Implementierer

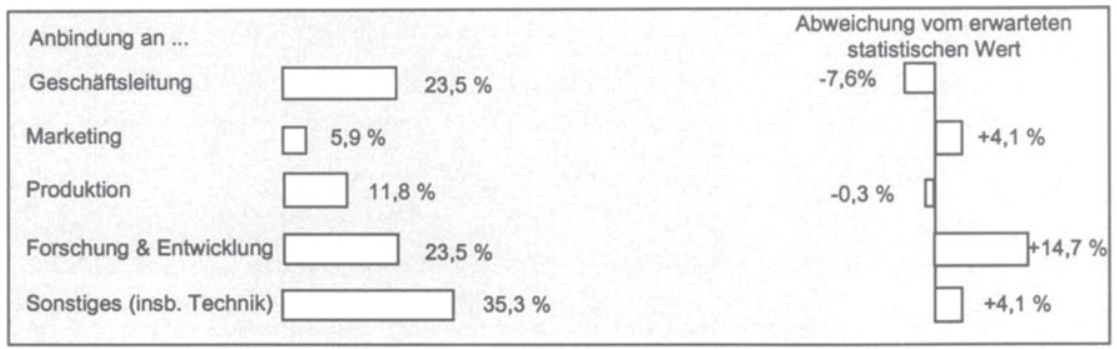

\section{Autokratische Ad hoc-Externalisierer}

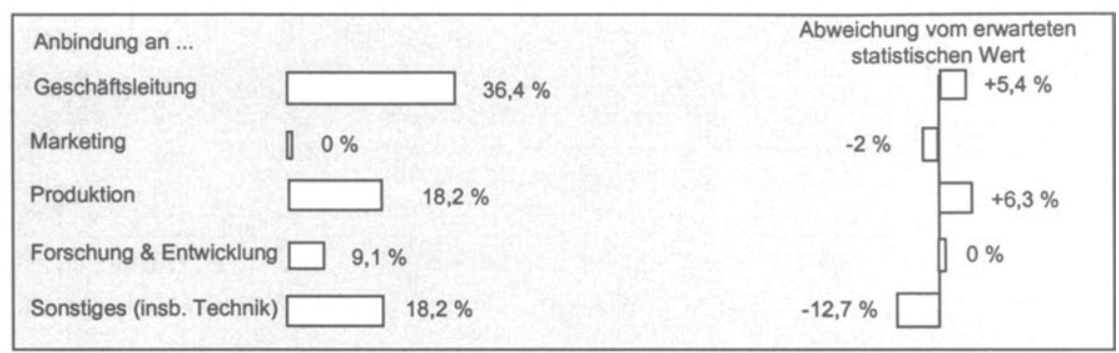

Frühe integrierte Internalisierer

\begin{tabular}{|c|c|c|c|c|}
\hline \multirow{2}{*}{$\begin{array}{l}\text { Anbindung an ... } \\
\text { Geschaftsleitung }\end{array}$} & & \multirow[b]{2}{*}{$34,5 \%$} & \multicolumn{2}{|c|}{$\begin{array}{l}\text { Abweichung vom erwarteten } \\
\text { statistischen Wert }\end{array}$} \\
\hline & & & & $+3,1 \%$ \\
\hline Marketing & $3,4 \%$ & & & $+1,4 \%$ \\
\hline Produktion & $3,4 \%$ & & $-8,6 \%$ & \\
\hline Forschung \& Entwicklung & $6,9 \%$ & & $-2,1 \% \square$ & \\
\hline Sonstiges (insb. Technik) & & $27,6 \%$ & $-3,8 \%$ & \\
\hline
\end{tabular}

Abb. 35b: Organisatorische Anbindung der Maßnahmen differenziert nach Implementierungstypen

Schließlich werden bei den Frühen integrierten Internalisierern alle organisatorischen Maßnahmen überdurchschnittlich häufig einsetzt. Workshops werden mit der höchsten positiven Abweichung von 23,5\% wiederholt eingesetzt. Dadurch wird deutlich, daß dieses Cluster zum einen sehr früh eigene Erfahrungen ge- 
macht hat und zum anderen ein System integriert bzw. integriert hat, welches Schritt für Schritt unter Beteiligung der Mitarbeiter entstanden ist. Die Einrichtung eines eigenständigen Geschäftsbereiches sowie die Anbindung an die Geschäftsleitung signalisieren bei diesem Cluster die insgesamt hohe Bedeutung der Implementierung der Rücknahme- und Recyclingsysteme.

Die Unternehmenskultur als weiteres Element der Herstellerpotentiale bringt die gemeinsam geteilten Werte der Mitarbeiter zum Ausdruck und wird als Makroperspektive des Führungsverhaltens gesehen. Sie dient der Reduktion der Komplexität und Ungewißheit bei der Aufgabenbewältigung im Unternehmen. ${ }^{507}$ Insbesondere bei neuen Aufgaben und damit notwendigen Änderungen - wie die Implementierung eines Rücknahme- und Recyclingsystems - kann die Kultur Handlungsweisen und damit die Umsetzung und Durchsetzung auf der Unternehmensebene steuern. ${ }^{508}$

Voraussetzung für die Untersuchung des Einflusses der Unternehmenskultur auf das Implementierungsverhalten bei Rücknahme- und Recyclingsystemen ist zunächst die Ableitung von Merkmalen zur Beschreibung der Kultur. ${ }^{509}$ Dabei bieten sich für die Zielsetzung der vorliegenden Untersuchung die jeweiligen Kulturausprägungen der Außenorientierung, Innenorientierung, Personen- sowie Ökologieorientierung an. Hersteller mit einer vergleichsweise starken Außenorientierung

507 Vgl. zu dieser Auffassung Frese, E., Grundlagen der Organisation, a.a.O., S. 148ff. und Schein, E. H., Organisational Culture and Leadership, San Francisco, Washington, London 1985, S. $5 \mathrm{ff}$.

Meffert unterscheidet zwei grundlegende Forschungsrichtungen im deutschsprachigen Raum: die objektivistische Kulturforschung (die Unternehmung hat eine Kultur) sowie die individualistisch-subjektivistische Kulturforschung (die Unternehmung ist eine Kultur). Vgl. Meffert, H., Marketing-Management, a.a.O., S. 426f.

Die Beeinflußbarkeit der Kultur ist zwar generell gegeben, wird jedoch insbesondere kurzfristig als sehr schwierig angesehen. Schein erläutert individualbezogene Mechanismen, die die Kultur verändern können. Vgl. Schein, E. H., Organisational Culture and Leadership, a.a.O., S. 223ff. Individualbezogene Größen werden im Kapitel C 1.5 erläutert. Kultur wird demzufolge auch im Sinne eines Corporate Behavior als verhaltensorientierter Bestandteil einer Corporate Identity (Unternehmensidentität) gesehen. Vgl. Birkigt, K., Stadler, M.M., Funck, H.J., Corporate Identity, 5. Aufl., Landsberg/Lech 1992.

509 Meffert, Hafner und Poggenpohl leiten Grundorientierungen der Unternehmenskultur ab, die Indikatoren für die Kulturschwerpunkte und -stärke eines Unternehmens sind. Vgl. Meffert, H., Hafner, K., Poggenpohl, M., Unternehmenskultur und Unternehmensführung - Ergebnisse einer empirischen Untersuchung, in: Meffert, H., Wagner, H. (Hrsg.), Marktorientierte Führung in Stagnation und Rezession, Arbeitspapier Nr. 43 der Wissenschaftlichen Gesellschaft für Marketing und Unternehmensführung e.V., Münster 1988, S. 9ff. Diese Grundorientierungen (z.B. Kostenorientierung, Leistungsorientierung) sind teilweise allerdings nicht überschneidungsfrei zu der wettbewerbsbezogenen Strategieausrichtung. 
richten sich an gesellschaftlichen Belangen, internationalen Aspekten sowie möglichen Kooperationspartnern aus und zeichnen sich durch eigenes Engagement in Verbänden aus. ${ }^{510}$

Die Innenorientierung von Unternehmen ließe sich demgegenüber durch eine Innovations-, Technik- oder Kernkompetenzorientierung abbilden. Diese Dimensionen stellen vor allem auf produktbezogene Merkmale ab, durch die sich Hersteller von ihren Wettbewerbern unterscheiden. Empirische Untersuchungen haben in der Vergangenheit versucht, den Zusammenhang zwischen einer besonderen Innovationsfähigkeit oder technologischen Kompetenz mit bestimmten Unternehmensmerkmalen wie beispielsweise der Größe oder Organisationsstruktur herzustellen. Die Ergebnisse zeigten, daß oftmals kleine oder horizontal miteinander verknüpfte Unternehmen überdurchschnittlich innovativ sind. ${ }^{511}$ Hervorgehoben werden u.a. Geschwindigkeitsvorteile und die Fähigkeit dieser Unternehmen zur Schaffung von Know-how-Transferbeziehungen. Eine ausgeprägte Fokussierung auf die eigene Kernkompetenz läßt demgegenüber nicht auf eine eindeutige Einflußrichtung bei der Implementierung von Rücknahme- und Recyclingsystemen schließen, da sowohl die Internalisierung als auch die Externalisierung Möglichkeiten für den Aufbau bzw. Ausbau eigener Kernkompetenzen bieten.

Als weiteres Merkmal einer Unternehmenskultur ist die personenbezogene Ausrichtung zu analysieren, die in der verhaltenswissenschaftlichen und betriebswirtschaftlichen Literatur unter unterschiedlichen Schwerpunkten diskutiert wird. Einige Autoren fordern beispielsweise die Überordnung der Mitarbeiterbelange über diejenigen der Kunden („Employee first, Customer second“), ${ }^{512}$ während andere

510 Bronder und Pritzel weisen darauf hin, daß in Netzwerken neben einem fundamentalen Fit (Synergiepotentiale) und dem strategischen Fit (Übereinstimmung von strategischen Zielsetzungen) ein kultureller Fit zwischen den Netzwerkpartnern für den Erfolg wichtig ist. Sie zeigen eine Reihe von personalpolitischen Aktivitäten auf, die den kulturelien Fit beeinflussen können: Zuweisung von Führungskräften, Transfermöglichkeiten, Eigenständigkeiten, Kompetenz und Unvoreingenommenheit, Loyalität sowie Karriere- und Vergütungspläne. Vgl. Bronder, Chr., Pritzel, R., Strategische Allianzen zur Steigerung der Wettbewerbsfăhigkeit, a.a.O., S. 29f.

Meffert und Netzer weisen darauf hin, daß das Vorhandensein einer gemeinsamen "MetaIdentität" (i.S. gemeinsam getragener Normen und Wertvorstellungen) die Voraussetzung für Vertrauen zwischen den Netzwerkpartnern ist, weiches wiederum die Senkung von Transaktionskosten ermöglicht. Vgl. Meffert, H., Netzer, F., Formen strategischer Netzwerke und Implikationen für das Marketing, a.a.O., S. 9f.

511 Kotabe, M., Swan, K. S., The role of strategic alliances in high-technology new product development, in: Strategic Management Journal, Nr. 16, 1995, S. 631.

512 Schlesinger und Heskett sehen die Grundprämisse für den Erfolg eines Servicegeschäftes in der Qualität des Bedienungs- und Verkaufspersonals und fordern dementsprechend, das BeIrene Giesen-Netzer and Universität Münster - 978-3-631-75058-2 
die Kundenorientierung im Sinne einer Einbeziehung des Kunden in die Leistungserstellung - vor allem bei Dienstleistungen - postulieren. ${ }^{513}$

Schließlich ist das Merkmal der Ökologieorientierung als Teil der Unternehmenskultur zu untersuchen. ${ }^{514}$ Die Ausprägungen dieses Merkmals können in Zusammenhang mit den Ausführungen zur Umweltstrategie gesehen werden. ${ }^{515} \mathrm{Bei}$ einer eher offensiven Strategie werden sich die Hersteller bezüglich ihrer Ökologieorientierung weniger auf Gesetze und Verordnungen berufen, da bereits gehandelt wird, bevor es der Gesetzgeber verlangt. Darüber hinaus werden offensive Unternehmen sich ex definitione früher als der Wettbewerb mit Umweltschutz befassen, d.h. bezüglich ihrer umweltschutzbezogenen Aktivitäten sich weniger am Wettbewerb orientieren. Die folgende Basishypothese faßt die Überlegungen zusammen:

$\mathrm{H}_{\text {POT2: }}$ Die Ausprägungen der Unternehmenskultur beeinflussen das jeweilige Implementierungsverhalten der Hersteller.

Abbildung 36 zeigt den Zusammenhang zwischen der Kulturausprägung der Hersteller und den Implementierungstypen. Bei der Analyse der F-Werte fällt auf, daß lediglich vier der vorliegenden neun Variablen signifikante Mittelwertunterschiede aufweisen. ${ }^{516}$ Daher muß die Basishypothese $\mathrm{H}_{\text {POT2 }}$ abgelehnt werden, obwohl folgende Tendenzaussagen einen Kultur-Implementierung-Zusammenhang aufzeigen.

triebssystem um diese herum auszuformen. Vgl. Schlesinger, L. A., Heskett, J. L., Dem Kunden dienen - das müssen viele Dienstleister erst noch lernen, in: Harvard Manager, Heft 2, 1992, S. 107.

Kricsfalussy setzt die Kundenorientierung der Mitarbeiterorientierung gleich, da jeder Mitarbeiter als Kunde der vorgelagerten Wertschöpfungsstufe zu sehen ist. Durch diese Sichtweise wächst die Motivation und Einsicht der Mitarbeiter, sich auch gegenüber dem externen Kunden marktgerecht $\mathrm{zu}$ verhalten. Vgl. Kricsfalussy, A., Kundenorientierung = Mitarbeiterorientierung?!, in: zfo, Heft 2, 1997, S. 101.

Vgl. zur Bedeutung der Ökologieorientierung als Erfolgsfaktor für ein Umweltmanagement, Meffert, H., Kirchgeorg, M., Marktorientiertes Umweltmanagement, S. 422ff.

Vgl. Kapitel C 1.4.2.2.

516 Eine explorative Faktorenanalyse ergab vier Faktoren: Außen-, Innen-, Personen- und Okologieorientierung. 61,4\% der Varianz der Variablen kann durch diese Lösung erklärt werden. Vgl. ausgewählte Ergebnisse im Anhang 2.8.

Hinsichtlich der Operationalisierung der Kultur herrscht in der Literatur nicht zuletzt aufgrund der Multidimensionalität des Konstruktes Uneinigkeit. 

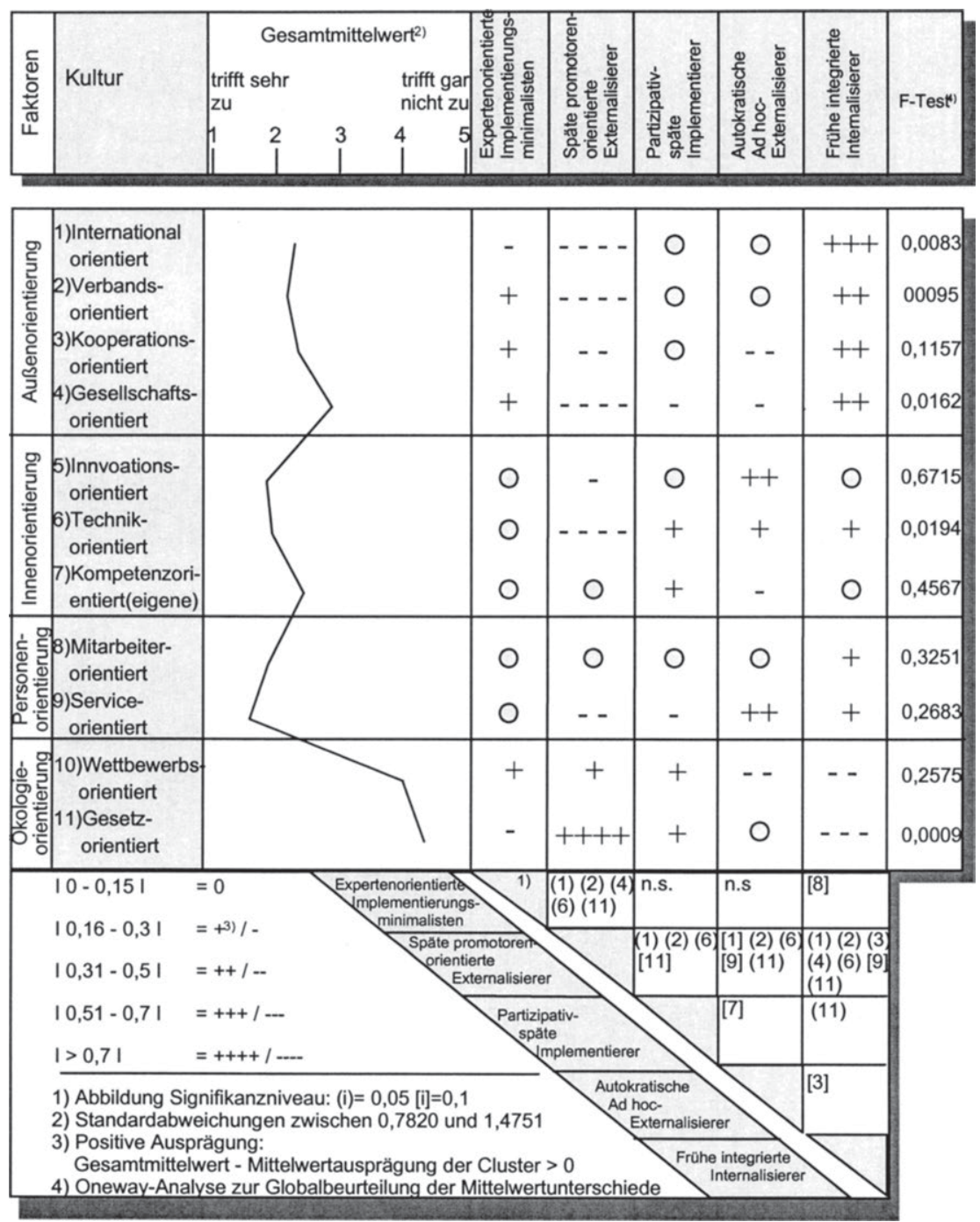

Abb. 36: Zusammenhang zwischen Kultur und Implementierungstypen

Die Außenorientierung wird durch die vier Variablen der internationalen Ausrichtung, der Verbands-, der Kooperations- sowie der Gesellschaftsorientierung beschrieben. Die Frühen integrierten Internalisierer weisen dabei deutliche po- 
sitive Abweichungen auf. Wird die Außenorientierung gemeinsam mit den Ergebnissen des Zusammenhangs zwischen der Unternehmensgröße und den Implementierungstypen betrachtet, nimmt die Außenorientierung offenbar mit zunehmender Unternehmensgröße zu (und umgekehrt). ${ }^{517}$ Eine Einbeziehung externer Faktoren und externer Anspruchsgruppen bei der Implementierung von Rücknahme- und Recyclingaktivitäten läßt auf ein integriertes Vorgehen schließen.

Bei der Innenorientierung sind die Autokratischen Ad hoc-Externalisierer diejenigen Hersteller, die positive Abweichungen hinsichtlich der Innovationsorientierung und der Technikorientierung aufweisen. Damit scheinen sich die innovationsfördernden Strukturen insbesondere bei kleinen und mittelständischen Betrieben auch bei dieser Untersuchung wiederzufinden. Eine kurze Implementierungsdauer mit einem relativ stark ausgeprägtem innovationsfördernden Bottom-upPrinzip und hoher Verantwortungsübernahme auf Geschäftsleitungsebene bei diesem Cluster versprechen dabei die erwähnten Geschwindigkeitsvorteile und Know-how-Transferbeziehungen.

Eine hohe Personenorientierung könnte zunächst auf einen erhöhten Anteil Partizipativ-später Implementierer schließen lassen. Die F-Werte weisen jedoch auf eine ungenügende Trennung der Mittelwerte der Cluster hin. Darüber hinaus zeigen die Partizipativ-späten Implementierer einen lediglich durchschnittlichen Wert bei der Mitarbeiterorientierung und eine negative Abweichung von 0,2 vom Gesamtmittelwert bei der Serviceorientierung. Eine Ursache dieses Ergebnisses kann in dem methodischen Vorgehen der Arbeit gesucht werden, das einen psychologischen Effekt bei den Antwortpersonen auslöst. So erscheint es plausibel, daß alle Hersteller angeben, sehr mitarbeiter- und serviceorientiert zu sein, da es sich um Attribute handelt, die sozial erwünscht sind. ${ }^{518}$

517 Dieser Zusammenhang wird von Dyllick als öffentliche Exponiertheit bezeichnet, die u.a. von der Unternehmensgröße bestimmt wird. Vgl. Dyllick, T., Management der Umweltbeziehungen, in: Die Unternehmung, Nr. 3, 1988, S. 194.

Auffällig sind die sehr stark abweichenden negativen Ausprägungen der Außenorientierung bei den Späten promotorenorientierten Externalisierern, die überwiegend aus kleinen Unternehmen bestehen. Auch dieses bestätigt den positiven Zusammenhang zwischen Unternehmensgröße und Außenorientierung. 
Die Frühen integrierten Internalisierer lehnen sich hinsichtlich ihrer Ökologieorientierung wenig an Gesetz und Wettbewerbern an. Das stimmt mit der frühzeitigen Implementierung von Rücknahme- und Recyclingsystemen dieses Clusters überein. Hingegen orientieren sich die Späten promotorenorientierten Externalisierer wesentlich stärker an Gesetzen und Wettbewerbern. ${ }^{519}$ Das Implementierungsverhalten der Hersteller dieses Clusters, die eine defensive Umweltstrategie verfolgen und zudem relativ spät mit der Implementierung von Rücknahme- und Recyclingsystemen beginnen, erscheint dazu konsistent.

Bei der Analyse des Einflusses der Informationssysteme auf das Implementierungsverhalten als weiteres Unternehmemenspotential der Hersteller soll der Einsatz von ökonomischen und ökologischen Informations-, Planungs-, Steuerungsund Kontrollinstrumenten untersucht werden. ${ }^{520}$ Dabei werden diese Systeme heute i.d.R. mit dem Einsatz der elektronischen Datenverarbeitung gekoppelt. Vorteile dieser EDV-gestützten Systeme liegen in der schnelleren und flexibleren Organisation der Unternehmensressourcen. ${ }^{521}$

Bei den ökonomischen Informationssystemen werden aufgrund der bisher überwiegenden Kostenorientierung bei Rücknahme- und Recyclingsystemen vermutlich Kostenvergleiche diejenigen Systeme sein, die bei allen Herstellern gleichermaßen am häufigsten zum Einsatz kommen. ${ }^{522}$ Bei den ökologiebezogenen In-

519 Statistisch signifikante Unterschiede bestehen jedoch lediglich hinsichtlich der Gesetzesorientierung, bei der sich die Späten promotorenorientierten Externalisierer gegenüber allen anderen Clustern deutlich abgrenzen. Insgesamt jedoch fällt auf, daß die Mittelwerte bei den beiden Variablen mit Werten von 3,67 und 4,05 sehr niedrig ausfallen. Dieses ist angesichts der Stichprobenzusammensetzung (überwiegend Pioniere) bei der Implementierung von Rücknahme- und Recyclingsystemen nicht weiter überraschend.

520 Diese Analyse weist bereits auf Kapitel $\mathrm{C} 2$ hin, in dem die Wirkungen untersucht und die ökonomischen und ökologischen Zielgrößen diskutiert werden.

Meffert erläutert hinsichtlich der Anpassung der Systeme im globalen Wettbewerb die Notwendigkeit der Angleichung der Managementsysteme bei den weltweit kooperierenden Einheiten, um den Umfang der strukturellen Koordination zu verringern. Dazu ist es jedoch notwendig, die Standardisierungsvoraussetzungen zu überprüfen, da insbesondere hinsichtlich strategischer Entscheidungen eine flexible Handhabung strategischer Informationen notwendig ist, um Wettbewerbsvorteile abzusichern. Vgl. Meffert, H., Marketing-Management, a.a.O., S. 290f. Dieser Gedankengang ist ebenso auf das entstehende Netzwerk bei der Implementierung eines Rücknahme- und Recyclingsystems zu übertragen.

Individualbezogene Anreizsysteme werden in Kapitel C 1.5 näher analysiert. leitet, übertriebene Informationsanforderungen zu stellen. Vgl. Meffert, H., MarketingManagement, a.a.O., S. 371. 
formationssystemen wurden grundsätzlich ${ }^{523}$ bereits frühzeitig Checklisten ${ }^{524}$ und produktbezogene Ökobilanzen ${ }^{525}$ diskutiert und eingesetzt. ${ }^{526}$ Aufgrund der relativ einfachen Umsetzbarkeit ist anzunehmen, daß bei den betrachteten Herstellern wenn auch im unterschiedlichen Ausmaß - überwiegend Checklisten zum Einsatz kommen. Der empirischen Analyse wird folgende Basishypothese vorangestellt:

Нотз: Die rücknahme- und recyclingsystembezogenen Anpassungen der Informationssysteme beeinflussen das jeweilige Implementierungsverhalten der Hersteller.

Der Einsatz von ökonomischen und ökologischen sowie (gleichzeitig) EDVgestützten Informationssystemen bei den befragten Herstellern wird aus Abbildung 37 ersichtlich. Eine Analyse der F-Werte zeigt, daß sich die Cluster hinsichtlich des Einsatzes solcher Systeme signifikant unterscheiden. Eine Ausnahme bildet die Anwendung von Kostenvergleichen bei Rücknahme- und Recyclingsystemen, die mit einem Mittelwert von 1,9 über alle Implementierungstypen hinweg sehr intensiv eingesetzt werden. Damit kann die Hypothese Н $_{\text {Poтзa }}$ bestätigt werden. Dieses Ergebnis deckt sich mit den Ergebnissen der Analyse der ökonomischen Zielsetzungen der Unternehmen, bei der die Kostenwirtschaftlichkeit an

Dutz und Femerling stellen Ansätze einer (Pozeß)Kostenrechnung zur Erfassung und Verrechnung von Entsorgungskosten vor. Vgl. Dutz, E, Femerling, Chr., Prozeßmanagement in der Entsorgung: Ansätze und Verfahren, in: DBW, Heft 2, 1994, S. 233ff. Horneber stellt änsätze einer Lebenszykluskostenrechnung vor, Horneber, M., Innovatives Entsorgungsmanagement, a.a.O., S. $211 \mathrm{ff}$.

${ }^{523}$ "Grundsätzlich" bedeutet in diesem Zusammenhang, daß diese Instrumente allgemein zur ökologiebezogenen Problemlösung herangezogen wurden, unabhängig von der zugrunde gelegten Problemstellung der Implementierung von Rücknahme- und Recyclingsystemen.

Vgl. einige beispielhafte Checklisten in: Winter, G., Das umweltbewußte Unternehmen, München 1987.

Die Verwendung von Checklisten verfolgt den Zweck, alle relevanten Informationen für die Analyse umweltrelevanter Probleme zu erfassen und anhand von Maßnahmenkatalogen Gestaltungsempfehlungen abzuleiten. Vgl. Meffert, H., Kirchgeorg, M., Marktorientiertes Umweltmanagement, a.a.O., S. $159 f$.

525 Eine Umweltbilanz stellt physische Inputs den Outputs gegenüber. Die erste Stufe ist eine Sachbilanz, die die Datenerfassung sicherstellt, die zweite Stufe ist die Wirkungsbilanz, die Umweltwirkungen einzuschätzen sucht und die dritte Stufe bildet die Bewertungs- und Verbesserungsbilanz, die Handlungsempfehlungen ableitet. Vgl. Meffert, H., Kirchgeorg, M., Marktorientiertes Umweltmanagement, a.a.O., S. $163 \mathrm{ff}$.

526 Arndt und Günter monieren, daß (computergestützte) betriebliche Umweltinformationssysteme bisher noch im unzureichenden Maße umgesetzt werden und versuchen, Gründe für diesen Befund zu eruieren. Gleichzeitig geben Sie einen Überblick über betriebliche Umweltinformationssysteme. Vgl. Arndt, H.- K., Günther, O., Betriebliche Umweltinformationssysteme: ein Überblick, in: UWF, Heft 1, 1996, S. 11-16 
erster Stelle steht. ${ }^{527}$ Bei dem Einsatz ökologischer Informationssysteme werden etwas häufiger Checklisten als Ökobilanzen durchgeführt.
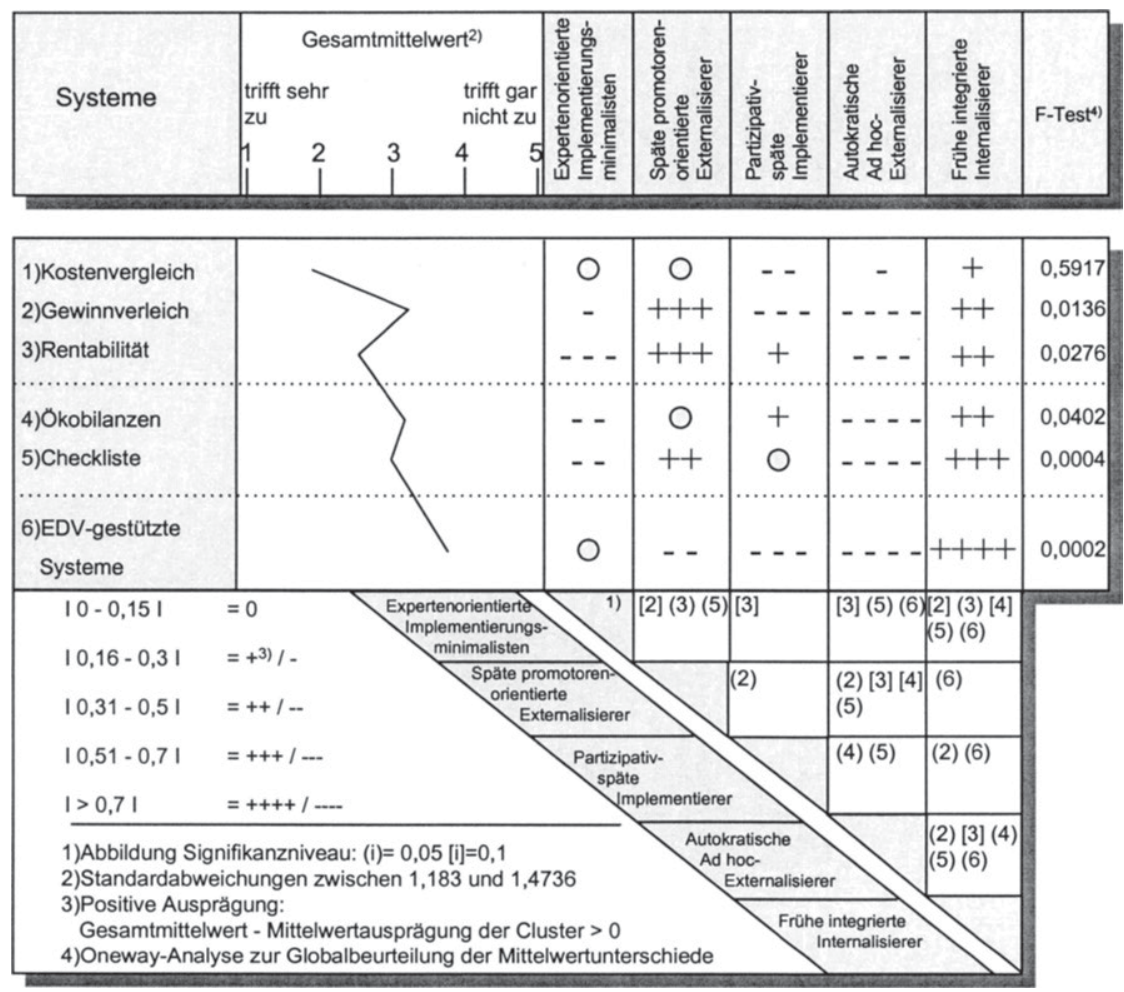

\section{Abb. 37: Zusammenhang zwischen Informationssystemen und Implementierungstypen}

Bei den Frühen integrierten Internalisierern sowie bei den Späten promotorenorientierten Implementierern werden Informationssysteme häufiger eingesetzt als dieses statistisch zu erwarten wäre. Die Rolle der Zeitdimensionen der Implementierungsaktivitäten für den Einsatz von Informationssystemen wird durch dieses Ergebnis unterstützt. Offenbar sind diejenigen Hersteller, die frühzeitig mit der Implementierung des Rücknahme- und Recyclingsystems angefangen haben, beim Einsatz solcher Planungs-, Kontroll- und Steuerungsinstrumente am weite-

527 Vgl. Kapitel B 1.3. 
sten fortgeschritten. Gleichzeitig bilden derartige Systeminfrastrukturen scheinbar eine wichtige Voraussetzung, um Rücknahme- und Recyclingsysteme frühzeitig in inrer Gesamtheit zu schließen. Zudem erstreckt sich die Sammlung und Analyse von Daten über einen gewissen Zeitraum, so daß die relativ lange Dauer der Implementierung erklärbar ist. ${ }^{528}$ Der höhere Standardisierungsgrad bei den Frühen integrierten Internalisierern, der in der Koordination über Richtlinien und Handbücher zum Ausdruck kommt, erscheint vor diesem Hintergrund plausibel. Auch bei den Späten promotorenorientierten Externalisierern ist der Zeithorizont relativ lang und der Einsatz von Richtlinien und Handbüchern überdurchschnittlich hoch ausgeprägt.

EDV-Systeme werden von den Frühen integrierten Internalisierern signifikant häufiger eingesetzt (Mittelwert von 2,76) als von allen anderen Clustern. Insgesamt überrascht jedoch die geringe Nutzung von EDV-gestützten Systemen über alle Cluster hinweg (Mittelwert von 3,7). Daran wird deutlich, daß beim Einsatz von EDV-Systemen bei der Implementierung und Gestaltung von Rücknahme- und Recyclingsystemen offensichtlich noch große Barrieren für die befragten Hersteller bestehen. ${ }^{529}$ Dies mag zum einen darin begründet liegen, daß hinsichtlich der Erfassung und Bewertung von ökologischen Daten wie auch hinsichtlich deren ökonomischer Zuordenbarkeit eine große Unsicherheit besteht. Zum anderen existieren wenig standardisierte Softwarepakete, welche die gewünschten ökonomischen und ökologischen Daten zu liefern vermögen.

528 Im Rahmen der Informationsbeschaffung ist nicht nur ein Mengengerüst der relevanten Inputund Outputgrößen zu beschaffen, sondern darüber hinaus wird ein Wertgerüst benötigt, um die ökologische Schädlichkeit und die Kostenwirkungen beurteilen zu können. Diese Quantifizierung ist eine hoch komplexe und zeitaufwendige Aufgabe. Vgl. Steven, M., Effizienz von betrieblichen Entsorgungsprozessen, a.a.O., S.124f.

Einen möglichen Ansatz zur Bewertung von ökologischen Wirkungen der Produkte geben Kreikebaum und Türk. Vgl. Kreikebaum, H., Türk, R., Ein Ansatz zur Bewertung der ökologischen Wirkungen von Produkten, in: ZfB-Ergänzungsheft 2, 1993, S. 119-138.

529 Auch der Einsatz von Ökobilanzen ist mit einem Mittelwert von 3,2 eher als gering zu bezeichnen. Lediglich die Frühen integrierten Internalisierer sowie die Partizipativ-späten Implementierer setzen dieses Informationssystem häufiger ein. Dieses ist vermutlich auf den recht hohen zeitlichen und finanziellen Aufwand bei deren Erstellung zurückzuführen. 


\subsection{Einflußfaktoren der Durchsetzung von Rücknahme- und Recycling- systemen auf der Individualebene}

\subsubsection{Komponenten der Durchsetzung}

Die Analyse der Einflußfaktoren auf der Individualebene ist von besonderer praktischer Relevanz, da die Gründe für einen Abbruch von Implementierungsprozessen häufig nicht im sachlogischen Bereich, sondern im Widerstandsverhalten von Individuen liegen. ${ }^{530}$ Dementsprechend fordern einige Autoren, daß unmittelbar nach der Verabschiedung der Strategie durch die Geschäftsleitung die Vermittlung der Strategieinhalte (Kennen und Verstehen-Komponente) im Vordergrund stehen müßte, ${ }^{531}$ während andere Autoren die Durchsetzung und Umsetzung als Prozesse sehen, die parallel ablaufen. ${ }^{532}$

Durch die Modifizierung des Konzepts der Rücknahme- und Recyclingsysteme ${ }^{533}$ sowie durch die Umsetzungsmaßnahmen auf der Unternehmensebene können auf der einen Seite Anforderungen an die Mitarbeiter gesenkt werden, ${ }^{534}$ während auf der anderen Seite die Durchsetzungsaufgaben Leistungsniveausteigerungen bei den betroffenen Mitarbeitern hinsichtlich der rücknahme- und recy-

530 Vgl. dazu z.B. Hilker, J., Marketingimplementierung, a.a.O., S. 307; Linn, N., Die Implementierung vertikaler Kooperationen, a.a.O., S. 2 und Kirsch, W., Esser, W. M., Gabele, E., Das Management des geplanten Wandels von Organisationen, a.a.O., S. 15ff. Kirsch, Esser und Gabele kommen zu dem Ergebnis, daß bei $45,2 \%$ der befragten Unternehmen die Reorganisationsprozesse am erklärten Widerstand der Betroffenen scheitern und bei 43,5\% mangelndes Anpassungsvermögen der Betroffenen zum Abbruch der Implementierungsprozesse führten. In einem gesonderten Kapitel gehen sie daher auf die Aktoren des geplanten Wandels ein. Vgl. ebenda, S. $187 \mathrm{ff}$.

531 Kolks identifiziert dabei zwei Zielgruppen: Im ersten Schritt ist die Strategie den Führungskräften zu vermitteln, die die Implementierungsverantwortung tragen sollen, und im zweiten Schritt sind die betroffenen Mitarbeiter über die wesentlichen Konzeptinhalte zu informieren. Vgl. Kolks, U., Strategieimplementierung, a.a.O., S. $114 \mathrm{ff}$.

Vgl. Hilker, J., Marketingimplementierung, a.a.O., S. 307.

533 Dies deutet darauf hin, daß nicht nur eine Kontextanpassung im Unternehmen, sondern auch eine Konzeptanpassung stattfinden muß, damit eine erfolgreiche Implementierung von Rücknahme- und Recyclingsystemen stattfinden kann. Reiß spricht in diesem Zusammenhang von einer "Implementierung durch Kontext-Anpassung" und einer "Implementierung durch Konzept-Anpassung“, die eine „Benutzergerechtigkeit“ sicherstellen soll. Vgl. Reiß, M., Implementierung, in: Corsten, H., Reiß, M. (Hrsg.), Handbuch Unternehmensführung: Konzepte - Instrumente - Schnittstellen, Wiesbaden 1995, S. $196 \mathrm{ff}$.

Beispielsweise kann die Konzeption des Rücknahme- und Recyclingsystems verändert werden, indem nur ausgewählte Produkte zurückgenommen werden. Damit werden der Koordinationsaufwand gesenkt und die Mitarbeiter entlastet.

Über Neueinstellungen können beispielsweise das noch fehlende rücknahme- und recyclingsystemspezifische Wissen enworben und somit die Mitarbeiter entlastet werden. Da diese Option kaum von den betrachteten Unternehmen (Mittelwert 3,94) eingesetzt wurde, wurde sie in den vorherigen Aussagen nicht weiter berücksichtigt. 
clingsystemspezifischen Aufgaben ermöglichen sollen (vgl. Abb. 38). ${ }^{535}$ Die Unternehmenskultur als verhaltensbeeinflussende Variable nimmt allerdings eine Sonderstellung im Implementierungsprozeß ein, da sie sich nur langfristig verändern läßt.

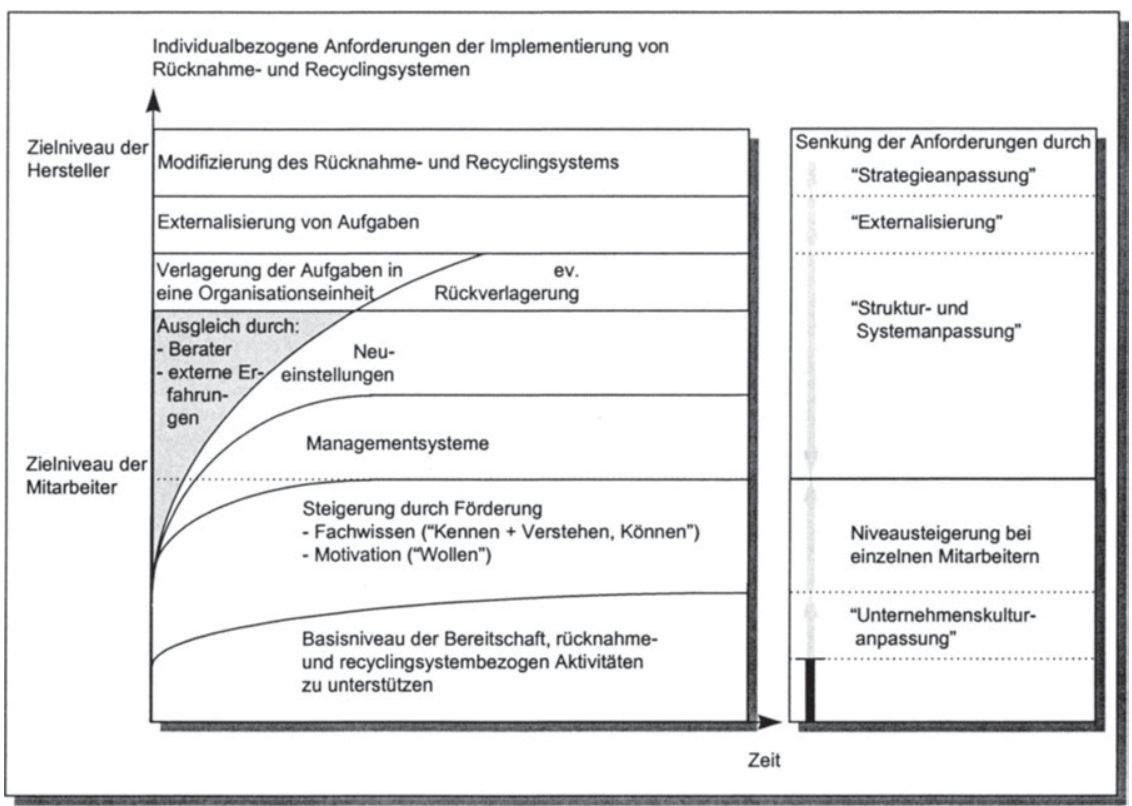

Abb. 38: Zusammenwirken der Umsetzung und Durchsetzung zur Erreichung der Anforderungen der Implementierung von Rücknahme- und Recyclingsystemen (In ähnlicher Form in: Hilker, J., Marketingimplementierung: Grundlagen und Umsetzung am Beispiel ostdeutscher Unternehmen, Wiesbaden 1993, S. 309)

Insbesondere an die Führung ${ }^{536}$ der Herstellerunternehmen ergeben sich mit der Implementierung von Rücknahme- und Recyclingsystemen aus zwei Gründen be-

535 Vgl. Hilker, J., Marketingimplementierung, a.a.O., S. 308ff.

Verhaltensänderungen können generell erzielt werden durch: 1. Änderung der Bedingungen, die das Verhalten auslösen, 2. Änderung der Umwelt, in der das Verhalten stattfindet, 3. Änderung der Konsequenzen, die auf das Verhalten folgen. Vgl. zur genaueren Analyse von Lernprozessen und Verhaltensänderungen Zimbardo, P. G., Psychologie, 4. neubearb. Aufl., Berlin u.a. 1983, S. $211 \mathrm{ff}$.

536 Zur Anwendung kommt hier der verhaltensbezogene Führungsbegriff, der als personenbezogene Handlung von Führenden auf Geführte zu einem zielentsprechenden Handeln veranlassen soll. Vgl. dazu die Ausführungen in Kapitel A 3. 
sondere Anforderungen. ${ }^{537}$ Der erste Grund besteht darin, daß von der Implementierung von Rücknahme- und Recyclingsystemen i.d.R. mehrere Systempartner betroffen sind, die ein Netzwerk bilden. Diese Netzwerkstrukturen erfordern aufgrund der hohen Komplexität eine größere Verantwortungsübernahme und vor allem politisches und diplomatisches Geschick zur Entwicklung und Stabilisierung der interorganisationalen Beziehungen. ${ }^{538}$ Dazu bedarf es Verhandlungen über die Grenzen des Netzwerks und jedes einzelnen Netzwerkunternehmens, da strategische Programme nicht mehr mittels Weisungen, sondern über eine gemeinsame Konsensfindung durchgesetzt werden müssen. Neben diesen eher persönlichkeitsbezogenen Fähigkeiten bedarf es besonderer kognitiver und fachlicher Fähigkeiten, da zusätzlich zu den Kontingenzen des eigenen Unternehmens Kontingenzen auf der Netzwerkebene berücksichtigt werden müssen („thinking in networks"). ${ }^{539}$ Tabelle 8 bringt zum Ausdruck, welche Kriterien sich zusätzlich verändern, wenn ein Unternehmen zu einem Netzwerkunternehmen wird. ${ }^{540}$

537 Bereits ohne die Besonderheiten des spezifischen Implementierungsobjektes „Rücknahmeund Recyclingsysteme" werden an die Führung von Implementierungsprozessen besondere Anforderungen gestellt. Kirsch, Esser und Gabele erläutern neun Forderungen an das Management: 1. Das Schaffen eines entsprechenden Klimas, 2. Die systematische Planung, 3. Die Abstimmung der Reorganisationsprobleme auf das Potential der beteiligten Aktoren, 4. Die Steuerung der Reorganisation durch eine strategische Planung, 5. Die Handhabung von Kopplungen in Reorganisationsprozessen, 6. Die Berücksichtigung der strukturellen und personellen Gegebenheiten, 7. Die systematische Einbeziehung flankierender und den Prozeß vorantreibender Maßnahmen, 8. Die Entwicklung eines Anpassungssystems bzw. einer Projektorganisation und 9. Der Aufbau eines projektbezogenen Ausbildungssystems. Vgl. Kirsch, W., Esser, W. M., Gabele, E., Das Management des geplanten Wandels von Organisationen, a.a.O., S. $48 \mathrm{ff}$.

Bonoma identifiziert drei Fehler, die das Management im Umgang mit Neuerungen begeht: 1 . Strukturen werden hingenommen, ohne diese zu hinterfragen (Management by Assumption), 2. Übertragung von Aufgaben an Abteilungen, die diese Aufgaben nicht lösen können (Strukturelle Widersprüche) und 3. Prioritäten werden nicht deutlich gesetzt (Globale Mittelmäßigkeit). Vgl. Bonoma, T. V., Der Marketing-Vorsprung, a.a.O., S. $70 \mathrm{ff}$. und S. $88 f$.

Sydow begründet dies mit der Notwendigkeit des Ausbalancierens verschiedener Interessen, der Tolerierung multipler Loyalitätsanforderungen sowie der Schaffung einer Vertrauensbasis. Vgl. Sydow, J., Netzwerkorganisation, a.a.O., S. 632.

Kieser stellt den Zusammenhang zwischen Loyalität bzw. Commitment und Unternehmenskultur her. Während die Kultur die geteilten Werte im Unternehmen umfaßt, entsteht Loyalität bzw. Commitment durch eine hohe Übereinstimmung zwischen Werten des Individuums und der Unternehmenskultur. Vgl. Kieser, A., Loyalität und Commitment, in: Kieser, A., Reber, G., Wunderer, R. (Hrsg.), Handwörterbuch der Führung, 2. Aufl., 1995, S. $1350 f$.

Vgl. Mattsson, L. G., Management of Strategic Change in a "Markets-as-Networks“ Perspective, in: Pettigrew, A. M. (Hrsg.), The Management of Strategic Change, Oxford 1987, S. 253 f.

Sydow spricht in diesem Zusammenhang von einer Mehrebenenbetrachtung des Management (Unternehmens- und Netzwerkinteressen). Darüber hinaus muß das Management eine veränderte Vorstellung von „Wachstum“ erlangen, da durch Quasi-Externalisierungen kleinere Einheiten entstehen, die jedoch mehr Einfluß/Macht besitzen und für die Durchsetzung von hoher Bedeutung sein können. Vgl. Sydow, J., Unternehmensnetzwerke, a.a.O., S. 166f. Eine veränderte Sichtweise vom Wachstum wird auch im Rahmen des Sustainable DevelopmentIrene Giesen-Netzer and Universität Münster - 978-3-631-75058-2 
Dieses "Denken in Netzwerken“ deutet bereits auf den zweiten Grund der besonderen Anforderungen hin, die bei der Implementierung eines Rücknahme- und Recyclingsystems an die Führung gestellt werden. Das „Denken in Netzwerken“ wird noch erweitert zum „Denken in Kreisläufen“ (von der Durchlaufwirtschaft zur Kreislaufwirtschaft), welches eine wesentliche Änderung der Denkstrukturen erfordert. ${ }^{541}$

\begin{tabular}{|c|c|c|}
\hline & Einzelunternehmen & Netzwerkunternehmen \\
\hline $\begin{array}{l}\text { Betrachtungsfokus der Reor- } \\
\text { ganisation }\end{array}$ & intraorganisational & interorganisational \\
\hline Gegenstand des Management & $\begin{array}{l}\text { Wertschöpfungskette des ein- } \\
\text { zelnen Unternehmens }\end{array}$ & $\begin{array}{l}\text { unternehmensübergreifendes } \\
\text { Wertschöpfungssystem }\end{array}$ \\
\hline Ziele & $\begin{array}{l}\text { so viele Kernkompetenzen wie } \\
\text { möglich zur Erzielung des } \\
\text { "competitive advantage" }\end{array}$ & $\begin{array}{l}\text { so viele Kernkompetenzen wie } \\
\text { nötig zur effektiven Nutzung } \\
\text { des "collaborative advantage" }\end{array}$ \\
\hline Managementaufgabe & $\begin{array}{l}\text { Erfolg des einzelnen Unter- } \\
\text { nehmens sicherstellen }\end{array}$ & $\begin{array}{l}\text { Netzwerk bilden und zum Erfolg } \\
\text { führen }\end{array}$ \\
\hline $\begin{array}{l}\text { Auswahl von Kooperations- } \\
\text { partnern }\end{array}$ & $\begin{array}{l}\text { unter machtpolitischen Ge- } \\
\text { sichtspunkten }\end{array}$ & $\begin{array}{l}\text { nach komplementären Kern- } \\
\text { kompetenzen }\end{array}$ \\
\hline Interesse der Kooperation & Sicherstellung des Status Quo & $\begin{array}{l}\text { Weiterentwicklung der Partner- } \\
\text { schaft }\end{array}$ \\
\hline Art der Partner & $\begin{array}{l}\text { Aufbau von Branchen-Know- } \\
\text { how }\end{array}$ & $\begin{array}{l}\text { Nutzung der Erfahrungen von } \\
\text { Partnern anderer Branchen }\end{array}$ \\
\hline
\end{tabular}

Tab. 8: Veränderung der Denkweise vom Einzelunternehmen zum Netzwerkunternehmen (in Anlehnung an: Padberg, A., Partnerschaften entlang der Wertschöpfungskette, Formelle und informelle Netze von Einzelunternehmen: Eigene Schwächen durch Zusammenarbeit mit anderen kompensieren, in: Blick durch die Wirtschaft, 11.3.1996, S. 11)

Dementsprechend muß die Führung ein anderes "Verständnis“ (Kennen und Verstehen - Komponente) von Altprodukten erlangen. Vor der Implementierung von Rücknahme- und Recyclingsystemen wurden die Altprodukte i.d.R. als Gegen-

Konzepts verlangt, das ein rein quantitatives Wachstum durch ein qualitatives Wachstum zu ersetzen sucht.

540 Padberg, A., Partnerschaften entlang der Wertschöpfungskette, a.a.O., S.11.

541 Albach differenziert drei für das Umweltmanagement notwendige Denkweisen: 1. Wissenschaftliches Denken, das eine Analyse von Wirkungsnetzen (Ursache - (Haupt- und Neben)wirkungen) ermöglicht, 2 . Systemdenken bzw. vernetztes Denken, das eine Berücksichtigung von Teilsystemen beinhaltet und 3. OKonomisches Denken, welches ein wirtschaftliches Abwägen zwischen Alternativen unter Berücksichtigung ihrer Wirkungen und Interssenlagen umfaßt. Vgl. Albach, H., Umweltmanagement als Führungsaufgabe, in: ZFB, Heft 12, 1994, S. $1572 \mathrm{ff}$. 
stände betrachtet, die nicht mehr im Einflußbereich der Hersteller lagen und daher keiner Verantwortungsübernahme bedurften. Darüber hinaus entstand lediglich ein Entsorgungsproblem, wenn Altprodukte wieder zum Herstellerunternehmen gelangten. Rücknahme- und Recyclingsysteme erfordern demgegenüber ein Denken, das zum einen eine Verantwortungsübernahme für die Altprodukte impliziert und zum anderen eine Wertschätzung ("die Altprodukte sind noch wertvoll“) der Altprodukte beinhaltet. Dieses Umdenken ist jedoch nicht nur bei den Führungskräften, sondern auch bei den Mitarbeitern erforderlich. Die Rolle der Führungskräfte wird hervorgehoben, da sie mit ihrem Verhalten das Verhalten der Mitarbeiter beeinflussen. Dies gilt um so mehr, als bei der Implementierung von Rücknahme- und Recyclingsystemen Mitarbeiterängste bestehen, die bei anderen Implementierungsobjekten in geringerem Umfang vorliegen. So kann durchaus die Gefahr bestehen, im Unternehmen nicht ernst genommen zu werden, da umweltbezogene Aktivitäten oft nicht profitabel erscheinen und damit ein wichtiger extrinsischer Anreiz entfällt.

Vor diesem Hintergrund erscheint es wichtig, ob und ggf. welche Aufgabenschwerpunkte die Führungskräfte bei der Durchsetzung des Rücknahme- und Recyclingsystems setzen bzw. welche Aufgaben als wichtig erkannt werden. ${ }^{542}$ Diese Aufgaben orientieren sich an den Durchsetzungszielen der drei Ebenen „Kennen und Verstehen“, „Können“ und „Wollen“. 543

$\mathrm{H}_{\mathrm{IND} 1}$ : Die Schwerpunktsetzung bei den Durchsetzungsaufgaben des Rücknahme- und Recyclingsystems hat Einfluß auf die Bildung der Implementierungstypen.

Die Durchsetzungsaufgaben konzentrieren sich dabei zunächst auf die „Kennen und Verstehen“-Komponente, d.h. auf regelmäßige Informationen der Mitarbeiter über Implementierungsschritte in verständlicher Sprache. ${ }^{544}$ Bei der

542 Bartlett und Ghoshal sehen die Aufgaben des Topmanagement heute weniger in der Verhaltenskontrolle als vielmehr in der Entwicklung von breiten Fähigkeiten und Perspektiven. Den mitarbeiterbezogenen Durchsetzungsaufgaben geben sie dabei Vorrang vor Umsetzungsaufgaben und Strategiefindung. Die Instrumente sehen sie in einer verständlichen (und gemeinsamen) Zielformulierung, in einer Beteiligung der gesamten Organisation sowie in der Erreichung meßbarer Ergebnisse. Vgl. Bartlett, Chr. A., Ghoshal, S., Die wahre Aufgabe des Topmanagement heute, a.a.O., S. $56 \mathrm{ff}$.

Vgl. dazu die Ausführungen in Kapitel B 1.3.

544 Vgl. Kolks, U., Strategieimplementierung, a.a.O., S. 189.

Wottawa und Gluminski erläutern im Rahmen der sozial-kognitiven Lerntheorie, daß die Merkmale der beobachtbaren Person (in diesem Fall der Mitarbeiter), z.B. die Wahrnehmungsmöglichkeit und -kapazität oder frühere Erfahrungen für Aufmerksamkeitsprozesse und damit für eine erfolgreiche Organisationsentwicklung.- und übertragen auch auf die Durchset-

Irene Giesen-Netzer and Universität Münster - 978-3-631-75058-2 
„Können“-Komponente sind organisatorische Gegebenheiten anzupassen sowie persönliche Fähigkeiten der Mitarbeiter zu fördern. ${ }^{545}$ Die Anpassung der organisatorischen Gegebenheiten betrifft die Erfüllung von Umsetzungsaufgaben, während die Fähigkeiten der Mitarbeiter vor allem durch die Vermittlung von Fachkenntnissen gefördert werden können. Weitere Möglichkeiten zur Beeinflussung der Können-Komponente bestehen in der täglichen Konfrontation mit dem Rücknahme- und Recyclingsystem ("Learning by doing") sowie in der Förderung der Kommunikation ${ }^{546}$ zwischen den betroffenen Mitarbeitern. ${ }^{547}$ Die „Wollen“Komponente (Motivation) ${ }^{548}$ beinhaltet schließlich die Förderung der positiven Einstellung zu Rücknahme- und Recyclingsystemen ${ }^{549}$ sowie den Abbau von Einwendungen gegen diejenigen Personen, die das Rücknahme- und Recyclingsystem implementieren wollen. ${ }^{550}$ Schließlich hilft auch der Abbau von Verunsicherungen bei der Übernahme neuer Aufgaben, das „Wollen" und damit die Unterstützung der Aktivitäten bei der Implementierung des Rücknahme- und Recyclingsystems der Mitarbeiter sicherzustellen. ${ }^{551}$

Abbildung 39 zeigt im Mittelwertprofil deutlich, daß die diskutierten Durchsetzungsaufgaben mit Werten zwischen 2 und 2,5 insgesamt einen wichtigen Stellenwert einnehmen. Lediglich der Abbau von Einwendungen gegen Personen, die

zung von Konzepten - wichtig sind. Vgl. Wottawa, H., Gluminski, I., Psychologische Theorien für Unternehmen, a.a.O., S. 240f.

Die Informationsvermittlung muß jedoch nicht grundsätzlich formal durch gezielte Veranstaltungen erfolgen, sondern gerade die informale Informationsweitergabe stellt ein wichtiges Instrument dar. Vgl. ebenda, S. 116. Vgl. dazu Kapitel A 3.

In einer empirischen Untersuchung von Kolks wurde die Förderung der Kommunikation als ein wichtiger Erfolgsfaktor - z.T. sogar als der zentrale Erfolgsfaktor - zur Durchsetzung von Strategien erkannt. Vgl. Kolks, U., Strategieimplementierung, a.a.O., S. 194 f.

Derartige Maßnahmen wären vor allem bei den Frühen integrierten Internalisierern sowie den Partizipativ-späten Implementierern zu erwarten, da diese ein hohes Maß an Interaktion bzw. Mitarbeiterorientierung auszeichnet.

Die Motivationspsychologie geht davon aus, daß die Motivation zum einen direkt auf das Verhalten einwirkt und zum anderen indirekt als Verstärker des Handelns wirkt. Dementsprechend zeigen motivierte Personen mehr Handlungen und zeigen diese eher, schneller und stärker als unmotivierte Personen. Vgl. Zimbardo, P. G., Psychologie, a.a.O., S. 345.

Vgl. dazu die Ausführungen zur Einstellungsakzeptanz in Kapitel B 1.3.

Strategische Planer gelten unternehmensintern häufig als "Theoretiker", die vom Geschäft nichts verstehen. Diesen Akzeptanzbarrieren kann begegnet werden, indem zum einen Mitarbeiter der Linie mit an der Planung arbeiten können und/oder die Planer auch Mitarbeiter der Linie waren oder nur für eine begrenzte Zeit als Planer tätig sind. Vgl. Kolks, U., Strategieimplementierung, a.a.O., S. $188 f$.

Bewährtes Verhalten in vertrauten Aufgabenbereichen ist eine bedeutende emotionale Barriere bei der Durchsetzung von Neuerungen. Vgl. Wottawa, H., Gluminski, I., Psychologische Theorien für Unternehmen, a.a.O., S. 294. 
das Rücknahme- und Recyclingsystem einrichten, wird mit einem Wert von 3,2 nur mit mittlerer bis kaum wichtiger Bedeutung eingestuft. Dies liegt vermutlich darin begründet, daß die Implementierung der Rücknahme- und Recyclingsysteme überwiegend von internen Mitarbeitern durchgeführt wird und externe Berater im Gegensatz zur Implementierung anderer Objekte - kaum eine Rolle spielen. Darüber hinaus befinden sich die befragten Führungskräfte selbst in der Rolle der "Implementierer" und nehmen vermutlich entsprechende Einwendungen gegen ihre eigene Person weniger wahr.

Bei der Betrachtung der F-Werte fällt auf, daß sich die Cluster nur bei drei Variablen bemerkenswert unterscheiden. Lediglich die Frühen integrierten Internalisierer unterscheiden sich gegenüber den Autokratischen Ad hoc-Externalisierern bei den ersten 5 Variablen signifikant. Damit kann die Basishypothese $H_{\text {IND1 }}$ nur eingeschränkt bestätigt werden kann.

Die Frühen integrierten Internalisierer sind diejenigen Hersteller, die in der Erfüllung der „Kennen und Verstehen“- sowie „Können“- Komponente einen besonderen Schwerpunkt sehen. Eine Ausnahme bildet die Bedeutung der verständlichen Sprache der Planungen. Dieser Befund erscheint plausibel, da neben dieser Variablen auch die Variablen der "Wollen“- Komponente nur eine durchschnittliche bis leicht unterdurchschnittliche Ausprägung haben. Aufgrund des frühen Anfangszeitpunktes der Implementierung der Rücknahme- und Recyclingsysteme sehen diese Hersteller demzufolge kaum besonderen Bedarf zur Förderung der Motivation. Das Verständnis des Problembereiches ist zudem bereits offenbar so groß, daß derartige Hemmnisse i.d.R. schon beseitigt sind. ${ }^{552}$ Auch die Ausführungen zu der umweltbezogenen Strategie und der Ökologieorientierung dieses Clusters verdeutlichen diesen Zusammenhang.

552 Zu unterscheiden ist jedoch der "gewöhnliche“ Motivationsbedarf, der unabhängig von der Implementierung besteht. 

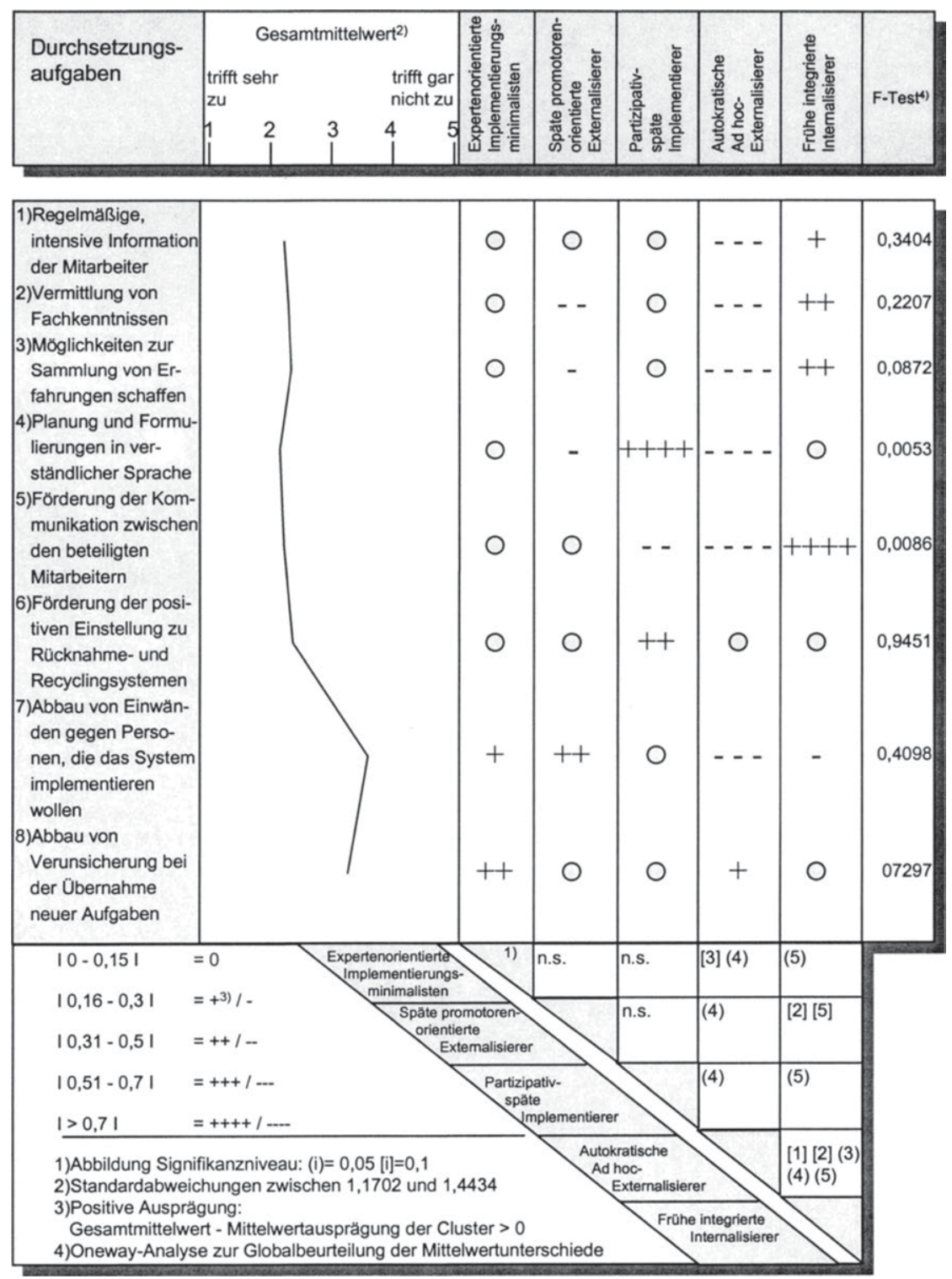

\section{Abb. 39: Zusammenhang zwischen Schwerpunktsetzungen bei den Durchsetzungsaufga- ben und Implementierungstypen}


Dieser Sachverhalt stellt sich bei den Partizipativ-späten Implementierern anders dar, die bei der Variablen „Planungen und Formulierungen in verständlicher Sprache" einen besonderen Schwerpunkt setzen. Darüber hinaus ist die Ausprägung der Förderung einer positiven Einstellung zu Rücknahme- und Recyclingsystemen höher als dieses statistisch zu erwarten wäre. Dieses Ergebnis legt den Zusammenhang zu den Partizipationsdimensionen der Implementierung offen. Aufgrund der hohen Mitbestimmungsmöglichkeiten besitzen diese Durchsetzungsaufgaben augenscheinlich hohe Priorität. ${ }^{53}$ Dies gilt hinsichtlich der „Kennen und Verstehen"- Komponente aufgrund des späten Anfangszeitpunktes ebenso wie für die "Können"- und „Wollen"- Komponenten aufgrund der insgesamt hohen Mitarbeiterorientierung dieses Clusters. Andererseits ist der Intensitätsgrad der Implementierung des Rücknahme- und Recyclingsystems bei den Partizipativspäten Implementierern nur mittelmäßig hoch ausgeprägt, so daß relativ wenige Abteilungen und Mitarbeiter intensiv in den Implementierungsprozeß einbezogen sind. Daher ist es plausibel, daß die Mitarbeiterinformation („Kennen und Verstehen") und die Vermittlung der Fachkenntnisse nur auf durchschnittlichem Niveau verharren, während ein besonderer Wert auf eine verständliche Sprache bei der Kommunizierung von Planungen gelegt wird. ${ }^{554}$

Die Priorisierung bei den Durchsetzungsaufgaben im Cluster der Autokratischen Ad hoc-Externalisierer ist signifikant niedriger ist als statistisch zu erwarten wäre. Dieses Ergebnis kann insbesondere mit dem relativ hohen Externalisierungsgrad, dem Führungsstil sowie der kurzen Dauer der Implementierungsaktivitäten dieses Clusters erklärt werden. Demzufolge fallen die beschriebenen Durchsetzungsaufgaben bei einem hohen Externalisierungsgrad in kleinerem Umfang an bzw. haben nur eine geringe Priorität bei den Führungskräften, da viele Aufgaben von Dritten erfüllt werden. Darüber hinaus benötigt eine konsequente Erfüllung der Durchführungsaufgaben offenkundig einen gewissen Zeitraum.

553 Die Einbeziehung von Betroffenen in den Zielbildungsprozeß und in die Entscheidungen eignet sich dazu, das Umweltbewußtsein „am Ursprung" zu beeinflussen und damit eine positive Einstellung zum Rücknahme- und Recyclingsystem zu erlangen. Minsel und Bente leiten noch weitere Rahmenbedingungen ab, die eine Beeinflussung des Umweltbewußtseins ermöglichen (z.B. Aktualisieren der Diskussion um Umweltbelastungen). Vgl. Minsel, W. W., Bente, G., Pädagogik und Verhaltensmodifikation als Strategie zur Veränderung des Umweltbewußtseins, in: Fietkau, H.- J., Umweltlernen: Veränderungsmöglichkeiten des Umweltbewußtseins; Modelle - Erfahrungen, Königstein im Taunus 1981, S. 178. 


\subsubsection{Instrumente zur Durchsetzung von Rücknahme- und Recycling- systemen}

Vor diesem Hintergrund stellt sich die Frage, mit welchen Instrumenten Individuen hinsichtlich der Implementierung eines Rücknahme- und Recyclingsystems gefördert werden können und welchen Einfluß der Einsatz solcher Instrumente auf das Implementierungsverhalten hat.

Den Instrumenten zur Förderung des „Kennen und Verstehens" sowie des "Könnens" wurde eine explorative Faktorenanalyse vorangestellt, die zwei Faktoren mit einem erklärten Varianzanteil von $49,8 \%$ ergab. ${ }^{555}$ Die zwei Faktoren lassen sich als theoretisch-analytische und direkte mitarbeiterbezogene Instrumente bezeichnen. Unter theoretisch-analytische Instrumente fallen Auswertungen von Fachberichten oder anderen Marktdaten sowie deren Verbreitung im Unternehmen. Auch können externe Referenzprojekte (d.h. bereits bestehende Rücknahme- und Recyclingsysteme anderer Unternehmen) im Sinne von benchmarks analysiert sowie eine systematische Analyse eigener Erfahrungen durchgeführt werden, um diese z.B. anschließend in Form von innerbetrieblichen Berichten (z.B. Umläufe, Werkszeitschriften) zu verbreiten. Direkte mitarbeiterbezogene Instrumente können in Schulungen der Mitarbeiter bestehen. Präferieren Hersteller eher Maßnahmen des "Training on the Job“, kommen eigene Pilotprojekte, Job Rotation und die Einweisung bzw. das Coaching der Mitarbeiter in Frage. ${ }^{556}$

Die Instrumente zur Förderung des "Wollens" werden unter dem Begriff der Anreizsysteme diskutiert ${ }^{557}$ und können in extrinsische und intrinsische Instrumente

555 Vgl. dazu die explorative Faktorenanalyse im Anhang 2.9.

556 Coaching soll im Sinne der Förderung von Mitarbeitern in Unternehmen durch Vorgesetzte verstanden werden. Im Rahmen der Organisationsentwicklung - zumeist aufgrund von wahrgenommenem Zeitdruck- spielt Coaching eine besondere Rolle, da durch eine gemeinsame Situationsanalyse zwischen Vorgesetzten und Mitarbeitern eine Lösung erarbeitet werden soll. Zur ausführlichen Erläuterung des Coaching vgl. Angermeyer, H. Chr., Coaching - eine spezielle Form der Beratung, in: zfo, Heft 2, 1997, S. 105ff.

Mit der direkten Einweisung von Mitarbeitern können nach empirischen Erkenntnissen zum einen Unsicherheiten abgebaut werden, und zum anderen behalten die Mitarbeiter von selbst Erlerntem $40 \%$ mehr Inhalte als von dem, was innen durch Wort und Bild vermittelt wird. Vgl. Kolks, U., Strategieimplementierung, a.a.O., S. 119.

557 Anreizsysteme im engeren Sinne sind solche Instrumente, die ausschließlich mit Blick auf die Steigerung der Motivation der Mitarbeiter eingesetzt werden, während bei Anreizsystemen im weiteren Sinne auch solche betriebswirtschaftlichen Instrumente einbezogen werden, die unter Motivationsgesichtspunkten positive Nebeneffekte haben. Vgl. Frese, E., Grundlagen der Organisation, a.a.O., S.22. So hat die Teilnahmemöglichkeit an Seminaren nicht nur den Effekt, daß das Fachwissen der Mitarbeiter gefördert wird, sondern auch den Nebeneffekt, daß die Aufgabeninhalte dadurch eine Aufwertung erfahren und die (intrinsische) Motivation steigt.

Irene Giesen-Netzer and Universität Münster - 978-3-631-75058-2 
unterschieden werden. Extrinsische Faktoren sind solche Faktoren, die Bedingungen des Arbeitsvollzuges darstellen wie z.B. finanzielle Anreize. Intrinsische Faktoren beziehen sich hingegen auf die Arbeit selbst, d.h. auf die Aufgabeninhalte. ${ }^{558}$ Intrinsischen Faktoren wird dabei eine besonders wichtige Rolle zugesprochen, da diese in der Literatur häufig als "Zufriedenheitsstifter" gelten. ${ }^{559}$

Die Geschäftsleitung als Vorbild im Umgang mit Rücknahme- und Recyclingsystemen nimmt eine Doppelfunktion ein. So ist die Rolle von Vorbildern neben der Motivationssteigerung einerseits insbesondere für eine erfolgreiche Organisationsentwicklung andererseits von Bedeutung, da eine Person durch Beobachtung modellhaften Verhaltens von Vorgesetzten ein spezifisches Wissen akquiriert. Dieses "Lernen am Modell“ ermöglicht es, den Prozeß des Lernens erheblich zu beschleunigen und kostspielige Fehler zu verringern. Folgende Basishypothese liegt der empirischen Überprüfung zugrunde:

$\mathrm{H}_{\mathrm{IND2}}$ : Die Einsatzhäufigkeit von Instrumenten zur Förderung der Komponenten der Individualebene unterscheidet sich signifikant hinsichtlich der Implementierungstypen.

Als wichtigstes Instrument zur Förderung des „Kennens und Verstehens" wird mit einem Mittelwert von 2,1 die Auswertung von eigenen Erfahrungen im Rahmen der theoretisch-analytischen Instrumente eingeschätzt (vgl. Abb. 40). Flankierend werden häufig eigene Pilotprojekte im Rahmen der direkten mitarbeiterbezogenen Instrumente durchgeführt (Mittelwert 2,4).

Hagen betont, daß ein Anreiz nur dann seine Wirkung zeigen kann, wenn eine klare (und vom Mitarbeiter wahrgenommene) Beziehung zwischen der in Aussicht gestellten Belohnung und der erwarteten Leistung besteht. Vgl. Hagen, R., Anreizsysteme zur Strategiedurchsetzung, Spardorf 1985, S. 132. Zur detaillierten Analyse der Komponenten von Anreizsystemen vgl. ebenda, S. 88ff.

Vgl. z.B. Wottawa, H., Gluminski, I., Psychologische Theorien für Unternehmen, a.a.O., S. $187 \mathrm{ff}$. und S. $216 \mathrm{ff}$. oder vgl. Wagner, H., Städler, A., Führung, Grundlagen, Prozesse und Konzeptionen der Mitarbeiterführung in Unternehmungen, 2., überarb. Aufl., Münster 1989, S. 50.

Hagen weist auf die Verknüpfung von ex- und intrinsischen Faktoren hin. Er erläutert, daß ein klassischer extrinsischer Reiz wie Geld intrinsische Folgen haben kann. Vgl. Hagen, R., Anreizsysteme zur Strategiedurchsetzung, a.a.O., S. 133. Motivationstheorien erklären, welche spezifischen Faktoren den Menschen zur Arbeit motivieren (Inhaltstheorien), während Prozeßtheorien erklären, wie Arbeitsverhalten gesteuert werden kann. Vgl. ebenda, S. 29.

Nach Herzberg existieren Zufriedenheitsstifter (Motivatoren), die bei positiver Ausprägung Arbeitszufriedenheit erzeugen. Dabei handelt es sich um Faktoren, die arbeitsintrinsischen Charakter haben. Unzufriedenheitsstifter (Hygienefaktoren) führen bei negativer Ausprägung zu Arbeitsunzufriedenheit. Dies sind extrinsische Faktoren. Vgl. zu dieser Interpretation Wagner, H., Städler, A., Führung, a.a.O., S. 49ff. 

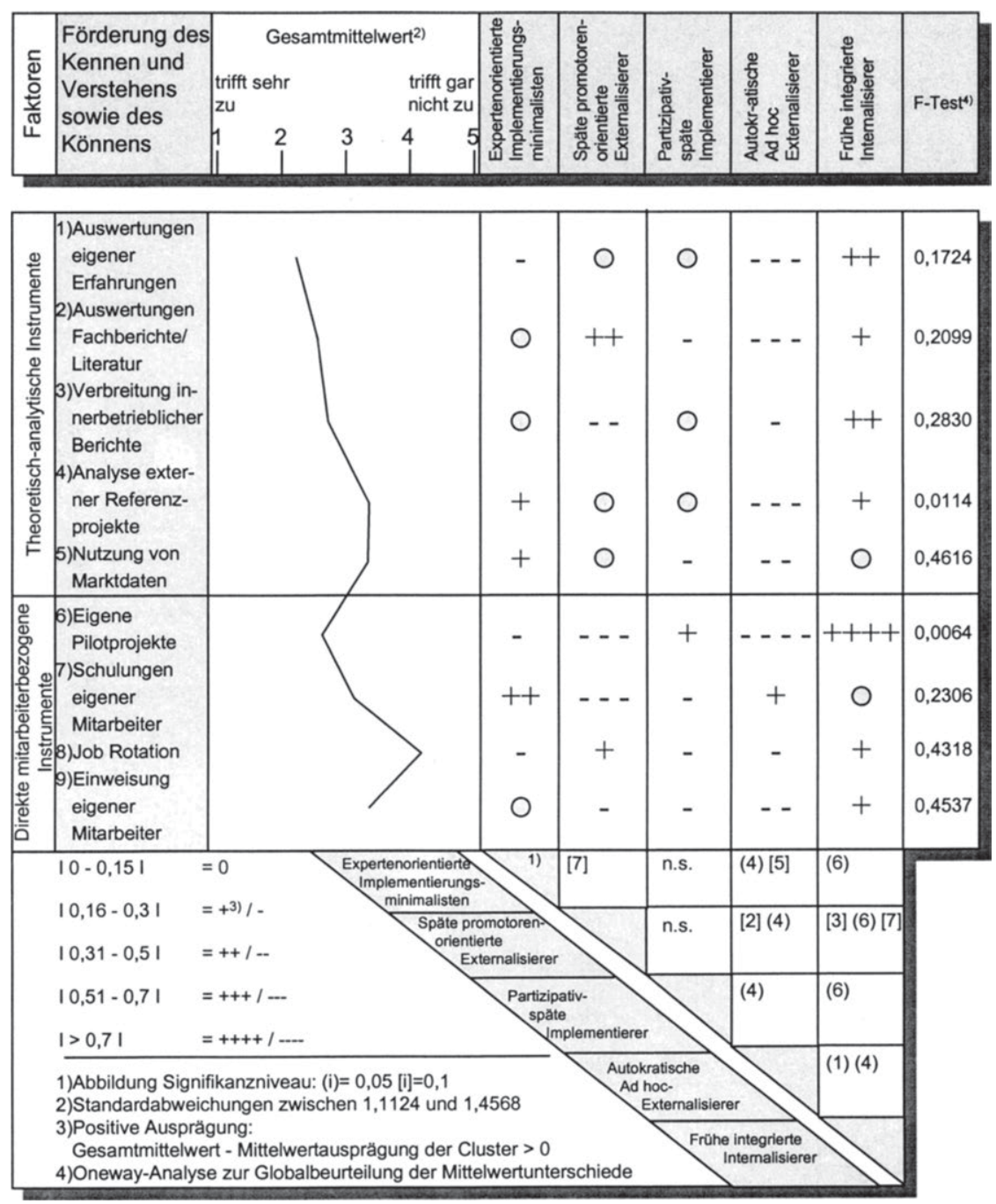

Abb. 40: Zusammenhang zwischen Förderung des „Kennens und Verstehens“ und „Könnens“" bei den Mitarbeitern und Implementierungstypen

Pilotprojekte werden von den Frühen integrierten Internalisierern angesichts des clustertypisch hohen Internalisierungsgrades deutlich am häufigsten eingesetzt. Im Zusammenhang mit den obigen Überlegungen zum Stellenwert von 
Durchsetzungsaufgaben erscheint es plausibel, daß die Frühen integrierten Internalisierer theoretisch-analytische Instrumente intensiv einsetzen. Auch die Auseinandersetzung mit den eigenen Erfahrungen in Form von Auswertungen, die innerhalb des Unternehmens weiterverbreitet und ggf. durch sekundärstatistische Informationen ergänzt werden, korrespondiert mit dem überdurchschnittlich hohen Einsatz von Handbüchern zur Koordination der Implementierungsaktivitäten, der das Implementierungsverhalten dieser Hersteller kennzeichnet.

Bei den Expertenorientierten Implementierungsminimalisten werden insbesondere Schulungen als direktes mitarbeiterbezogenes Instrument durchgeführt. Darüber hinaus setzt dieses Cluster ebenfalls die Analyse externer Referenzprojekte sowie die Nutzung von Marktdaten stärker ein als statistisch zu erwarten wäre. Da das Implementierungsverhalten der Expertenorientierten Implementierungsminimalisten grundsätzlich auf den überdurchschnittlich hohen Einsatz externer Informationsquellen schließen läßt, um Expertenwissen im eigenen Unternehmen aufzubauen, kann dieses Ergebnis nicht überraschen. Zudem können Schulungen genau in Inhalt und Umfang auf ausgewählte Mitarbeiter (Experten) abgestimmt werden.

Autokratische Ad hoc-Externalisierer setzen signifikant weniger theoretischanalytische Instrumente sowie eigene Pilotprojekte ein als der Stichprobendurchschnitt. Offensichtlich besteht durch Externalisierungsaktivitäten bei diesem Cluster weder ein großes Interesse an der Nutzung von externen Quellen noch an der Analyse und Durchführung interner Maßnahmen. Mit Bezug auf die Interventionsebene legt dieses Cluster ähnlich wie die Expertenorientierten Implementierungsminimalisten viel Wert auf die Ernennung von Fachexperten. Daher besteht eine - wenn auch geringe - positive Abweichung hinsichtlich des Einsatzes von Schulungen der Mitarbeiter, wodurch ein Mindestmaß an Fachwissen abgesichert werden kann.

Die Partizipativ-späten Implementierer weisen einen eher durchschnittlichen bzw. unterdurchschnittlichen Instrumenteeinsatz zur Förderung des „Kennens und Verstehens" sowie des „Könnens" auf. Eine Erklärung dieses Befundes bewegt sich einerseits zwischen der Innenorientierung, die auf die Auswertung und Verbreitung eigener Erfahrungen schließen läßt, und dem späten Beginn der Pilotphase andererseits, der keinen besonders ausgeprägten Einsatz theoretisch- 
analytischer Maßnahmen zuläßt, da ein fachspezifisches internes und externes Informationssystem Zeit zum Aufbau benötigt. Ähnliches gilt für die direkten mitarbeiterbezogenen Instrumente, die erst mit zeitlicher Verschiebung zielgerichtet eingesetzt werden können, dann aber von relativ hoher Bedeutung sein dürften, da in diesem Cluster eine hohe Mitarbeiterorientierung vorherrscht.

Bezüglich der Instrumente zur Förderung des "Wollens" ergeben die F-Werte eine signifikante $(\alpha=0,1)$ Unterscheidung der Implementierungstypen (vgl. Abb. 41). ${ }^{560}$ Damit kann die Tendenzhypothese $H_{\text {IND2 }}$ insgesamt bestätigt werden, obwohl die Instrumente zur Förderung des "Kennens und Verstehens“ keine so deutlichen Ergebnisse liefern können wie diejenigen zur Förderung des "Wollens“.

Der Einsatz von Anreizinstrumenten ist bei den Frühen integrierten Internalisierern höher als statistisch zu erwarten wäre. Damit wird die Bedeutung der integrierten Vorgehensweise bei den Interventions- und Partizipationsdimensionen sowie der Stellenwert der Intensitätsdimensionen für den Einsatz derartiger Instrumente verdeutlicht. Frühe integrierte Implementierer setzen zum einen intrinsische Instrumente intensiv ein. Zum anderen wenden sie auch extrinsische Faktoren an, da motivierte Mitarbeiter bei einem hohen Internalisierungsgrad sehr wichtig sind. Auch die Geschäftsleitung als Vorbild nimmt bei den Frühen integrierten Internalisierern einen überdurchschnittlich hohen Stellenwert ein, um ein integriertes Implementierungsvorgehen sicherzustellen, welches die Mitarbeiter in allen drei Komponenten befähigt bzw. motiviert.

560 Eine Ausnahme bildet dort der Einsatz des umweltorientierten Vorschlagwesens, der ein Signifikanzniveau von ca. $11 \%$ erreicht. 

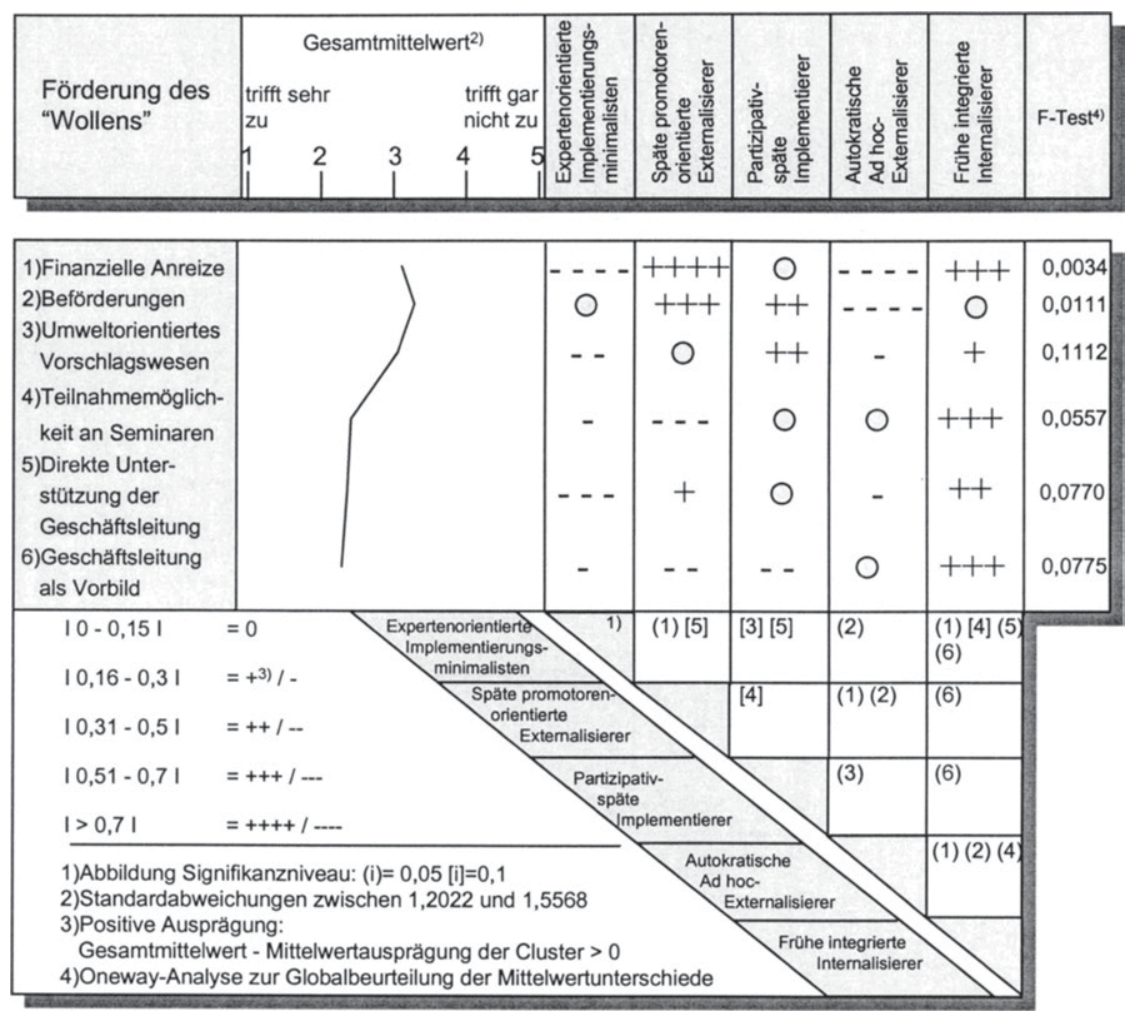

Abb. 41: Zusammenhang zwischen Förderung des „Wollens“ bei den Mitarbeitern und Implementierungstypen

Bei den Partizipativ-späten Implementierern kommt häufiger als statistisch erwartet ein umweltorientiertes Vorschlagwesen zur Anwendung. Auch Beförderungen werden zur Motivation eingesetzt, die anderen Instrumente werden aber nur durchschnittlich oder sogar unterdurchschnittlich häufig verwendet. Vor dem Hintergrund der relativ geringen Priorisierung der Durchsetzungsaufgaben in diesem Cluster wird dieses Ergebnis jedoch verständlich. ${ }^{561}$ Die Späten promotorenorientierten Externalisierer setzen insbesondere finanzielle Anreize, Beförderungen und die direkte Unterstützung der Geschäftsleitung als Instrumente ein, welche die „Wollen“- Komponente der Individualebene unterstützen sollen. Damit wird 
die Konzentration dieses Implementierungstyps auf wenige Mitarbeiter bzw. Promotoren verdeutlicht. Geht ein Hersteller promotorenorientiert vor, ist es demnach sinnvoll, diese Promotoren, deren Kreis sich auf wenige Führungskräfte beschränkt, durch gezielte Anreize zu motivieren, ihrer Rolle weiterhin gerecht zu werden. ${ }^{562}$

Aus der Analyse der Einflußfaktoren der Individualebene mit den drei Komponenten „Kennen und Verstehen“, „Können“ und „Wollen“ geht insgesamt hervor, daß in großen Teilen signifikante Unterschiede hinsichtlich der Implementierungstypen bestehen. Allerdings scheinen insbesondere netzwerkbezogene und externe unternehmensbezogene Einflußfaktoren eine wichtige Rolle bei der Implementierung von Rücknahme- und Recyclingsystemen zu spielen.

Im Anschluß an die Analyse der Einflußfaktoren erfolgt eine Untersuchung der Erfolgswirkungen der identifizierten Implementierungstypen, d.h. es wird der Frage nachgegangen, ob und ggf. in welchen Wirkungsgrößen sich die Implementierungstypen unterscheiden. Damit verändert sich der Fokus der vorliegenden Untersuchung: Waren bei der Analyse der Einflußfaktoren die Implementierungstypen die abhängigen Variablen („Welchen Einfluß haben die Faktoren auf das Implementierungsverhalten?"), sind im folgenden die jeweiligen Implementierungstypen die unabhängigen Variablen („Welche Auswirkungen hat das Implementierungsverhalten auf den Erfolg?"). ${ }^{563}$

562 Mit einer gezielten Förderung von "Schlüsselmanagern“ kann der Erfolg der Implementierung maßgeblich beeinflußt werden. Vgl. Kolks, U., Strategieimplementierung, a.a.O., S. 207. Als solche Schlüsselmanager können Fach- und Machtpromotoren eingestuft werden. Vgl. Meffert, H., Marketing-Management, a.a.O., S. 374.

Hauschildt und Kirchmann untersuchen einen weiteren Promotorentyp, den Prozeßpromotor, der aufgrund der Organisationskenntnis in der Lage ist, Barrieren des "Nicht-Dürfens" zu überwinden. Sie bestätigen empirisch zum einen die Erkenntnisse von Witte: 1. Die Existenz von Promotoren und deren Arbeitsteilung wirkt sich positiv auf Innovationsprozesse aus, 2. Das Gespann von Macht- und Fachpromotor erzielt grundsätzlich bessere Ergebnisse als die Struktur ohne Promotoren oder als eine einseitige Promotorenstruktur. Zum anderen stellen sie fest, daß eine Troika (Macht-, Fach-, und Prozeßpromotor) den Innovationsprozeß am erfolgreichsten zu unterstützen vermag. Vgl. Hauschildt, J., Kirchmann, E., Arbeitsteilung im Innovationsmanagement, Zur Existenz und Effizienz von Prozeßpromotoren, in: zfo, Heft 2, 1997, S. 68ff. 


\section{Erfolgswirkungen der Implementierung von Rücknahme- und Recy- clingsystemen}

Im Zusammenhang mit dem mehrdimensionalen Zielbezug ${ }^{564}$ der Implementierung von Rücknahme- und Recyclingsystemen wurden zunächst Implementierungsziele sowie implementierungsobjektspezifische (ökologische und ökonomische) Ziele diskutiert. ${ }^{565}$

\subsection{Implementierungserfolg}

Die Erreichung der Durchsetzungsziele auf der Netzwerkebene ist - neben den Ausprägungen in den Implementierungsdimensionen der Hersteller - insbesondere vor dem Hintergrund zu untersuchen, ob und ggf. mit welchen Kooperationspartnern die befragten Hersteller ein Netzwerk bilden ${ }^{566}$ Gleichzeitig stellt sich die Frage, ob der herstellerspezifische Führungsstil Auswirkungen auf das Verhalten gegenüber Systempartnern hat und damit die Ziele auf der Netzwerkebene beeinflußt. Bei der Analyse des Umsetzungserfolgs auf der Unternehmensebene sind vorwiegend interne unternehmensbezogene Einflußfaktoren zu diskutieren, da diese Aufschluß über genaue herstellerspezifische Gegebenheiten geben können ${ }^{567}$ Dazu gehören neben den Anpassungen der Unternehmenspotentiale die Diskussion der Strategieausgestaltung, die erheblichen Einfluß auf das Implementierungsverhalten bei Rücknahme- und Recyclingsystemen hat. Die Erreichung der individualbezogenen Durchsetzungsziele wird schließlich insbesondere in Zusammenhang mit der Partizipationsdimension zu analysieren sein. Den individualbezogenen Durchsetzungszielen wird in der Gesamtbetrachtung des Implementierungserfolgs häufig ein hoher Stellenwert eingeräumt. Zum Teil

\footnotetext{
564 Im Rahmen der organisatorischen Gestaltung von Rücknahme- und Recyclingsystemen wurde der mehrdimensionale Zielbezug als konstitutives Merkmal von Rücknahme- und Recyclingsystemen herausgearbeitet. Vgl. Kapitel B 1.2.

Kolks bezeichnet die Messung des Zielerreichungsgrades einer Strategie als Ergebniskontrolle. Davon grenzt er die Durchführungskontrolle (Wird die Strategie realisiert, die verabschiedet wurde?) sowie die Prämissenkontrolle (Gelten noch die gleichen Prämissen, die als Grundlage zur Herleitung der Ziele und Strategie dienten?) ab. Vgl. Kolks, U., Strategieimplementierung, a.a.O., S. 55. Der Schwerpunkt der vorliegenden Arbeit liegt bei der Ergebniskontrolle, da diese letztlich Aussagen darüber zu geben vermag, ob das Rücknahme- und Recyclingsystem erfolgreich implementiert wurde.

Bezüglich derjenigen Unternehmen, die als Partner der Hersteller bei der Erfüllung der Aufgaben fungieren können, lassen sich Zulieferer, Handel, Verwerter und Wettbewerber unterscheiden. Die Auswahl ist von Relevanz, da die Partner den Erfolg eines Rücknahme- und Recyclingsystems mit beeinflussen. Neben den genannten Systempartnern können auch Verbände und spezielle Arbeitsgruppen insbesondere bei der Planung von Branchenlösungen wichtige Beratungs- und Koordinationsfunktionen übernehmen.
}

Vgl. Kapitel C 1.4.2. 
fordern Autoren, daß zunächst Maßnahmen auf der Individualebene ergriffen werden, bevor die Unternehmenspotentiale angepaßt werden. ${ }^{568}$ Dies wird damit begründet, daß die betroffenen Mitarbeiter als zentrale Erfolgsfaktoren die Implementierung durchführen. ${ }^{569}$ Vor diesem Hintergrund wird der empirischen Analyse folgende Basishypothese vorangestellt:

$\mathrm{H}_{\text {WIR1 }}$ Die Implementierungstypen unterscheiden sich hinsichtlich der Erfolgswirkungen bei der Erreichung der Implementierungsziele.

Bei der Analyse der Erreichung der Implementierungsziele zeigen die F-Werte der Abbildung 42, daß sich die Implementierungstypen hinsichtlich 9 (von 14) Variablen auf einem Signifikanzniveau von $10 \%$ unterscheiden. Damit kann die Hypothese $H_{\text {WIR1 }}$ weitgehend bestätigt werden.

Bei den horizontalen Durchsetzungszielen auf der Netzwerkebene fällt auf, daß eine vertrauensvolle Zusammenarbeit zwischen den Systempartnern über alle Cluster hinweg mit einem Mittelwert von 2,0 gut erreicht wird. Dieses deutet zum einen auf die sorgfältige Auswahl der Systempartner und zum anderen auf die hohe Bedeutung des Vertrauens innerhalb eines Netzwerkes hin.

568 Kolks erläutert, daß Interdependenzen zwischen Durchsetzungs- und Umsetzungszielen vorliegen und schließt daraus: Implementierungsmaßnahmen sollten zu Beginn eher Durchsetzungscharakter haben, während der Umsetzungsschwerpunkt im Laufe der Implementierung zunimmt. Vgl. Kolks, U., Strategieimplementierung, a.a.O., S. 259.

569 Bartlett und Ghoshal kritisieren die „Strategiegläubigkeit" vieler Unternehmen und fordern stattdessen eine stärkere Orientierung auf Führungsprozesse und Mitarbeiter. Vgl. Bartlett, Chr. A., Ghoshal, S., Die wahre Aufgabe des Topmanagement heute, a.a.O., S. 56ff. 

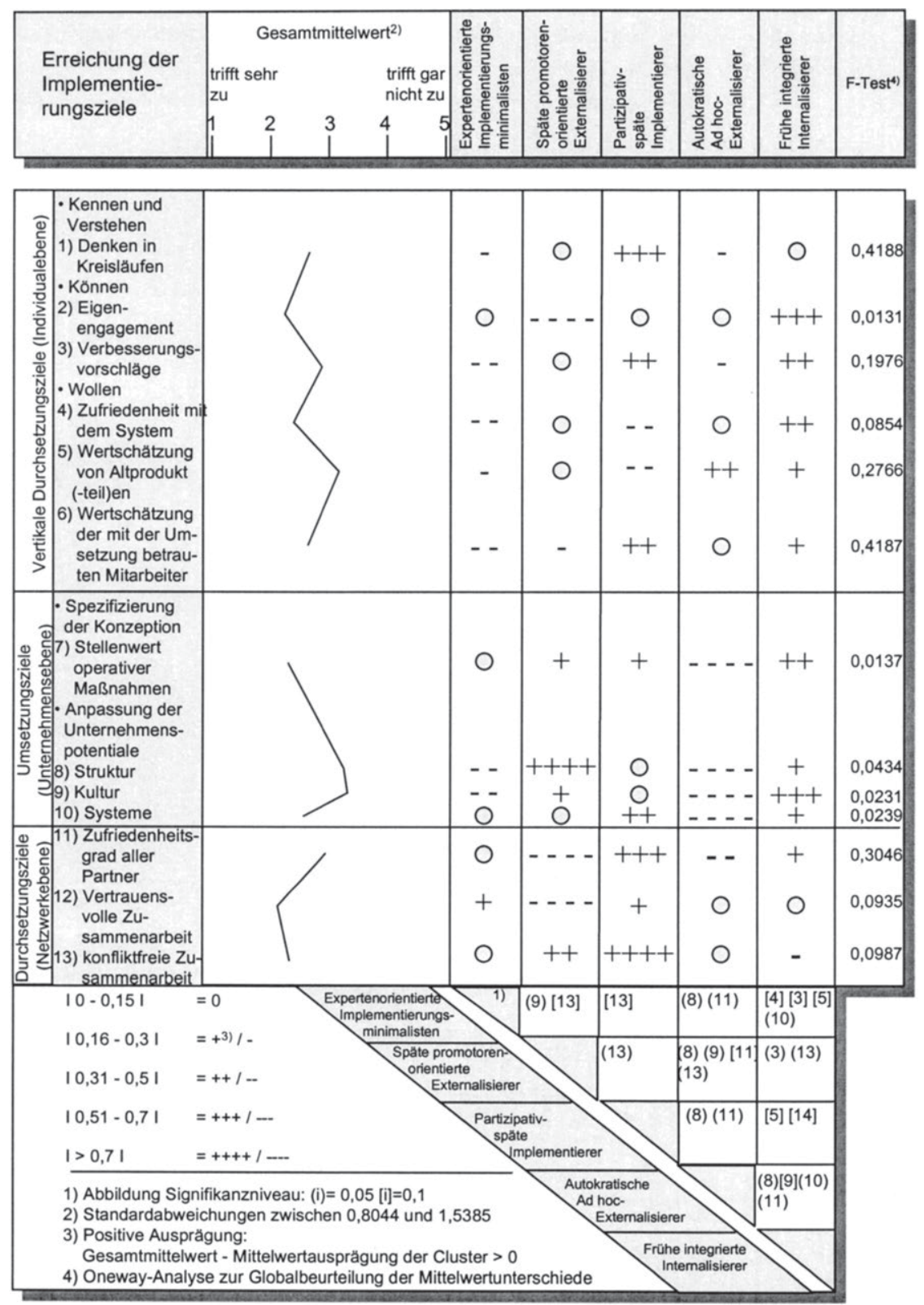

Abb. 42: Erreichung der Implementierungsziele

Irene Giesen-Netzer and Universität Münster - 978-3-631-75058-2

Downloaded from PubFactory at 01/11/2019 08:44:49AM 
Die Partizipativ-späten Implementierer erreichen die Durchsetzungsziele der Netzwerkebene insgesamt signifikant besser als die anderen Cluster. Damit wird deutlich, daß der (hersteller)interne Führungsstil z.T. Rückschlüsse auf das Verhalten innerhalb des Netzwerkes zuläßt. Demzufolge sind die Partizipativ-späten Implementierer relativ offen gegenüber den Systempartnern und beziehen deren Vorschläge in ihre Entscheidungen und Handlungsweisen ein. Es erscheint daher plausibel, daß dieses Cluster einen hohen Zufriedenheitsgrad und eine relativ konfliktfreie Zusammenarbeit ${ }^{570}$ sowie ein vertrauensvolles Verhältnis mit den Systempartnern erreicht. ${ }^{51}$ Zudem beteiligen sich die Partizipativ-späten Implementierer zu $76,4 \%$ an kooperativen Systemen, ${ }^{572}$ arbeiten also i.d.R. mit mehreren unterschiedlichen Systempartnern zusammen. ${ }^{573}$ Dieses läßt den Schluß zu, daß die Bedeutung der Ziele auf der Netzwerkebene für die Implementierung der Rücknahme- und Recyclingsysteme von diesem Cluster als hoch eingestuft wird.

Die Frühen integrierten Internalisierer hingegen implementieren häufig (hersteller)eigene Rücknahme- und Recyclingsysteme. Deren Partner sind daher relativ häufig Verwerter, die in einem lieferantenähnlichen Auftragsverhältnis zu den Herstellern stehen und ausgewählte Aufgaben übernehmen. Folglich liegen die Ausprägungen bei der Erreichung der netzwerkbezogenen Durchsetzungsziele in diesem Cluster nur im Mittelwert.

Bei den Umsetzungszielen auf der Unternehmensebene erreichen die Frühen integrierten Internalisierer sowie die Späten promotorenorientierten Externalisierer diese Ziele signifikant häufiger als erwartet. Daraus kann in Verbindung

570 Modelle der Konfliktnutzung gehen davon aus, daß Konflikte nicht immer etwas Negatives, den Unternehmenserfolg Beeinträchtigendes sind. Konstruktive Konflikte können im Gegensatz zu destruktiven Konflikten sogar im Sinne der Zielerreichung genutzt werden. Eine Suche nach Kompromissen ist dabei oft der falsche Weg, da dadurch Frustration und Aggressionen gegenüber den anderen beteiligten Personen entstehen. Vgl. zu einer Theorieübersicht zu Konfliktmodellen Wottawa, H., Gluminski, I., Psychologische Theorien für Unternehmen, a.a.O., S. $115 \mathrm{ff}$.

571 Schul, Little und Pride stellen in zwei Studien fest, daß (in Franchisesystemen) die wahrgenommene Konfliktintensität negativ mit der Partizipation an systembezogenen Entscheidungen, der Unterstützung und Betreuung der Franchisenehmer durch die Systemzentrale sowie der Formulierung von Richtlinien und Anweisungen verknüpft ist. Vgl. Schul, P.L., Pride, W. M., Little Jr., T. E., The Impact of Channel Leadership on Intrachannel Conflict, in: Journal of Marketing, 1983, Nr. 3, S. 31.

Dieser Wert ist der höchste im Vergleich zu den anderen Clustern.

573 Opportunistisches Verhalten in Netzwerken kann durch spezifische Investitionen aller Partner vermindert werden. Dadurch ergibt sich auch ein Eigeninteresse am Gesamterfolg. Vgl. Hansen, U., Raabe, T., Dombrowsky, B., Die Gestaltung des Konsumgüterrecycling als strategische Netzwerke, a.a.O., S. 67. 
mit ihren Externalisierungsaktivitäten geschlossen werden, daß die Späten promotorenorientierten Externalisierer ihren Zielen entsprechend nur relativ geringe interne Anpassungen zu vollziehen haben und dadurch schnelle Umsetzungserfolge erzielen können. Dies gilt zum einen für strukturelle Anpassungsmaßnahmen, bei denen dieses Cluster angibt, rücknahme- und recyclingsystembezogene Aufgaben überwiegend durch die Integration in bestehende Linienstellen zu bewältigen. ${ }^{574}$ Zum anderen trifft eine ähnliche Argumentation für die Zielerreichung hinsichtlich der Anpassung der anderen Unternehmenspotentiale und der Spezifizierung der Konzeption zu. ${ }^{575}$ Darüber hinaus deutet die eher als defensiv einzustufende Umweltstrategie dieses Clusters darauf hin, daß die Implementierung des Rücknahme- und Recyclingsystems für das Unternehmen keine übergeordnete Relevanz besitzt. ${ }^{576}$

Bei den Frühen integrierten Internalisierern werden hingegen aufgrund der hoch ausgeprägten Intensitätsdimension viele Anpassungsmaßnahmen notwendig. ${ }^{577}$ Allerdings zeigt sich, daß dieses Cluster seine (hohen) gesetzten unternehmensbezogenen Umsetzungsziele relativ gut erreicht hat, da es bereits früh mit den Implementierungsaktivitäten begonnen hat. Die gleichzeitige Verfolgung einer offensiven Umweltstrategie belegt zudem die hohe Bedeutung von rücknahme- und recyclingsystembezogenen Aktivitäten für das unternehmerische Handeln dieses Clusters.

Der Umsetzungserfolg auf der Unternehmensebene wird bei den Autokratischen Ad hoc-Externalisierern deutlich unterdurchschnittlich erreicht. Die Ursachen könnten darin liegen, daß die Hersteller in der Erwartung einer Rücknahmeverordnung ${ }^{578}$ die unternehmensbezogenen Implementierungsmaßnahmen erst dann

$57444,4 \%$ der befragten Späten promotorenorientierten Externalisierer gaben an, eine Integration der Aufgaben in die bestehende Linie vorzunehmen.

575 Bei der Frage nach dem Einsatz von Informationssystemen geben die Späten promotorenorientierten Externalisierer beispielsweise an, Gewinn- und Rentabilitätsvergleiche relativ häufig durchzuführen. Auch Checklisten werden in diesem Cluster relativ intensiv eingesetzt. Daher ist eine Anpassung der bestehenden Informationssysteme notwendig. Da dieses Cluster aber erst relativ spät mit Implementierungsaktivitäten angefangen hat, konnte das hier angestrebte Ziel noch nicht ganz erreicht werden.

576 Aus dieses Ausführungen wird deutlich, daß das Erreichen der Implementierungsziele wesentlich von der zugrunde liegenden subjektiven Zielsetzung der Hersteller abhängt, also immer eine individuelle Betrachtungsweise darstellt.

$\mathrm{Vgl}$. jeweils die deutlich überdurchschnittlichen Ausprägungen bei den Einflußfaktoren der Struktur, Kultur und den Systemen in Kapitel C 1.4.2.3. 
ergreifen, wenn die Verordnung in Kraft tritt. Diese Argumentation wird dadurch gestützt, daß sich dieses Cluster häufig in Branchenlösungen engagiert und in der Realisationsphase wenige Abteilungen sehr intensiv einbezieht.

Bei den vertikalen Durchsetzungszielen auf der Individualebene fällt auf, daß die Führungskräfte insbesondere das Eigenengagement und die Globalzufriedenheit der Mitarbeiter mit den Rücknahme- und Recyclingsystemen über alle Cluster hinweg mit Mittelwerten von 2,1 und 2,3 klar am positivsten beurteilen. Gleichzeitig differenzieren sich die Implementierungstypen hinsichtlich dieser Variablen deutlich gegeneinander. So geben insbesondere die Frühen integrierten Implementierer - erheblich über den statistisch zu erwartenden Werten - an, daß die Mitarbeiter sehr engagiert sind und die Zufriedenheit hoch ist. Insgesamt erreicht dieses Cluster die Durchsetzungsziele auf der Individualebene besser als die anderen Cluster. Dieses Ergebnis zeigt, daß eine integrierte Vorgehensweise Erfolge auf der Individualebene ermöglicht. Diese Befund erscheint konsitent zu der relativ langen Zeitdauer, in der dieses Cluster bereits Erfahrungen mit den Systemen machen konnte.

Bei den Partizipativ-späten Implementierern sind lediglich die Variablen "Denken in Kreisläufen“, „Verbesserungsvorschläge“ und „Wertschätzung der mit der Umsetzung betrauten Mitarbeiter" deutlich positiver ausgeprägt als bei den anderen Clustern. Hingegen werden die "Zufriedenheit mit dem System" sowie die „Wertschätzung von Altproduktteilen“ mit Werten angegeben, die klar unter dem Durchschnitt liegen. Dieses differenzierte Bild zeigt, daß dieses Cluster offenbar noch nicht genügend zeitlichen Vorlauf hatte und das Rücknahme- und Recyclingsystem dementsprechend noch einige Anfangsschwierigkeiten zu überwinden hat. Gleichzeitig sind jedoch die Mitarbeiter für die Rücknahme- und Recyclingsysteme bereits soweit sensibilisiert, daß sie die "Kennen und Verstehen"- Komponente erfüllen, während sie bei den anderen Komponenten der Individualebene ("Können“, "Wollen“) ein uneinheitliches Bild aufweisen. Die Priorisierung der Durchsetzungsaufgaben mit durchschnittlichen bzw. überdurchschnittlichen Werten unterstützt diese Argumentation. 


\section{2 Ökologischer und ökonomischer Erfolg}

Neben den Implementierungszielen gilt es, die Ziele der Rücknahme- und Recyclingsysteme als Objekt der Implementierung auf deren Erreichung zu überprüfen. Bei der Untersuchung der ökologischen Ziele ist zu vermuten, daß sich durch die Implementierung von Rücknahme- und Recyclingsystemen die Erfassungsquoten für die Altprodukte über alle Cluster hinweg im Vergleich zu der Situation, als die Rücknahme- und Recyclingsysteme noch nicht eingerichtet waren, erhöht haben. Dennoch fällt die Erfassungsquote wahrscheinlich nicht so positiv aus, wie die Hersteller ursprünglich geplant haben. ${ }^{579}$

Bei den Verwertung von Altprodukten ${ }^{580}$ ist ebenfalls davon auszugehen, daß diese sich über alle Implementierungstypen hinweg erhöht hat, da hier geringere Anforderungen als bei der Verwendung von Altprodukten an die Unternehmen gestellt werden ${ }^{581}$ Beispielsweise müssen für eine Verwertung von Altprodukten weder die Funktionsfähigkeit sichergestellt noch Qualitätskontrollen der Altprodukt(teil)e durchgeführt werden. Bezüglich einer Weiterverwertung werden die Ausprägungen bei den Implementierungstypen vermutlich ebenfalls kaum differieren, da die betrachteten Hersteller hierbei zum einen nicht mehr mit den Sekundärrohstoffen aus den Altprodukten konfrontiert werden und daher keine weiteren Aktivitäten nach der Verwertung der Altprodukte notwendig werden. Zum anderen wird die Weiterverwertung sicherlich in allen untersuchten Implementierungstypen durchgeführt, da diese Recyclingart eine übliche Recyclingpraxis darstellt. Differenzierter kann demgegenüber eine Analyse der Verwendungsziele und der Wiederverwertung erfolgen. Daher werden folgende Basishypothesen formuliert:

\begin{tabular}{|ll|}
\hline$H_{\text {WIR2a }}$ & $\begin{array}{l}\text { Zwischen den Implementierungstypen bestehen signifikante Unterschiede hinsichtlich der } \\
\text { Erreichung der Verwendungsziele. }\end{array}$
\end{tabular}

579 Die Vermutung wird aufgrund von der Verfasserin geführter Gespräche mit den Herstellern aufgestellt. Als Ursachen lassen sich zum einen die enormen Zeitverzögerungen bei der Rückgabe und die bereits eingangs skizzierte hohe Unsicherheit zukünftiger Entwicklungen aufführen. Auch können unrealistische Zielsetzungen seitens der Hersteller dazu führen, daß die ökologischen Ziele nicht erreicht werden.

580 Corsten und Götzelmann weisen im Zusammenhang von Verwertungszielen darauf hin, daß eine Verwertung der Abfälle zu einer Verlängerung der Verweildauer von Stoffen im Wirtschaftskreislauf führt. Damit wird einerseits eine temporäre Entlastung des Primärrohstoffverbauchs und andererseits eine temporäre Entlastung des Entsorgungsaufkommens erzielt. Vgl. Corsten, H., Götzelmann, F., Abfallvermeidung und Reststoffverwertung, a.a.O., S. $106 f$.

581 Dennoch sind auch hier vielfältige Voraussetzungen zu erfüllen. Neben der Erfassung muß beispielsweise eine möglichst sortenreine Trennung und Reinigung der Stoffraktionen erfolgen. Vgl. dazu beispielsweise die detaillierten Ausführungen der Altfensteraufbereitung in der FREI-Fallstudie, a.a.O. 
$\begin{array}{|ll|}H_{\text {WIR2b }} & \begin{array}{l}\text { Zwischen den Implementierungstypen bestehen signifikante Unterschiede hinsichtlich der } \\ \text { Erreichung der Wiederverwertungsziele. }\end{array}\end{array}$

Bei der ökologischen Zielerreichung der Hersteller, die in Abbildung 43 dargestellt wird, zeigt sich, daß sich die Implementierungstypen lediglich hinsichtlich der Wiederverwertung (F-Wert von 0,0064) und sich die Frühen integrierten Internalisierer hinsichtlich der Wiederverwendung von Altprodukt(teil)en im Kundendienst unterscheiden. Daher kann die Hypothese HwiR2b bestätigt werden, während die Basishypothese $\mathrm{H}_{\text {WIR2a }}$ nur in der Tendenz bestätigt werden kann.
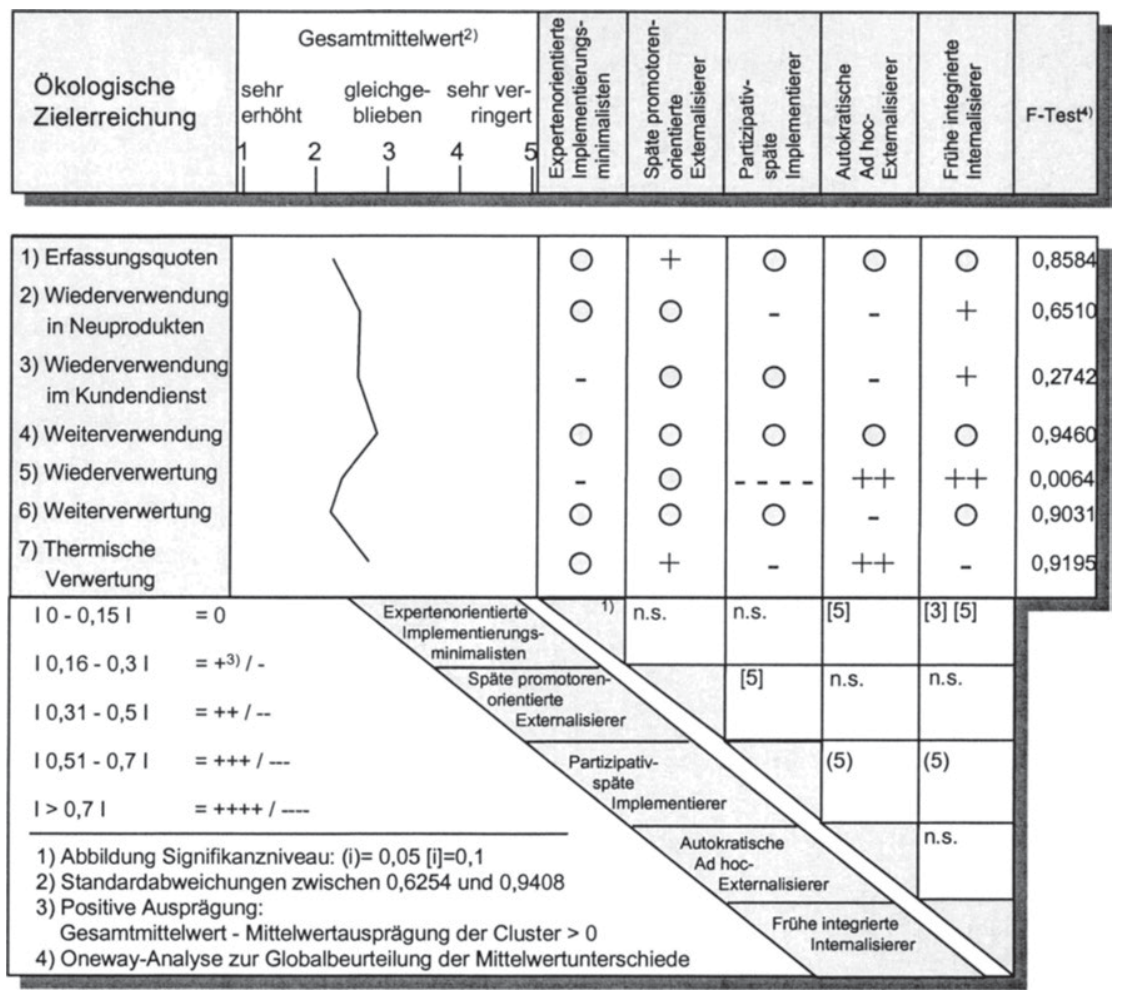

\section{Abb. 43: Ökologische Zielerreichung}

Annahmegemäß haben sich die Erfassungsquoten (Mittelwert von 2,3) sowie die Verwertungsquoten (Mittelwert von 2,3 bzw. 2,4) bei den Herstellern seit Implementierung der Rücknahme- und Recyclingsysteme erhöht. Verwendungen von 
Altproduktteilen in der Neuproduktion sowie im Kundendienst haben mit Werten von 2,8 dagegen kaum zugenommen. Daran wird klar, daß der Erhaltung von Altproduktwerten - bis auf einige Ausnahmen - bisher kaum Interesse beigemessen wird, sondern lediglich Stofffraktionen als Sekundärmaterialien gewonnen werden. Eine leicht positive Abweichung zeigen hier die Frühen integrierten Internalisierer, d.h. bei diesem Implementierungstyp wurden mehr Altprodukt(teil)e in der Neuproduktion sowie im Kundendienst eingesetzt als bei den übrigen Clustern. Dieses scheint angesichts der Internalisierungsaktivitäten und der häufig anzutreffenden organisatorischen Gestaltung der Rücknahme- und Recyclingsysteme in Form von herstellereigenen Systemen plausibel. Durch die Internalisierungsaktivitäten sind Hersteller demzufolge in der Lage, die Funktion wie auch die Qualität der Altproduktteile sicherzustellen, um diese im Produktionsprozeß oder Kundendienst erneut einzusetzen.

Bei den Frühen integrierten Internalisierern und Autokratischen Ad hocExternalisierern hat sich die Wiederverwertung mehr erhöht als bei den anderen Clustern. Dieses kann mit den Ausprägungen der Intensitätsdimension der Implementierung dieser Cluster begründet werden, die beide in der Realisationsphase viele Abteilungen intensiv in die Implementierung der Rücknahme- und Recyclingsysteme einbeziehen. Dieses ist auch bei einer Wiederverwertung der Fall. In dieser Weise ist beispielsweise der Einkauf unmittelbar betroffen, da in diesem Bereich die entsprechenden Primärrohstoffe durch die Sekundärrohstoffe ersetzt werden. Die Qualitätskontrolle hat die Aufgabe, die Sekundärrohstoffe zu prüfen, während in der Produktion auf die veränderten Anforderungen des Materials reagiert werden muß. Diese Argumentation wird durch die Tatsache gestützt, daß bei Branchenlösungen - die insbesondere bei Autokratischen Ad hoc-Externalisierern vorliegen $^{582}$ - die Hersteller z.T. verpflichtet werden, die entstehenden Recyclate den Verwertern abzunehmen, um eine Funktionsfähigkeit des Systems sicherzustellen. ${ }^{583}$

Bei der Erreichung der ökonomischen Ziele sind diejenigen der einzelnen Hersteller $^{544}$ von den Zielen des Gesamtsystem des Netzwerkes bzw. aller beteilig-

\footnotetext{
582 Vgl. Kapitel C 1.3.

583 Vgl. z.B. FREI-Fallstudie, a.a.O.

584 Rautenstrauch leitet eine Formel her, die der herstellerseitigen Entscheidungsfindung hinsichtlich der ökonomischen Effizienz Rechnung tragen soll. Danach müssen die Kosten für die Nutzung, Erfassung und den Einsatz der Altprodukte sowie die Fertigungskosten beim Einsatz Irene Giesen-Netzer and Universität Münster - 978-3-631-75058-2
} 
ten Partner zu unterscheiden. Wird ein Rücknahme- und Recyclingsystem auf Grundlage von Verträgen zwischen Hersteller und Verwerter implementiert, so kann die Kostensituation bei relativ geringen Erfassungsquoten für die Hersteller durchaus befriedigend sein, da häufig nur dann vertraglich vereinbarte Zahlungen für die vorher festgelegten Altprodukte (in Stück oder in Tonnen) fällig werden, wenn diese auch anfallen. ${ }^{585}$ Diese Situation ist jedoch gleichzeitig für den Verwerter recht unbefriedigend, da dieser die Kapazitäten, die eventuell aufgrund dieses Vertrages aufgebaut wurden, auslasten will, dieses aber bei geringen Erfassungsquoten nicht leisten kann. ${ }^{586}$ Damit wird deutlich, daß die Erreichung der ökonomischen Ziele - neben der Ausgestaltung der Implementierungsdimensionen - insbesondere von den institutionellen Ausgestaltungsformen der Rücknahme- und Recyclingsysteme bestimmt wird. ${ }^{587}$ Der empirischen Überprüfung liegt folgende Basishypothese zugrunde:

$\mathrm{H}_{\text {WIR3 }}$ Zwischen den Implementierungstypen bestehen signifikante Unterschiede hinsichtlich der Erreichung der ökonomischen Ziele.

Wird das Ergebnis der ökonomischen Zielerreichnung in Abbildung 44 betrachtet, fällt auf, daß die Hersteller im Gesamtdurchschnitt die Erreichung der Image- und Profilierungsziele sowie die Kostenziele als diejenigen Ziele angeben, die sie am ehesten erreicht haben. Gewinn-, Umsatz- und Kapazitätsauslastungsziele werden hingegen mit Mittelwerten von 3,5 bis 3,6 nur schlecht erreicht. Dies ist angesichts der hinter den herstellerseitigen Erwartungen zurückbleibenden

von Sekundärrohstoffen kleiner sein als die Kosten der Primärstoffe bis zum Einsatz in der Produktion und die Fertigungskosten beim Einsatz von Primärstoffen zuzüglich der Kosten für die Abfallbehandlung abzüglich der Erlöse des Recycling. Vgl. Rautenstrauch, C., Betriebliches Recycling, a.a.O., S. $96 f$.

Vgl. z.B. Bauknecht-Fallstudie, a.a.O. Dem versuchen die Verwerter entgegenzuwirken, indem sie beispielsweise die Altprodukte bündeln, d.h. sie nehmen Großgeräte von verschiedenen Herstellern zurück oder sie versuchen, die Verwertungsverfahren so zu gestalten, daß sie möglichst verschiedene Produktgruppen zusammenfassen können.

586 Bei dieser Argumentation wird von Subventionen, die für ausgewählte Recyclingverfahren gewährleistet werden, abstrahiert. Nichtsdestoweniger beeinflussen Subventionen die ökonomische Zielerreichung der Unternehmen.

587 Aus volkswirtschaftlicher Sicht wird dieser Zusammenhang in Principal-Agent-Modellen diskutiert. Aufgrund einer Informationsasymmetrie zwischen Herstellern (Principals) und Verwertern (Agents) versucht der Hersteller, das mit der Rücknahmeverpflichtung verbundene Risiko auf den Verwerter zu übertragen. Die Informationsasymmetrien bestehen neben dem Zeitpunkt der Rücknahme und Veränderungen der Materialzusammensetzung sowie -qualităt insbesondere in oft starken Schwankungen der Daten auf den Sekundärmärkten. Gleichzeitig sind die Kosten der Verwerter von Umfang und Auslastungen der bereitgestellten Kapazităten, Produktionsfunktionen und Inputs abhängig, während die Erlöse vom unsicheren Sekundärmarkt und dem Entgelt des Herstellers abhängen. Vgl. dazu im Detail Clausen, H., Wacker, H., Okonomische Modellierung von Rücknahmepflichten, a.a.O., S. $43 \mathrm{f}$. 
Erfassungsquoten und der unterausgelasteten Kapazitäten verständlich, die nur bei einer vertraglichen Vereinbarung zwischen Herstellern und Verwertern eine geringere Rolle spielen. ${ }^{588}$ Diese Aussagen gelten gleichermaßen für alle Implementierungstypen. Nur hinsichtlich der Image- und Profilierungsziele sowie der Erfahrungsziele zeigen sich bei der Betrachtung der F-Werte $(\alpha=0,1)$ signifikante Unterschiede. Daher kann die Basishypothese $H_{\text {WIR3 }}$ lediglich in Teilen angenommen werden.

Die Frühen integrierten Internalisierer erreichen die Image- und Profilierungsziele sowie die Erfahrungsziele besser als dieses statistisch zu erwarten wäre. Zum einen kann diese Überlegung mit den Pionieraktivitäten begründet werden, da dieses Cluster deutlich früher als die anderen Hersteller aktiv wird. ${ }^{589}$ Zum anderen scheinen Kunden bei Systemen, die der Hersteller selbst durchführt („alles aus einer Hand"), weniger skeptisch zu sein, da Verwertungsunternehmen nicht immer eine gute Reputation genießen. Darüber hinaus ist aufgrund des hohen Anteils von Vertrauens- und Erfahrungseigenschaften ${ }^{590}$ von Rücknahme- und Recyclingsystemen die Skepsis der Kunden bei der herstellerseitigen Implementierung solcher Systeme in besonderem Maße zu berücksichtigen. ${ }^{591}$ Dieser Umstand sollte insbesondere bei Herstellern, die viele Aktivitäten internalisieren, dazu

588 Demzufolge beklagen auch Verwerter die noch fehlende Durchsetzung des Kreislaufwirtschaftsgesetzes. Vgl. Kook, W., Umsetzung des Kreislaufwirtschaftsgesetzes aus Sicht der Rethmann AG \& Co, a.a.O., S. 27.

589 Vgl. zu der Diskussion von Pionier- und Folgerstrategien Remmerbach, K.-U., Markteintrittsentscheidungen, a.a.O., S. 58ff. und die dort angegebene Literatur.

Meffert und Kirchgeorg diskutieren im Zusammenhang mit dem noptimalen " ökologieorientierten Profilierungszeitpunkt primäre und sekundäre Marktwiderstände, die die kundenseitigen und wettbewerbsseitigen Barrieren bei der Einführung von neuen Produkten erfassen. Vgl. Meffert, H., Kirchgeorg, M., Marktorientiertes Umweltmanagement, a.a.O., S. $231 \mathrm{f}$.

Diese Diskussion kann in Anlehnung an die Diskussion von Eigenschaften von Dienstleistungen geführt werden. Bei Suchkomponenten (Search Qualities) kann der Kunde anhand von Indikatoren die Leistung im Vorfeld der Inanspruchnahme beurteilen. Bei der Erfahrungskomponente (Experience Qualities) ist der Kunde in der Lage, die Leistung aufgrund von Erfahrungen zu beurteilen. Dagegen sind Vertrauenskomponenten (Credence Qualities) nicht durch den Kunden beurteilbar. Vgl. Zeithaml, V. A., How Consumer Evaluation Processes differ between Goods and Services, in: Donnelly, J. H., George, W. R., (Hrsg.), Marketing of Services, Chicago 1981, S. 186ff. und vgl. Meffert, H., Bruhn, M., Dienstleistungsmarketing: Grundlagen, Konzepte, Methoden; mit Fallbeispielen, Wiesbaden 1996, S. 200.

Kirchgeorg stuft die Rückgabefähigkeit eines Gebrauchsgutes als Erfahrungseigenschaft ein, da ein Produktverwender erst am Ende der Lebensdauer Erfahrung mit dem Rückgabemodus machen kann. Das Leistungsmerkmal der Recyclingfähigkeit hingegen ist als Vertrauenseigenschaft einzustufen, da der Kunde i.d.R. keinen Einblick in die Recyclingprozesse der Hersteller oder Verwerter erhält. Vgl. Kirchgeorg, M., Unternehmensstrategische Gestaltungsprobleme von Stoffkreisläufen, a.a.O., S. $117 \mathrm{f}$. 
führen, daß sie besondere Sorgfalt - im Sinne eines integrierten Vorgehens - zeigen.

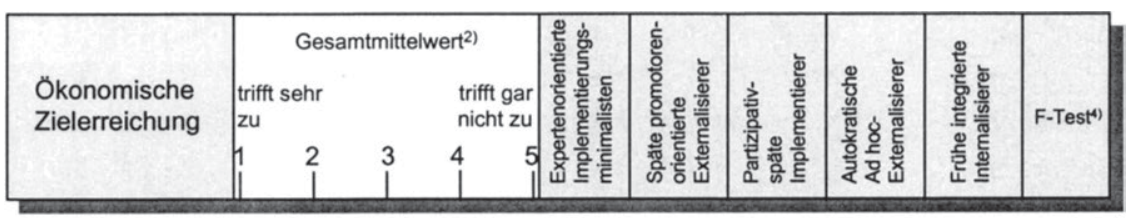

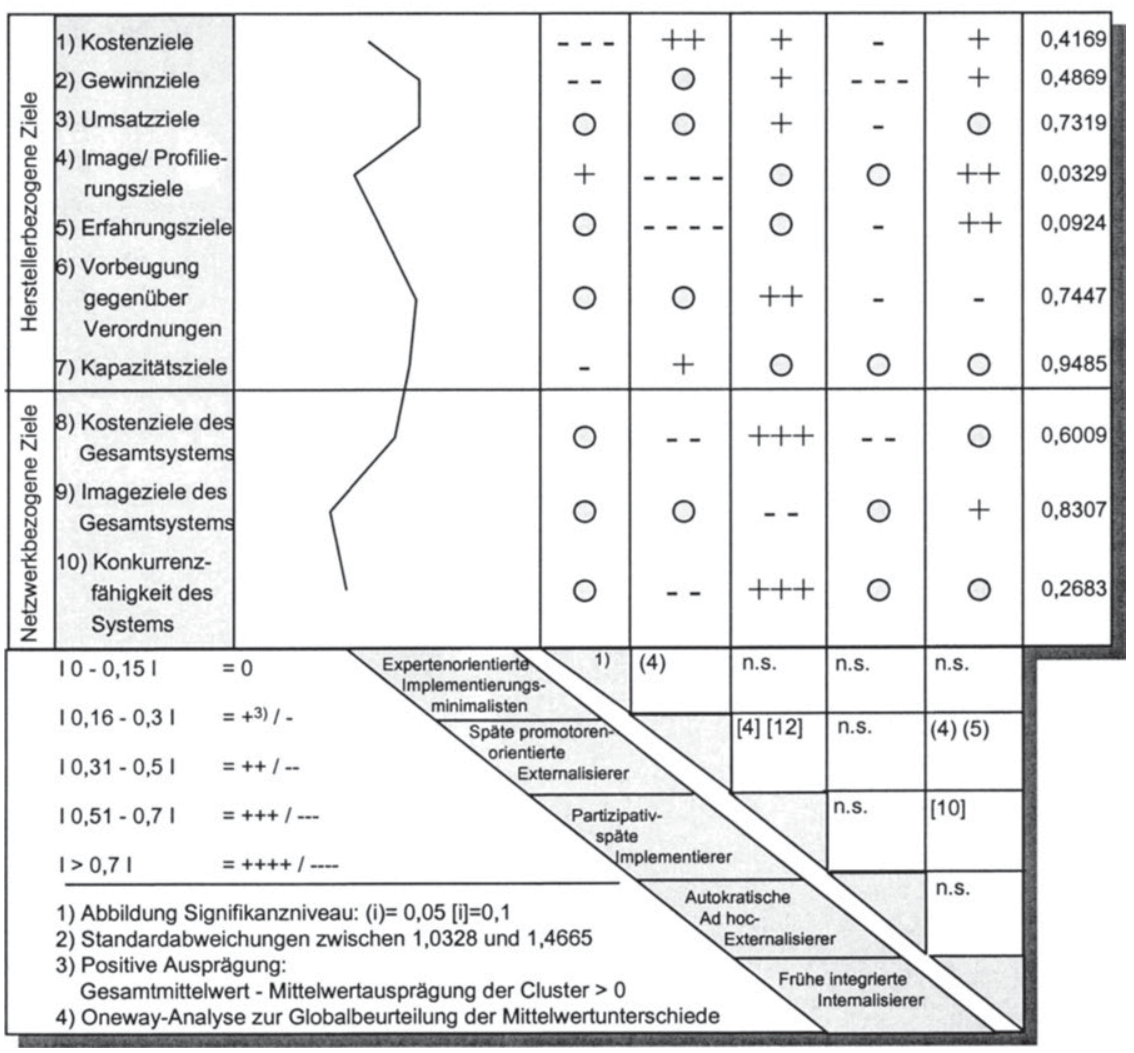

\section{Abb. 44: Ökonomische Zielerreichung}

Hinsichtlich der Beurteilung der Konkurrenzfähigkeit des gesamten Rücknahmeund Recyclingsystems beurteilen die Partizipativ-späten Implementierer ihr System optimistischer als die anderen Implementierungstypen. Bei diesem Cluster 
fällt jedoch insgesamt auf, daß es die Zielerreichung relativ positiv beurteilt. Dies kann darin begründet liegen, daß sich die Partizipativ-späten Implementierer überwiegend noch in der Planungsphase befinden und daher nur Vermutungen über die ökonomische Zielerreichung abgeben konnten. ${ }^{592}$

Bei den Späten-promotorenorientierten Externalisierern wurden die (herstellerseitigen) Kostenziele überdurchschnittlich gut erreicht, wobei die Kostenziele im Gesamtsystem des Netzwerkes unterdurchschnittlich gut erreicht wurden. Dieser Befund zeigt, daß durch die Externalisierungsaktivitäten dieses Implementierungstyps die Risiken auf die Verwerter abgewälzt werden konnten. ${ }^{593}$ Zudem erreichen die Späten promotorenorientierten Externalisierer Image- und Profilierungsziele sowie Erfahrungsziele weniger gut. Ursachen liegen vermutlich darin, daß die Hersteller zum einen wenig mit Aktivitäten werben, die sie nicht selbst durchführen, noch diese Aktivitäten anderweitig besonders herausstellen und kaum eigene Erfahrungen mit dem Recycling sammeln können. Am Ergebnis der Späten promotorenorientierten Externalisierer wird ersichtlich, daß zu dem Zeitpunkt der Befragung eine "Externalisierungsstrategie" in Form einer vertraglichen Vereinbarung zwischen Herstellern und Verwertern durchaus erfolgversprechend ist, jedoch fraglich bleibt, ob sich dieses Verhalten auch in einer langfristigen Perspektive bewährt.

Damit ist die Analyse der Erfolgswirkungen des Implementierungsverhaltens von Herstellern langlebiger Gebrauchsgüter abgeschlossen. Eine Zusammenfassung der zentralen Ergebnisse und die Ableitung von Implikationen der Untersuchung für die Praxis und die Forschung schließen sich im Schlußkapitel der Untersuchung an.

$59253 \%$ der befragten Partizipativ-späten Implementierer befinden sich in der Planungsphase. $\mathrm{Vgl}$. Anhang 1.7. Darüber hinaus beurteilten nur 5 Hersteller dieses Clusters die okonomische Effektivität, da die anderen Hersteller des Clusters sich nicht in der Lage sahen, diese Zielgrößen zu beurteilen. 


\section{Zusammenfassung und Implikationen der Untersuchungsergebnisse über Rücknahme- und Recyclingsysteme}

\section{Zusammenfassende Würdigung der Untersuchungsergebnisse}

Der in Theorie und Praxis gleichermaßen intensiv diskutierte Entwurf des Kreislaufwirtschaftsgesetzes bildete den Anlaß, sich mit den Aktivitäten von Unternehmen auseinanderzusetzen, die mit diesem Gesetz in unmittelbarem bzw. mittelbaren Zusammenhang stehen. Im Mittelpunkt der vorliegenden Arbeit stand das Verhalten von Herstellern langlebiger Gebrauchsgüter bei der Implementierung von Rücknahme- und Recyclingsystemen. Angesichts der Vielfalt unterschiedlicher beobachtbarer Ausgestaltungsformen wurde zunächst eine Systematisierung von Rücknahme- und Recyclingsystemen vorgenommen. Anschließend erfolgte eine Operationalisierung des Implementierungsverhaltens durch sogenannte Implementierungsdimensionen, die sich in intensitätsbezogene, hierarchiebezogene und zeitliche Dimensionen gliedern ließen. Auf Grundlage dieser Implementierungsdimensionen konnten herstellerseitige Implementierungstypen gebildet werden, die sich hinsichtlich der Ausprägungen in ihrem Verhalten mit hoher diskriminatorischer Güte voneinander unterschieden.

Das Vorliegen unterschiedlicher Implementierungstypen wurde zum Anlaß genommen, mögliche Erklärungsansätze für diesen Tatbestand zu gewinnen. Erkenntnisse aus kontingenztheoretischen Arbeiten bildeten die Grundlage für die Ableitung netzwerk-, unternehmens- und individualbezogener Einflußfaktoren, deren Wirkung auf die Ausprägung der Implementierungstypen Inhalt der Analyse war. Die Untersuchung zeichnete sich durch hohe Komplexität aus, da sich Rücknahme- und Recyclingsysteme durch unternehmens- und funktionsübergreifende Eigenschaften auszeichnen und darüber hinaus in ihrem Anspruch eine Abkehr von der Durchlauf- zur Kreislaufwirtschaft fordern. Aus einer Vielzahl relevanter Einflußfaktoren werden im folgenden einige besonders prägnante Faktoren zu einer abschließenden Synopse verdichtet.

In Abbildung 45 werden die institutionellen Ausgestaltungsformen als netzwerkbezogener Einflußfaktor und der Status des Rücknahme- und Recyclingsystems mit den Implementierungstypen in Verbindung gebracht. ${ }^{594}$ Es wird deutlich,

594 Eine detaillierte Analyse zeigt die Kreuztabelle über den Status der Rücknahme- und Recyclingsysteme in bezug auf die Implementierungstypen. Vgl. Anhang 1.7.

Irene Giesen-Netzer and Universität Münster - 978-3-631-75058-2 
daß sich die Implementierungstypen hinsichtlich ihrer Ausprägungen deutlich voneinander unterscheiden. Nahezu 30 Prozent der untersuchten Herstellerunternehmen befinden sich als Frühe integrierte Internalisierer bereits in der Realisationsphase und haben Rücknahme- und Recyclingsysteme häufig als herstellereigenes Rücknahme- und Recyclingsystem implementiert. Zusätzlich lassen sich bei diesem Implementierungstyp auch Kooperationen beobachten.

Im Gegensatz zu diesem Cluster weisen die noch überwiegend im Planungsstadium befindlichen Späten promotorenorientierten Externalisierer weitaus „marktnähere" Formen wie beispielsweise eine vertragsmäßige Abgabe der Rücknahme- und Recyclingaktivitäten an Verwerter auf. Eine intensivere Form der vertraglichen Kooperation wählen die Expertenorientierten Implementierungsminimalisten, die mengenmäßig das zweitgrößte Cluster der Stichprobe bilden. Auffällig ist das „Überspringen" einer Pilotphase, da eine nach den eigenen Wünschen angestrebte Lösung von den anderen Kooperationspartnern - i.d.R. Verwertungsunternehmen - vor der endgültigen Implementierung zu testen ist. Hier wird deutlich, daß das Durchlaufen einer Testphase dem typischen Kennzeichen der "Aktivitätsminimierung" dieses Clusters widerspricht.

Das anteilmäßig kleinste Cluster der Autokratischen Ad hoc-Externalisierer hat die Rücknahme- und Recyclingsysteme in Zusammenarbeit mit Unternehmen derselben Branche bereits in das Stadium der Realisation gebracht. Angesichts der geringen mengenmäßigen Relevanz dieses Implementierungstyps für die Gesamtstichprobe ist zu vermuten, daß für die Beantwortung der Fragen der empirischen Analyse nur das jeweils führende bzw. für Öffentlichkeitsbelange zuständige Unternehmen stellvertretend für die Unternehmen der Branchenkooperation bestimmt wurde. Deutlich weniger stark an Zeitvorteilen orientiert stellen sich schließlich die Partizipativ-späten Implementierer dar, die ihre Implementierungsaktivitäten über Mischsysteme organisieren. 


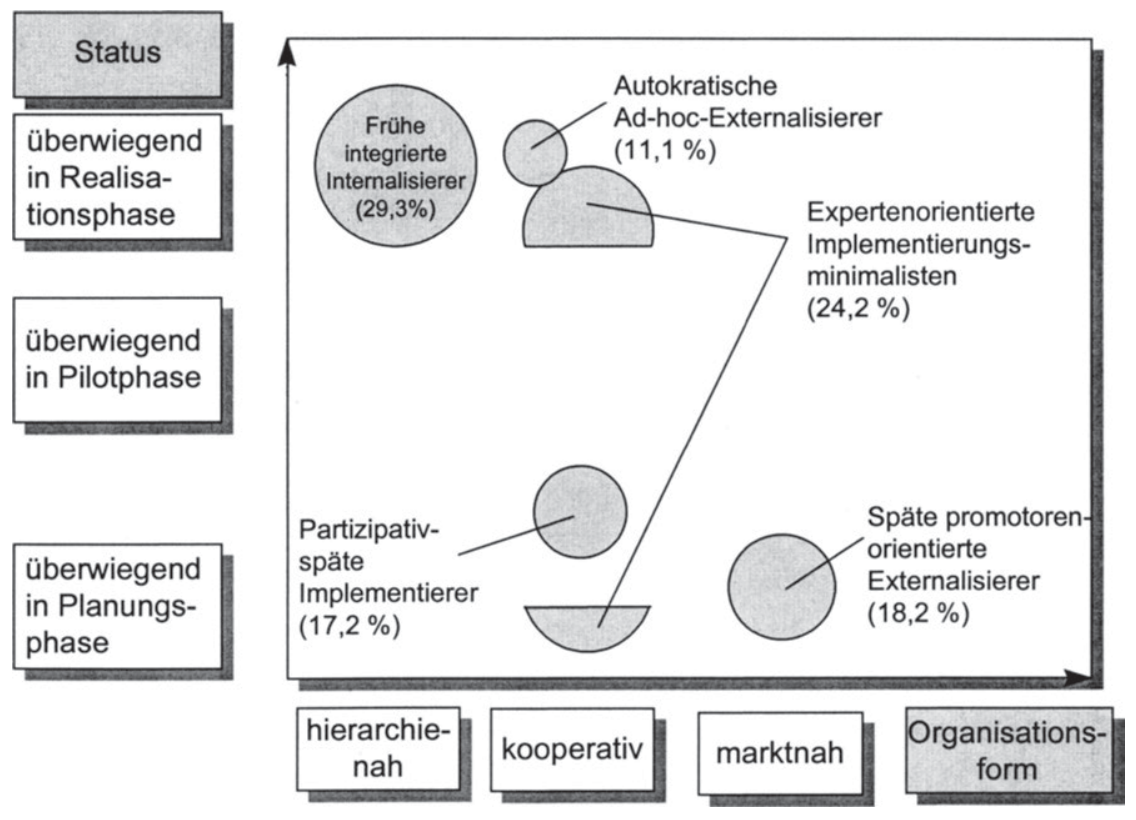

Der Kreisdurchmesser entspricht dem Prozentanteil des Clusters an der Gesamtstichprobe.

Abb. 45: Postionierung der Implementierungstypen anhand des Status der Implementierung und der institutionellen Ausgestaltungsformen von Rücknahme- und Recyclingsystemen

Neben den eher formalen Einflußfaktoren auf der Netzwerkebene galt das Augenmerk auch Variablen auf der Ebene der einzelnen Herstellerunternehmung, die ihrerseits in die Unternehmens- und Individualebene unterteilt wurde. Von besonderem Interesse für eine entscheidungsorientierte Interpretation des Implementierungsverhaltens erwies sich die Analyse der Strategiewahl der Unternehmen. In der Strategie-Implementierungs-Synopse (vgl. Abbildung 46) werden die Implementierungstypen nach ihrer wettbewerbsstrategischen Ausrichtung und der Umweltstrategie klassifiziert. Die Verbindung der Umweltstrategie mit den Wettbewerbsvorteilsdimensionen „Kostenführerschaft" und "Qualitätsführerschaft" sowie der Marktabdeckung ermöglicht vor dem Hintergrund hoch signifikanter Wir- 
kungszusammenhänge ein tieferes Verständnis des Implementierungsverhaltens. ${ }^{595}$

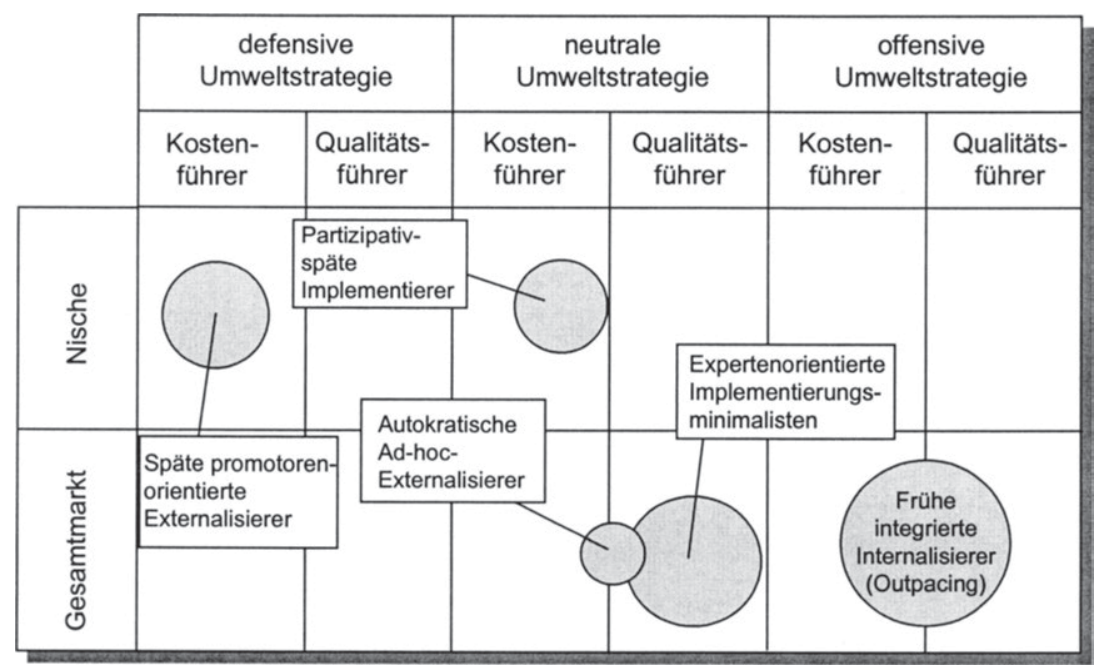

Der Kreisdurchmesser entspricht dem Prozentanteil des Clusters an der Gesamtstichprobe.

\section{Abb. 46: Synoptische Darstellung des Strategie-Implementierungs-Zusammenhangs}

Deutlich wird zunächst der Einfluß der Stichprobenauswahl auf die Ausprägung der Umweltstrategie, da lediglich ein kleiner Anteil der erfaßten Unternehmen eine defensive Umweltstrategie verfolgt. Offenbar stehen Hersteller, die als Kostenführer Marktnischen besetzen, Umweltaspekten zögerlich bis abwartend gegenüber und versuchen, die im Zusammenhang mit Rücknahme- und Recyclingsystemen entstehenden Kosten und Risiken über Verträge mit Verwertern abzuwälzen. Das Verhalten dieser Späten promotorenorientierten Externalisierer erscheint auch angesichts der Unternehmensgröße nicht überraschend, da es sich überwiegend um kleine Unternehmen handelt. ${ }^{596}$ Demgegenüber streben Hersteller, die eine offensive Umweltstrategie verfolgen, sowohl Kosten- als auch Qualitätsvorteile an

\footnotetext{
595 An dieser Abbildung wird die Kritik an der Strategiesystematisierung von Porter deutlich. So befindet sich die Wahl über die Marktabdeckung nicht auf der gleichen Entscheidungsebene wie die Wahl der Kosten- oder Qualitätsführerschaft. So entscheidet ein Unternehmen, ob es Qualitäts- oder Kostenführer werden und ob es einen Nischenmarkt oder den Gesamtmarkt abdecken möchte.
} 
und lassen bei der Implementierung von Rücknahme- und Recyclingsystemen eine integrierte Vorgehensweise erkennen (Frühe integrierte Internalisierer). Offenbar ermöglichen die Gesamtmarktausrichtung und die Unternehmensgröße ${ }^{597}$ nicht nur die Verfolgung einer Outpacing-Strategie, sondern darüber hinaus auch die Internalisierung rücknahme- und recyclingsystembezogener Aktivitäten.

Die Ergebnisse der Analyse lassen schließlich deutlich werden, daß mehr als die Hälfte der untersuchten Hersteller im Vergleich zu den zuvor genannten Extremausprägungen eher eine neutrale Umweltstrategie aufweisen, wenngleich der Mittelwert absolut betrachtet bei 2,09 liegt. Die Expertenorientierten Minimalisten verfolgen eine qualitätsorientierte Gesamtmarktabdeckung und ergänzen diese Ausrichtung durch gezieltes Expertenwissen um umweltbezogene Aspekte. Die eindeutige Qualitätsausrichtung korrespondiert mit einer hohen Produktkomplexität und einem vergleichsweise hohen Anteil von Vermietung und Leasing als Absatzform, der für dieses Cluster kennzeichnend ist. Die Unternehmensgröße 83,3\% dieser Hersteller rechnen zu den Großunternehmen - ermöglicht die Ausrichtung des Leistungsprogramms auf den Gesamtmarkt.

Eine Gesamtmarktausrichtung in Verbindung mit niedrigerer Qualitätsorientierung führt demgegenüber zu einem deutlich unterschiedlichen Implementierungsverhalten, das sich im Cluster der Autokratischen Ad hoc-Externalisierer widerspiegelt. Eine Betrachtung der Partizipativ-späten Implementierer läßt schließlich die Vermutung laut werden, daß Nischenpositionen gewisse zeitliche Freiheitsgrade hinsichtlich rücknahme- und recyclingbezogener Implementierungsaktivitäten gewähren. Offenbar sind Nischenanbieter in geringerem Umfang der Kritik öffentlicher Anspruchsgruppen ausgesetzt und befinden sich bei umweltbezogenen Aktivitäten daher nicht in einem so hohen Legitimationszwang wie Anbieter mit einer stärkeren Gesamtmarktorientierung. Im Gegensatz zur Nische der Späten promotorenorientierten Externalisierer weisen die Partizipativ-späten Implementierer jedoch ein etwas höheres Maß an Qualitätsorientierung auf. ${ }^{588}$

597 Knapp 70 \% dieser Unternehmen zählen zu den Großunternehmen (>500 Mitarbeiter) der zugrunde liegenden Stichprobe. Vgl. Kapitel C 1.4.1.2.

598 Dennoch darf nicht übersehen werden, daß die Ausprägung der Qualitătsführerschaft über alle betrachteten Unternehmen bei dem Wert von 1,6 liegt. Der Interpretation der einzelnen Cluster liegt allerdings eine relative Betrachtung zugrunde. 
Neben der Analyse der Einflußfaktoren auf das Implementierungsverhalten der Hersteller bei Rücknahme- und Recyclingsystemen wurden die Erfolgswirkungen des Implementierungsverhaltens untersucht. Ausgangspunkt bildete die Frage der Zielerreichung der untersuchten Hersteller in bezug auf ökonomische, ökologische und Implementierungsziele.

In Abbildung 47 sind die verdichteten Erfolgswirkungen als relative Zielerreichungsgrade wiedergegeben. ${ }^{599}$ Eine Betrachtung der Ergebnisse läßt zunächst einen positiven Zusammenhang zwischen dem relativen Implementierungserfolg und dem relativen ökonomischen Erfolg deutlich werden. Diese fundamentale - und bereits häufig in der Literatur theoretisch hergeleitete - Erkenntnis läßt die Interpretation $\mathrm{zu}$, daß sich umfangreiche Implementierungsaktivitäten seitens der Hersteller letztlich positiv auf die Erreichung ökonomischer Ziele auswirken, die mit dem Objekt der Implementierung verfolgt werden. Die Cluster der Autokratischen Ad hoc-Externalisierer und Frühen integrierten Internalisierer markieren die Extrempunkte dieses Zusammenhangs.

Theoretische Überlegungen, die einen Mißerfolg bei gut implementierten, jedoch schlecht konzeptionierten Strategien bzw. Objekten vorhersagen, ${ }^{600}$ können ebenfalls bestätigt werden. Das Cluster der Partizipativ-späten Implementierer weist zwar sowohl einen relativ hohen Implementierungs- als auch ökonomischen Erfolg auf, vermag aber die „inhaltlichen ökologischen“ Ziele des Rücknahme- und Recyclingsystems schlechter als der Durchschnitt zu erreichen. In dieser Tatsache spiegeln sich auch die mitunter recht diffusen Ergebnisse zur organisatorischen Ausgestaltung der Rücknahme- und Recyclingsysteme in diesem Cluster wider.

599 Der Ermittlung der einzelnen Werte, die in dem dreidimensionalen Achsenkreuz abgetragen sind, lag eine Auswertung der positiven bzw. negativen Abweichungen von den Clustermittelwerten zugrunde. Grundlage waren dabei die mit Plus- und Minuszeichen hervorgehobenen Abweichungen aus den Abbildungen 42, 43 und 44. Der für Cluster III (Partizipativ-späte Implementierer) ermittelte Wert kommt beispielsweise wie folgt zustande: 18 Pluszeichen minus 4 Minuszeichen $=14$ Pluszeichen (14 Punkte $=$ Achsenwert) beim Implementierungserfolg . Diese Art der Berechnung impliziert die Möglichkeit der gegenseitigen Kompensation einzelner Variablenausprägungen. Damit wird die realiter bestehende Unterschiedlichkeit auf der Variablenebene zugunsten einer höheren Anschaulichkeit auf einer hoch aggregierten Ebene in diesem Fall aufgegeben. Zudem muß die Subjektivität der Befragten insbesondere bei Fragen der Zielerreichung kritisch in die Interpretation der Ergebnisse einfließen. 


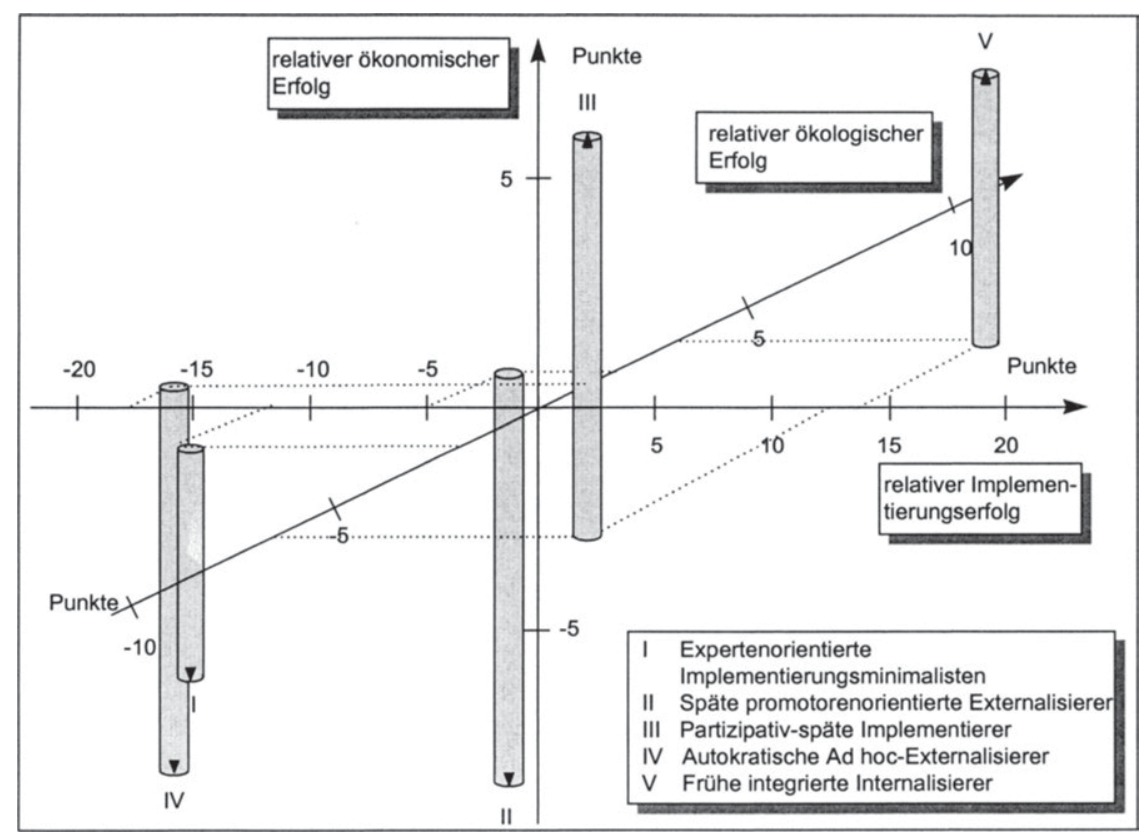

Abb. 47: Positionierung der Implementierungstypen anhand der relativen Erfolgswirkungen

Eine Betrachtung der Expertenorientierten Implementierungsminimalisten verdeutlicht, daß eine abwehrende Grundhaltung bei vorgegebenen umweltbezogenen Minimalanforderungen in keiner der hier erfaßten Erfolgsdimensionen zielführend ist. Als Empfehlung kann der Satz gelten: „Wenn ich etwas mache, dann mache ich es richtig!" Eine Analyse der Späten promotorenorientierten Externalisierer verdeutlicht demgegenüber, daß auch eine Erreichung der ökologischen Ziele - in diesem Fall vor allem Erfassungsquoten und thermische Verwertung - durch Vertragslösungen als ökonomischer Mißerfolg und Implementierungsmißerfolg gewertet werden kann. Dies gilt insbesondere für die Zusammenarbeit mit den Systempartnern, die offenbar nicht zufriedenstellend ist.

\section{Implikationen für Hersteller langlebiger Gebrauchsgüter}

Im Verlauf der empirischen Untersuchung wurde deutlich, daß Rücknahme- und Recyclingsysteme vielfältige Ausgestaltungsformen annehmen können und vor dem Hintergrund ihres branchenspezifischen Kontextes und zahlreicher weiterer 
Einflußfaktoren zu beurteilen sind. Allein die Bildung von fünf Implementierungstypen verdeutlicht die Heterogenität des Untersuchungsobjekts und verbietet eine allzu allgemeine Ableitung von Schlußfolgerungen für die „richtige“ Implementierung von Rücknahme- und Recyclingsystemen. Gleichzeitig konnte die Erfolgsrelevanz der Implementierung nachgewiesen werden, so daß einerseits eine stärkere Einbeziehung von Implementierungsaspekten bereits bei der Planung von Rücknahme- und Recyclingsystemen erforderlich scheint, andererseits jedoch auch Hinweise für Erfolgspfade bei bereits implementierten Systemen gegeben werden können.

Für große Herstellerunternehmen, deren Leistungsprogramm vor allem aus langlebigen Gebrauchsgütern mit hohem Produkteinzelwert besteht und die über angemessene finanzielle Ressourcen verfügen, scheint ein eigenes Rücknahmeund Recyclingsystem erfolgversprechend. Zum einen bieten sich hohe Wertschöpfungspotentiale, so daß die Erreichung ökonomischer Ziele aussichtsreich erscheint. Zum anderen bestehen in Absatzformen wie dem Leasing und der Vermietung Ansatzpunkte zur Kontrolle der Produktnutzung und damit die Möglichkeit, die ökologischen Verwendungsziele zu erreichen. Zugleich erwachsen aus kundenbindungsorientierten Absatzformen Möglichkeiten zur ökologiebezogenen Imageverbesserung. Voraussetzung für die Erreichung der ökonomischen Ziele ist allerdings ein stufenweiser Implementierungsprozeß, der unter maßgeblichem Einfluß der Geschäftsführung für eine zielorientierte Umsetzung und Durchsetzung des Rücknahme- und Recyclingsystems steht.

Für kleine Unternehmen, die ein Rücknahme- und Recyclingsystem für ein auf eine Marktnische abgestimmtes Leistungsangebot zu planen haben, erweist sich die Externalisierung von damit zusammenhängenden Aktivitäten - i.d.R. an Verwertungsunternehmen - als ein möglicher erfolgsträchtiger Weg. Einbußen müssen dann jedoch bei Image-, Profilierungs- und Erfahrungszielen in Kauf genommen werden, während sich Kostenziele durchaus in befriedigendem Ausmaß erreichen lassen. Zudem können diese Unternehmen gewisse Freiheitsgrade hinsichtlich des Zeithorizonts der Implementierung für sich nutzen.

Die empirische Untersuchung hat darüber hinaus gezeigt, daß sich die häufig bei mittelgroßen Unternehmen zu findenden Branchenlösungen ökonomisch als relativ nachteilig erweisen. Als Ursachen sind vor allem ein zu geringer Wert des ein- 
bezogenen Produktes sowie der hohe organisatorische Aufwand zu vermuten, der offenbar nicht den erhofften Entlastungseffekt für die einzelnen Herstellerunternehmen zeigt. Zudem gehen mit einer Branchenlösung i.d.R. Abnahmeverpflichtungen von Recyclaten durch die Hersteller einher. Für die Erreichung der ökologischen Zielgrößen - hier insbesondere Verwertungsziele - bieten sich hingegen gute Ansatzpunkte.

Bei bereits bestehenden Rücknahme- und Recyclingsystemen gilt es, das Implementierungsverhalten im Sinne eines geplanten organisatorischen und vor allem ökologischen Wandels schrittweise so zu ändern, daß höhere Zielerreichungsgrade resultieren. Im Zuge der theoretischen und empirischen Analyse wurde deutlich, daß jedwedes Implementierungsverhalten stets im Kontext des Gesamtunternehmens zu sehen ist. Änderungen dieses Verhaltens sind daher umfassender Natur und mitunter überaus trägen Wirkungsprozessen unterworfen. An den Autokratischen Ad hoc-Externalisierern sowie den Expertenorientierten Implementierungsminimalisten wird beispielhaft deutlich, daß diese Hersteller den Änderungsbedarf offenbar zu gering einschätzen und mit einem „ruckartigen" Vorgehen kaum erfolgreiche Rücknahme- und Recyclingsysteme erreichen können. Demgegenüber erscheint es angesichts der untersuchten Verhaltens- und Erfolgswirkungen angemessen, die Frühen integrierten Internalisierer als Leitbild einer erfolgreichen Implementierung des komplexen Objektes der Rücknahme- und Recyclingsysteme zu bezeichnen. Gleichwohl ist eine derartige Empfehlung vor dem Hintergrund der kontextbezogenen Merkmale der in diesem Cluster zusammengefaßten Herstellerunternehmen zu relativieren. Zu nennen seien beispielhaft der Volumenstrom und die qualitative Beschaffenheit der Altprodukte sowie die Existenz von Sekundärmärkten bzw. mögliche Einsatzfelder für Altprodukt(teil)e ${ }^{601}$

\section{Auswirkungen auf die zukünftige theoretische und empirische For- schung}

Im Zuge der Analyse des herstellerbezogenen Implementierungsverhaltens von Rücknahme- und Recyclingsystemen wurde deutlich, daß sich angesichts der notwendigen Fokussierung auf ausgewählte Teilaspekte der Problemstellung zahlreiche Ansatzpunkte für weiterführende Forschungsarbeiten bieten. Dies gilt

601 Vgl. Schwarz, E. J., Unternehmensnetzwerk im Recycling-Bereich, a.a.O., S. 61f.; Falke, R., Verantwortung für das Ganze tragen, a.a.O., S. 33f. 
sowohl in inhaltlicher als auch methodischer Hinsicht. Folgende Aspekte sollen in diesem Zusammenhang besonders herausgestellt werden:

1. Die Sichtung und Auswertung der implementierungsspezifischen Literatur ergab eine relativ deutliche Dreiteilung der Anwendungsfälle bzw. Implementierungsobjekte in informationstechnologische Systeme, Strategien und - mit einigem Abstand - Marketing. Im Sinne einer stärkeren Akzentuierung der bisher gänzlich unterrepräsentierten Behandlung umweltbezogener Implementierungsobjekte sind weitere praxisbezogene Forschungsarbeiten wünschenswert, deren Erkenntnisse Unternehmen eine stärker analytische Durchdringung komplexer ökologiebezogener Problemstellungen ermöglichen. Dies gilt um so mehr, als insbesondere die Implementierung immer wieder als zentrales Praxisproblem identifiziert wird.

2. Da an einem Rücknahme- und Recyclingsystem grundsätzlich mehrere Marktbzw. Systempartner beteiligt sein können, liegt zusätzlich die Erfassung der Perspektive von Lieferanten, Handelsunternehmen, Verwertern und nicht zuletzt Konsumenten nahe. In Form einer Spiegelbilduntersuchung ließen sich Aussagen über Gemeinsamkeiten und Unterschiede in Einstellungs- und Verhaltensdimensionen der am Rücknahme- und Recyclingsystem Beteiligten gewinnen, so daß die Grundlage für eine bessere Zielerreichung insbesondere in den ökologischen und ökonomischen Zielen geschaffen wird.

3. Die Operationalisierung der Zielgrößen hat zum einen das Problem der Einbeziehung ökologischer Dimensionen in "traditionelle" ökonomische Ziele offenbart. Zum anderen wurde die Schwierigkeit der Vorgabe ökologischer Ziele deutlich. Während sich das ökologische Oberziel der Vermeidung aus leicht ersichtlichen Gründen einem Maximierungsstreben entzieht, stellen andere Zielgrößen wie Erfassungsquoten oder Verwendungs- und Verwertungsquoten Größen dar, deren betriebswirtschaftliche Bewertung vom jeweiligen Kontext abhängig ist. Auch hier versagen klassische Maximierungsansätze an Interdependenzen zwischen den ökologiebezogenen Zielsetzungen untereinander sowie den Wechselwirkungen mit ökonomischen Zielen. Eine Bewertung ökologischer Zielgrößen ${ }^{602}$ hat in der betriebswirtschaftlichen Diskussion bisher zu

602 Ist es nicht möglich, Ziele zu operationalisieren, wird auch von einem Zielsetzungsdefekt gesprochen, d.h. es existiert keine operationale, eindimensionale Zielfunktion. Ursachen können Irene Giesen-Netzer and Universität Münster - 978-3-631-75058-2 
keiner befriedigenden Lösung geführt und erfordert einen stärker interdisziplinär ausgerichteten Ansatz, der vor allem die Bereiche des Ingenieurwesens, der Chemie und Biologie mit der Betriebswirtschaftslehre integriert.

4. Implementierungsbezogene Forschungsergebnisse belegen immer wieder den hohen Stellenwert individualbezogener Durchsetzungsaufgaben im Rahmen der Implementierung. Diese Aussage muß für den Kontext umweltbezogener Problemstellungen verstärkt werden, da gerade die Komponenten des "Kennens und Verstehens", „Könnens" und "Wollens" in diesem Bereich in hohem Maße von persönlichem Involvement der Betroffenen getragen werden. Beispielhaft sei auf die Abkehr von der Durchlauf- zur Kreislaufwirtschaft hingewiesen. Für die weitere Erforschung intra- und interpersonaler Aspekte bei der Implementierung von Rücknahme- und Recyclingsystemen bietet sich ein weites Anwendungsfeld für eine stärkere Vernetzung der Betriebswirtschaftslehre mit dem Gebiet der Psychologie. ${ }^{603}$

5. Der hier vorgelegte empirische Forschungsansatz bereitet Grund für weitere methodische Anwendungen. Zum einen ist eine in regelmäßigen Abständen durchzuführende Längsschnittanalyse mit den in der Untersuchungsstichprobe enthaltenen Herstellerunternehmen vorstellbar, die wertvolle Erkenntnisse über die weiteren Entwicklungen der Implementierung geben könnte. Zum anderen ließe eine Ausweitung des Stichprobenumfangs die Ableitung von Aussagen zu, deren Aussagekraft sich durch eine höhere Allgemeingültigkeit auszeichnete.

Die Ausführungen zur Erfolgswirkung der Implementierung von Rücknahme- und Recyclingsystemen sind abschließend aus dem betriebswirtschaftlichen Kontext herauszulösen und auf die Ebene einer ökologisch-ethischen Betrachtung zu überführen. Unternehmen sind als Elemente des sozialen Gesamtsystems aufgerufen, zu einer dauerhaften Entwicklung beizutragen und dieser Verpflichtung durch das Anstreben einer Kreislaufwirtschaft näherzukommen. Damit sind insbesondere die ökologischen Zielsetzungen vor dem Hintergrund der obengenannten

inbesondere darin liegen, daß die Zielgröße nicht festliegt bzw. mehrere konfliktäre Ziele zu beachten sind oder deren Ausmaß nicht festliegt. Vgl. Adam, D., Planung und Entscheidung, a.a.O., S. 11 f. 
Problematik zu überdenken und mit den ökonomischen Zielen in ein möglichst konsistentes Zielsystem zu überführen. Daher sind die von den Herstellerunternehmen bereits eingeschlagenen Pfade der Implementierung als wichtiger Schritt in diese Richtung zu begrüßen. 


\section{Anhangverzeichnis}

Anhang 1: Ergänzende Abbildungen und Erläuterungen

1.1 Ziele bei der Gestaltung von Rücknahme- und Recyclingsystemen in der Automobilbranche

1.2 Ziele bei der Gestaltung von Rücknahme- und Recyclingsystemen in der Elektro- und Elektronikindustrie

1.3 Ziele bei der Gestaltung von Rücknahme- und Recyclingsystemen in der Möbelindustrie

1.4 Ziele bei der Gestaltung von Rücknahme- und Recyclingsystemen in der Bauzubehör- und Chemiebranche

1.5 Ausprägungen der Gesamtmittelwerte der clusterbildenden Variablen

1.6 Beispielhafte Ausprägungen von ausgewählten strukturellen, koordinationsbezogenen und kulturellen Merkmalen bei verschiedenen Ausgestaltungsformen von Rücknahme- und Recyclingsystemen .. 242

1.7 Status der Rücknahme- und Recyclingsysteme. 244

Anhang 2: Statistik. 245

2.1 Design und Methodik der empirischen Untersuchung 245

2.2 Beispielhafte Operationalisierung der Zeitdimensionen "Timing" und "Zeithorizont" 249

2.3 Schrittweise Agglomeration der 5-Cluster-Lösung.......................... 250

2.4 Klassifikationsmatrix der schrittweisen Diskriminanzanalyse........... 251

2.5 Explorative Faktoranalyse zur Absatzmarktsituation ...................... 252

2.6 Explorative Faktoranalyse zur Beschaffungsmarktsituation ............ 253

2.7 Signifikanzen der Implementierungstypen hinsichtlich der Produktmerkmale.

2.8 Explorative Faktoranalyse zur Unternehmenskultur ........................ 256

2.9 Explorative Faktoranalyse zu individualbezogenen Komponenten des „Kennen und Verstehens" sowie des „Könnens"

3.1 Auszüge aus dem Fragebogen der persönlichen Befragung .......... 258

3.2 Entwurf eines Mitarbeiterfragebogens 270 


\section{Anhang 1: Ergänzende Abbildungen und Erläuterungen}

1.1 Ziele bei der Gestaltung von Rücknahme- und Recyclingsystemen in der Automobilbranche

\section{Automobilindustrie}

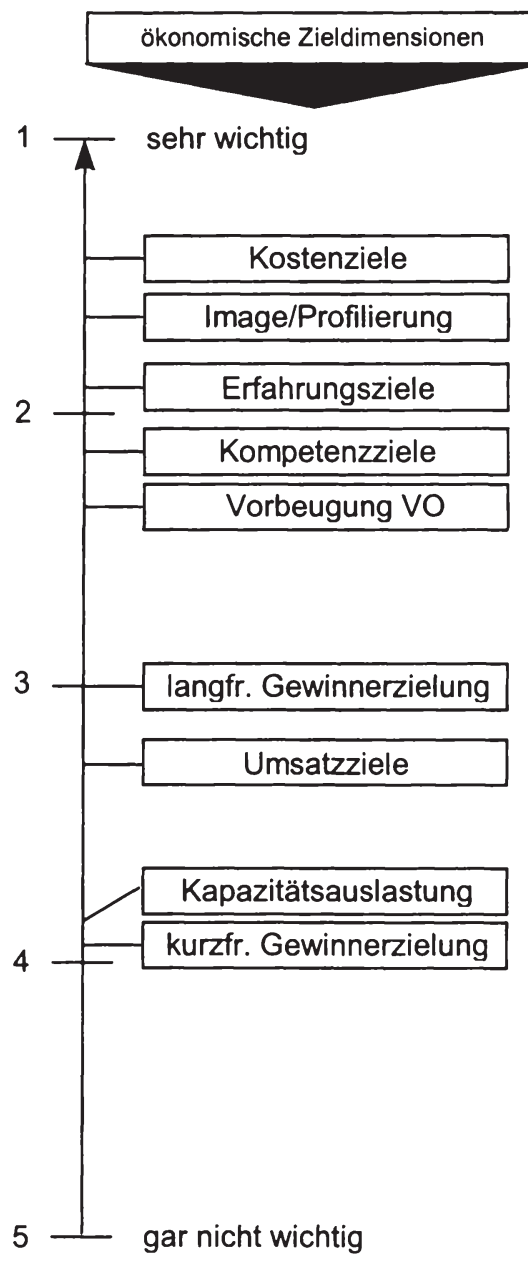

ökologische Zieldimensionen

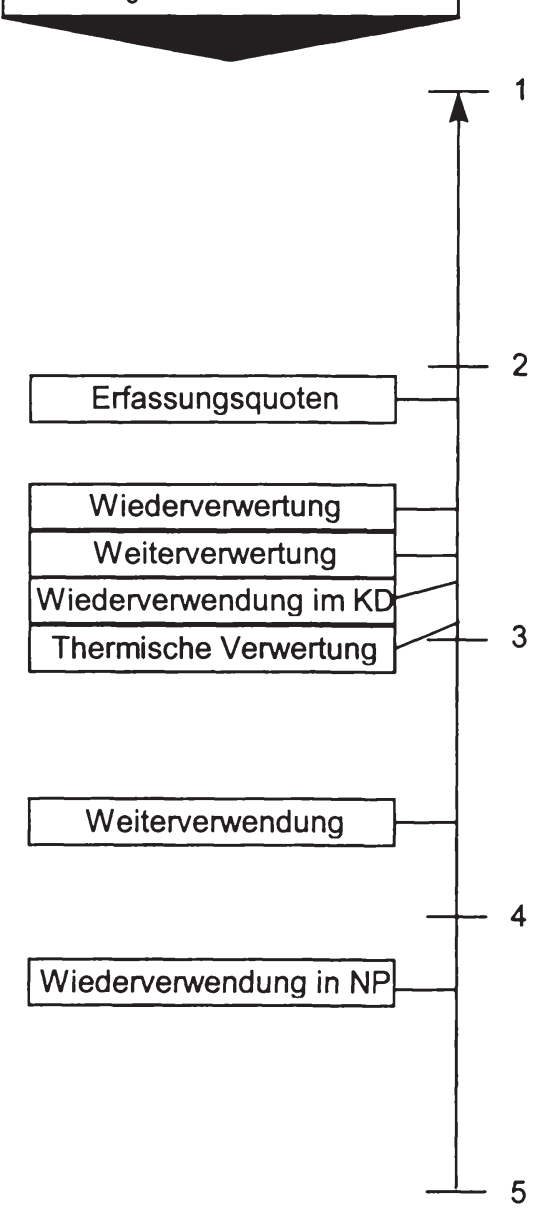


1.2 Ziele bei der Gestaltung von Rücknahme- und Recyclingsystemen in der Elektro- und Elektronikindustrie

\section{Elektro-/Elektronikindustrie}
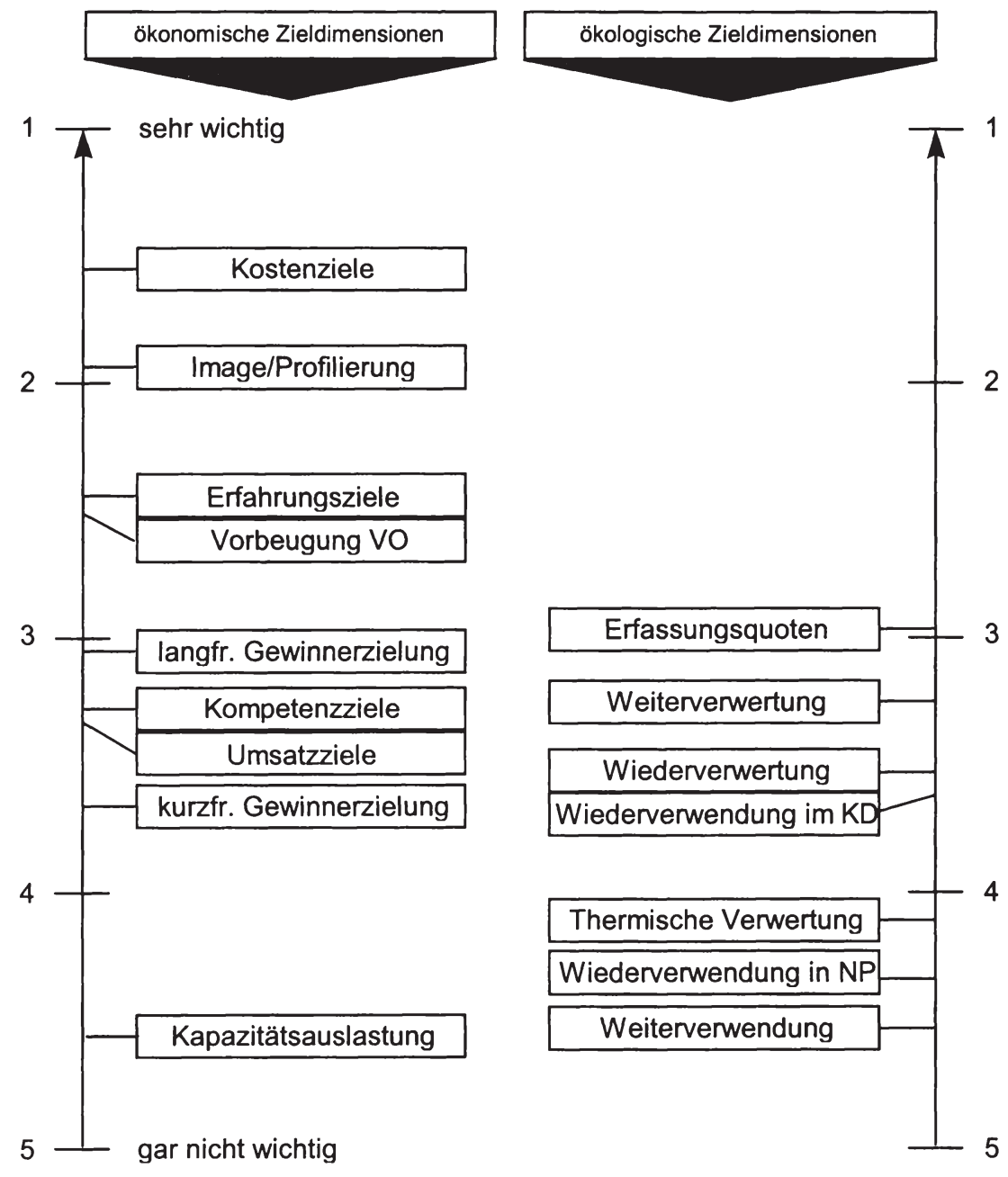
1.3 Ziele bei der Gestaltung von Rücknahme- und Recyclingsystemen in der Möbelindustrie

\section{Möbelindustrie}

ökonomische Zieldimensionen

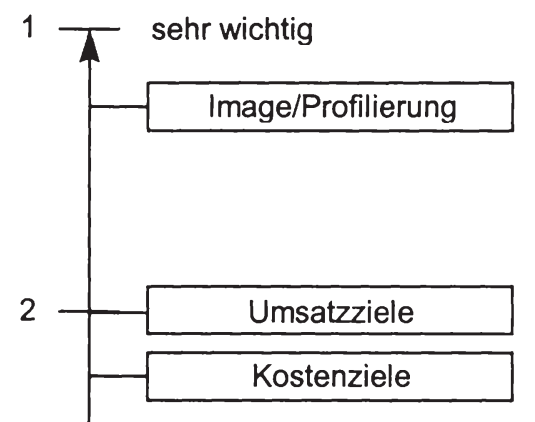

31 \begin{tabular}{|c|}
\hline langfr. Gewinnerzielung \\
\hline Kompetenz/Erfahrung \\
\hline Vorbeugung VO \\
\hline
\end{tabular}

kurzfr. Gewinnerzielung

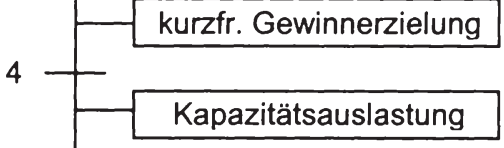

ökologische Zieldimensionen

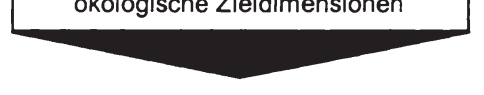

1

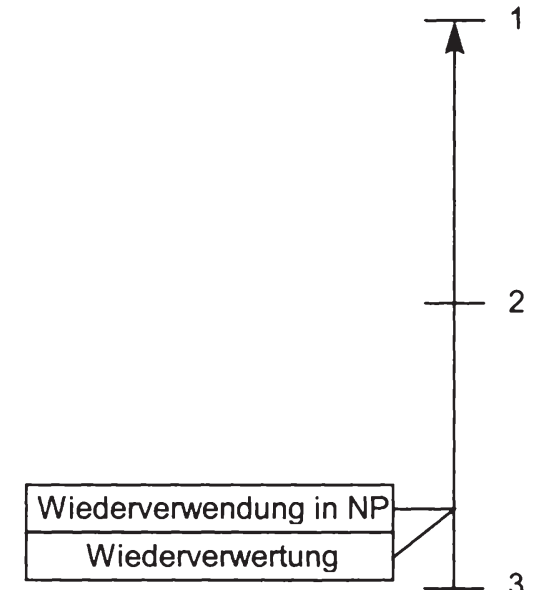

Thermische Verwertung

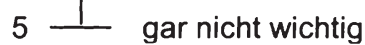


1.4 Ziele bei der Gestaltung von Rücknahme- und Recyclingsystemen in der Bauzubehörund Chemiebranche

\section{Bauzubehör/Chemie}
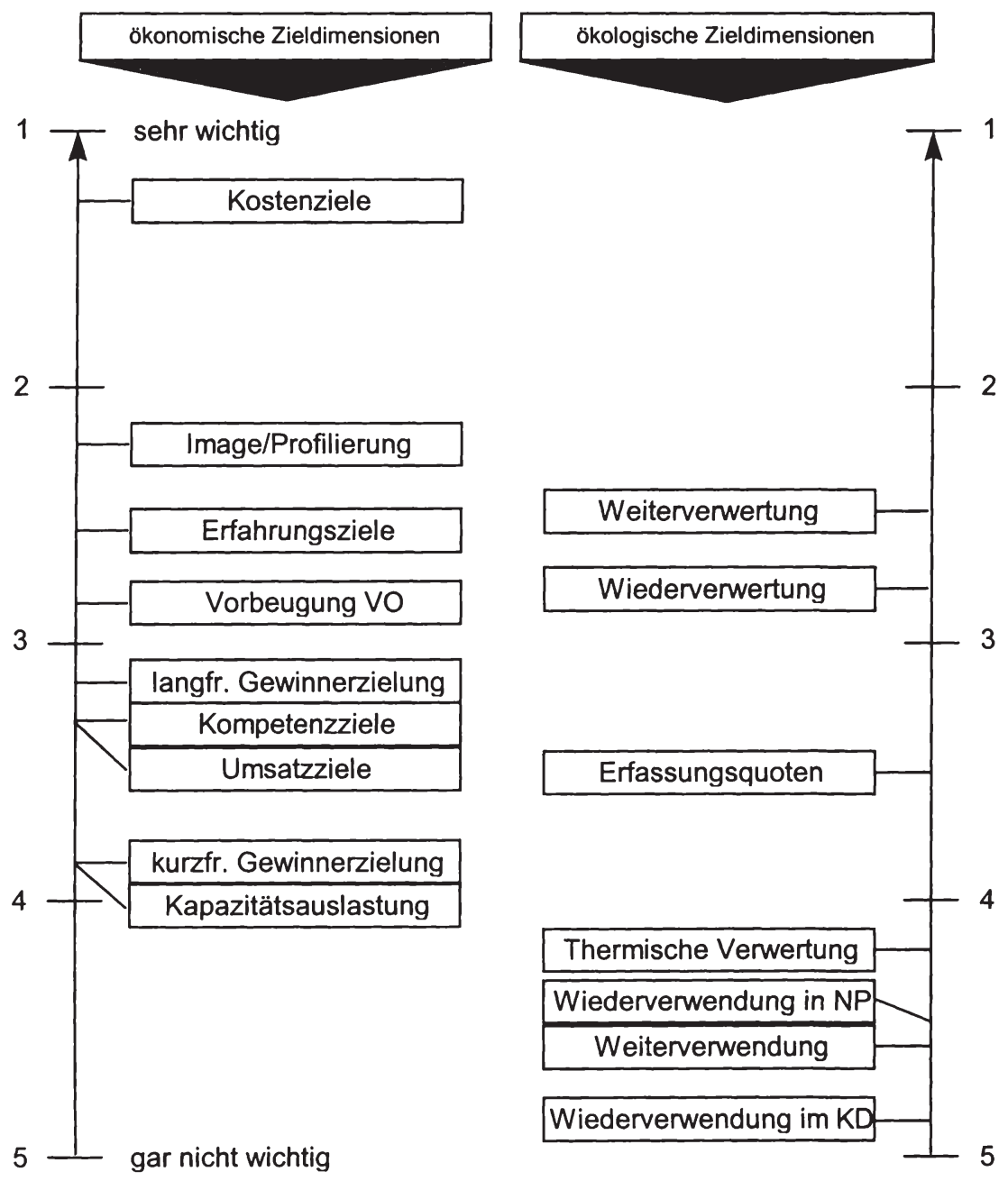
1.5 Ausprägungen der Gesamtmittelwerte der clusterbildenden Variablen

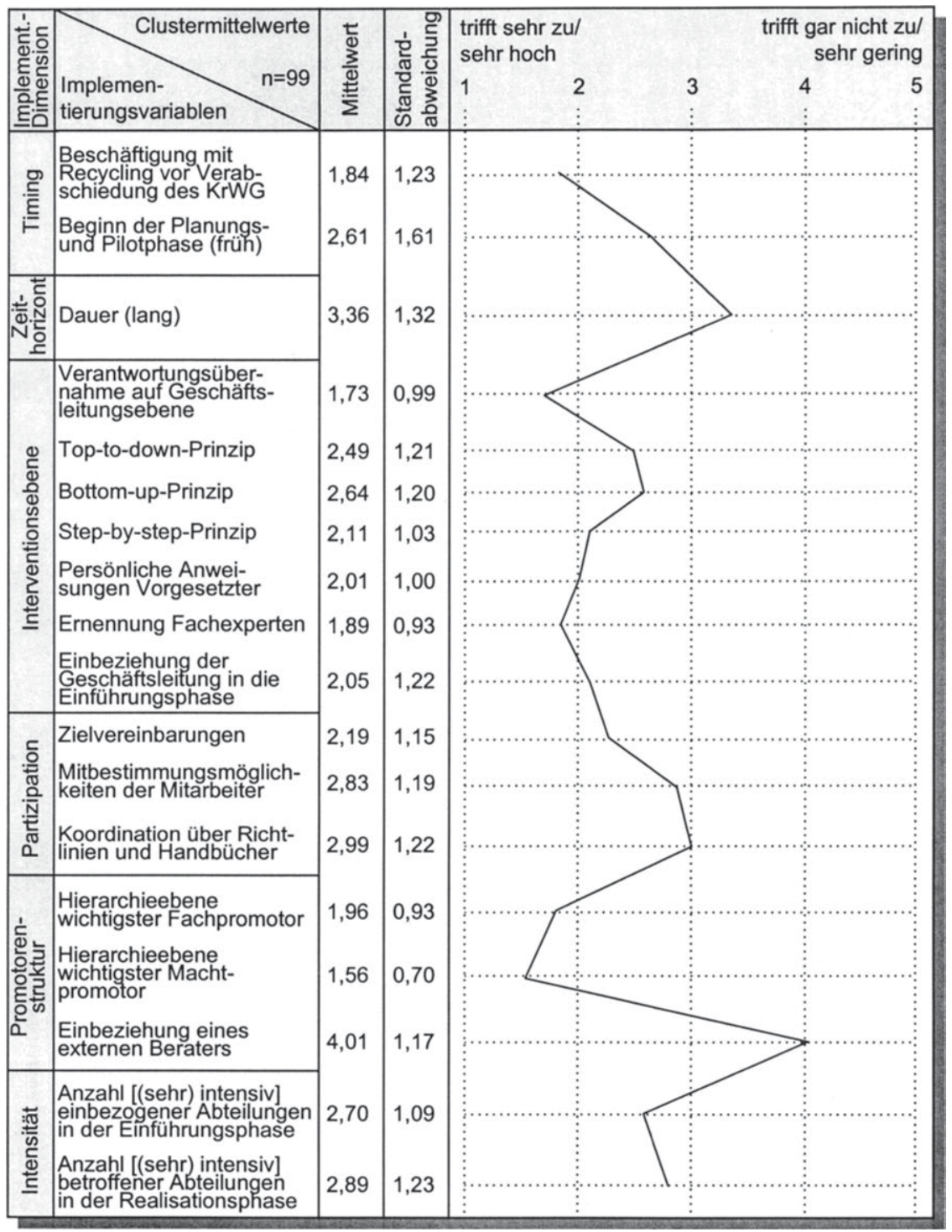


1.6 Beispielhafte Ausprägungen von ausgewählten strukturellen, koordinationsbezogenen und kulturellen Merkmalen bei verschiedenen Ausgestaltungsformen von Rücknahmeund Recyclingsystemen

\begin{tabular}{|l|c|c|c|c|}
\hline \multicolumn{1}{|c|}{ Formen } & $\begin{array}{l}\text { eigenes Sy- } \\
\text { stem }\end{array}$ & $\begin{array}{l}\text { Branchen- } \\
\text { kooperation }\end{array}$ & $\begin{array}{l}\text { Gemeinsame } \\
\text { Entwicklung } \\
\text { mit Verwer- } \\
\text { tern }\end{array}$ & $\begin{array}{l}\text { Vertrag mit } \\
\text { Verwertern }\end{array}$ \\
\hline $\begin{array}{l}\text { hohr) zu } \\
\text { von Entreffen }\end{array}$ & 1 & 0,6 & 0,81 & 0,96 \\
\hline $\begin{array}{l}\text { Machtstruaktisation } \\
\text { verteilt }\end{array}$ & 0,23 & 0,25 & 0,36 & 0,26 \\
\hline hohe Offenheit & 0,08 & 0,27 & 0,64 & 0,87 \\
\hline $\begin{array}{l}\text { hohe Stabilität 1 } \\
\text { (kein Wechsel der } \\
\text { Systempartner) }\end{array}$ & 0,91 & 1 & 1 & 0,93 \\
\hline $\begin{array}{l}\text { hohe Stabilität 2 } \\
\text { (Aufgaben können } \\
\text { von anderen über- } \\
\text { nommen werden) }\end{array}$ & 0,58 & 0,26 & 0,73 & 0,33 \\
\hline $\begin{array}{l}\text { intensive } \\
\text { Kommunikation, } \\
\text { Erfahrungsaustausch }\end{array}$ & 0,54 & 0,6 & 0,82 & 0,33 \\
\hline $\begin{array}{l}\text { geringes Konfliktni- } \\
\text { veau }\end{array}$ & 0,42 & 0,4 & 0,6 & 0,4 \\
\hline
\end{tabular}

Merkmale treffen am meisten zu (Zeilenmaximum)

Wird das strukturelle Merkmal der Zentralisation betrachtet, kann festgestellt werden, daß bei der Einrichtung eines herstellereigenen Systems die Zentralisation zu hundert Prozent angegeben wird, d. $h$. alle wichtigen Entscheidungen werden zentral getroffen. ${ }^{1}$ Diese Ausprägung verwundert nicht, da das Unternehmen überwiegend alle Tätigkeiten selbst durchführt und daher selbst entscheidet. Mit einem Wert von $96 \%$ geben auch die Unternehmen an, die ein marktähnliches System haben, daß die Zentralisation stark ausgebildet ist. Auch hier ist durch das Auftagsverhältnis klar, daß die Herstellerunternehmen (fast) alle wichtigen Entscheidungen bezüglich des Rücknahme- und Recyclingsystems selbst treffen. Bei der Branchenlösung geben immerhin noch $60 \%$ an, daß die Entscheidungen zentral getroffen werden. Dieses liegt in der Regel daran, daß einige Unternehmen sich bei der Bildung des Rücknahme- und Recyclingsystems sehr stark engagieren und dann auch Entscheidungen treffen.

$1 \quad$ Bei der Implementierung eines eigenen Rücknahme- und Recyclingsystems werden einige ausgewählte Aufgaben externalisiert, z.B. die Verwertung von Schadstoffen. Daher sind auch hier Systempartner beteiligt, die jedoch keine tragende Rolle bei der Gestaltung und der Implementierung des Rücknahme- und Recyclingsystems gespielt haben.

Irene Giesen-Netzer and Universität Münster - 978-3-631-75058-2 
Eine Gleichverteilung der Machtstruktur liegt überwiegend in allen Systemen überwiegend nicht vor. Dieses strukturelle Merkmal trifft mit $36 \%$ am ehesten bei den Rücknahme- und Recyclingsystemen zu, die in Gemeinschaft zwischen Herstellerunternehmen und Verwertern entwickelt werden. Hier haben aufgrund der Integration der Verwerter diese ein gewisses Mitspracherecht. Bei der vertraglichen Lösung und bei der Lösung in Gemeinschaft zwischen Herstellern und Verwertern liegt eine hohe Offenheit der Rücknahme- und Recyclingsysteme vor. Dieses liegt zum einen darin begründet, daß in diesen organisatorischen Formen die Spezifität des Verwertens relativ gering ist und damit kein spezifischer Wettbewerbsvorteil vorliegt. Zum anderen ist bei einem vertraglichen Verhältnis die Bindung der Partner nur auf den Vertragsinhalt beschränkt und kann jederzeit wechseln.

Bei der Stabilität (1) des Unternehmens, die durch die Häufigkeit des Wechsels der Systempartner operationalisiert wurde, sind alle untersuchten Systeme nach dem Bekunden der Hersteller erstaunlich stabil. Das mag darin begründet liegen, daß die befragten Hersteller gute Erfahrungen mit den implementierten Rücknahme- und Recyclingsystemen gemacht haben. Wird die Stabilität (2) daran gemessen, ob andere Systempartner Aufgaben mitübernehmen können, wenn ein Systempartner ausfällt, werden differenziertere Ergebnisse deutlich. 73\% der befragten Hersteller, die gemeinsam mit Verwertern ein Rücknahme- und Recyclingsystem implementieren und $58 \%$ der Hersteller die ein eigenes System durchführen, erklären, daß dieses möglich ist. Der Know-howZuwachs im eigenen Unternehmen oder in der Zusammenarbeit mit den Verwertern macht es möglich, daß diese Aufgaben durchaus an andere Verwerter (oder andere beteiligte Systempartner) weitergegeben werden können.

Die Kommunikation, die durch den intensiven Erfahrungsaustausch operationalisiert wurde, ist bei einem System, das zwischen Herstellern und Verwertern gebildet wurde, sowie bei einer Branchenlösung am intensivsten ausgeprägt. Auch dieses läßt sich gut erklären, weil bei einem gemeinsamen System das Interesse aller Partner auf gemeinsame Zielerfüllung groß ist.

Schließlich ist das Konfliktniveau bei einem gemeinsamen System von Herstellern und Verwertern am höchsten. Auch hier ist aufgrund von intensiver Zusammenarbeit und hoher Kommunikationsintensität klar, daß sich vielfältiger Diskussionsbedarf ergibt, der mit (durchaus konstruktiven) Konflikten verbunden sein kann. 


\subsection{Status der Rücknahme- und Recyclingsysteme}

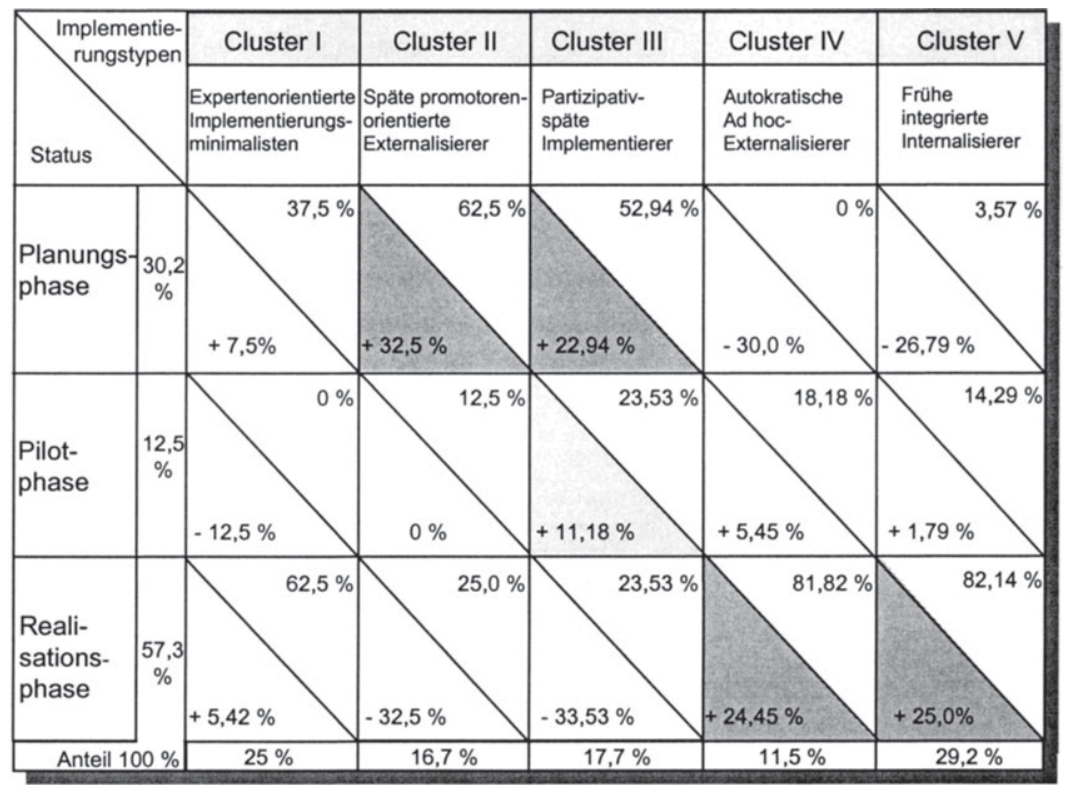

\begin{tabular}{|c|c|c|c|c|}
\hline $\begin{array}{l}\text { Chi-Quadrat-Test } \\
\text { gemäß: }\end{array}$ & $\begin{array}{l}\text { Signifikanz- } \\
\text { niveau: }\end{array}$ & $x$ & $x \%$ & $0-5 \%$ \\
\hline Pearson & 0,00004 & $\begin{array}{l}\text { tatsächlicher } \\
\text { Anteil des }\end{array}$ & $\begin{array}{l}\text { Abweichung } \\
\text { vom }\end{array}$ & $5,1-10 \%$ \\
\hline \multirow{2}{*}{$\begin{array}{l}\text { Likelihood } \\
\text { Ratio }\end{array}$} & \multirow[t]{2}{*}{0,00000} & $\begin{array}{l}\text { Implemen- } \\
\text { tierungstyps }\end{array}$ & $\begin{array}{l}\text { erwarteten } \\
\text { statistischen }\end{array}$ & $10,1-20 \%$ \\
\hline & & & Wert & $>20 \%$ \\
\hline
\end{tabular}




\section{Anhang 2: Statistik}

\subsection{Design und Methodik der empirischen Untersuchung}

Die empirische Grundlage der zu überprüfenden Hypothesen liefert eine primärstatistische Erhebung in Form einer mündlichen Befragung, die das Institut für Marketing im Auftrag der Bundesstiftung Umwelt konzipiert und durchgeführt hat. Es wurden von Mai 1995 bis Februar 1996101 persönliche Interviews mit Herstellern aus der Elektro- und Elektronikbranche, der Automobilindustrie, der Möbelindustrie sowie der Bauzubehörbranche geführt. ${ }^{2}$ Zuvor wurden 1500 Hersteller $^{3}$ zunächst mit der Bitte um Mitwirkung angeschrieben. Diejenigen Hersteller, die eine Zusage gegeben hatten, wurden anschließend telefonisch kontaktiert, um zum einen die Eignung für ein Interview festzustellen und zum anderen ggf. Termine abzusprechen. Die Eignung bezog sich auf die Feststellung, ob die Hersteller bereits Erfahrungen mit der Gestaltung und Implementierung von Rücknahme- und Recyclingsystemen gemacht hatten. Dabei erwies sich die Erreichung der angestrebten Anzahl von ca. 100 mündlichen Interviews als relativ schwierig, da einerseits der Fokus auf Hersteller langlebiger Gebrauchsgüter gelegt wurde und andererseits viele Unternehmen noch keine Erfahrungen mit Rücknahme- und Recyclingsystemen hatten, ${ }^{4}$ obwohl die Inkraftretung des Kreislaufwirtschaftsgesetzes im Jahre 1996 bevorstand.

Die Zielgruppe der kontaktierten Hersteller war jeweils die Geschäftsleitung bzw. der Vorstand, wobei alle Personen namentlich bekannt waren. Diese Vorgehensweise wurde gewählt, damit diese den jeweils zuständigen Fachexperten benennen konnten. Überraschend war dabei, daß sich viele Geschäftsleiter selbst zum Interview bereit erklärten mit der Begründung, daß dieses Thema „Chefsache" sei. $^{5}$

Die Stichprobenstruktur zeigt die Branchenstruktur sowie die Mitarbeiterzahl der befragten Hersteller und ist in nachfolgender Tabelle wiedergegeben. Daraus wird deutlich, daß überwiegend Hersteller der Elektro- und Elektronikbranche sowie der Bauzubehörbranche und Chemiebranche sowie mittlere und große Hersteller befragt wurden.

$2 \quad$ Die 101 Interviews wurden von der Verfasserin und speziell geschulten Mitarbeitern des Marktforschungsunternehmens "Institut für Sozialforschung und Kommunikation“, Bielefeld, durchgeführt.

3 Die 1500 Adressen wurden von der AZ Direct Marketing Bertelsmann GmbH bezogen. Diese wurden zufallsgestützt quotiert und entsprechend der Grundgesamtheit der in den Branchen insgesamt verfügbaren Adressen ausgewählt.

$4 \quad$ Aufgrund dieser Erfahrungen wird unterstellt, daß es sich bei den befragten Unternehmen um Pioniere bezüglich der Gestaltung und Implementierung von Rücknahme- und Recyclingsystemen handelt.

522 Interviews der persönlichen Befragungen wurden mit der Geschäftsleitung (oder Mitgliedern der Geschäftsführung, Direktoren oder Inhabern sowie einem Prokuristen) durchgeführt. 


\begin{tabular}{|l|c|}
\hline Branchen & in Prozent \\
\hline Automobil & 11,9 \\
\hline Möbel & 11,9 \\
\hline Elektro- und Elektronikgerăte & 35,7 \\
\hline Bauzubehör und Chemie & 28,7 \\
\hline Metall- u. Maschinenbau & 9,8 \\
\hline sonstige & 2 \\
\hline
\end{tabular}

\begin{tabular}{|l|c|}
\hline Größenklassen & in Prozent \\
\hline 20 bis 50 Beschäftigte & 13,9 \\
\hline 51 bis 500 Beschäftigte & 30,7 \\
\hline 501 bis 2000 Beschäftigte & 10,9 \\
\hline über 2000 Beschäftigte & 44,6 \\
\hline
\end{tabular}

\section{Struktur der empirischen Befragung der Hersteller langlebiger Gebrauchsgüter}

Der Datenerfassung lag ein 43-seitiger Fragebogen mit 85 Fragen zugrunde. Diese enthielten insbesondere Themenkomplexe aus sechs Bereichen:

1. Produktbezogene und stoffbezogene Fragestellungen ${ }^{6}$,

2. Strategie- und Zielvariablen,

3. Situationsvariablen,

4. Organisiertheit ${ }^{7}$ der Rücknahme- und Recyclingsysteme,

5. Implementierungsvariablen und

6. Erfolgsvariablen.

Die Dauer der Interviews betrug je nach Erklärungsbedarf des Interviewten zwischen zweieinhalb und vier Stunden, wobei zum großen Teil geschlossene Fragestellungen zur Anwendung kamen, da diese eine relativ problemlose und zeiteffiziente Bearbeitung gewährleisten. Die Mehrzahl der Variablen wurde dabei auf 5 -stufigen bipolaren Ratingskalen ${ }^{8}$ erhoben, um eine Überführung in

$6 \quad$ Viele Hersteller produzieren mehr als ein Produkt und haben dementsprechend mehrere produktbezogene Rücknahme- und Recyclingsysteme implementiert. Aufgrund der Komplexităt der Fragestellung wurden die Hersteller daher gebeten, dasjenige Produkt (und damit Rücknahme- und Recyclingsystem) zu diskutieren, das den höchsten Umsatzanteil erbringt.

7 Sydow untersucht in Anlehnung an organisationstheoretische Erkenntnisse die Organisiertheit strategischer Netzwerke und unterscheidet dabei: 1. Den Umfang (die Größe) des Netzwerkes, 2. Die Funktionsteilung, 3. Die Dichte, 4. Die Diversität, 5. Die Zentralităt, 6. die Multiplexität, 7. Die Konnektivität, 8. Die Interdependenz, 9. Die Redundanz, 10. Die Stabilität, 11. Die Offenheit und 12. Die Sichtbarkeit strategischer Netzwerke. Vgl. Sydow, J., Strategische Netzwerke, a.a.O., S. 83ff. Diese Merkmale wurden auf die Problemstellung der Rücknahmeund Recyclingsysteme übertragen.

8 Obwohl es sich bei einer derartigen Befragung um ordinal skalierte Daten handelt, d.h. es können Rangwerte mit Ordinalzahlen, jedoch ohne gleichgroße Abschnitte und ohne natürlichen Nullpunkt, bestimmt werden, soll hier von Intervallskalierung ausgegangen werden. Dieses geschieht, um die Daten multivariat auswerten zu können. Dabei wird dem in der Literatur üblichen Vorgehen gefolgt. Vgl. Backhaus, K., u.a., Multivariate Analysemethoden, a.a.O., S. Irene Giesen-Netzer and Universität Münster - 978-3-631-75058-2 
multivariate Analysemethoden zu ermöglichen. ${ }^{9}$ Zusätzlich wurden offene Fragen gestellt, damit den spezifischen Erfahrungen der Hersteller Rechnung getragen werden konnte.

Um die Vollständigkeit und Verständlichkeit der Fragestellungen zu gewährleisten, fanden im Vorfeld 12 Testinterviews statt. Aufgrund dessen wurde der Fragebogen angepaßt und in der endgültigen Fassung ins Feld gebracht. Auf der Grundlage der Interviews und weiteren Gesprächen wurden mit 6 Herstellern Fallstudien ${ }^{10}$ erarbeitet. Weiterhin führte das Institut für Marketing einen eintägigen Workshop zu produktbezogenen Rücknahme- und Recyclingsystemen durch. ${ }^{11}$

Für die statistische Datenauswertung wurde SPSS for windows 6.01 (Superior Performing Statistical Software) herangezogen. ${ }^{12}$ Die Analyse der Daten erfolgte unter Rückgriff auf verschiedene uni, bi- und multivariate Verfahren. ${ }^{13}$ Das in Kapitel B vorgelegte Modell der Implementierung von Rücknahme- und Recyclingsystemen wurde mit der konfirmatorischen Faktoranalyse, die der Kausalanalyse zugerechnet wird, überprüft.

Zusätzlich kamen die multivariaten Verfahren der Cluster- sowie der Diskriminanzanalyse zur Anwendung. In Kapitel C - bei der Untersuchung der Einflußfaktoren und Erfolgswirkungen der Imple-

XIIIff. Meffert führt dazu aus, daß die Abstände zwischen den Rangplätzen von den Befragten bei entsprechend graphischer Darstellung als konstant betrachtet werden und damit die mathematischen Voraussetzungen von Intervallskalen erfüllen und daher den Einsatz entsprechender statistischer Operationen erlauben. Vgl. Meffert, H., Marketingforschung und Käuferverhalten, a.a.O., S. 185.

9 Die relativ geringe Fallzahl von 101 Fällen vermag dem Anspruch der Repräsentativität nicht zu genügen. Daher ist zu berücksichtigen, daß die Analyse lediglich Tendenzaussagen beinhaltet, die jedoch erste Implikationen für eine weitergehende Forschung und für die praxisorientierte Implementierung von Rücknahme- und Recyclingsystemen ermöglichen.

10 Die Fallstudien wurden mit den Firmen Rank Xerox (Kopierer), Siemens-Nixdorf (Computer), Fenster Recycling Initiative (PVC-Fenster), Bauknecht (Hausgeräte), Volkswagen (Automobil) und Kaldewei (Badewannen) erstellt. Diese Unternehmen wurden ausgewählt, da zum einen bereits intensive Erfahrungen mit ihren implementierten Rücknahme- und Recyclingsystemen bestanden und sie zum andern eine weite Branchenabdeckung lieferten. Darüber hinaus wurden bei diesen Herstellern sehr unterschiedliche organisatorische Formen der Rücknahmeund Recyclingsystemen gewählt. Siemens-Nixdorf und Rank Xerox haben überwiegend eigene Systeme, bei FREI handelt es sich um ein Branchensystem, während Bauknecht und Kaldewei eher die Marktlösung über einen Verwerter gewählt haben.

11 Die Ergebnisse des Workshops wurden dokumentiert in: Meffert, H., Wagner, H., Backhaus, K. (Hrsg.), Marktorientierte Unternehmensführung in der Kreislaufwirtschaft - Kostenmanagement oder Wettbewerbsprofilierung, Dokumentationspapier Nr. 106 der Wissenschaftlichen Gesellschaft für Marketing und Unternehmensführung e.V., Münster 1996.

12 Dieses Programmpaket ist im lokalen Netzwerk des wirtschaftswissenschaftlichen Fachbereichs der Universität verfügbar.

13 Zu einer Darstellung wesentlicher Charakteristika der verschiedenen Verfahren hinsichtlich Aufbau und Einsatzmöglichkeiten vgl. Meffert, H., Marketingforschung und Käuferverhalten, a.a.O., S. 243ff. und Fahrmeir, L., Hamerle, A. (Hrsg.), Multivariate statistische Verfahren, Berlin, New York, de Gruyter 1984. 
mentierung - wurden Aussagen überwiegend aufgrund von kontingenzanalytischen Verfahren getroffen. $^{14}$

Die konfirmatorische Faktoranalyse ist im Gegensatz zur explorativen Faktoranalyse, die eine Hypothesen bildende, Faktor erzeugende und damit "erforschende Faktoranalyse" ist, eine Hypothesen testende, bestätigende Faktoranalyse. ${ }^{15}$ Dieses Verfahren ermöglicht die Prüfung auf Basis theoretisch abgeleiteter Hypothesen über die Anzahl von Faktoren (Implementierungsdimensionen) eines komplexen Konstrukts (Implementierung von Rücknahme- und Recyclingsystemen), die Beziehungen zwischen den Faktoren sowie die Beziehungen zwischen den Faktoren und ihren Indikatoren. ${ }^{16}$ Daher besteht eine hohe Eignung der konfirmatorischen Faktoranalyse zur Überprüfung der Implementierungsdimensionen.

$14 \quad$ Auf eine ausführliche Erläuterung der Verfahren soll im Rahmen dieser Arbeit grundsătzlich verzichtet werden, da die Verfahren in einer Vielzahl von betriebswirtschaftlichen Analysen als Standardverfahren eingesetzt werden. Es erfolgt lediglich eine kurze Darstellung der zentralen Grundlagen bei der Interpretation der Ergebnisse in den verschiedenen Kapiteln.

Vgl. Ost, F., Faktorenanalyse, in: Fahrmeir, L., Hamerle, A. (Hrsg.), Multivariate statistische Verfahren, Berlin, New York, de Gruyter 1984, S. $639 \mathrm{ff}$.

16

Vgl. Backhaus, K. u.a., Multivariate Analysemethoden, a.a.O., S. $409 \mathrm{ff}$. 
2.2 Beispielhafte Operationalisierung der Zeitdimensionen „Timing“ und „Zeithorizont“

\begin{tabular}{|c|c|c|c|}
\hline Unternehmen & $\begin{array}{c}\text { Beginn der } \\
\text { Planungsphase }\end{array}$ & $\begin{array}{c}\text { Beginn der Pilot- } \\
\text { phase }\end{array}$ & $\begin{array}{c}\text { Beginn der } \\
\text { Realisationsphase }\end{array}$ \\
\hline a & 1995 & 1996 & 1997 \\
\hline b & 1990 & 1992 & 1993 \\
\hline c & & 1994 & 1995 \\
\hline d & 1993 & & 1994 \\
\hline e & 1991 & 1995 & \\
\hline $\mathbf{f}$ & 1995 & & \\
\hline
\end{tabular}

\begin{tabular}{|c|c|c:c|}
\hline Zeitpunkt & Wert & Zeitdauer & \\
\hline$\leq 1992$ & (sehr früh) & 2 Jahre & $\begin{array}{c}1 \\
\text { (sehr lang) }\end{array}$ \\
\hline 1993 & 2 & 4 Jahre & 2 \\
\hline 1994 & 3 & 3 Jahre & 3 \\
\hline 1995 & 4 & 2 Jahre & 4 \\
\hline$\geq 1996$ & (sehr spät) & 1 Jahr & $\begin{array}{c}5 \\
\text { (sehr kurz) }\end{array}$ \\
\hline
\end{tabular}

\begin{tabular}{|c|c:c|c:c|}
\hline $\begin{array}{c}\text { Zeitdimensionen } \\
\text { Unternehmen }\end{array}$ & Timing & Wert & Zeithorizont & Wert \\
\hline a & 1995 & 4 & 2 Jahre & 4 \\
\hline b & 1990 & 1 & 3 Jahre & 3 \\
\hline c & 1994 & 3 & 1 Jahr & 5 \\
\hline d & 1993 & 2 & 1 Jahr & 5 \\
\hline e & 1991 & 1 & 4 Jahre & 2 \\
\hline f & 1995 & 4 & - & - \\
\hline
\end{tabular}




\subsection{Schrittweise Agglomeration der 5-Cluster-Lösung}

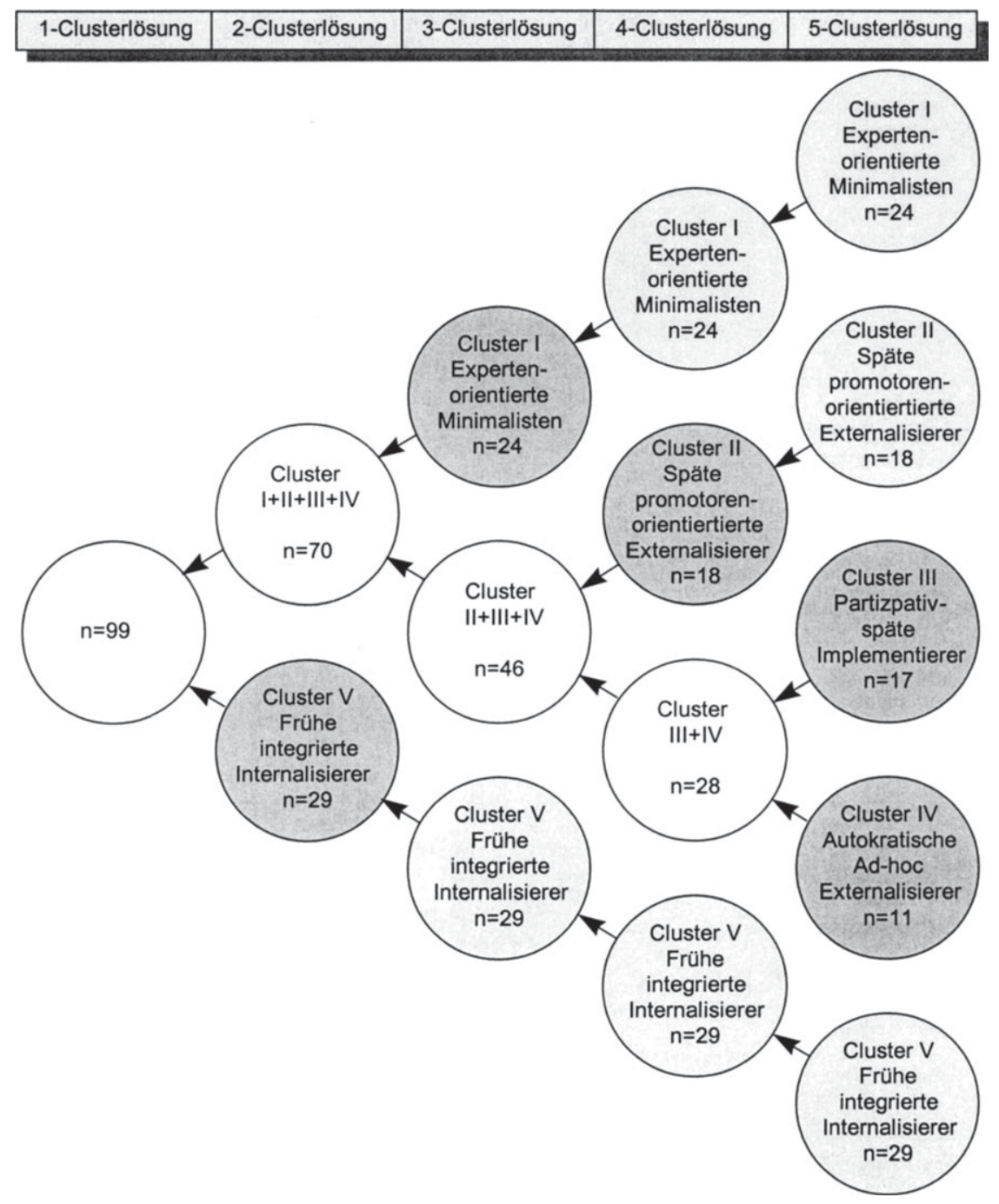




\subsection{Klassifikationsmatrix der schrittweisen Diskriminanzanalyse}

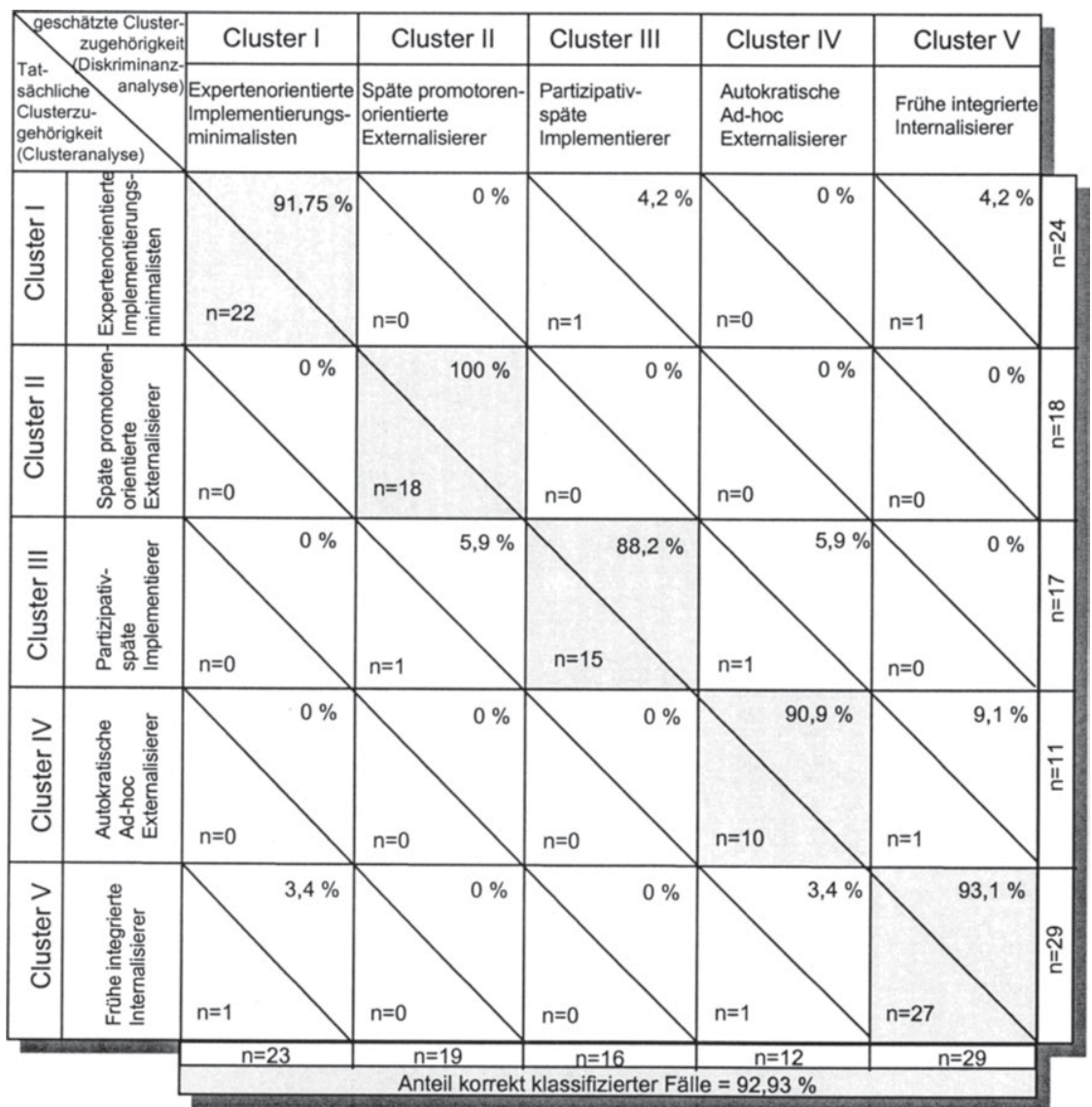




\subsection{Explorative Faktoranalyse zur Absatzmarktsituation}

\section{Verfahrensspezifikationen}

Analysis number 1 Pairwise deletion of cases with missing values

Extraction 1 for analysis 1, Principal Components Analysis (PC)

$\begin{array}{lllll} & \text { Mean } & \text { Std Dev } & \text { Cases } & \text { Label } \\ \text { F17A } & 2,50505 & 1,17259 & 99 & \text { Gesättigt_Markt } \\ \text { F17B } & 1,68687 & 0,93292 & 99 & \text { Preissens_Kunde } \\ \text { F17C } & 1,54545 & 0,79889 & 99 & \text { Qualität_Kunde } \\ \text { F17D } & 2,77083 & 1,17410 & 96 & \text { Recycl_Kunde } \\ \text { F17E } & 2,86957 & 1,28574 & 92 & \text { Material_Kunde } \\ \text { F17F } & 2,85227 & 1,32647 & 88 & \text { Umwelt_Handel } \\ \text { F17G } & 1,52525 & 0,98295 & 99 & \text { Preiskampf } \\ \text { F17H } & 2,55670 & 1,35377 & 97 & \text { Ausland_Wettber } \\ \text { F17I } & 2,00000 & 1,22685 & 98 & \text { Prod_gleich } \\ \text { F22 } & 3,79000 & 1,53277 & 99 & \text { Gebrauchtmarkt }\end{array}$

Final Statistics:

$\begin{array}{lllllll}\text { Variable } & \text { Communality } & * & \text { Factor } & \text { Eigenvalue } & \text { Pct of Var } & \text { Cum Pct } \\ \text { F17A } & , 70880 & * & 1 & 1,80745 & 18,1 & 18,1 \\ \text { F17B } & , 66371 & * & 2 & 1,43675 & 14,4 & 32,4 \\ \text { F17C } & , 63228 & * & 3 & 1,27232 & 12,7 & 45,2 \\ \text { F17D } & , 69747 & * & 4 & 1,02276 & 10,2 & 55,4 \\ \text { F17E } & , 71237 & * & 5 & 1,00589 & 10,1 & 65,5 \\ \text { F17F } & , 66290 & * & & & & \\ \text { F17G } & , 67104 & * & & & & \\ \text { F17H } & , 24115 & * & & & & \\ \text { F171 } & , 76678 & * & & & & \\ \text { F22 } & , 78866 & * & & & & \end{array}$

Rotated Factor Matrix:

$\begin{array}{llllll} & \text { Factor 1 } & \text { Factor 2 } & \text { Factor 3 } & \text { Factor 4 } & \text { Factor 5 } \\ \text { F17A } & , 76746 & & & & \\ \text { F17B } & , 67699 & & -, 64793 & & \\ \text { F17C } & & , 66496 & , 76119 & & \\ \text { F17D } & & , 79350 & & & \\ \text { F17E } & & , 45398 & & & \\ \text { F17F } & & & & \\ \text { F17G } & , 74874 & & & , 86270 \\ \text { F17H } & & & & \end{array}$




\subsection{Explorative Faktoranalyse zur Beschaffungsmarktsituation}

\section{Verfahrensspezifikationen}

Analysis number 1 Pairwise deletion of cases with missing values

Extraction 1 for analysis 1, Principal Components Analysis (PC)

$\begin{array}{lllll} & \text { Mean } & \text { Std Dev } & \text { Cases } & \text { Label } \\ \text { F81A } & 1,91954 & 1,31378 & 87 & \text { lange Verträge } \\ \text { F81B } & 2,51136 & 1,57564 & 88 & \text { Gem_Entwicklung } \\ \text { F81C } & 2,82022 & 1,59944 & 89 & \text { eigenes_Know_How } \\ \text { F81D } & 2,47126 & 1,45346 & 87 & \text { stand_Vorprodukt } \\ \text { F81E } & 2,83133 & 1,33270 & 83 & \text { Leistungsanspr } \\ \text { F81F } & 2,59524 & 1,26223 & 84 & \text { Konzentration } \\ \text { F81G } & 2,81176 & 1,62198 & 85 & \text { Rohstofferzeuger }\end{array}$

Final Statistics:

$\begin{array}{lllllll}\text { Variable } & \text { Communality } & * & \text { Factor } & \text { Eigenvalue } & \text { Pct of Var } & \text { Cum Pct } \\ \text { F81A } & , 61784 & * & 1 & 1,86836 & 26,7 & 26,7 \\ \text { F81B } & , 66136 & * & 2 & 1,49523 & 21,4 & 48,1 \\ \text { F81C } & , 61132 & * & 3 & 1,05327 & 15,0 & 63,1 \\ \text { F81D } & , 47161 & * & & & & \\ \text { F81E } & , 72672 & * & & & & \\ \text { F81F } & , 56278 & * & & & & \\ \text { F81G } & , 76524 & * & & & & \end{array}$

Rotated Factor Matrix:

$\begin{array}{llll} & \text { Factor } 1 & \text { Factor } 2 & \text { Factor } 3 \\ \text { F81A } & , 67854 & & \\ \text { F81B } & , 76106 & & \\ \text { F81C } & , 74871 & & \\ \text { F81D } & & , 67117 & \\ \text { F81E } & & & , 84997 \\ \text { F81F } & & , 67748 \\ \text { F81G } & & , 87205 & \end{array}$




\subsection{Signifikanzen der Implementierungstypen hinsichtlich der Produktmerkmale}

$$
\begin{aligned}
& \text { I = Expertenorientierte Implementierungsminimalisten } \\
& \text { II }=\text { Spăte promotorenorientierte Externalisierer } \\
& \text { III = Partizipativ-spăte Implementierer } \\
& \text { IV = Autokratische Ad hoc-Externalisierer } \\
& \text { V = Frühe integrierte Internalisierer }
\end{aligned}
$$
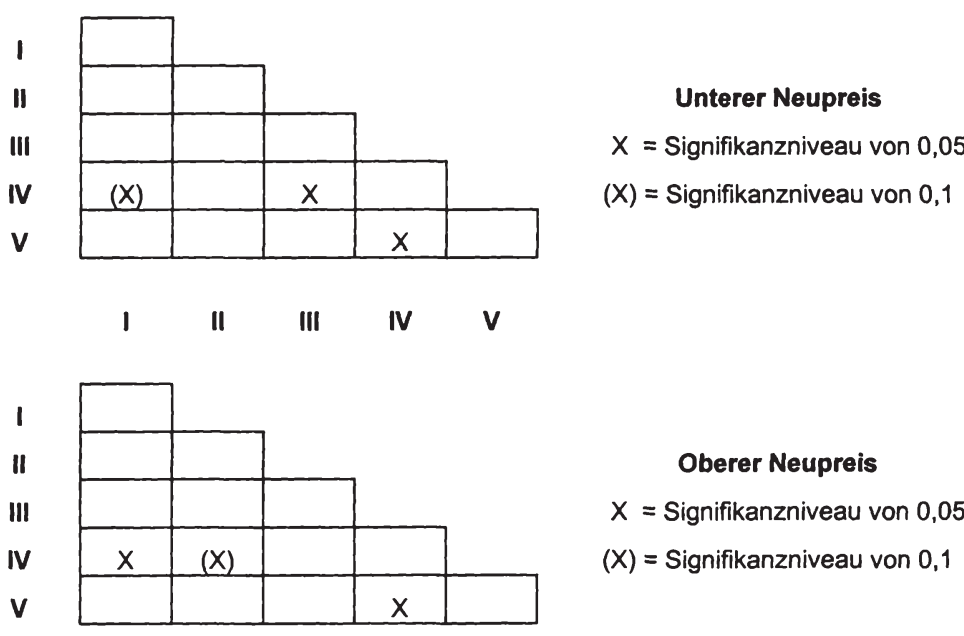

$$
\begin{gathered}
\text { Oberer Neupreis } \\
X=\text { Signifikanzniveau von } 0,05 \\
(X)=\text { Signifikanzniveau von } 0,1
\end{gathered}
$$

I II III IV V

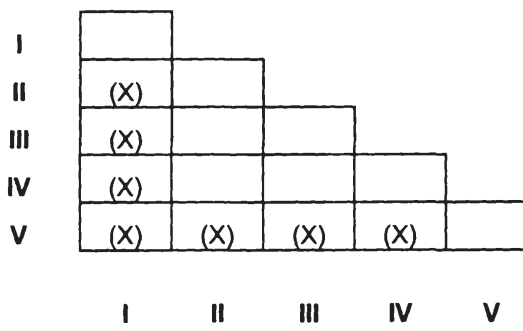


1 = Expertenorientierte Implementierungsminimalisten

$\|$ = Späte promotorenorientierte Externalisierer

III = Partizipativ-späte Implementierer

$\mathrm{IV}=$ Autokratische Ad hoc-Externalisierer

$V=$ Frühe integrierte Internalisierer

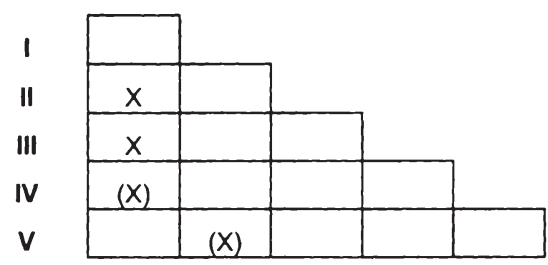

$$
\begin{gathered}
\text { Verkauf } \\
X=\text { Signifikanzniveau von } 0,05 \\
(X)=\text { Signifikanzniveau von } 0,1
\end{gathered}
$$

I II III IV V

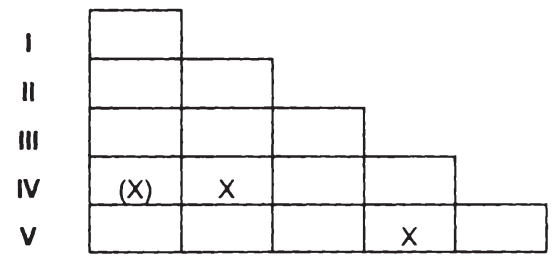

$$
\begin{aligned}
& \text { Fremd-/Selbstproduktion } \\
& X=\text { Signifikanzniveau von } 0,05 \\
& (X)=\text { Signifikanzniveau von } 0,1
\end{aligned}
$$

I II III IV V

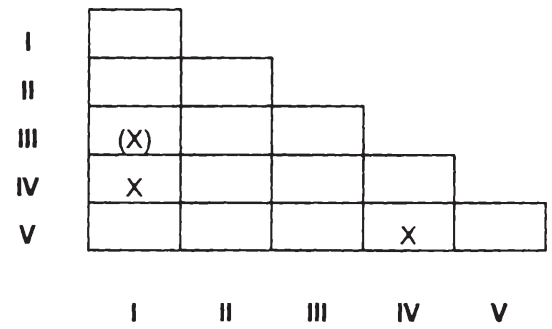

\section{Anzahl Komponenten}

$X=$ Signifikanzniveau von 0,05

$(X)=$ Signifikanzniveau von 0,1 


\subsection{Explorative Faktoranalyse zur Unternehmenskultur}

\section{Verfahrensspezifikationen}

Analysis number 1 Pairwise deletion of cases with missing values

Extraction 1 for analysis 1 , Principal Components Analysis (PC)

$\begin{array}{lllll} & \text { Mean } & \text { Std Dev } & \text { Cases } & \text { Label } \\ \text { F14C } & 1,73000 & 0,93046 & 99 & \text { Innovationsorientiert } \\ \text { F14G } & 1,78571 & 1,02796 & 98 & \text { technikorientiert } \\ \text { F141 } & 1,80000 & 0,77850 & 99 & \text { mitarbeiterorientiert } \\ \text { F14J } & 2,18000 & 1,14044 & 99 & \text { kompetenzorientiert } \\ \text { F14K } & 1,61000 & 0,95235 & 99 & \text { serviceorientiert } \\ \text { F14L } & 3,66000 & 1,26507 & 99 & \text { wettbewerbsorient. } \\ \text { F14M } & 4,03000 & 1,28279 & 99 & \text { gesetzorientiert } \\ \text { F14N } & 2,86000 & 1,25545 & 99 & \text { gesellschaftsorient. } \\ \text { F140 } & 2,26000 & 1,48813 & 99 & \text { internationalorient. } \\ \text { F14P } & 2,11000 & 1,26247 & 99 & \text { verbandsorientiert } \\ \text { F14Q } & 2,44444 & 1,34181 & 99 & \text { kooperationsorient. }\end{array}$

Final Statistics:

$\begin{array}{lllllll}\text { Variable } & \text { Communality } & * & \text { Factor } & \text { Eigenvalue } & \text { Pct of Var } & \text { Cum Pct } \\ \text { F14C } & , 41198 & * & 1 & 2,78469 & 25,3 & 25,3 \\ \text { F14G } & , 64156 & * & 2 & 1,51413 & 13,8 & 39,1 \\ \text { F141 } & , 71106 & * & 3 & 1,37250 & 12,5 & 51,6 \\ \text { F14J } & , 63954 & * & 4 & 1,08495 & 9,9 & 61,4 \\ \text { F14K } & , 54930 & * & & & & \\ \text { F14L } & , 65956 & * & & & & \\ \text { F14M } & , 60845 & * & & & & \\ \text { F14N } & , 50874 & * & & & & \\ \text { F14O } & , 72494 & * & & & & \\ \text { F14P } & , 62065 & * & & & & \end{array}$

Rotated Factor Matrix:

$\begin{array}{lllll} & \text { Factor 1 } & \text { Factor 2 } & \text { Factor 3 } & \text { Factor 4 } \\ \text { F14C } & & , 52929 & & \\ \text { F14G } & & , 77752 & & \\ \text { F141 } & & , 75363 & & , 83413 \\ \text { F14J } & & & , 79497 & , 59591 \\ \text { F14K } & & , 65488 & \\ \text { F14L } & & & & \\ \text { F14M } & & & & \\ \text { F14N } & , 46105 & & \\ \text { F140 } & , 67118 & & \\ \text { F14P } & , 71244 & & \\ \text { F14Q } & , 75374 & & \end{array}$


2.9 Explorative Faktoranalyse zu individualbezogenen Komponenten des „Kennen und Verstehens" sowie des „Könnens"

\section{Verfahrensspezifikationen}

Analysis number 1 Pairwise deletion of cases with missing values

Extraction 1 for analysis 1, Principal Components Analysis (PC)

$\begin{array}{lllll} & \text { Mean } & \text { Std Dev } & \text { Cases } & \text { Label } \\ \text { F64A } & 2,17778 & 1,25937 & 90 & \text { Fachwiss_Erfahrung } \\ \text { F64B } & 2,62222 & 1,27709 & 90 & \text { Literatur } \\ \text { F64C } & 2,77778 & 1,32205 & 90 & \text { int_Berichte } \\ \text { F64D } & 3,12222 & 1,44431 & 90 & \text { Referenzprojekte } \\ \text { F64E } & 2,43333 & 1,49193 & 90 & \text { Pilotprojekte } \\ \text { F64G } & 3,08889 & 1,26885 & 90 & \text { Marktdaten } \\ \text { F64H } & 3,03333 & 1,42569 & 90 & \text { Schulung } \\ \text { F64I } & 4,19101 & 1,10655 & 89 & \text { Job_Rotation } \\ \text { F64J } & 3,54444 & 1,37523 & 90 & \text { Coaching }\end{array}$

Final Statistics:

$\begin{array}{lllllll}\text { Variable } & \text { Communality } & * & \text { Factor } & \text { Eigenvalue } & \text { Pct of Var } & \text { Cum Pct } \\ \text { F64A } & , 58414 & * & 1 & 3,05826 & 34,0 & 34,0 \\ \text { F64B } & , 65021 & * & 2 & 1,42582 & 15,8 & 49,8 \\ \text { F64C } & , 47985 & * & & & & \\ \text { F64D } & , 34826 & * & & & & \\ \text { F64E } & , 31024 & * & & & & \\ \text { F64G } & , 36149 & * & & & & \\ \text { F64H } & , 53524 & * & & & & \\ \text { F64I } & , 54239 & * & & & & \\ \text { F64J } & , 67226 & * & & & & \end{array}$

Rotated Factor Matrix:

$\begin{array}{lll} & \text { Factor 1 } & \text { Factor 2 } \\ \text { F64A } & , 75580 & \\ \text { F64B } & , 79944 & \\ \text { F64C } & , 66229 & \\ \text { F64D } & , 49254 & \\ \text { F64E } & & , 46895 \\ \text { F64G } & , 51748 & \\ \text { F64H } & & , 69853 \\ \text { F64I } & & , 72962 \\ \text { F64J } & & , 81987\end{array}$




\section{Anhang 3: Fragebögen}

\subsection{Auszüge aus dem Fragebogen der persönlichen Befragung}

1. Das Kreislaufwirtschaftsgesetz wird im Herbst 1996 in Kraft treten. Inwieweit hat die Verabschiedung des Gesetzes Ihr Unternehmen veranlaßt, sich mit der Rücknahme und mit dem Recycling Ihrer Produkte auseinanderzusetzen?

$\begin{array}{ccccc}\text { sehr } & & & \text { gar nicht } \\ 1 & 2 & 3 & 4 & 5 \\ \square & \square & \square & \square & \square\end{array}$

2. Inwieweit haben Sie sich schon vor der Verabschiedung des Gesetzes mit dem Recycling beschäftigt?

sehr

1

12

23

34

gar nicht

$\square$

$\square \quad \square$

3. Erwarten Sie aufgrund des Kreislaufwirtschaftsgesetzes für lhre Branche den Erlaß einer spezifischen Rücknahmeverordnung?

Ja

Nein

9. Bitte geben Sie an, wieviel Prozent der gesamten Absatzmenge des Produkts Ihr Unternehmen in Deutschland als Konsumgüter und/oder als Investitionsgüter (Business to Business- Geschäft) verkauft?

Konsumgüter:

Investitionsgüter:

Gesamt:

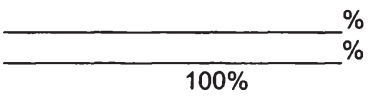

12. Produkte können von Kunden gekauft, gemietet oder geleast werden. Bitte geben Sie für das ausgewählte Produkt an, wieviel Prozent der gesamten Absatzmenge im Inland auf diese Vermarktungsformen entfallen.

Verkauf:

Vermietung:

Leasing:

Gesamtabsatz:

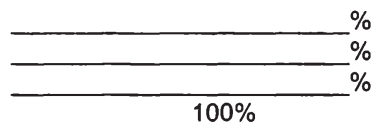

13. Wenn Sie einmal den Endabgabepreis betrachten, den der Verwender Ihrer Produkte bezahlen muß, wie hoch ist der Einstiegspreis des ausgewähiten Produktes und wie hoch ist der Preis bei der aufwendigsten Ausstattung dieses Produktes?

Einstiegspreis:

Preis der aufwendigsten Produktausstattung: DM DM 
14. Wenn Sie die längerfristige Grundausrichtung Ihres Unternehmens in dem Geschäftsbereich betrachten, dem das betrachtete Produkt zugeordnet ist, wie stark treffen folgende Verhaltensweisen zu?

Das Unternehmen...

versucht sich am Markt mit preisaggressivsten Angeboten Wettbewerbsvorteile zu schaffen

erhebt den Anspruch, Qualitätsführer im Markt zu sein

profiliert sich am Markt mit den innovativsten Produktleistungen

strebt im Umweltschutz eine Vorreiterfunktion in der Branche an

geht auf Kundenwünsche sehr individuell ein

konzentriert sich auf einen Nischenmarkt

setzt in der Produktion die modernsten Techniken ein

verfolgt konsequent Kostensparprogramme

ist mitarbeiterorientiert

konzentriert sich ausschließlich auf eigene Kompetenzen

versucht durch Serviceleistungen Wettbewerbsvorteile zu erzielen

orientiert sich beim Umweltschutz an Wettbewerbern

wartet mit Umweltschutzmaßnahmen, bis konkrete gesetzliche Vor-

schriften erlassen werden

engagiert sich für gesellschaftliche Aufgaben

expandiert auf internationalen Märkten

ist in Verbänden engagiert

nutzt Kooperationen zur Erzielung von Wettbewerbsvorteilen

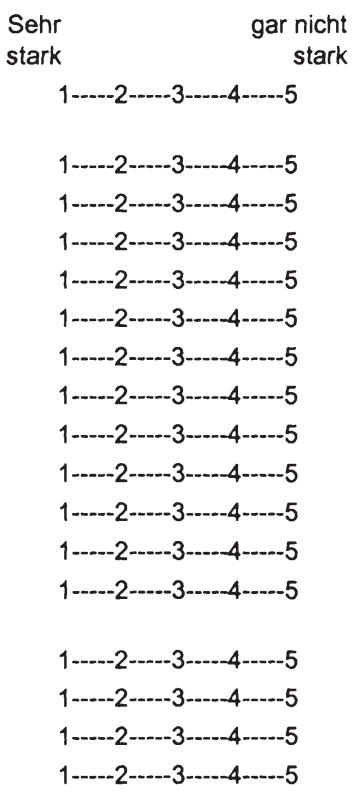

17. Wenn Sie die Absatzmarktsituation für Ihre Produkte einschätzen, wie zutreffend sind folgende Situationsmerkmale?

Der Markt ist in hohem Maße gesättigt

Kunden sind sehr preissensibel

Kunden stellen sehr hohe Qualitätsanforderungen

Kunden sind bereit, Rücknahme- und Recyclingaktivitäten zu unterstützen

Kunden akzeptieren zunehmend Produkte aus Recyclingmaterialien

Der Handel verlangt zunehmend umweltgerechte Produkte und Serviceleistungen

Es herrscht ein aggressiver Preiskampf

Wettbewerbsfähigkeit wird durch ausländische Anbieter gefährdet

Die stoffliche Zusammensetzung der am Markt angebotenen Produkte ist die gleiche

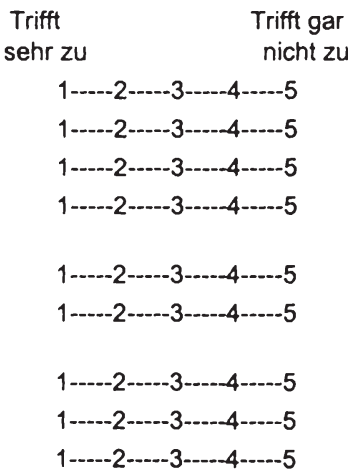


22. In welchem Ausmaß existiert für Ihr Produkt ein Gebrauchtmarkt?

$$
\begin{array}{r}
\text { sehr } \\
1
\end{array}
$$

$$
2
$$$$
3
$$

4

gar nicht

$\begin{array}{llll}1 & 0 & 5 & 5 \\ & \square & \square & \square\end{array}$

23. Betrachten wir nun einmal das Produkt nach seiner spezifischen Beschaffenheit. Wieviele Bauteile oder Komponenten werden bei der Montage des Produktes in etwa zusammengesetzt?

Anzahl der Bauteile oder Komponenten: ca.

24. Wieviel Prozent aller Komponenten, die für das Produkt benötigt werden, stellen Sie selbst her und wieviel beziehen Sie von Lieferanten?

Anteil Selbstproduktion:

Anteil des Zukaufs von Lieferanten:

Gesamt:

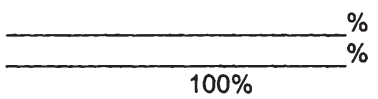

33. Wir möchten jetzt auf Ihr Rücknahme- und Recyclingsystem zu sprechen kommen. Welche Aussagen auf dieser Liste beschreiben den derzeitigen „Status“ Ihres Rücknahmeund Recyclingsystems bezüglich des von uns ins Auge gefaßten Produkts am besten?

- Wir befinden uns in der Planungsphase und nehmen derzeit noch keine Produkte zurück

$0 \quad$ Wir befinden uns bereits im Pilotversuch und nehmen im begrenzten Maße Produkte zurück

O Wir haben das Rücknahme- und Recyclingsystem bereits regulär eingeführt, d.h. wir können Produkte in vollem Maße zurücknehmen

34. Bitte geben Sie das Jahr an, wann Sie mit der Planungsphase begonnen haben.; und in welchem Jahr haben Sie mit der Pilotphase und wann mit der regulären Betriebsphase begonnen?

Planungsbeginn:

19

Beginn der Pilotphase:

19

Beginn der regulären Betriebsphase:

19 
35. Jetzt lese ich Ihnen einige Ziele vor, und Sie sagen mir bitte jeweils an Hand dieser Skala, wie stark es Ihnen darauf ankommt, diese Ziele mit Ihrem Rücknahme- und Recyclingsystem zu erreichen?

\begin{tabular}{|c|c|}
\hline & $\begin{array}{r}\text { gar nicht } \\
\text { stark }\end{array}$ \\
\hline Kostenwirtschaftlichkeit des Systems & 1-----2-----3-----4-----5 \\
\hline Kurzfristige Gewinnerzielung & 1-----2-----3-----4------5 \\
\hline Langfristige Gewinnerzielung & 1-----2-----3-----4-----5 \\
\hline Erschließung neuer Umsatzmöglichkeiten & 1-----2-----3-----4-----5 \\
\hline Erlangung von Imagevorteilen beim Kunden & 1-----2-----3-----4-----5 \\
\hline Erhöhung der Kundenbindung & $1----2----3----4----5$ \\
\hline Profilierung gegenüber der Konkurrenz & 1-----2-----3-----4-----5 \\
\hline Vorbeugung gegenüber drohenden Rücknahmeverordnungen & $1-----2-----3-----4-----5$ \\
\hline $\begin{array}{l}\text { Reduzierung von Haftungsrisiken durch Übernahme der gesamten } \\
\text { Produktverantwortung }\end{array}$ & 1-----2-----3-----4-----5 \\
\hline Aufbau von eigenen Kompetenzen im Recyclingbereich & 1-----2-----3-----4-----5 \\
\hline Sammlung von Erfahrungen im Umgang mit Altprodukten & $1----2-\cdots--3-\cdots---4-\cdots-5$ \\
\hline Erschließung neuer Absatzchancen auf Sekundärrohstoffmärkten & 1-----2-----3-----4-----5 \\
\hline Auslastung von Recyclingkapazitäten & 1-----2-----3-----4-----5 \\
\hline Entwicklung innovativer Recyclingtechnologien & $1-----2-----3----4-----5$ \\
\hline $\begin{array}{l}\text { Verkürzung von Produktionszeiten durch Wiedereinsatz von Ge- } \\
\text { brauchtteilen }\end{array}$ & 1-----2-----3------4-----5 \\
\hline Senkung von Deponiekosten für nicht verwertbare Abfälle & 1-----2-----3-----4-----5 \\
\hline Erzielung hoher Erfassungsquoten für die eigenen Altprodukte & 1-----2-----3-----4-----5 \\
\hline \multicolumn{2}{|l|}{ Wiedereinsatz von Gebrauchtteilen } \\
\hline - für die eigenen Neuprodukte & 1-----2-----3-----4-----5 \\
\hline - für den Kundendienst als Ersatzteile & $1-\cdots--2-----3-\cdots--4-\cdots--5$ \\
\hline EinsatzNerkauf von Gebrauchtteilen außerhalb des Unternehmens & 1-----2-----3------4-----5 \\
\hline Wiederverwertung von Stofffraktionen außerhalb des Unternehmens & 1-----2-----3-----4-----5 \\
\hline Förderung der thermischen Verwertung & $1-----2-----3-----4-----5$ \\
\hline Förderung einer brancheneinheitlichen Lösung & $1----2-----3----4----5$ \\
\hline Förderung einer EU-weiten Lösung & $1----2----3----4----5$ \\
\hline
\end{tabular}


42. Auf dieser Liste stehen verschiedene Möglichkeiten der Organisation eines Rücknahmeund Recyclingsystems. Sagen Sie mir bitte jeweils, ob diese Möglichkeit auf Ihr System zutrifft!

\begin{tabular}{|l|l|lr|}
\hline 1 & $\begin{array}{l}\text { Wir haben uns einer Lösung angeschlossen, in der mehrere Wettbewerber } \\
\text { gemeinsam ein System entwickeln }\end{array}$ & $0 \mathrm{ja}$ & 0 nein \\
\hline 2 & $\begin{array}{l}\text { Wir haben ein unternehmenseigenes System entwickelt, in dem wir überwie- } \\
\text { gend selbst alle Aktivitäten durchführen und kontrollieren }\end{array}$ & $0 \mathrm{ja}$ & 0 nein \\
\hline 3 & Wir haben mit Verwertungsunternehmen gemeinsam ein System entwickelt & $0 \mathrm{ja}$ & 0 nein \\
\hline 4 & $\begin{array}{l}\text { Wir sind mit Wettbewerbern und/oder Verwertern Mitglied in einem Gemein- } \\
\text { schaftsunternehmen }\end{array}$ & $0 \mathrm{ja}$ & 0 nein \\
\hline 5 & $\begin{array}{l}\text { Wir haben mit Verwertungsunternehmen einen Vertrag abgeschlossen, ohne } \\
\text { eine intensive, weiterführende Kooperation einzugehen }\end{array}$ & $0 \mathrm{ja}$ & 0 nein \\
\hline
\end{tabular}

51. Wenn Sie das Gesamtsystem in dieser Form betrachten, in welchem Maße bringt Ihr Unternehmen Ressourcen ein?

\begin{tabular}{|c|c|}
\hline & $\begin{array}{l}\text { in sehr } \\
\text { hohem Ausmaß }\end{array}$ \\
\hline Beziehungen zu politischen Behörden/Institutionen & 1-----2-----3-----4-----5 \\
\hline Beziehungen zu Experten & $1-\cdots--2-----3----4-----5$ \\
\hline Finanzielle Ressourcen & 1----2-----3-----4-----5 \\
\hline Personelle Kapazitäten & $1-----2-----3-----4-----5$ \\
\hline Räumliche Kapazitäten & 1-----2-----3-----4-----5 \\
\hline Annahmestellen für Altprodukte & 1-----2-----3-----4-----5 \\
\hline Rückführungslogistik & 1-----2-----3-----4-----5 \\
\hline Lagerhaltungsflächen & 1-----2------3-----4-----5 \\
\hline Informationssysteme & 1-----2-----3------4-----5 \\
\hline Demontage-Know-How & 1-----2------3-----4-----5 \\
\hline Zugang zu Sekundärrohstoffmärkten & 1-----2-----3-----4-----5 \\
\hline Öffentlichkeitsarbeit & 1-----2-----3-----4-----5 \\
\hline
\end{tabular}

53. Ob und wie intensiv sind die einzelnen Funktionsbereich Ihres Unternehmens bei der Gestaltung des Rücknahme- und Recyclingsystems beteiligt? (Planungsphase)

Einkauf / Beschaffung
Forschung und Entwicklung
Produktion/Technik
Umweltschutz
Finanzierung
Controlling
Marketing
Vertrieb
Kundendienst
Offentlichkeitsarbeit

sehr intensiv 
54. Wer sind „die Väter" des Projektes?

a. Denken Sie bitte an bis zu drei Personen, die das System in Ihrem Unternehmen von seiner fachlichen Seite maßgeblich ausgearbeitet haben.

Aus welcher Abteilung kommen diese Personen, und auf welcher Hierarchieebene sind diese angesiedelt?
1. Person
2. Person
3. Person
O Marketing
O Marketing
O Marketing
O Produktion/Technik
O Produktion/Technik
O Produktion/Technik
O Umweltschutz
O Umweltschutz
O Umweltschutz
O Produktentwicklung
O Produktentwicklung
O Produktentwicklung

\section{Person}

O Geschäftsführung

O Bereichsleitung

O Fachleitung

O Abteilungsleitung

O sonstige
2. Person

O Geschäftsführung

O Bereichsleitung

O Fachleitung

O Abteilungsleitung

O sonstige
3. Person

○ Geschäftsführung

O Bereichsleitung

O Fachleitung

O Abteilungsleitung

O sonstige

b. Wenn Sie einmal daran denken, welche Personen in Ihrem Unternehmen die Schlüsselentscheidungen des Rücknahme- und Recyclingsystems getroffen haben, aus welchen Abteilungen kommen diese und auf welcher Hierarchieebene sind diese angesiedelt?

\section{Person}

O Marketing

O Produktion/Technik

O Umweltschutz

O Produktentwicklung
2. Person

O Marketing

O Produktion/Technik

O Umweltschutz

O Produktentwicklung
3. Person

O Marketing

O Produktion/Technik

O Umweltschutz

O Produktentwicklung

\section{Person}

O Geschäftsführung

O Bereichsleitung

O Fachleitung

O Abteilungsleitung

$O$ sonstige
2. Person

○eschäftsführung

O Bereichsleitung

O Fachleitung

O Abteilungsleitung

O sonstige
3. Person

O Geschäftsführung

O Bereichsleitung

O Fachleitung

O Abteilungsleitung

O sonstige 
55. Wenn Sie einmal die Auswirkungen der Einrichtung des Rücknahme- und Recyclingsystems auf die einzelnen Funktionsbereiche Ihres Unternehmens betrachten, wie stark hat sich die Umsetzung auf die folgenden Bereiche ausgewirkt? (Realisationsphase)

\begin{tabular}{|c|c|}
\hline & $\begin{array}{l}\text { sehr } \\
\text { intensiv }\end{array}$ \\
\hline Einkauf / Beschaffung & 1-----2-----3-----4-----5 \\
\hline Forschung und Entwicklung & 1----2----3-----4----5 \\
\hline Produktion/Technik & 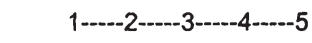 \\
\hline Umweltschutz & 1-----2-----3-----------5 \\
\hline Finanzierung & 1-----2-----3-----4-----5 \\
\hline Controlling & 1-----2-----3-----4----5 \\
\hline Marketing & 1-----2-----3-----4-----5 \\
\hline Vertrieb & 1-----2-----3-----4----5 \\
\hline Kundendienst & 1-----2-----3-----4-----5 \\
\hline Öffentlichkeitsarbeit & 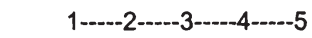 \\
\hline
\end{tabular}

63. Wenn Sie die geplanten Veränderungen betrachten wie wichtig sind dann folgende Vorgehensweisen für die Umsetzung der Maßnahmen in Ihrem Unternehmen?

Direkte Verantwortungsübernahme auf der Geschäftsleitungsebene Klare Anweisungen über die Hierarchie von „oben nach unten“ (Top-to-down-Prinzip)

Systematisches Herunterbrechen von Teilaufgaben auf einzelne Abteilungen unter Einbeziehung der Mitarbeiter (Bottom-up-Prinzip)

Schrittweise Umsetzung der geplanten Maßnahmen

Ernennung eines verantwortlichen Fachexperten im Unternehmen

Einbeziehung eines externen Beraters bei der Umsetzung

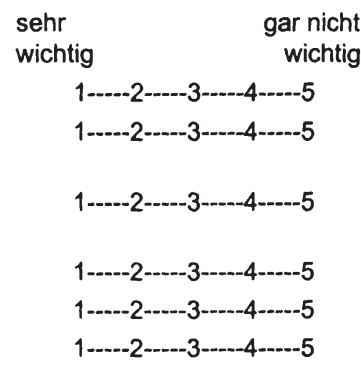

64. In welchem Maße nutzt Ihr Unternehmen die folgenden Instrumente, um das Fachwissen der betroffenen Mitarbeiter über die mit der Umsetzung des Rücknahme- und Recyclingsystems verbundenen Maßnahmen zu steigern?

Systematische Auswertung der bisherigen Erfahrungen

Systematische Auswertung von Fachberichten/Literatur Innerbetriebliche Verbreitung von Berichten über Recyclingsysteme Besichtigung und Analyse externer Referenzprojekte Sammlung von Erfahrungen durch eigene Pilotprojekte Nutzung von Marktdaten Schulung der Mitarbeiter Job-Rotation Coaching einzelner Mitarbeiter gar nicht

$$
\begin{aligned}
& \text { 1----2----3----4----5 } \\
& \text { 1-----2-----3-----4--.-.5 } \\
& \text { 1----2----3-----4---5 } \\
& \text { 1----2----3----4----5 } \\
& \text { 1.-.--2-----3-----4-----5 } \\
& \text { 1----2-----3-----4----.5 } \\
& \text { 1-----2----3-----4----5 } \\
& \text { 1----2----3-----4----5 } \\
& \text { 1-----2-----3-----4----5 }
\end{aligned}
$$


65. Wie intensiv nutzen Sie die folgenden Vorgehensweisen, um Mitarbeiter für die Umsetzung der Rücknahme- und Recyclingsysteme zu motivieren?

\begin{tabular}{|c|c|c|}
\hline \multicolumn{3}{|c|}{$\begin{array}{ll}\text { sehr } & \text { gar } n i \\
\text { stark } & \end{array}$} \\
\hline Umweltorientiertes Vorschlagwesen & \multicolumn{2}{|c|}{ 1----2-----3-----4----5 } \\
\hline Gewährung von finanziellen Anreizen & \multicolumn{2}{|c|}{ 1----2----3-----4----5 } \\
\hline Berufliche Entfaltungschancen/Beförderung in Aussicht stellen & \multicolumn{2}{|c|}{ 1-----2-----3-----4-----5 } \\
\hline Direkte Unterstützung durch die Geschäftsleitung (Bsp. Gespräche) & \multicolumn{2}{|c|}{ 1-----2-----3-----4-----5 } \\
\hline Geschäftsleitung als Vorbild im Umgang mit Umweltschutz & \multicolumn{2}{|c|}{ 1-----2-----3-----4----5 } \\
\hline $\begin{array}{l}\text { Teilnahmemöglichkeit an umweltbezogenen Seminaren und Vorträ- } \\
\text { gen }\end{array}$ & \multicolumn{2}{|c|}{$1-\cdots--2-\cdots--3-\cdots--4-\cdots--5$} \\
\hline \multicolumn{3}{|c|}{$\begin{array}{l}\text { 66. Welche der folgenden organisatorischen Maßnahmen haben Sie innerhalb Ihres Unte } \\
\text { nehmens für die Umsetzung des Rücknahme- und Recyclingsystems ergriffen? }\end{array}$} \\
\hline Einrichtung von Workshops & $0 \mathrm{ja}$ & 0 nein \\
\hline Einrichtung von Projektteams- bzw. Gruppen & 0 ja & 0 nein \\
\hline Einrichtung einer neuen Funktion/Linienstelle & $0 \mathrm{ja}$ & 0 nein \\
\hline Integration in bestehende Linienstellen & 0 ja & 0 nein \\
\hline Einrichtung eines Stabes & $0 \mathrm{ja}$ & 0 nein \\
\hline Bildung eines eigenständigen Unternehmensbereiches & 0 ja & 0 nein \\
\hline
\end{tabular}

\section{Wie wird diese Stelle angebunden?}

\begin{tabular}{|c|c|c|}
\hline Anbindung an... & & \\
\hline die Geschäftsleitung & $0 \mathrm{ja}$ & 0 nein \\
\hline das Marketing & 0 ja & 0 nein \\
\hline die Produktion/Technik & $0 \mathrm{ja}$ & 0 nein \\
\hline die Forschung und Entwicklung & $0 \mathrm{ja}$ & 0 nein \\
\hline Sonstiges & $0 \mathrm{ja}$ & 0 nein \\
\hline
\end{tabular}


68. Welchen Stellenwert nehmen die folgenden unternehmensbezogenen Aufgaben bei der Umsetzung des Rücknahme- und Recyclingsystems ein?

\begin{tabular}{|c|c|}
\hline & $\begin{array}{lr}\text { sehr } & \text { gar nicht } \\
\text { wichtig } & \text { wichtig }\end{array}$ \\
\hline Ausarbeitung von operativen Maßnahmen & 1-----2----3-----4----5 \\
\hline Ermittlung der Funktionsbereiche & 1-----2-----3-----4-----5 \\
\hline Bereitstellung geeigneter Führungskräfte und Mitarbeiter & 1-----2-----3-----4-----5 \\
\hline $\begin{array}{l}\text { Ermittlung des Informationsbedarfs für die Planung, Steuerung und } \\
\text { Kontrolle }\end{array}$ & 1------2-----3------4-----5 \\
\hline Anpassung der Organisationsstruktur & 1-----2-----3-----4-----5 \\
\hline Anpassung der Unternehmenskultur bzw. -philosophie & 1-----2-----3-----4-----5 \\
\hline Analyse der Auswirkungen auf den Wettbewerb/Marktstellung & 1-----2-----3------4-----5 \\
\hline $\begin{array}{l}\text { Handhabung von Konflikten, die mit der Einrichtung von Kreisläufen } \\
\text { verbunden sind }\end{array}$ & 1-----2-----3-----4-----5 \\
\hline Klare personelle Aufgabenverteilung & $1----2-----3----4----5$ \\
\hline Analyse des Zeitbedarfs der Implementierung & 1-----2-----3---.-4-----5 \\
\hline
\end{tabular}

69. Wie intensiv werden die folgenden Informations- bzw. Kontrollsysteme bei der Implementierung des Rücknahme- und Recyclingsystems genutzt?

Kostenvergleich

sehr

Gewinnvergleich

Rentabilität

Ökobilanzen

Checklisten

Handbücher (z.B. zur Demontage)

EDV-gestützte Systeme

$$
\begin{aligned}
& \text { gar nicht } \\
& \text { 1-----2-----3-----4-----5 } \\
& \text { 1-----2-----3-----4-----5 } \\
& \text { 1-----2-----3-----4-----5 } \\
& \text { 1-----2-----3-----4-----5 } \\
& \text { 1-----2-----3-----4-----5 } \\
& \text { 1-----2-----3-----4-----5 } \\
& \text { 1-----2-----3-----4-----5 }
\end{aligned}
$$

70. Wodurch und wie stark werden die Implementierungsaktivitäten in Ihrem Unternehmen koordiniert?

Durch persönliche Anweisungen der Vorgesetzten

Durch konkrete Festlegung von Zielvereinbarungen zwischen Vorgesetzten und Mitarbeitern

Durch Mitbestimmungsmöglichkeiten der Mitarbeiter an wichtigen Entscheidungen

Koordination durch Richtlinien, Handbücher u.s.w. sehr intensiv gar nicht

$$
\begin{gathered}
\text { 1-----2-----3----4-----5 } \\
\text { 1-----2-----3----4----5 } \\
\text { 1-----2-----3-----4-----5 } \\
\text { 1-----2-----3-----4-----5 }
\end{gathered}
$$


71. Wie groß ist Ihrer Meinung nach der Handlungsbedarf in folgenden Punkten, um Fähigkeits- und Willensbarrieren bei den Mitarbeitern zu überwinden?

\begin{tabular}{|c|c|}
\hline & $\begin{array}{l}\text { sehr } \\
\text { groß }\end{array}$ \\
\hline Regelmäßige und intensive Information der Mitarbeiter & 1-----2-----3-----4-----5 \\
\hline Vermittlung von Fachkenntnissen durch Coaching/Schulungen & $1-----2-----3-----4----5$ \\
\hline $\begin{array}{l}\text { Möglichkeiten schaffen, daß Mitarbeiter Erfahrungen sammeln kön- } \\
\text { nen }\end{array}$ & 1-----2-----3-----4-----5 \\
\hline Planungen und Formulierungen in verständlicher Sprache & $1-----2-----3-----4-----5$ \\
\hline Förderung der Kommunikation zwischen den beteiligten Mitarbeitern & $1-----2-----3-----4-----5$ \\
\hline Förderung der positiven Einstellung zur Umweltproblematik & 1-----2-----3-----4-----5 \\
\hline $\begin{array}{l}\text { Abbau von Einwänden seitens der betroffenen Mitarbeiter gegen } \\
\text { Personen, die den Kreislauf implementieren wollen }\end{array}$ & 1-----2-----3-----4-----5 \\
\hline $\begin{array}{l}\text { Abbau von Verunsicherung bei Mitarbeitern, die neue Aufgaben } \\
\text { übernehmen müssen }\end{array}$ & 1-----2-----3-----4-----5 \\
\hline
\end{tabular}

73. Wie würden Sie aus Ihrer Sicht den Implementierungserfolg auf Mitarbeiterebene kennzeichnen? Wie stark treffen folgende Aussagen zu?

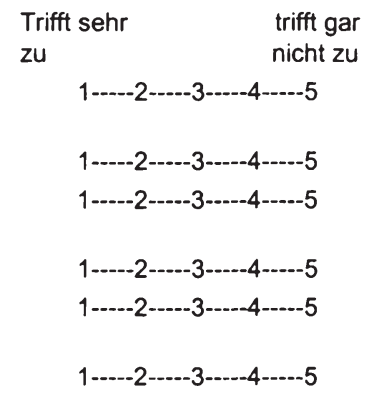


74. Wie stark wurden durch die Einrichtung des Rücknahme- und Recyclingsystems folgende abfallwirtschaftliche Zielgrößen beeinflußt? Vergleichen Sie mit der Zeit, als Sie das System noch nicht eingerichtet hatten.

Erfassungsquote von eigenen Altprodukten

Wiedereinsatz von Gebrauchtteilen

- für die eigenen Neuprodukte

- für den Kundendienst als Ersatzteile

Einsatz bzw. Verkauf von Gebrauchtteilen außerhalb des eigenen Unternehmens

Anteil der stofflichen Verwertung zum Einsatz der Stofffraktionen im eigenen Unternehmen

Anteil der stofflichen Verwertung zum EinsatzNerkauf außerhalb des Unternehmens

Energetische/Thermische Verwertung

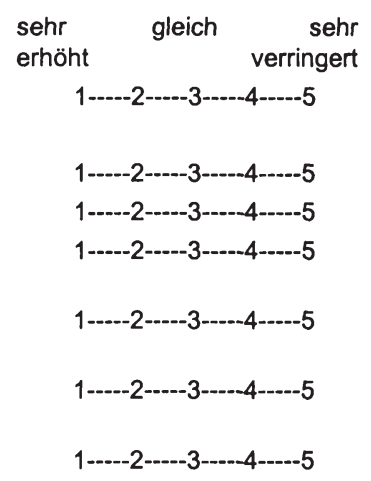

78. Wenn Sie einmal den betriebswirtschaftlichen Erfolg der Einrichtung des Rücknahmeund Recyclingsystems Ihres Unternehmens bewerten, in welchem Maße konnten Sie die folgenden Ziele erreichen?

Kostenwirtschaftlichkeit

Erschließung neuer Umsatzmöglichkeiten

Imagevorteile beim Kunden

Erzielung eines Erfahrungsvorsprungs gegenüber Hauptwettbewerbern

Reduzierung von Haftungsrisiken durch Übernahme der Produktverantwortung

$$
\begin{aligned}
& \text { voll gar nicht } \\
& \text { erreicht erreicht } \\
& \text { 1-----2----3-----4-----5 } \\
& \text { 1-----2----3-----4-----5 } \\
& \text { 1----2----3-----4----5 } \\
& \text { 1-----2-----3-----4-----5 } \\
& \text { 1-----2-----3------4-----5 }
\end{aligned}
$$

80. Wenn Sie nicht nur den Erfolg Ihres Unternehmens betrachten, sondern das Gesamtsystem zusammen mit den Partnern sehen, inwieweit sind die folgenden Zielsetzungen erreicht worden?

Auslastung der vorhandenen Recyclingkapazitäten

voll gar nicht

erreicht erreicht

Kostenwirtschaftlichkeit des Gesamtsystems

Gutes Image des Systems bei den Kunden

Konkurrenzfähigkeit des Gesamtsystems

Konfliktfreie Zusammenarbeit mit Systempartnern

Vertrauensvolle Zusammenarbeit mit Systempartnern

$$
\begin{aligned}
& \text { 1-----2-----3-----4----5 } \\
& \text { 1-----2-----3----4----5 } \\
& \text { 1-----2----3-----4----5 } \\
& \text { 1-----2----3-----4----5 } \\
& \text { 1----2-----3----4----5 } \\
& \text { 1-----2-----3-----4----5 }
\end{aligned}
$$


81. Wenn Sie die Situation auf den Beschaffungsmärkten betrachten, wie zutreffend sind die folgenden Aussagen?

\begin{tabular}{|c|c|}
\hline $\begin{array}{l}\text { trifft } \\
\text { sehr zu }\end{array}$ & $\begin{array}{l}\text { trifft gar } \\
\text { nicht zu }\end{array}$ \\
\hline \multicolumn{2}{|c|}{ 1-----2-----3-----4-----5 } \\
\hline \multicolumn{2}{|c|}{ 1-----2-----3-----4-----5 } \\
\hline \multicolumn{2}{|c|}{ 1-----2----3----4-----5 } \\
\hline 1--. & ----5 \\
\hline $1--$. & $4-\cdots--5$ \\
\hline $1-$ & 4---5 \\
\hline
\end{tabular}

Wir arbeiten mit Zulieferern im Rahmen von langfristigen Verträgen zusammen

Wir entwickeln mit unseren Zulieferern gemeinsam Komponenten und Vorprodukte

Grundsätzlich verfügen wir über das Know-how, einen Großteil der Vorprodukte selbst zu fertigen

Es gibt nur wenige Zulieferer, die unseren Leistungsansprüchen gerecht werden

Der Beschaffungsmarkt ist starken Konzentrationstendenzen unterworfen

Unser Unternehmen bezieht direkt von Rohstofferzeugern Vorprodukte 


\subsection{Entwurf eines Mitarbeiterfragebogens}

1. In welcher Abteilung sind Sie tätig und seit wann?

O Einkauf 0 Kundendienst

O Marketing 0 Finanzen

O Produktion 0 Technik seit:

O Demontage 0 Vertrieb

○ Montage 0 Qualitätskontrolle

O Forschung und Entwicklung 0 Sonstiges

Welche Funktion üben Sie in Ihrer Abteilung aus?

○ Geschäftsleitung

○ Bereichsleitung

O Fachleiter-/Abteilungsleiterebene

O technische/r oder kaufmännische/r Angestellte/r ohne Personalverantwortung

O gewerbliche/r Arbeitnehmer/in

O Azubi

Nennen Sie bitte Aufgaben, mit denen Sie normalerweise beschäftigt sind? 
3. In welcher Weise hat die Umsetzung eines Rücknahme- und Recyclingsystems Ihre Aufgaben verändert bzw. welche Aufgaben haben Sie übernommen? Bitte geben Sie konkrete Beispiele an!

4. Wenn Sie an die Aufgaben denken, die sich durch das Rücknahme- und Recyclingsystem geändert haben bzw. neu hinzugekommen sind, wie zutreffend sind folgende Aussagen? Bitte stufen Sie Ihre Antworten zwischen "1=trifft sehr zu“ und „5=trifft gar nicht zu" ein!

Trifft sehr Trifft gar zu nicht zu

Ich werde auf Veränderungen gut vorbereitet

Neue/ Veränderte Aufgaben sind klar strukturiert

Ich habe genügend Freiheit, wie ich diese Aufgaben erledige

Beschlossene Änderungen werden zielstrebig durchgeführt

Neue Arbeitsabläufe sind gut organisiert

Es gibt hilfreiche unternehmensinterne Vorschriften

Ich arbeite häufig mit unseren Systempartnern (z.B. Verbände,

Verwerter) zusammen

Durch die Zusammenarbeit der Systempartner lernen alle Beteilig-

$$
\begin{aligned}
& \text { 1-----2-----3-----4-----5 } \\
& \text { 1-----2----3----4----5 } \\
& \text { 1-----2----3----4----5 } \\
& \text { 1----2-----3----4---5 }
\end{aligned}
$$

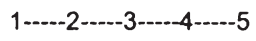

$$
\begin{aligned}
& \text { 1----2-----3----4----5 } \\
& \text { 1----2-----3---- } 4 \\
& \text { 1-----2-----3-----4-----5 } \\
& \text { 1-----2-----3-----4----5 }
\end{aligned}
$$

Kooperationen motivieren alle, die Rücknahme- und Recyclingsysteme schneller umzusetzen 
5. Aus welcher Motivation heraus beschäftigen Sie sich mit dem Rücknahme- und Recyclingsystem? Bitte stufen Sie Ihre Antworten zwischen „1=trifft sehr zu“ und „5=trifft gar nicht zu" ein!

Trifft sehr Trifft gar

Anweisung von oben

zu nicht zu

Neues Gebiet, was mich fachlich fordert

$1-----2-----3-----4-----5$

Neues Gebiet, das meine Arbeit bereichert

1----2-----3----4----5

1----2----3----4----5

Aussicht auf Beförderung

Aussicht auf Prämie

Aussicht auf persönliche Anerkennung

Umweltschutz interessierte mich schon immer

1-----2----3----4----5

1-----2----3----4----5

1----2-----3----4-----5

1----2----3-----4----5

„Müll“" wird ein Rohstoff der Zukunft sein und damit wird die Bedeu-

$1----2----3----4-----5$

tung der umweltbezogenen Arbeiten steigen

Durchführung von Recyclingkreisläufen ist auch für mich und meine

Familie wichtig

$1----2-\cdots--3-\cdots--\cdot-\cdot-5$

6. Wie wichtig sind Ihnen folgende Informationen über das Rücknahme- und Recyclingsystem? Sind Sie zufrieden mit den zur Verfügung gestellten Informationen in Ihrem Unternehmen? Bitte stufen Sie Ihre Antworten zwischen 1 und 5 ein!

$\begin{array}{llll}\text { sehr } & \text { gar nicht } & \text { sehr } & \text { gar nicht } \\ \text { wichtig } & \text { wichtig zufrieden } & \text { zufrieden }\end{array}$

Informationen darüber, welche Produkte in 1-----2-----3-----4-----5 1-----2-----3---------5 das Rücknahme- und Recyclingsystem einbezogen werden

Informationen darüber, wie recycelt wird (z.B. WiedereinsatzNerbrennung)

Informationen über ökologische Zielgrößen wie z.B. Rücklaufquoten

Informationen über ökonomische Zielgrößen (z.B. Kosten und Erlöse)

Informationen über beteiligte externe Partner (z.B. Verwertungsunternehmen, Verbände)

1----2-----3-----4----5

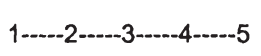

1----2----3----4----5

1-----2-----3----4-----5

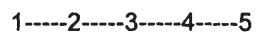

Informationen über Gesetze/ Verordnungen und gesetzliche Auflagen

Informationen über die stoffliche Zusammensetzung der Produkte
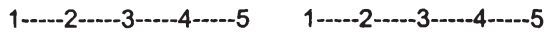

1----2-----3----4-----5

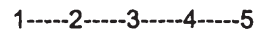


7. Wie werden Sie über ihr eigenes Rücknahme- und Recyclingsystem informiert? Bitte stufen Sie Ihre Antworten zwischen „1=trifft sehr zu“ und „5=trifft gar nicht zu“ ein!

$\begin{array}{ll}\text { Trifft sehr } & \text { Trifft gar } \\ \text { nut } & \text { nicht }\end{array}$

Gespräche mit Vorgesetzten

1-----2-----3-----4-----5

Gespräche mit Kollegen

$1----2----3----4-----5$

Interne Hausmitteilungen

1----2-----3----4----5

Interne Informationsveranstaltungen

1----2-----3----4----5

Interne Schulungen

1----2----3-.--4----5

Sonstiges

1-----2-----3-----4-----5

8. Wenn Sie Fragen zum Rücknahme- und Recyclingsystem haben, an wen wenden Sie sich in erster Linie (Abteilung und Hierarchieebene)?

1. Abteilung:

Ebene:

2. Abteilung:

Ebene:

3. Abteilung:

Ebene:

4. Abteilung:

Ebene:

9. Hatten Sie die Möglichkeit, Vorschläge zu machen, die das Rücknahme- und Recyclingsystem zu verbessern halfen?

$\square$ nein

$\square$ ja Wenn ja, über welchen Weg war das möglich (z.B. Vorschlagwesen, direkte Gespräche)?

Wenn ja, warum haben Sie Vorschläge gemacht? 
10. Welche Probleme sehen Sie bei der Umsetzung des Rücknahme- und Recyclingsystems? Bitte stufen Sie Ihre Antworten zwischen „1=sehr große" und „5=gar keine" ein!

Hohe Kosten des Aufbaus von Rücknahme- und Recyclingsyste-

Sehr große gar keine

men

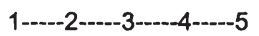

Mangelnde Gewinnaussichten

$1----2----3----4----5$

Fehlende Rücklaufmengen

$1----2----3----4----5$

Fehlendes Personal

$1----2-----3-----4-----5$

Fehlendes Fachwissen

$1----2----3----4----5$

Fehlende Abnehmer für die Recyclingstoffe/produkte

$1----2----3-----4-----5$

Sonstige Probleme:

$1-----2-----3-----4----5$

Sonstige Probleme:

$1----2----3----4----5$

11. Wie intensiv verfolgt ihr Unternehmen aus Ihrer Sicht die folgenden Ziele mit dem Rücknahme- und Recyclingsystem. Bitte stufen Sie Ihre Antworten zwischen „1=sehr intensiv" und „5=gar nicht" ein!

Gewinnerzielung

Erlangung von Imagevorteilen beim Kunden

Erlangung von Konkurrenzvorteilen

Vorbeugung gegenüber gesetzlichen Maßnahmen

Sammlung von Erfahrungen mit Altprodukten

Aufbau neuer Geschäftsfelder

Senkung der Deponiekosten für nicht verwertbare Abfälle

Wiedereinsatz von Gebrauchtteilen

Rückführung von Altprodukten zur Gewinnung von „neuen“ Ressourcen

Förderung einer brancheneinheitlichen Lösung, d.h. zwischen Wettbewerbern und ggf. Verwertern und/ oder Verbänden

Förderung einer EU-weiten Lösung

Vervollständigung der Umweltschutzstrategie des eigenen Unternehmens

Erfüllung von Forderungen, die an das Unternehmen von verschiedenen Gruppen (z.B. Greenpeace, Anwohner) gestellt werden
Sehr intensiv gar nicht

$$
\begin{aligned}
& \text { 1-----2-----3-----4----5 } \\
& \text { 1----2-----3----4-----5 } \\
& \text { 1----2----3-----4----5 } \\
& \text { 1----2-----3-----4----5 } \\
& \text { 1----2----3--------5 } \\
& \text { 1----2-----3-----4----5 } \\
& \text { 1-----2----3-----4----5 } \\
& \text { 1-----2-----3-----4----5 } \\
& \text { 1----2----3-----4----5 } \\
& \text { 1-----2-----3-----4-----5 } \\
& \text { 1-----2-----3-----4-----5 } \\
& \text { 1----2-----3-----4-----5 } \\
& \text { 1-----2-----3-----4-----5 }
\end{aligned}
$$


12. Wie schätzen Sie die Bedeutung des Rücknahme- und Recyclingsystems im Gesamtzusammenhang Ihres Unternehmens ein?

sehr große

$$
1
$$

$\square$

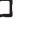

2

$\square$

3

4

gar keine Bedeutung

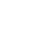

Wird die Bedeutung eher

steigen

$\square \quad$ sinken

$\square \quad$ gleich bleiben

13. Wenn Sie an die Zeit denken, bevor das Rücknahme- und Recyclingsystem in Ihrem Unternehmen existierte, hat Sie das Rücknahme- und Recyclingsystem für Umweltbelange sensibilisiert?

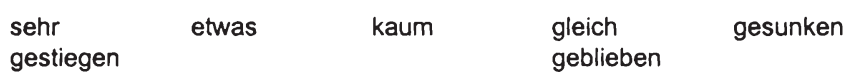

\section{Demographie}

Seit wie vielen Jahren arbeiten Sie für Ihre Firma?
o weniger als 2 Jahre
o 2-5Jahre
o 6-10 Jahre
o mehr als 10 Jahre

Haben Sie vorher schon in einer anderen Funktion/Abteilung gearbeitet? Wenn ja, in welcher und wie lange?

o nein

O ja Wenn ja, in welcher? (Jahre) (Jahre)

Angaben über Geschlecht und Alter?

O weiblich

o männlich (Jahre) 


\section{Literaturverzeichnis}

Adam, D.,

AgPU

(Arbeitsgemeinschaft PVC und Umwelt

Hrsg.),

Albach, $\mathrm{H}$.,

Andritzky, K.,

Angermeyer, H. Chr.,

Ansoff, H. I.,

Ansoff, H. I.,

Stewart, H. M.,

Arndt, H.-K.,

Günther, O.,

Backhaus, K.,

Backhaus, K.,

Backhaus, K., u.a.,

Bamberg, G.,

Coenenberg, A. G.,

Barbier, E. B.,
Planung und Entscheidung: Modelle - Ziele - Methoden, mit Fallstudien und Lösungen, 4., vollst. überarb. u. erw. Aufl., Wiesbaden 1996

Renaissance eines Klassikers, in: Zukunftsfähiges Wirtschaften, Wettlauf mit der Zeit, Reporter spezial, Heft 2, 1995, S. 10ff.

Umweltmanagement als Führungsaufgabe, in: ZFB, Heft 12, 1994, S. 1567-1579

Die Operationalisierbarkeit von Theorien zum Konsumentenverhalten, in: Schriften zum Marketing, Band 4, Berlin 1976

Coaching - eine spezielle Form der Beratung, in: zfo, Heft 2, 1997, S. 105-109

Implanting strategic Management, Englewood Cliffs u.a. 1984

Strategies for a Technology-based Business, in: Harvard Business Review, Nov.-Dec. 1967, S. 71-83

Betriebliche Umweltinformationssysteme: ein Überblick, in: UWF, Heft 1, 1996, S. 11-16

Investitionsgütermarketing, 5., enw. und überarb. Aufl., München 1997

Marketingschnittstellen, in: Specht, G. (Hrsg.), Marketingschnittstellen, Stuttgart 1989, S. 287-304

Multivariate Analysemethoden: Eine anwendungsorientierte Einführung, 8., verb. Aufl., Berlin, u.a.1996

Betriebswirtschaftliche Entscheidungslehre, 9. überarb. Aufl., München 1996

The Concept of Sustainable Development, in: Environmental Conservation, No. 2, 1987, S.101-110 
Bartlett, Chr. A., Ghoshal, S.,

Bean, A. S., u.a.,

Beyss, B.,

Birkigt, K.,

Stadler, M. M.,

Funck, H. J.,

Bergs, S.,

Bleymüller, J.,

Bonoma, T. V.,

Bonus, $H_{\text {., }}$

Bonus, $\mathrm{H}$.,

Bonus, $\mathrm{H}$.,
Die wahre Aufgabe des Topmanagement heute, in: Harvard Business Manager, Heft 2, 1995, S. 56-65

Structural and Behavioral Correlates of Implementation in U.S. Business Organisations, in: Schultz, R. L., Slevin, D. P. (Hrsg.), Implementing Operations Research/ Management Science, New York, London, Amsterdam 1975, S. 77-132

Akzeptanz neuer Personalbeurteilungssysteme, Mannheim 1983

Corporate Identity, 5. Aufl., Landberg/Lech 1992

Optimalität bei Cluster-Analysen: Experimente zur Bewertung numerischer Klassifikationsverfahren, Münster 1981

Multivaritate Analyse für Wirtschaftswissenschaftler, Manuskript, Münster 1989

Der Marketing-Vorsprung: wie man Marketingstrategien sofort erfolgreich in die Praxis umsetzt, Landsberg 1986

Institutionen und Institutionelle Ökonomik, Anwendungen für die Umweltpolitik, Volkswirtschaftliche Diskussionsbeiträge, Nr. 231, Münster 1996

Umweltschutz und Wettbewerb aus ökonomischer Sicht, in: 12. Trierer Kolloquium zum Umwelt- und Technikrecht, Umweltschutz und Wettbewerb, Trier 1996, S. 11-34

Bei der Umweltpolitik geht es letzt - endlich um die Freiheit, in: Handelsblatt, 27./28.9.1996, S. 2 
Brenk, A.,

Bronder, Chr., Pritzl, R.,

Bundesminister für Umwelt, Naturschutz und Reaktorsicherheit, Bonn, Stand vom 20. Februar 1996

Bundesminister für Bundesrat verabschiedet Kreislaufwirtschaftsgesetz, Umwelt, Naturschutz Pressemitteilung Nr. 49/94, Bonn 1994 und Reaktorsicherheit,

Bundesminister für Entwurf über die Entsorgung von Altautos, Bonn, Stand Umwelt, Naturschutz vom 27. Januar 1994 und Reaktorsicherheit,

Bundesminister für Umwelt, Naturschutz und Reaktorsicherheit,

Bundesminister für Gesetz zur Vermeidung von Rückständen, Verwertung von Sekundärrohstoffen und Entsorgung von Abfällen (Kreislaufwirtschafts- und Abfallgesetz - KrW-/ AbfG), Bonn im März 1993

Umwelt, Naturschutz

Entwurf der Verordnung über die Vermeidung, Verringerung und Verwertung von Abfällen gebrauchter elekund Reaktorsicherheit, trischer und elektronischer Geräte (Elektronik-SchrottVerordnung), Bonn, Stand vom 15. Oktober 1992

Bundesminister für Weg frei für das Inkraftreten der deutschnen Umwelt, Naturschutz Altautoverordnung, Pressemitteilung vom 20.1.98, S. 1und Reaktorsicherheit, 3

Burgdorf, P., 3
Computerwiederverwendung und -recycling: Erfahrun-
gen bei Siemens-Nixdorf, Vortrag 1995 3
Computerwiederverwendung und -recychir
gen bei Siemens-Nixdorf, Vortrag 1995

Byars, L. L.,

Moderne umweltpolitische Konzepte: Sustainable Development und ökologisch-soziale Marktwirtschaft, Diskussionspapier Nr. 3 des Instituts für Verkehrswissenschaft an der Universität Münster, Münster 1991

Strategische Allianzen zur Steigerung der Wettbewerbsfähigkeit, in: io Mangement Zeitschrift, Heft 5, 1991, S. 27-30

Entwurf der Verordnung über die Entsorgung von Geräten der Informationstechnik (IT-Geräte-Verordnung),

Strategic Management: Planning and Implementation, Conceps and Cases, Second Edition, New York u.a. 1987 
Ceyp, M.,

Chandler, A. D.,

Clausen, $\mathrm{H}$., Wacker, $\mathrm{H}$.,

Clausen, $\mathrm{H}$., Wacker, H.,

Clauß, G., Finze, F.-R., Partzsch, L., Clauss, M., Corsten, H., Götzelmann, F.,

Corsten, H., Götzelmann, F.,

Der Rat von Sachverständigen für Umweltfragen,
Ókologieorientierte Profilierung im vertikalen Marketing dargestellt am Beispiel der Elektrobranche, Frankfurt am Main 1996

Strategy and Structure, Cambridge, Mass. 1962

Rücknahmeverpflichtungen als intertemporäres Allokationsproblem, in: Jahrbuch für Wirtschaftswissenschaften, Bd. 48, Heft 1, 1998 (im Druck)

Ökonomische Modellierung von Rücknahmeverpflichtungen, in: : Herbold, R., u.a. (Hrsg.), Unterwegs in die Kreislaufwirtschaft, interdisziplinäre Beiträge, IWT-Papier 16, Institut für Wissenschafts- und Technikforschung, Universität Bielefeld, 1997, S. 33-45 (zugleich Volkswirtschaftlicher Diskussionsbeitrag Nr. 241, Westfälische WilhelmsUniversität Münster)

Statistik für Soziologen, Pädagogen und Mediziner, Band I: Grundlagen, Fankfurt am Main 1994

Die Strategie der Implementierung in der Unternehmung, Freiburg 1989

Abfallvermeidung und Reststoffverwertung - eine produkt- und verfahrensorientierte Analyse, in: Betriebswirtschaftliche Forschung und Praxis, 1992, Heft 2, S. 103-119

Das Kooperationsprinzip in umweltpolitischer und unternehmerischer Sicht, in: WISU, Heft 5, 1991, S. 377- 381

Umwelt-Gutachten 1996: Zur Umsetzung einer dauerhaft-umweltgerechten Entwicklung, Stuttgart 1996 
Der Rat von

Sachverständigen für

Umweltfragen,

Deutscher Bundestag, Zustimmungsbedürttige Verordnung über die Entsorgung von Altautos und die Anpassung straßenverkehrsrechtlicher Vorschriften, Drucksache 13/5998 vom 7.11 .96

Diehl, J. M.,

Dienstbach, H.,

Dienstbach, H.,

Donckels, R. von

Drobek, F. K.,

Dubois, A.,

Dutz, E.,

Femerling,Chr.,

Dyckhoff, H., Jacobs, R.,

Dyllick, T.,

Eckert, W.,

Umwelt-Gutachten 1994: Für eine dauerhaft-umweltgerechte Entwicklung, Stuttgart 1994

Varianzanalyse, Frankfurt am Main 1977

Dynamik der Unternehmensorganisation, Wiesbaden 1972

Die Anpassung der Unternehmungs-Organisation, München 1968

Profil und Determinanten der Netzbildung bei Kleinund Mittelunternehmen in Belgien, in: Zeitschrift für Klein- und Mittelunternehmen, Heft 1/4, 1993, S. 37-46

Public-Private-Partnership am Beispiel der Abfallverwertungsanlage Augsburg, in: Energiewirtschaftliche Tagesfragen, Heft 5, 1994, S. 273-275

Changing Activity Structures - A new View of Make-orBuy Decisions, in: Institut de Recherche de l'Entreprise (Hrsg.), Business Networks in an international Context: Recent Research Developments, Proceedings of the 8th IMP Conference, 1992, S.70-79

Prozeßmanagement in der Entsorgung: Ansätze und Verfahren, in: DBW, Heft 2, 1994, S. 221-245

Organisation des Umweltschutzes in Industriebetrieben, in: ZfB, 1994, Heft 6, S. 717-735

Management der Umweltbeziehungen, in: Die Unternehmung, 1988, Nr. 3, S. 190-205

Ökologieorientierte Strategische Beschaffungsbeziehungen, Wiesbaden 1996 
Engelhardt, W. H., Kleinaltenkamp, M., Reckenfelderbäumer, M.,

Evan, W. M., Olk, P.,

Fahrmeir, L., Hamerle, A.,

Fahrmeir, L., Hamerle, A.,

Falke, R.,

Fischer, M., u.a.,

Förster, F., u.a.,

Frank, G.,

Frese, E.,

Frese, E.,

Frese, E.,

Fritz, W.,
Leistungsbündel als Absatzobjekte, Ein Ansatz zur Überwindung der Dichotomie von Sach- und Dienstleistungen, in: ZfbF, Heft 5, 1993, S. 395-426

R\&D Consortia: A New U.S. Organisational Form, in: Sloan Mangement Review, Nr. 1, 1990, S. 37-46

Diskriminanzanalyse, in: Fahrmeir, L., Hamerle, A. (Hrsg.), Multivariate statistische Verfahren, Berlin, New York, de Gruyter 1984, S. 301-370

Varianz- und Kovarianzanaylse, in: Fahrmeir, L, Hamerle A. (Hrsg.), Multivariate statistische Verfahren, Berlin, New York, de Gruyter 1984, S. 155-210

Verantwortung für das Ganze tragen, in: Beschaffung aktuell, Heft 2, 1993, S. 33-34

Marketing und neuere ökonomische Theorie: Ansätze zu einer Systematisierung, in: BFuP, Heft 4, 1993, S. 444-470

Der LISREL-Ansatz der Kausalanalyse und seine Bedeutung für die Marketing-Forschung, in: ZfB, Heft 4, 1984, S. 346-367

Strategische Partnerschaften in mittelständischen Unternehmen, Wiesbaden 1994

Grundlagen der Organisation: Konzept - Prinzipien Strukturen, 6., überarb. Aufl., Wiesbaden 1995

Organisationstheorie: historische Entwicklung, Ansätze, Perspektiven, 2. überarb. und erw. Aufl., Wiesbaden 1992

Organisationstheorie: Stand und Aussagen aus betriebswirtschafticher Sicht, Wiesbaden 1990

Marketing-Management und Unternehmenserfolg: Grundlagen und Ergebnisse einer empirischen Untersuchung, 2., überarb. und erg. Aufl., Stuttgart 1995 
Galbraith, J. R., Kazanjian, R. K.,

Gebert, D.,

Gilbert, X., Strebel, P. J.,

Gils, M. R. van,

Greenley, G. E.,

Grimmeisen, M.,

Hagen, R.,

Hall, D. J.,

Saias, M. A.,

Hansen, U., Jeschke, K.,

Hansen, U., Raabe, T., Dombrowsky, B., Hauch, S.,

Hauschildt, J., Kirchmann, E.,
Strategy Implementation, Structure, Systems and Process, 2. Aufl., St. Paul u.a. 1986

Organisationsentwicklung, Probleme des geplanten organisatorischen Wandels, in: Bolte, K. u.a. (Hrsg.), Sozioökonomie, Bd. 6, Stuttgart 1974

Outpacing Strategies, in: IMEDE, Perspektives for Managers, Nr. 2, 1985

Interorganisationational relations and networks, in: Drenth, P. J. D., U.a. (Hrsg.), Handbook of work and organisational psychology, Chinchester u.a. 1984, S. 1073-1100

The Strategic and Operational Planning of Marketing, London u.a. 1986

Perspektiven eines Implementierungscontrolling, in: zfo, Heft 5, 1995, S. 290-296

Anreizsysteme zur Strategiedurchsetzung, Spardorf 1985

Strategy follows Structure!, in SMJ, Nr. 2, 1980, S. 149163

Nachkaufmarketing, in: Marketing ZFP, Heft 2, 1992, S. 88-97

Die Gestaltung des Konsumgüterrecycling als strategische Netzwerke, in: UWF, Heft 1, 1995, S. 62-69

Die Rolle des Handels bei der Durchsetzung neuer Konzepte, in: Kreislaufwirtschaft statt Abfallwirtschaft: Optimierte Nutzung und Einsparung von Ressourcen durch OKo-Leasing und Servicekonzepte, Hockerts, K., u.a., (Hrsg.), Ulm 1994, S. 177-180

Hauschildt, J., Kirchmann, E., Arbeitsteilung im Innovationsmanagement, Zur Existenz und Effizienz von Prozeßpromotoren, in: zfo, Heft 2, 1997, S. $68 \mathrm{ff}$ 
Heinen, E.,

Heinen, E.,

Herzberg, F.,

Mausner, B.,

Snydermann, B. B.,

Hesterley W. S.,

Liebeskind, J.,

Zenger, T. R.,

Hilker, J.,

Hockerts, K., u.a.

Hoffmann, F.,

Kreder, M.,

Horneber, M.,
Betriebswirtschaftliche Führungslehre, ein entscheidungsorientierter Ansatz, in: Heinen, E. (Hrsg.), Entscheidungsorientierte betriebswirtschaftliche Studien, Bd. 2, Wiesbaden 1978

Einführung in die Betriebswirtschaftslehre, 6. verb. und erw. Aufl., Wiesbaden 1977

The Motivation to work, New York, London, Sydney 1959

Organisationational economics: An impending revolution in organization theory?, in: Academy of Management Review, 1990, S. 402-420

Marketingimplementierung: Grundlagen und Umsetzung am Beispiel ostdeutscher Unternehmen, Wiesbaden 1993

Servicekonzepte als Element einer öko-effizienten Kreislaufwirtschaft, in: Kreislaufwirtschaft statt Abfallwirtschaft: optimierte Nutzung und Einsparung von Ressourcen durch Öko-Leasing und Servicekonzepte, in: Bayreuther Initiative für Wirtschaftsökologie e.V., Universität Bayreuth (Hrsg.), Schriftenreihe, Band 1, Ulm 1994, S. 3-14

Situationsabgestimmte Strukturform: Ein Erfolgspotential der Unternehmung, in: ZfbF, Nr.6, 1985, S. 455485

Innovatives Entsorgungsmanagement: Methoden und Instrumente zur Vermeidung und Bewältigung von Umweltbelastungsproblemen, in: Pfeiffer, W. (Hrsg.), Innovative Unternehmensführung: Planung, Durchführung und Kontrolle von Innovationen, Band 24, Göttingen 1995

Implementing Strategy, New York 1984

Hrebiniak, L. G.

Joyce, W. F., 
Hungenberg, $H_{\text {., }}$

Jaeger, K.,

Jarillo, J. C.,

Kaas, K. P.,

Kaas, K. P.,

Kaufmann, $H_{\text {., }}$

Pape, $H_{\text {., }}$

Kempf, T.,

Kern, W.,

Kieser, A. (Hrsg.)

Kieser, A.,

Kieser, A.,

Kubicek, $H_{\text {., }}$

Kieser, A.,

Kubicek, $H_{\text {., }}$
Zentralisation und Dezentralisation: strategische Entscheidungsverteilung in Konzernen, Wiesbaden 1995

Eine ökonomische Theorie des Recycling, in: Kyklos, Heft 29, 1975, S. 660-667

On strategic networks, in: Strategic Management Journal, Heft1, 1988, S. 31-41

Marketing und Neue Institutionenökonomik, in: Kaas, K. P. (Hrsg.), Kontrakte, Geschäftsbeziehungen, Netzwerke: Marketing und neue Institutionenökonomik, Frankfurt am Main 1995, S. 1-17

Marketing zwischen Markt und Hierarchie, in: Kaas, K. P. (Hrsg.), Kontrakte, Geschäftsbeziehungen, Netzwerke: Marketing und neue Institutionenökonomik, Frankfurt am Main 1995, S. 19-42

Clusteranalyse, in: Fahrmeir, L., Hamerle, A. (Hrsg.), Multivariate statistische Verfahren, Berlin, New York, de Gruyter 1984 , S. $371-472$

Unterstützung und Widerstand, Macht und Konflikt bei Organisationsänderungen, Berlin 1984

Die Zeit als Dimension betriebswirtschaftlichen Denkens und Handelns, in: DBW, Heft 1, 1992, S. 41-58

Organisationstheorien, 2. überarb. Aufl., Stuttgart/ Berlin/ Köln 1995

Loyalität und Commitment, in: Kieser, A., Reber, G., Wunderer, R. (Hrsg.) Handwörterbuch der Führung, 2. Aufl., 1995, S. 1442-1455

Organisation, 3. Aufl., Berlin, New York 1992

Organisationstheorien I, Wissenschaftstheoretische Anforderungen und kritische Analyse klassischer Ansätze, in: Bolte, K. M., u.a. (Hrsg.), Sozioökonomie, Band 514/l, Stuttgart u.a. 1978 
Kirchgeorg, M.,

Kirchgeorg, M.,

Kirchgeorg, M.,

Kirchgeorg, M.,

Kirchgeorg, M.,

Kirchgeorg, M.,

Giesen-Netzer, I.,

Kirchgeorg, M.,

Giesen-Netzer, I.,

Kirsch, W.,

Esser, W. M.,

Gabele, E.,
Kreislaufwirtschaft - Neue Herausforderungen für das Marketing, in: Marketing ZFP, Heft 4, 1995, S. 232-248

Unternehmensstrategische Gestaltungsprobleme von Stoffkreisläufen - eine theoriegeleitete empirische Analyse von kreislaufspezifischen Strategien und Strukturen, im Druck 1997

Ökologieorientiertes Unternehmensverhalten: Typologien und Erklärungsansätze auf empirischer Grund-

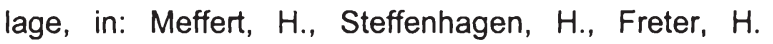
(Hrsg.), Schriftenreihe Unternehmensführung und Marketing, Band 24, Wiesbaden 1990

Kreislaufwirtschaft - neue Herausforderungen an das Marketing, Meffert, H., Wagner, H., Backhaus, K. (Hrsg.), Arbeitspapier Nr. 92 der Wissenschaftlichen Gesellschaft für Marketing und Unternehmensführung e.V., Münster 1995

Ziele, Strategien und Organisationskonzepte von Recycling- und Rücknahmesystemen für langlebige Gebrauchsgüter, in: Meffert, H., Wagner, H., Backhaus, K. (Hrsg.), Marktorientierte Unternehmensführung in der Kreislaufwirtschaft - Kostenmanagement oder Wettbewerbsprofilierung, Dokumentationspapier Nr. 106 der Wissenschaftlichen Gesellschaft für Marketing und Unternehmensführung e.V., Münster 1996, S. 1125

WSK-Infobrief, Institut für Marketing, Projektgruppe Wertschöpfungskreislaufe (Hrsg.), Ausgabe 2, 1995

WSK-Infobrief, Institut für Marketing, Projektgruppe Wertschöpfungskreislaufe (Hrsg.), Ausgabe 4, 1996

Das Management des geplanten Wandels von Organisationen, Stuttgart 1974 
Klein, $\mathbf{K}$.

Kleinaltenkamp, M.,

Knoblich, $H_{\text {., }}$

Knopf, R. H.,

Knopf, R. H., u.a.,

Kolks, U.,

Kolks, U.,

Koog, W.,

Kopp, M.,

Kotabe, M.,

Swan, K. S.,
Die Konsultation externer Berater in Innovationsprozesse: Ein Beitrag zur empirischen Theorie der Unternehmung, München 1972

Recycling - Strategien, Berlin 1985

Die typologische Methode in der Betriebswirtschaftslehre, in: WiSt, Heft 4, 1972, S. 141-147

Dimensionen des Erfolgs von Reorganisationsprozessen, Mannheim 1976

Die Effizienz von Reorganisationsprozessen aus der Sicht der Praxis, München 1976

Strategieimplementierung: ein anwenderorientiertes Konzept, Wiesbaden 1990

Praktische Probleme und organisatorische Lösungsansätze der Strategieimplementierung - Dokumentation einer explorativen Studie, Arbeitspapier Nr.1 des Lehrstuhls BWL II der Justus-Liebig-Universität Gießen, Gießen 1988

Umsetzung der Kreislaufwirtschaft aus der Sicht der Rethmann AG \& Co, in: Meffert, H., Wagner, H., Backhaus, K. (Hrsg.), Marktorientierte Unternehmensführung in der Kreislaufwirtschaft - Kostenmanagement oder Wettbewerbsprofilierung, Dokumentationspapier Nr. 106 der Wissenschaftlichen Gesellschaft für Marketing und Unternehmensführung e.V., Münster 1996, S. $26-30$

Hypothesenformulierung in der Absatzforschung, in: Betriebswirtschaftliche Schriften, Heft 60, Berlin 1972 The role of strategic alliances in high-technology new product development, in: Strategic Management Journal, Nr. 16, 1995, S. 631. 
Kotler, P.,
Bliemel, F.,

Kotler, P.,

Krcal, H.-Chr.

Kreikebaum, H.,

Kreikebaum, $\mathrm{H}$., Türk, R.,

Kricsfalussy, A.,

Laakmann, K., Müller, N.,

Levitt, T.,

Lehner, J. M.,

Linn, N.,
Marketing-Management, Analyse, Planung, Umsetzung und Steuerung, 8. vollst. neu bearb. und erw. Aufl., Stuttgart 1995

Marketing-Management - Analysis, Planning, Implementation and Control, 7. Aufl., Englewood Cliffs 1991

Wirkungsbeziehungen produktbezogener Umweltschutzmaßnahmen als Beweggrund zwischenbetrieblicher Zusammenarbeit, in: UWF, Heft 4, 1995, S. 22-32

Strategische Unternehmensplanung, 5. Aufl., Stuttgart, Berlin, Köln 1993

Ein Ansatz zur Bewertung der ökologischen Wirkungen von Produkten, in: ZfB-Ergänzungsheft 2, 1993, S. 119138

Kundenorientierung = Mitarbeiterorientierung?!, in: zfo, Heft 2, 1997, S. 99-103

Umfrageergebnisse zu Zielen, Strategien und Maßnahmen deutscher Unternehmen in der Rezession, in: Meffert, H., Wagner, H., Backhaus, K. (Hrsg.), Marketing in der Rezession, Arbeitspapier Nr. 82 der Wissenschaftlichen Gesellschaft für Marketing und Unternehmensführung e.V., Dokumentation des Workshops vom 17. Juni 1993, Münster 1993, S. 37-48

Marketing Imagination, Die unbegrenzte Macht des Marketing, Landberg/Lech 1984

Implementierung von Strategien: Konzeption unter Berücksichtigung von Unsicherheit und Mehrdeutigkeit, Wiesbaden 1996

Die Implementierung vertikaler Kooperationen: theoretische Konzeption und erste empirische Ergebnisse zum Prozeß der Ausgliederung logistischer Teilaufgaben, Frankfurt am Main u.a. 1989 
Matschke, M.,

Lemser, B.,

Mattsson, L. G.,

Meffert, $H_{\text {., }}$

Meffert, H.,

Meffert, $H_{\text {., }}$

Meffert, H.,

Meffert, H.,

Meffert, H.,
Entsorgung als betriebliche Grundfunktion, in: Betriebswirtschaftliche Forschung und Praxis, Heft 2, 1992, S. 85-101

Management of Strategic Change in a "Markets-asNetworks" Perspektive, in: Pettigrew, A. M. (Hrsg.), The Management of Strategic Change, Oxford 1987, S. 234-260

Marketing:

Grundlagen

marktorientierter Unternehmensführung:

Konzepte-InstrumentePraxisbeispiele; mit neuer Fallstudie VW-Golf, 8., vollst. neubearb. und erw. Aufl., Wiesbaden 1998

Marketing-Management: Analyse, Strategie, Implementierung, Wiesbaden 1994

Erfolgreiches Marketing in der Rezession: Strategien und Maßnahmen in engeren Märkten; mit Erkenntnissen aus einer umfassenden Untersuchung, Wien 1994

Marketingforschung und Käuferverhalten, 2. vollst. überarb. und erw. Aufl., Wiesbaden 1992

Ökologieorientierte Marketing- und Werbestrategie der Marke Opel, Hamburg 1990

Stand und Perspektiven des Umweltmanagement in der betriebswirtschaftlichen Forschung und Lehre, in: Weber, J. (Hrsg.), Umweltmanagement, Aspekte einer umweltbezogenen Unternehmensführung, Stuttgart 1997, S. 5-28 
Meffert, H.,

Meffert, H.,

Meffert, H., Bruhn, M.,

Meffert, $H$., Bruhn, M.,

Meffert, $H$., Hafner, K., Poggenpohl, M.,

Meffert, $H_{\text {., }}$ Kirchgeorg, M., Meffert, $H$., Kirchgeorg, M.,
Sustainable Development: Thesen zur betriebswirt-

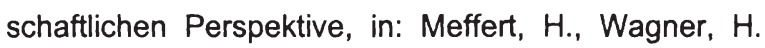
(Hrsg.), Sustainable Development als Leitbild der umweltbewußten Unternehmensführung, Dokumentation einer Vortragsveranstaltung am 22. Juni 1992 an der Westfälischen Wilhelms-Universität zu Münster, in: Schriftenreihe der Wissenschaftlichen Gesellschaft für Marketing und Unternehmensführung, Münster 1992, S. $23-49$

Anforderungen an die Strategie-Implementierung, in: Meffert, H. (Hrsg.), Strategische Unternehmemensführung und Marketing, Wiesbaden 1988

Dienstleistungsmarketing: Grundlagen, Konzepte, Methoden; mit Fallbeispielen, Wiesbaden 1996

Das Umweltbewußtsein von Konsumenten - Ergebnisse einer empirischen Untersuchung in Deutschland im Längsschnittvergleich, in: Meffert, $H$., Wagner, $H$., Backhaus, K. (Hrsg.), Arbeitspapier Nr. 99 der Wissenschaftlichen Gesellschaft für Marketing und Unternehmensführung, Münster 1996

Unternehmenskultur und Unternehmensführung Ergebnisse einer empirischen Untersuchung, in: Meffert, H., Wagner, H. (Hrsg.), Marktorientierte Führung in Stagnation und Rezession, Arbeitspapier Nr. 43 der Wissenschaftlichen Gesellschaft für Marketing und Unternehmensführung e.V., Münster 1988

Ökologisches Marketing, Erfolgsvoraussetzungen und Gestaltungsoptionen, in: UWF, Heft 1, 1995, S. 18-27

Marktorientiertes Umweltmanagement: Grundlagen und Fallstudien, 3. überarb. und erw. Aufl., Stuttgart 1997 
Meffert, H., Kirchgeorg, M.,

Meffert, $H$., Kirchgeorg, M., Giesen-Netzer, I., Meffert, $H$., Netzer, F.,

Meffert, $H_{\text {., }}$ Wagner, $H_{\text {., }}$ Backhaus, K. (Hrsg.),

Meurer, J.,

Meyer, M.,

Miles, R. E., Snow, Ch. C., Miller, D., Friesen, P. H.,
Ziele und Strategien zur Gestaltung von produktbezogenen Rücknahme- und Recyclingsystemen - ausgewählte Forschungsergebnisse einer Hersteller- und

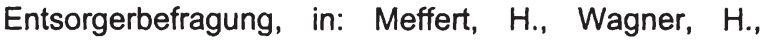
Backhaus, K. (Hrsg.), Arbeitspapier Nr. 107 der Wissenschaftlichen Gesellschaft für Marketing und Unternehmensführung, Münster 1997

Die meisten mittelständischen Betriebe haben sich noch nicht auf die Veränderungen vorbereitet, in: Blick durch die Wirtschaft, 17.7.1996, S. 8

Formen strategischer Netzwerke und Implikationen für das Marketing, in: Meffert, H., Wagner, H., Backhaus, K. (Hrsg.), Arbeitspapier Nr. 109 der Wissenschaftlichen Gesellschaft für Marketing und Unternehmensführung e.V., Münster 1997

Marktorientierte Unternehmensführung in der Kreislaufwirtschaft - Kostenmanagement oder Wettbewerbsprofilierung, Dokumentationspapier Nr. 106 der Wissenschaftlichen Gesellschaft für Marketing und Unternehmensführung e.V., Münster 1996

Führung von Franchisesystemen: Erklärungsansätze, Verhaltens- und Erfolgswirkungen auf Grundlage einer empirischen Führungstypologie, Wiesbaden 1997

Ökonomische Organisation der Industrie: Netzwerkarrangements zwischen Markt und Unternehmung, Wiesbaden 1995

Organisational Strategy, Strucure and Process, New York 1978

Momentum and Revolution in Organisational Adaptation, in: Academy of Management Journal, Nr. 4, 1980 , S. $591 \mathrm{ff}$ 
Minsel, W. W, Bente, G.,

Mintzberg, $H_{\text {., }}$

Mintzberg, H.,

Mintzberg, $H_{\text {., }}$ McHugh, A.,

Monhemius, K. Ch.,

Müller, H.,

Müller, N.,

Narver, J. C., Slater, S. F.,

o.V.,

o.V.,

o. V.,
Pädagogik und Verhaltensmodifikation als Strategie zur Veränderung des Umweltbewußtseins, in: Fietkau, H.J., Umweltlernen: Veränderungsmöglichkeiten des Umweltbewußtseins; Modelle - Erfahrungen, Königstein im Taunus 1981, S. 149-186

Strategie als Handwerk, in: Strategie: Die brillanten Beiträge der weltbesten Experten, Montgomery, C., Porter, M. E. (Hrsg.), Wien 1996, S. 459-476

Patterns in strategy Formation, in: Management Science, Nr. 9, 1978, S. 934-948

Strategy Formation in Adhocracy, in: Administrative Science Quarterly, Juni 1985, S. 160- 197

Umweltbewußtes Kaufverhalten von Konsumenten: ein Beitrag zur Operationalisierung, Erklärung und Typologie des Verhaltens in Kaufsituationen, Frankfurt am Main u.a. 1992

Industrielle Abfallbewältigung, Entscheidungsprobleme aus theoretischer Sicht, Wiesbaden 1991

Marketingstrategien in High-Tech-Märkten: Typologisierung, Ausgestaltungsformen und Einflußfaktoren auf der Grundlage strategischer Gruppen, Frankfurt am Main u.a. 1995

Becoming more Market Oriented: An Exploratory Study of the Programmatic and Market-Back Approaches, Report Nr. 91-128 des Marketing Science Institute, Cambridge, Mass. 1991

Altautoverordnung sorgt für neuen Wirbel, in: Die Welt, 7.3.1998, Online-Archiv 0307wi16.html

Computerrecycling, Edelschrott, in: Stern, Heft 25, 1995, S. 40-46

Gute, teure Technik ist schwer verkäuflich, in: Handelsblatt, 14.6.1995, S. B1 
o.V.,

o.V.,

o. V.,

o. V.,

Ost, F.,

Padberg, A.,

Pearce, D.,

Turner, R. K.,

Pfeifer, A.,

Schmidt, P.,

Picot, A.,

Pfohl, H.-Chr.,

Kunz, T.,

Linn, N.,

Porter, M. E.,

Porter, M. E.,
Italiens Regierung subventioniert den Autokauf, in: Frankfurter Allgemeine Zeitung, 2.1.1997, S. 9

Neue Wege bei der Demontage von Monitoren durch flexible Recyclingprozesse, in: Handelsblatt, 10.3.1995, S. 7

Opel zahlt Abwrackprämie, in: Handelsblatt, 21.3.1995, S. 23

Schnittstellenfrei, in: Ernährungswirtschaft, Heft 2, 1995, S. 17-23

Faktorenanalyse, in: Fahrmeir, L., Hamerle, A. (Hrsg.), Multivariate statistische Verfahren, Berlin, New York, de Gruyter 1984, S. 575-662

Partnerschaften entlang der Wertschöpfungskette, Formelle und informelle Netze von Einzelunternehmen: Eigene Schwächen durch Zusammenarbeit mit anderen kompensieren, in: Blick durch die Wirtschaft, 11.3.1996, S. 11

Economics of Natural Resources and the Environment, New York/ Harvester Wheatsheaf 1990

Lisrel: Die Analyse komplexer Strukturgleichungsmodelle, Stuttgart, New York 1987

Transaktionskostenansatz in der Organisationstheorie: Stand der Diskussion und Aussagewert, in: DBW, Heft 42,1982, S. $267-284$

Implementierung, Forschungsansätze und Ergebnisse, in: Veröffentlichungen des Fachgebiets Unternehmensführung, Nr. 5, Technische Hochschule Darmstadt 1988 The Competitive Advantage of Nations, London, Basingstoke 1990

Competitive Advantage, Creating and Sustaining Superior Performance, New York, London 1985 
Porter, M .E.,

Pues, C.,

Raabe, T.,

Rasche, Chr.,

Rautenstrauch, C.,

Reiß, M.,

Remmerbach, K. U., Markteintrittsentscheidungen: eine Untersuchung im

Richardson, G. B., The Organisation of Industry, in: Economic Journal, No.

Ruchay, D., Rahmen der strategischen Marketingplanung unter besonderer Berücksichtigung des Zeitaspektes, Wiesbaden 1988 2, 1972, S. 883-896

Competitive Strategy, Techniques für Analyzing Industry and Competitors, New York 1980

Marketing in der Entsorgungswirtschaft, in: IfM-News, Meffert, H. (Hrsg.), Heft 2, 1995, S. 13-17

Die Elektronik-Schrott-Verordnung: Perspektiven einer aktiven, herstellerseitigen Redistributionspolitik, in: Jahrbuch der Absatz- und Verbrauchsforschung, 1993, S. 283-309

Wettbewerbsvorteile durch Kernkompetenzen: ein ressourcenorientierter Ansatz, Wiesbaden 1994

Betriebliches Recycling, eine Literaturanalyse, in: ZfBErgänzungsheft 2, 1993, S. 87-104

Implementierung, in: Corsten, H., Reiß, M. (Hrsg.), Handbuch Unternehmensführung: Konzepte - Instrumente - Schnittstellen, Wiesbaden 1995, S. 291-301

Kreislaufwirtschaft und Rücknahmeverordnungen Umweltpolitische Ziele und Status der Umsetzung, in: Marktorientierte Unternehmensführung in der Kreislaufwirtschaft - Kostenmanagement oder Wettbewerbsprofilierung?, in: Meffert, H., Wagner, H. Backhaus, K. (Hrsg.), Marktorientierte Unternehmensführung in der Kreislaufwirtschaft - Kostenmanagement oder Wettbewerbsprofilierung?, Dokumentationspapier Nr. 106 der Wissenschaftlichen Gesellschaft für Marketing und Unternehmensführung e.V., Münster 1996, S. 5-10 
Sattelberger, T.,

Schanz, G.,

Schanz, G.,

Schierenbeck, H.,

Schein, E. H.,

Schlesinger, L. A., Heskett, J. L.,

Schnaars, St. P.,

Schneidewind, U.,

Scholz, Chr.,

Schreiner, M.,

Schreyögg, G.,

Schubert, W., Küting, K.,
Die lernende Organisation im Spannungsfeld von Strategie, Struktur und Kultur, in: Die lernende Organisation: Konzepte für eine neue Qualität der Unternehmensentwicklung, Sattelberger, T. (Hrsg.), 3. Aufl., Wiesbaden 1996

Methodologie für Betriebswirte, 2., überarb. und erw. Aufl., Stuttgart 1988

Zwei Arten von Empirismus, in: ZfbF, 1975, S. 307-331

Grundzüge der Betriebswirtschaftslehre, 11. völlig überarb. Aufl., München, Wien, Oldenburg 1993

Organisational Culture and Leadership, San Francisco, Washington, London 1985

Dem Kunden dienen - das müssen viele Dienstleister erst noch lernen, in: Harvard Manager, Heft 2, 1992, S. 106-116

When Entering Growth Markets, Are Pioneers better than Poachers?, in: Business Horizons, March-April 1986, S. $27-36$

Ökologisch orientierte Kooperationen aus betriebswirtschaftlicher Sicht, in UWF, Heft 4, 1995, S. 16-21

Strategisches Management: ein integrativer Ansatz, Berlin/ New York 1987

Umweltmanagement in 22 Lektionen: ein ökonomischer Weg in eine ökologische Wirtschaft, 2. überarb. Aufl., Wiesbaden 1991

Unternehmensstrategie, Grundfragen einer Theorie strategischer Unternehmensführung, Berlin, New York 1984

Unternehmenszusammenschlüsse, München 1981 
Schul, P.L.,

Pride, W. M.,

Little Jr., T. E.,

Schulte-Zurhausen, Organisation, München 1995

M.,

Schwarz, E. J.,

Short, J. E.,

Venkatraman, N.,

Someren, van T. C. R. Einführung von Umweltmanagementsystemen: das

Staehle, W. H.,

Stahel, W.,

Stahlmann, V.,

Steinle, C.,

Steven, M.,

Stölzle, W., Wachstumsmodell, in: UWF, Heft 6, 1994, S. 58-64

The Impact of Channel Leadership on Intrachannel Conflict, in: Journal of Marketing, 1983, Nr. 3, S. 21-34

Unternehmensnetzwerke im Recycling-Bereich, Wiesbaden 1994

Beyond Business Redesign: Redefining Baxter's Business Network, in: Sloan Management Review, Nr. 4, 1992, S. 7-21

Management: eine verhaltenswissenschaftliche Perspektive, 7. Aufl., München 1994

Langlebigkeit von Gütern, Materialrecycling, Ressourceneffizienz, in: Hockerts, K., u.a. (Hrsg.), Kreislaufwirtschaft statt Abfallwirtschaft: optimierte Nutzung und Einsparung von Ressourcen durch ÖkoLeasing und Servicekonzepte, Ulm 1994, S. 93-102

Umweltorientierte Materialwirtschaft: das Optimierungskonzept für Ressourcen, Recycling und Rendite, Wiesbaden 1988

Führungskonzepte und ihre Implementation, in: Kieser, A., u.a. (Hrsg.), Handwörterbuch der Führung, Stuttgart 1987, S. $576-590$

Effizienz von betrieblichen Entsorgungsprozessen, in: Betriebswirtschaftliche Forschung und Praxis, Heft 2, 1992, S. 120-135

Ansätze zur Erfassung der Umweltschutzkosten in der betriebswirtschaftlichen Kostenrechnung, in: Zeitschrift für Umweltpolitik und Umweltrecht, Heft 4, 1990, S. 379-412 
Stölzle, W., Jung, K. P.,

Ströbele, W.,

Sydow, J.,

Sydow, J.,

Sydow, J.,

Szeliga, M.,

Tacke, W.,

Voegele, A.,

Wächter, F.,
Strategische Optionen der Entsorgungslogistik zur Realisierung von Kreislaufwirtschaftskonzepten, in: UWF, Heft 1, 1994, S. 31-36

Die Nutzung regenerierbarer natürlicher Ressourcen, in: WISU, Heft 3, 1990, S. 183-190

Strategische Netzwerke: Evolution und Organisation, Wiesbaden 1992

Unternehmungsnetzwerke, in: Corsten, H., Reiß, M. (Hrsg.), Handbuch Unternehmensführung: Konzepte Instrumente - Schnittstellen, Wiesbaden 1995, S. 159169

Netzwerkorganisation, Interne und externe Restrukturierung von Unternehmungen, in: WiSt, Heft 12, 1995, S. $629-634$

Push und Pull in der Markenpolitik: ein Beitrag zur modellgestützten Marketingplanung am Beispiel des Reifenmarktes, Frankfurt am Main u.a. 1995

Stagnation und Rezession aus der Sicht von Unternehmen, Auswirkungen, Entwicklungen, Probleme und Problemlösungen, in: Meffert, H., Wagner, H. (Hrsg.), Marktorientierte Führung in Stagnation und Rezession, Arbeitspapier Nr. 10 der Wissenschaftlichen Gesellschaft für Marketing und Unternehmensführung e.V., Dokumentation des 5 . Münsteraner Führungsgesprächs, Münster 1983, S. 33-49

Entsorgungslogistik: Von der logistischen Kette zum logistischen Kreis, in: Beschaffung aktuell, Heft 11, 1993, S. 39-40

Durchsetzbarkeit im Handel und logistische Probleme der Redistribution, in: Hockerts, K., u.a. (Hrsg.), Kreislaufwirtschaft statt Abfallwirtschaft: optimierte Nutzung und Einsparung von Ressourcen durch ÖkoLeasing und Servicekonzepte, Ulm 1994, S. 193-196 
Wagner, $H$.,

Städler, A.,

Weiland, R.,

Weltkommission für Umwelt und Entwicklung

Wicke, L.,

Wildemann, $H_{\text {., }}$

Williamson, O. E.,

Winter, G.,

Witte, E.,

Wöhe, G.,

Wottawa, H., Gluminski, I.,

Zeithaml, V. A.,
Führung, Grundlagen, Prozesse und Konzeptionen der Mitarbeiterführung in Unternehmungen, 2., überarb. Aufl., Münster 1989

Rücknahme- und Entsorgungspflichten in der Abfallwirtschaft, eine institutionenökonomische Analyse der Automobilbranche, Wiesbaden 1995

Unsere gemeinsame Zukunft, der Brundtland-Bericht der Weltkommission für Umwelt und Entwicklung, Hauff, V. (Hrsg.), Greven 1987

Umweltökonomie: eine praxisorientierte Einführung, 2. Erw. u. aktualisierte Aufl., München 1989

Wiedmann, K. W. u.a., Die Überprüfung unternehmerischer Zielsysteme als Voraussetzung eines effizienten Öko-Marketing, in: Ökologisches Marketing, Brand, A., u.a., (Hrsg.), Frankfurt am Main, New York 1988

Kooperationen über die Wertschöpfungskette, in: Corsten, H., Reiß, M. (Hrsg.), Handbuch Unternehmensführung: Konzepte - Instrumente - Schnittstellen, Wiesbaden 1995, S. 743-751

Markets and hierarchies: Analysis and antitrust implication. Free Press, New York, London 1975

Das umweltbewußte Unternehmen, München 1987

Organisation für Innovationsentscheidungen, Göttingen 1973

Einführung in die Allgemeine Betriebswirtschaftslehre, 17. überarb. und erw. Aufl., München 1990

Psychologische Theorien für Unternehmen, in: Schuler, H. (Hrsg.), Schriftenreihe Wirtschaftspsychologie, Göttingen 1995

How Cusumer Evaluation Processes differ between Goods and Services, in: Donnelly, J. H., George, W. R., (Hrsg.), Marketing of Services, Chicago 1981, S. 186ff. 
Zentralverband

Elektrotechnik- und

Elektroindustrie e.V.

(Hrsg.)

Zeyer, U.,

Ziems, D., Koschay, V.,

Zikmund, W. G., Stanton, W. J.,

Zimbardo, P. G.

Zimmermann, H.,
Lösungskonzept der deutschen Elektroindustrie für die Verwertung und Entsorgung elektrotechnischer und elektronischer Geräte, ZVEI-Memorandum zum Entwurf einer "Elektronik-Schrott-Verordnung", Frankfurt am Main 1993

Zeitaspekte der Implementierung aktueller Managementkonzepte, in: Zeitschrift für Organisation, Heft 5, 1995, S. 283-289

Innerbetriebliche Entsorgungslogistik, in: UWF, Heft 1, 1996, S. 37-42

Recycling Solid Wastes: A Channels-of-distribution Problem, in: Journal of Marketing, Nr. 3, 1971, S. 34-39

Psychologie, 4. neubearb. Aufl., Berlin u.a. 1983

Automobilrecycling im Verbund, PREUSSAG-Konzept eines flächendeckenden Netzes von Kfz-Demontagezentren, in: UWF, Heft 4, 1995, S. 44-47 


\section{SCHRIFTEN ZUM MARKETING}

Band 1 Friedrich Wehrle: Strategische Marketingplanung in Warenhäusern. Anwendung der Portfolio-Methode. 1981. 2. Auflage. 1984.

Band 2 Jürgen Althans: Die Übertragbarkeit von Werbekonzeptionen auf internationale Märkte. Analyse und Exploration auf der Grundlage einer Befragung bei europaweit tätigen Werbeagenturen. 1982

Band 3 Günter Kimmeskamp: Die Rollenbeurteilung von Handelsvertretungen. Eine empirische Untersuchung zur Einschätzung des Dienstleistungsangebotes durch Industrie und Handel. 1982.

Band 4 Manfred Bruhn: Konsumentenzufriedenheit und Beschwerden. Erklärungsansätze und Ergebnisse einer empirischen Untersuchung in ausgewählten Konsumbereichen. 1982.

Band 5 Heribert Meffert (Hrsg.): Kundendienst-Management. Entwicklungsstand und Entscheidungsprobleme der Kundendienstpolitik. 1982.

Band 6 Ralf Becker: Die Beurteilung von Handelsvertretern und Reisenden durch Hersteller und Kunden. Eine empirische Untersuchung zum Vergleich der Funktionen und Leistungen. 1982.

Band 7 Gerd Schnetkamp: Einstellungen und Involvement als Bestimmungsfaktoren des sozialen Verhaltens. Eine empirische Analyse am Beispiel der Organspendebereitschaft in der Bundesrepublik Deutschland. 1982.

Band 8 Stephan Bentz: Kennzahlensysteme zur Erfolgskontrolle des Verkaufs und der MarketingLogistik. Entwicklung und Anwendung in der Konsumgüterindustrie. 1983.

Band 9 Jan Honsel: Das Kaufverhalten im Antiquitätenmarkt. Eine empirische Analyse der Kaufmotive, ihrer Bestimmungsfaktoren und Verhaltenswirkungen. 1984.

\section{SCHRIFTEN ZU MARKETING UND MANAGEMENT}

Band 10 Matthias Krups: Marketing innovativer Dienstleistungen am Beispiel elektronischer Wirtschaftsinformationsdienste. 1985.

Band 11 Bernd Faehsler: Emotionale Grundhaltungen als Einflußfaktoren des Käuferverhaltens. Eine empirische Analyse der Beziehungen zwischen emotionalen Grundhaltungen und ausgewählten Konsumstrukturen. 1986.

Band 12 Ernst-Otto Thiesing: Strategische Marketingplanung in filialisierten Universalbanken. Integrierte Filial- und Kundengruppenstrategien auf der Grundlage erfolgsbeeinflussender Schlüsselfaktoren. 1986.

Band 13 Rainer Landwehr: Standardisierung der internationalen Werbeplanung. Eine Untersuchung der Prozeßstandardisierung am Beispiel der Werbebudgetierung im Automobilmarkt. 1988.

Band 14 Paul-Josef Patt: Strategische Erfolgsfaktoren im Einzelhandel. Eine empirische Analyse am Beispiel des Bekleidungsfachhandels. 1988. 2. Auflage. 1990.

Band 15 Elisabeth Tolle: Der Einfluß ablenkender Tätigkeiten auf die Werbewirkung. Bestimmungsfaktoren der Art und Höhe von Ablenkungseffekten bei Rundfunkspots. 1988.

Band 16 Hanns Ostmeier: Ökologieorientierte Produktinnovationen. Eine empirische Analyse unter besonderer Berücksichtigung ihrer Erfolgseinschätzung. 1990.

Band 17 Bernd Büker: Qualitätsbeurteilung investiver Dienstleistungen. Operationalisierungsansätze an einem empirischen Beispiel zentraler EDV-Dienste. 1991.

Band 18 Kerstin Ch. Monhemius: Umweltbewußtes Kaufverhalten von Konsumenten. Ein Beitrag zur Operationalisierung, Erklärung und Typologie des Verhaltens in der Kaufsituation. 1993. 
Band 19 Uwe Schürmann: Erfolgsfaktoren der Werbung im Produktlebenszyklus. Ein Beitrag zur Werbewirkungsforschung. 1993.

Band 20 Ralf Birkelbach: Qualitätsmanagement in Dienstleistungscentern. Konzeption und typenspezifische Ausgestaltung unter besonderer Berücksichtigung von Verkehrsflughäfen. 1993.

Band 21 Simone Frömbling. Zielgruppenmarketing im Fremdenverkehr von Regionen. Ein Beitrag zur Marktsegmentierung auf der Grundlage von Werten, Motiven und Einstellungen. 1993.

Band 22 Marcus Poggenpohl: Verbundanalyse im Einzelhandel auf der Grundlage von Kundenkarteninformationen. Eine empirische Untersuchung von Verbundbeziehungen zwischen Abteilungen. 1994.

Band 23 Kai Bauche: Segmentierung von Kundendienstleistungen auf investiven Märkten. Dargestellt am Beispiel von Personal Computern. 1994.

Band 24 Ewald Werthmöller: Räumliche Identität als Aufgabenfeld des Städte- und Regionenmarketing. Ein Beitrag zur Fundierung des Placemarketing. 1995.

Band 25 Nicolaus Müller: Marketingstrategien in High-Tech-Märkten. Typologisierung, Ausgestaltungsformen und Einflußfaktoren auf der Grundlage strategischer Gruppen. 1995.

Band 26 Nicolaus Henke: Wettbewerbsvorteile durch Integration von Geschäftsaktivitäten. Ein zeitablaufbezogener wettbewerbsstrategischer Analyseansatz unter besonderer Berücksichtigung des Einsatzes von Kommunikations- und Informationssystemen (KIS). 1995.

Band 27 Kai Laakmann: Value-Added Services als Profilierungsinstrument im Wettbewerb. Analyse, Generierung und Bewertung. 1995.

Band 28 Stephan Wöllenstein: Betriebstypenprofilierung in vertraglichen Vertriebssystemen. Eine Analyse von Einflußfaktoren und Erfolgswirkungen auf der Grundlage eines Vertragshändlersystems im Automobilhandel. 1996.

Band 29 Michael Szeliga: Push und Pull in der Markenpolitik. Ein Beitrag zur modellgestützten Marketingplanung am Beispiel des Reifenmarktes. 1996.

Band 30 Hans-Ulrich Schröder: Globales Produktmanagement. Eine empirische Analyse des Instrumenteeinsatzes in ausgewählten Branchen der Konsumgüterindustrie. 1996.

Band 31 Peter Lensker: Planung und Implementierung standardisierter vs. differenzierter Sortimentsstrategien in Filialbetrieben des Einzelhandels. 1996.

Band 32 Michael H. Ceyp: Ökologieorientierte Profilierung im vertikalen Marketing. Dargestellt am Beispiel der Elektrobranche. 1996.

Band 33 Mark Unger: Die Automobil-Kaufentscheidung. Ein theoretischer Erklärungsansatz und seine empirische Überprüfung. 1998.

Band 34 Ralf Ueding: Management von Messebeteiligungen. Identifikation und Erklärung messespezifischer Grundhaltungen auf der Basis einer empirischen Untersuchung. 1998.

Band 35 Andreas Siefke: Zufriedenheit mit Dienstleistungen. Ein phasenorientierter Ansatz zur Operationalisierung und Erklärung der Kundenzufriedenheit im Verkehrsbereich auf empirischer Basis. 1998.

Band 36 Irene Giesen-Netzer: Implementierung von Rücknahme- und Recyclingsystemen bei Gebrauchsgütern. 1998. 


\title{
Efficient Consumer Response (ECR)
}

\author{
3., aktualisierte und erweiterte Auflage
}

\section{Basisstrategien und Grundtechniken, zentrale Erfolgsfaktoren sowie globaler Implementierungsplan}

Frankfurt/M., Berlin, Bern, New York, Paris, Wien, 1997, 3., akt. u. erw. Aufl. 1998. 287 S., zahlr. Abb. u. Fallbeispiele

Europäische Hochschulschriften: Reihe 5, Volks- und Betriebswirtschaft. Bd. 2062 ISBN 3-631-33228-9 • br. DM 65.-*

Dieses Buch hat richtungsweisende Akzente in der ECR-Diskussion gesetzt und sich innerhalb kürzester Zeit als Standardwerk in Praxis und Wissenschaft etabliert. Auch in der nun vorliegenden dritten, aktualisierten und erweiterten Auflage soll der immer wichtiger werdende ECR-Ansatz, der eine partnerschaftlich angelegte Zusammenarbeit zwischen Handel und Industrie zum Wohle des Verbrauchers postuliert, in seiner Gesamtheit vorgestellt und analysiert werden.

"Mit dieser Grundlagenarbeit, die mit keiner Seite die wertvolle Zeit des Lesers verschwendet, ist es dem Autor nicht nur gelungen, ECR umfassend, kritisch und fundiert zu untersuchen, sondern es konnten vielmehr auch wichtige Lösungsvorschläge und Anregungen geliefert werden, die den Unternehmen von großem Wert sein dürften."

Alexander M. Lintner, Geschäftsführer Roland Berger \& Partner

„Eine sehr detaillierte und gleichermaßen verständliche und eingängige Ausarbeitung." Lebensmittel Zeitung

"Ich bin froh, diese Schrift in Händen zu haben."

Prof. Dr. Lothar Müller-Hagedorn, Universität Köln

"Dieses Werk ist für unsere Mitglieder sicherlich von groBem Interesse."

Angelika Wiesgen-Pick, Geschäftsführerin Bundesverband der Deutschen Spirituosenindustrie e.V., Bonn

"Umfassend, verständlich und auf Deutsch."

manager magazin

"Ein wichtiger Beitrag zum Thema ECR, der sicher vielen Managern eine wichtige Entscheidungshilfe sein kann."

Prof. Dr. H.-P. Liebmann, Universität Graz

"Eine hervorragende Arbeit. Nicht nur phantastisch präsentiert, sondern auch inhaltlich ausgezeichnet."

Wolfgang K. A. Disch, Herausgeber MARKETING JOURNAL, Hamburg

Aus dem Inhalt: ECR-Historie - ECR-Basisstrategien und Grundtechniken · Zentrale ECR-Erfolgsfaktoren - Globaler ECR-Implementierungsplan

Frankfurt/M - Berlin - Bern - New York - Paris - Wien

Auslieferung: Verlag Peter Lang AG

Jupiterstr. 15, CH-3000 Bern 15

Telefax (004131) 9402131

*inklusive Mehrwertsteuer

Preisänderungen vorbehalten 
Irene Giesen-Netzer and Universität Münster - 978-3-631-75058-2 Downloaded from PubFactory at 01/11/2019 08:44:49AM via free access 